\title{
Transformation organischer Schadstoffe und abiotische Bildung von Huminstoffen in Böden durch oberflächeninduzierte Reaktionen an Tonmineralen
}

\author{
Dissertation \\ zur Erlangung des Doktorgrades \\ der Mathematisch-Naturwissenschaftlichen Fakultäten \\ der Georg-August-Universität zu Göttingen
}

vorgelegt von

Diplom-Geograph Ulf Birkel

aus Trier 
D 7

Referent: Prof. Dr. Gerhard Gerold

Korreferent: PD Dr. Jürgen Niemeyer

Tag der mündlichen Prüfung: 01.02.2001 


\section{Vorwort}

Diese Dissertation entstand im Rahmen des Teilprojektes A6 „Grenzflächenreaktivität, Aufbau und Zerfall von Mikroaggregaten“ des von der Deutschen Forschungsgemeinschaft geförderten Sonderforschungsbereiches 468 ,Wechselwirkungen an geologischen Grenzflächen“ und des Projektes,,Aufbau und Zerfall von Bodenaggregaten, Reaktivität von Bodenmineralen an der Grenzfläche Mineral-Porenraum“ (DFG-Fördernummer GE 431/14-1). Der Deutschen Forschungsgemeinschaft sei für die finanzielle Unterstützung gedankt.

Ich danke Herrn Prof. Dr. Gerhard Gerold für die Vergabe dieses Themas, den freien Raum, den er mir zur eigenen Schwerpunktsetzung ließ und für seine umfassende Unterstützung.

Herrn PD Dr. Jürgen Niemeyer sei vielmals gedankt für anregende Diskussionen und die Übernahme des Korreferates.

Für die Unterstützung bei den zahlreichen Analysen und die Bereitstellung der Geräte seien Herrn Bodo Seeger vom Max-Planck-Institut für Experimentelle Medizin Göttingen, Frau Dr. Kirsten Techmer und Herrn PD Dr. Martin Dietzel vom Sediment-Petrographischen Institut der Georg-August-Universität Göttingen, Herrn Dr. Hanns-Peter Brückner und Frau Emmi Krings vom Fachbereich VI Geographie/Geowissenschaften - Abteilung Geologie der Universität Trier, Herrn PD Dr. Habicher vom Institut für Organische Chemie der TU Dresden, Herrn Griep-Raming von der AG Organische Chemie der Universität Oldenburg und Herrn Dr. Andreas Reimer sowie Frau Birgit Roering vom Institut und Museum für Geologie und Paläontologie Göttingen gedankt.

Für ihre Unterstützung bei den Laborarbeiten bedanke ich mich bei Sandra Gehrt, Claudia Burkhard, Nicole Walter, Marika Zai, Christian Ripping und Robert Karsten.

Für eine kritische Durchsicht des Manuskriptes, zahlreiche fruchtbare Hinweise und die gute Zusammenarbeit innerhalb des Projektes danke ich Frau Annette Dathe, Herrn PD Dr. Niemeyer, Herrn Dr. Siegfried Eins, Herrn Dr. Jürgen Grotheer, und Herrn Tom Gallé.

Meinen Eltern sei für die langjährige Unterstützung während des Studiums und der Anfertigung der Dissertation gedankt.

Schließlich möchte ich meiner Frau Elvira danken für die Zeit, die sie mir zum Anfertigen der Arbeit ließ, als auch für ihre Geduld und Bemühungen, mich daran zu erinnern, dass es neben der wissenschaftlichen Arbeit auch noch andere Dinge im Leben gibt. 


\section{Inhaltsverzeichnis}

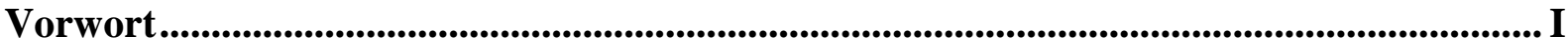

Verzeichnis der Abkürzungen ............................................................................................................. VI

Verzeichnis der Abbildungen ........................................................................................................... VII

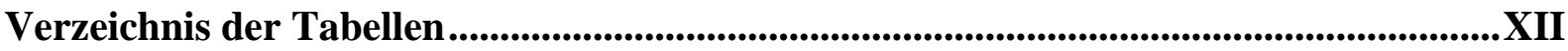

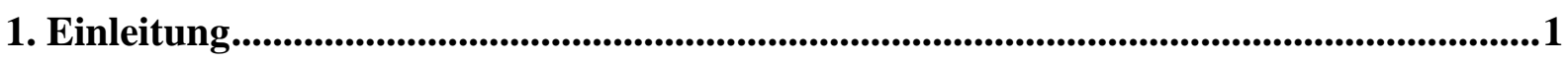

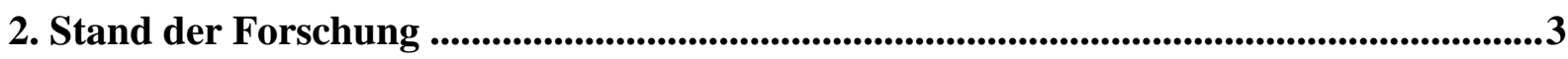

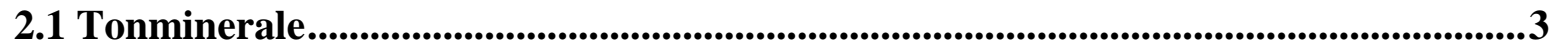

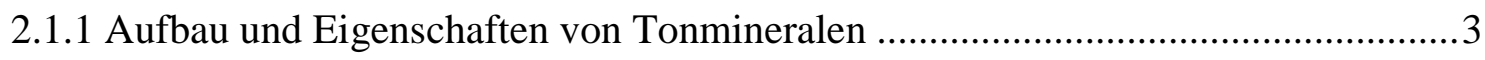

2.1.1.1 Adsorptive Eigenschaften von Tonmineralen..........................................6

2.1.2 Smektite als reaktive Tonmineralgruppe .....................................................

2.1.2.1 Ursachen der katalytischen Eigenschaften von

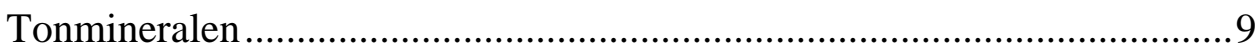

2.1.3 Tonminerale als Brønsted-Säuren................................................................. 10

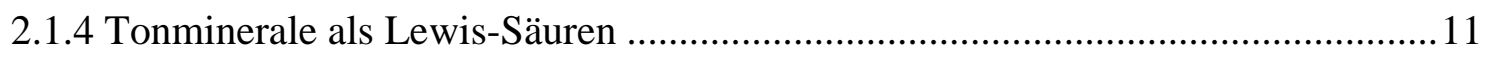

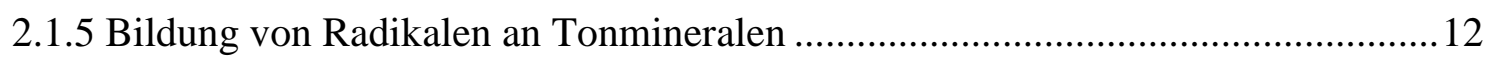

2.1.5.1 Bildung von anorganischen Radikalen ................................................ 13

2.1.5.2 Bildung von organischen Radikalen .................................................. 13

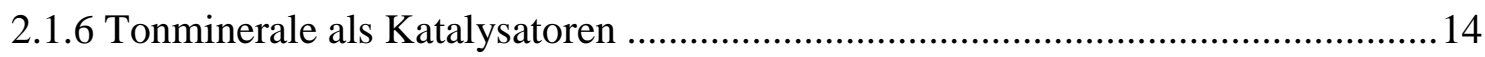

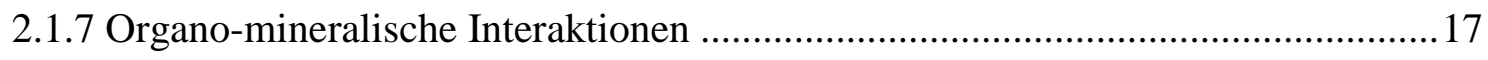

2.2 Organische Substanz in Böden ............................................................................17

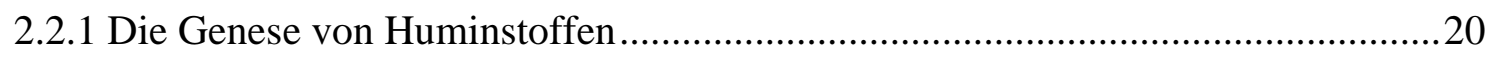

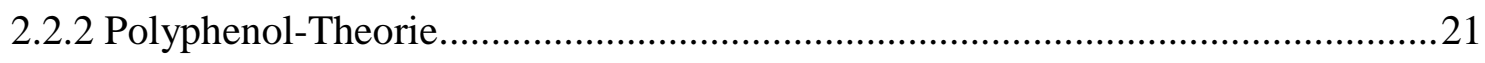

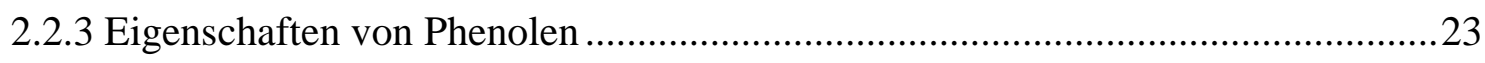

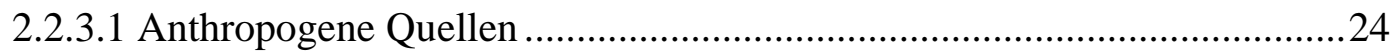

2.2.3.2 Natürliche Quellen ..............................................................................24

2.2.4 Verhalten und Reaktionsprozesse von Phenolen im Boden .................................25

2.2.5 Oxidation und Transformation von Phenolen durch Radikale ..............................26

2.2.5.1 Brenzkatechin und Pyrogallol ............................................................. 30

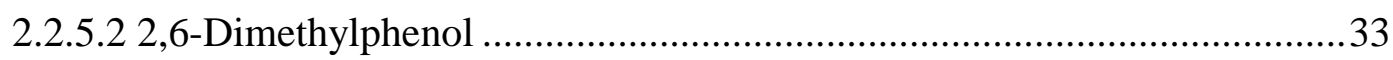

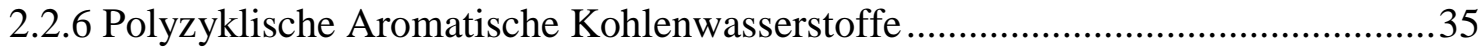

2.2.7 Verhalten und Reaktionsprozesse von PAK im Boden ......................................... 35

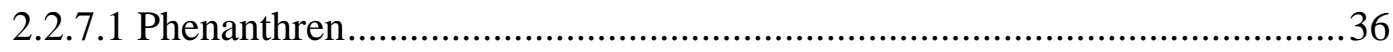

3. Zielsetzung ........................................................................................................................38

3.1 Konzeptionelle Grundlagen und Hypothesen..............................................................38

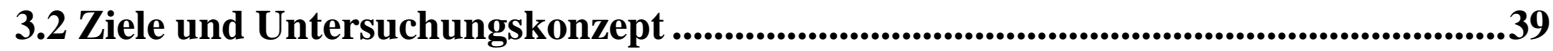

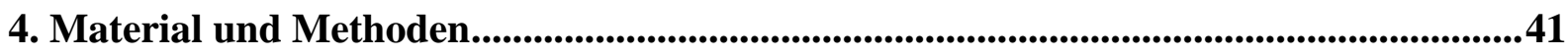

4.1 Belegung und Probenvorbreitung ..............................................................................41 


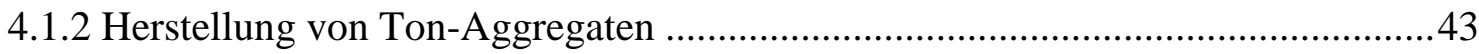

4.1.3 Durchführung der Belegung mit Phenolen ..........................................................43

4.1.4 Ergebnisse der Belegung von Tonmineralen mit Phenolen .................................44

4.1.5 Belegung der Tonminerale mit Phenanthren .....................................................46

4.1.6 Extraktion organischer Beläge von Tonmineraloberflächen ...............................47

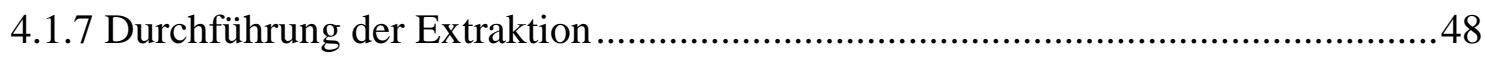

4.2 Rasterelektronen-Mikroskopie ....................................................................................................49

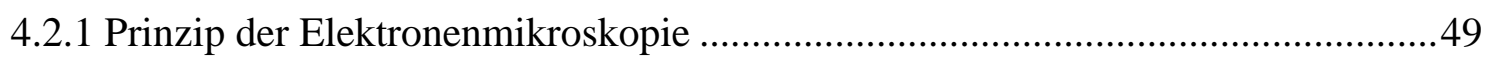

4.2.1.1 Rückstreu- und Sekundärelektronen ......................................................51

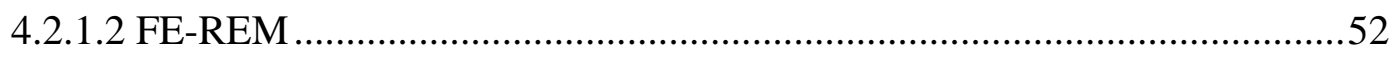

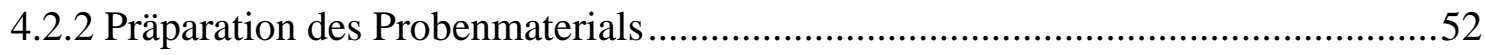

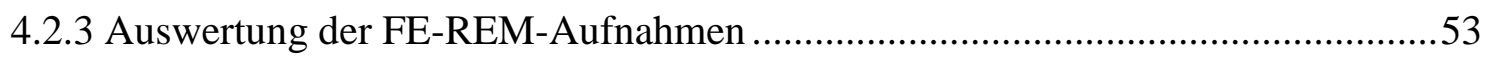

4.2.4 Diskussion der rasterelektronenmikroskopischen

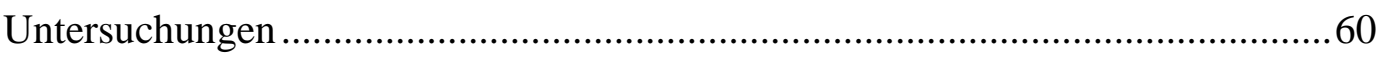

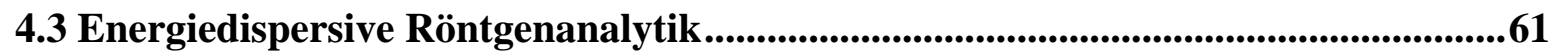

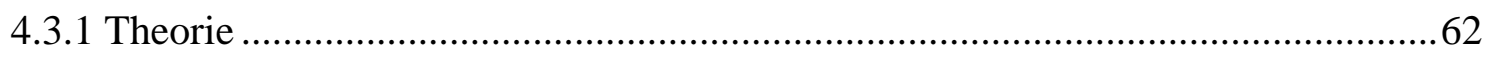

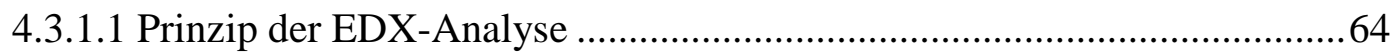

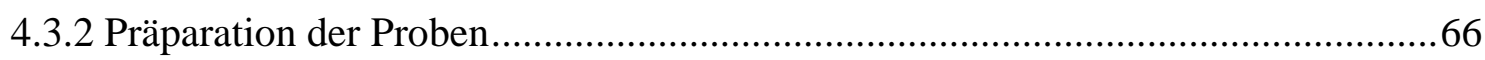

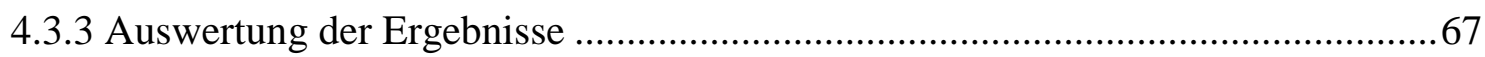

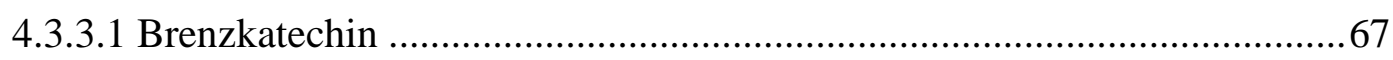

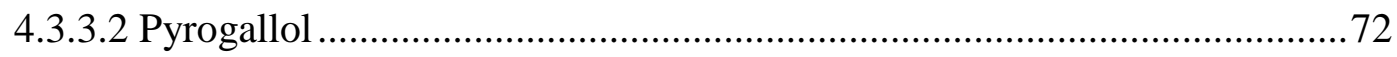

4.3.4 Hinweise auf den Reaktionsmechanismus ...................................................... 74

4.3.5 Diskussion der Resultate der Röntgenmikrobereichsanalysen .............................81

4.4 Qualitative Nachweise mit nasschemischen Methoden ............................................83

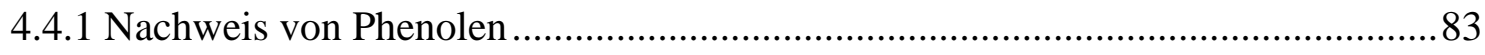

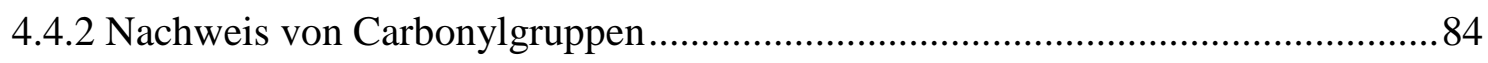

4.4.2.1 Nachweis von Aldehyden und Ketonen................................................ 84

4.4.2.2 Nachweis von Carbonylgruppen mit

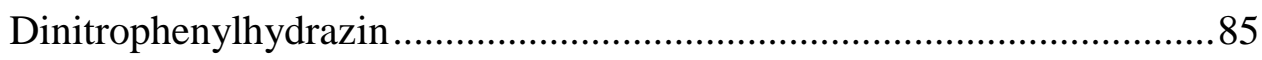

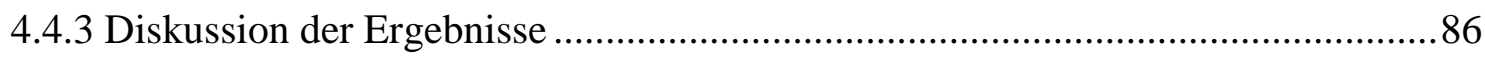

4.5 High Performance Liquid Chromatographie.....................................................8.87

4.5.1 Analytische und präparative Chromatographie ……........................................ 88

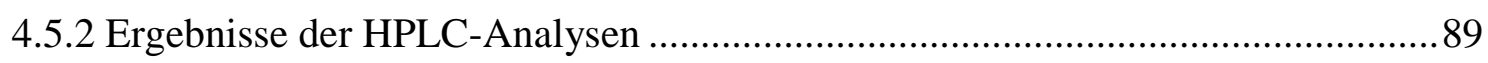

4.5.2.1 Reaktionsprodukte des Brenzkatechins ..................................................89

4.5.2.2 HPLC-MS der Reaktionsprodukte des Brenzkatechins ........................... 91

4.5.2.3 Reaktionsprodukte des Pyrogallols .......................................................92

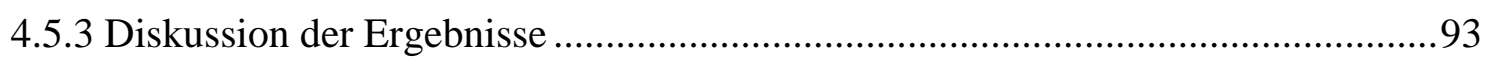

4.6 Fourier-Transform Infrarot Spektroskopie ...................................................................94

4.6.1 Physikalische Grundlagen der Infrarot-Spektroskopie ........................................ 94

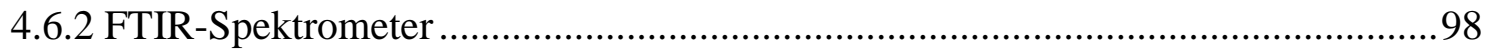

4.6.3 Präparation der Proben für die FT-IR-Spektroskopie ........................................ 98

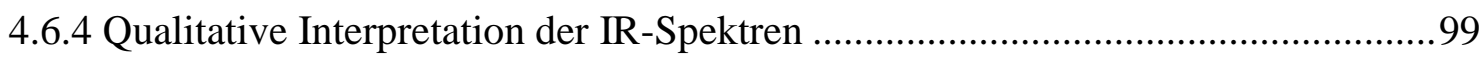

4.6.5 Absorptionsbereiche organischer Verbindungen ............................................ 100 


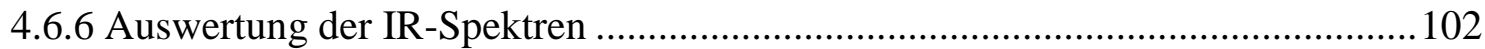

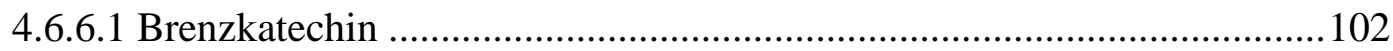

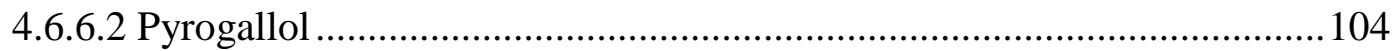

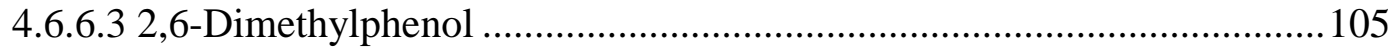

4.6.6.4 Extrahierte Überzüge des mit Brenzkatechin belegten

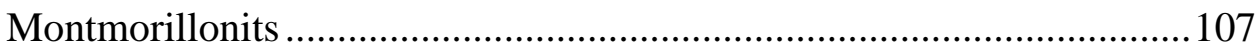

4.6.6.4.1 Fraktionen 1-5 der chromatographisch getrennten

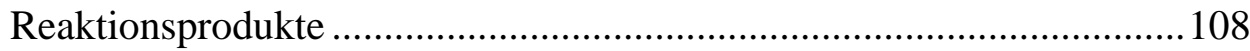

4.6.6.4.2 Fraktionen 6-10 der chromatographisch getrennten Reaktionsprodukte ….................................................... 110

4.6.6.5 Extrahierte Überzüge des mit Pyrogallol belegten

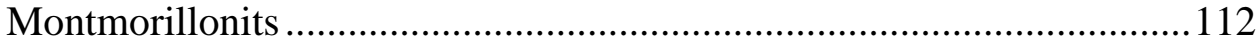

4.6.6.6 Extrahierte Überzüge des mit 2,6-Dimethylphenol

belegten Montmorillonits..................................................................... 115

4.6.6.7 Extrahierte Überzüge des mit Phenanthren bzw.

Brenzkatechin und Phenanthren belegten

Montmorillonits

4.6.7 Vergleich der IR-Spektren mit Spektren bodenbürtiger

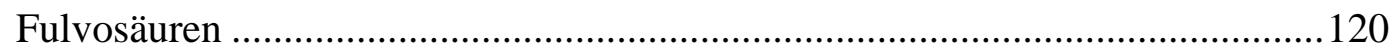

4.6.7.1 IR-Absorptionsbereiche von Fulvo- und Huminsäuren .........................120

4.6.8 Diskussion der IR-spektroskopischen Untersuchungen....................................123

$4.7{ }^{13}$ C-NMR Spektroskopie.........................................................................................125

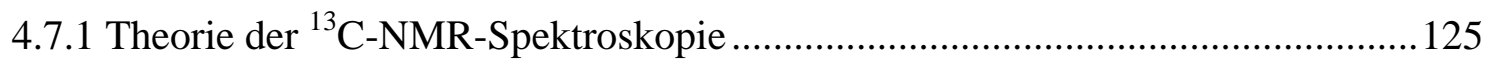

4.7.2 Chemische Verschiebungen in der ${ }^{13} \mathrm{C}-\mathrm{NMR}$-Spektroskopie..............................128

4.7.3 Präparation der Proben für die ${ }^{13} \mathrm{C}$-NMR-Spektroskopie ................................... 130

4.7.4 ${ }^{13} \mathrm{C}$-NMR spektroskopische Untersuchungen .................................................. 130

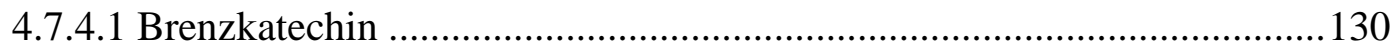

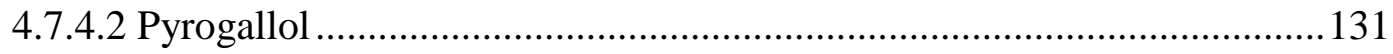

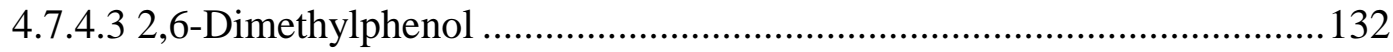

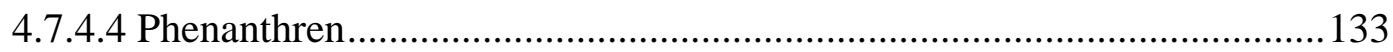

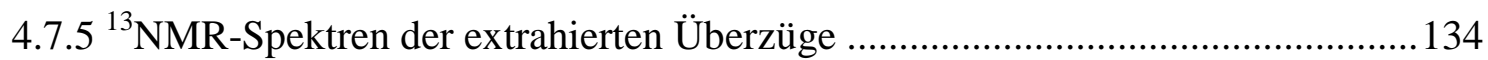

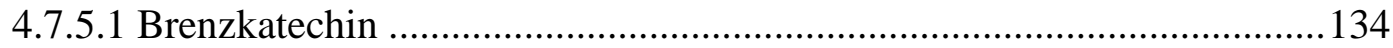

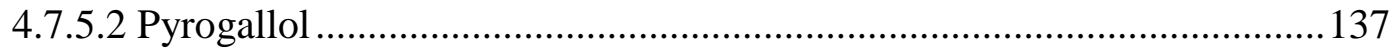

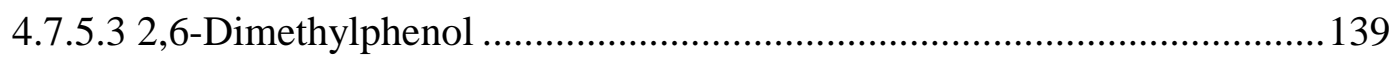

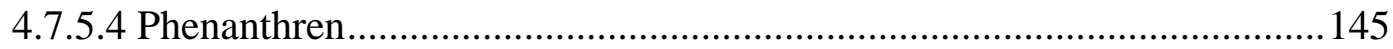

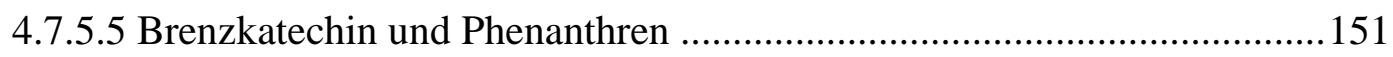

4.7.5.6 Pyrogallol und Phenanthren ................................................................... 154

4.7.6 Diskussion der Ergebnisse der ${ }^{13} \mathrm{C}$-NMR spektroskopischen

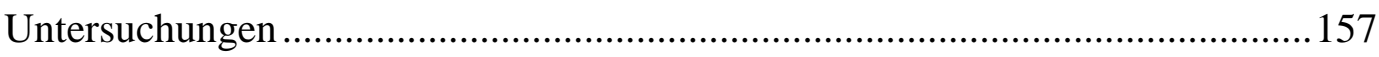

4.8 Massenspektrometrische Untersuchungen .........................................................159

4.8.1 Matrix Assisted Laser Desorption Ionization Time of Flight

Mass Spectrometry 
4.8.1.1 Funktionsprinzip des MALDI-

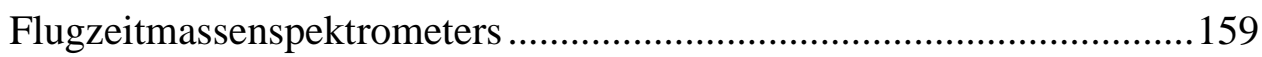

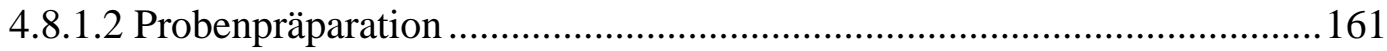

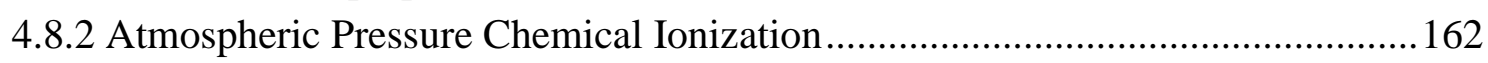

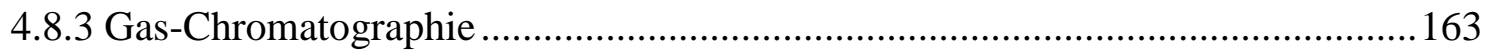

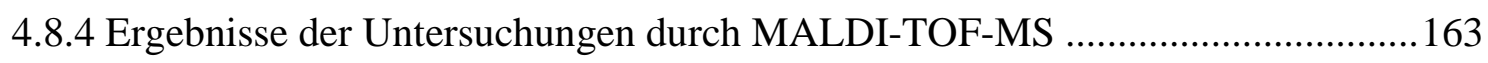

4.8.4.1 Brenzkatechin auf Na-Montmorillonit................................................... 163

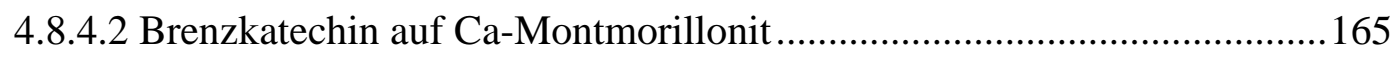

4.8.5 Ergebnisse der Untersuchungen mittels GC-MS ............................................. 167

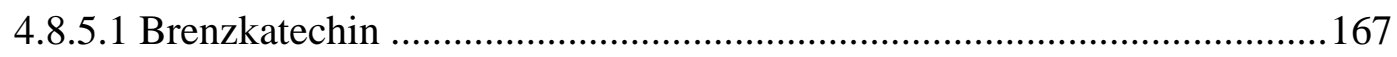

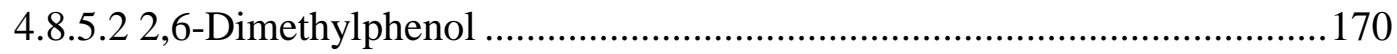

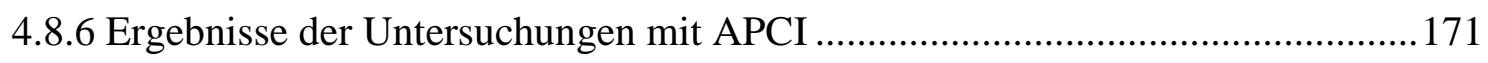

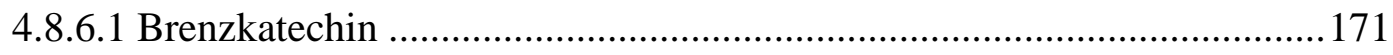

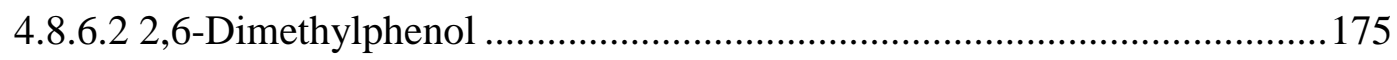

4.8.7 Diskussion der Ergebnisse der massenspektrometrischen

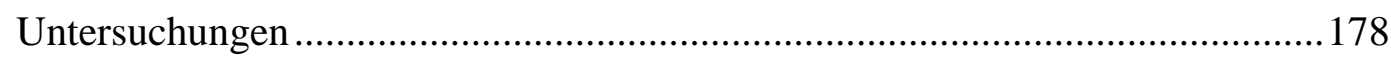

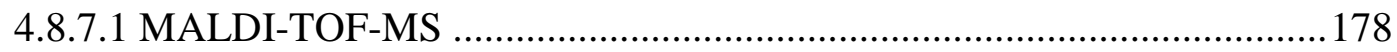

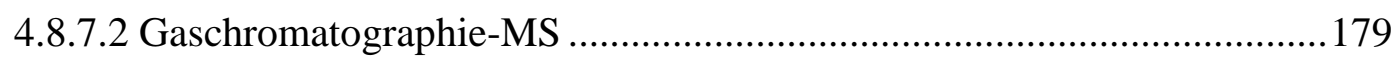

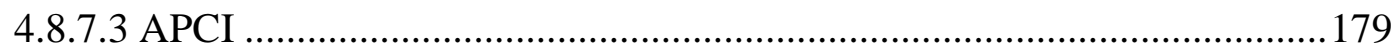

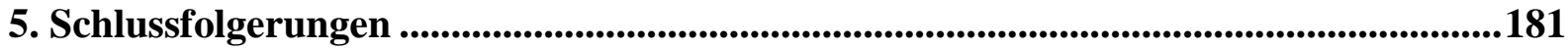

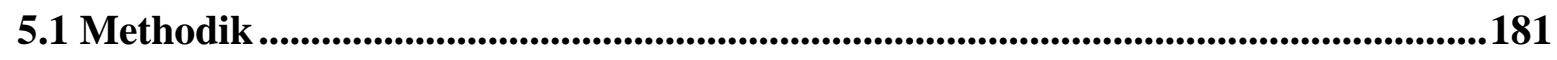

5.2 Verhalten von Organika an Tonmineralen ......................................................182

5.3 Relevanz und Konsequenzen der erzielten Ergebnisse .........................................186

6. Zusammenfassung ...................................................................................................................................187

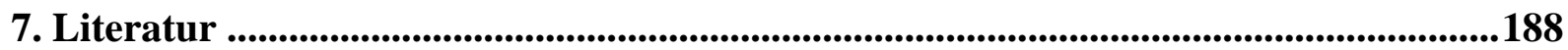




\section{Verzeichnis der Abkürzungen}

\begin{tabular}{|c|c|c|}
\hline APCI & $=$ & Atmospheric Pressure Chemical Ionization \\
\hline${ }^{\circ} \mathrm{C}$ & $=$ & Grad Celsius \\
\hline CI & $=$ & chemische Ionisation \\
\hline $\mathrm{cm}$ & $=$ & Zentimeter \\
\hline EI & $=$ & Elektronenstoßionisation \\
\hline $\mathrm{Da}$ & $=$ & Dalton \\
\hline DFG & $=$ & Deutsche Forschungsgemeinschaft \\
\hline DMSO & $=$ & Dimethylsulfoxid \\
\hline DPQ & $=$ & Diphenoquinone \\
\hline EDX & $=$ & Elektronen-dispersive Röntgenanalytik \\
\hline $\mathrm{eV}$ & $=$ & Elektronenvolt \\
\hline FE-REM & $=$ & Feldemissions-Rasterelektronenmikroskop \\
\hline FTIR & $=$ & Fourier-Transform-Infrarot-Spektroskopie \\
\hline g & $=$ & Gramm \\
\hline GC-MS & $=$ & Gaschromatographie-Massenspektrometrie \\
\hline $\mathrm{GPa}$ & $=$ & Gigapascal \\
\hline $\mathrm{h}$ & $=$ & Stunde \\
\hline HPLC & $=$ & High Performance Liquid Cromatography \\
\hline i.p. & $=$ & in-plane \\
\hline IR & $=$ & Infrarot-Spektroskopie \\
\hline KV & $=$ & Kilovolt \\
\hline $\mathrm{KBr}$ & $=$ & Kaliumbromid \\
\hline MALDI-TOF-MS & $=$ & $\begin{array}{l}\text { Matrix Assisted Laser Desorption Ionization-Time of Flight- } \\
\text { Mass Spectrometry }\end{array}$ \\
\hline mbar & $=$ & Millibar \\
\hline $\mathrm{mg}$ & $=$ & Milligramm \\
\hline $\mathrm{mm}$ & $=$ & Millimeter \\
\hline $\mathrm{nm}$ & $=$ & Nanometer \\
\hline NMR & $=$ & Nuclear Magnetic Resonance \\
\hline o.o.p. & $=$ & out-of-plane \\
\hline PAK & $=$ & polyzyklischer aromatischer Kohlenwasserstoff \\
\hline PE & $=$ & Primärelektronen \\
\hline Ppm & $=$ & parts per million (chemischer Verschiebungswert) \\
\hline RE & $=$ & Rückstreuelektronen \\
\hline REM & $=$ & Rasterelektronenmikroskop \\
\hline SE & $=$ & Sekundärelektronen \\
\hline SFB & $=$ & Sonderforschungsbereich \\
\hline TMS & $=$ & Tetramethylsilan \\
\hline $\mathrm{u}$ & $=$ & Molmasse \\
\hline$\mu \mathrm{m}$ & $=$ & Mikrometer \\
\hline $\mathrm{z}$ & $=$ & Ordnungszahl \\
\hline
\end{tabular}




\section{Verzeichnis der Abbildungen}

Abbildung 1: Die umweltrelevanten Funktionen von Tonen (RAE \& PARKER 1998)........................................ 3

Abbildung 2: Aufbau der Dreischicht-Tonminerale (nach JASMUND \& LAGALY 1993)................................

Abbildung 3: Interaktionen von Organika mit Bodenkolloiden (JOHNSTON 1996)..........................................5

Abbildung 4: Schematischer Darstellung der Mikrostruktur eines Smektits, A: große, mehr oder weniger kontinuierliche Räume, B: kleine, isolierte Räume, C: interlamellarer

Raum, D: Kontaktregion interagierender Tonmineralstapel mit elektrischen Doppelschichten (PUSCH 1998).

Abbildung 5: Adsorptionsplätze von Organika an einem Tonmineral (BEDIOUI 1995)....................................6

Abbildung 6: Adsorption von Organika an Tonmineralen (YARIV 1996). ...................................................... 7

Abbildung 7: Sorptionsplätze für Organika an den Kanten der Tonminerale (SCHOONHEYDT 1995).

Abbildung 8: Adsorptionsisothermen für Huminstoffe an Montmorillonit, gesättigt mit verschiedenen Kationen (GRAUER 1989).

Abbildung 9: Transformationsprozesse von Xenobiotika in Böden (RUGGIERO 1999).................................. 9

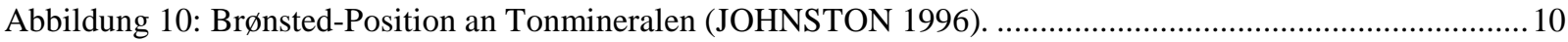

Abbildung 11: Abhängigkeit der Azidität eines Tonminerals am Beispiel von Kaolinit vom Wassergehalt (VOUDRIAS \& REINHARD 1986)............................................................................. 11

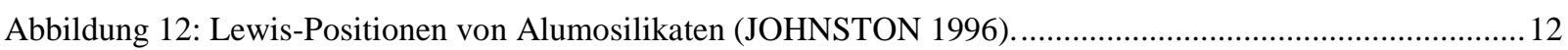

Abbildung 13: Chelatisierung von Phenolen mit Eisen (nach MILLER et al. 1990)........................................... 14

Abbildung 14: Reaktion von Brenzkatechin an Kaolinit und Dimethylcarbonat (FU et al. 1998)......................16

Abbildung 15: Struktur einer Humisäure (SCHULTEN \& SCHNITZER 1997)............................................. 19

Abbildung 16: Vereinfachte Struktur einer Fulvosäure (MORILL 1982). .......................................................20

Abbildung 17: Schema der Bildung von Huminstoffen im Boden (KEREN \& SPARKS 1995).........................20

Abbildung 18: Phenylpropan-Bausteine der Lignine (nach STEVENSON 1994)..........................................21

Abbildung 19: Modifikation des Lignins durch Demethylierung und Oxidation (STEVENSON 1994). ..............21

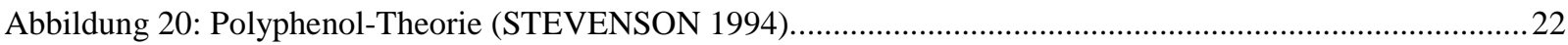

Abbildung 21: Oxidation von Hydrochinon (ZIECHMANN \& MÜLLER-WEGENER 1990)...........................22

Abbildung 22: Adsorption von Phenolen an Tonmineralen (YARIV 1996). .................................................25

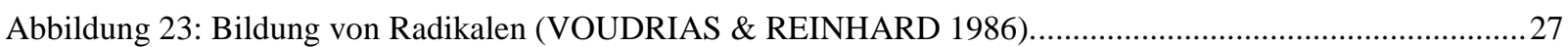

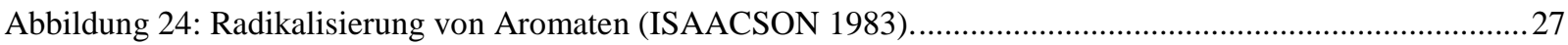

Abbildung 25: Radikalbildung bei Hydrochinon (WANG et al. 1983) . ...................................................28

Abbildung 26: Oxidation von Hydrochinon zu p-Benzochinon (THOMPSON \& MOLL 1973).........................28

Abbildung 27: Oxidation an trockenem Montmorillonit (THOMPSON \& MOLL 1973)....................................28

Abbildung 28: Reaktion von freien Radikalen mit Aromaten (DRAGUN \& HELLING 1985)...........................29

Abbildung 29: Polymerisation von aromatischen Radikalen (DRAGUN \& HELLING 1985)...........................29

Abbildung 30: Zerstörung von freien Radikalen (DRAGUN \& HELLING 1985).............................................29

Abbildung 31 : Radikalischer Reaktionsmechanismus (VOUDRIAS \& REINHARD 1986)................................30 
Abbildung 32: Oxidation des Brenzkatechins zum o-Benzochinon (PARK et al. 1999)..................................30

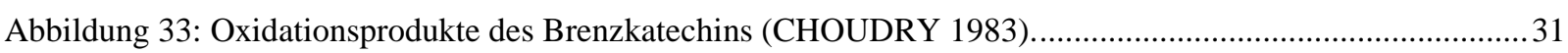

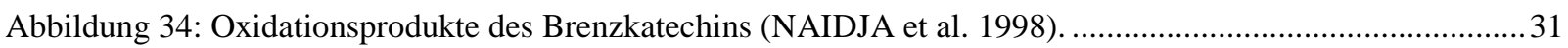

Abbildung 35: Oxidation und Polymerisation von Phenolen (ARAKI et al. 1992)..........................................32

Abbildung 36: Abbau von Brenzkatechin und Protokatechussäure (MORILL 1982)........................................32

Abbildung 37: Polymerisation von 2,6-Dimethylphenol (nach HAY 1967). ....................................................33

Abbildung 38: Radikalische Kopplung von 2,6-Dimethylphenol (HAY 1967)...............................................33

Abbildung 39: Polymerbildung aus 2,6-Dimethylphenolmonomeren (nach IONESCU \& MIHIS 1997).

Abbildung 40: Enzymatische oxidative Polymerisation von 2,6-Dimethylphenol (nach UYAMA et al. 1998). .34

Abbildung 41: Mikrobielle Degradation des Phenanthrens (CERNIGLIA 1993). 36

Abbildung 42: Beeinflussung des Abbaus von Phenanthren durch die Gegenwart von Ton (ORTEGACALVO \& SAIZ-JIMENEZ 1998) 37

Abbildung 43: Theorie der Entstehung von Huminstoffen (nach Stevenson 1994).........................................38

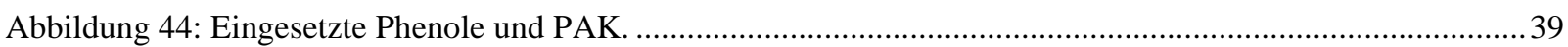

Abbildung 45: Resultate der Belegung von Na-Montmorillonit mit Phenolen...............................................44

Abbildung 46: Ergebnisse der Belegung des Ca-Montmorillonits mit Phenolen. ..........................................45

Abbildung 47: Nachweis der Abhängigkeit der Reaktion vom Sauerstoff (BIRKEL 1997). ..............................46

Abbildung 48: Schematische Darstellung eines Rasterelektronenmikroskops (BARKOW 1994)........................50

Abbildung 49: Abhängigkeit der Ausbreitung der PE innerhalb der Probe aufgrund von U0 und Z

(BARKOW 1994). .51

Abbildung 50: FE-REM-Aufnahme eines unbelegten Na-Montmorillonits. .53

Abbildung 51: FE-REM-Aufnahme eines mit Brenzkatechin belegten Na-Montmorillonits mit schleierförmigen Objekten auf der Oberfläche (siehe Markierungen). ................................................54

Abbildung 52: Ausschnittvergrößerung (Vergrößerungsstufe 33000-fach).

Abbildung 53: FE-REM-Aufnahme des mit Brenzkatechin belegten Montmorillonits (Ausschnittvergrößerung, Vergrößerungsstufe 67000 -fach) .....................................................................56

Abbildung 54: Übersichtsaufnahme einer mit Pyrogallol belegten Aggregatprobe..........................................57

Abbildung 55: Ausschnittvergrößerung der FE-REM-Aufnahme (Bildmitte). ...............................................58

Abbildung 56: Ausschnittvergrößerung der FE-REM-Aufnahme (rechte Bildhälfte).......................................59

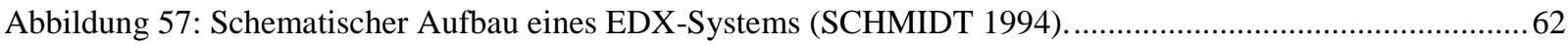

Abbildung 58: Prinzip derRöntgenmikrobereichsanalyse (SCHMIDT 1994) ................................................63

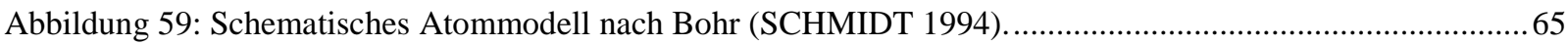

Abbildung 60: Schematische Darstellung des Elektronenbeschusses (BARKOW 1994). ................................65

Abbildung 61: Freisetzung der charakteristischen Röntgenstrahlung: linke Abbildung: Herausschlagen eines Elektrons aus der K-Schale; rechte Abbildung: Übergang eines Elektrons der Lauf die K-Schale und das dadurch emittierte Röntgenquant (BARKOW 1994)...........................66

Abbildung 62: REM-Aufnahme des mit Brenzkatechin belegten Montmorillonits (Pulverpräparat)...................68

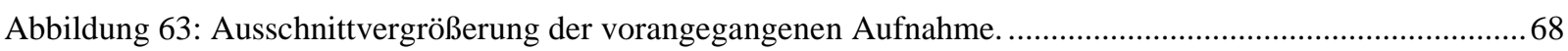

Abbildung 64: REM-Aufnahme des mit Brenzkatechin belegten Montmorillonits...........................................69 
Abbildung 65: EDX-Scan eines mit Brenzkatechin belegten Na-Montmorillonits. ........................................70

Abbildung 66: EDX-Analyse des mit Brenzkatechin belegten Montmorillonits.................................................71

Abbildung 67: EDX-Analyse des mit Brenzkatechin belegten Montmorillonits (Ausschnittvergrößerung).

Abbildung 68: Auftretende Elemente der EDX-Analyse eines mit Pyrogallol belegten Montmorillonits

Abbildung 69: EDX-Analyse des mit Pyrogallol belegten Montmorillonits (Scanlinie: gelb, Kohlenstoff: rot, mineralische Oberfläche (blau). ............................................................................. 74

Abbildung 70: EDX-Analyse der mit Brenzkatechin belegten Montmorillonitoberflächen.................................75

Abbildung 71: EDX-Scan (C: rot/Fe: grün) des mit Brenzkatechin belegten Montmorillonits...........................76

Abbildung 72: EDX-Analyse der mit Brenzkatechin belegten Montmorillonitoberflächen.................................77

Abbildung 73: REM/EDX-Scan (C: rot/Fe: grün) des mit Brenzkatechin belegten Montmorillonits...................78

Abbildung 74: EDX-Scan des mit Brenzkatechin belegten Montmorillonits. .....................................................79

Abbildung 75: EDX/REM-Analyse des mit Brenzkatechin belegten Montmorillonits.....................................80

Abbildung 76: EDX-Analyse des mit Brenzkatechin belegten Montmorillonits (verschobene Scanlinie).

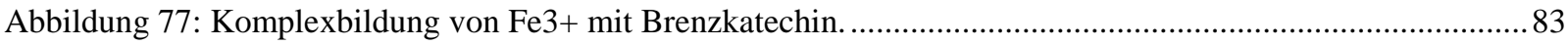

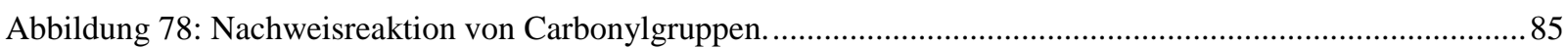

Abbildung 79: Chromatogramm extrahierter Überzüge nach Belegung mit Brenzkatechin. .90

Abbildung 80: Chromatogramm der extrahierten Überzüge des mit Pyrogallol belegten Montmorillonits

Abbildung 81: Chromatogramm extrahierter Überzüge des mit 2,6-Dimethylphenol belegten Montmorillonits.

Abbildung 82: Mögliche Schwingungsformen eines dreiatomigen, linearen Moleküls (GÜNZLER \& HEISE 1996). 96

Abbildung 83: Mögliche Schwingungsformen eines dreiatomigen, linearen Moleküls mit unsymmetrischer Massenverteilung (GÜNZLER \& HEISE 1996). .96

Abbildung 84: Mögliche Schwingungsformen eines dreiatomigen, gewinkelten Moleküls (GÜNZLER \& HEISE 1996). 97

Abbildung 85: Gruppenfrequenzen im IR-Spektrum (GOTTWALD \&WACHTER 1997).............................100

Abbildung 86: C-H-Schwingungsformen am aromatischen Ring (GÜNZLER \& HEISE 1996)........................ 101

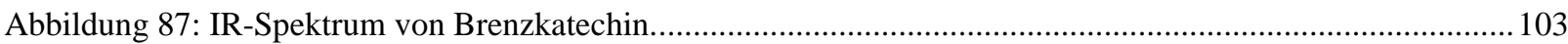

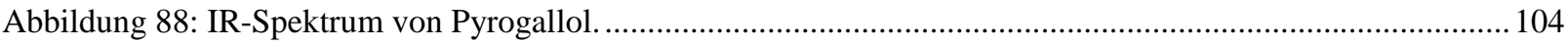

Abbildung 89: Substitutionsbanden (dC-H o.o.p.) von Benzolderivaten (GÜNZLER \& HEISE 1996)............... 105

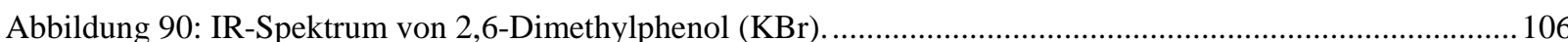

Abbildung 91: IR-Spektrum der extrahierten Überzüge des mit Brenzkatechin belegten Montmorillonits (schwarz: Brenzkatechin Standard; blau: extrahierte Überzüge).

Abbildung 92: IR-Spektren der Fraktionen 1-5 (schwarz: Fraktion 1, blau: Fraktion 2, rot: Fraktion 3, grün: Fraktion 4, rosa: Fraktion 5).

Abbildung 93: IR-Spektren der Fraktionen 6-10 (blau: Frakt. 6, rot: Frakt. 7, grün: Frakt. 8, rosa: Frakt. 9, schwarz: Frakt. 10). 
Abbildung 94: IR-Spektrum der extrahierten Überzüge des mit Pyrogallol belegten Montmorillonits (schwarz: Pyrogallol Standard; blau: extrahierte Überzüge).

Abbildung 95: IR-Spektren der Fraktionen 1-5 (schwarz: Fraktion 1, blau: Fraktion 2, rot: Fraktion 3, grün: Fraktion 4, rosa: Fraktion 5, türkis: Fraktion 6)

Abbildung 96: IR-Spektrum der extrahierten Überzüge des mit 2,6-Dimethylphenol belegten Montmorillonits (schwarz: Pyrogallol Standard; blau: extrahierte Überzüge).

Abbildung 97: IR-Spektren der Fraktionen 1-6 (schwarz: Fraktion 1, blau: Fraktion 2, rot: Fraktion 3, grün: Fraktion 4, rosa: Fraktion 5, türkis: Fraktion 6)

Abbildung 98: IR-Spektren der Fraktionen 7-13 (rosa: Fraktion 7, türkis: Fraktion 8, braun: Fraktion 9, schwarz: Fraktion 10, blau: Fraktion 11, rot: Fraktion 12, grün: Fraktion 13).

Abbildung 99: IR-Spektren von Brenzkatechin (schwarz), Phenanthren (blau) und der extrahierten Überzüge des mit Brenzkatechin (grün) sowie mit Brenzkatechin und Phenanthren belegten Montmorillonits (rot).

Abbildung 100: Vergleich der IR-Spektren bodenbürtiger Fulvosäuren (Stevenson 1994, verändert) mit den gewonnenen Reaktionsprodukten des Brenzkatechins...

Abbildung 101: Vergleich der IR-Spektren bodenbürtiger Fulvosäuren (Stevenson 1994, verändert) mit den gewonnenen Reaktionsprodukten des Pyrogallols.

Abbildung 102: Vergleich der IR-Spektren bodenbürtiger Fulvosäuren (Stevenson 1994, verändert) mit den gewonnenen Reaktionsprodukten des 2,6-Dimethylphenols.

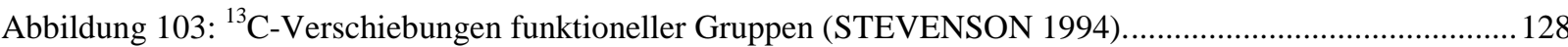

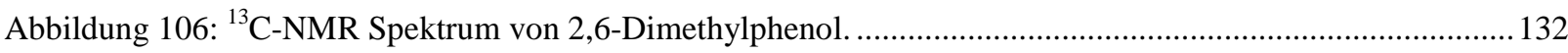

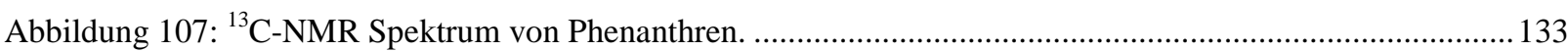

Abbildung 108: ${ }^{13} \mathrm{C}-\mathrm{NMR}$ Spektrum extrahierter Überzüge des mit Brenzkatechin belegtem Na-

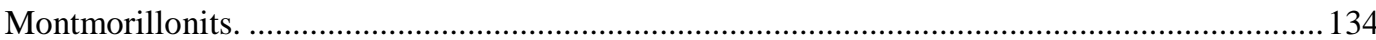

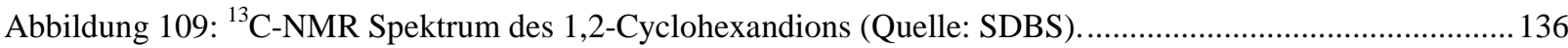

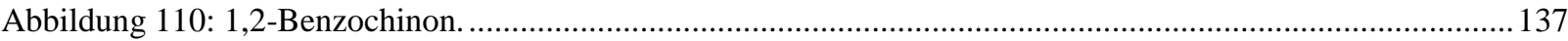

Abbildung 111: ${ }^{13} \mathrm{C}-\mathrm{NMR}$ Spektrum der extrahierten Überzüge des mit Pyrogallol belegten Ca-

Montmorillonits

Abbildung 112: ${ }^{13} \mathrm{C}-\mathrm{NMR}$ Spektrum der extrahierten Überzüge des mit 2,6-Dimethylphenol belegten

Na-Montmorillonits.

Abbildung 113: ${ }^{13} \mathrm{C}-\mathrm{NMR}$ Spektrum der extrahierten Überzüge des mit 2,6-Dimethylphenol belegten

Na-Montmorillonits (Ausschnittvergrößerung).

Abbildung 114: Bildung von PPO aus 2,6-Dimethylphenol (nach Ikeda et al. 1996).

Abbildung 115: Oxidatives Polymerisationsprodukt (DPQ) aus 2,6-Dimethylphenol (Ikeda et al. 1996).

Abbildung 116: Polymer aus 2,6-Dimethylphenol-Monomeren (IKEDA et al. 1996).

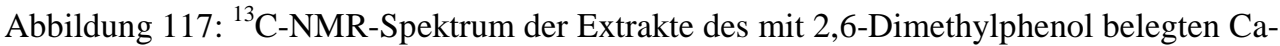
Montmorillonits.

Abbildung 118: ${ }^{13} \mathrm{C}-\mathrm{NMR}$-Spektrum der extrahierten Überzüge des mit 2,6-Dimethylphenol belegten

Ca-Montmorillonits (Ausschnittvergrößerung)

Abbildung 119: ${ }^{13} \mathrm{C}-\mathrm{NMR}-$ Spektrum von Na-Montmorillonit belegt mit Phenanthren.

Abbildung 120: ${ }^{13} \mathrm{C}-\mathrm{NMR}-$ Spektrum von Ca-Montmorillonit belegt mit Phenanthren........ 


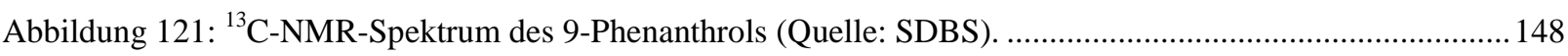

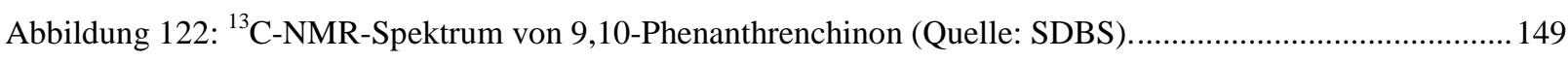

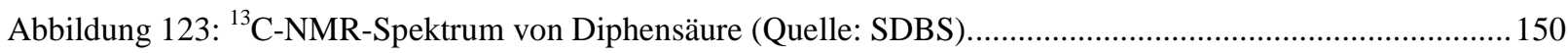

Abbildung 125: Na-Montmorillonit belegt mit Phenanthren und Brenzkatechin. ............................................ 153

Abbildung 126: ${ }^{13} \mathrm{C}-\mathrm{NMR}-$ Spektrum der extrahierten Überzüge des mit Phenanthren und Brenzkatechin belegten Ca-Montmorillonits

Abbildung 127: ${ }^{13}$ NMR-Spektrum Extrakte von Ca-Montmorillonit belegt mit Phenanthren und Pyrogallol.

Abbildung 128: Prozess der Desorption und Ionisation beim MALDI (Quelle: Abteilung Massenspektrometrie, Organische Chemie Uni Bielefeld). 160

Abbildung 129: Schematische Darstellung eines TOF-Massenspektrometers (WU \& ODOM 1998)

Abbildung 130: Funktionsprinzip eines APCI (Quelle: Mass Spectrometry Facility, Institut für Organische Chemie, Universität Heidelberg)

Abbildung 131: Verteilung der Massengewichte des mit Brenzkatechin belegten Na-Montmorillonits. 163

Abbildung 132: Zerfall langkettiger Aliphate durch Laserbeschuss. 165

Abbildung 133: Massenspektrum des mit Brenzkatechin belegten Ca-Montmorillonits 166

Abbildung 134: Polymerisation von 2,6-Dimethylphenol (nach IKEDA et al. 1996)....

Abbildung 135: Dimer aus zwei 2,6-Dimethylphenol-Molekülen mit der Molmasse 240 u (Quelle: SDBS).

Abbildung 136: Polymerisation des 2,6-Dimethylphenols (nach IKEDA et al. 1996). 176

Abbildung 137: Polymerisationsprozess des 2,6-Dimethylphenols vom Dimer zum Heptamer. 176

Abbildung 138: Bildung eines Dimers aus 2,6-Dimethylphenol mit der Molmasse $254 \mathrm{u}$. 177

Abbildung 139: Bildung von Oligomeren mit den Molmassen 374 und $495 \mathrm{u}$.

Abbildung 140: Bildung phenolischer Radikale und deren anschließende Polymerisation

(VOUDRIAS \& REINHARD 1986)

Abbildung 141: Theorie der Entstehung von Huminstoffen (nach Stevenson 1994). 186 


\section{Verzeichnis der Tabellen}

Tabelle 1: Vorkommen und Herkunft funktioneller Gruppen in Huminstoffen (nach ZIECHMANN \& MÜLLER-WEGENER 1990).

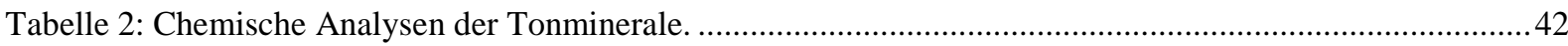

Tabelle 3: Belegung von Phenolen und Phenanthren auf Tonminerale. ........................................................43

Tabelle 4: Bestimmung der C-Gehalte von unbelegten und belegten Tonproben. ........................................45

Tabelle 5: Sorption von Phenolen in Luft oder Stickstoff (nach SAWHNEY 1985)......................................47

Tabelle 6: Ergebnisse des Phenol-Nachweises mit FeCl3-Lösung. ............................................................8 83

Tabelle 7: Nachweis von Aldehyden und Ketonen mit Schiff' schem Reagenz...............................................8

Tabelle 8: Nachweis von Aldehyden und Ketonen nach mehrstündiger Reaktionszeit...................................85

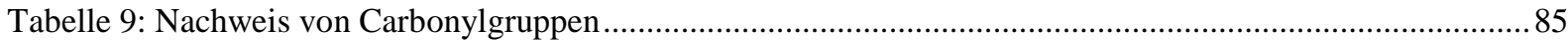

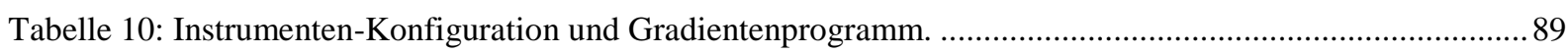

Tabelle 11: Ergebnisse der HPLC-MS-Analyse der extrahierten Überzüge der Belegung mit Brenzkatechin

Tabelle 12: Verteilung der Symmetrierassen von Normalschwingungen des Benzols und monosubstituierter Derivate (nach GÜNZLER \& HEISE 1996).................................................98

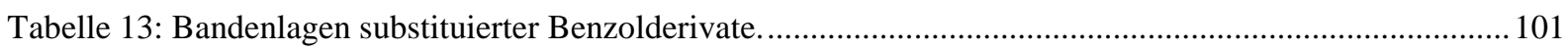

Tabelle 14: Absorptionsbanden von Huminstoffen (nach Stevenson 1994)...............................................102

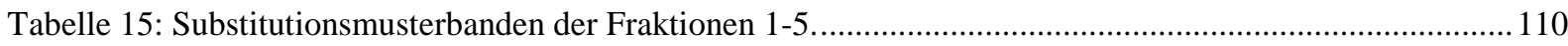

Tabelle 16: 13C-Verschiebungen von Fulvo- und Huminsäuren (nach STEVENSON 1994). ........................ 129

Tabelle 17: Brenzkatechin, Verschiebungswerte und Zuordnungen (Quelle: SDBS). .................................. 130

Tabelle 18: Pyrogallol, Verschiebungswerte und Zuordnungen (Quelle: SDBS)....................................... 132

Tabelle 19: 2,6-Dimethylphenol, Verschiebungswerte und Zuordnungen (Quelle: SDBS)............................133

Tabelle 20: Zuordnung der chemischen Verschiebungen für Phenanthren (Quelle: SDBS). ............................ 133

Tabelle 21: Übersicht der chemischen Verschiebungen. ........................................................................... 135

Tabelle 22: Übersicht der chemischen Verschiebungen und ihre Zuordnungen (Quelle: SDBS).

Tabelle 23: Übersicht der chemischen Verschiebungen. ...................................................................... 138

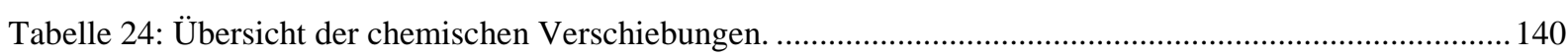

Tabelle 25: Chemische Verschiebungen des Extraktes des mit 2,6-Dimethylphenol belegten Na-Montmorillonits (entnommen aus Abb. 112+Abb. 113)........................................................ 142

Tabelle 26: Chemische Verschiebungen des Reaktionsproduktes und Zuordnungen..................................... 143

Tabelle 27: Übersicht der chemischen Verschiebungen und Zuordnungen.................................................. 146

Tabelle 28: Übersicht der chemischen Verschiebungen und ihre Zuordnungen.......................................... 148

Tabelle 29: Verschiebungswerte des 9-Phenanthrols und Zuordnungen. ......................................................... 149

Tabelle 30: Verschiebungswerte des 9,10-Phenanthrenchinons und ihre Zuordnungen................................... 150

Tabelle 31: Chemische Verschiebungen der Diphensäure und Zuordnung. .............................................. 150 
Tabelle 32: Übersicht der chemischen Verschiebungen und ihre Zuordnungen.

Tabelle 33: Übersicht der chemischen Verschiebungen und ihre Zuordnungen. 156

Tabelle 34: Übersicht der Massengewichte und Signalintensitäten der Probe W2 164

Tabelle 35: Übersicht der Massengewichte und Signalintensitäten der Probe A1. 167

Tabelle 36: Ergebnisse für Reaktionsprodukte mit der Molmasse 178 (Quelle: NIST). 168

Tabelle 37: Resultate der Datenbankrecherche für Verbindungen mit der Molmasse 214. 169

Tabelle 38: Verbindungen mit der Molmasse 256. 171

Tabelle 39: Verbindungen mit der Molmasse $168 \mathrm{u}$. 172

Tabelle 40: Verbindungen mit der Molmasse $214 \mathrm{u}$ 173

Tabelle 41: Verbindungen mit der Molmasse $246 \mathrm{u}$ 174

Tabelle 42: Verbindungen mit der Molmasse $322 \mathrm{u}$ 174

Tabelle 43: Verbindungen mit der Molmasse $370 \mathrm{u}$. 175 


\section{Einleitung}

Der Boden stellt aufgrund seiner Filter- und Pufferfunktionen im terrestrischen Bereich die bedeutendste Senke für anthropogene Stoffeinträge dar. Durch Sorptions- oder Bindungsprozesse können Xenobiotika zurückgehalten werden, so dass ihre Verbreitung gehemmt und die Entfaltung ihres eventuell toxischen Potenzials reduziert oder gar verhindert wird. Durch chemische Transformation oder Degradation, aufgrund bisher zumeist biotischen Faktoren zugesprochenen Prozessen, vermögen Böden als echte Senken für organische Schadstoffe zu fungieren. In den Boden eingetragene Xenobiotika werden durch Adsorption und zum Teil durch Einbindung in Huminstoffe dauerhaft festgelegt.

Bisher wurde zumeist davon ausgegangen, dass die natürliche organische Substanz des Bodens, gebildet durch die Aktivität von Mikroorganismen, die alleinige Verantwortung für die chemische Umsetzung und Festlegung organischer Schadstoffe trägt, wenn von photooxidativen Prozessen an der Oberfläche von Böden abgesehen wird. Der mineralischen Fraktion des Bodens, insbesondere den Tonmineralen, an die der Großteil der Huminstoffe über tonorganische Komplexe gebunden ist, wurde in den Boden- und Umweltwissenschaften im Hinblick auf eine Transformation von Organika nur in sehr geringem Umfang Beachtung geschenkt. Im Gegensatz dazu machte sich die industrielle Verfahrenstechnik die katalytische Aktivität von Tonmineralen seit Beginn des 20. Jahrhunderts in einem breiten Spektrum chemisch-technischer Anwendungen zu Nutze.

Vor diesem Hintergrund ergab sich die Frage, ob und in welcher Weise auch Tonminerale im Boden einen Beitrag bei der Genese von Huminstoffen und der Transformation organischer Schadstoffe zu leisten vermögen. Aufgrund der hohen Komplexität natürlicher Huminstoffe, deren chemische Struktur bisher nur sehr begrenzt aufgeklärt werden konnte und der Problematik der weitreichenden chemischen Modifizierung bei der Gewinnung solcher natürlicher organischer Substanzen, war es somit sinnvoll, chemisch definierte organische Substanzen relativ einfacher Struktur einzusetzen. Die Verwendung von Bausteinen des Lignins - eine hochmolekulare Verbindung und Hauptbestandteil von Pflanzen und somit auch der $\mathrm{Hu}-$ minstoffe - erschien dazu angebracht. Phenole, wie Brenzkatechin, Pyrogallol und 2,6Dimethylphenol stellen solche Ligninbruchstücke dar. Darüber hinaus bieten sie den Vorteil, gleichzeitig Schadstoffcharakter aufzuweisen, womit nicht nur ihre eventuell eintretende Transformation als Modell für die Bildung von Huminstoffen, sondern auch der Umsetzung organischer Schadstoffe angesprochen werden könnte. Als weitere definierte, charakteristische Stoffklasse, vorwiegend anthropogenen Ursprungs, bieten sich die polyzyklischen aromatischen Kohlenwasserstoffe (PAK) an. Diese zeichnen sich, mit Zunahme des Kondensationsgrades, durch eine relative Trägheit bzw. Inertie gegenüber Transformationsreaktionen aus. Ein niedrig kondensierter PAK (Phenanthren) wurde ausgewählt, um das Verhalten solcher, durch hochspezialisierte Organismen abbaubarer Substanzen, in Gegenwart von, als mineralische Katalysatoren wirkenden, Tonen zu beobachten. 
Somit sollte die Voraussetzungen erfüllt sein, anhand eines relativ einfachen, definierten Versuchsaufbaus biomimetische Ab-, Um- und Aufbauprozesse organischer Substanzen auf der Oberfläche von Tonmineralen zu beobachten und die Reaktionsprodukte zu charakterisieren, um so Aussagen über die Bedeutung der Tonminerale bei der Genese von Huminstoffen bzw. bei der Transformation von Xenobiotika zu treffen. 


\section{Stand der Forschung}

\subsection{Tonminerale}

„It seems reasonable to suggest, that catalytic reactions at clay surfaces in soils and in sediments play a much more important role in conversions and alterations of organic compounds adsorbed at their surfaces than one would conclude from the literature where almost all such changes are attributed to biological agency“" (MORTLAND 1970).

Tonminerale verfügen über eine Vielzahl von umweltrelevanten Eigenschaften (PARKER \& RAE 1998, PINNAVAI \& MORTLAND 1986), so dass es von großem Interesse erscheint, diese Merkmale, auch unter dem Gesichtspunkt, dass Tonminerale einen bedeutenden Anteil an der Zusammensetzung von Böden haben, zu untersuchen (Abbildung 1).

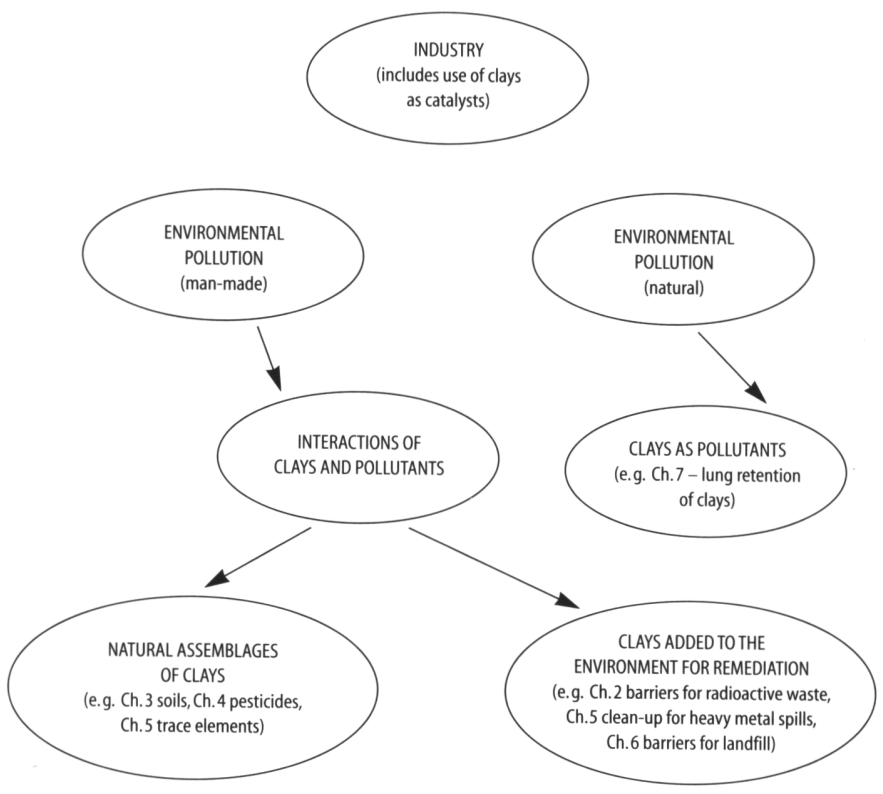

Abbildung 1: Die umweltrelevanten Funktionen von Tonen (RAE \& PARKER 1998).

\subsubsection{Aufbau und Eigenschaften von Tonmineralen}

Tonminerale als Verwitterungsprodukte von Glimmern bzw. Verwitterungsneubildungen der Kalifeldspäte sind mehr oder weniger gut kristallisierte OH-haltige Alumosilikate. Sie bestehen aus Schichten dichtgepackter O- und $\mathrm{OH}-$ Liganden, in deren Zwischenräume Kationen ( $\mathrm{Si}, \mathrm{Al}, \mathrm{Fe}$ ) eingelagert sind. Aufgrund der geringeren Größe von Zentralkationen, wie Silizium oder zum Teil auch Aluminium, sind diese von vier Sauerstoffatomen (Koordinationszahl 4), die größeren Kationen, wie Al, Fe und Mg, dagegen sind von sechs Sauerstoff- oder OHLiganden (Koordinationszahl 6) umgeben. Aus dieser unterschiedlichen Koordination ergeben sich Tetraeder $\left(\mathrm{Si}_{1} \mathrm{AlO}_{4}\right)$ und Oktaeder $\left[(\mathrm{Al}, \mathrm{Mg}, \mathrm{Fe})(\mathrm{O}, \mathrm{OH})_{6}\right]$ (SCHACHTSCHABEL et al. 1992). Die Tetraeder sind über gemeinsame Sauerstoffionen zu Schichten verknüpft. In der Ebene sind die Tetraeder zu einem Netzwerk aus Sechserringen verbunden. Die Tetraederschicht ist an eine Oktaederschicht kondensiert (JASMUND \& LAGALY 1993). Ein aus ei- 
ner Abfolge von Tetraeder und Oktaeder aufgebautes Tonmineral wird als ZweischichtMineral bezeichnet, hierzu gehören die Kaolinite. Die hingegen aus einer Abfolge von Tetraeder-Oktaeder-Tetraeder aufgebauten Minerale werden als Dreischicht-Minerale bezeichnet, dazu gehört die Gruppe der Smektite mit Montmorillonit und Nontronit (Abbildung 2).

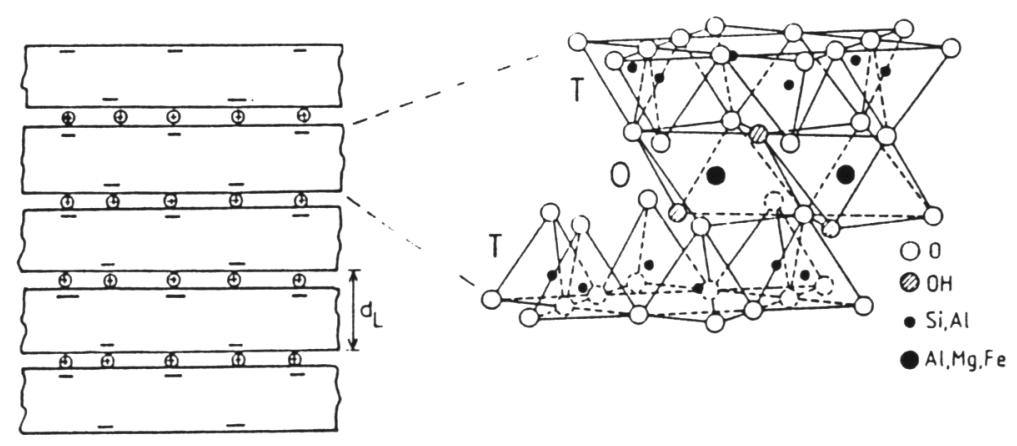

Abbildung 2: Aufbau der Dreischicht-Tonminerale (nach JASMUND \& LAGALY 1993).

Das eigentliche Tonmineralblättchen wird durch etwa 10-40 solcher Silikatschichten gebildet. Montmorillonite verfügen über viele Eigenschaften, deren Diversität liegt primär in der Struktur der Tonminerale begründet. Die Idealformel wird durch den folgenden Term beschrieben:

$$
\left(\mathrm{Al}_{2-\mathrm{x}} \mathrm{Mg}_{\mathrm{x}}\right)\left(\mathrm{Si}_{4-\mathrm{y}} \mathrm{Al}_{\mathrm{y}}\right) \mathrm{O}_{10}(\mathrm{OH})_{2} \mathrm{M}^{+}{ }_{\mathrm{x}+\mathrm{y}} \times \mathrm{nH}_{2} \mathrm{O} \text {, }
$$

wobei $\mathrm{M}$ das jeweilige Zwischenschichtkation, $\mathrm{x}$ und $\mathrm{y}$ die entsprechenden oktaedrischen bzw. tetraedrischen Substitutionen (wobei $x>y$ ) beschreiben (ORTEGO et al. 1991). Durch den isomorphen Ersatz von Zentralkationen bei der Entstehung von Tonmineralen mit entsprechender Größe, jedoch geringerer Ladung bei den Tetraedern $\mathrm{Al}^{3+}$ anstelle von $\mathrm{Si}^{4+}$ bzw. bei den Oktaedern $\mathrm{Mg}^{2+}$ statt $\mathrm{Al}^{3+}$ kommt es zu einem Defizit an positiver Ladung und folglich zu einem permanenten negativen Ladungsüberschuss. Zum Ausgleich dieser negativen Ladung werden Kationen in den Zwischenschichten eingelagert (Abbildung 2). Im Normalfall handelt es sich bei den Zwischenschichtkationen der Smektit-Gruppe um Erdalkali- (Ca, Mg) und Alkalimetallionen ( $\mathrm{Na}, \mathrm{K})$, die in hydratisiertem Zustand vorliegen. Die Zwischenschichtkationen und die an der äußeren Oberfläche liegenden Gegenionen sind austauschbar gebunden. Bei den Smektiten können diese leicht gegen andere anorganische oder organische Kationen, sofern sie über entsprechende Ladungen verfügen (z.B. $\mathrm{Cu}^{2+}$ oder $\mathrm{Fe}^{3+}$ ), austauschen (SOMA \& SOMA 1989, TRIBUTH \& LAGALY 1986).

Die in dieser Arbeit untersuchten Montmorillonite gehören als Dreischicht-(2:1)Minerale der Gruppe der Smektite an. Diese Lagerstättentone werden oft als Bentonite bezeichnet. Lagerstättentone enthalten neben den Tonmineralen zumeist eine Reihe weiterer Minerale, oft sind diese in bestimmten Korngrößenfraktionen angereichert. Bentonite können neben den Smektiten Glimmer, Illite, Aber auch Kaolinit, Chlorite, Wechsellagerungsverbindungen, Feldspäte, Quarz und Carbonate enthalten. Der Gehalt an Eisenoxiden und organischem Material ist gering. Die am häufigsten zur Katalyse herangezogenen Tonminerale sind die Smektite: $\mathrm{Na}^{+}$-Montmorillonit $(\mathrm{SWy}-2)$ aus Bentonitlagerstätten in Wyoming und $\mathrm{Ca}^{2+}$ - 
Montmorillonit (SAz-1) aus Arizona in den USA. Wyoming-Bentonit verfügt über einen hohen oktaedrischen Aluminiumgehalt (KOMADEL et al. 1997).

Bentonite (Smektite) werden aufgrund ihrer physikalischen und chemischen Eigenschaften in einem breiten Spektrum technischer Anwendungen (TRIBUTH \& LAGALY 1986) eingesetzt:

- Chemische Industrie: Katalysatoren, Adsorptionsmittel, Träger für Insektizide und Fungizide, Füllstoffe, Trockenmittel, Bindemittel für Öl

- $\quad$ Pharmazeutische Industrie: Grundlagen für Salben und Kosmetika

- Mineralölindustrie: Raffination, Entfärbung, Reinigung von Mineralölen, Katalysatoren zur Ölkrackung

- Lebensmittelindustrie: Schönung von Wein, Bier, Reinigung und Entfärbung von Fetten

- Papierindustrie: Abwasserbehandlung

- $\quad$ Reinigungsmittel: Waschmittel, Poliermittel, Seifenzusatz

- $\quad$ Landwirtschaft: Bodenverbesserung, Pelletierung, Adsorptionsmittel, Güllebindung

- $\quad$ Erzgewinnung: Pelletiermittel

- Gießerei-Industrie: Formsandbinder

- Bohrindustrie: Bohrspülflüssigkeiten

- $\quad$ Bauindustrie: Schlitzwandbau, Abdichtungen, Gleitmittel, Betonzusatz

- Keramik: Plastifizierung keramischer Massen

- $\quad$ Teer, Farben, Lacke: Emulgierung, Thixotropie, Verdickung, Antiabsetzmittel.

Die Probleme der Kontamination der Umwelt durch Luftverschmutzung, die Deponierung von Abfällen und die Ausbringung von Pestiziden auf der Erdoberfläche beziehen Tone und Tonminerale als bedeutende Bestandteile der Pedosphäre ein (Abbildung 3).

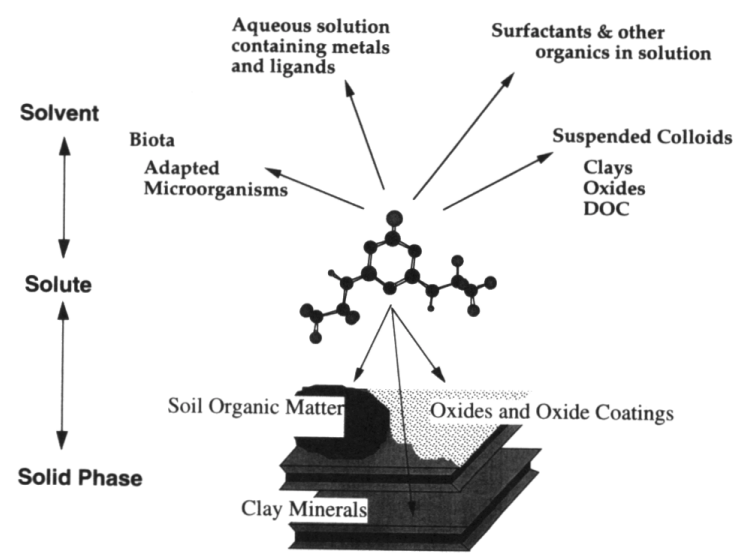

Abbildung 3: Interaktionen von Organika mit Bodenkolloiden (JOHNSTON 1996).

Die reaktiven Eigenschaften von Tonmineralen sind durch ihren strukturellen und chemischen Aufbau charakterisiert. Hieraus ist ersichtlich, dass es von großer Wichtigkeit ist, den Aufbau und den sich daraus ableitenden Chemismus von Tonmineralen zu verstehen. 


\subsubsection{Adsorptive Eigenschaften von Tonmineralen}

Dreischicht-Minerale verfügen über eine große spezifische, innere und äußere Oberfläche mit $700-800 \mathrm{~m}^{2} / \mathrm{g}$ (MORILL 1982). Somit bieten sie einer großen Zahl und Variation von Molekülen die Möglichkeit zur Einlagerung in die Zwischenschichten und zur Adsorption an den Mineraloberflächen (Abbildung 4).

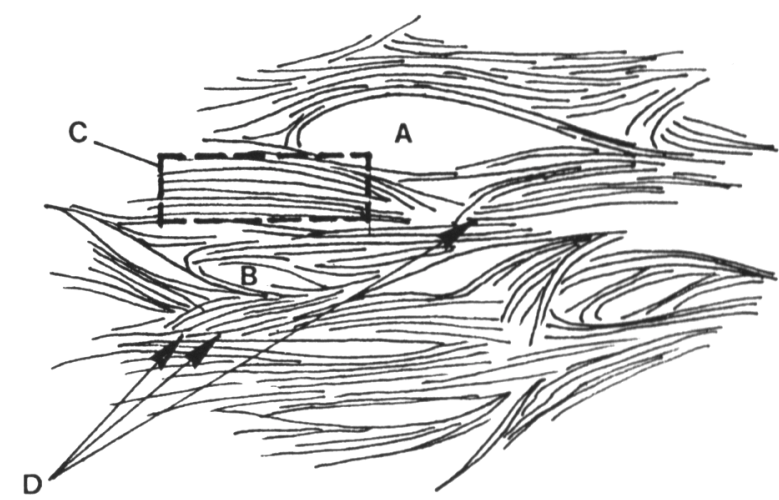

Abbildung 4: Schematischer Darstellung der Mikrostruktur eines Smektits, A: große, mehr oder weniger kontinuierliche Räume, B: kleine, isolierte Räume, C: interlamellarer Raum, D: Kontaktregion interagierender Tonmineralstapel mit elektrischen Doppelschichten (PUSCH 1998).

Durch ihre hohe negative Oberflächenladung verfügen Montmorillonite über eine hohe Austauschkapazität (80-120 meq/100 g), die permanente negative Ladung wird durch anorganische und organische Kationen kompensiert. Das an den Austauschkationen koordinierte Wasser spielt dabei eine wichtige Rolle in der Interaktion mit ungeladenen organischen Verbindungen, da deren polare Gruppen mit dem Wasser um die die Kationen umgebenden Ligandenplätze konkurrieren. Dort kann es zu Kation-Dipol-Wechselwirkungen (Abbildung 5), beispielsweise bei der Bindung von ungeladenen organischen Polymeren an der Tonmineraloberfläche kommen (WANG 1986).

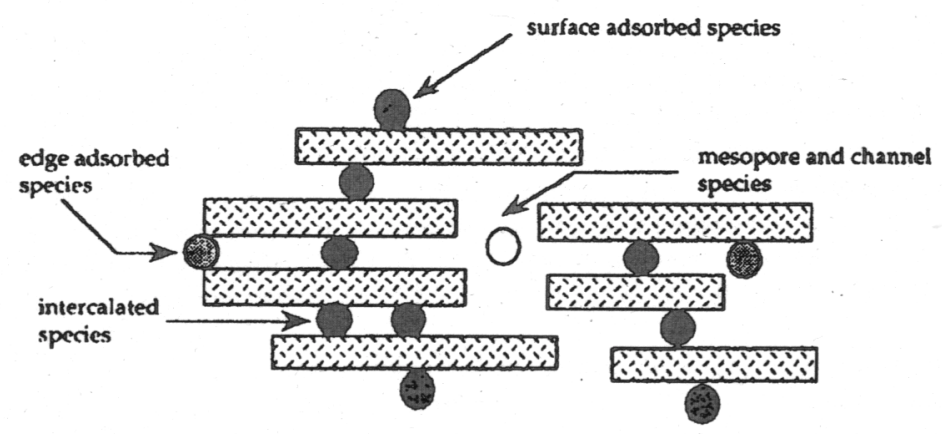

Abbildung 5: Adsorptionsplätze von Organika an einem Tonmineral (BEDIOUI 1995).

Der Adsorption an Oberflächen von Bodenbestandteilen ist in einem reaktiven System wie dem des Bodens besondere Bedeutung beizumessen. Jeder Mechanismus, der für einen heterogen-katalytischen Prozess in Frage kommt, basiert darauf, dass die Adsorption der primäre Schritt in der Konversion eines reaktiven Moleküls in ein aktives Stadium ist, sie ist Bestand- 
teil vieler katalytischer Bodenreaktionen (COLLINS et al. 1995). Die Oberfläche von Schichtsilikaten weist eine große Zahl aktiver Adsorptionszentren verschiedener Natur auf (HERMOSIN et al. 1993, JOHNSTON 1996, LI \& GUPTA 1994):

- Neutrale Siloxan-Oberflächen

- Isomorphe Substitutionsplätze

- Die Kationenaustauschplätze besetzende Metallkationen

- Die austauschbaren Kationen umgebende Wassermoleküle

- Hydrophobe Plätze

- Kanten und Störungen an Tonmineralen, an denen Silanol- und Aluminolgruppen an der Oberfläche anstehen.

Die Hauptzentren der Adsorption an Tonmineralen sind Si-OH-, Al-OH- und $\mathrm{Mg}-\mathrm{OH}-$ Gruppen (Abbildung 6).

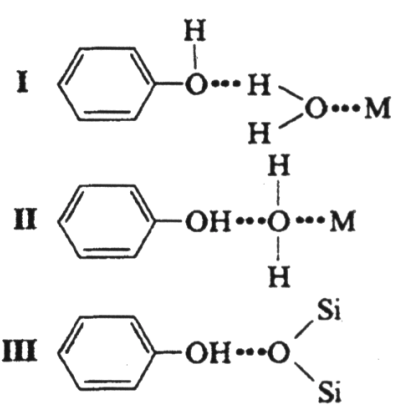

Abbildung 6: Adsorption von Organika an Tonmineralen (YARIV 1996).

Insbesondere die Kanten der Tonmineralstapel sind bevorzugte Sorptionsplätze für anorganische und organische Verbindungen (MORTLAND 1986). Der Grund dafür liegt in den dort „frei“" zugänglichen Si-OH- und Al-OH-Gruppen (Abbildung 7).

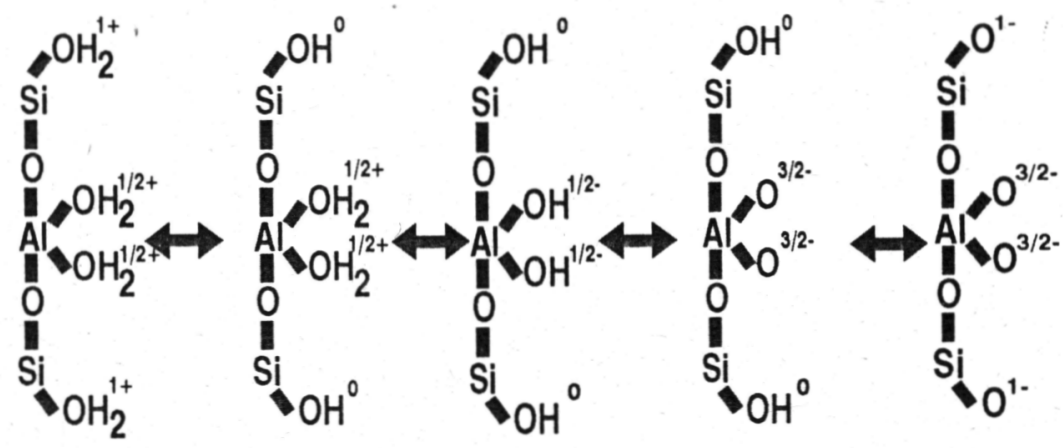

Abbildung 7: Sorptionsplätze für Organika an den Kanten der Tonminerale (SCHOONHEYDT 1995).

Bentonite sind starke Adsorbenten organischer Substanz (BACKHAUS et al. 1995, LAGALY 1994). In Böden sind Tonminerale immer mit organischer Substanz assoziiert. Die organische Substanz ist an den äußeren Oberflächen und in den Zwischenschichten der Tone gebunden (CHAHI et al. 1996, VIOLANTE et al. 1999). Die Adsorption von organischer Substanz an der Oberfläche von Tonmineralen kann die Bildung von stabilen Adsorptionskomplexen 
stoppen und als erster Schritt der katalytischen Konversion von organischen Substanzen angesehen werden:

1. Abbau zu niedermolekularen Produkten mit partieller oder kompletter Desorption, 2. sich anschließende Transformation in höhermolekulare Produkte durch Addition und Polymerisation und mögliche

3. nachfolgende Desorption sowie teilweiser Austausch (ZUBKOVA 1989).

Die planaren Oberflächen von Montmorilloniten sind Sauerstoff-Oberflächen. Die Adsorption organischer Substanzen an Tonmineralen hängt von der Austauschkapazität ab, von der Stärke der negativen Ladung, der Spezifikation der Adsorptionsplätze und der Natur der Kationen am Austauschkomplex (Abbildung 8).

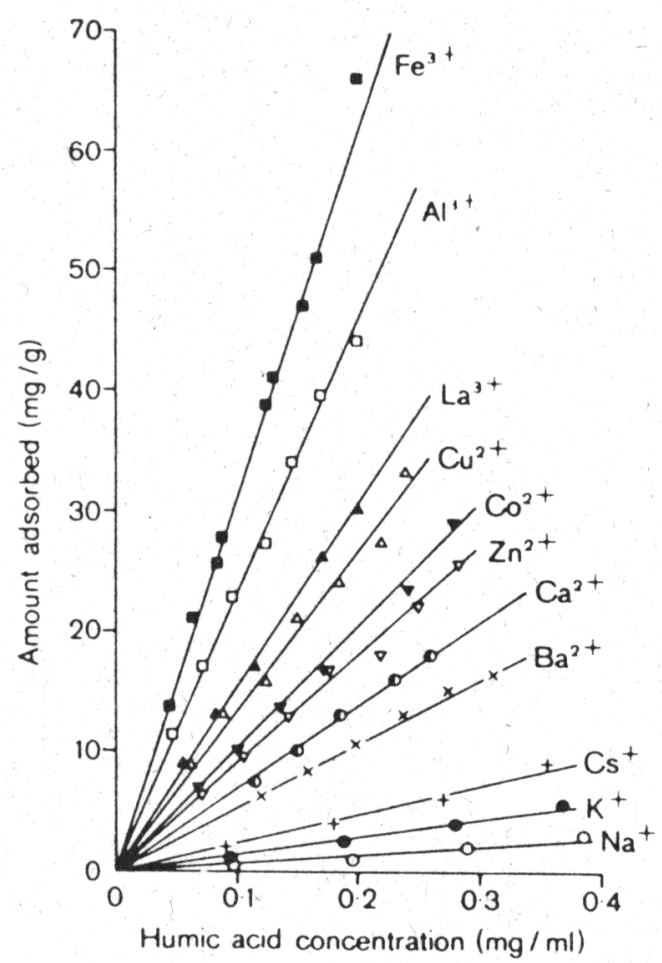

Abbildung 8: Adsorptionsisothermen für Huminstoffe an Montmorillonit, gesättigt mit verschiedenen Kationen (GRAUER 1989).

Organische Substanzen mit hoher Polarität bilden zwei oder mehr Molekülschichten zwischen den Tontaktoiden. Viele organische Pestizide polarer Natur werden zwischen zwei TetraederSchichten adsorbiert (Zwischenschicht-Adsorption), diese sind dann nicht mehr aktiv und zumeist gut vor mikrobiellem Abbau geschützt (bound residues).

An Tonmineralen werden bevorzugt die niedrigmolekularen Huminstofffraktionen mit hohem spezifischen Carboxylgehalt sorbiert (GRAUER 1989). An Tonmineralen adsorbierte organische Substanz kann jedoch auch die Eigenschaften mineralischer Oberflächen verändern (EVANKO \& DZOMBAK 1999). Beispielsweise erfolgt bei der Adsorption von Xenobiotika (Fluorochinoloncarbonsäuren-Derivate) in den Zwischenschichten eine Aufweitung dieser Schichten (NOWARA et al. 1997). Die Interkalation von natürlichen Huminsäuren in die 
Zwischenschichten des Montmorillonits beginnt bei pH-Werten unter 6 und führt erst bei $\mathrm{pH}<4$ zu einer nennenswerten Schichtaufweitung (BART 1979).

\subsubsection{Smektite als reaktive Tonmineralgruppe}

Smektite verfügen über einzigartige Eigenschaften, welche von keinen anderen natürlich vorkommenden Mineralen gezeigt werden. In Böden sind sie ubiquitär als Montmorillonite weit verbreitet. Aufgrund dieser Merkmale stellt die Gruppe der Smektite bereits seit Beginn der 70er Jahre eine immer wieder im Hinblick auf katalytische Interaktionen untersuchte anorganische Komponente des heterogenen Mediums Boden dar (ADAMS et al. 1983, BALLANTINE et al. 1983, FRIPIAT \& CRUZ-CUMPLIDO 1974, KÜRSCHNER et al. 1998, MORTLAND 1970, MORTLAND 1980, OCCELLI 1987, PINNAVAIA 1983). Die reaktiven Fähigkeiten dieser Tonmineralgruppe sind das Resultat einer extrem kleinen Kristallgröße, von Variationen in der internen chemischen Zusammensetzung, einer hohen Kationenaustauschkapazität, einer großen chemisch aktiven Oberfläche, von Variationen der austauschbaren Kationen und der Oberflächenladung, sowie von Interaktionen mit anorganischen und organischen Verbindungen. In den folgenden Kapiteln soll im einzelnen auf die Ursachen der Reaktivität von Tonmineralen genauer eingegangen werden.

\subsubsection{Ursachen der katalytischen Eigenschaften von Tonmineralen}

Tonminerale, Metalloxide und Huminstoffe sind eine komplexe Mischung von Bodenkomponenten, die auf abiotischem Wege eine Vielzahl von Reaktionen mit Xenobiotika (Abbildung 9) eingehen können (HUANG 1990, NAIDJA et al. 2000).

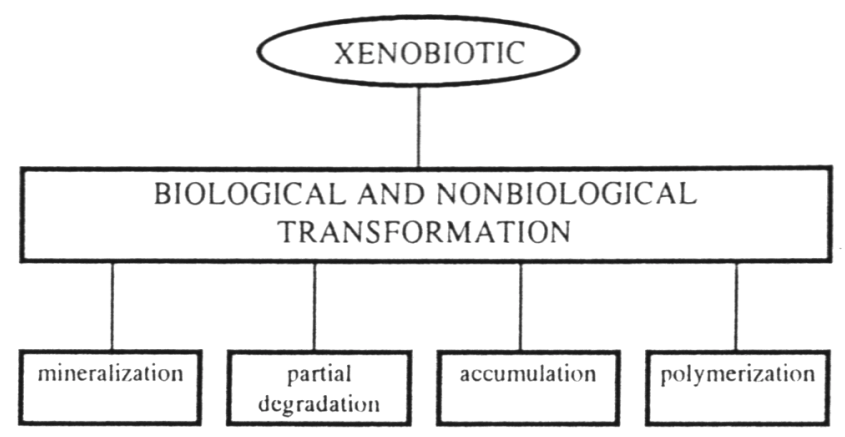

Abbildung 9: Transformationsprozesse von Xenobiotika in Böden (RUGGIERO 1999).

Chemische Prozesse wie Hydrolyse, Oxidation und Polymerisation werden durch Tonminerale sowie Mn- und Fe-Oxide katalysiert (BRINDLEY \& LEMAITRE 1987, SHINDO 1994, RUGGIERO 1999). Die Reaktivität der Tonminerale rührt vor allem von den funktionellen Gruppen an deren Oberflächen her. Die Oberflächenchemie der Minerale ist primär von der Natur ihrer funktionellen Gruppen bestimmt (NEWMAN 1987). Durch Hydrolyse, Elektronentransfer und Radikalbildung können insbesondere organische Verbindungen an Tonmine- 
raloberflächen umgesetzt werden (RUPERT et al. 1987). Die Hauptursachen der Aktivität von Tonmineralen sind vor allem ihre Eigenschaft, als Säuren zu fungieren und durch Elektronentransfer-Reaktionen von adsorbierten Verbindungen zum Mineral Radikale zu bilden. Die häufigsten Reaktionen an Oberflächen von Montmorilloniten basieren auf deren Säureeigenschaften (CLARK et al. 1995). Die starke Oberflächenazidität vieler Alumosilikate kann die Katalyse adsorbierter organischer Verbindungen bewirken (HEIMAN 1993, HELSEN 1982). Es gibt zwei allgemeine Arten von azidischen Spezies, einerseits die, die als Protonendonatoren für basische Moleküle fungieren können. Darüber hinaus jene, die Elektronenpaare von basischen Molekülen, Koordinationsbindungen bildend, akzeptieren können. Die ersteren werden als Brönsted-Säure bezeichnet, die zweiten als Lewis-Säuren. Im Folgenden wird auf die strukturellen Gründe für diese Eigenschaften eingegangen.

\subsubsection{Tonminerale als Brønsted-Säuren}

Laut Definition sind Brønsted-Säuren Stoffe, die Protonen (Wasserstoffionen) abgeben können. Alumosilikate verfügen über protonische Zentren des Brønsted-Typs (ZUBKOVA 1989). Die Brönsted-Azidität der Tonminerale setzt sich aus mehreren Quellen zusammen (Abbildung 10): schwach azidische SiOH-Gruppen an den Kanten der Tonminerale oder an Oberflächendefekten der basalen Schichten, stark azidische, verbrückte Hydroxylgruppen. Weiterhin an den Mineraloberflächen polarisierte Wassermoleküle, insbesondere die, welche mit polyvalent kompensierenden Kationen wie $\mathrm{Ca}^{2+}$ und $\mathrm{Al}^{3+}$ assoziiert sind (BROWN \& RHODES 1997, SOLOMON \& HAWTHORNE 1983). Vor allem feuchte und luftrockene Tone besitzen eine Brønsted-Azidität McBRIDE (1985). Die Brønsted-Azidität gründet sich in der Dissoziation von adsorbierten Wassermolekülen nach folgender Gleichung:

$$
\left[\mathrm{M}\left(\mathrm{H}_{2} \mathrm{O}\right)_{\mathrm{x}}\right]^{\mathrm{n}+} \Leftrightarrow\left[\mathrm{M}(\mathrm{OH})\left(\mathrm{H}_{2} \mathrm{O}\right)\right]^{(\mathrm{n}-1)+}+\mathrm{H}^{+} \text {. }
$$

Die Ausrichtung des Gleichgewichtes ist abhängig von der Art der austauschbaren Kationen $\mathrm{M}^{+}$. Je höher die Ladung der Kationen und je kleiner deren Radien sind, desto größer ist der polarisierende Effekt auf die sie umgebenden Wassermoleküle (CAIRNS-SMITH 1986). Wird das Ladungs-/Radius-Verhältnis kleiner sinkt die Azidität. Die Azidität steigt somit in der Reihenfolge $\mathrm{Al}^{3+}, \mathrm{Mg}^{2+}>\mathrm{Ca}^{2+}>\mathrm{Li}^{+}>\mathrm{Na}^{+}>\mathrm{K}^{+}$an.

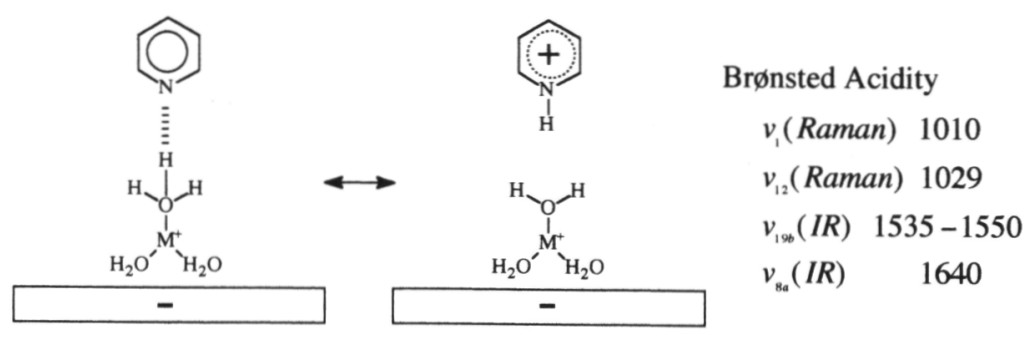

Abbildung 10: Brønsted-Position an Tonmineralen (JOHNSTON 1996).

Neben der Wertigkeit der Ladung der Zwischenschichtkationen ist die Fähigkeit der Tonminerale Protonen zu liefern sehr stark vom Wassergehalt beeinflusst (Abbildung 11). Bei hohen 
Wassergehalten ist der polarisierende Effekt der Kationen insgesamt auf eine relativ große Zahl von $\mathrm{H}_{2} \mathrm{O}$-Molekülen verteilt, die Brønsted-Azidität ist relativ gering. Tonminerale mit Wassergehalten von weniger als $5 \%$ können eine Azidität an ihrer Oberfläche entwickeln, die der einer 90 \%-igen Schwefelsäure entspricht (SOMA \& SOMA 1989).

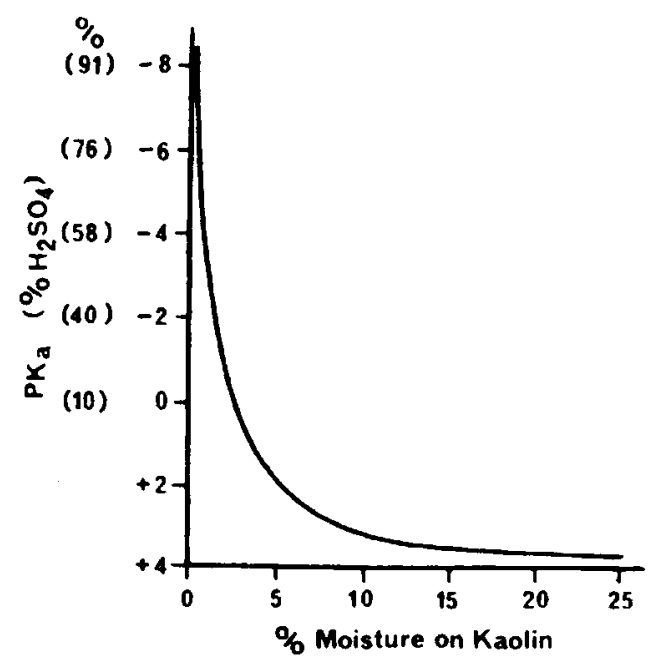

Abbildung 11: Abhängigkeit der Azidität eines Tonminerals am Beispiel von Kaolinit vom Wassergehalt (VOUDRIAS \& REINHARD 1986).

Die Reaktionsbedingungen in Montmorilloniten können somit konzentrierter Schwefelsäure entsprechen (COZENS et al. 1993). Nimmt der Wassergehalt weiter ab, bis auch die letzten Spuren sorbierten Wassers entfernt sind, sinkt gleichzeitig die Azidität. Die dazu noch vorhandene sehr schwache Brønsted-Azidität rührt von strukturellen Hydroxylgruppen an planaren und Kantenflächen der Mineralschichten her. Dazu zählen die schwachen azidischen Silanolgruppen $(\mathrm{SiOH})$ an Kristallkanten der Mineralschichten oder basaler Oberflächendefekte (SOLOMON \& HAWTHORNE 1983). Dass die Seitenflächen der Tonminerale über einen wesentlichen Einfluss auf deren Reaktivität verfügen, kann durch Behandlung der Tonmineraloberflächen mit Na-Metaphosphat veranschaulicht werden. Phosphate blockieren die funktionellen Gruppen an den Tonmineralkanten, daraufhin ist im Anschluss eine wesentlich geringere katalytische Aktivitäten an den Tonmineraloberflächen zu beobachten (WANG \& HUANG 1987). Die Protonen liefernde Fähigkeit dieser funktionellen Gruppen wird durch die Anwesenheit benachbarter elektronenanziehender Aluminiumionen noch verstärkt (THENG 1981).

\subsubsection{Tonminerale als Lewis-Säuren}

Neben der Eigenschaft, Protonen abzugeben, können Tonminerale über ihre aprotischen Zentren auch als Elektronenakzeptoren fungieren (ZUBKOVA 1989). 


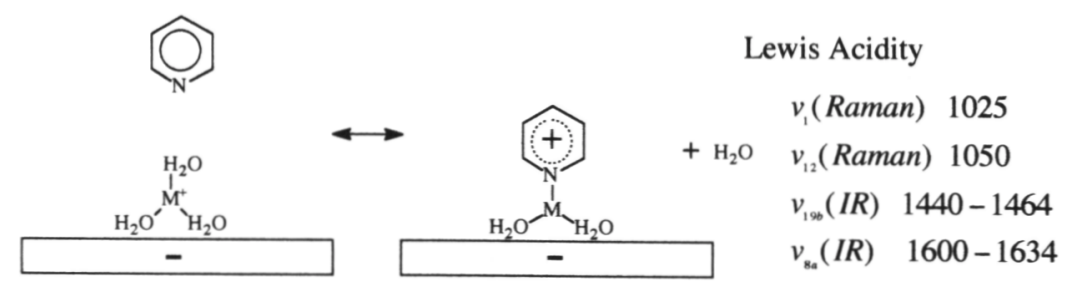

Abbildung 12: Lewis-Positionen von Alumosilikaten (JOHNSTON 1996).

Damit reagieren sie gemäß Definition als Lewis-Säuren. Die dominierenden, für die LewisAzidität an Tonmineraloberflächen verantwortlichen Ionen sind normalerweise ungesättigt koordinierte $\mathrm{Al}^{3+}$-Ionen, exponiert an den Mineralkanten der Schichtsilikate oder dehydrierte, oligomere Oxyaluminium-Kationen. In Anwesenheit von $\mathrm{H}_{2} \mathrm{O}-$ Molekülen sind diese LewisPositionen hydriert und ihre Azidität maskiert, falls jedoch $\mathrm{Al}^{3+}$-Ionen elektronegative Substituenten besitzen, wie beispielsweise Halogen- oder Silikationen, können die koordinierten Wassermoleküle ausreichend polarisiert werden und so starke Brønsted-Säuren bilden (SCHULTZ et al. 1987). Als weitere Ursache der Lewis-Azidität können Ionen der Übergangsmetalle $\left(\mathrm{Fe}^{2+}, \mathrm{Fe}^{3+}, \mathrm{Cu}^{2+}\right)$ als strukturelle ,Verunreinigungen“ in der Kristallstruktur bzw. in den Zwischenschichten der Tonminerale eingelagerte Kationen fungieren. Durch Elektronenübergänge zwischen adsorbierten organischen Molekülen und diesen Kationen können Redoxreaktionen initiiert werden:

$$
\begin{aligned}
& \left(\mathrm{M}^{\mathrm{n}+}\right)_{\text {layer }}+\mathrm{RH} \rightarrow\left(\mathrm{M}^{(\mathrm{n}-1)+}\right)_{\text {layer }}+\mathrm{R} \bullet+\mathrm{H}^{+} \\
& \left(\mathrm{M}^{\mathrm{n}+}\right)_{\text {gallery }}+\mathrm{Ar} \rightarrow\left(\mathrm{M}^{(\mathrm{n}-1)+}\right)_{\text {gallery }}+\mathrm{Ar} \bullet^{+},
\end{aligned}
$$

wobei RH: Kohlenwasserstoff, Ar: aromatischer Rest (SAWHNEY 1985).

Zusätzlich zur Freisetzung von Protonen können durch diese Elektronenübergänge Radikale der an der Oberfläche der Tonminerale adsorbierten organischen Moleküle gebildet werden. Die Lewis-Säure-Aktivität der Tonminerale bewirkt die Fähigkeit zu Friedel-CraftsAlkylierung sowie zur Diels-Alder-Reaktion (BROWN \& RHODES 1997).

\subsubsection{Bildung von Radikalen an Tonmineralen}

Die oxidierende Kraft von Montmorilloniten ist nach SOLOMON \& HAWTHORNE (1983) größtenteils auf das in den Oktaedern der Silikatschichten enthaltene strukturelle $\mathrm{Fe}^{3+}$ zurückzuführen. An unvollständig koordinierten Al-Ionen, die an Mineralkanten der Alumosilikate auftreten, oder in Gegenwart von Oxyaluminiumionen können gleichfalls Ladungsübergänge stattfinden. Durch den Ladungstransfer werden aus adsorbierten organischen Molekülen mit niedrigen Ionisierungspotentialen, wie z.B. aromatische Kohlenwasserstoffe, Radikalkationen gebildet. Nach ISAACSON \& SAWHNEY (1983) kann die Oberflächenazidität der Tonminerale aber auch in Abwesenheit von Übergangsmetallen zur Bildung hochreaktiver Radikalkationen führen. Tonminerale bilden, insbesondere nach einer Dehydrierung, radikalische Zentren aus (KANNAN \& PITCHUMANI 1997). Dies sind vor allem Silanoxyl-Radikale, welche durch Entfernung von Wassermolekülen von oberflächlichen Silanolgruppen entstehen. Sie 
treten isoliert oder verbunden über Peroxy-Bindungen auf $\left(\mathrm{O}_{3} \mathrm{Si}-\mathrm{O}-\mathrm{O}-\mathrm{SiO}_{3}\right)$. Diese Radikale können auch zur Entstehung von Superoxid-Radikalen $\left(\mathrm{O}_{2}{ }^{-}\right)$führen. Durch die Stärke dieser Radikale sind Tonminerale in der Lage, die meisten organischen Substrate in ihre Radikalkationen zu oxidieren (BALOGH et al. 1993).

\subsubsection{Bildung von anorganischen Radikalen}

Tonminerale können als Oxidanten oder Reduktanten in einer Vielzahl organischer Reaktionen fungieren. Die primären Quellen dieser Aktivität sind vor allem $\mathrm{Fe}^{2+}$ - und $\mathrm{Fe}^{3+}$-Ionen, aber auch andere Kationen der Übergangsmetalle. Diese treten als austauschbare Spezies, als isomorphe Substituenten oder als an die Tonminerale gebundene Oxide auf. Es erfolgt ein Transfer von Elektronen der adsorbierten Moleküle zu oktaedrischen Positionen der Metallionen an den Schichtkanten oder an basalen Oberflächen der Tonminerale.

Bildung von Hydroxyl-Radikalen $(\bullet \mathrm{OH})$ in der Haber-Weiss-Reaktion an Fe-Oxiden (AUST 1995):

$$
\begin{aligned}
& \mathrm{O}_{2} \bullet^{-}+\mathrm{Fe}^{3+} \rightarrow \mathrm{O}_{2}+\mathrm{Fe}^{2+} \\
& 2 \mathrm{O}_{2} \bullet^{-}+2 \mathrm{H}^{+} \rightarrow \mathrm{H}_{2} \mathrm{O}_{2}+\mathrm{O}_{2} \\
& \mathrm{Fe}^{2+}+\mathrm{H}_{2} \mathrm{O}_{2} \rightarrow \bullet \mathrm{OH}+\mathrm{OH}^{-}+\mathrm{Fe}^{3+} .
\end{aligned}
$$

Bei der oxidativen Degradation organischer Verbindungen durch $\mathrm{H}_{2} \mathrm{O}_{2}$ wird in Gegenwart von Eisen ein Hydroxyl-Radikal gebildet:

$$
\mathrm{Fe}^{2+}+\mathrm{H}_{2} \mathrm{O}_{2} \rightarrow \mathrm{Fe}^{3+}+\mathrm{OH}^{-}+\bullet \mathrm{OH}
$$

Mit diesem Radikal reagieren die sorbierten organischen Verbindungen (DO \& YEH 1995):

$$
\mathrm{RH}+\bullet \mathrm{OH} \rightarrow \mathrm{R} \bullet+\mathrm{H}_{2} \mathrm{O}
$$

zu organischen Radikalen.

\subsubsection{Bildung von organischen Radikalen}

Die Bildung von organischen Radikalkationen kann durch den Transfer von Elektronen von elektronenreichen Zentren adsorbierter organischer Moleküle zu elektronendefizitären Plätzen oder Spezies erfolgen. Die elektronenspendenden Zentren können $\pi$-Elektronenwolken aromatischer oder ungesättigter Gruppen sein. Der Oxidationsprozess kann in der Bildung von Ladungs-Transfer-Komplexen resultieren, wobei das transferierte Elektron zwischen der mineralischen Seite und der organischen Spezies geteilt wird bzw. der Transfer vollständig unter Bildung eines Radikalkations erfolgt. Ladungs-Transfer-Komplexe können auch zwischen Lewis-Plätzen und koordinierten aromatischen Verbindungen mit niedrigen Ionisierungspotentialen gebildet werden, bei Dissoziation der Komplexe werden freie Radikalkationen gebildet. Die potentiellen Elektronenakzeptoren sind auch Übergangsmetalle auf der Oberfläche der Minerale oder im Kristallgitter adsorbierter Sauerstoff sowie chemisorbierter molekularer Sauerstoff oder Sauerstoffradikalanionen.

Die Bildung von Hydroxyl-Radikalen $(\bullet \mathrm{OH})$ erfordert eine Elektronenreduktion von $\mathrm{O}_{2}$ zum Superoxid $\left(\mathrm{O}_{2}^{-} \cdot\right)$, Wasserstoffperoxid $\left(\mathrm{H}_{2} \mathrm{O}_{2}\right)$ bis schließlich zum $\bullet \mathrm{OH}$ bevor die Oxidation 
des Biomoleküls eintritt:

$$
\begin{aligned}
& \mathrm{RH}+\bullet \mathrm{OH} \rightarrow \mathrm{R} \bullet+\mathrm{H}_{2} \mathrm{O} \\
& \mathrm{R} \bullet+\mathrm{O}_{2} \rightarrow \mathrm{ROO} \bullet \\
& \mathrm{ROO} \bullet+\mathrm{RH} \rightarrow \mathrm{ROOH}+\mathrm{R} \bullet
\end{aligned}
$$

Einige Biomoleküle, darunter das Pyrogallol regieren direkt mit Dioxygen unter Bildung von $\mathrm{O}_{2} \bullet$ und/oder $\mathrm{H}_{2} \mathrm{O}_{2}$ :

$$
\begin{aligned}
& \mathrm{RH}_{2}+\mathrm{O}_{2} \rightarrow \mathrm{RH} \bullet+\mathrm{O}_{2}^{-}+\mathrm{H}^{+} \\
& \mathrm{RH}_{2}+\mathrm{O}_{2} \rightarrow \mathrm{R}+\mathrm{H}_{2} \mathrm{O}_{2} .
\end{aligned}
$$

Solche Reaktionstypen werden als Autoxidationen bezeichnet. Diese sind definiert als unkatalysierte Oxidation einer Substanz, die dem Sauerstoff der Luft ausgesetzt ist. Um jedoch eine höhere Umsetzungsrate zu erreichen, ist ein Katalysator, wie z.B. Übergangsmetalle notwendig. Eisen und Kupfer sind die vorherrschenden Übergangsmetalle in der Natur (MILLER et al. 1990). Durch Ladungsübergänge vom Phenol zum Eisen wird dieses oxidiert (Abbildung 13).

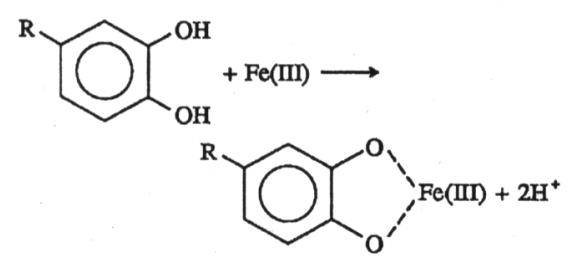

Abbildung 13: Chelatisierung von Phenolen mit Eisen (nach MILLER et al. 1990).

Die Radikalkationen und die Sauerstoffspezies sind in Clustern auf der Mineraloberfläche vorhanden. Weiterhin können Elektronen der Minerale an elektronendefizitäre Verbindungen wie Chinone abgegeben werden, es werden korrespondierende Radikalanionen gebildet. Eine Radikalbildung benachbarter organischer Komponenten sowie eine Dimerisierung ist die Folge (SOLOMON \& HAWTHORNE 1983).

\subsubsection{Tonminerale als Katalysatoren}

Tonminerale dienen durch ihre physikalischen und chemischen Eigenschaften bereits seit vielen Jahrzehnten in zahlreichen chemisch-technischen Synthesen als Katalysatoren. Einen umfangreichen Überblick über die Verwendung von Tonmineralen als Katalysatoren bieten BIRKEL \& NIEMEYER (1998) und VACCARI (1999). Sie wurden in der petrochemischen Industrie zur Herstellung von Treibstoffen (IZUMI et al. 1992), von Additiven (BREEN et al. 1985) sowie der industriellen Synthese zur Darstellung von organischen Verbindungen (CORMA \& WOJCIECHOWSKI 1985, MORIKAWA 1993) verwendet. Ihre Wirksamkeit als Katalysatoren bei der Reduktion von Stickoxid in Kraftfahrzeugabgasen wird derzeit untersucht (Anonymus 1995). Als weiterführende Übersichten sind die Veröffentlichungen von IZUMI et al. (1992), ORTEGO et al. (1991), THENG (1981) und ZUBKOVA (1989) zu nennen. 
Ein Überblick über die Vielzahl der neueren Forschungen zur Reaktivität von Tonmineralen zeigt, dass im Hinblick auf die Katalyse in chemisch-technischen Synthesen als auch unter Umweltgesichtspunkten den Tonmineralen ein immer höherer Stellenwert eingeräumt wird.

Zur Verwendung des in der Literatur häufig benutzten Begriffes „Katalysator“ bleibt anzumerken, dass dieser Ausdruck im genauen Sinne der Definition nicht unproblematisch erscheint, da die Oberfläche von Tonmineralen während der an ihr ablaufenden Reaktionen chemische und physikalische Veränderungen erfährt (THENG 1981, RHODES \& BROWN 1995, KRETZSCHMAR et al. 1997). In Bezug auf die zitierten Literaturquellen wird der Begriff aus Gründen der Übersichtlichkeit jedoch beibehalten.

Die heterogene Katalyse organischer Reaktionen durch anorganische Feststoffe (Tonminerale) hat in den letzten Jahren eine steigende Bedeutung erfahren. Diese Materialien sind kostengünstig, umweltfreundlich und wiederverwendbar (JNANESHWARA et al. 1999).

- Für das Kracken von Kohlenwasserstoffen werden Tonminerale als Katalysatoren verwendet. Um Kohlenwasserstoffe zu kracken benötigt man einen sauren Katalysator um Carbonium-Ionen auf seiner Oberfläche zu produzieren. Solche Katalysatoren sind natürliche Tonminerale (CORMA \& WOJCIECHOWSKI 1985). Zu Beginn des 20. Jahrhunderts wurde das HOUDRY-Verfahren zur Herstellung hochoktaniger Treibstoffe eingeführt und dieses bis in die 60er Jahre hinein angewendet.

- Montmorillonite werden als „solid acids“ zur Katalyse organischer Reaktionen (SHARMA et al. 1994) eingesetzt.

- Tonminerale sind effiziente Katalysatoren der direkten Konversion von Alkoholen und Phenolen zu Tetrahydropyranyl (CAMPELO 1994).

- Tone sind effiziente Katalysatoren für die Diels-Alder-Synthese der Cycloaddition organischer Verbindungen (Cyclopentadien mit chiralen Acrylaten) sowie der ClaisenReaktion (CATIVIELA 1993a,b).

- Die Addition von Thiolen an Olefine (Styrol) erfolgt durch Katalyse an Tonmineralen über radikalische Intermediäre (KANNAN \& PITCHUMANI 1997).

- Rearrangement von Hopenen zu Terpenen erfolgt durch toninduzierte Katalyse (HAUKE et al. 1993).

- Ringöffnung von Oxiranen durch Thiophenole (MAITI et al. 1993).

- Katalytische Debutylierung von Butylphenolen (SALEH \& MAHMOUD 1998).

- Isomerisation von Hexen (KÜRSCHNER et al. 1998).

- Michael-Addition von Carbonyl-Verbindungen durch Montmorillonit (POUPAERT et al. 1999).

- Friedel-Crafts-Alkylierung von Benzol (VACCARI 1998).

- Synthese von Alkylphenolen und Bisphenolen mit Al-Montmorillonit (TATEIWA et al. 1995).

- Oxidative Kopplung von Phenolen zur von Bi- und Tertaarylen (SARTORI et al. 1992). 
- Tone als Katalysatoren der Transesterisierung von langkettigen Alkoholen, ungesättigten Alkoholen und Phenolen (PONDE et al. 1998). Selektiver Schutz der funktionellen Gruppen von Aldehyden, Ketonen und Hydroxylgruppen. Tone stabilisieren energiereiche Intermediäre und können die Energie in ihrer Gitterstruktur aufnehmen, diese Energie kann in Form von chemischer Energie abgegeben werden. Die Alkylierung von Alkoholen, eine der fundamentalsten Reaktionen in der organischen Chemie, kann an Tonmineralen ablaufen.

- Die Methylierung von Brenzkatechin zu Guajacol (Abbildung14, Verwendung in Duftstoffen, Pharmazeutika) wird durch Tonminerale initiiert (LI et al. 1998).

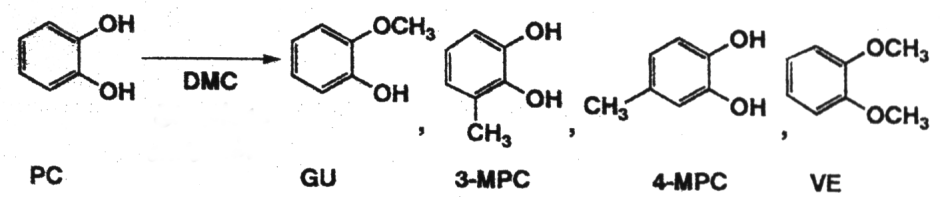

Abbildung 14: Reaktion von Brenzkatechin an Kaolinit und Dimethylcarbonat (FU et al. 1998).

- Oxidation von Alkoholen und Nitration von Phenolen durch Montmorillonit (BEKASSY et al. 1998).

- Abiotische Transformation von TNT in Montmorillonitsuspensionen (BRANNON et al. 1998).

- Montmorillonit als Katalysator der Bildung seltener Saccharide (SHARMA et al. 1994).

- Tonminerale wirken am Prozess der Erdölgenese mit SOLOMON (1968).

- Na-Montmorillonit bewirkt eine Dimerisation von Aminosäuren, gefolgt von der Bildung cyclischer Anhydride sowie eine Verlängerung von Peptidketten. Oligomerisation kleiner Oligopeptide sowie Heterooligomerisation wurden beobachtet (BUJDAK \& RODE 1996, BUJDAK et al. 1996).

- Aminosäuren wie das Alanin und das Glycin wurden durch Katalyse an Montmorilloniten zu Peptiden umgewandelt (SON et al. 1998). Es wurden Tripeptide gebildet.

- Acetylierung von Alkoholen, Phenolen und Amine mit einem Essigsäureanhydrid. Acylierung von Alkoholen, Thiolen, Phenolen und Aminen mit Acetylchlorid und Benzoylchlorid (LI \& LI 1998).

- Oxidative Kopplung von 2-Naphtol durch Tonminerale (KANTAM et al. 1998).

- Alkylierung von Toluol mit Benzoylchlorid und Benzoylalkohol (CSERI et al. 1995).

- Alkylierung von Phenol mit Methanol zur Synthese von Kresolen und Xylenolen (VELU \& SWAMY 1996).

Die breite Anwendung der Tonmineralen in chemisch-technischen Anwendungen macht zwingend darauf aufmerksam, dass es naheliegend ist, auch das Verhalten von Tonmineralen im Boden auf ihre reaktiven Eigenschaften hin zu untersuchen. 


\subsubsection{Organo-mineralische Interaktionen}

Die organische Substanz, vor allem die Huminstoffe des Bodens und das sind in landwirtschaftlich genutzten Böden 70-80 \% des Grundkörpers der organischen Substanz (SCHULTEN \& SCHNITZER 1997), bilden zum größten Teil mit den Tonmineralen des Bodens tonorganische Komplexe. In diesen Komplexen sind 60 bis $90 \%$ des organischen Kohlenstoffs gebunden (ZIECHMANN \& MÜLLER-WEGENER 1990). Die Hauptanteile der tonorganischen Komplexe sind polyaromatischer und polysaccharidischer Natur (SHEVCHENKO \& BAILEY 1998).

Die Komplexbildung erfolgt über diverse Bindungsformen (EVANGELOU 1998):

1. Anionen- und Ligandenaustausch an Tonmineralkanten

2. Kationen- oder Wasserbrücken an basalen Tonoberflächen

3. H-Brückenbindungen an der Siloxanschicht

4. Van-der-Waals Kräfte

5. Festlegung in Kristallporen

6. Adsorption in Zwischenschichträumen.

Aus diesem Sachverhalt wird ersichtlich, dass Ton-Huminstoffkomplexe von sehr großer Bedeutung hinsichtlich der chemischen und physikalischen Eigenschaften eines Bodens sind. Die organische Substanz hat großen Einfluss auf die Stabilität des Bodengefüges, sie bewirkt die Kohäsion der Mineralpartikel und trägt zur Aggregierung der Bodenmatrix bei(BUONDONNO 1994, GU \& DONER 1992).

Daraus ergeben sich mehrere Fragestellungen:

- Was sind die Gründe für die umfassende Bindung organischer Substanz an die Tonfraktion?

- Werden organische Substanzen nur adsorptiv und kovalent an Tonminerale gebunden oder erfahren diese auch eine chemische Transformation?

- Welche Rolle spielen Tone somit bei der Genese von Huminstoffen?

- Wie verhalten sich Tonminerale und tonorganische Komplexe gegenüber in den Boden eingebrachter Xenobiotika?

Um einen Beitrag zur Klärung dieser Fragen zu leisten, sind der Aufbau und die Eigenschaften von organischer Substanz sowie der Tonminerale darzustellen.

\subsection{Organische Substanz in Böden}

Die organische Substanz des Bodens ist eine komplexe Mischung aus relativ unzersetzten pflanzlichen und tierischen Rückständen und komplexen polymeren Huminstoffen, gebildet aus mikrobiellen oder chemischen Degradationsprozessen. Die Klassifikation unterscheidet in zwei Gruppen: Nichthumin- und Huminstoffe. Nichthuminstoffe bestehen aus unveränderten Rückständen der Degradation pflanzlicher, tierischer und mikrobieller Rückstände, wie Cellulose, Stärke, Proteine, Chitin und Fette (KÖGEL-KNABNER 1997b). Die Huminstoffe hin- 
gegen sind pigmentierte, hochmolekulare Polyelektrolyte, die durch sekundäre Synthesen umgewandelt wurden (ANDREUX 1982). Sie sind charakterisiert durch hohe Anteile an sauerstoffreichen funktionellen Gruppen (Tabelle 1), wie $\mathrm{COOH}$ von Carbonsäuren, phenolische und enolische $\mathrm{OH}-$ Gruppen, alkoholisches $\mathrm{OH}$ und das $\mathrm{C}=\mathrm{O}$ von Chinonen (GRAUER 1989, STEVENSON 1994). Die Ausgangssubstanzen der Huminstoffe sind außerordentlich zahlreich und die Kombinationsmöglichkeiten sind astronomisch hoch. Es ist daher schlüssig, dass der Aufbau der Huminstoffe standortabhängig ist und das diese Stoffe auch an einem bestimmten Standort keine einheitlichen Verbindungen darstellen. Huminstoffe terrestrischen Ursprungs haben einen verhältnismäßig hohen Anteil an aromatischem Kohlenstoff. Phthalsäure, Salicylsäuregruppen sowie o-Diphenole spielen hier als Liganden eine wichtige Rolle (GRAUER 1989).

Tabelle 1: Vorkommen und Herkunft funktioneller Gruppen in Huminstoffen (nach ZIECHMANN \& MÜLLER-WEGENER 1990).

\begin{tabular}{|c|c|c|}
\hline & funktionelle Gruppe & Herkunft \\
\hline$-\mathrm{OH}$ & Hydroxygruppe & $\begin{array}{c}\text { Phenole (Ligninfragmente), Alkohole (Sei- } \\
\text { tenkette der Coniferylalkohole u.a.) }\end{array}$ \\
\hline$-\mathrm{COOH}$ & Carboxyl-Gruppe & Carbonsäuren (Aminosäuren) \\
\hline$>\mathrm{C}=\mathrm{O}$ & $\begin{array}{c}\text { Carbonyl-Gruppe (zumeist } \\
\text { in Chinonen) }\end{array}$ & Oxidationsprodukte der Phenole \\
\hline$-\mathrm{O}-$ & Ätherbrücken & Kohlenhydrate, Lignine \\
\hline$-\mathrm{OCH}_{3}$ & Methoxyl-Gruppe & Lignine \\
\hline$-\mathrm{NH}_{2}$ & Aminogruppe & (biogene) Amine, Aminosäuren \\
\hline & Heterozyklischer Stickstoff & Heterozyklen z.B. Melanine \\
\hline
\end{tabular}

Aufgrund ihrer Komplexität sind Huminstoffe relativ stabil gegenüber weiterem Abbau. Huminstoffe bestehen aus Alkanen mit $\mathrm{C}_{14}$ bis $\mathrm{C}_{36}$ und Fettsäuren gleichen Kohlenstoffgehalts. Dazu kommen Polysaccharide, Saccharide und Glukoronsäuren. Sie werden weiter unterteilt in Fulvosäuren, Huminsäuren und Humine. Diese drei Fraktionen verfügen über ähnliche Strukturen, unterscheiden sich jedoch in ihrer Molmasse und in ihren funktionellen Gruppen. Die Huminstoffe werden hinsichtlich ihres Löslichkeitsverhaltens in mehrere Fraktionen unterschieden:

- Humine: in kalter Natronlauge unlöslich, schwarz gefärbte Endprodukte des Humifizierungsprozesses, C-Gehalt $>60 \%$. Humine enthalten hochmolekulare aromatische Polymere mit phenolischen und COOH-Gruppen wie bei den Huminsäuren (MORILL 1982).

- Huminsäuren: Huminsäuren enthalten Polysaccharide, Proteine, Phenole und Metalle, die chemisch oder physikalisch an einen komplexen aromatischen Kern gebunden sind (Abbildung 15). Das Grundgerüst bilden Alkylaromate, die kovalent an aliphatische Ketten gebunden sind. Sie werden als ,building blocks“ bezeichnet (SCHULTEN \& SCHNITZER 1997). 


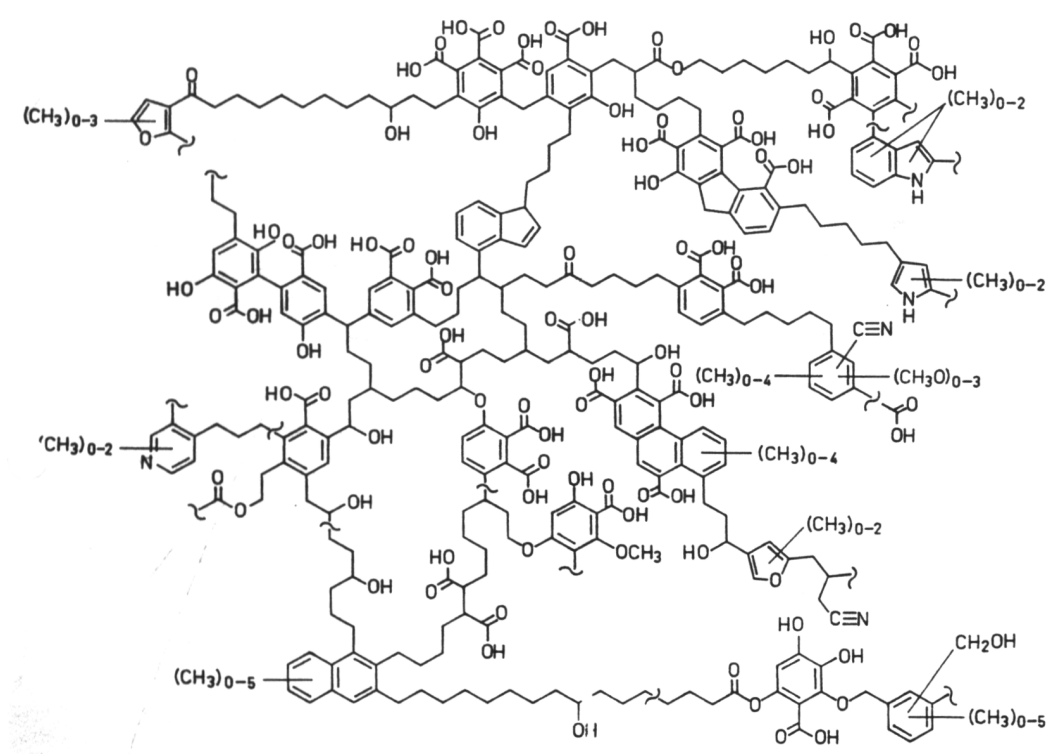

Abbildung 15: Struktur einer Huminsäure (SCHULTEN \& SCHNITZER 1997).

Huminsäuremolekülen wird eine Struktur polymerer Micellen mit zwei- oder dreiwertigen Phenolen und sich wiederholenden Einheiten verbunden über -O-, -NH-, -Nund -S-Bindungen sowie stabile OH-Gruppen und Chinonbindungen nachgesagt. Durch Pyrolyse-Massenspektrometrie konnte gezeigt werden, dass Kohlehydrate, Phenole, Ligninmonomere, Lignindimere, Lipide, Alkylaromate und N-enthaltende Verbindungen die Hauptkomponenten der Huminsäure sind (SCHULTEN \& SCHNITZER 1997). Huminsäuren enthalten zudem stabile organische Radikale (bis zu $10^{18}$ Radikale/g). Sie sind alkalilöslich, säurefällbar; hoher Aromatenanteil, sie werden weiter unterschieden in: Die molaren Massen von Fulvosäuren liegen im Bereich von 500 bis 2000 Dalton (Da). Huminsäuren erreichen molare Massen bis 100.000 Da.

Braunhuminsäuren: koagulieren nicht in alkalischer Lösung in Gegenwart eines Elektrolyten, tiefbraune Farbe, C-Gehalt 50-60 \%.

Grauhuminsäuren: Koagulation in Anwesenheit eines Elektrolyts, grauschwarz, C-Gehalt zwischen 58 und $62 \%$.

Hymatomelansäuren: alkalilöslich, fällbar in Säure, löslich in Alkohol, braune Farbe, CGehalt 58-62\%.

- Fulvosäuren: alkalilöslich, nicht säurefällbar, höherer Polysaccharidgehalt, gelbgelbbraun gefärbt, Molekulargewicht 800-9000, C-Gehalt 43-52 \%. Fulvosäuren sind relativ niedrig-molekulares Material mit einem erhöhten Anteil Sauerstoff enthaltender funktioneller Gruppen als Huminsäuren. Fulvosäuren bestehen vorwiegend aus phenolischen und Benzolcarboxylsäure-Gruppen (Abbildung 16), die über kovalente und $\mathrm{H}$ Brücken-Bindungen zu stabilen polymeren Molekülen verbunden sind (MORILL 1982). 


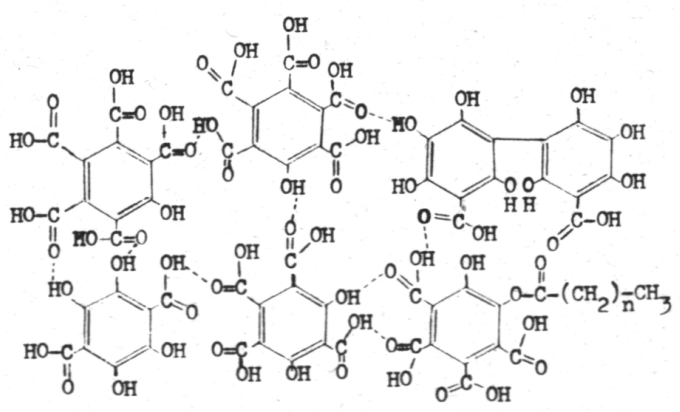

Abbildung 16: Vereinfachte Struktur einer Fulvosäure (MORILL 1982).

\subsubsection{Die Genese von Huminstoffen}

Huminstoffe stammen primär von höheren Pflanzen und Bodenorganismen ab. Die Kernstruktur der Huminstoffe besteht aus oxidiertem Lignin (BOLLAG et al. 1998, SHEVCHENKO et al. 1999). Sie setzen sich aus Teilen von Abbauprodukten von Pflanzenbestandteilen, Autolyseprodukten der Mikroorganismenzellen und von extrazellulären Metaboliten der von Mikroorganismen zersetzten organischen Verbindungen (Zucker, Aminosäuren, Phenole etc.) zusammen. Diese unterliegen sowohl enzymatischer Oxidation, Autoxidation und nicht-enzymatischer katalytischer Oxidation (HAYES 1991). Letztere führt laut WANG et al. (1983) zur Bildung von Huminstoffen.

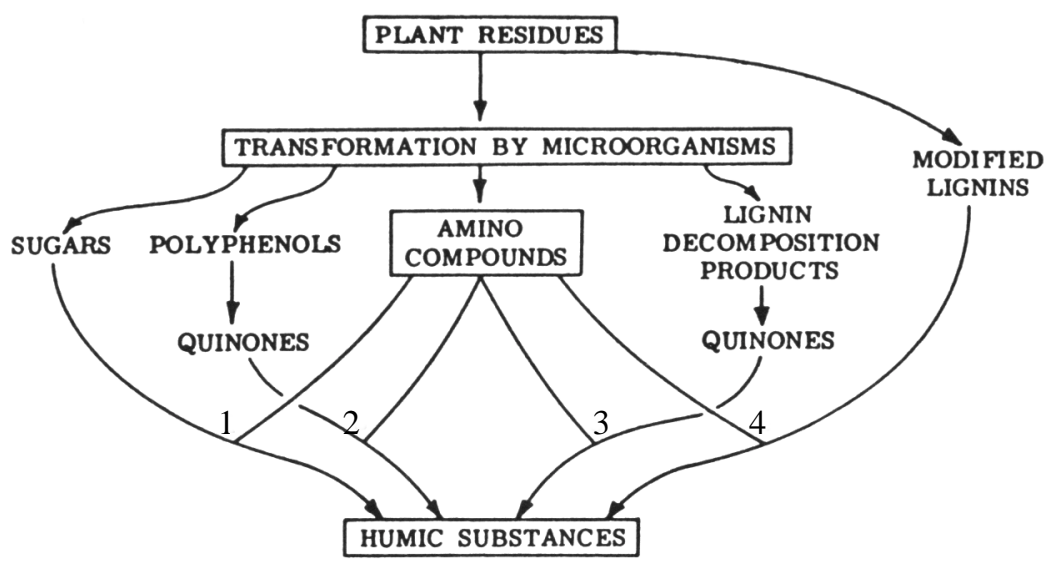

Abbildung 17: Schema der Bildung von Huminstoffen im Boden (KEREN \& SPARKS 1995).

In Abbildung 17 werden die verschiedenen Reaktionswege zur Bildung von Huminstoffen durch mikrobiellen Abbau pflanzlicher Rückstände über die Freisetzung von Teilabbauprodukten bis hin zur Neubildung organischer Substanz (KEREN \& SPARKS 1995) dargestellt. Der Gehalt an aromatischen Strukturen in Huminstoffen wird in älteren Quellen mit 50-80 \% angegeben, FILIP et al. (1981) gehen von einer Zusammensetzung der Huminsäuren zu $45 \%$ aus humusähnlichen Melaninen und zu mindestens $20 \%$ aus verschiedenen Phenolen aus. Neuere Arbeiten hingegen erachten jedoch einen Gehalt von $25 \%$ als Obergrenze (SCHUMACHER 1995). Zum Prozess der Genese von Huminstoffen existieren mehrere 
Theorien. Die „klassische“ Theorie von WAKSMAN (1932) besagt, dass Huminstoffe aus modifizierten Ligninen gebildet werden (Abbildung 17, Reaktionsweg 4). Lignine setzen sich aus Phenylpropan-Einheiten zusammen (Abbildung 18):

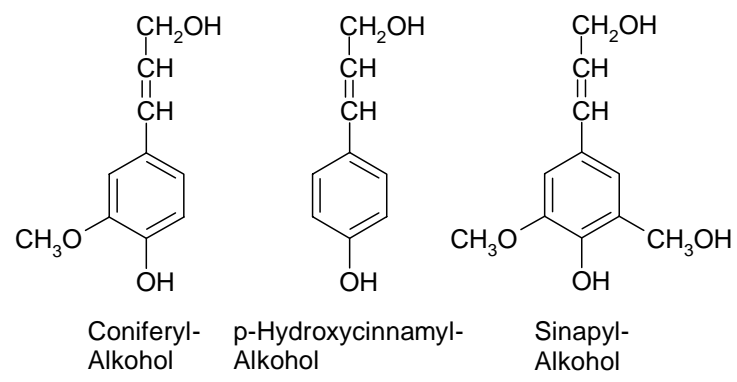

Abbildung 18: Phenylpropan-Bausteine der Lignine (nach STEVENSON 1994).

Die Umwandlung von Ligninen in Huminstoffe ist charakterisiert durch den Verlust der Lignine an Methylgruppen (-OCH 3$)$. Nach SANGER et al. (1997) werden die zuvor abgeschirmten phenolischen $\mathrm{OH}-\mathrm{Gruppen}$ zugänglich und eine Oxidation zu Carboxylgruppen $(\mathrm{COOH})$ tritt ein (Abbildung 19).

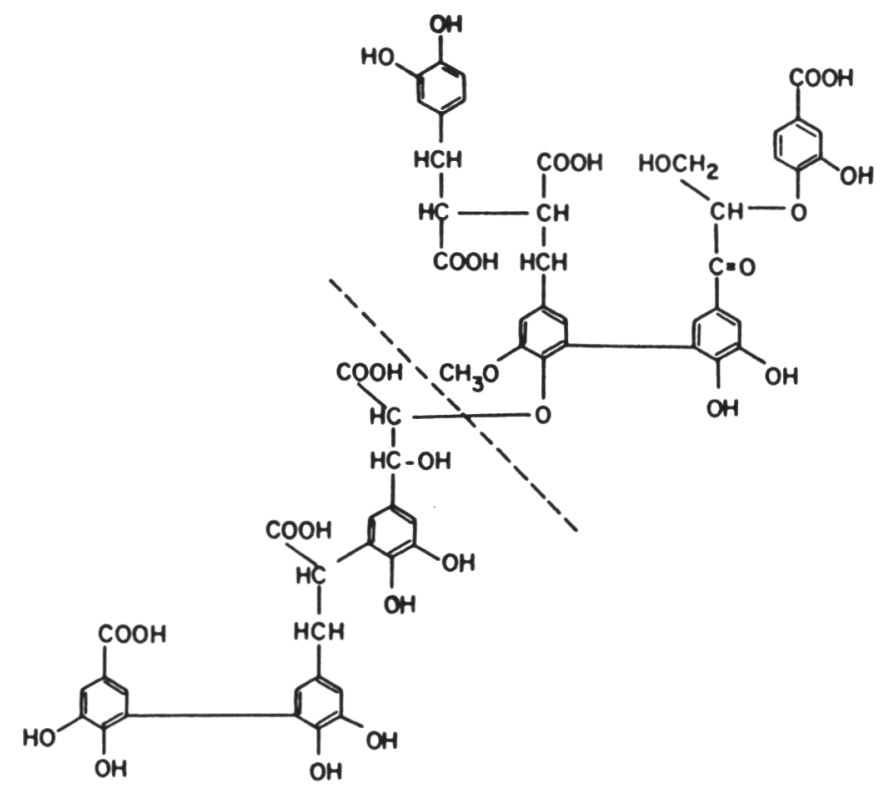

Abbildung 19: Modifikation des Lignins durch Demethylierung und Oxidation (STEVENSON 1994).

Die bei der Demethylierung des Lignins entstandenen o-Dihydroxybenzole unterliegen einer Oxidation zu Chinonen. Diese wiederum gehen Kondensationsreaktionen beispielsweise mit weiteren Chinonen oder Aminoverbindungen ein.

\subsubsection{Polyphenol-Theorie}

Die zur Zeit weitverbreitete und anerkannte Theorie zur Genese von Huminstoffen ist die Polyphenol-Theorie (Abbildung 17, Reaktionsweg 2/3). Sie beschreibt die Genese von $\mathrm{Hu}$ minstoffen durch zwei Reaktionspfade. Zum einen werden phenolische Aldehyde und Säuren aus Ligninen während des mikrobiellen Angriffs freigesetzt und durch Enzyme, beispielswei- 
se Phenoloxidasen ligninabbauender Pilze, zu Chinonen oxidiert. Diese bilden sowohl in Gegenwart als auch in Abwesenheit von Aminoverbindungen huminstoffähnliche Makromoleküle. Als zweiter Reaktionsweg wird angenommen, dass Polyphenole aus anderen Kohlenstoffquellen (z.B. Zellulose) durch Mikroorganismen enzymatisch zu Chinonen oxidiert und in Huminstoffe umgeformt werden (Abbildung 20). Das Ausgangssubstrat der PolyphenolTheorie besteht aus niedermolekularen organischen Verbindungen, die durch Kondensation und Polymerisation zu großen höhermolekularen Verbindungen reagieren (BOLLAG et al. 1998).

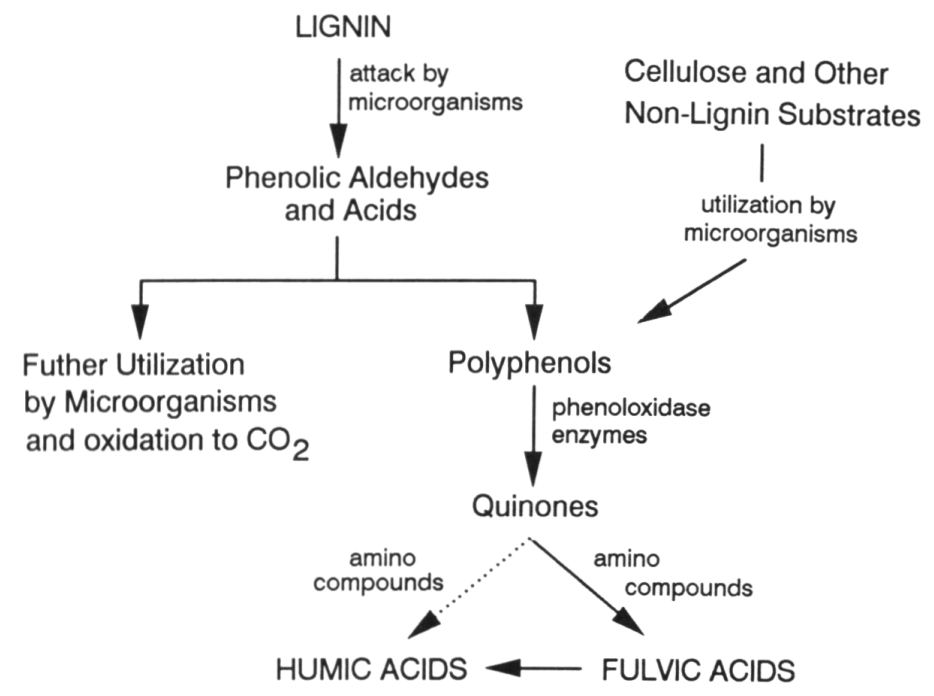

Abbildung 20: Polyphenol-Theorie (STEVENSON 1994).

Der Humifizierungsprozess gliedert sich nach ZIECHMANN \& MÜLLER-WEGENER (1990) in mehrere Phasen (Abbildung 21):

1. metabolische Phase: partieller mikrobieller Abbau hoch- und höhermolekularer Substanzen führt zu humifizierbarem Material,

2. Radikalphase: In aromatischen Strukturen vollzieht sich die Bildung von Radikalen, eingeleitet durch elektrophilen Angriff eines Elektronenakzeptors (LOVLEY et al. 1996). Am Beispiel des Hydrochinons verdeutlicht: der erste Reaktionsschritt ist die Ionisation des Phenols (1), gefolgt von elektrophilem Angriff (2) durch folgende Elektronenübergänge:

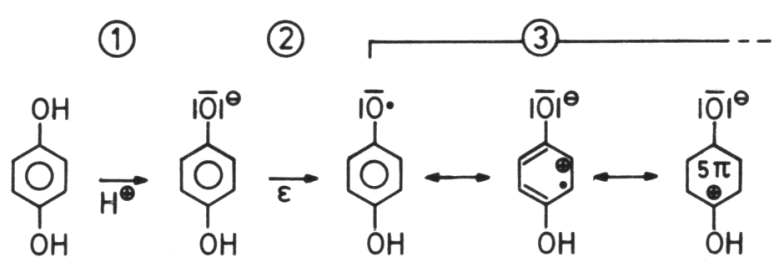

Abbildung 21: Oxidation von Hydrochinon (ZIECHMANN \& MÜLLER-WEGENER 1990).

3. Im letzten Schritt kommt es zur Bildung mesomerer O- und C-Radikale des Phenols. Abschließend erfolgt die Aufnahme nicht-aromatischer Ausgangsstoffe in die $\mathrm{Hu}$ - 
minstoffmatrix durch Umsetzung von Nichthuminstoffen mit den reaktionsfähigen Huminsäurevorstufen.

Organische Verbindungen wie beispielsweise Phenole, Kohlehydrate, Aminosäuren, Amine, Peptide und Enzyme setzen sich zu stabilen Produkten um. Durch die Reaktivität der Radikale kommt es zur Ausbildung neuer kovalenter Bindungen: C-C-Bindungen durch Reaktion zweier C-Radikale oder C-O-C-Bindungen durch Umsatz von C- mit O-Radikalen. In der letzten Phase kommt es zur Ausbildung eines Huminstoffsystems durch intermolekulare Wechselwirkungen zu Ladungsübertragungs- oder Elektronen-Donator-Akzeptor-Komplexen, welche zu mesomerie-stabilisierten Zuständen der organischen Substanz führen. Die Radikale werden durch Vermehrung der mesomeren Grenzzustände als Folge der Teilchenvergrößerung der humifizierten Substanzen stabilisiert und somit reaktionsträger (ZIECHMANN \& MÜLLER-WEGENER 1990). Primäre Ausgangsstoffe sind aromatische, leicht in Radikale überführbare Verbindungen. Zahlreiche mono-, di- und trihydroxy-substituierte Phenole, wie Brenzkatechin, Hydrochinon und Pyrogallol (FLAIG 1988), stellen Bausteine für die Synthese zu Huminstoffen dar. Deren Oxidation resultiert in der Bildung von Chinonen. Diese können miteinander oder mit anderen organischen Molekülen (Aminosäuren, Amine, Zucker etc.) zu Polymeren kondensieren. Es kommt zur Entstehung polymerer, braun bis schwarz gefärbter Produkte, die chemische Grundeigenschaften aufweisen, die denen natürlicher Huminstoffe gleichen.. Diese Reaktionen gelten als erste Schritte der Humifizierung (SHINDO \& HUANG. 1995a,b, ZIECHMANN \& MÜLLER-WEGENER 1990). Aus diesem Grunde werden Phenole auch als Huminsäure-Vorstufen bezeichnet (WANG \& WU 1977, WANG et al. 1983, WANG 1986, HASSELMANN 1994).

Im Rahmen dieser Arbeit wurden drei Phenole als Untersuchungsobjekte von Modellsubstanzen für Huminsäure-Vorstufen ausgewählt: Pyrogallol, 2,6-Dimethylphenol und Brenzkatechin. Letzteres stellt auch ein Degradationsprodukt des mikrobiellen Abbaus des Polyzyklischen Aromatischen Kohlenwasserstoffs Phenanthren dar. Die biotische Degradation dieses PAK ist durch zahlreiche Untersuchungen und Veröffentlichungen nachgewiesen und detailliert beschrieben (Kapitel 2.2.7.1). Aus diesen Gründen bietet sich das Phenanthren ebenfalls auf eine mögliche chemische Transformation durch abiotische Katalyse an Tonmineralen hin zu untersuchen.

\subsubsection{Eigenschaften von Phenolen}

Phenole sind durch Hydroxylgruppen subsituierte Derivate des Benzols. Sie stellen organische Säuren mit hoher Wasserlöslichkeit und niedrigem Dampfdruck dar (DUNNIVANT \& SCHWARZENBACH 1992). Ihre relativ hohe Reaktivität führt leicht zur Oxidation und photolytischer Transformation. Phenolische Verbindungen besitzen Schadstoffcharakter. Unter den 129 Chemikalien, die nach der U.S.-Umweltschutzbehörde (Environmental Protection Agency) die in der Umwelt auftretenden Hauptschadstoffe darstellen, sind 11 phenolische Verbindungen aufgelistet (KOWALSKA et al. 1994). Phenole sind ab Konzentrationen von 1 
$\mathrm{mg}^{-1}$ toxisch gegenüber wasserlebenden Organismen (HANCOCK \& DEAN 1997). Ihre toxische Wirkung auf den menschlichen Organismus liegt in der Depression der Funktionen des zentralen Nervensystems sowie den blut- und leberschädigenden Eigenschaften als Protoplasmagift. Die Aufnahme von $1 \mathrm{~g}$ Phenol wirkt bei Menschen tödlich (KOCH 1995).

\subsubsection{Anthropogene Quellen}

Phenole gelangen über zahlreiche Quellen in die Umwelt. Anthropogene Quellen sind die chemische Industrie, für die USA und Westeuropa wurden die Produktionsmengen 1989 auf jährlich 2,8 Millionen Tonnen geschätzt, allein die in der Bundesrepublik produzierte Menge betrug im Jahr 1974270.000 t. Von der Gesamtproduktion werden 60 \% zu Kunststoffen und Weichmachern verarbeitet (KOCH 1995). Ein geschätzter Anteil von 2,5 \% der Gesamtmenge gelangt in die Umwelt (BLUME 1992). Anwendung finden Phenole als Kresole in der Herstellung von: Harzen, Lösungsmitteln und Desinfektionsmitteln, von denen $60 \%$ Phenole als wirksame Komponente enthalten. Hydrochinon wird in der Filmentwicklung, zur Herstellung von Antioxidationsmitteln, Polymerisationshemmern, Stabilisatoren, in der Gummiverarbeitung und in der Papierindustrie verwendet. Als Styrole kommen sie in der Kunststoffproduktion zur Anwendung (BLUME 1992). Phenole werden in der Herstellung synthetischer Farbstoffe verwendet und sind Bestandteil von Schmierölen. Weitere anthropogene Emissionsquellen sind die Verdunstung und unvollständige Verbrennung von Treibstoffen, Emissionen aus Steinkohlenteer und industriellen Abwässern sowie die Ausbringung von Pflanzenschutzmitteln (Insektizide, Herbizide) und die Anwendung von Holzschutzanstrichen (Chlorphenole). Diese werden im Boden nur mäßig gebunden, sie bilden mit Tonmineralen über H-Brücken Polymere. Durch ihre Dechlorierung entstehen weniger toxische Polymerisate (BLUME 1992).

\subsubsection{Natürliche Quellen}

Phenole und ihre Ether sind in der Natur weitverbreitet. Neben anthropogenen Emissionen werden Phenole auch durch natürliche Quellen freigesetzt. Durch den mikrobiellen Abbau tierischer und pflanzlicher Biomasse gelangt ein breites Spektrum phenolischer Verbindungen in die Umwelt (BLUME 1992). Phenole kommen natürlich in Oberflächen- und Grundwässern als Degradationsprodukte des Lignins sowie als Algenausscheidungen vor. Phenolische Verbindungen sind vor allem Bestandteile hochmolekularer Lignine. Als Hauptbestandteile der Pflanzenmasse fallen Lignine sowohl im Wald als auch als Ernterückstand auf Ackerflächen in großen Mengen an und werden durch Mikroorganismen wie Basidiomyceten, Ascomyceten und Bakterien in niedermolekulare phenolische Spaltprodukte zerlegt (ZIECHMANN \& MÜLLER-WEGENER 1990). Ebenso fungieren Tannine, die aus polykondensierten Phenolen bestehen, bei ihrem biotischen Abbau als Quellen für Phenole (Van OLPHEN 1991). Insbesondere Derivate 2- und 3-wertiger Phenole können natürlichen Ursprungs sein, sie sind Bestandteil natürlicher Stoffkreisläufe. Katechole, charakteristisch für viele vaskuläre Pflanzen und Pilze, treten in der Umwelt als phenolische Methoxyleinheiten des Lignins sowie 
durch Hydroxylierung der Orthoposition von einfacheren Phenolen durch Phenoloxidasen auf (LARSON \& HUFNAL 1980). Phenole sind primäre Komponenten der Huminstoffe (POSPISIL 1994). Als diese spielen sie eine wichtige Rolle bei der Bildung von Huminstoffen (Humifizierung).

\subsubsection{Verhalten und Reaktionsprozesse von Phenolen im Boden}

Bereits in den 70er Jahren wurde durch KUNC \& STOTZKY (1977) der Abbau von Ligninen, die Spaltung aromatischer Strukturen, der reaktionsbeschleunigende Abbau von Aldehyden (Oxidation der Aldehydgruppe zur Carboxylform) in Gegenwart von Montmorillonit beobachtet und damals einem Anstieg der mikrobiellen Aktivität durch Beeinflussung des pHWertes, der Pufferkapazität des Bodens und der Veränderung des Kationenverhältnisses im Boden zugeschrieben. HANCOCK \& DEAN (1997) beobachteten in Bodenproben nach Zusatz von Phenolen eine Zunahme der Aromatizität. Phenole zeigen bei Adsorption an Tonen ihren amphoteren Charakter. Sie können Protonen abgeben oder annehmen. Folgende Konfigurationen der phenolischen Gruppe im Zwischenschichtraum der Tonminerale treten auf (Abbildung 22).

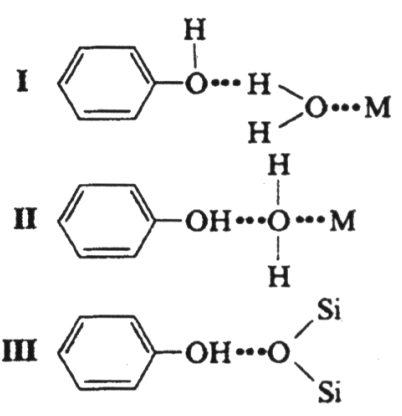

Abbildung 22: Adsorption von Phenolen an Tonmineralen (YARIV 1996).

Neben der Entstehung von Huminstoffen durch biologische Prozesse wird nun auch deren Bildung durch heterogene katalytische Effekte von anorganischen Bodenkomponenten vermutet. Die gesteigerte Huminstoffbildung in Anwesenheit von Tonmineralen kann als Effekt auf den mikrobiellen Metabolismus oder auf einer direkten katalytischen Wirkung der Tonminerale basieren. Eine radikalisch initiierte oxidative Polymerisation von Phenolen zu polyzyklischen Dimeren und Trimeren wurde bei mehreren natürlichen Tonen beobachtet (PAL \& PAL 1996). Für xenobiotische Phenole ergaben Modellversuche, dass sie kovalente Bindungen mit natürlichen phenolischen Huminstoff-Vorläufern im Boden eingehen und bei der Polymerisation mit in das Huminstoff-Makromolekül inkorporiert werden (SCHEUNERT 1999, DEC \& BOLLAG 2000, PARK et al. 2000). Die oxidative Polymerisation von Phenolen in Böden ist ein bedeutender Prozess der Bildung von Huminstoffen (WANG \& HUANG 2000). Die Polymerisation kann neben enzyminduzierter Katalyse auch durch anorganische Katalysatoren herbeigeführt werden. Weder die Reaktionsmechanismen, noch die Natur der Reaktionsprodukte der durch mineralische Oberflächen sowie Enzyme katalysierten Polyphenoltransformation sind vollständig verstanden (NAIDJA et al. 1998). 
Der in der Literatur häufig beschriebene „browning effect“ von Tonmineralen auf eine Lösung von Pyrogallol wurde oft als Beispiel für die abiotische Synthese von Huminstoffen (Humifizierung) an anorganischen Oberflächen beschrieben (WANG 1983). Die Untersuchung der Struktur der so gebildeten Huminsäuren zeigte, dass deren geringe Kristallinität als Beweis für eine nicht-enzymatische, durch heterogene chemische Katalyse geförderte Entstehung anzusehen ist (WANG et al. 1983). Heteronukleare aromatische Polymere werden durch Kopplung und subsequente Oxidation von Radikalkationen, die durch eine Elektronenoxidation des aromatischen Rings entstehen, gebildet. Wenn die Oligomere redoxaktiv sind können sie oxidiert werden und weiter aneinander koppeln (FAGUY et al. 1995). Die durch Metalloxide bzw. Tonminerale oxidierte Substanz kann in der Entstehung freier Radikale oder Phenoxonium-Ionen resultieren. Beide Spezies können kovalente Bindungen mit den Huminstoffen des Bodens durch eine oxidative Kopplungsreaktion eingehen (HANCOCK \& DEAN 1997). Die Bildung von Charge-Transfer-Komplexen und Radikalionen ist auch in natürlichen Böden durch Phenole und aromatische Amine zu beobachten. $\mathrm{Fe}^{3+}$-Ionen fungieren als Akzeptorstellen für Elektronen. Durch Huminstoffe kann die Reduktion von Fe(III) stimuliert werden, das in die Struktur von Tonmineralen und in kristalline Formen der Fe(III)-oxide eingebaut ist (LOVLEY et al. 1998). Die Bildung von Radikalionen wurde durch ESRSpektroskopie nachgewiesen (KLÖPFFER 1996, PARK et al. 2000).

\subsubsection{Oxidation und Transformation von Phenolen durch Radikale}

Oxidative Kopplung von Phenolen untereinander erfolgt durch biotische oder abiotische Prozesse (BOLLAG et al. 1998). Phenole wie Brenzkatechin sind gegenüber abiotischen Abbauwegen sehr empfänglich. In alkalischem Milieu werden Katechole kovalent an oberflächliche Silanolgruppen der Tonminerale in Form von zyklischen Siloxanen gebunden. Von einem in der Silikatstruktur inkorporierten Kation ( $\mathrm{Zn}, \mathrm{Al}, \mathrm{Fe}, \mathrm{Cu}$ ) kann jeweils ein Elektron zu an der Tonmineraloberfläche adsorbierten Sauerstoffmolekülen transferiert werden (WANG et al. 1983) und zur Bildung von Radikalen mit nachfolgender Polymerisation führen. In einem ersten Schritt werden organische Verbindungen zu instabilen freien Radikalen oxidiert. Diese reagieren mit anderen Molekülen zu radikalischen Intermediären, die dann weiter reagieren können. Dadurch erfolgt eine Bildung von Huminstoffvorläufern sowie eine direkte Reaktion mit natürlicher, im Boden enthaltener organischer Substanz (BOLLAG et al. 1992). Radikale entstehen auch, wenn Phenole an Tonmineraloberflächen adsorbiert werden (CORNELIS \& LAZLO 1986). Durch Verunreinigungen des Tons mit bereits Radikale enthaltender organischer Substanz, Übergangsmetallkationen in der Kristallstruktur bzw. Zwischenschichten und durch Hydroperoxylradikale $(\bullet \mathrm{OOH})$ können Radikale an den Tonoberflächen gebildet werden. Hydroxylradikale $(\bullet \mathrm{OH})$ als freie Radikale treten bei oxidativer Transformation von Phenolen mit Nicht-Übergangsmetall-Kationen auf (SAWHNEY et al. 1984). Die oxidative Polymerisation von Phenolen durch Tonminerale wird durch zwei Mechanismen hervorgerufen: 
1. durch die Reaktion von Phenolen mit sauerstoffenthaltenden Radikalen, die an Tonmineraloberflächen entstanden sein können und

2. durch die Reaktion von Phenolen mit sauerstoffenthaltenden Radikalen, die an Tonmineraloberflächen entstanden sein können und durch die Bildung von Radikalkationen aromatischer Moleküle in Anwesenheit von mit bestimmten Übergangsmetallionen belegten Austauscherplätzen der Tonminerale.

Fe und Al als Spurenelemente in Schichtsilikaten sowie an der Oberfläche von Tonmineralen chemisorbierter Sauerstoff sind Katalysatoren der frei-radikalischen Oxidation (Abbildung 23). Diese wird als Entfernung von Elektronen einer chemischen Spezies definiert, sie tritt bei organischen Verbindungen in zwei Varianten auf:

- heterolytische oder polare Reaktion: ein elektrophiles Agens attackiert organische Moleküle und trennt dabei ein Elektronenpaar ab,

- homolytische oder frei-radikalische Oxidation: ein Agens trennt nur ein Elektron von organischen Verbindungen ab (DRAGUN \& HELLING 1985).

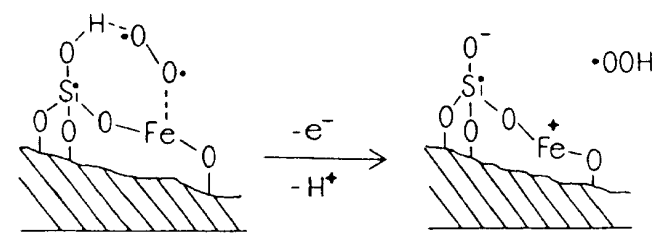

Abbildung 23: Bildung von Radikalen (VOUDRIAS \& REINHARD 1986).

Zum Erhalt der Ladungsneutralität wird von einer oberflächlichen Silanolgruppe ein Proton abgegeben, gefolgt von Rehydrierung oder Rearrangement der Oberfläche (LARSON \& HUFNAL 1980). Bei der von der Tonmineraloberfläche freigesetzten Sauerstoffspezies handelt es sich um Hydroxyperoxyl-Radikale $(\bullet \mathrm{OOH})$. In wässrigem Milieu treten zusätzlich Superoxid-Radikalanionen $\left(\mathrm{O}_{2}^{-}\right)$auf. Beide Radikalarten setzen sich in aquatischer Umgebung zu $\mathrm{O}_{2}$ und $\mathrm{H}_{2} \mathrm{O}_{2}$ um und attackieren adsorbierte oder in Lösung befindliche Katechole. In Gegenwart von leicht oxidierbaren oder reduzierbaren Kationen $\left(\mathrm{Cu}^{2+}, \mathrm{Fe}^{3+}\right.$ etc. $)$ wird das $\mathrm{H}_{2} \mathrm{O}_{2}$ katalytisch unter der Bildung von Hydroxy- und Hydroxyperoxyl-Radikalen zersetzt:

$$
\mathrm{H}_{2} \mathrm{O}_{2}+\mathrm{M}^{\mathrm{n}+} \rightarrow \cdot \mathrm{OH}+\mathrm{HO}^{-}+\mathrm{M}^{\mathrm{n}+1} \mathrm{H}_{2} \mathrm{O}_{2}+\mathrm{M}^{\mathrm{n}+1} \rightarrow \cdot \mathrm{OOH}+\mathrm{H}^{+}+\mathrm{M}^{\mathrm{n}+} \text {. }
$$

Daneben können aus Hydroperoxylradikalen auch Hydroxylradikale $(\bullet \mathrm{OH})$ entstehen (ISAACSON 1983). Hydroxylradikale gehören mit zu den stärksten Oxidantien und sind in der Lage, die meisten natürlichen organischen Spezies zu attackieren, indem sie an Phenole addieren oder H-Atome abspalten und so phenolische Radikale bilden (Abbildung 24). Diese können anschließend zu Polymeren koppeln (McBRIDE 1994).

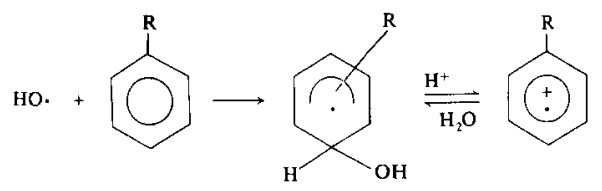


In Abwesenheit elektrophiler Substituenten reagieren phenolische OH-Gruppen wie schwache Basen, mit steigendem pH-Wert dissoziieren sie zu Anionen. Bei der Oxidation werden die Anionen in Semichinone mit O- und C-Radikalen umgewandelt. Der Sauerstoff fungiert als Elektronenakzeptor, und es kommt zur Bildung mesomerer Radikale des Hydrochinons (Abbildung 25).

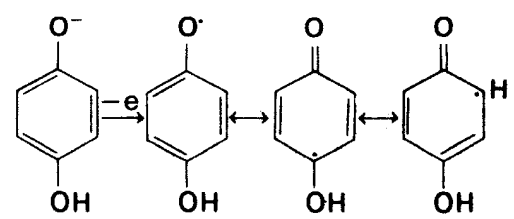

Abbildung 25: Radikalbildung bei Hydrochinon (WANG et al. 1983).

Das an der Tonmineraloberfläche adsorbierte $\mathrm{O}_{2}$ führt über den nachfolgenden Elektronentransfer zur Ausbildung von Radikalen:

$$
\begin{gathered}
\mathrm{O}_{2}+2 \mathrm{e}^{-} \rightarrow 2 \mathrm{O}^{-} \\
\mathrm{O}_{2}+4 \mathrm{e}^{-} \rightarrow 2 \mathrm{O}^{2-} \bullet .
\end{gathered}
$$

Die folgenden Abbildungen (26 und 27) zeigen den Ablauf der Transformation von Hydrochinon zu p-Benzochinon an Tonmineralen. Die Anwesenheit von Sauerstoff führt zur Bildung von Hydroxylradikalen und zur Oxidation des Hydochinons zu p-Benzochinon (Abbildung 26).

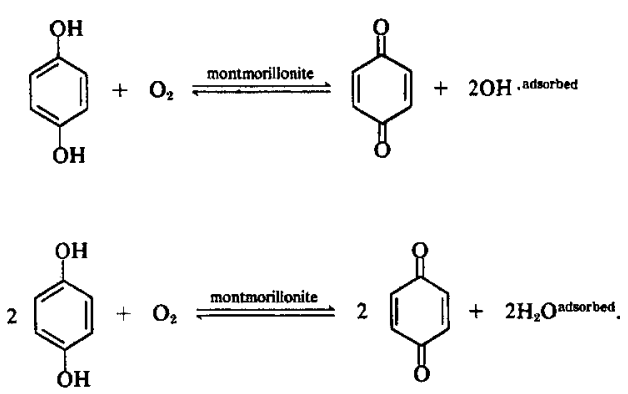

Abbildung 26: Oxidation von Hydrochinon zu p-Benzochinon (THOMPSON \& MOLL 1973).

Diese Reaktion läuft bevorzugt in feuchtem Milieu ab, während der in der folgenden Abbildung dargestellte Reaktionsmechanismus primär an trockenen Tonmineraloberflächen unter Mitwirkung von Austauschkationen (Abbildung 27) Hydrochinon zu p-Benzochinon durch Hydroxylradikale oxidiert (THOMPSON \&MOLL 1973).

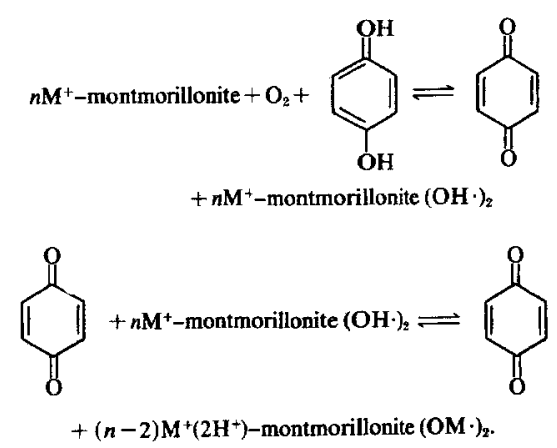

Abbildung 27: Oxidation an trockenem Montmorillonit (THOMPSON \& MOLL 1973). 
Nach DRAGUN \& HELLING (1985) kann die Bildung von Radikalkationen an den Oberflächen von mit Übergangsmetallen gesättigten Smektiten, durch Elektronentransfer von aromatischen Spezies zu Übergangsmetallkationen $\left(\mathrm{Cu}^{2+}, \mathrm{Fe}^{3+}\right)$ erfolgen. Auf diese Weise wurde die Polymerisation von Phenolen bis hin bis zu Tetrameren beobachtet (ISAACSON 1983). Die frei-radikalische Oxidation besteht häufig aus zwei oder mehr Schritten; im ersten Schritt erfolgt die reversible Bildung eines freien Radikals durch Entfernung eines einzelnen Elektrons aus einem Molekül. Dies wird ausgelöst durch Dissoziation von Molekülen mit schwachen kovalenten Bindungen oder durch Transfer von Elektronen von Molekülen zu Übergangsmetallen mit unvollständig besetzten Orbitalen (Fe, Al). Die meisten freien Radikale sind hoch reaktiv und reagieren mit der nächstmöglich verfügbaren Spezies (DRAGUN \& HELLING 1985). Bei niedriger Gesamtkonzentration der Radikale reagieren sie dann zumeist mit organischen Molekülen (Abbildung 28).

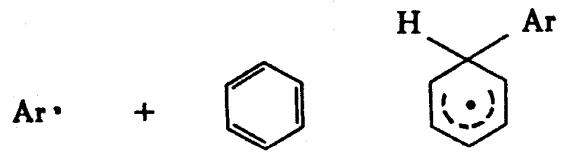

Abbildung 28: Reaktion von freien Radikalen mit Aromaten (DRAGUN \& HELLING 1985).

Bei diesem Schritt attackiert das aromatische Radikal (Ar•). Aufgrund von Resonanzeffekten ist das Zwischenprodukt relativ stabil, es kann mit einem anderen organischen Molekül eine höhermolekulare, aber chemisch sehr ähnliche Spezies bilden. Die Wiederholung einer solchen Reaktion führt über die Bildung von Oligomeren hin zu Polymeren (Abbildung 29).

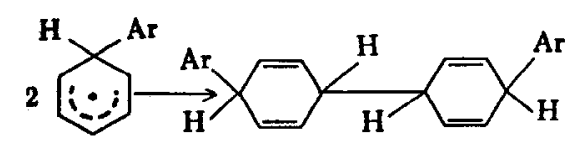

Abbildung 29: Polymerisation von aromatischen Radikalen (DRAGUN \& HELLING 1985).

Als abschließender Reaktionsschritt der frei-radikalischen Oxidation (Abbildung 30) erfolgt die Zerstörung des freien Radikals durch einfache Kopplung, durch Disproportionierung oder Abstrahierung in Anwesenheit einer Spezies die Wasserstoff abspalten kann.

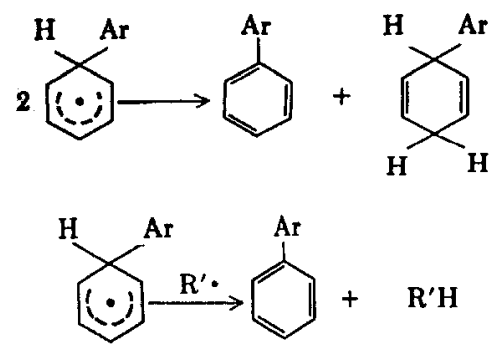

Abbildung 30: Zerstörung von freien Radikalen (DRAGUN \& HELLING 1985).

Die folgende Abbildung (31) zeigt den Reaktionsmechanismus der radikalischen Oxidation und Polymerisation von Phenolen durch Elektronentransfer am Beispiel eines mit $\mathrm{Cu}-$ 
Kationen in den Zwischenschichten belegten Tonminerals. Durch Elektronenübergänge vom adsorbierten organischen Molekül zum Tonmineral entsteht aus diesem ein Radikal, dessen genaue Struktur durch Mesomerie nicht eindeutig festzulegen ist. Dieses Radikal reagiert nun unter Protonenabgabe mit weiteren adsorbierten Phenolen zu höhermolekularen Reaktionsprodukten (VOUDRIAS \& REINHARD 1986).

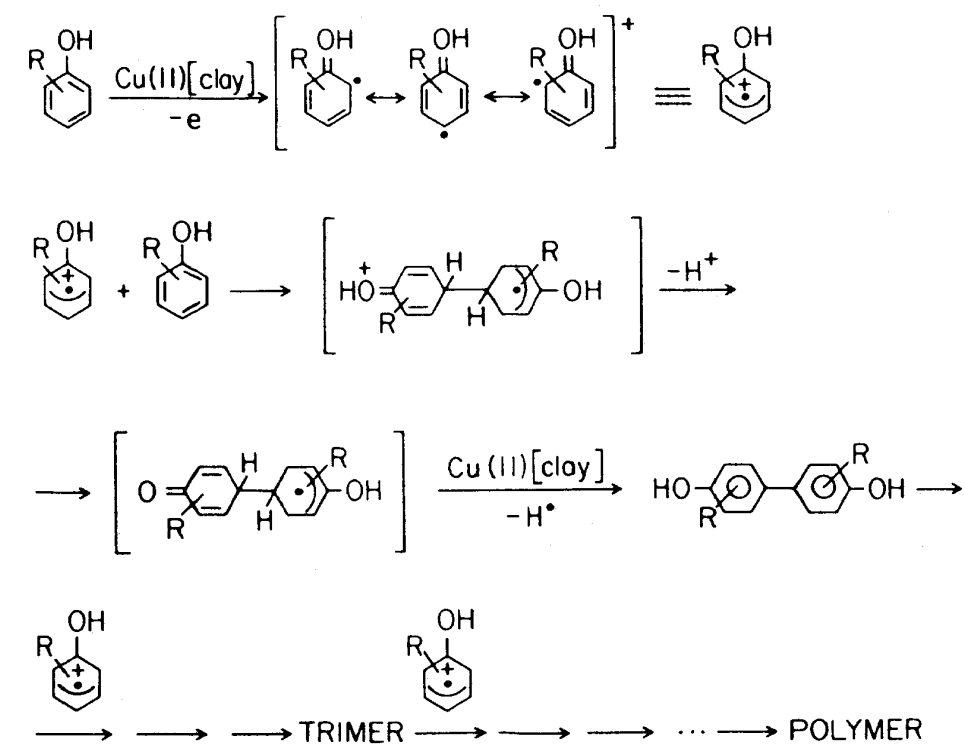

Abbildung 31 : Radikalischer Reaktionsmechanismus (VOUDRIAS \& REINHARD 1986).

\subsubsection{Brenzkatechin und Pyrogallol}

Brenzkatechin und Pyrogallol stammen aus der Degradation von Ligninen und stellen charakteristische Modellhuminsäuren dar (FLAIG 1988, WANG \& HUANG 2000). Die Bildung phenolischer Hydroxylgruppen ist ein Schritt, der zum Abbau aromatischer Anteile im Ligninmolekül, zu aliphatischen Verbindungen als auch zu Möglichkeiten der Bildung höhermolekularer Anteile durch oxidative Polymerisation führen kann (BOLLAG et al. 1998). Die Oxidation von Phenolen verläuft zu den entsprechenden Oxidationsprodukten, den Chinonen. Für das Brenzkatechin (1,2-Dihydroxyphenol) ergeben sich dabei folgende Strukturen (Abbildung 32).

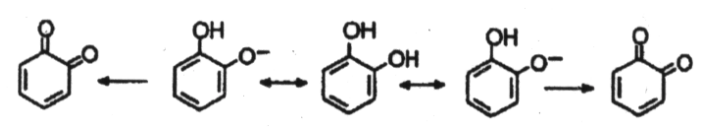

Abbildung 32: Oxidation des Brenzkatechins zum o-Benzochinon (PARK et al. 1999).

Das o-Benzochinon kann über H-Brücken an ein weiteres Chinon koppeln bzw. es kommt bei Zuführung von $\mathrm{OH}^{-}$-Ionen zur Verschiebung des Gleichgewichts in Richtung der Bildung eines aromatischen Radikals (Abbildung 33). 


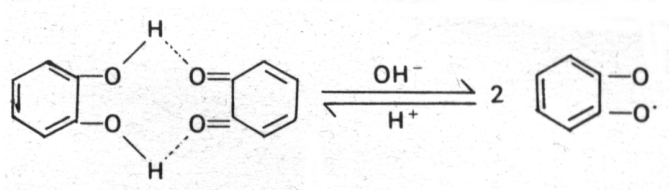

Abbildung 33: Oxidationsprodukte des Brenzkatechins (CHOUDRY 1983).

NAIDJA et al. (1998) beschreiben nach enzymatischer Katalyse des Brenzkatechins mit Tyrosinase bzw. der Oxidation mittels Birnessit die folgenden Oxidationsprodukte (Abbildung $34)$.

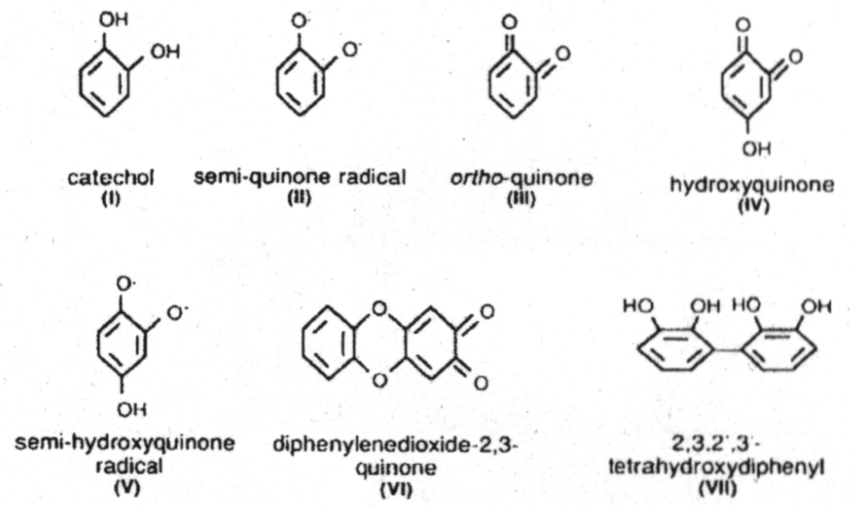

Abbildung 34: Oxidationsprodukte des Brenzkatechins (NAIDJA et al. 1998).

Die Kopplung mit anderen Brenzkatechin-Molekülen oder die Reaktion mit Sauerstoff führt zur Entstehung polymerisierter, intensiv gefärbter Verbindungen (VOUDRIAS \& REINHARD 1986). Das Hydroxyl-Radikal reagiert mit Brenzkatechin über die Abspaltung von Wasserstoff oder Addition zum aromatischen Ring zu einem resonanz-stabilisierten Radikal. Das entstehende Zwischenprodukt ist aufgrund von Resonanzeffekten relativ stabil, es kann jedoch mit anderen aromatischen Ringstrukturen ein größeres, chemisch sehr ähnliches Produkt bilden. Die Wiederholung dieses Prozesses kann über die Bildung von Oligomeren (Dimeren, Trimeren) bis hin zu Polymeren führen (VOUDRIAS \& REINHARD 1986, ARAKI et al. 1992, BOLLAG et al. 1998). Es können zwei Transformationsmechanismen ablaufen: einerseits eine o-Chinon-Kopplung und zu des weiteren die Kopplung durch freie Radikale unter nukleophiler Addition zu chinoiden Oligomeren. Brenzkatechin-Moleküle treten als Phenoxidanionen, die durch nukleophile Addition zu o-Chinonen koppeln auf. Im folgenden Schritt koppeln die oxidierten Verbindungen untereinander (Abbildung 35). Bei Anwesenheit von Cosubstraten gehen diese über Kreuzkopplungen mit in die Verbindungen ein (PARK et al. 1999). 
Phenol Oxidation:

(1) First stage hydrogen abstraction:

$$
\begin{aligned}
& \mathrm{HO}-\mathrm{O}-\mathrm{O} \stackrel{\text { (Step b) }}{\longrightarrow} \mathrm{HO}-(\mathrm{O}) \text { or } \cdot \mathrm{O}-\mathrm{O}-\mathrm{O}-\mathrm{O} \\
& \mathrm{HO}-\mathrm{O}-\mathrm{CH}_{3} \stackrel{\text { (Step b) }}{\longrightarrow} \mathrm{HO}-\mathrm{CH}_{2} \text {. }
\end{aligned}
$$

(?) Radical coupling:

$$
\begin{aligned}
& 2 \mathrm{HO}-\mathrm{O} \cdot \mathrm{O}-\mathrm{O}-\mathrm{CH}_{2} \longrightarrow \mathrm{O}-\mathrm{O}-\mathrm{CH}_{2}-\mathrm{CH}_{2}-\mathrm{O}-\mathrm{OH} \\
& 2 \mathrm{HO}-\mathrm{O}-\mathrm{O}-\mathrm{O}-\mathrm{O}-\mathrm{O}-\mathrm{O} \text { (poiymerization) }
\end{aligned}
$$

(3) Second stage hydrogen abstraction:

$$
\begin{aligned}
& \text { (O)- (O) } \\
& \mathrm{HO}-(\mathrm{O})-\mathrm{CH}_{2}-\mathrm{CH}_{2}-\mathrm{O}-\mathrm{OH} \stackrel{(\mathrm{Step} \mathrm{b})}{\longrightarrow} \mathrm{HO}-\mathrm{O}-\mathrm{CH}=\mathrm{CH}-\mathrm{O}-\mathrm{OH} \\
& \stackrel{(\text { Step b) }}{\longrightarrow} 0 \mathrm{CH}-\mathrm{CH}=\mathrm{C}=0
\end{aligned}
$$

Abbildung 35: Oxidation und Polymerisation von Phenolen (ARAKI et al. 1992).

Brenzkatechin kann jedoch nicht nur durch Oxidation und radikalische Prozesse zu Polymeren reagieren, sondern es kann auch ein Abbau des Phenols zu Aldehyden und Carbonsäuren erfolgen, der somit in einer Aufspaltung der aromatischen Strukturen resultiert (MORILL 1982, CERNIGLIA 1984, WANG \& HUANG 2000).

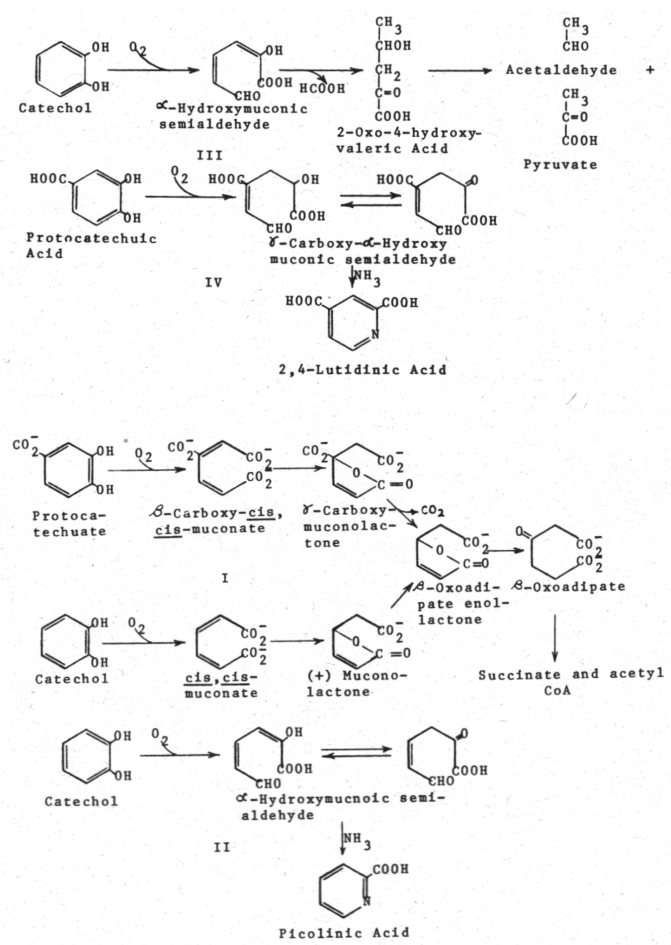

Abbildung 36: Abbau von Brenzkatechin und Protokatechussäure (MORILL 1982).

Die Oxidation erfolgt über den ortho-Pfad, was eine Spaltung der Bindung zwischen den CAtomen der beiden Hydroxylgruppen zu cis,cis-Muconsäure oder über den meta-Pfad mit 
Spaltung der Bindung der substituierten C-Atome verläuft (Abbildung 36).

\subsubsection{2,6-Dimethylphenol}

2,6-Dimethylphenol ist wie Brenzkatechin und Pyrogallol ein Monomer des Lignins. In chemisch-technischen Synthesen werden 2,6-Dimethylphenol-Monomere zur Herstellung von hochwertigen Kunststoffen (PPO: Poly(2,6-dimethyl-1,4-oxyphenylen) (polyphenylenoxid)) verwendet. Die oxidative Kopplung von 2,6-Dimethylphenol wurde schon 1967 durch HAY beschrieben. Es erfolgt eine Polymerisation zu Polyphenylenethern, begleitend werden Diphenochinone gebildet (Abbildung 37).

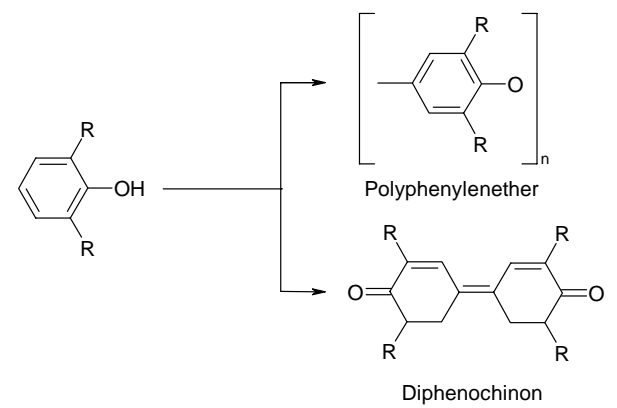

Abbildung 37: Polymerisation von 2,6-Dimethylphenol (nach HAY 1967).

Das Polymer wird bevorzugt bei kleinen Substituenten gebildet, steigt die Größe der Substituenten (propyl- oder butyl-) an, entsteht eher Diphenochinon. Die Reaktion des 2,6Dimethylphenols zu Polymeren verläuft durch eine Radikalbildung, in der oligomere Phenoxy-Radikale eine head-to-tail-Kopplungsreaktion eingehen (Abbildung 38).

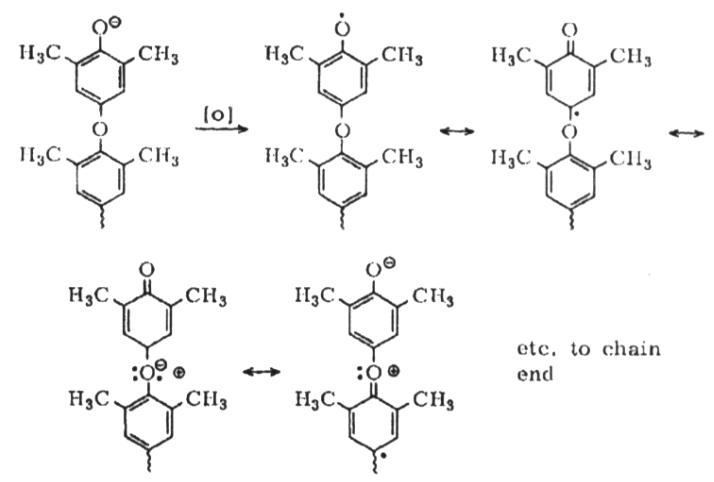

Abbildung 38: Radikalische Kopplung von 2,6-Dimethylphenol (HAY 1967).

Während des Herstellungsverfahrens nutzte man bisher Kupfer/Amin-Katalysatoren. In neueren Arbeiten (IKEDA et al. 1996) wurde die Polymerisation von 2,6-Dimethylphenol durch Katalyse mittels Enzyme untersucht. Die Untersuchungen ergaben, dass Phenole und Alkylphenole durch Peroxidasen oder Laccase eine oxidative Polymerisation erfahren (Abbildung 39). 


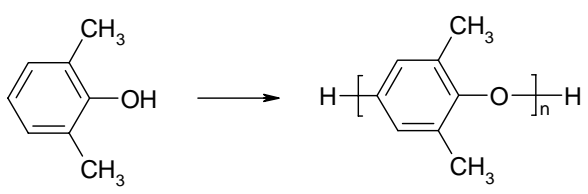

Abbildung 39: Polymerbildung aus 2,6-Dimethylphenolmonomeren (nach IONESCU \& MIHIS 1997).

Die Enzyme bewirken über die Freisetzung von $\mathrm{H}_{2} \mathrm{O}_{2}$ die Bildung phenolischer Radikale. Das Phenol-Monomer wird durch eine Elektronen-Oxidation durch die Oxireduktase in drei radikalische Intermediäre gleicher Resonanzstrukturen überführt (Abbildung 40). Die Intermediäre Ia und $\mathrm{Ib}$ reagieren zu einem Dimer (II). Dieses Dimer wird durch Eliminierung von $\mathrm{CO}_{2}$ zum Phenylenoxid (Dimer III). Im Fortsetzungsstadium erfolgt eine radikalische Kopplung zwischen der Radikalspezies des Monomers und des Polymers, was in einer um eine Moleküleinheit verlängerte Polymereinheit resultiert (UYAMA et al. 1998).

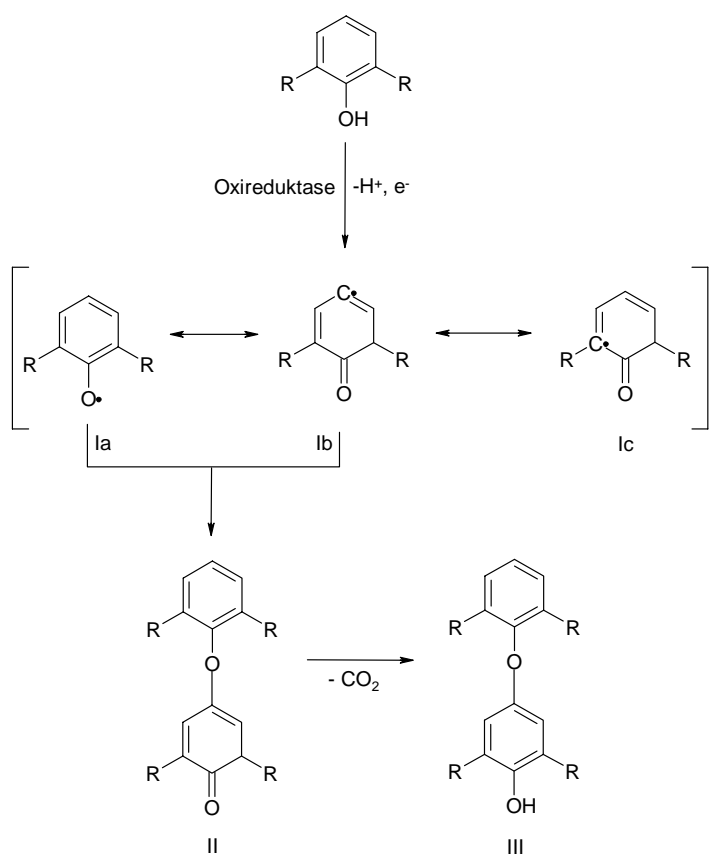

Abbildung 40: Enzymatische oxidative Polymerisation von 2,6-Dimethylphenol (nach UYAMA et al. 1998).

Somit konnte eine Alternative zur Herstellung von Phenolharzen, ohne den bis dahin notwendigen Einsatz von umweltbelastendem Formaldehyd, aufgezeigt werden.

Zusammenfassend bleibt festzuhalten, dass an Tonmineraloberflächen adsorbierte Phenole durch Elektronentransfers zu in den Zwischenschichten sorbierten Metallkationen zu Radikalkationen reagieren bzw. durch an der Tonmineraloberfläche gebundenen Sauerstoff über dessen Radikale oxidiert und/oder selbst zu Radikalen werden. Im Falle von Benzol und seinen Derivaten führen diese Reaktionsmechanismen zur Bildung von Polymeren. Brenzkatechin, Pyrogallol und 2,6-Dimethoxyphenol werden durch Montmorillonit katalysiert, es erfolgt eine Kreuzkopplung der Xenobiotika. Während die Umsetzung von Pyrogallol vollständig erfolgt, werden die beiden anderen Phenole nur teilweise oxidiert (PAL et al. 1994). Das Maß der Oxidation nimmt dabei mit der Anzahl der Hydroxylgruppen der Moleküle zu (WANG et al. 
1983). Die Reaktion verläuft über Radikale mit unterschiedlich delokalisiertem ungepaarten Spin, über Chinone bis zu kombinierten C-C- oder C-O-Verbindungen. Es werden Dimere, Trimere und Polymere bei der Reaktion eines C-Radikals mit einem O-Radikal gebildet. Als weitere Reaktionen folgen die Disproportionierung des Radikals oder Verlust des Protons. Die Radikal-Kationen weisen ein Elektronendefizit auf und können daher Elektronen aufnehmen. Die durch Polymerisation entstandenen Huminsäuren stellen dann ein optimal stabilisierendes System von Elektronen-Donator-Akkzeptor-Komplexen dar. Dies ist nicht nur für die Entstehung von Huminstoffen, sondern auch für die Reaktion mit Xenobiotika etc. von Bedeutung (SAWHNEY et al. 1984, POSPISIL 1994). Die an der Tonmineraloberfläche gebildeten Radikale reagieren jedoch nicht nur mit Phenolen, sondern auch mit anderen Monomeren, sie können mit N-enthaltenden Molekülen über C-C-Brücken oder die Kopplung von aromatischen Ringen Verbindungen eingehen. In Anwesenheit polymerisierter Chinone gehen diese ebenfalls in eine Reaktion ein (WANG et al. 1983). Ein weiterer wichtiger Aspekt der durch Tonminerale katalysierten Prozesse ist außerdem, dass diese unter sehr moderaten Bedingungen, was Temperatur-, Feuchte- und pH-Verhältnisse betrifft, ablaufen (BOYD \& MORTLAND 1986).

\subsubsection{Polyzyklische Aromatische Kohlenwasserstoffe}

Polyzyklische Aromatische Kohlenwasserstoffe (PAK) besitzen ein Grundgerüst aus zwei oder mehreren kondensierten Benzolringen. Neben ihrem natürlichen Vorkommen in der organischen Substanz der Böden entstehen PAK bei der unvollständigen Verbrennung organischer Energieträger (Holz, Kohle, Öl etc.) und gehören zu den weitest verbreiteten organischen Schadstoffen (STEVENSON 1994). Der Eintrag in den Boden erfolgt nach Bindung an Staubpartikel über die Luft. In Deutschland werden die jährlichen Einträge in die Umwelt zwischen 500 bis 1000 t geschätzt (BLUME 1992). PAK bestehend aus 4 und mehr Benzolringen verfügen über eine karzinogene und mutagene Wirkung. Aufgrund der hohen Umweltrelevanz hat die amerikanische Umweltbehörde (EPA) 16 PAK in eine Liste der besonders gefährdenden Verbindungen aufgenommen, darunter befindet sich auch das Phenanthren (THIEM 1994).

\subsubsection{Verhalten und Reaktionsprozesse von PAK im Boden}

Polyzyklische aromatische Kohlenwasserstoffe werden stark an die Bodenmatrix sorbiert (CERNIGLIA 1993). Dabei erfolgt bevorzugt eine Adsorption an die organische Substanz des Bodens. Je hydrophober und unpolarer der PAK, desto stärker ist die Sorption. Der größte Teil der PAK ist fast ausschließlich in der Humusauflage und in den A-Horizonten der Böden angereichert. Polyzyklische aromatische Kohlenwasserstoffe entstehen in Böden auch auf natürlichem Wege unter reduzierenden Bedingungen. Der Anteil der PAK an der organischen Substanz des Bodens wird zwischen 10 und $25 \%$ angenommen (STEVENSON 1994). 


\subsubsection{Phenanthren}

Phenanthren wird als Schadstoff in Böden, Flusssedimenten, Schlämmen nachgewiesen, es ist toxisch gegenüber Diatomeen, Gastropoden, Muscheln und Fischen. Phenanthren gehört mit Anthracen und Naphtalen $\mathrm{zu}$ den biologisch inaktiven PAK (MÜNCNEROVA \& AUGUSTIN 1994). Der PAK weist folgende chemisch-physikalischen Eigenschaften auf (EMUNDTS 1997):

- Molgewicht: 178,23

- Löslichkeit: $1.000 \mu \mathrm{g} / 1$

- Schmelzpunkt: $101^{\circ} \mathrm{C}$

- Kochpunkt: $340^{\circ} \mathrm{C}$.

Phenanthren ist als angular anneliertes PAK stabiler als linear annelierte Systeme (z.B. Anthracen. Neben der erwähnten starken Bindung der PAK an die Huminstoffe, werden PAK im Boden durch Mikroorganismen abgebaut (MARSCHNER 1997). Der initiale Schritt beim Abbau von Phenanthren durch Mikroorganismen ist immer eine Ringaktivierung durch die Einführung von molekularem Sauerstoff durch eine Dioxygenase und die Bildung von cisDihydrodiolen (Abbildung 41). Diese werden weiter zu Dihydroxy-Verbindungen dehydriert (KÖHLER 1992).

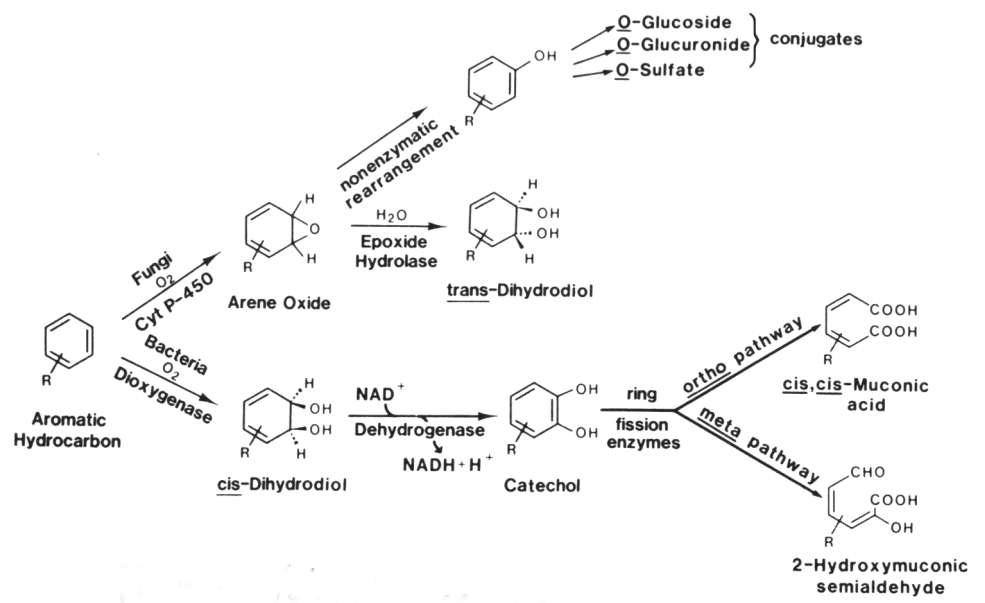

Abbildung 41: Mikrobielle Degradation des Phenanthrens (CERNIGLIA 1993).

Phenanthren lässt sich leicht zum entsprechenden Chinon (9,10-Phenanthrenchinon) oxidieren. Außerdem ist eine Reduktion zur Dehydro-Verbindung leicht möglich $(9,10-$ Dihydrophenanthren). Dies liegt in der besonderen Reaktivität der 9,10-Positionen des Phenanthrens begründet, die sich aus der niedrigen Resonanzstabilisierung durch den dritten Benzolring ergibt. Elektrophile aromatische Substitutionen finden bei Phenanthren am leichtesten in der 9-Position statt, oft ergeben sich disubstituierte Produkte. Der mikrobielle Abbau von Phenanthren produziert Metabolite wie Phenole, Chinone und trans-Hydrodiole (SUTHERLAND 1992). Der erste Schritt im fungalen Abbau ist die Epoxidation eines unsubstituierten PAK mit Entstehung eines instabilen Arenoxids und der Isomerisation zu Phenolen. Phenolbildende PAK sind Phenanthren, Anthracen, Naphtalen, Fluoranthen und Ben- 
zo[a]pyren. Beim Abbau durch Pilze wird Phenanthren zu Monohydroxylderivaten und Chinonen abgebaut (x-Hydroxyphenanthren, 9,10-Phenanthrenchinon und 2,2'-Diphensäure) (BEZALEL et al. 1997, CASILLAS et al. 1996, HAMMEL 1992), daneben treten Methoxyphenanthrene auf (SACK et al. 1997). Als Endprodukt des mikrobiellen Abbaus von Anthracen und Phenanthren entsteht Brenzkatechin (CERNIGLIA 1993). Der Tongehalt des Bodens ist bei der Bindung von PAK von keinem signifikanten Einfluss (BLUME 1992).

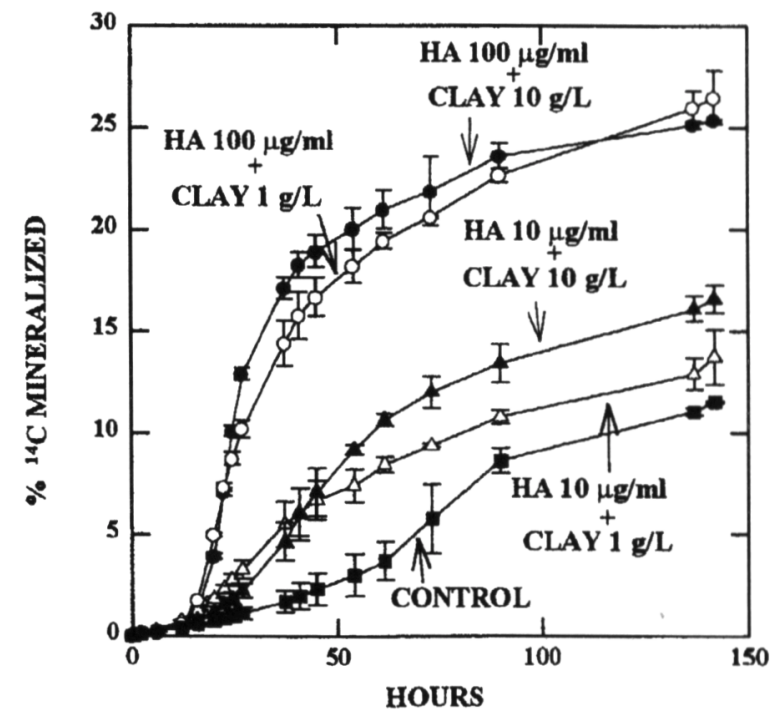

Abbildung 42: Beeinflussung des Abbaus von Phenanthren durch die Gegenwart von Ton (ORTEGA-CALVO \& SAIZ-JIMENEZ 1998).

Jedoch wurde in Gegenwart von Huminsäuren und Montmorillonit eine Stimulation der Degradation von Phenanthren (Abbildung 42) beobachtet (ORTEGA-CALVO \& SAIZJIMENEZ 1998).

Das breite Spektrum der in der Literatur beschriebenen katalytischen Aktivität von Tonmineralen zeigt den Forschungsbedarf, der hinsichtlich der Bedeutung der mineralischen Fraktion des Bodens in der Transformation und Festlegung von natülicher als auch xenobiotischer Organika besteht. 


\section{Zielsetzung}

\subsection{Konzeptionelle Grundlagen und Hypothesen}

Die Ausführungen des vorangegangenen Kapitels machen deutlich, dass zur Untersuchung des Verhaltens organischer Substanzen an mineralischen Oberflächen besondere Anforderungen an die konzeptionelle Herangehensweise und die Analytik gestellt werden. Organische Substanzen aus natürlichen Stoffkreisläufen sowie anthropogen eingebrachte Xenobiotika werden in Böden durch

- Adsorption,

- Festlegung an mineralischen Oberflächen (Chemisorption),

- Biotische und abiotische Transformationsprozesse,

- Einbau in die organische Substanz des Bodens

festgelegt bzw. modifiziert. Im Rahmen des Teilprojektes A6 „Grenzflächenreaktivität, Aufbau und Zerfall an Mikroaggregaten“ des SFB 468 sollten die, eventuell an der Grenzfläche Porenraum-Matrix auf der Nano- bis Mikroskala ablaufenden Prozesse qualitativ erfasst werden, um Aussagen hinsichtlich der Auswirkung solcher Prozesse auf Transformation und Festlegung von Organika, natürlicher und xenobiotischer Herkunft, im Boden zu treffen. Im methodisch-analytischen Teil dieser Arbeit wurde versucht, Antworten auf diese Aufgabenstellung zu erhalten. Diese Fragestellungen beinhalten die Bedeutung von Tonmineralen bei der abiotischen Katalyse (TADJERPISHEH 1986) phenolischer Verbindungen (PAL et al. 1994, WANG \& HUANG 1994) zu zumindest huminstoff-ähnlichen Substanzen (BOSETTO et al. 1994/1995/1997, BIGI et al. 1999, D’ACQUI et al. 1998, NAKAZAWA et al. 1995, THENG 1976) sowie bei der Transformation und Festlegung von Xenobiotika in Böden (RIGHI et al. 1995, SAWHNEY et al. 1996, ZIELKE \& PINNAVAIA 1989). Ziel ist es, die Hypothese der aktuellen Huminstoffgenese durch biotische Prozesse (Abbildung 43) dahingehend zu prüfen, ob auch die abiotische Aktivität von Tonmineralen einen Beitrag zur Transformation von Organika im Boden und somit zur Bildung von Huminstoffen leistet.

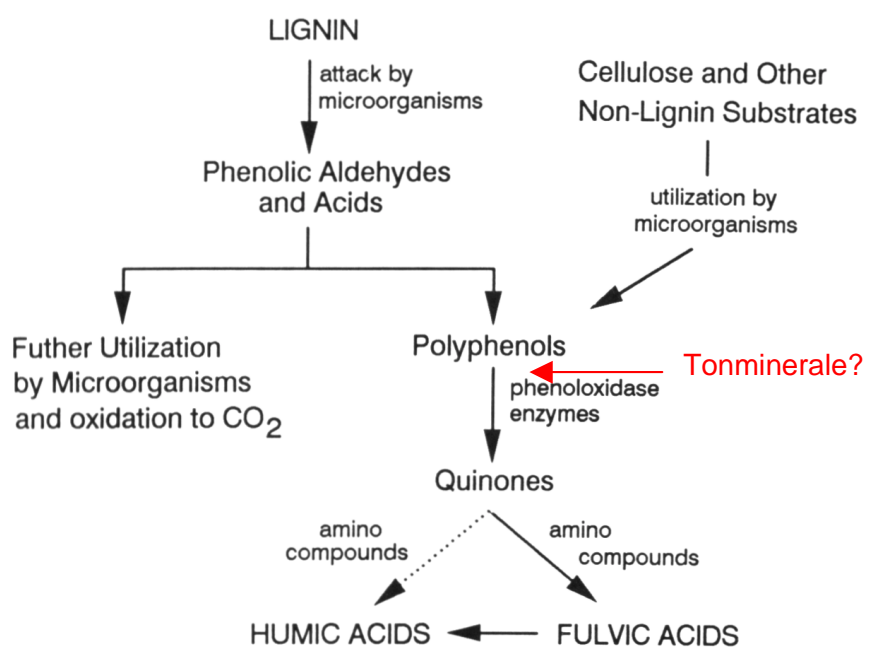

Abbildung 43: Theorie der Entstehung von Huminstoffen (nach Stevenson 1994). 
Anhand von Modellsubstanzen ist zu prüfen, ob durch physikochemische Prozesse an Tonmineraloberflächen Organika so fest in der organischen Substanz bzw. an den Tonmineralen festgelegt werden, dass sie als nicht-extrahierbare Rückstände (NER, bound residues) (ALEXANDER 1995, HATZINGER \& ALEXANDER 1995) angesehen werden können. Die Bildung von gebundenen Rückständen in der Bodenmatrix wird nach SCHEUNERT (1999) durch

- Einlagerung in die Zwischenschichten von quellfähigen Tonmineralen (Interkallationskomplexe),

- Einlagerung in Hohlräume von Huminpolymeren der organischen Bodensubstanz (Sequestrierung),

- kovalente Bindung an natürlichen, phenolischen Huminstoff-Vorläufern (z.B. Brenzkatechin) und Inkorporation in die entstehenden Huminstoffe durch Copolymerisation angenommen.

\subsection{Ziele und Untersuchungskonzept}

Das vorrangige Ziel dieser Arbeit ist es, zu untersuchen, ob an mineralischen Bodenbestandteilen abiotische Transformationsprozesse einfacher organischer Verbindungen - die einerseits als Modellsubstanzen für Huminsäuremonomere anzusehen sind und andererseits die Funktion organischer Schadstoffe innehaben - initiiert werden können. Dazu werden drei Phenole und ein polyzyklischer aromatischer Kohlenwasserstoff (Abbildung 44) durch Sublimation aus der Gasphase bzw. durch Kontamination aus alkoholischer Lösung auf den Oberflächen von Montmorilloniten abgelegt.<smiles>Oc1ccccc1O</smiles>

Brenzkatechin<smiles>Oc1cccc(O)c1O</smiles>

Pyrogallol<smiles>Cc1cccc(C)c1O</smiles>

2,6-Dimethylphenol<smiles>c1ccc2c(c1)ccc1ccccc12</smiles>

Phenanthren Abbildung 44: Eingesetzte Phenole und PAK.

In einer ersten Phase werden die Morphologie und die Verteilung organischer Überzüge auf den Oberflächen von Tonmineralen und ihre Charakterisierung mit Hilfe der Rasterelektronenmikroskopie zu untersuchen sein.

Durch sich anschließende Elementkartierungen der mit Organika belegten Oberflächen wird angestrebt, eventuelle Korrelationen in der Verteilung chemischer Elemente der organischen Überzüge und des mineralischen Untergrundes festzustellen, um somit gegebenenfalls Rückschlüsse auf mögliche Reaktionswege und -prozesse ziehen zu können.

Mit Hilfe chromatographischer Analyse- und Trennverfahren sollen jeweils die Anzahl der abiotisch gebildeten Reaktionsprodukte der von den Oberflächen der Tonminerale extrahier- 
ten organischen Überzüge bestimmt und getrennt in einzelnen Fraktionen weitergehenden qualitativen Analysen zugeführt werden.

Durch Anwendung nasschemischer Nachweisreaktionen sollen, den aufwändigeren Analysemethoden vorgeschaltete, Vorproben als Kontrollen einer eingetretenen chemischen Transformation der auf den mineralischen Oberflächen sublimierten Organika eingesetzt werden.

Zur detaillierten qualitativen Bestimmung der Zusammensetzung und Struktur der durch eventuell eingetretene physikochemische Prozesse an Tonmineralen gebildeten Reaktionsprodukte sollen spektroskopische (FTIR-Spektroskopie, ${ }^{13}$ C-NMR-Spektroskopie) und massenspektrometrische Verfahren herangezogen werden.

Durch die Kombination aller angewendeten Methoden soll neben dem Nachweis abiotischer Reaktionen von Organika an Tonmineralen nicht zuletzt Aussagen über die Funktion von Tonen bei der Genese von Huminstoffen und beim Einbau von Xenobiotika in die organische Substanz des Bodens treffen zu können. 


\section{Material und Methoden}

\subsection{Belegung und Probenvorbreitung}

Zum Nachweis der katalytischen Eigenschaften von Tonmineralen wurde ein im Versuchsaufbau unkompliziertes und in der Durchführung sehr schnelles Verfahren gewählt, um zu testen, ob und in welchem Ausmaß Reaktionen von organischen Verbindungen an Tonmineraloberflächen zu beobachten sind. Das hier zur Gewinnung von Reaktionsprodukten der katalytischen Aktivität von Tonmineralen verwendete „trockene“ Verfahren ermöglicht die Belegung von Tonmineraloberflächen ohne die Berücksichtigung von, bei anderen Verfahren auftretenden Problemen. Diese Methode wurde den in der Literatur häufig beschriebenen nasschemischen Versuchsansätzen, in denen phenolische Lösungen mit Tonmineralen versetzt und anschließend die Reaktionsprodukte analysiert werden, aus mehreren Gründen vorgezogen:

1. Der vorrangige Aspekt bei der bestehenden Zielsetzung war, unter anderem auch infrarotspektroskopische Messungen durchzuführen. Dieses Analyseverfahren weist jedoch eine sehr hohe Empfindlichkeit gegenüber eventuell im Probenmaterial enthaltenen Wasser auf. Dies würde in einer starken Absorption und Störung der Spektreninformation der zu analysierenden Substanzen resultieren. Allein aus diesem Grunde sind Verfahren, in denen wässrige Lösungen verwendet werden zu vermeiden, da das an Tonmineraloberflächen adsorbierte bzw. in den Zwischenschichten eingelagerte Wasser nur durch Trocknung bei hohen Temperaturen $\left(\geq 110^{\circ} \mathrm{C}\right)$ bzw. in langwierigen Trocknungsprozessen bei Umgebungstemperaturen zu entfernen wäre. Dies würde jedoch zumindest zu einer Veränderung der an der Tonmineraloberfläche gebundenen Reaktionsprodukte führen.

2. Die in wässrigen Lösungen ablaufenden hydrolytischen Prozesse komplizieren eine Abschätzung der katalytischen Aktivität von Tonmineralen. In Dispersionen aus Ton und gelösten Organika wären Reaktionen, ausgelöst durch die Reaktivität der Tonminerale, nicht eindeutig von anderen in wässrigen Dispersionen stattfindenden Vorgängen zu trennen.

3. Gerade bei Verwendung von nasschemischen Verfahren handelt es sich häufig um langfristigere Versuchsansätze, in denen die Versuchsdauer mehrere Wochen erreichen kann (WANG et al. 1983). Dadurch nimmt die Gefahr der Verfälschung der Versuchsergebnisse durch auftretenden mikrobiellen Ab- bzw. Umbau der eingesetzten Organika deutlich zu.

Zur Durchführung der Belegung von Tonmineralen wurden Brenzkatechin, Pyrogallol und 2,6-Dimethylphenol als Huminsäure-Vorstufen ausgewählt. Deren chemische Eigenschaften und ihr Verhalten im Boden wurde bereits in Kapitel 2.2.3 dargestellt. Diese Phenole neigen, abgesehen vom Pyrogallol in wässriger Lösung nicht zur, unter Phenolen häufig zu beobachtenden, Autoxidation (z.B. Hydrochinon). Phenole mit benachbarten Hydroxylgruppen wie 
Brenzkatechin sind gegenüber der Autoxidation fast inert (LARSON \& HUFNAL 1980). Durch Belegung der Tonminerale mit Phenolen durch Sublimation aus der Gasphase wurde die Gefahr der Autoxidation und Hydrolyse weitestgehend vermieden.

Die zur Belegung mit Phenolen und PAK verwendeten Tonminerale wurden vom Clay Minerals Repository der Clay Minerals Society, University of Missouri, USA. bezogen. Es handelt sich um zwei Bentonite, einen Na-Montmorillonit (SWy-2, Crook County, Wyoming, USA) und einen Ca-Montmorillonit (SAz-1, Apache County, Arizona, USA). Die chemische Zusammensetzung der Tonminerale wurde freundlicherweise von Herrn W.D. Johns, Kurator des Clay Minerals Repository - Department of Geology, University of Columbia-Missouri zur Verfügung gestellt (Tabelle 2).

Tabelle 2: Chemische Analysen der Tonminerale.

\begin{tabular}{|c|c|c|c|}
\hline & SWy-1 & $(=S W y-2)$ & SAz-1 \\
\hline & $\mathrm{CL}$ & $\mathrm{MH}$ & $\mathrm{MH}$ \\
\hline $\mathrm{SiO}_{2}$ & 62,9 & 62,9 & 60,4 \\
\hline $\mathrm{Al}_{2} \mathrm{O}_{3}$ & 19,3 & 19,6 & 17,6 \\
\hline $\mathrm{TiO}_{2}$ & 0,16 & 0,09 & 0,24 \\
\hline $\mathrm{Fe}_{2} \mathrm{O}_{3}$ & 3,85 & 3,35 & 1,42 \\
\hline $\mathrm{FeO}$ & 0,12 & 0,32 & 0,08 \\
\hline $\mathrm{MnO}$ & 0,01 & 0,006 & 0,099 \\
\hline $\mathrm{MgO}$ & 2,8 & 3,05 & 6,46 \\
\hline $\mathrm{CaO}$ & 1,8 & 1,68 & 2,82 \\
\hline $\mathrm{Na}_{2} \mathrm{O}$ & 1,54 & 1,53 & 0,063 \\
\hline $\mathrm{K}_{2} \mathrm{O}$ & 0,56 & 0,53 & 0,19 \\
\hline \multicolumn{4}{|l|}{$\mathrm{Li}_{2} \mathrm{O}$} \\
\hline $\mathrm{P}_{2} \mathrm{O}_{5}$ & 0,06 & 0,049 & 0,02 \\
\hline $\mathrm{S}$ & & 0,05 & \\
\hline $\mathrm{F}(\S)$ & 0,111 & & 0,287 \\
\hline \multicolumn{4}{|l|}{$\mathrm{F}\left({ }^{\circ}\right)$} \\
\hline ign. loss & 5,1 & & \\
\hline-550 & & 1,59 & 7,54 \\
\hline $550-1000$ & & 4,47 & 2,37 \\
\hline $\mathrm{CO}_{2}$ & 1,33 & & \\
\hline total & 99,54 & 99,22 & 99,3 \\
\hline $\begin{array}{l}\text { CL: Clemency; MH: Haydn } \\
\mathrm{F}(\S) \text { : fluor by Thomas, not }\end{array}$ & yur in & in ignition & \\
\hline
\end{tabular}




\subsubsection{Herstellung von Ton-Aggregaten}

Die Herstellung von Ton-Aggregaten erfolgte unter der Prämisse, kleine Objekte zu präparieren, die unter anderem zur elektronenmikroskopischen Untersuchung und zur Verwendung bei der elektronendispersiven Röntgenanalytik (Kapitel 4.2/4.3) Vorteile gegenüber Pulverpräparaten aufgrund ihrer höheren Stabilität und Zugänglichkeit bieten.

Dazu wurden die entsprechenden Tone mit destilliertem Wasser in verschließbaren Flaschen im Verhältnis $3 \mathrm{~g}$ Ton auf $40 \mathrm{~g}$ Wasser gemischt, $5 \mathrm{~min}$ im Utraschallbad dispergiert und 20 min im Überkopfschüttler geschüttelt. Die so gewonnenen Suspensionen wurden in Porzellanschälchen ausgestrichen. Bei Raumtemperatur erfolgte im Zeitraum von zwei Tagen, unter viermaligem Wiederbefeuchten mit destilliertem Wasser, die Aggregierung der Dispersion.

\subsubsection{Durchführung der Belegung mit Phenolen}

Die Belegung der Tonminerale erfolgte in Anlehnung an die Versuchsvorschrift von SAWHNEY et al. (1984): 2 g Ton wurde auf einem Uhrglas gleichmäßig durch Verstreichen mit einem Spatel auf diesem verteilt, wobei darauf zu achten war, dass der Ton dadurch nicht zu stark zusammengepresst wurde um eine möglichst große, einer Reaktion zur Verfügung stehende Oberfläche zu erhalten. Zur Belegung des Bentonits wurde jeweils 0,5 g des entsprechenden Phenols in Porzellantiegel gefüllt (Tabelle 3).

Tabelle 3: Belegung von Phenolen und Phenanthren auf Tonminerale.

\begin{tabular}{|l|c|}
\hline \multicolumn{1}{|c|}{ Phenol/PAK } & Ton \\
\hline Brenzkatechin & Na-Montmorillonit (SWy-2) \\
\hline Pyrogallol & Na-Montmorillonit (SWy-2) \\
\hline 2,6-Dimethylphenol & Na-Montmorillonit (SWy-2) \\
\hline Brenzkatechin & Ca-Montmorillonit (SAz-1) \\
\hline Pyrogallol & Ca-Montmorillonit (SAz-1) \\
\hline 2,6-Dimethylphenol & Ca-Montmorillonit (SAz-1) \\
\hline Phenanthren & Na-Montmorillonit (SWy-2) \\
\hline Phenanthren & Ca-Montmorillonit (SAz-1) \\
\hline
\end{tabular}

Alle Behältnisse wurden zuvor mit Methanol und Hexan gereinigt und anschließend im Muffelofen bei $800^{\circ} \mathrm{C}$ ausgeglüht. Als Reaktionsgefäße dienten mit Deckeln verschließbare Dosen aus Weißblech ( $\varnothing$ etwa $30 \mathrm{~cm}$ ). Jeweils ein Uhrglas mit dem entsprechenden Ton und ein Tiegel mit Phenol wurden in der Dose platziert. Anschließend wurde die Dose mit einem Deckel verschlossen und in einem eigens dafür vorgesehenen Wärmeschrank mit integriertem Abzug, um eventuell austretende toxische Phenoldämpfe (2,6-Dimethylphenol) zu vermeiden, gestellt. Zur Sublimation des Phenols auf die Tonmineraloberfläche wurde eine Equilibrierungsdauer von $24 \mathrm{~h}$ bei einer Temperatur von $50^{\circ} \mathrm{C}$ festgelegt. Die Temperatur wurde gewählt, um die gewünschte Reaktion dadurch zu beschleunigen, dass das die Phenole langsam in die Dampfphase übergehen und sich an den Oberflächen der Tonminerale niederschlagen. Man erreicht damit einen intensiveren und gleichmäßigen Kontakt der so feinverteilten Organika auf den Tonmineralen. Höhere Temperaturen sind nicht $\mathrm{zu}$ empfehlen, da sonst eine thermische Zersetzung der Ausgangssubstanzen die Folge wäre. Nach 24 h wurden die Dosen 
aus dem Wärmeschrank genommen und unter einen Abzug gestellt. Dort wurden die Deckel geöffnet und die Phenolquelle entfernt und zur Entsorgung gesammelt. Die Tonproben auf den Uhrgläsern verblieben für weitere $24 \mathrm{~h}$ in den Metalldosen unter dem Abzug um überschüssiges, an den Tonen kondensiertes Phenol verflüchtigen zu lassen.

\subsubsection{Ergebnisse der Belegung von Tonmineralen mit Phenolen}

Nach 48 h wurden die Uhrgläser aus den Reaktionsgefäßen entnommen und optisch ausgewertet. Die Belegung des Na-Montmorillonits (SWy-2) mit Phenolen führte zu folgenden Resultaten (Abbildung 45).

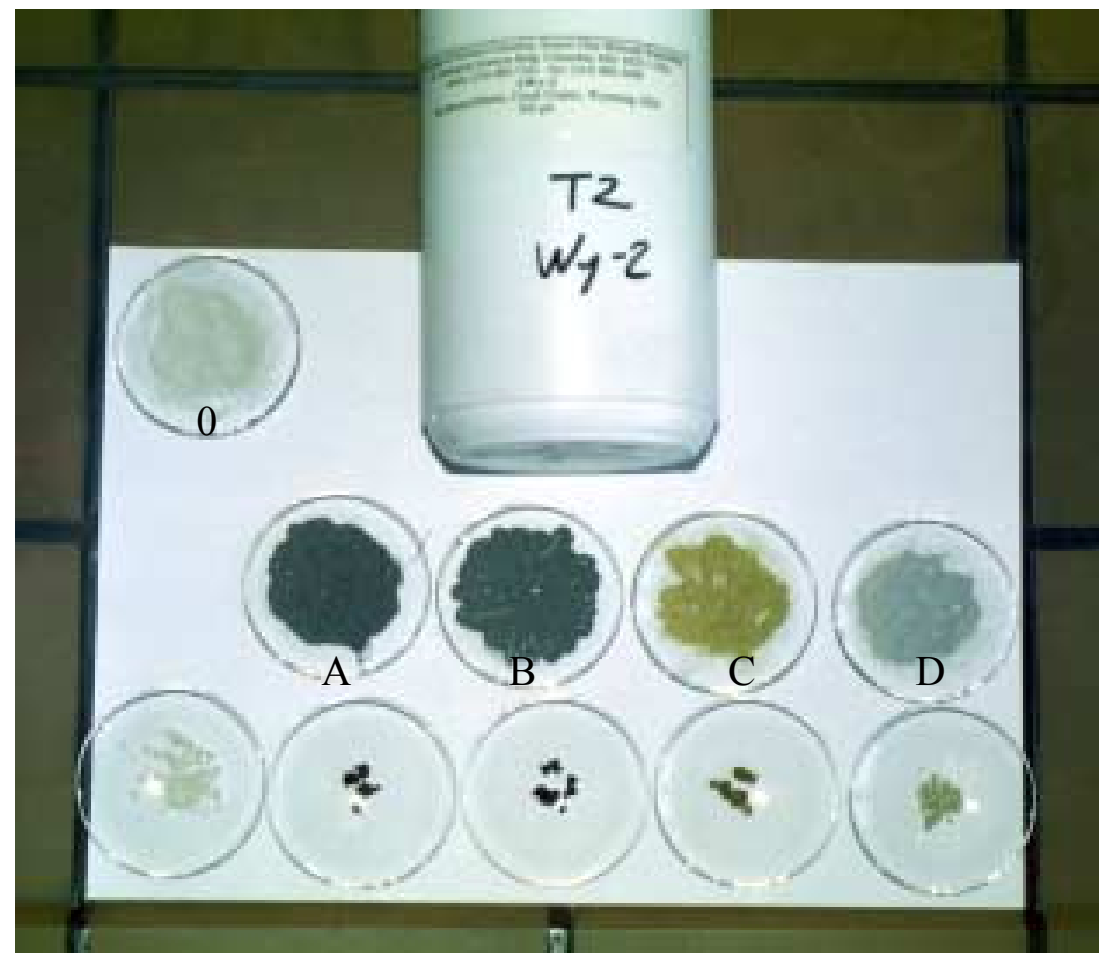

Abbildung 45: Resultate der Belegung von Na-Montmorillonit mit Phenolen.

Die Proben des mit Phenolen belegten Na-Montmorillonits weisen deutliche Verfärbungen auf. Im Vergleich zum unbelegten Ton (Abbildung 45: Probe 0) sind bei den Proben A (Brenzkatechin) und B (Pyrogallol) eine intensive Schwarzbraun- bzw. Braunfärbung des Tons zu beobachten. Der mit 2,6-Dimethylphenol belegte Montmorillonit (Probe C) zeigt hingegen eine leuchtend orangerote Färbung. Anhand dieser Verfärbungen ist abzuleiten, dass der Kontakt der Phenole mit dem Ton zu einer chemischen Reaktion der Organika an der Tonmineraloberfläche geführt hat.

In einer Versuchsparallele wurde die Belegung eines Ca-Montmorillonits (SAz-1) mit Phenolen durchgeführt. Auch hier weisen die Oberflächen des Tons nach der Belegung bei allen Proben wiederum deutliche Verfärbungen auf (Abbildung 46). Diese reichen von dunkelbraun bei der mit Brenzkatechin versetzten Probe (A) über hellbraun bei Pyrogallol (B) bis rotorange bei 2,6-Dimethylphenol (C). 


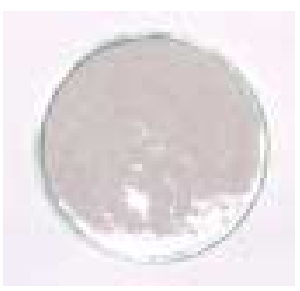

Probe 0: SAz-1, unbelegt.

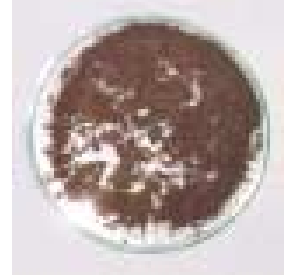

Probe A: SAz-1, belegt mit Brenzkatechin.

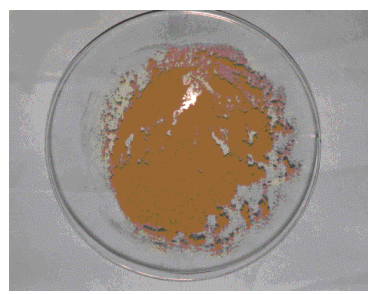

Probe B: SAz-1, belegt mit Pyrogallol.

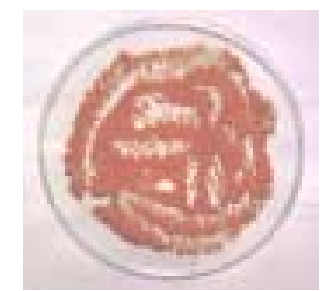

Probe C: SAz-1, belegt mit 2,6Dimethylphenol.

Abbildung 46: Ergebnisse der Belegung des Ca-Montmorillonits mit Phenolen.

Auch die Belegung des Ca-Montmorillonits mit Organika führte somit $\mathrm{zu}$ einer deutlich sichtbaren Reaktion. Die hier im Vergleich mit den Resultaten der Belegung des NaMontmorillonits leicht abweichenden Farbtöne, insbesondere bei Pyrogallol (B) sind jedoch kein Hinweis auf eine geringere Reaktivität des Ca-Montmorillonits; es handelt sich lediglich um eine geringere Konzentration der beaufschlagten Organika auf der Tonmineraloberfläche. Zur quantitativen Bestimmung der durch die verwendete Methode der Belegung von Tonen durch Sublimation von Phenolen aus der Gasphase tatsächlich auf den Tonen abgelegten Mengen an Organika wurden unbelegte und belegte Tonproben in einem CN-Analysator (LECO CHN-1000) analysiert und der Kohlenstoffgehalt bestimmt. Die Ergebnisse sind in Tabelle 4 aufgeführt.

Tabelle 4: Bestimmung der C-Gehalte von unbelegten und belegten Tonproben.

\begin{tabular}{|l|c|c|c|c|}
\hline \multicolumn{1}{|c|}{ Probe } & $\begin{array}{c}\text { Einwaage } \\
{[\mathbf{m g}]}\end{array}$ & C-Gehalt [\%] & C-Gehalt [mg] & $\begin{array}{c}\text { C-Gehalt [mg] } \\
\text { abzgl. blank }\end{array}$ \\
\hline Na-Montmorillonit, unbelegt & 49,8 & 0,328 & 0,163344 & 0,163344 \\
\hline Na-Montmorillonit, unbelegt & 49,6 & 0,366 & 0,181536 & 0,181536 \\
\hline Na-Montm+Brenzkatechin 1 & 49,87 & 2,218 & 1,1061166 & 0,9336766 \\
\hline Na-Montm+Brenzkatechin 1 & 50,49 & 2,622 & 1,3238478 & 1,1514078 \\
\hline Na-Montm+Brenzkatechin 2 & 50,84 & 2,571 & 1,3070964 & 1,1346564 \\
\hline Na-Montm+Brenzkatechin 2 & 50,25 & 2,382 & 1,196955 & 1,024515 \\
\hline Na-Montm+2,6-DMP 1 & 50,31 & 0,902 & 0,4537962 & 0,2813562 \\
\hline Na-Montm+2,6-DMP 1 & 50,26 & 0,973 & 0,4890298 & 0,3165898 \\
\hline Na-Montm+2,6-DMP 2 & 50,94 & 1,177 & 0,5995638 & 0,4271238 \\
\hline Na-Montm+2,6-DMP 2 & 49,56 & 1,206 & 0,5976936 & 0,4252536 \\
\hline Na-Montm+Pyrogallol 1 & 50,04 & 0,616 & 0,3082464 & 0,1358064 \\
\hline Na-Montm+Pyrogallol 1 & 50,81 & 0,607 & 0,3084167 & 0,1359767 \\
\hline Na-Montm+Pyrogallol 2 & 50,8 & 0,465 & 0,23622 & 0,06378 \\
\hline Na-Montm+Pyrogallol 2 & 49,78 & 0,51 & 0,253878 & 0,081438 \\
\hline Na-Montm+Phenanthren 1 & 50,32 & 0,902 & 0,4538864 & 0,2814464 \\
\hline Na-Montm+Phenanthren 1 & 49,83 & 0,874 & 0,4355142 & 0,2630742 \\
\hline Na-Montm+Phenanthren 2 & 50,24 & 0,94 & 0,472256 & 0,299816 \\
\hline Na-Montm+Phenanthren 2 & 50,51 & 0,969 & 0,4894419 & 0,3170019 \\
\hline
\end{tabular}

Die Analysen zeigen, dass der Na-Montmorillonit unbelegt einen C-Gehalt von etwa 0,3\% aufweist. Nach der Belegung mit Organika steigen die Kohlenstoffgehalte auf Werte zwischen 2,2 und 2,6\% für das Brenzkatechin, 0,5 bis 0,6 \% bei Pyrogallol und 0,9 bis 1,2\% für das 2,6-Dimethylphenol an. Die Sublimation von Phenolen ist somit sehr effektiv für das 
Brenzkatechin, die geringe Ausbeute im Falle des Pyrogallols hängt mit dem deutlich höheren Schmelzpunkt (Pyrogallol: 133-134 C, Brenzkatechin: $105^{\circ} \mathrm{C}$ ) zusammen.

\subsubsection{Belegung der Tonminerale mit Phenanthren}

Aufgrund des hohen Schmelzpunktes des Phenanthrens konnte dieses nicht wie die Phenole durch Sublimation aus der Gasphase auf den Ton aufgebracht werden. Aus diesem Grunde wurde das Phenanthren in Methanol gelöst (10 mg Phenanthren auf $10 \mathrm{ml}$ Methanol) und mit einer Pasteur-Pipette so auf den Ton auftropft, dass eine gleichmäßige Durchfeuchtung der Probe erreicht wurde. Zur Verflüchtigung des Lösemittels wurden die Proben bei Raumtemperatur $\left(\sim 20^{\circ} \mathrm{C}\right)$ über Nacht unter dem Abzug stehen gelassen. Die weitere Belegung und Extraktion erfolgte entsprechend der für die Phenole angewendeten Methode.

Nach der Belegungsphase konnte bei den mit Phenanthren belegten Tonproben optisch nur eine leichte Graufärbung der Tonmineraloberfläche beobachtet werden (Abbildung 45, Probe D). Intensive Verfärbungen konnten in keiner Versuchsreihe festgestellt werden. Eine eventuell eingetretene Bildung von Transformationsprodukten des Phenanthrens konnte, im Gegensatz zu den mit Phenolen belegten Proben zuerst einmal nicht beobachtet werden, daher ist auf die spektroskopischen Analysen (Kapitel 4.6 und 4.7) zu verweisen.

Frühere Versuche (BIRKEL 1997) zeigten, dass die Reaktion der sublimierten Phenole vom Sauerstoffgehalt der Umgebungsatmosphäre abhängig ist. Dazu wurden die entsprechend belegten Parallelen statt in Metalldosen in einen dickwandigen Glaskolben gestellt und dieser mittels Vakuumpumpe evakuiert (Abbildung 47).

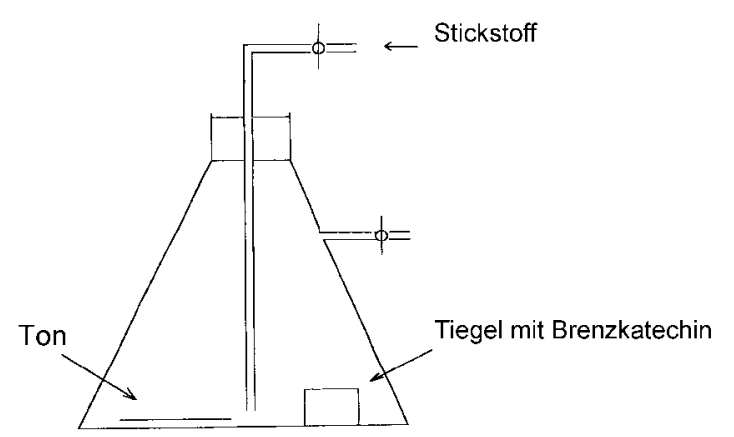

Abbildung 47: Nachweis der Abhängigkeit der Reaktion vom Sauerstoff (BIRKEL 1997).

Anschließend wurde der Behälter mit reinem Stickstoff (4.6) gespült. Die Evakuierung und Spülung mit $\mathrm{N}_{2}$ wurde jeweils zweimal wiederholt. Das Reaktionsgefäß wurde mit zur Vermeidung von eventuell auftretenden Autoxidationseffekten mit Aluminiumfolie völlig lichtgeschützt abgedeckt. Der Kolben wurde anschließend auf ein Sandbad gestellt und entsprechend den bereits vorab beschriebenen Versuchsbedingungen $24 \mathrm{~h}$ lang bei einer Temperatur von $50^{\circ} \mathrm{C}$ erwärmt. Nach Ablauf der Versuchsdauer wurde das Reaktionsgefäß vom Sandbad genommen, die Aluminiumfolie entfernt und die Ton-Probe einer Sichtkontrolle unterzogen. Als ein Ergebnis dieser Versuchreihe zeigte sich, dass im Vergleich zur Belegung unter Nor- 
malatmosphäre, nur eine sehr leichte schwärzlichgraue Färbung des Tons zu beobachten war, was auf eine nur schwache Reaktion der Phenole an der Tonoberfläche zurückzuführen ist. Daraus kann geschlossen werden, dass der Sauerstoff der Luft für die Sorption bzw. Reaktion von Organika an Tonmineralen (Tabelle 5) unbedingt notwendig ist (SAWHNEY 1985).

Tabelle 5: Sorption von Phenolen in Luft oder Stickstoff (nach SAWHNEY 1985).

\begin{tabular}{|c|c|c|c|c|c|}
\hline \multirow{2}{*}{ Kation } & \multicolumn{2}{|c|}{ o-Methylphenol } & \multicolumn{2}{c|}{ o-Chlorphenol } & m-Methylphenol \\
\cline { 2 - 6 } & Luft & Stickstoff & Luft & Stickstoff & Luft \\
\hline $\mathrm{Na}$ & 4,5 & $\mathrm{nn}$ & $\mathrm{nn}$ & 0,20 & 3,2 \\
\hline $\mathrm{Ca}$ & 17,2 & 0,05 & 17,3 & 0,40 & 11,6 \\
\hline $\mathrm{Al}$ & 10,2 & 0,53 & 13,6 & 1,1 & 7,0 \\
\hline $\mathrm{Fe}$ & 12,2 & 0,75 & 14,5 & 1,1 & 8,5 \\
\hline
\end{tabular}

nn: nicht nachweisbar

Diese Ergebnisse geben deutliche Hinweise auf die an Tonmineralen ablaufenden Reaktionsmechanismen (Kapitel 2.1.5). Der angenommenen Reaktionsmechanismus, dass der an der Oberfläche adsorbierte Sauerstoff für die Oxidation des Brenzkatechins an Tonmineraloberflächen verantwortlich ist, wurde letztendlich dadurch bestätigt, dass nach Öffnung des Reaktionsgefäßes und damit erfolgendem Kontakt der Probe mit dem Sauerstoff der Luft eine rasche, innerhalb von 10-20min eintretende intensive Schwarzfärbung der Tonmineraloberfläche einsetzte. Hieraus kann somit abgeleitet werden, dass der (Luft-)Sauerstoff den wohl bedeutendsten Faktor in der Oxidation des Brenzkatechins stellt. Infolgedessen wird der durch VOUDRIAS \& REINHARD (1986) beschriebene Reaktionsmechanismus, der die Bildung von Radikalen an der Tonmineraloberfläche in Anwesenheit von Sauerstoff mit Radikalisierung der Phenole und anschließender Oxidation und Polymerisation zu neuen Verbindungen mit entsprechenden chemischen Eigenschaften beschreibt, bestätigt. Die trotzdem noch zu beobachtende schwache Reaktion des Brenzkatechins an der Tonmineraloberfläche in $\mathrm{N}_{2}$ Atmosphäre kann zum einen damit erklärt werden, dass noch geringe Mengen von adsorbiertem Sauerstoff, trotz mehrfachem Evakuieren und Spülen mit Stickstoff, an den Tonmineraloberflächen verblieben sind und für die Initiierung der Reaktion zur Verfügung standen. Diese Hypothese wird auch von SOLOMON \& HAWTHORNE (1983) gestützt, die ebenfalls eine vollständige Entfernung des an Tonmineraloberflächen gebundenen Sauerstoffs aufgrund von Chemisorption negieren.

\subsubsection{Extraktion organischer Beläge von Tonmineraloberflächen}

Das klassische Verfahren zur Extraktion von Huminstoffen mit Natronlauge (SCHNITZER 1982, STEVENSON 1994) wurde zur Entfernung der organischen Überzüge aus zwei Gründen nicht angewendet:

1. aufgrund der hohen Aggressivität des Extraktionsmittels ist davon auszugehen, dass auch in einer als Schutzgas wirkenden $\mathrm{N}_{2}$-Atmosphäre (SCHUMACHER 1995) die Bildung von Artefakten nicht zu vermeiden wäre. Qualitative Aussagen zu den entstandenen Reaktionsprodukten wären so nicht mehr uneingeschränkt möglich. 
2. im Hinblick auf die beabsichtigten infrarotspektroskopischen Untersuchung der Extrakte bedeutet die Verwendung von wässrigen Lösungen $(\mathrm{NaOH})$, dass durch Wasser induzierte, die Spektreninterpretation stark störende Absorptionsbanden zu erwarten wären.

Zur Vermeidung dieser, die Aussagefähigkeit der IR-Spektren beeinflussenden Effekte bieten sich wasserfreie organische Extraktionsmittel an. Zur Extraktion von Brenzkatechin und dessen Reaktionsprodukten erweist sich Methanol $\left(\mathrm{CH}_{3} \mathrm{OH}\right)$ nach SAWHNEY et al. (1984) als sehr gut geeignet, da es als polar protisches Lösungsmittel aufgrund seiner nukleophilen und elektrophilen Eigenschaften Wasserstoffbrücken auszubilden und somit Anionen als auch Kationen zu solvatisieren vermag. Aceton hingegen als dipolar aprotisches Lösemittel verfügt über keine Hydroxylgruppen, kann somit keine H-Brücken ausbilden und vermag dadurch Anionen nur schlecht zu solvatisieren (EHLERS 1989). Die bessere Eignung von Methanol als Extraktionsmittel gegenüber Aceton ist in der praktischen Anwendung schon dahingehend zu erkennen, dass das Methanol in der Lage ist, wesentlich größere Anteile der organischen Verbindungen von der Tonoberfläche zu lösen, was durch eine stärkere Entfärbung des Rückstandes (Ton) nach der Zentrifugation zu beobachten war.

\subsubsection{Durchführung der Extraktion}

Zur Extraktion wurden je 2,0 g des entsprechend belegten Montmorillonits mit $40 \mathrm{ml}$ Methanol (Merck LiChroSolv) in Glasflaschen mit einem Volumen von $50 \mathrm{ml}$ gefüllt. Diese wurden verschlossen und 15 min bis zur völligen Dispergierung im Überkopfschüttler geschüttelt. Anschließend erfolgte zur Unterstützung der Lösung der organischen Verbindungen von den Tonmineralen eine 15-minütige Dispergierung im Ultraschallbad. Zur Abtrennung des Lösemittels von der Tonfraktion wurde $30 \mathrm{~min}$ bei $20^{\circ} \mathrm{C}$ mit $3.000 \mathrm{Upm}$ zentrifugiert. Der Überstand wurde mittels Pipette abgezogen und durch einen Polyamid-Filter mit 0,2 $\mu \mathrm{m}$ Porenweite filtriert. Die so gewonnenen Extrakte wurden in Braunglasfläschchen aufgefangen und bis zur weiteren Verwendung lichtgeschützt und gekühlt aufbewahrt.

Anzumerken ist, dass auch das Methanol als Extraktionsmittel nicht in der Lage war, alle organischen Reaktionsprodukte von der Tonmineraloberfläche vollständig zu entfernen (TIPTON \& GERDOM 1992). Dies zeigte sich nach erfolgter Extraktion und Zentrifugation, als immer noch eine intensive Färbung des Rückstands beobachtet werden konnte. Das die Sublimation von Pyrogallol auf der Tonmineraloberfläche zur Entstehung neuer Substanzen mit solch andersartigen Eigenschaften geführt hat, lässt sich schon daran erkennen, dass die Extraktion der farbigen Überzüge lediglich einen schwach gefärbten Teil der Reaktionsprodukte von den Tonmineralen entfernen konnte. Neben dem leicht zu extrahierenden Pyrogallol und weiteren leicht in Methanol löslichen Verbindungen verblieb der Großteil der intensiv gefärbten Substanz(en) auf dem Ton. Zahlreiche Versuchsserien mit verschiedensten Lösemitteln und Extraktionsverfahren (Soxhlett- und Mikrowellenextraktion) führten zu keinen befriedigenden Ergebnissen. Lediglich die Kombination von Mikrowellenextraktion mit Di- 
methylsulfoxid (DMSO) als Lösemittel lieferte als Ergebnis eine intensiv gefärbte Extraktlösung. Auf die Analyse dieses Extrakts wurde jedoch aufgrund der anzunehmenden starken Artefaktbildung des organischen Materials durch die extremen Extraktionsbedingungen $\left(100^{\circ} \mathrm{C}\right.$ bei mehrstündiger Extraktionsdauer), ebenso wie bei der klassischen Methode der Huminstoffextraktion mit Natronlauge, verzichtet. Aufgrund der sehr starken Bindung der entstandenen Reaktionsprodukte an die mineralische Komponente ist auf deren chemisorptive Festlegung zu schließen. Dieses Gesamtverhalten der entstandenen, nicht extrahierbaren Reaktionsprodukte weist somit auf Parallelen mit bodenbürtiger, nicht-extrahierbarer organischer Substanzen hin und kann als ein einfaches Modell für Ton-Humus-Komplexe aufgefasst werden (BIRKEL \& NIEMEYER 1998b).

\subsection{Rasterelektronen-Mikroskopie}

Die Rasterelektronen-Mikroskopie (REM) ist ein klassisches Verfahren zur Abbildung der Topographie von Festkörpern mit hoher Schärfentiefe und Ortsauflösung (GOLCHIN 1994, OHASHI \& NAKAZAWA 1996). Die in dieser Arbeit durchgeführten Untersuchungen mittels Rasterelektronenmikroskop hatten zum Ziel, zu überprüfen, ob die schon mit bloßem Auge zu erkennenden Verfärbungen nach der Sublimation von Organika auf den Tonmineralen bei hohen Vergrößerungen als Überzüge auf den Mineraloberflächen zu beobachten sind und somit Hinweise für die vermutete Polymerisation (Kapitel 2.2.5) der eingesetzten Phenole zu erhalten. Ein weiteres Ziel war, wenn solche Überzüge zu erkennen sind, nach Möglichkeit zu überprüfen, ob diese an bevorzugten Plätzen auf den Oberflächen angetroffen werden können.

\subsubsection{Prinzip der Elektronenmikroskopie}

Die Bestrahlung einer Probenoberfläche mit energiereichen Elektronen führt zur Auslösung von Sekundärelektronen und zu einer materialabhängigen Rückstreuung der einfallenden Primärelektronen. Zur Abbildung der Probenoberfläche wird der Durchmesser des anregenden Elektronenstrahls sehr stark verkleinert, so dass beim Auftreffen auf der Probenoberfläche der Strahldurchmesser nur noch bei 1-10 nm liegt. Synchron zur gerasterten Führung des primären Elektronenstrahls auf der Probenoberfläche wird der Elektronenstrahl einer Bildröhre über den Leuchtschirm gelenkt. Die Intensität dieses Schreibstrahls wird durch den gemessenen Strom der Sekundär- und Rückstreuelektronen gesteuert. Die Anzahl der durch die Wechselwirkung der Primärelektronen mit dem Probenmaterial entstehenden Sekundärelektronen hängt u.a. von der Oberflächentopographie ab, so dass auf dem Bildschirm ein direktes Abbild der Oberfläche entsteht (Abbildung 48). 


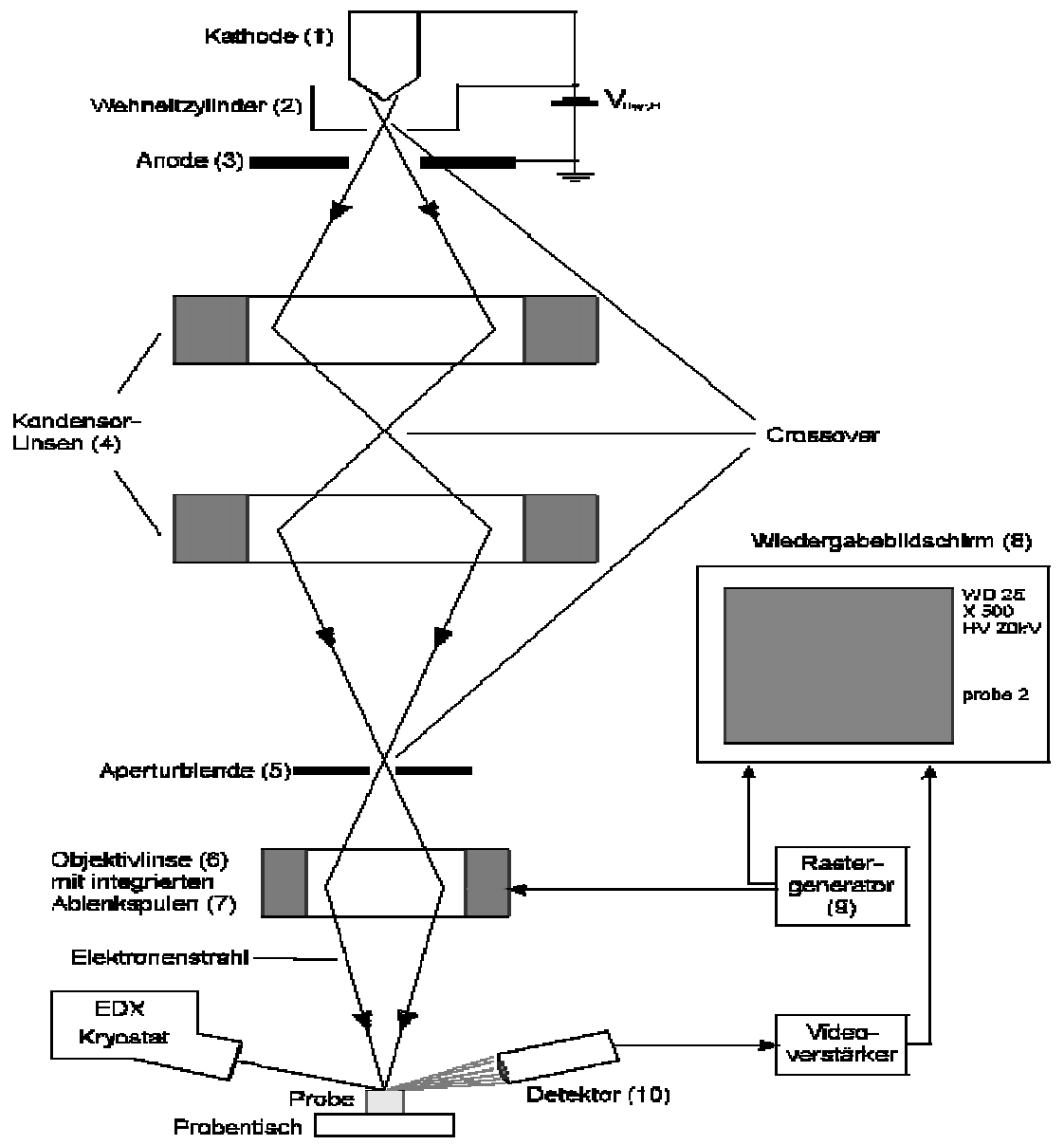

Abbildung 48: Schematische Darstellung eines Rasterelektronenmikroskops (BARKOW 1994).

Mit Hilfe der rückgestreuten Elektronen (BSE: backscattered electrons) ist zusätzlich auch eine Abbildung des Materialkontrastes möglich. Ein sehr fein gebündelter Strahl energiereicher Elektronen (1-30 keV) wird rasterförmig über die Probenoberfläche gelenkt. Diese Elektronen treten mit den Hüllen- Elektronen der Atome in der Probe in Wechselwirkung, wodurch viele unterschiedliche Prozesse ausgelöst werden können. U.a. verursacht der Elektronenbeschuss die Aussendung von niederenergetischen Sekundär- Elektronen, deren Anzahl vor allem von der Oberflächengeometrie des jeweiligen Emissionsortes bestimmt wird. Die Sekundär- Elektronen werden mit einer Zugelektrode abgesaugt und die Stärke dieses Sekundärelektronenstroms als Signal zur Intensitätssteuerung des Elektronenstrahls eines Bildschirmes herangezogen, dessen x-y-Führung synchron zur Probenabrasterung läuft. Dadurch entsteht eine Punkt-für-Punkt-Abbildung der Probenoberfläche. Die Ortsauflösung und damit auch die maximale förderliche Vergrößerung ist mehr als 10-mal so groß wie beim Lichtmikroskop (20000-fache, geräteabhängig bis 100000-fache Vergrößerung). Da die Abbildung ohne Linsen auskommt, werden die damit verbundenen Unzulänglichkeiten (i.e. stark eingeschränkter Tiefenschärfebereich und Verzeichnungsfehler) vermieden. So entstehen sehr plastisch wirkende Bilder der geometrischen Oberflächengestalt der Probe mit fast unbegrenzter Tiefenschärfe, die sowohl die Darstellung grober, schluchtenartiger Strukturen als auch die Aufzeichnung feinster Details bis in den Nanometerbereich erlaubt. REM eignet sich zur Aufklä- 
rung und Darstellung der Oberflächengestalt vom Millimeter- bis Nanometer-Strukturen und dient es als idealer Such-Monitor für EDX-Analysen (SCHMIDT 1994).

\subsubsection{Rückstreu- und Sekundärelektronen}

Rückstreuelektronen (RE) entsprechen in ihrem Energieniveau den Primärelektronen (PE) des Elektronenstrahles. Genaugenommen handelt es sich hierbei um PE die in die Probe eindringen und dort elastisch zurückgestreut werden, weshalb sich ihre Energie nicht verringert. Das bedeutet, sie behalten ihre durch die Beschleunigungsspannung erzeugte Geschwindigkeit bei und werden nur in ihrer Bewegungsrichtung abgelenkt. Die Eindringtiefe von ca. 5-10 mm der PE (die Ausbreitung erfolgt tropfenförmig) spielt eine wichtige Rolle in der Beurteilung der an einer EDX-Anlage angefertigten Analysen (Kapitel 4.3). Sie ist abhängig von der Ordnungszahl $(\mathrm{Z})$ der in der Probe enthaltenen Elemente und der Beschleunigungsspannung (Abbildung 49). Da diese RE allerdings aus tieferen Schichten des Tropfenvolumens aufgrund ihres hohen Energieniveaus stammen, erhält man durch sie keine genaue Abbildung der Probenoberfläche, sondern ein Bild aus den tieferen Lagen der Probe. Daher wird mit ihnen auch nicht die größtmögliche Auflösung am REM erreicht, weshalb sie zur Erzeugung von Bildern auch selten benutzt werden. Mit Sekundärelektronen hingegen werden bessere Auflösungen erzielt. SE entstehen, wenn einzelne Elektronen durch PE aus Atomen herausgeschlagen werden. Ihr Energieniveau ist kleiner $50 \mathrm{eV}$. SE, die im unteren Bereich des Tropfenvolumens erzeugt werden, werden auf ihrem Weg zur Probenoberfläche hin von der Probe absorbiert. Es gelangen also nur SE aus oberflächennahen Schichten (bei Metallen bis $1 \mathrm{~nm}$ ) zum Detektor des REM, wodurch man eine oberflächengetreuere Bildwiedergabe mit einer höheren Auflösung als mit RE erhält.
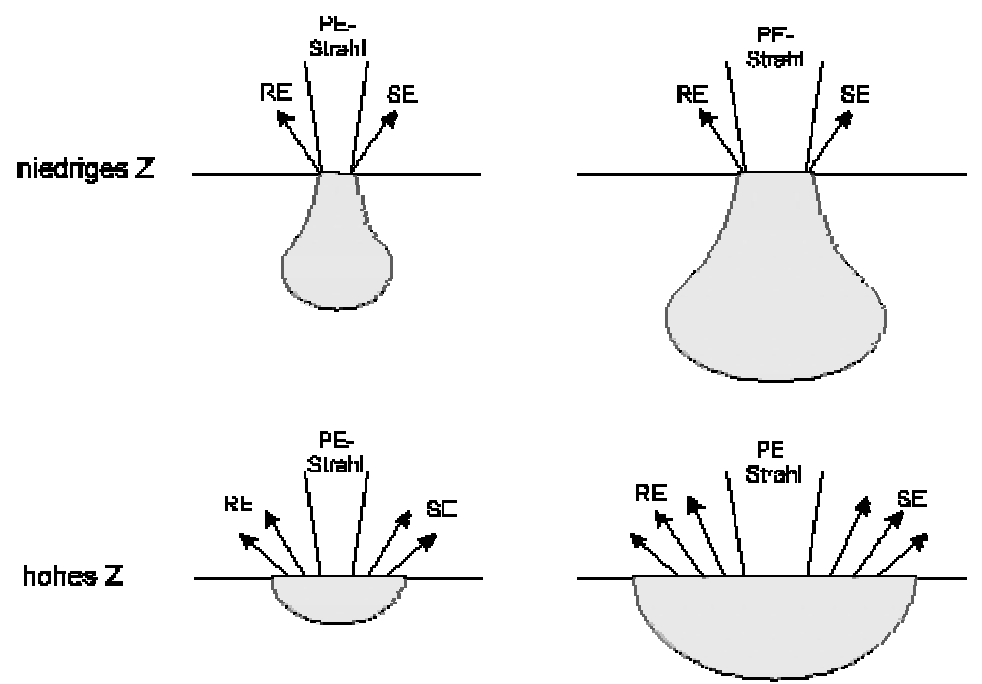

niedrigers $\mathrm{U}_{\text {. }}$

hoheri $\bigcup_{0}$

Abbildung 49: Abhängigkeit der Ausbreitung der PE innerhalb der Probe aufgrund von U0 und Z (BARKOW 1994). 


\subsubsection{FE-REM}

Rasterelektronenmikroskope mit konventioneller Kathode als Elektronenquelle erreichen hohe Auflösungen nur bei sehr hohen Beschleunigungsspannungen (25-40 kV). Daraus resultiert jedoch eine große Eindringtiefe der Primärelektronen mit dem damit verbundenen großen Wechselwirkungsvolumen in der Probe und der nicht optimalen Ortsaulösung für Rückstreuund Sekundärelektronen. Zusätzlich besteht die Gefahr, dass empfindliche Proben, hier auf mineralischer Oberfläche aufsitzendes organisches Material, durch die hohen Strahlenergien häufig verändert werden (HEINRICHS o.J.). Die bei der Untersuchung der organisch belegten Tonmineraloberflächen angestrebte hohe Ortsauflösung setzt hohe Anregungsenergien und damit eine leitfähige Beschichtung (Besputterung) mit nicht leitendenden Materialien voraus, um die in der Bildbetrachtung stark störenden Aufladungserscheinungen zu vermeiden. Mit diesen herkömmlichen Systemen ist eine Aufnahme unbeschichteter Proben mit hoher Auflösung nicht möglich. Um solches Probenmaterial mit einem Elektronenmikroskop untersuchen zu können ist es nötig ein System zu verwenden, welches mit niedrigen Beschleunigungsspannungen arbeitet. Das im SFB 468 genutzte Rasterelektronenmikroskop mit Feldemissions-Kathode (LEO Gemini 1530) ermöglicht hohe Auflösungen bis zu $3 \mathrm{~nm}$ bei $1 \mathrm{kV}$ (1 nm bei $20 \mathrm{kV}$ ) mit Vergrößerungen von 20- bis 900000-fach. Die Beschleunigungsspannungen liegen bei diesem System zwischen $200 \mathrm{~V}$ und $30 \mathrm{kV}$. Der Vorteil des verwendeten REM ist die Möglichkeit, unbehandelte Proben direkt, ohne eine vorherige Bedampfung des Objektes mit einer dünnen leitenden Schicht aus Gold - Kohlenstoff wäre im Hinblick auf die nachfolgend angestrebte Untersuchung mit einem gekoppelten EDX-System widersinnig - zu bedampfen. Dies liegt an der Fähigkeit des Systems mit sehr geringen Anregungsspannungen (ab $1 \mathrm{kV}$ ) zu arbeiten. Insbesondere die bei der Bildbetrachtung stark störenden Aufladungseffekte können bei Verwendung dieses Systems somit weitgehend vermieden werden.

\subsubsection{Präparation des Probenmaterials}

Die mit Phenolen bzw. Phenanthren belegten Tonminerale wurden sowohl als Pulver- als auch als Aggregatproben (Tonmineralaggregate ( $\mathrm{Na}^{+}$-Montmorillonit), $\varnothing$ ca. 2-3 mm) unter dem REM untersucht. Als Probenträger dienten Standardobjektträger aus Glas. Auf diese wurde etwas flüssiges „Leit-C“ aufgetragen und zu $1 \mathrm{~cm}$ großen Flächen ausgestrichen. Nach einer Trockenzeit von ca. 2 min wurde die Pulverproben auf die so präparierten Objektträger gestreut. Mit den Aggregatproben wurde in ähnlicher Weise verfahren, die einzelnen Aggregate wurden mit einer feinen Pinzette auf das Trägermaterial gesetzt und leicht angepresst. Überschüssiges, nicht auf den Objektträgern haftendes Material wurde mit Druckluft entfernt. Die Proben wurden in die Vakuumkammer des REM gegeben und diese für $2 \mathrm{~h}$ evakuiert. Die Durchführung der rasterelektronenmikroskopischen Untersuchungen wurden am im Rahmen des SFB 468 angeschafften Feldemissions-Rasterelektronenmikroskop vorgenommen. Zur elektronenmikroskopischen Aufnahme wurden das Sekundärelektronensignal verarbeitet um einen plastischen Eindruck der Oberflächenstrukturen zu erhalten. 


\subsubsection{Auswertung der FE-REM-Aufnahmen}

Das Ziel der Untersuchungen mit einem Rasterelektronenmikroskop mit einer FeldemissionsKathode war, zu überprüfen, ob die schon optisch beobachtbare Reaktion von sublimierten Phenolen auf den Oberflächen der Tonminerale zu erkennbaren Veränderungen geführt hat und eventuell eine Lokalisierung dieser Veränderungen erreicht werden kann. Dazu wurden sowohl unbelegte als auch mit Organika belegte Pulver- und Aggregatproben herangezogen und in mehreren Vergrößerungsstufen bis in den nano-skaligen Maßstabsbereich gescannt. Diese Proben wurden nicht besputtert um eine Modifikation der Probenoberfläche zu vermeiden. Die Beschleunigungsspannung lag bei 1,3 kV und der Abstand der Proben von der Kathode bei 8-9 $\mathrm{mm}$.

Eine typische REM-Aufnahme einer unbelegten Montmorillonitoberfläche repräsentiert die folgende Abbildung (50). Sie zeigt bei einer 12500-fachen Vergrößerung eine Oberfläche, die sich sowohl aus einzelnen charakteristischen Tonplättchen (Taktoide) als auch aus kompakten Agglomerationen mit Größen von $1 \mu \mathrm{m}$ bis zu mehreren Zehnern $\mu \mathrm{m}$ zusammensetzt.

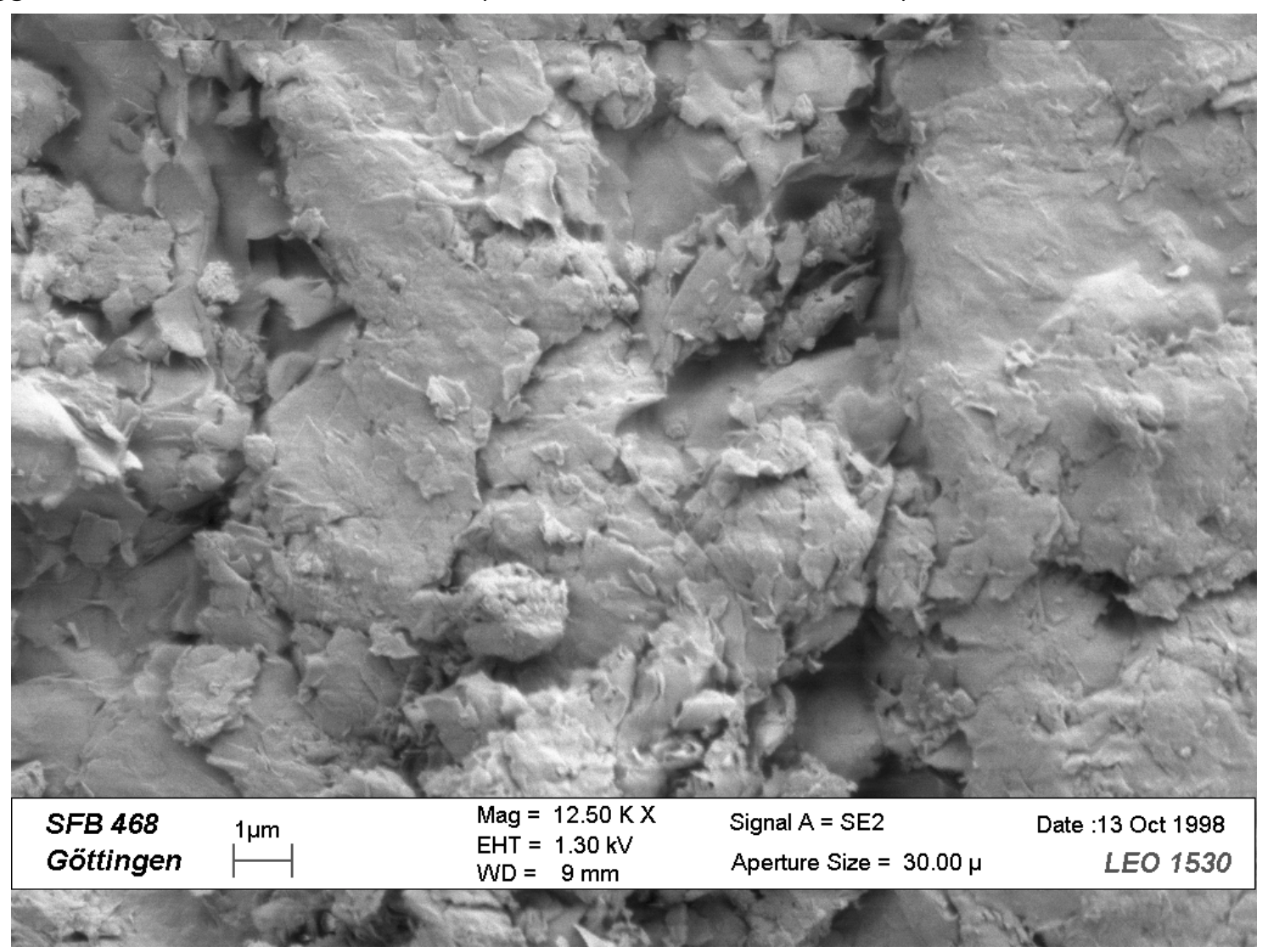

Abbildung 50: FE-REM-Aufnahme eines unbelegten Na-Montmorillonits.

Neben diesen Oberflächenmerkmalen sind auch bei höheren Vergrößerungsstufen bis in den nano-skalierten Maßstabsbereich keine zusätzlich auftretenden Objekte zu beobachten. Betrachtet man im Vergleich hierzu die REM-Aufnahme eines mit dem Phenol Brenzkatechin belegten Na-Montmorillonits (Abbildung 51), so fallen schon bei einer 7500-fachen VergröBerung, insbesondere auf der planaren Oberfläche von Tonmineralplättchen, Strukturen mit 
einer „unscharfen“ Gestalt auf, die als Schleier auf der Mineraloberfläche aufzusitzen scheinen (siehe Markierungen A).

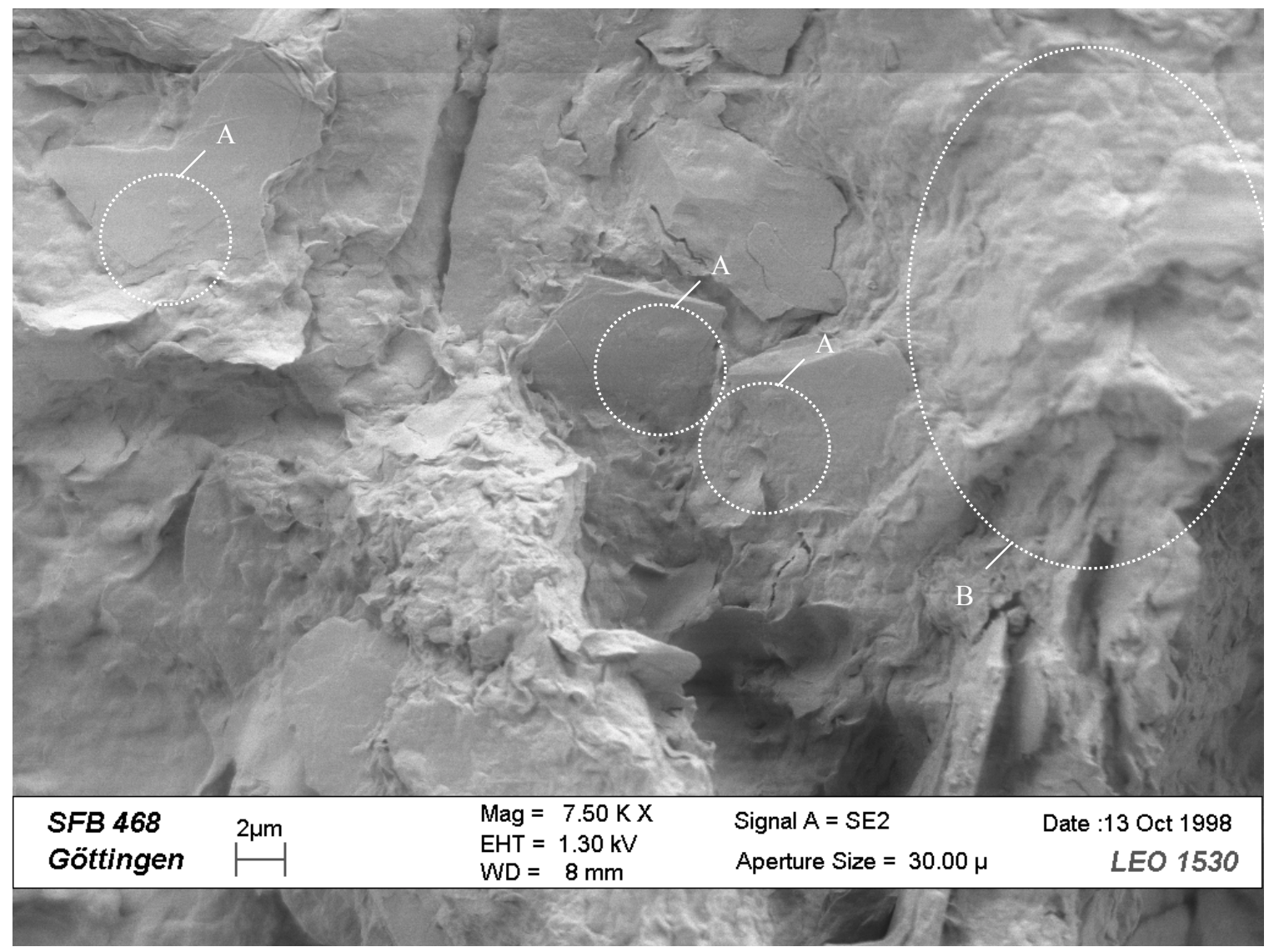

Abbildung 51: FE-REM-Aufnahme eines mit Brenzkatechin belegten Na-Montmorillonits mit schleierförmigen Objekten auf der Oberfläche (siehe Markierungen).

Bei eingehender Beobachtung eines solchen REM-Bildes fallen weitere, noch größere Struktureinheiten mit ähnlicher Gestalt auf, die weite Bereiche auf der Oberfläche in Form von Überzügen überdecken (siehe Markierung B). Diese Überzüge geben der Oberfläche eine konturarme Morphologie. Untersucht man diese hier noch recht klein erscheinenden Strukturen bei höheren Vergrößerungsstufen und führt zur Bildverbesserung eine Spreizung der Grauwertverteilung des Bildes sowie eine Filterung des Bildrauschens durch, so erhält man die folgenden Aufnahmen mit einer deutlich verbesserten Darstellung des Informationsgehaltes. Als zentrales Objekt wurde das aus mehreren Taktoiden aufgebaute Tonmineralplättchen aus der Bildmitte der vorangegangenen Aufnahme gewählt. Nach der Kontrastspreizung sind „blumenkohl“-artige Strukturen, die auf der Oberfläche des Tonminerals aufsitzen deutlich zu erkennen. 


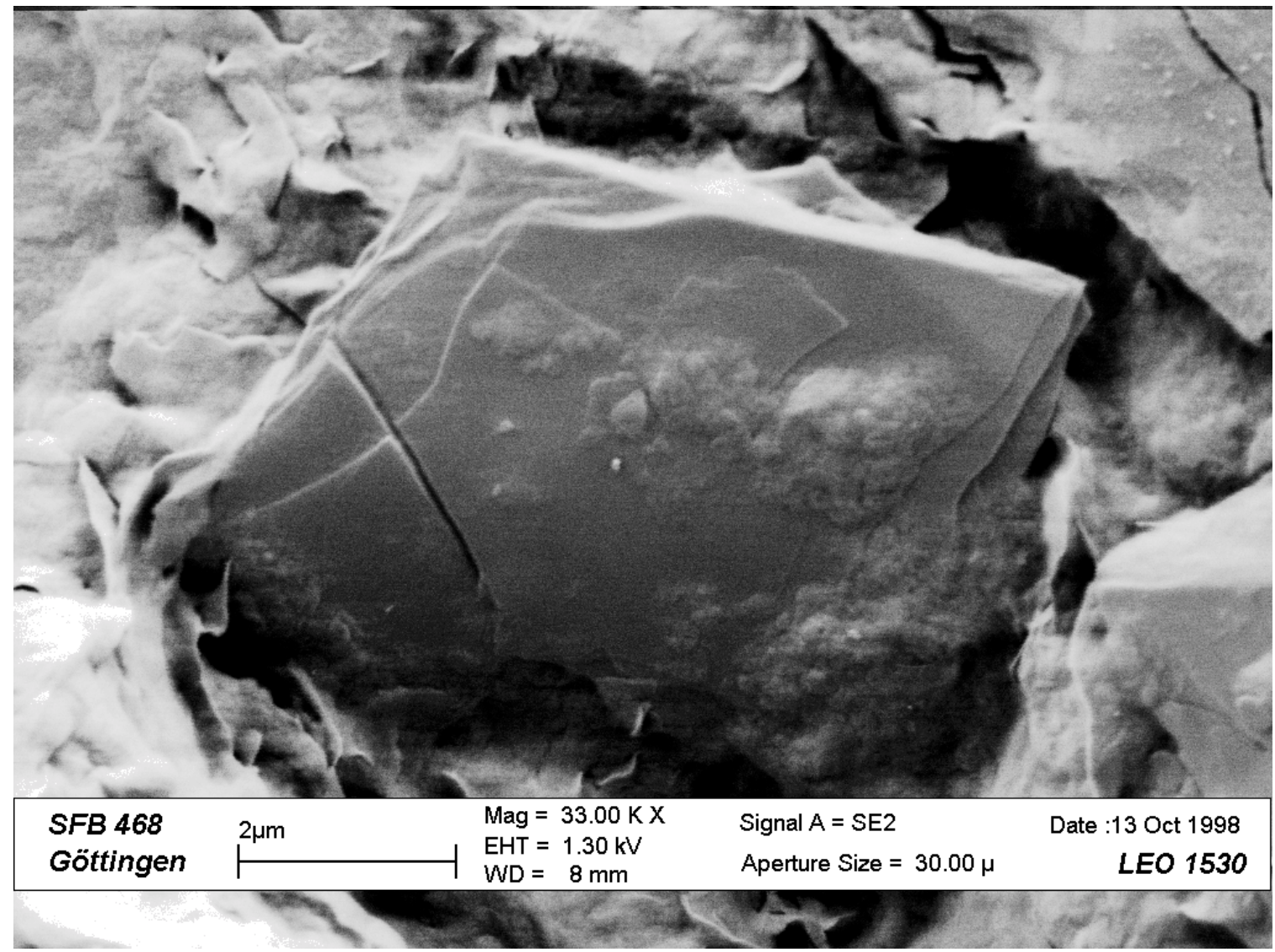

Abbildung 52: Ausschnittvergrößerung (Vergrößerungsstufe 33000-fach).

Aufgrund der Aufnahme eines Sekundärelektronenbildes werden diese Oberflächenstrukturen sehr plastisch wiedergegeben. Anhand des Bildmaßstabes ist zu erkennen, dass es sich bei den auftretenden Strukturen um recht große Objekte zwischen etwa 0,5 $\mu \mathrm{m}$ und $2 \mu \mathrm{m}$ handelt. Diese treten jedoch nicht nur, wie in Abbildung $52 \mathrm{zu}$ beobachten auf den Flächen der Tonminerale auf, sondern sind in wesentlich größerem Umfang an den Randbereichen der Minerale zu finden. Dort können sie durch die Überdeckung unterliegender mineralischer Oberflächenstrukturen eindeutig als Überzüge identifiziert werden. Im Gegensatz zur recht einförmig und regelhaften Oberfläche der Tonminerale zeigen diese Strukturen einen sehr heterogenen Formenschatz, der von den beschriebenen „,blumenkohl“-artigen Formen bis hin zu wattigen, schleierartigen Gespinsten zwischen einzelnen Tonmineralpartikeln reicht. Das gehäufte Auftreten an den Randbereichen der Minerale gibt erste Hinweise darauf, dass die Kanten von Tonmineralen bevorzugte Sorptionsplätze der hier gebildeten organischen Überzüge sind. 


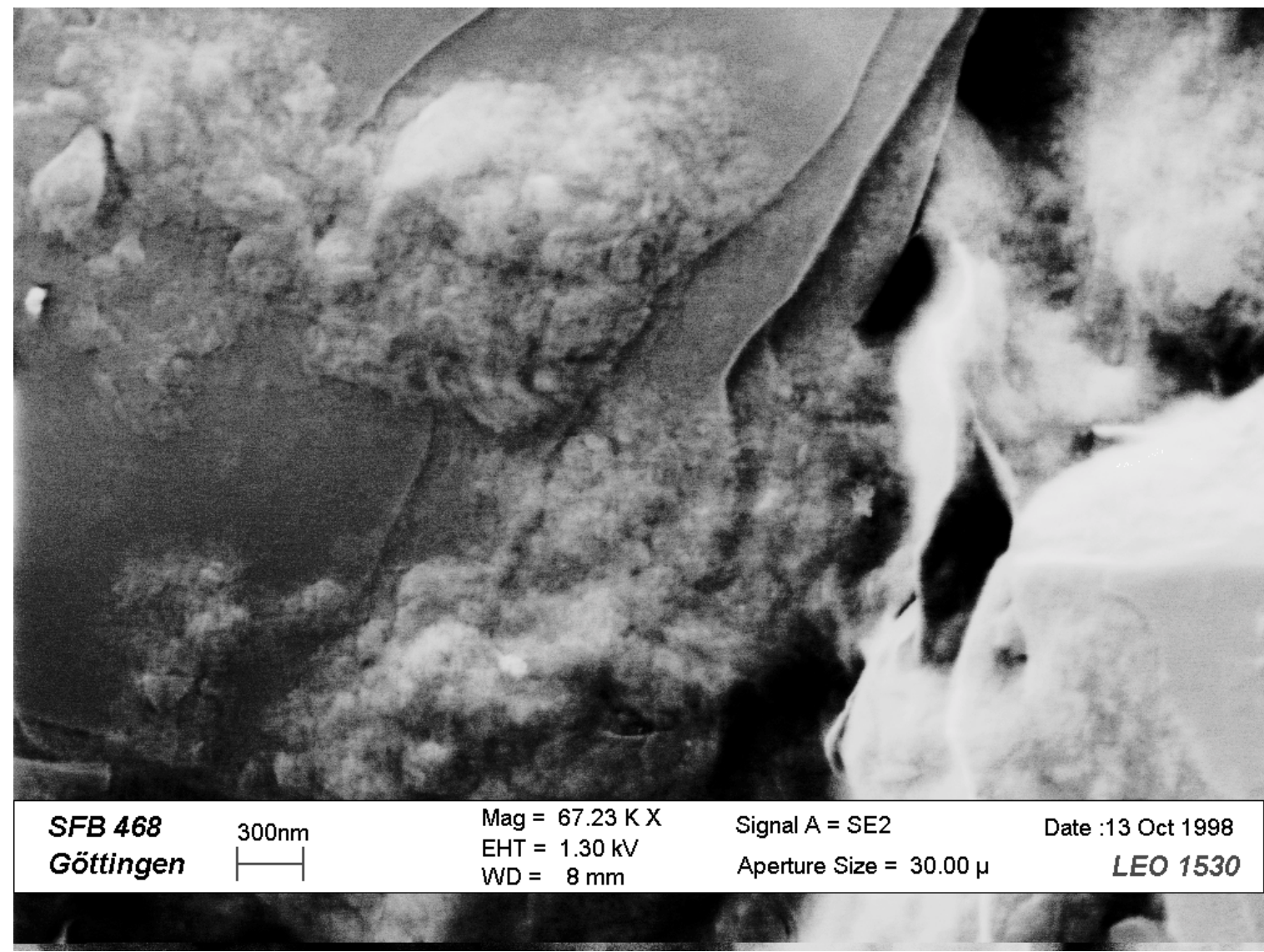

Abbildung 53: FE-REM-Aufnahme des mit Brenzkatechin belegten Montmorillonits (Ausschnittvergrößerung, Vergrößerungsstufe 67000-fach).

Die hochauflösende REM-Aufnahme (Abbildung 53) verdeutlicht nochmals den bereits beschriebenen schleierartigen Charakter dieser Strukturen mit gleichzeitigem Zugewinn an weiteren Informationen. So ist in diesem Bild zu erkennen, dass die aufgrund der Topographie der Oberfläche (Zuwendung zum Detektor) sehr stark reflektierenden Bereiche in der rechten Bildhälfte ebenfalls flächig von Überzügen bedeckt sind. Nochmals verstärkt wird auch der Eindruck, dass sich die Überzüge vorwiegend an den Kanten der Tonminerale gebildet haben und auf den besser einsehbaren Tonmineralflächen eine deutlich kleinere Zahl von Aufwachsungen mit geringeren Umfängen zu beobachten sind. Diese Beobachtung wird auch durch den in Kapitel 2.1 dargelegten Aufbau der Tonminerale gestützt, der den Tonmineralkanten aufgrund der dort besser zugänglichen Aluminium- und weiteren Metallatomen der inneren Mineralstruktur sowie der dort in größerem Umfang adsorbierten Metalloxide eine deutlich höhere Aktivität und Bindungskapazität aufweisen. Zudem spricht für die Adsorption an den Randbereichen von 2:1-Tonmineralen, dass deren Zwischenschichten aufgeweitet werden können und so ein partielles Eindringen organischer Substanz in den Zwischenschichtraum ermöglicht wird (Abbildung 5). Zur Stützung dieser Thesen und Beobachtungen sei auf die nachfolgend durchgeführten Untersuchungen mittels elektronendispersiver Röntgenanalytik in Kapitel 4.3 verwiesen. 
Untersucht wurden weiterhin die Oberflächen von Montmorilloniten, die mit dem Phenol Pyrogallol belegt wurden. Auch hier konnten, wie im Falle der Belegung mit Brenzkatechin, Überzüge auf den mineralischen Oberflächen beobachtet werden. Diese sind im REM-Bild (Abbildung 54) sehr deutlich in der Bildmitte zu erkennen und insbesondere dort, wo sich diese Überzüge auf die planaren Oberflächen der Tonmineralplättchen erstrecken (siehe Markierung A). Noch eindrücklicher stellen sich auf dieser Probe die gebildeten Überzüge auf der Oberfläche eines Minerals, vermutlich Carbonat (siehe Markierung B) dar, das sich durch seinen charakteristischen Habitus deutlich aus seiner Umgebung abhebt. In der unteren Bildmitte ist auf einem, ebenfalls als Verunreinigung auftretenden Fremdmineral (Markierung C) ein leichter, schleierartiger Überzug festzustellen.

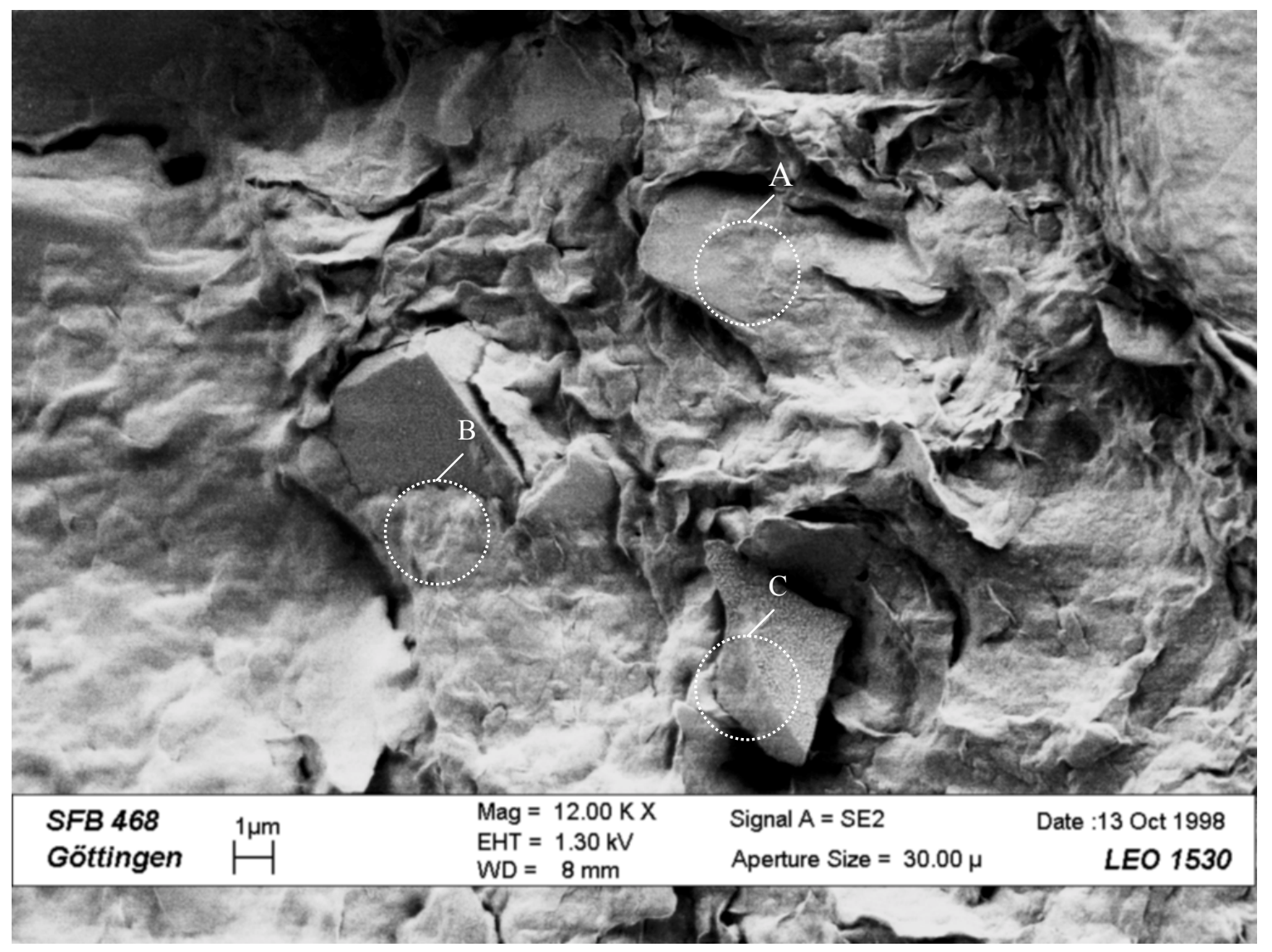

Abbildung 54: Übersichtsaufnahme einer mit Pyrogallol belegten Aggregatprobe.

Betrachtet man den unter B markierten Ausschnitt in einer höheren Vergrößerungsstufe $(65000 \times)$, so erhält man den gleichen optischen Eindruck der Struktur dieser Überzüge wie schon in der vorab beschriebenen Untersuchung der mit Brenzkatechin belegten Montmorillonitoberflächen. Sie können eindeutig als auf der Oberfläche aufsitzend identifiziert werden man erhält den optischen Eindruck, dass an der Grenze Überzug-Mineral eine „Beschattung“ eintritt. Die Struktur der Überzüge ist als heterogen zu bezeichnen, d.h. es wird kein Überzug von gleichmäßiger Mächtigkeit gebildet, sondern es sind Bereiche unterschiedlicher Dicke zu beobachten. 


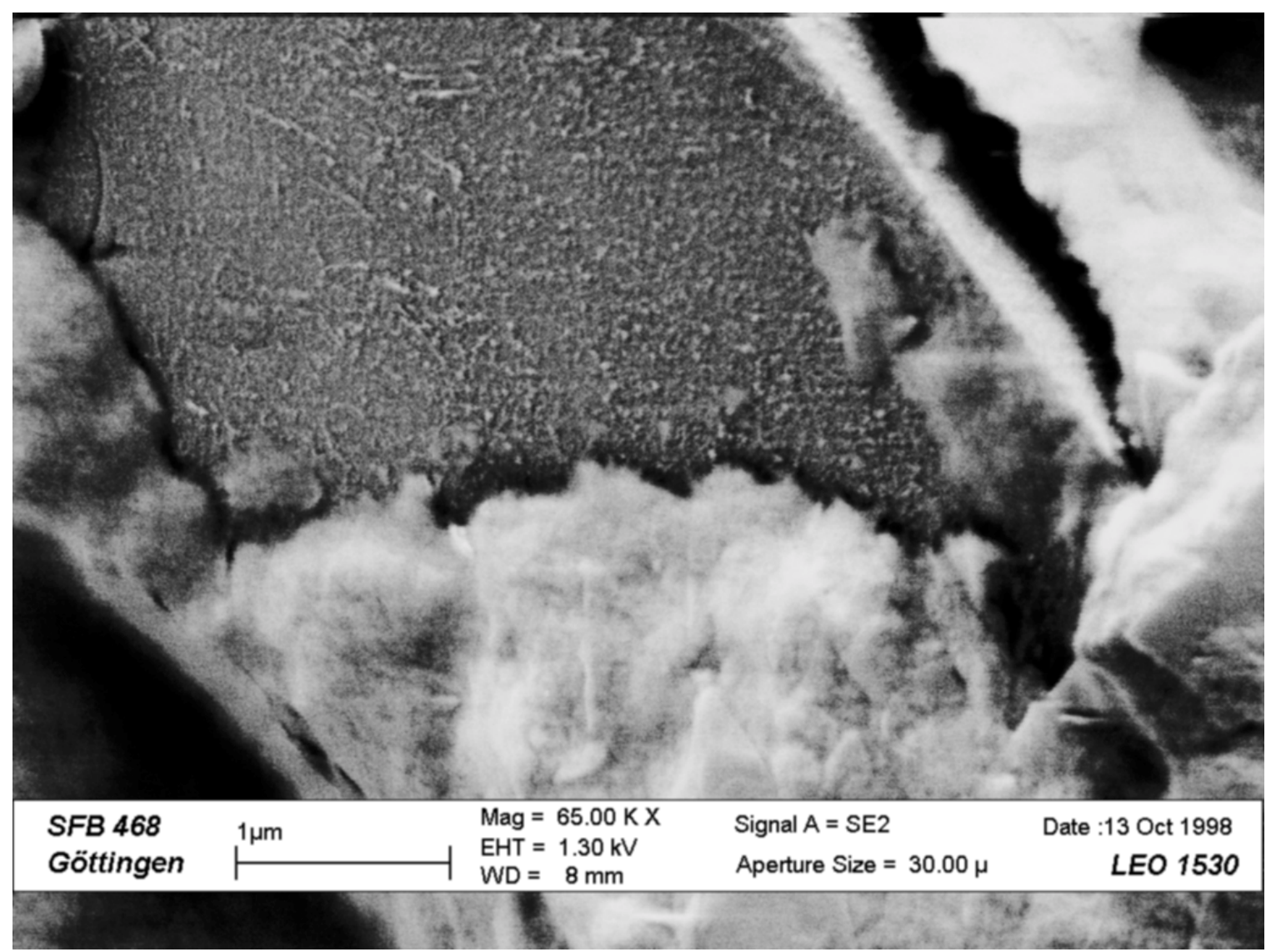

Abbildung 55: Ausschnittvergrößerung der FE-REM-Aufnahme (Bildmitte).

Das die Überzüge nicht nur stellenweise vorkommen, sondern auch teilweise eine flächendeckende Bedeckung der Tonmineraloberfläche stattfindet, ist in Abbildung 56 zu beobachten. Im markierten Bereich (B) ist zu erkennen, dass sich die Strukturen über das ganze Flächensegment erstrecken, was leicht an der bekannten „blumenkohl“-artigen Gestalt der Oberfläche erkannt werden kann. Es ist davon auszugehen, dass die Belegung des Montmorillonits mit Pyrogallol somit nicht nur zu einer Überdeckung des Tons mit dem Phenol geführt hat. Die weiträumige Bedeckung der anorganischen Oberfläche mit den charakteristischen Überzügen sowie das Auftreten von vereinzelten Strukturen auf den Flächen der Tonminerale spricht für einen Polymerisationsprozess, der zur Bildung von Reaktionsprodukten geführt hat, die in ihrer chemischen Zusammensetzung und in ihrem Aufbau bisher noch nicht weiter identifiziert wurden. 


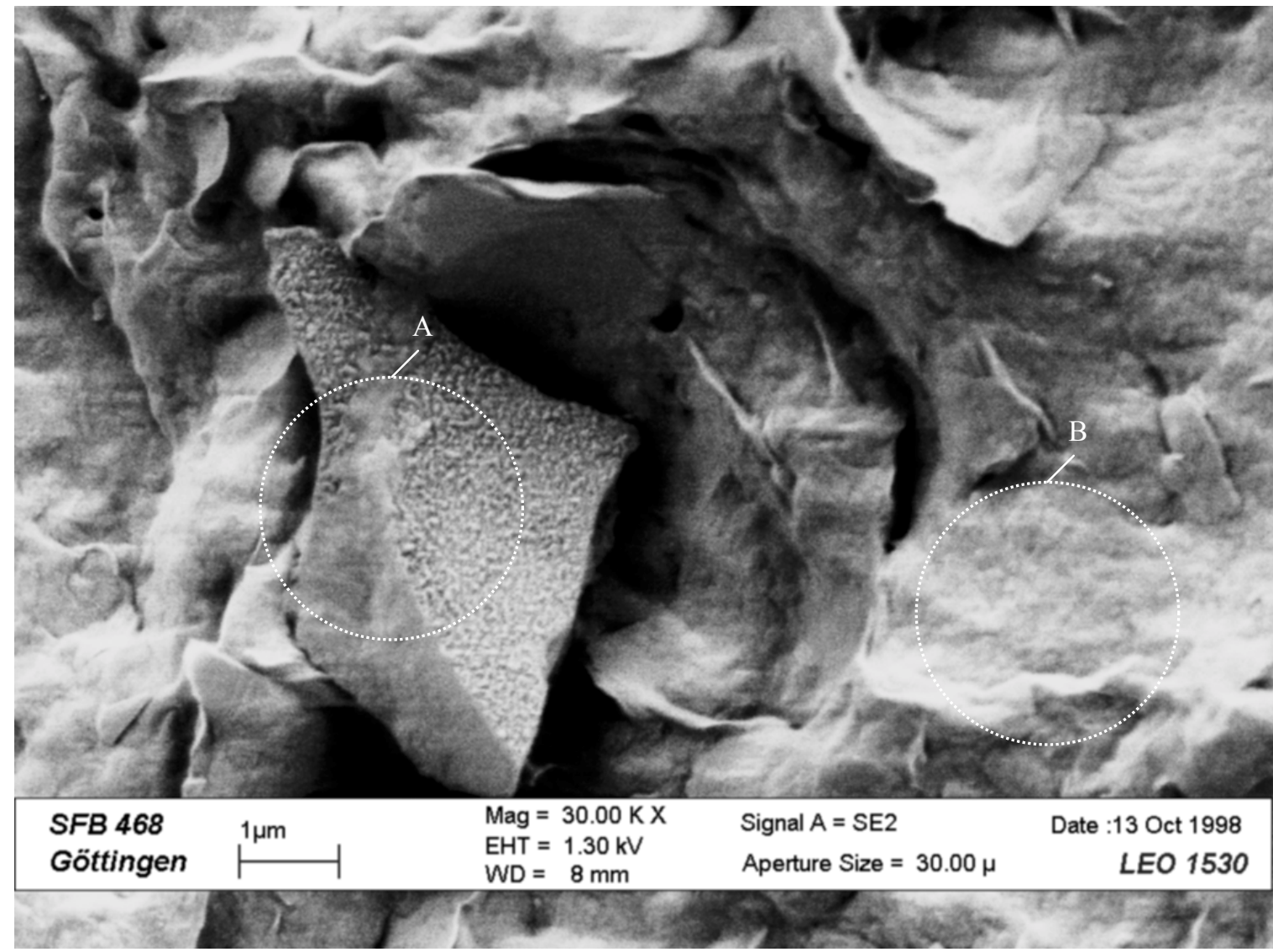

Abbildung 56: Ausschnittvergrößerung der FE-REM-Aufnahme (rechte Bildhälfte).

Die Auswertung von Probenmaterial, welches mit einem weiteren Phenol, i.e. 2,6Dimethylphenol, belegt war, erbrachte durch die Untersuchung mittels FE-REM keine darstellbaren Resultate. Auch hier ist von der Bildung von Polymerisationsprodukten des Phenols auszugehen, jedoch konnten keine, der Belegung mit Brenzkatechin bzw. Pyrogallol vergleichbaren Strukturen gefunden werden. Die Belegung von Tonmineraloberflächen mit dem polyzyklischen aromatischen Kohlenwasserstoff Phenanthren allein bzw. in Kombination mit einem Phenol führte leider ebenfalls zu keinen verwertbaren Ergebnissen. Es sollte jedoch aus diesem Grunde daraus nicht geschlossen werden, dass bei Belegung von Tonmineralen mit diesen Substanzen keine derartigen Strukturen gebildet werden können. Als Grund für die negativen Resultate kann auch eine unzureichende Probenpräparation nicht ausgeschlossen werden, da gerade bei diesen Proben stark störende Aufladungseffekte der Probenoberfläche eine Untersuchung mit hoher Ortsauflösung verhinderten. Da ein Besputtern der Proben mit Gold zur Vermeidung von Aufladungen wahrscheinlich zu einer Artefaktbildung auf der Probenoberfläche bzw. eine Störung der optischen Wahrnehmung der Oberflächenstrukturen geführt hätte, wurde auf eine weitere Untersuchungsreihe verzichtet. 


\subsubsection{Diskussion der rasterelektronenmikroskopischen Untersuchungen}

Es konnte nachgewiesen werden, dass die Belegung von Tonmineralen mit Organika, hier einfache aromatische Verbindungen, wie das Brenzkatechin und das Pyrogallol zur Bildung von neuen Strukturen auf der Oberfläche geführt hat. Da diese Überzüge nur bei organisch belegten Proben zu beobachten waren, ist somit daraus zu schließen, dass es sich hier um organische Strukturen handelt. Diese Strukturen überziehen die anorganische Oberfläche teilweise flächendeckend, treten aber auch als vereinzelte Formen auf. Auffällig erscheint bei der Betrachtung der gebildeten Überzüge, dass diese gehäuft an den Kanten der Tontaktoide lokalisiert sind. Diese Korrelation kann auf die bevorzugte Adsorption organischer Substanzen an Bruchkanten, Ecken, Verwerfungen etc. aufgrund der dort zugänglichen Zwischenschichten (ZIECHMANN 1996), Aluminium- und weiteren Metallatomen der Mineralstruktur der Tonminerale sowie durch bereits adsorbierte Metalloxide- und -hydroxide (ZIECHMANN \& MÜLLER-WEGENER 1990) zurückgeführt werden.

Die beobachteten Überzüge weisen einerseits eine heterogene innere Struktur auf, die auf ihre unterschiedliche Mächtigkeiten zurückzuführen sind. Andererseits können auch Flächen mit einer relativ gleichmäßigen Dicke festgestellt werden. Der unregelmäßige Aufbau der Überzüge resultiert für den Betrachter in einem plastischen Eindruck dieser Coatings mit einer charakteristischen „blumenkohl“-artigen, wattigen bis zu einer schleierförmigen Morphologie. Die Größe der entstandenen Strukturen reicht von mehreren Zehner Nanometern bis zu einigen Mikrometern Durchmesser.

Aus der Form der entstandenen Überzüge können Rückschlüsse auf eventuell abgelaufene Prozesse und Reaktionen gezogen werden. Geht man davon aus, dass eine anorganische, chemisch inerte Oberfläche mit Organika durch Sublimation belegt wird, so müsste die organische Substanz, hier die Phenole, homogen über die ganze Mineraloberfläche verteilt sein. Dieser These ist aufgrund der gewonnenen Untersuchungsergebnisse jedoch zu widersprechen. Die Häufung des Auftretens der organischen Überzüge an diskreten Orten und ihre unregelmäßige Verteilung über die Oberfläche spricht nicht nur für die bevorzugte Adsorption an bestimmten Bereichen der Tonminerale, sondern hiermit kann diesen Stellen eine Aktivität zugeschrieben werden. Dieses Ergebnis wird auch durch Arbeiten von SCHULTEN \& SCHNITZER (1997) gestützt: durch Modellierung von Huminstoffen konnte gezeigt werden, dass mineralische Oberflächen nicht vollständig von organischer Substanz bedeckt sind. Die enormen Größen der gebildeten Strukturen, geht man einmal von den Massegewichten der verwendeten Ausgangssubstanzen (Brenzkatechin: $110 \mathrm{u}$, Pyrogallol: $127 \mathrm{u}$ ) aus, zeigen, dass eine heftige Reaktion der Phenole an der Tonmineraloberfläche eingetreten ist. Da als Reaktanten nur die angesprochenen Phenole zur Verfügung standen, ist somit von einer Polymerisation dieser Ausgangssubstanzen auszugehen. Die Reaktion des Brenzkatechins bzw. des Pyrogallols führte zur Bildung hochpolymerer Reaktionsprodukte der betreffenden Aromaten. Die eingangs aufgestellte Hypothese, dass auf Tonmineraloberflächen abgelegte einfache organische Verbindungen durch die Aktivität von Tonmineralen einer abiotisch induzierten Ka- 
talyse unterliegen, die zur Bildung von polymerer Reaktionsprodukte führt, konnte somit bestätigt werden. Als Konsequenz daraus ist gleichfalls festzustellen, dass sich diese einfachen aromatischen Verbindungen, die in dieser Arbeit als Modellsubstanzen für Huminsäurebausteine angesprochen und eingesetzt wurden, entsprechend den in der Literatur dargestellten Bildungsmechanismen der Huminstoffgenese (i.e. Polyphenol-Theorie - STEVENSON 1994) verhalten haben. Durch die elektronenmikroskopischen Untersuchungen konnten somit Informationen über die Gestalt und Verteilung der schon nach der Belegung mit Phenolen optisch sehr anschaulichen Reaktionsergebnisse gewonnen werden und das vermutete Aufwachsen von Modellhuminstoffen auf Tonmineraloberflächen (Abbildung 53) beobachtet werden (BIRKEL et al. 2000b, BIRKEL et al. 2000c).

Aus diesen Beobachtungen heraus kann weiterhin angenommen werden, dass es durch Bildung von polymeren Organika zu kovalenter Verknüpfung von Tonteilchen bzw. -partikeln kommt. Weiterhin zeigen die Aufnahmen, dass die Huminstoffbildung nicht gleichmäßig verteilt über ein ganzes Tonmineralteilchen erfolgt, sondern Reaktionen nur an diskreten Stellen ablaufen. Dies steht im Widerspruch zum gängigen Modell, dass die Tonminerale in den obersten Horizonten der Böden gleichmäßig von einer Schicht organischen Kohlenstoffs überzogen sind (SCHACHTSCHABEL et al. 1992).

\subsection{Energiedispersive Röntgenanalytik}

Die in Kapitel 4.2 dargestellten hochaufgelösten elektronenmikroskopischen Aufnahmen von mit Organika belegten Tonmineralproben (Pulver- und Aggregatpräparate) zeigen etwa 1-2 $\mu \mathrm{m}$ große Objekte auf der Oberfläche von Tonmineralplättchen. Diese Strukturen weisen einen wattigen, blumenkohlartigen Habitus auf und sind nur bei den mit Phenolen belegten Proben zu beobachten, während bei dem mit Phenanthren versetzten Material keine derartigen Überzüge zu erkennen sind. Aus diesen vergleichenden Untersuchungen wurde daher abgeleitet, dass die Sublimation der Organika auf die Oberfläche von Tonmineralen und deren Aktivität zu einer Veränderung der Phenole hin zu Reaktionsprodukten mit einer sehr hohen Molekülgröße geführt haben kann. Der Nachweis, dass es sich bei diesen Strukturen tatsächlich um organische Coatings handelt, konnte mit den zur Verfügung stehenden Verfahren bislang nicht erbracht werden.

Energiedispersive Röntgenanalytik (EDX) eignet sich sehr gut zur Charakterisierung organischer Überzüge auf mineralischen Oberflächen (WALZAK et al. 1998). Aus diesem Grunde wurden in Zusammenarbeit mit der Abteilung Geologie des Fachbereichs Geowissenschaften der Universität Trier an einem Rasterelektronenmikroskop (Zeiss LEO 435VP) mit gekoppeltem EDX-System (Link eXL) Untersuchungen durchgeführt, um mit Hilfe dieses kohlenstoffsensitiven Systems nachzuprüfen, ob es sich bei den zu beobachtenden Strukturen tatsächlich um organische Strukturen handelt. 


\subsubsection{Theorie}

EDX ist ein Verfahren, das zur Analyse der oberflächennahen Bereiche von Festkörpern oder zur Charakterisierung von dünnen Schichten eingesetzt wird (FIEDLER \& EVERTS 1975). Die Informationstiefe des Verfahrens $(\mu \mathrm{m})$ liegt dabei wesentlich höher als bei den elektronen- und massenspektrometrischen Verfahren (nm). Neben der bildhaften Darstellung der Oberfläche von Proben kann gleichzeitig die elementare Zusammensetzung ausgewählter Bereiche bestimmt werden (Abbildung 57). Diese Technik ist sensitiv für Elemente ab 0,1 Gew.$\%$. Die Eindringtiefe reicht von 0,2 bis $8 \mu \mathrm{m}$, sie ist abhängig von der Energie des verwendeten Elektronenstrahls und der durchschnittlichen Atomzahl der Probe (WALZAK et al. 1998).

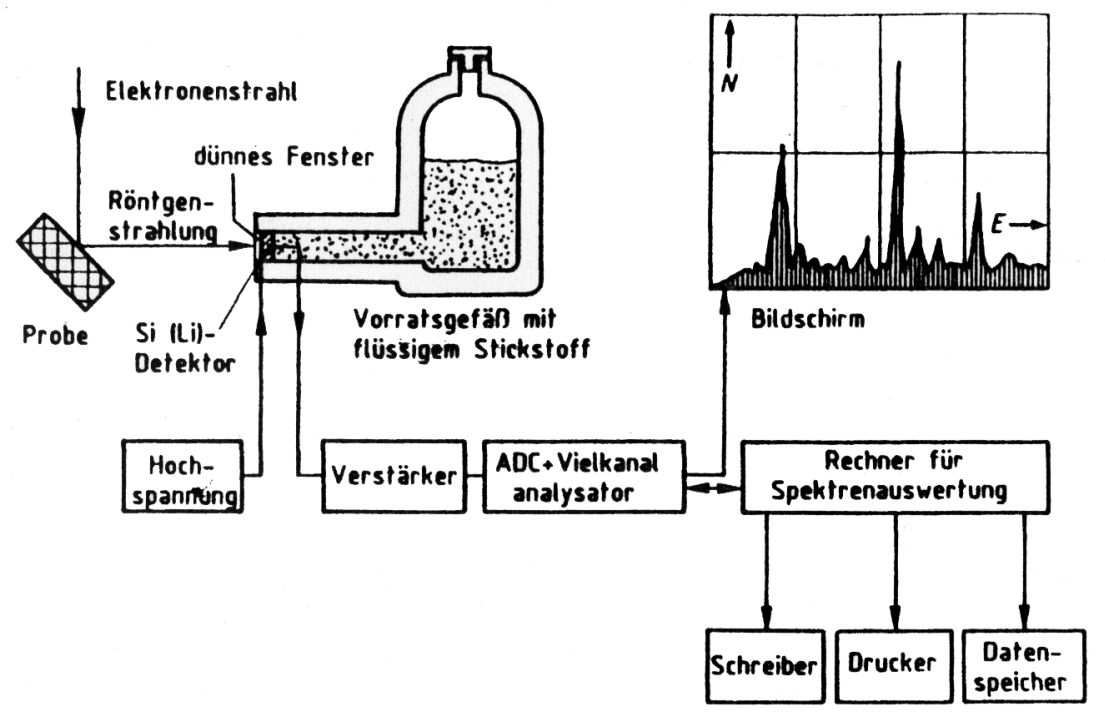

Abbildung 57: Schematischer Aufbau eines EDX-Systems (SCHMIDT 1994).

Beim EDX-Verfahren emittiert die zu untersuchende Probe, welche mit energiereichen Primärelektronen bestrahlt wird, charakteristische Röntgenstrahlung. Die Primärelektronen stoßen Elektronen aus kernnahen Schalen der Probenatome heraus. In die entstandenen Lücken fallen Elektronen aus weiter vom Atomkern entfernt liegenden Elektronenschalen. Die Energiedifferenz zwischen den beiden hierbei beteiligten Elektronenschalen kann als Konkurrenzprozess zur Augerionisation auch Röntgenstrahlung emittiert werden und ist für jedes Element charakteristisch. Die Auswertung der im Röntgenspektrum enthaltenen Spektrallinien erlaubt es, die Elementzusammensetzung der Probe zu identifizieren und über die Intensität auch zu quantifizieren. Hierzu wird die Röntgenstrahlung hinsichtlich ihrer Energie analysiert und die jeweilige Intensität der Spektrallinien gemessen (Abbildung 58). EDX wird häufig mit einem REM bzw. TEM kombiniert. Durch die Rasterung eines fein fokussierten Primärelektronenstrahls kann die Elementverteilung auf der Probenoberfläche mit hoher Ortsauflösung abgebildet werden (SCHMIDT 1994). 


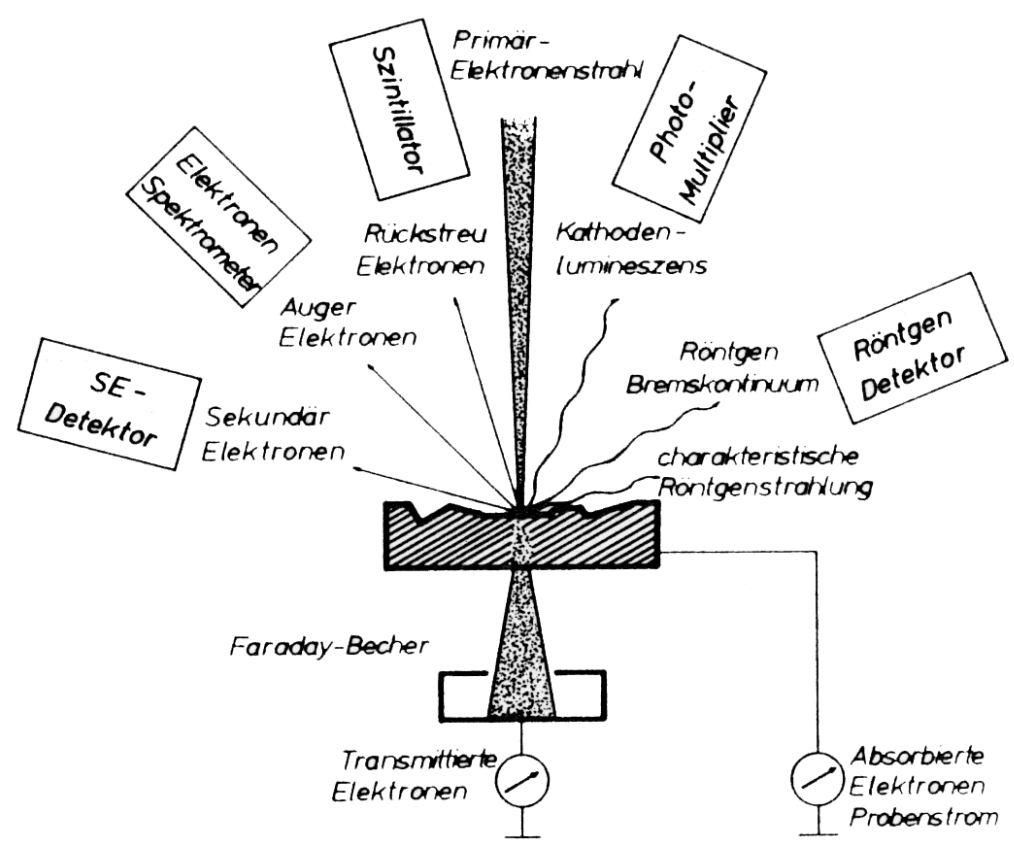

Abbildung 58: Prinzip der Röntgenmikrobereichsanalyse (SCHMIDT 1994).

Die Probe wird mit einem sehr fein gebündelten Strahl energiereicher Elektronen (15-25 keV) beschossen, wie er z.B. in einem Raster-Elektronenmikroskop zur Abbildung verwendet wird. Diese Elektronen dringen zum Teil tief in die Probe ein und können mit den Hüll-Elektronen von Atomen in tieferen Lagen der Probe (bis einige Mikrometer) in Wechselwirkung treten. Es kommt zur Freisetzung von Röntgenstrahlung. Dabei unterscheidet man die RöntgenBremsstrahlung und die charakteristische Röntgenstrahlung. Bei letzterer ist die Energie der Strahlung charakteristisch für das Atom, aus dem das Röntgenquant emittiert wurde. Separiert man die Röntgenstrahlen mit einem Energie-Spektrometer und misst ihre Intensität bei den jeweiligen Energien, so liefert jeweils die Strahlungsenergie das Indiz für die Art des Atoms und die Intensität ein Maß für die Konzentration, mit der die Atomart in der analysierten Probenschicht vertreten ist (BARKOW 1994). Man erhält einerseits eine qualitative Analyse über die Art der Elemente und ihre ortsaufgelöste Verteilung in der Probe. Andererseits kann über die Konzentration der Elemente quantitative Messungen durchgeführt werden. Die Nachweisempfindlichkeit für die Elemente nimmt aus zwei Gründen von leichten zu schweren Elementen kontinuierlich zu: Die Wahrscheinlichkeit für die Aussendung von chararakteristischer Röntgenstrahlung nimmt, ebenso wie ihre Durchdringungsfähigkeit und damit Nachweiswahrmöglichkeit, mit der Ordnungszahl zu. Der Nachweis von leichten Elementen (Kohlenstoff, Sauerstoff) in kleinen Konzentrationen ist schwierig und quantitativ unsicher. 


\subsubsection{Prinzip der EDX-Analyse}

EDX-Systeme ermöglichen in relativ kurzer Zeit (ca. 1-5 Minuten) eine Aussage über die chemische Zusammensetzung von Probekörpern. Um eine Energie-DispersiveRöntgenmikrobereichsanalyse durchführen zu können wird ein Gerät zur Erzeugung eines Elektronenstrahls benötigt. Daneben entsteht aber auch Röntgenstrahlung, die durch die Wechselwirkung von Elektronen mit Materie hervorgerufen wird. Diese Röntgenstrahlung besitzt eine für jedes chemische Element spezifische Energie, welche zur Identifikation der in einer Probe enthaltenen Elemente herangezogen werden kann. Bei einer EDX-Analyse ist nun hauptsächlich die direkte Anregung der Röntgenstrahlung von Interesse, die sich weiterhin in die unerwünschte Bremsstrahlung und in die für jedes Element charakteristische Röntgenstrahlung unterteilen lässt. Um die Vorgänge der Signalentstehung in der Probe verständlicher darstellen zu können, wird im folgenden kurz auf den Atomaufbau eingegangen.

In seinem Atommodell postulierte Bohr eine Kreisbewegung der Elektronen um den Atomkern, zur Erklärung des Gleichgewichts der Kräfte. Diese Theorie erfährt aber ihre Grenzen in der Folgerung, dass bei einer tatsächlich kreisförmigen Bewegung der Elektronen um den Atomkern diese als elektrische Ladungsträger ständig elektromagnetische Energie abstrahlen müssten und schließlich doch, aufgrund dieses Ungleichgewichtes, unweigerlich vom Kern angezogen werden würden. Die Widersprüche im Bohr'schen Modell konnten mit der Einführung mathematischer Modelle durch de Broglie, Heisenberg und Schrödinger gelöst werden. Aufgrund der besseren Darstellbarkeit wird hier bei der Erklärung der Signalentstehung auf das Atommodell nach Bohr zurückgegriffen. Nach diesem Atommodell können die Elektronen nur ganz bestimmte Energieniveaus besetzen, wobei jede Schale einem bestimmten Energieniveau entspricht. Das bedeutet, dass die Elektronen eines Atoms nicht jeden beliebigen Abstand vom Kern einnehmen und diese Energieniveaus als Schalen verstanden werden können auf denen sich die Elektronen bewegen. Weiterhin ist das Energieniveau der ersten, kernnächsten Schale am niedrigsten. Diese Energieniveaus sind durch die Periodennummer, die sogenannte Hauptquantenzahl grob festgelegt. Die einzelnen Elektronen einer Schale unterscheiden sich geringfügig in ihrer Energie. Um deren energetischen Niveauunterschied eindeutig zu definieren, kann man auf die Nebenquantenzahlen zurückgreifen. Die Energieunterschiede der einzelnen Elektronen einer Schale werden von der EDX-Anlage intern ermittelt (BARKOW 1994). Daraus kann gefolgert werden, dass ein Atom aus einem Kern und einer aus Schalen, auf denen die Elektronen verteilt sind, aufgebauten Hülle besteht (Abbildung 59). 


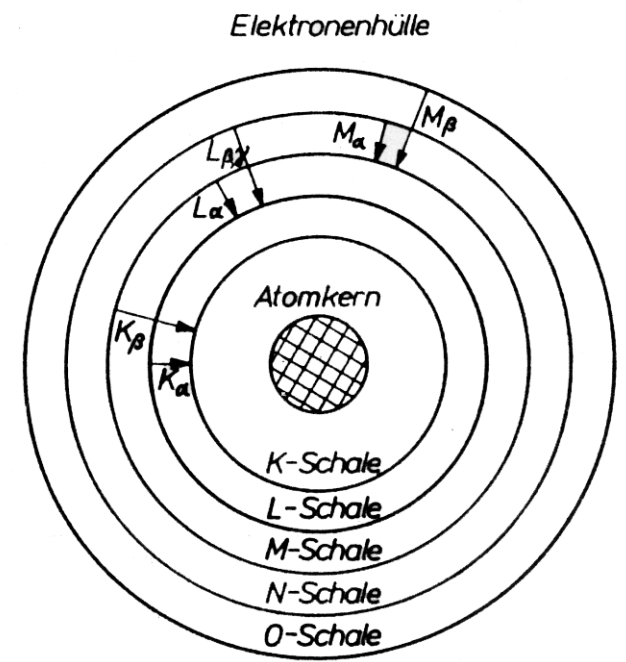

Abbildung 59: Schematisches Atommodell nach Bohr (SCHMIDT 1994).

Die Bremsstrahlung kommt durch die Abbremsung der Primärelektronen (PE) in der Probe durch das elektrische Feld des Atomverbandes zustande. Das elektrische Feld welches diese Bremsbeschleunigung bewirkt wird durch die Coulomb'schen Bindungskräfte der Atomkerne und ihren zugehörigen Elektronen hervorgerufen. Durchquert nun ein PE mit einer bestimmten Ausgangsenergie $E_{\max }\left(E_{\max }\right.$ entspricht $E_{\text {kin }}$ und wird durch die Beschleunigungsspannung erzeugt) dieses Feld, so wird ihm Energie entzogen, wodurch sich seine Geschwindigkeit verringert. Diese Verlustenergie wird zu ca. 99 \% in Wärme- und etwa $1 \%$ in Röntgenstrahlung umgewandelt, wobei die Energie der so entstandenen Röntgenphotonen der Verlustenergie der abgebremsten PE entspricht (Abbildung 60).

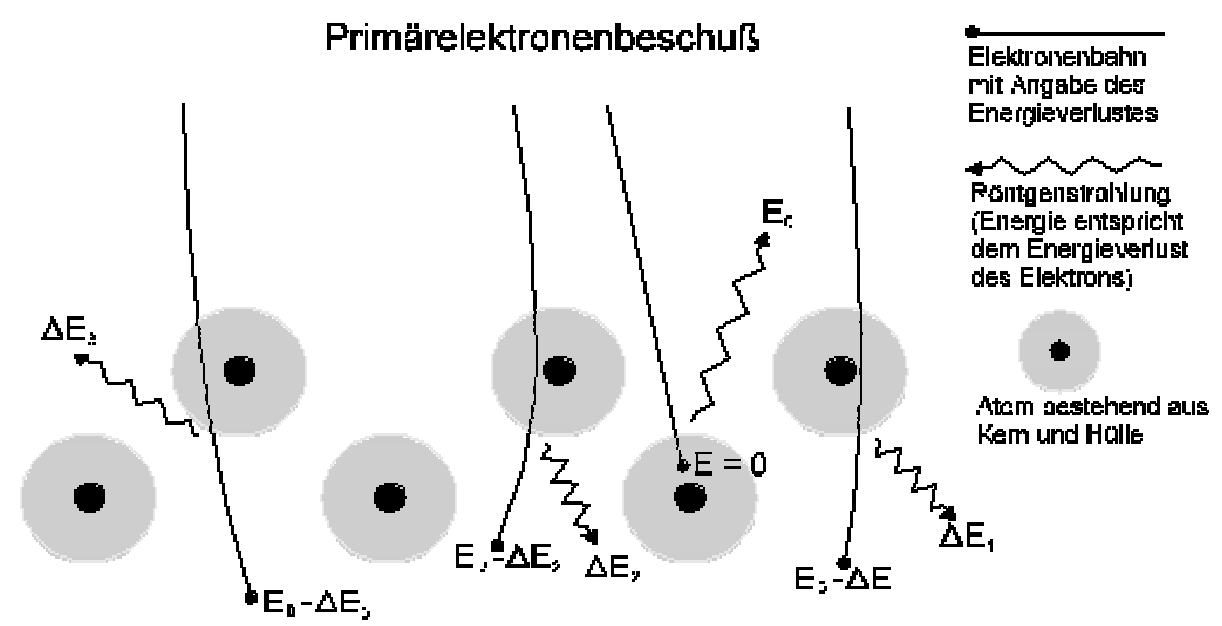

Abbildung 60: Schematische Darstellung des Elektronenbeschusses (BARKOW 1994).

Da bei Eintritt in die Probe nicht jedes PE gleich stark abgebremst wird, entsteht ein kontinuierliches Röntgenspektrum, dessen unterste Energiegrenze 0 und die Oberste $E_{\max }$ ist. Dass dabei keine ausgeprägten Peaks entstehen, die zur EDX-Analyse herangezogen werden könnten liegt daran, dass nur ca. 1\% der Verlustenergie in Röntgenstrahlung umgewandelt wird. Die charakteristische Röntgenstrahlung bildet die Grundlage zur Analyse der Proben mittels 
eines EDX-Systems. Diese Art der Strahlung entsteht, wenn durch den Beschuss mit Primärelektronen einzelne Elektronen aus den inneren Schalen der Atome (K-, L- und M-Schale) der Probe herausgeschlagen werden und die Atome somit ionisiert werden. Hierzu müssen die PE eine bestimmte Ionisierungsenergie aufweisen. Diese wird durch die Beschleunigungsspannung (in $\mathrm{kV}$ ) am REM eingestellt und entspricht der kinetischen Energie der PE. Die Ionisierungsenergie (in keV) muss immer größer sein als die Bindungsenergie der Elektronen des Atoms. Wird nun ein Elektron aus dem Atomverband herausgeschlagen, so entsteht eine Lücke die sofort von einem Elektron aus einer höherliegenden Schale besetzt wird. Da diese Schale aber ein geringeres Energieniveau besitzt als die Schale von der das Elektron kommt, muss dieses Energie verlieren (Abbildung 61). Diese Verlustenergie wird in Form von Röntgenquanten ausgesandt und ist für jedes Element charakteristisch. Mittels dieser Röntgenquanten lässt sich nun die chemische Zusammensetzung einer Probe ermitteln.

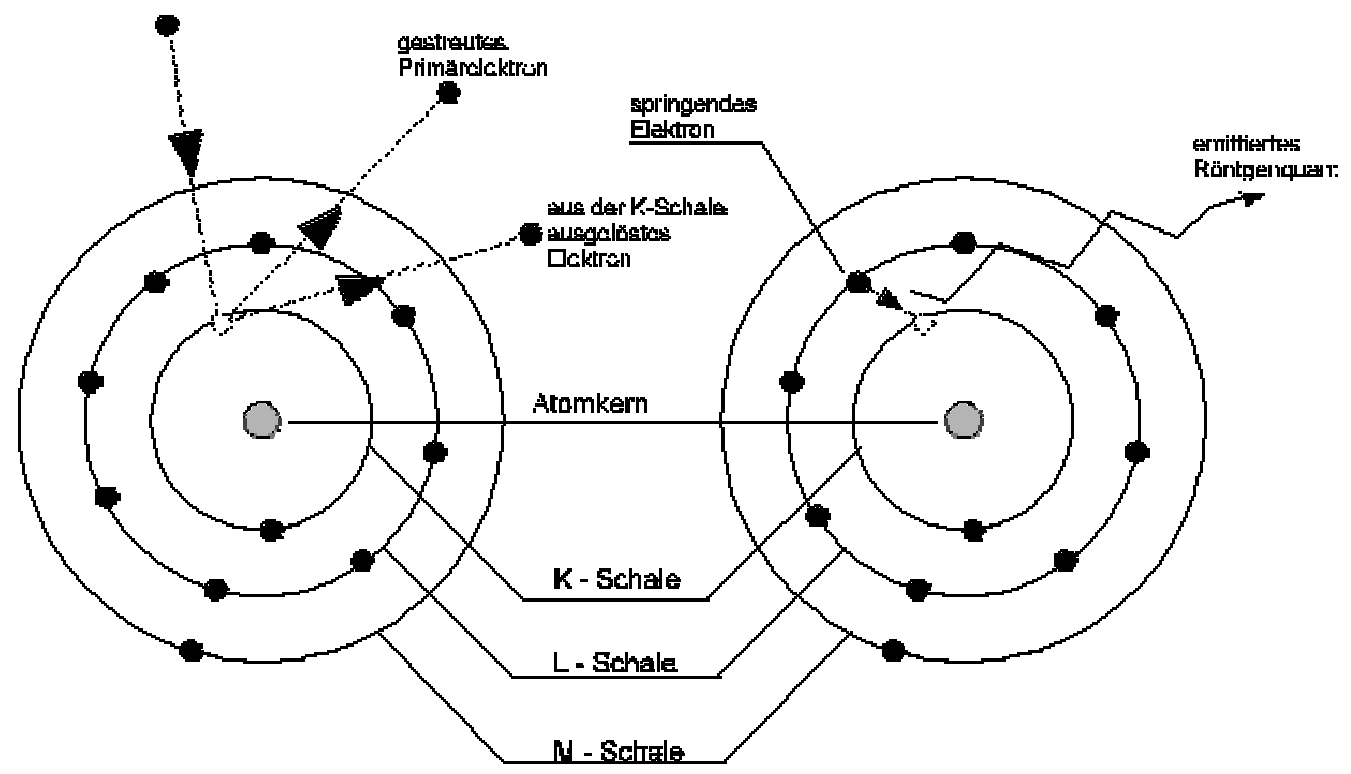

Abbildung 61: Freisetzung der charakteristischen Röntgenstrahlung: linke Abbildung: Herausschlagen eines Elektrons aus der K-Schale; rechte Abbildung: Übergang eines Elektrons der L- auf die K-Schale und das dadurch emittierte Röntgenquant (BARKOW 1994).

\subsubsection{Präparation der Proben}

Die Proben für die EDX-Analyse wurden, vergleichbar zur Präparation für die Untersuchung mittels Feldemissions-Rasterelektronenmikroskop (FEM) sowohl mit Organika belegten Pulver- als auch mit Aggregatproben (Na-Montmorillonit: $\varnothing$ ca. 2-3 mm) durchgeführt. Dazu wurden kleine Stiftteller (Cambridge-Typ) für die Elektronenmikroskopie mit flüssigem „Leit-C“ bestrichen und nach kurzer Trockenzeit ( 5 min) mit dem jeweiligen Probenmaterial versehen. Da es sich beim verwendeten REM/EDX-System im Gegensatz zum verwendeten FEM (Kapitel 4.2.1.2) um ein Gerät handelt, dass nur mit hohen Anregungsspannungen (>10 $\mathrm{kV}$ ) arbeitet, mussten die Proben, um die bei der Auswertung extrem störenden Aufladungs- 
erscheinungen zu vermeiden, mit einem leitenden Material zur Ableitung der entstehenden Ströme beschichtet werden. Dazu wurden die Proben mit einer dünnen Goldschicht (Dicke 8 $\mathrm{nm}$ ) bedampft. Zugleich wurde eine Parallele mit unbesputterten Proben bereitgestellt, da nach mündlicher Mitteilung von Dr. Brückner (Geologie, Universität Trier) es bei Proben mit geringen Kohlenstoffgehalten durch eine Goldbeschichtung zu einer Absorption des schwachen C-Signals kommen kann. Aus diesem Grunde wurde diese Probenserie auch vorzugsweise verwendet, da Tests ein doch relativ schwaches Kohlenstoff-Signal erwarten ließen. Die Messung der Proben erfolgte an einem REM (Zeiss LEO 435VP) mit gekoppelter EDXEinheit (Link eXL) der Abteilung Geologie der Universität Trier. Die Anregungsspannung betrug $20 \mathrm{kV}$, der Abstand vom Detektor zur Probe (WD) $19 \mathrm{~mm}$. Für optimale Ergebnisse wurden Rückstreuelektronen (RE) und Sekundärelektronen (SE) am Detektor (18\%: 82 \%) gemischt. Mittels EDX wurden Übersichts-Elementscans des Probenmaterials durchgeführt. Zusätzlich wurden die Proben speziell auf die Gehalte an den Elementen Kohlenstoff, Silizium und Eisen geprüft.

\subsubsection{Auswertung der Ergebnisse}

Diese Untersuchungsmethode bietet durch die Kopplung mit einem Elektronenmikroskop den Vorteil, dass in der mikroskopischen Aufnahme erkennbare Strukturen auf ihre Elementzusammensetzung hin untersucht werden können. Mit dem beschriebenen System wurden Linienscans über die ausgewählten Ausschnitte durchgeführt, wobei die Elemente Kohlenstoff (C) und Silizium (Si), die organischen Coatings bzw. die unbedeckte Tonmineraloberfläche repräsentierend, detektiert wurden.

\subsubsection{Brenzkatechin}

Als erste Versuchsreihe wurden sowohl Pulver- als auch Aggregatproben, die durch Sublimation mit Brenzkatechin belegt waren, untersucht. Dazu wurde die Oberfläche der entsprechenden Proben mehrmals mit dem Elektronenstrahl abgetastet, um so einen Überblick über die Elementverteilung auf der Tonmineraloberfläche zu erhalten. Die folgenden Abbildungen (62 und 63) zeigen zur Verdeutlichung in mehreren Vergrößerungsstufen die Verteilung von relativ strukturarmen dunkel erscheinenden Flächen und stärker reflektierenden Flächen mit einer deutlich heterogeneren Struktur. Die dunklen Flächen erscheinen dem Betrachter als Überzüge, die auf den darunter liegenden körnigen, in helleren Grautönen dargestellten Tonmineralplättchen aufzusitzen scheinen (siehe Ausschnittvergrößerung). 


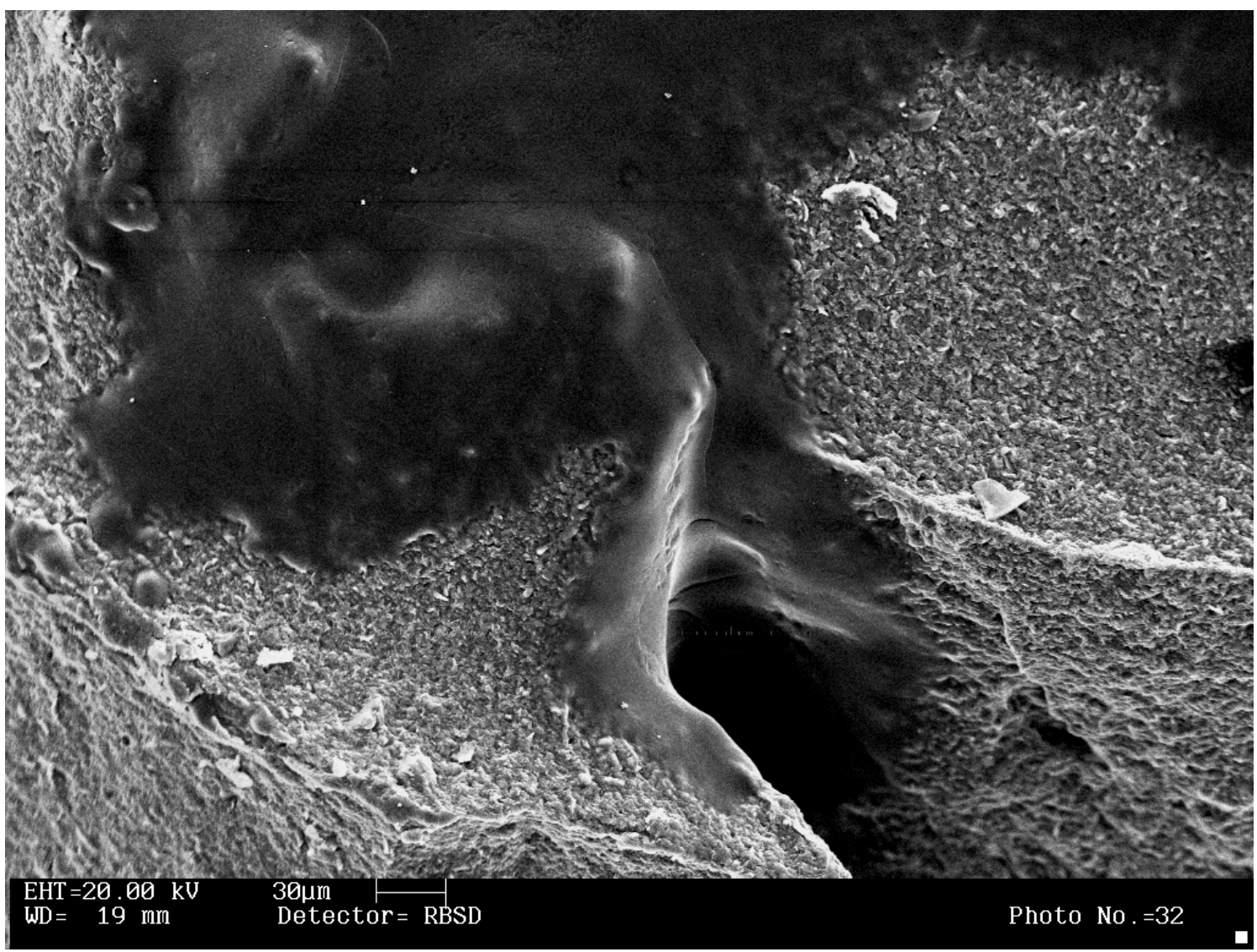

Abbildung 62: REM-Aufnahme des mit Brenzkatechin belegten Montmorillonits (Pulverpräparat).

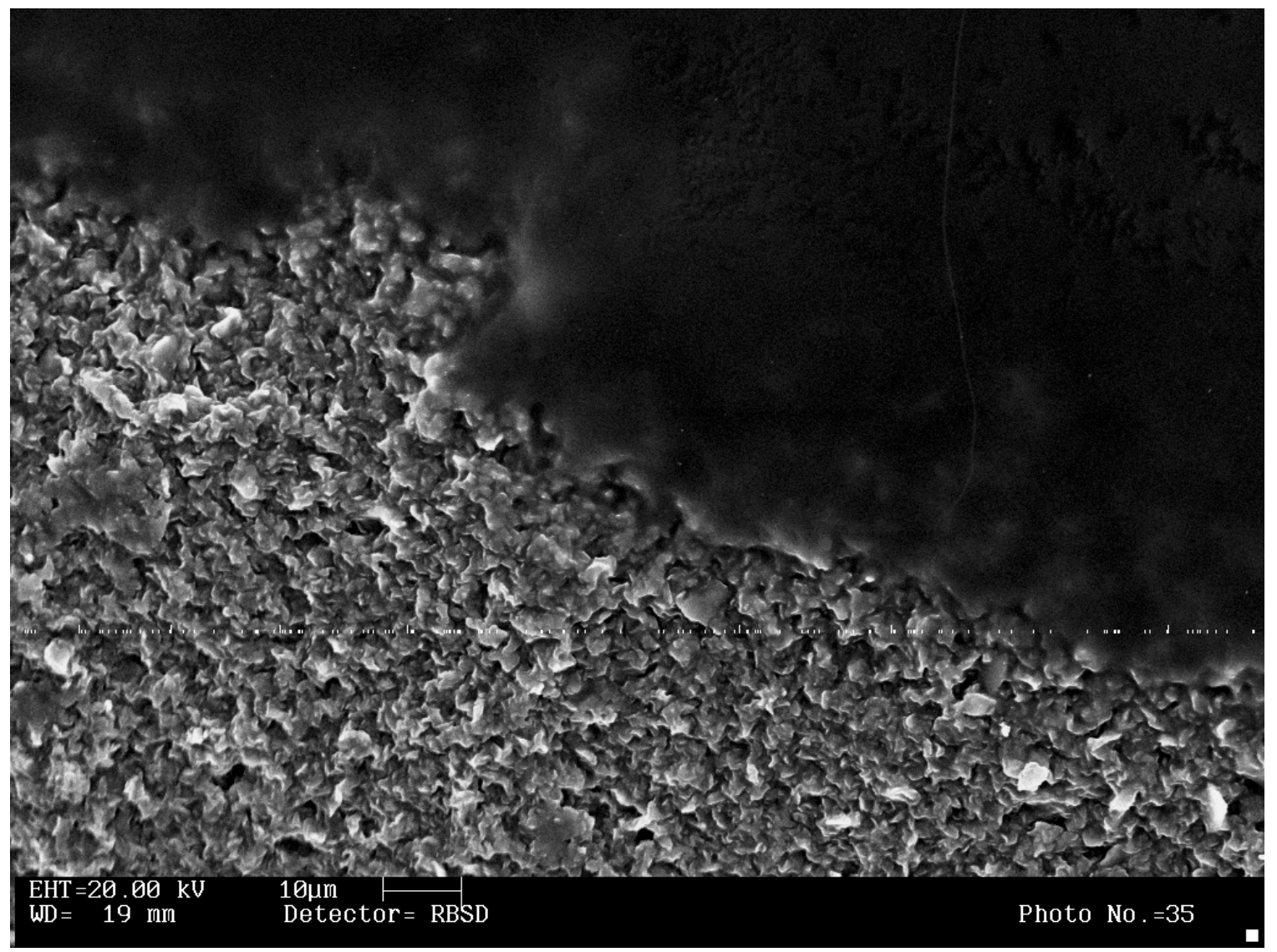

Abbildung 63: Ausschnittvergrößerung der vorangegangenen Aufnahme. 
In dieser Ausschnittvergrößerung des Randbereiches zwischen dunklen und heller erscheinenden Flächen ist eindeutig zu erkennen, dass die homogenen Flächen auf den Tonmineralplättchen, die eine durchschnittliche Größe von ca. $5 \mu \mathrm{m}$ aufweisen, aufliegen. Hier scheinen die Mineralpartikel gut erkennbar unter dem darüber liegenden Material durch.

Das REM-Bild des mit Brenzkatechin belegten Montmorillonits (Abbildung 64) zeigt die Aufnahme eines Aggregates, welches eine, abgesehen von Schrumpfrissen und blasigen Strukturen in der Bildmitte, eine unstrukturierte homogene Oberfläche aufweist. Auffallend war bei vielen REM-Aufnahmen des mit Brenzkatechin belegten Tons, dass, wie hier in der rechten oberen Bildhälfte besonders gut zu erkennen, ein samtiger, weitere Details auf der Aggregatoberfläche verdeckender Überzug zu beobachten ist.

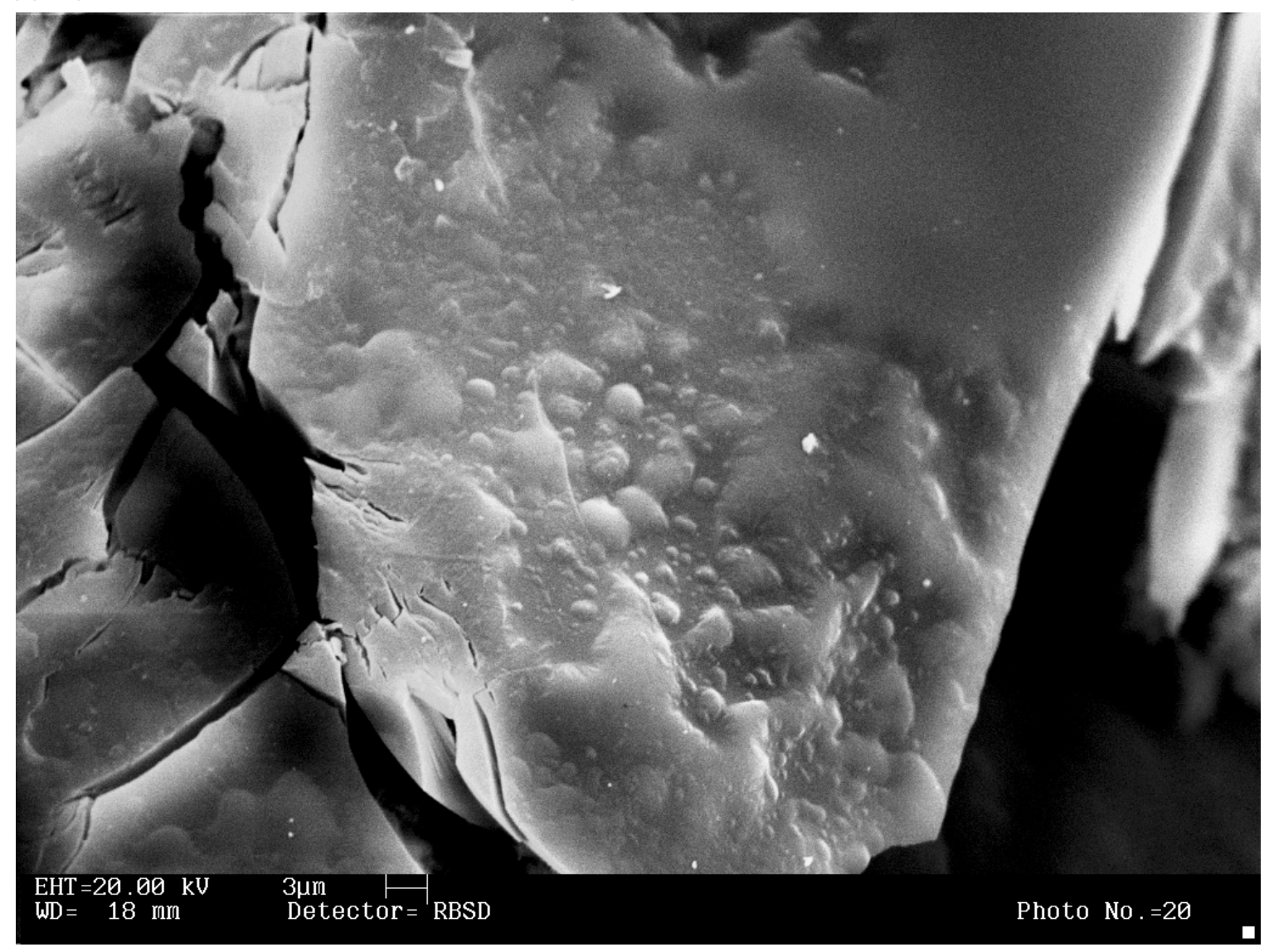

Abbildung 64: REM-Aufnahme des mit Brenzkatechin belegten Montmorillonits.

Der zur REM-Aufnahme zugehörige EDX-Scan der Elementverteilung auf der Oberfläche der Probe (Abbildung 65) lässt zu erkennen, dass neben den starken Signalen für Gold ( $\mathrm{Au}$ ) Peaks für Kohlenstoff $(\mathrm{C})$, Sauerstoff $(\mathrm{O})$ und Natrium $(\mathrm{Na})$ auftreten. Die Signale für die Elemente Kohlenstoff und Sauerstoff sind nach dem Gold, resultierend aus der Bedampfung des Probenmaterials, von höchster Intensität und zeigen, dass sich auf der Mineraloberfläche adsorbiertes organisches Material befindet. Durch den Nachweis dieser beiden Elemente wird die Vermutung, dass es sich bei den zu beobachtenden Überzügen, wie bereits im Kapitel 4.2.3 geschildert, um ein Aufwachsen organischer Hüllschichten bzw. Überzüge handelt, erhärtet. 


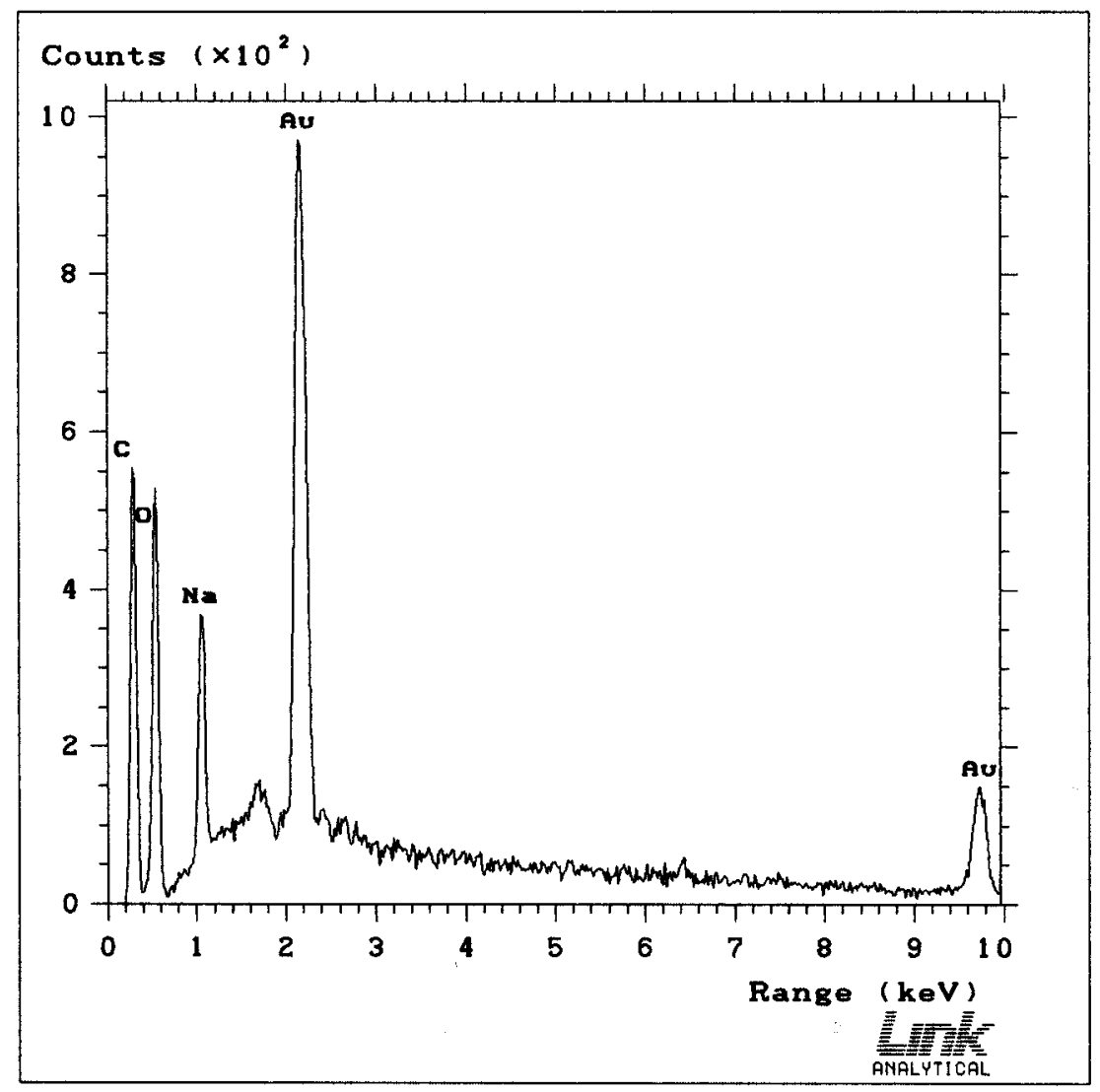

Abbildung 65: EDX-Scan eines mit Brenzkatechin belegten Na-Montmorillonits.

Der Kohlenstoff konnte durch EDX-Scans, im Gegensatz zu den unbelegten Proben, auf allen Mineraloberflächen in unterschiedlichen Intensitäten nachgewiesen werden. Trotz des Nachweises von Kohlenstoff und damit organischer Verbindungen auf der Mineraloberfläche konnte jedoch die Vermutung, dass es sich bei den in den REM-Aufnahmen als samtige mit relativ homogener Oberfläche erscheinenden Überzüge um die betreffenden organischen Strukturen handelt, noch nicht bestätigt werden. Um einen eindeutigen Nachweis zu erbringen, wurden Flächen auf den Proben gesucht, die eine heterogene Verteilung von, auf intensiv rückstreuenden Bereichen aufliegenden relativ strukturarmen und schwach rückstreuenden Oberflächen aufweisen, herausgesucht. An diesen Probenausschnitten wurden wiederum EDX-Scans durchgeführt, jedoch erfolgte die Analyse der Elementverteilung nicht flächenhaft, sondern durch die sich abwechselnden Bereiche wurde eine Scan-Linie gelegt und auf dieser Linie die Elementverteilung qualitativ erfasst. Die folgende REM-Aufnahme zeigt einen solchen Ausschnitt mit heterogenem Muster und die entsprechende, in gelb markierte Scan-Linie (Abbildungen 66). Entlang dieser Linie wurden die Elemente Kohlenstoff, organisches Material repräsentierend, und Silizium (Si) für anorganische, mineralische Oberflächen detektiert. 


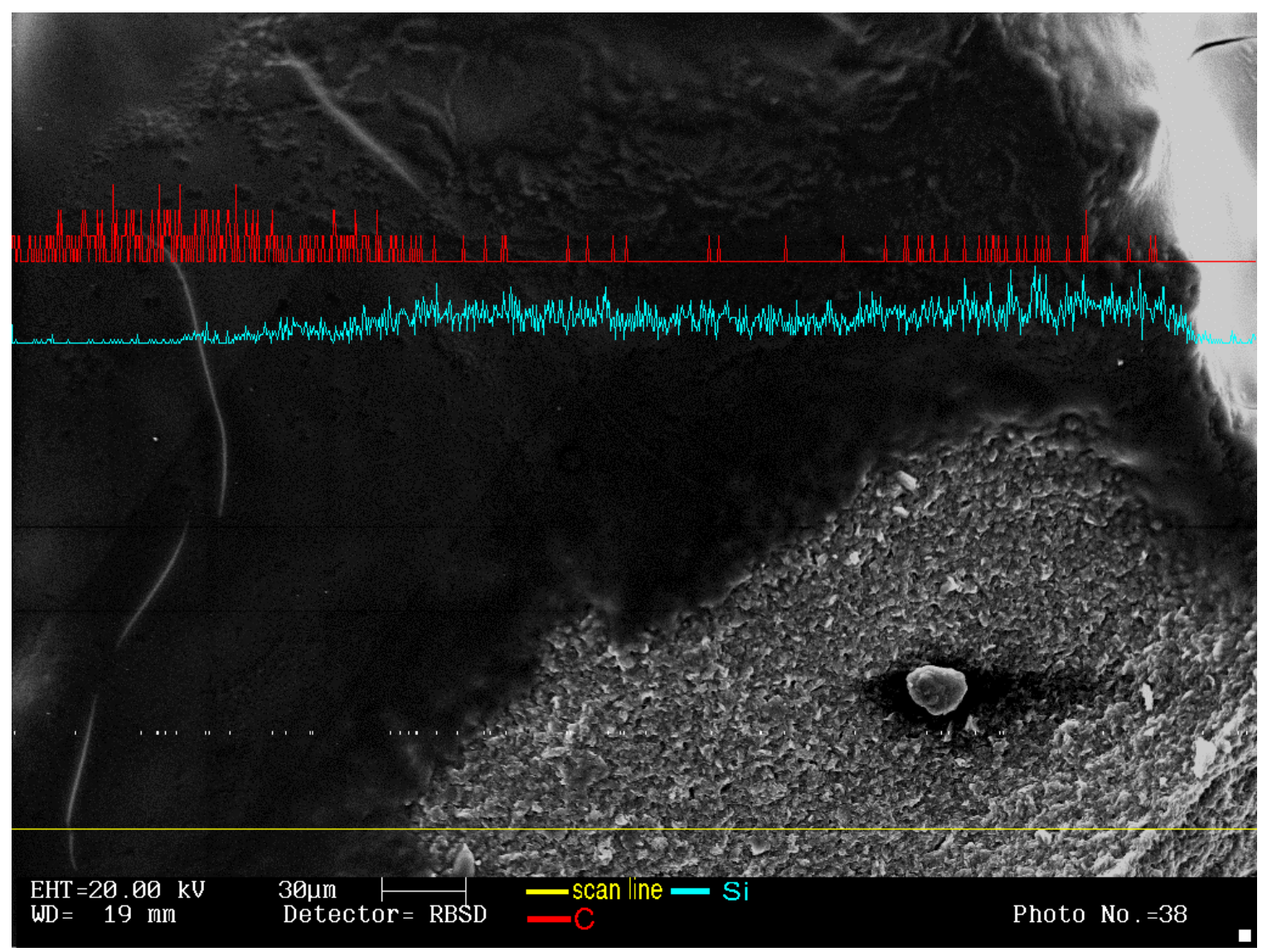

Abbildung 66: EDX-Analyse des mit Brenzkatechin belegten Montmorillonits.

Wie bereits anhand der Abbildungen 62 und 63 erläutert, ist auch hier, insbesondere in den Randbereichen zwischen den dunklen und den helleren Flächen wiederum zu erkennen, dass das stärker reflektierende heterogene Material durch die dunkleren Flächen durchscheint und somit einen Überzug auf der aus Montmorillonit bestehenden Probe nachweist. Führt man nun in dem Bereich sich abwechselnder hell und dunkel erscheinender Flächen einen Scan der Elemente Kohlenstoff und Silizium durch, so ist sehr schnell und eindeutig zu erkennen, dass im Bereich der dunkel erscheinenden Überzüge ein starkes Signal für Kohlenstoff zu beobachten ist, während bei der Abtastung der helleren, heterogeneren Flächen ein deutlicher Anstieg des Signals für Silizium auftritt.

In der Abbildung 67 ist deutlich zu erkennen, dass es sich bei den in der Aufnahme schwach reflektierenden und somit dunkel erscheinenden Bereichen, wie bereits vermutet, um organische Überzüge handelt. Durch das in roter Farbe gekennzeichnete Verteilungsmuster des Elements Kohlenstoff wird eindeutig nachgewiesen, dass in den dunkel erscheinenden Bereichen ein deutliches Signal hoher Intensität für das Element Kohlenstoff zu verzeichnen ist. Die in der Aufnahme hellgrau erscheinenden Bereiche der Oberfläche zeigen hingegen kein oder ein nur sehr schwaches Kohlenstoffsignal, während hier ein deutlicher Anstieg des Signals für das, rein mineralische Oberflächen charakterisierende Silizium anhand der in Blau markierten Linie zu beobachten ist. 


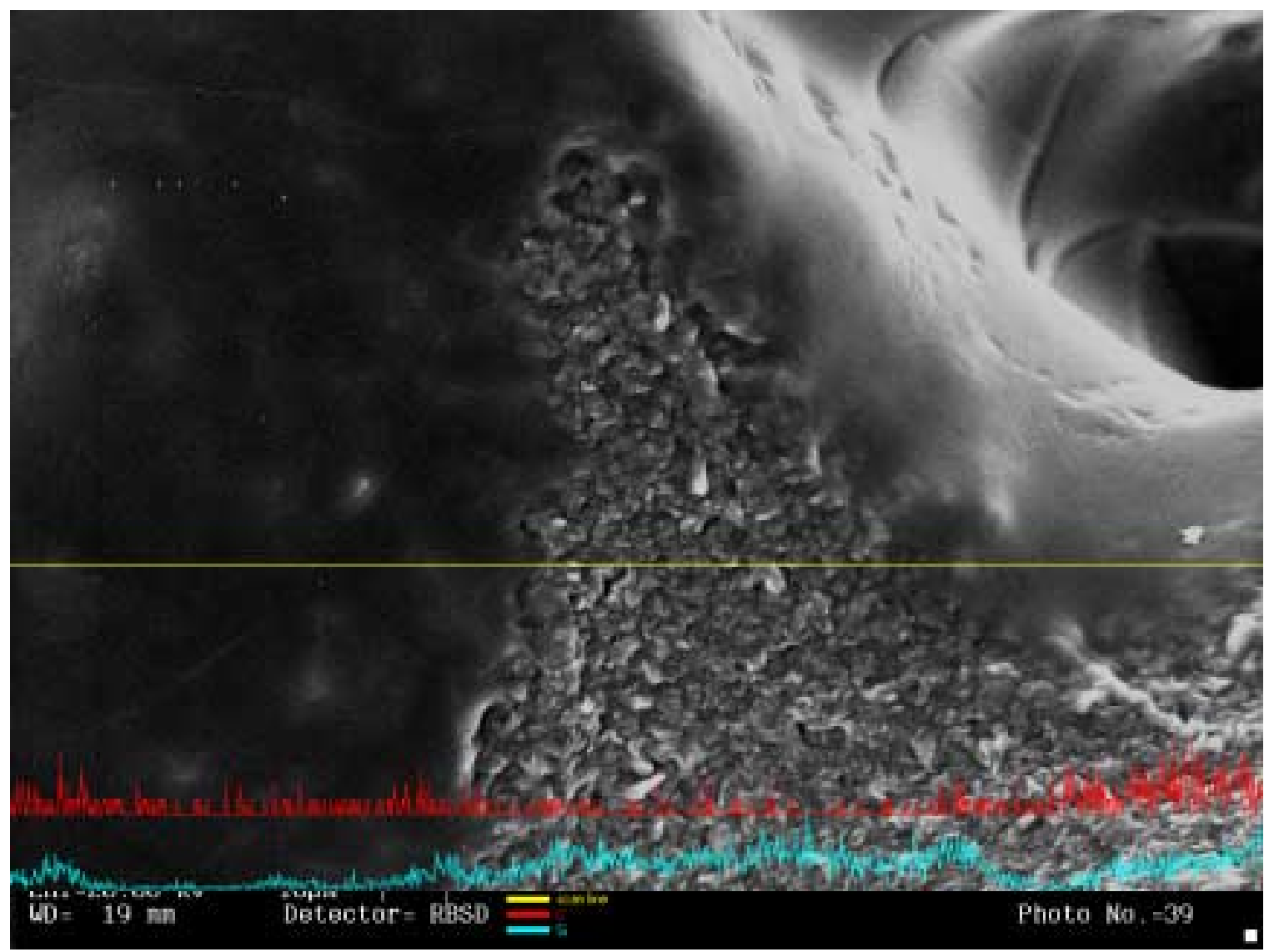

Abbildung 67: EDX-Analyse des mit Brenzkatechin belegten Montmorillonits (Ausschnittvergrößerung).

Aus diesen Beobachtungen können mehrere Schlüsse gezogen werden:

1. bei den in den REM-Aufnahmen schwach reflektierenden und somit dunkel erscheinenden strukturarmen Flächen handelt es sich eindeutig um organische Überzüge

2. die hell erscheinenden Bereiche sind aufgrund des intensiven Silizium-Signals als unbedeckte mineralische Flächen anzusprechen

3. aufgrund des Verteilungsmusters der Elemente Kohlenstoff und Silizium kann zudem nachgewiesen werden, dass die organischen Überzüge auf der mineralischen Unterlage aufsitzen. Dort, wo ein starkes Kohlenstoff-Signal zu verzeichnen ist kann nur ein sehr schwaches Signal für Silizium beobachtet werden und somit kann die Überlagerung der anorganischen mineralischen Oberfläche mit organischem Material angenommen werden.

\subsubsection{Pyrogallol}

Neben der Belegung von Tonmineralen mit Brenzkatechin wurden auch mit Pyrogallol belegte Proben mittels Röntgenmikrobereichsanalyse untersucht. Neben den Signalen für Kohlenstoff (C) und Silizium (Si) zeigt das Scannen der Elementverteilung (Abbildung 68) das Auftreten von Aluminium (Al), Calcium (Ca), Magnesium $(\mathrm{Mg})$ und Natrium $(\mathrm{Na})$ an. Die beiden Peaks für das Element Gold $(\mathrm{Au})$ resultieren aus der Bedampfung der Probenoberfläche. 


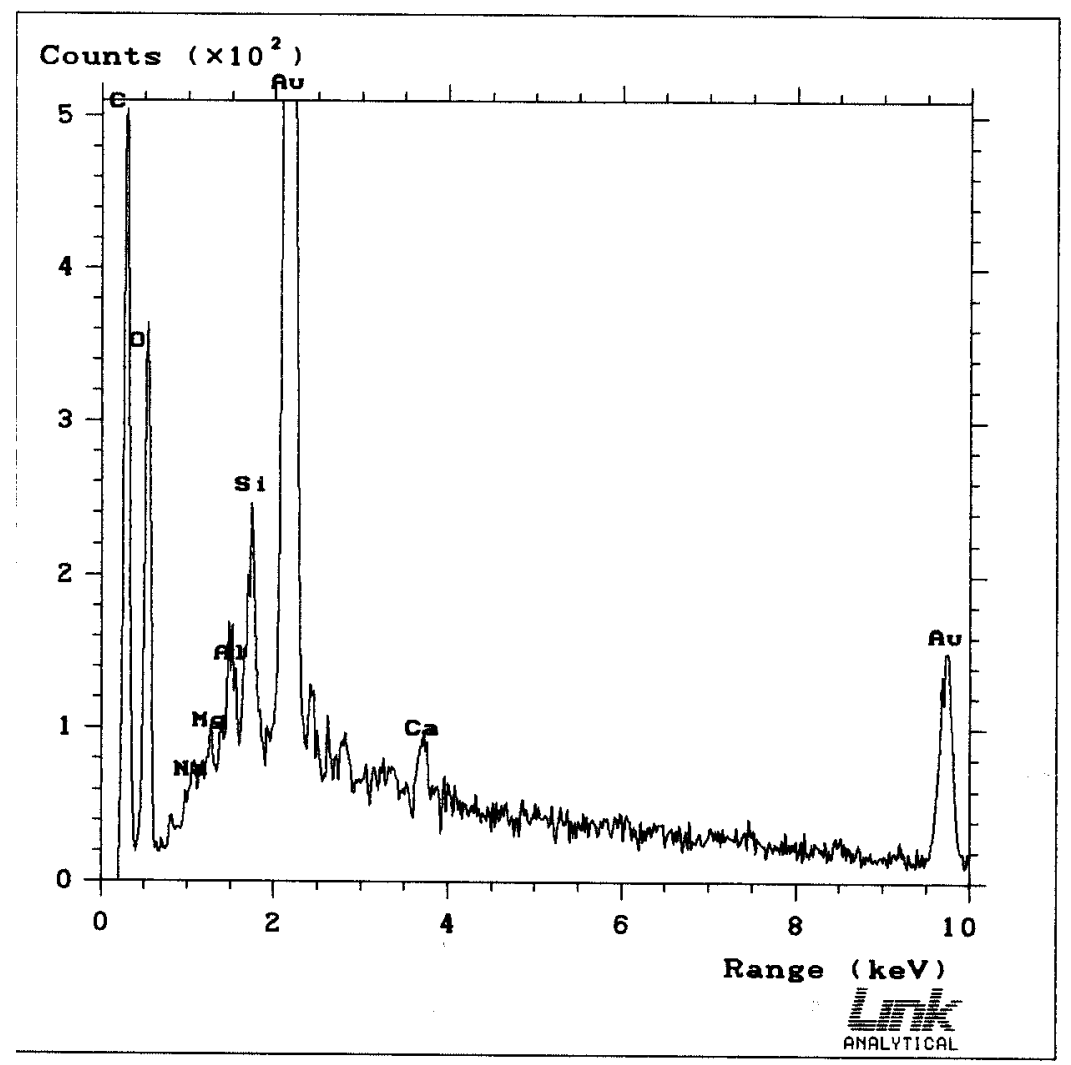

Abbildung 68: Auftretende Elemente der EDX-Analyse eines mit Pyrogallol belegten Montmorillonits.

Die Elemente Kohlenstoff und Sauerstoff weisen aufgrund ihrer relativ hohen Intensität das Auftreten organischer Verbindungen auf der Oberfläche der Tonminerale nach. Deren Signale konnten während Mikrobereichsanalysen unbelegter Proben nicht nachgewiesen werden und sind somit ein eindeutiges Indiz der Existenz organischen Materials auf den zuvor rein mineralischen Oberflächen. Die weiteren Elemente repräsentieren die anorganischen, mineralischen Anteile des Probenmaterials, i.e. den Montmorillonit. Dieser ist hauptsächlich aus den Elementen Aluminium, Silizium und Sauerstoff aufgebaut. Weitere Anteile sind Natrium, Calcium und Magnesium, die zum Teil aus den Zwischenschichten des Tonminerals herrühren, aber auch in die Kristallstruktur der Minerale eingebaut sein können (Kapitel 2.1.1). In Abbildung 69 ist zu erkennen, dass auch nach der Belegung mit diesem Phenol die Proben teilweise mit, im REM-Bild dunkel erscheinenden, Überzügen bedeckt sind. Die recht plastisch erscheinenden Überzüge liegen, auch hier gut erkennbar, auf der hell erscheinenden mineralischen Oberfläche auf. 


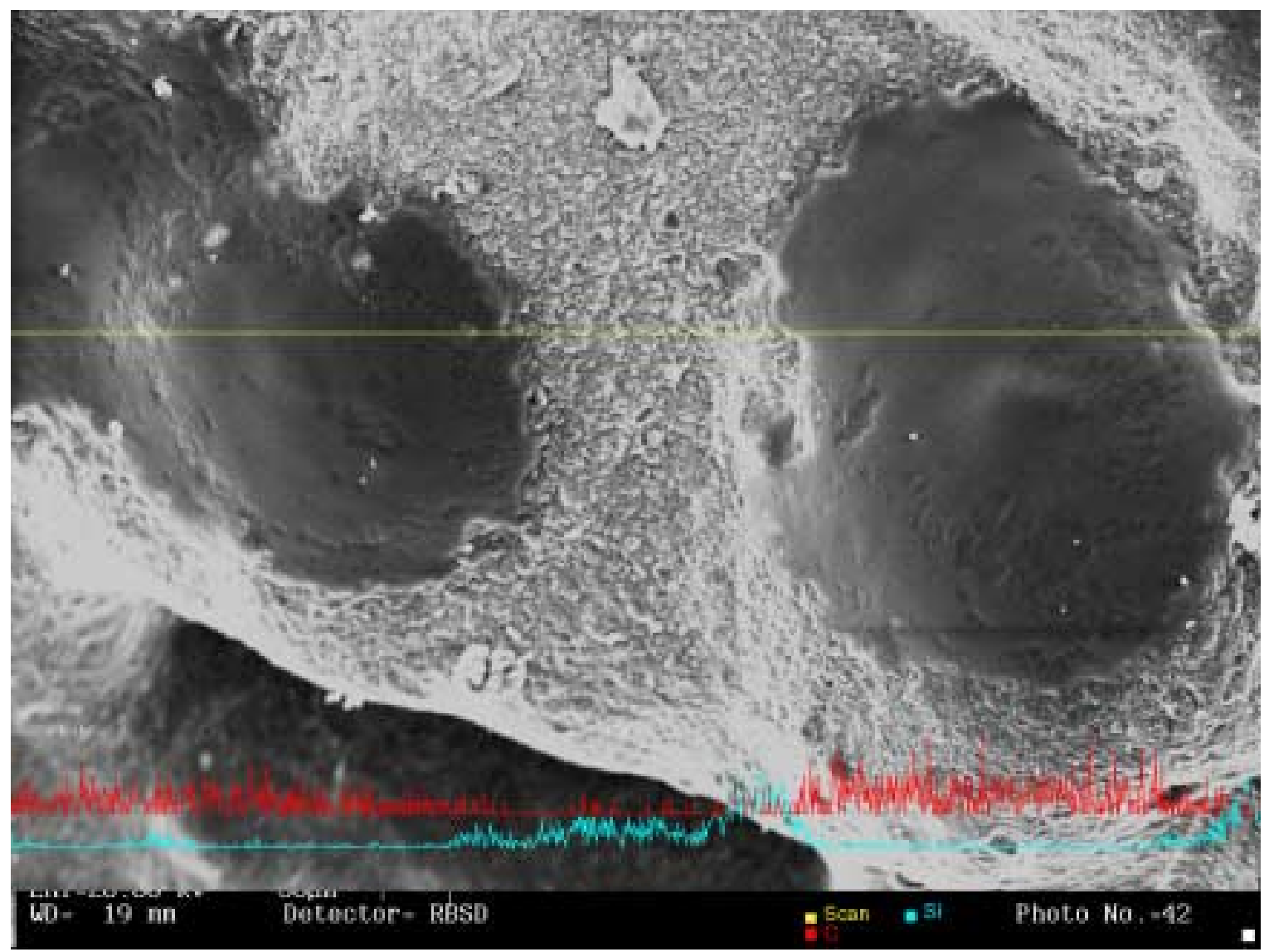

Abbildung 69: EDX-Analyse des mit Pyrogallol belegten Montmorillonits (Scanlinie: gelb, Kohlenstoff: rot, mineralische Oberfläche (blau).

Die durch die Bildmitte verlaufende Scanlinie zeigt, dass die dunkel erscheinenden Bereiche sehr gut mit dem Auftreten des Elements Kohlenstoff korrelieren. Gleichzeitig ist zu beobachten, dass außerhalb dieser Flächen das Signal für Silizium stark ansteigt und somit nicht nur die unbedeckte mineralische Oberfläche charakterisiert, sondern durch diesen Wechsel zusätzlich verdeutlicht, dass die organischen Flächen auf dem mineralischen Untergrund aufsitzen.

\subsubsection{Hinweise auf den Reaktionsmechanismus}

Für die Aktivität der Tonminerale hinsichtlich ihrer Fähigkeit organische Verbindungen zu katalysieren werden an die Oberfläche der Tonminerale gebundene Metalle, als adsorbierte Metallkationen oder Hydroxide bzw. in der Tonmineralstruktur eingebaute Metallionen (Eisen, Aluminium, Mangan) verantwortlich gemacht (Kapitel 2.1.2). Diese Metalle stellen reaktive Zentren der katalytischen Aktivität der Tonminerale dar und sollten somit in den Bereichen höherer Eisengehalte auch mit dem Auftreten organischer Überzüge korrelieren. Die in dieser Arbeit verwendeten Tonminerale enthalten laut Elementanalyse (Kapitel 4.1) in der Tat Eisen. Die Überlegung war nun, mit Hilfe der Röntgenmikrobereichsanalyse zu verifizieren, ob die Verteilung des Eisens an der Tonmineraloberfläche bzw. in Oberflächennähe auftretendes Eisen mit der angenommenen Verteilung organischen Materials an den Tonmineral- 
oberflächen zusammenhängt. Zur Überprüfung dieser Annahme wurden wiederum EDXAnalysen durchgeführt, wobei nun die Elemente Kohlenstoff $(\mathrm{C})$ und Eisen $(\mathrm{Fe})$ gescannt wurden.

Die Untersuchungen des mit Brenzkatechin belegten Montmorillonits führten nach einer erneuten Analyse der Elementverteilung zu folgenden Resultaten. Die Übersicht über die Verteilung der Elemente zeigt, dass neben den Peaks der bereits bekannten organischen $(\mathrm{C}, \mathrm{O})$ und mineralischen Bestandteile ( $\mathrm{Si}, \mathrm{Ca}, \mathrm{Na}, \mathrm{Mg}$ ) ein deutliches Signal des Elements Eisen (Fe) beobachten werden kann (Abbildung 70).

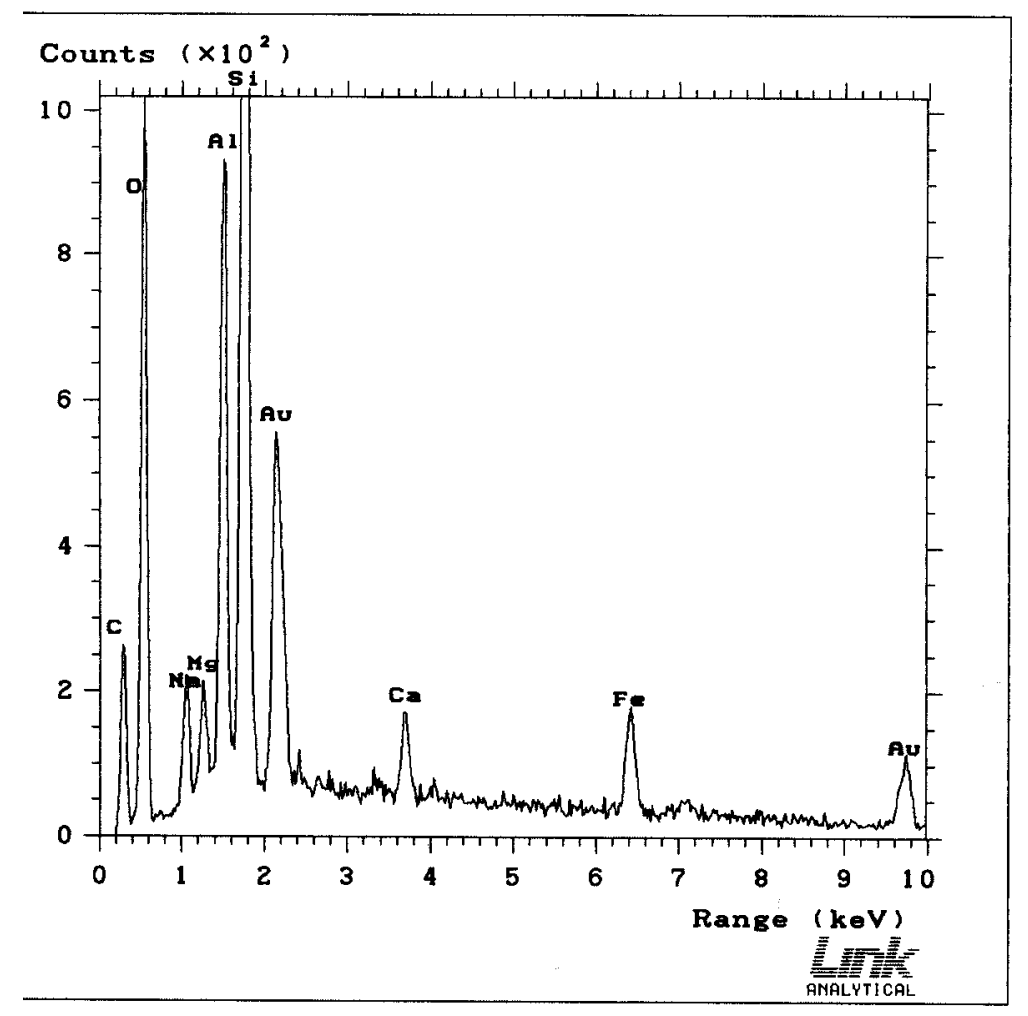

Abbildung 70: EDX-Analyse der mit Brenzkatechin belegten Montmorillonitoberflächen.

Die EDX-Analyse zeigt starke Signale für Silizium und Aluminium. Diese Peaks repräsentieren die Tonminerale. Der detektierte Sauerstoff stammt zum Teil ebenfalls aus der mineralischen Komponente, ist aber auch Bestandteil organischer Verbindungen. Dafür spricht auch das auftretende Signal für Kohlenstoff. Die auftretenden Signale für Calcium (Ca) und Magnesium (Mg) zeigen die Kationenbelegung des Montmorillonits an, können aber auch als Verunreinigungen in der Kristallstruktur des Tonminerals und als Beimengung auftreten. Das Element Mangan (Mn) kann neben Eisen sowohl als Bestandteil der Minerale als auch als Beimengung enthalten sein. Von Bedeutung hier ist aber die „Vergesellschaftung“ dieser beiden Elemente mit dem Kohlenstoff (C). Die linienhaft durchgeführten Elementscans zeigen eine deutliche Übereinstimmung des Auftretens der Elemente Kohlenstoff und Eisen. Daraus ist zu schließen, dass an der Oberfläche der Tonminerale befindliches Eisen, sei es als Mineralbestandteil etwa an Tonmineralkanten bzw. als an der Mineraloberfläche adsorbiertes Fe- 
Oxid oder Fe-Hydroxid, zumindest einen starken Sorptionspartner für organische Verbindungen, wenn nicht sogar einen bedeutenden Reaktionspartner bzw. -initiator darstellt. Die Rolle der Eisenoxide und -hydroxide bei physikochemischen Wechselwirkungen mit organischer Substanz (in Böden) ist hinlänglich bekannt (Kapitel 2.1.2.1).

Das Ergebnis ist in dieser Form jedoch von eingeschränkter Aussagekraft, da so nur festgestellt werden konnte, dass, neben den auftretenden Signalen für organisches Material auf der Probenoberfläche, zusätzlich auch noch Eisen in der Probe enthalten ist. Um Hinweise dahingehend zu erhalten, dass, wie vermutet, Signale für Eisen und Kohlenstoff örtlich gemeinsam auftreten, wurden mit dem verwendeten REM/EDX-System das organisch belegte Probenmaterial wiederum linienweise abgetastet und simultan die Verteilungskurven für die beiden betreffenden Elemente aufgezeichnet. Die folgenden REM-Aufnahme (Abbildung 71) zeigt die Oberfläche eines mit Brenzkatechin belegten Montmorillonits. Gut zu erkennen sind einzelne Tonmineralplättchen bzw. -flocken, die eine Größenverteilung von wenigen Mikrometern bis zu mehreren Zehnern von $\mu \mathrm{m}$ (Bildmitte) erreichen. Die Lage der Scanlinie wurde so gewählt, dass sowohl relativ ebene Oberflächen als auch die Kanten von Tonmineralplättchen abgetastet werden konnten. Der Grund hierfür war, zu untersuchen, ob die in der Literatur (Kapitel 2.1.2.1) beschriebene Häufung von Metallen, deren Hydroxiden bzw. von zugänglichem, in die Struktur der Tonminerale eingebautem, Eisen tatsächlich zu beobachten und gegebenenfalls eine Korrelation mit adsorbierter organischer Substanz feststellen zu können.

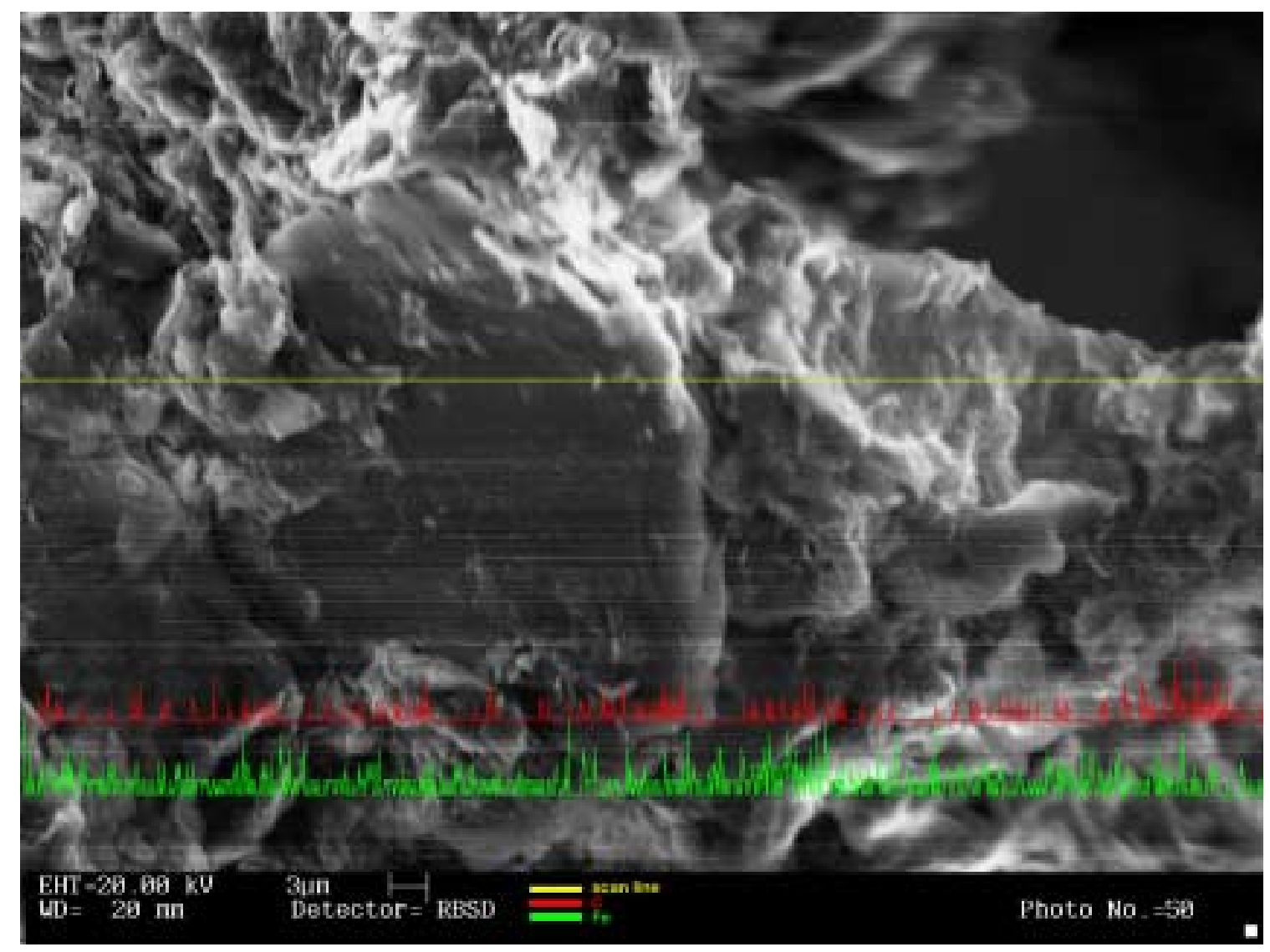

Abbildung 71: EDX-Scan (C: -rot/Fe: grün) des mit Brenzkatechin belegten Montmorillonits. 
Als sehr störend für die Bildauswertung hinsichtlich der Lokalisierung organischer Überzüge wirkt die allgemeine Unschärfe der Aufnahmen und die in horizontaler Richtung verlaufenden hellen Streifen. Dies liegt an den auftretenden Aufladungserscheinungen, hier leicht erkennbar durch die stark überstrahlten, hellen Flächen. Eine Reduzierung der Beschleunigungsspannung auf weniger als $20 \mathrm{kV}$ um diese Störungen zu minimieren, war systembedingt nicht möglich.

In Abbildung 71 ist allgemein betrachtet keine direkte Abhängigkeit des Kohlenstoff-Signals vom Gehalt an Eisen zu beobachten. In Bereichen höherer C-Konzentrationen sind zwar auch höhere Peaks für Eisen zu erkennen, in der Bildmitte und am rechten Bildrand, jedoch treten auch erhöhte Fe-Gehalte in Bereichen auf in denen kein Anstieg des Kohlenstoff-Signals festgestellt werden kann. Auffallend ist jedoch die Beobachtung, dass die Abtastung von Kanten eines Tonmineralplättchens bzw. einer Tonmineralflocke jedes Mal in einem Anstieg des Cals auch des Fe-Signals resultiert. Auf diesen Effekt wird im Verlauf dieses Kapitels noch genauer eingegangen. Aus diesem Grunde wurden weitere Mikrobereichsanalysen durchgeführt. Die folgende REM-Aufnahme (Abbildung 73) zeigt bei höherer Vergrößerungsstufe wiederum eine Montmorillonit-Probe, die durch Sublimation mit Brenzkatechin belegt wurde. Die Elementverteilung in der Übersicht (Abbildung 72) zeigt neben sehr hohen Intensitäten der Signale für Silizium und Sauerstoff die erwarteten Peaks für Kohlenstoff und Eisen an. Diese zeigen, dass die Probe sowohl organisches Material als auch Eisen enthält. Weitere, detektierte Elemente sind Aluminium, Natrium, Magnesium und Calcium.

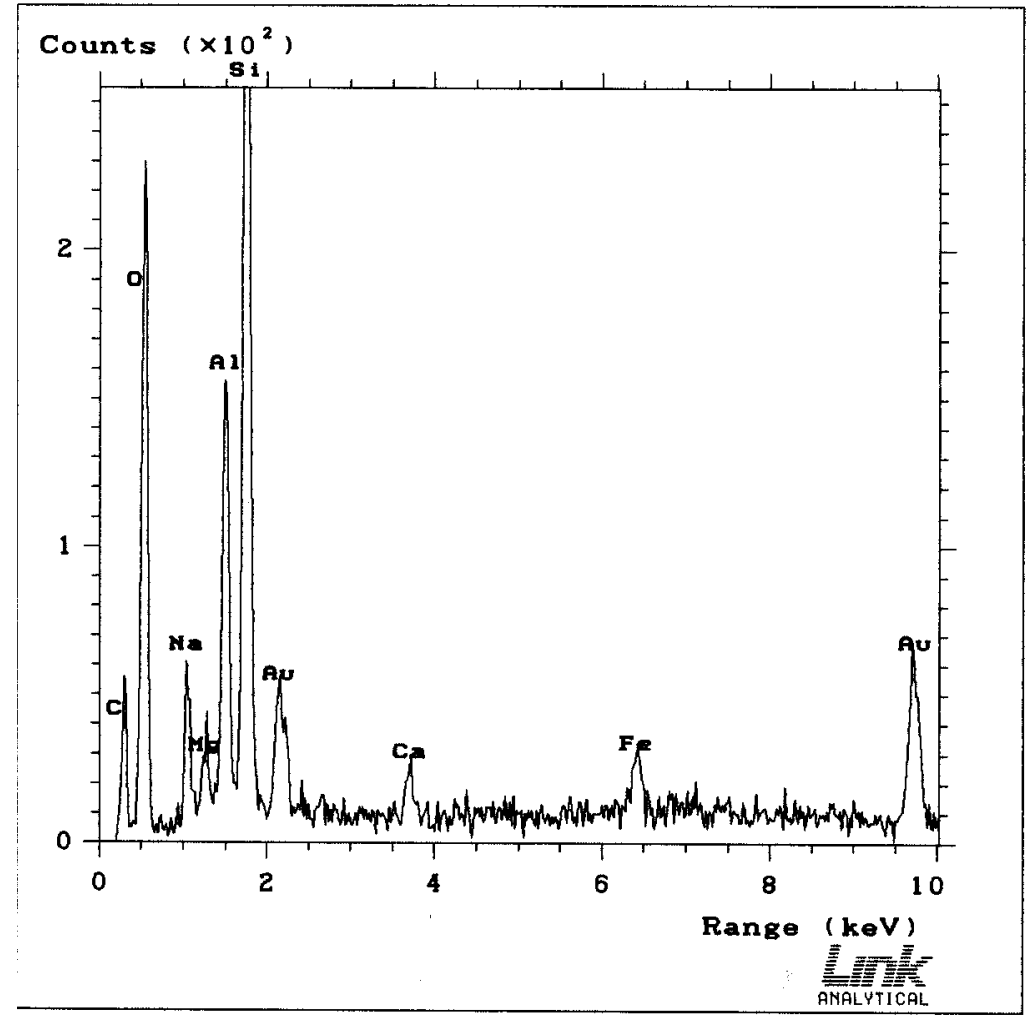

Abbildung 72: EDX-Analyse der mit Brenzkatechin belegten Montmorillonitoberflächen. 
Die Scanlinie der Mikrobereichsanalyse wurde hier ebenfalls so gewählt, dass ein repräsentativer Bildausschnitt erfasst wurde. Sowohl helle, dem Detektor zugewandte als auch dunkel erscheinende, dem Detektor abgewandte Flächen wurden berücksichtigt. Die Detektierung der Elemente Kohlenstoff und Eisen zeigt in dieser Aufnahme einen Anstieg des C-Signals bei gleichzeitiger Intensitätszunahme des Fe-Signals.

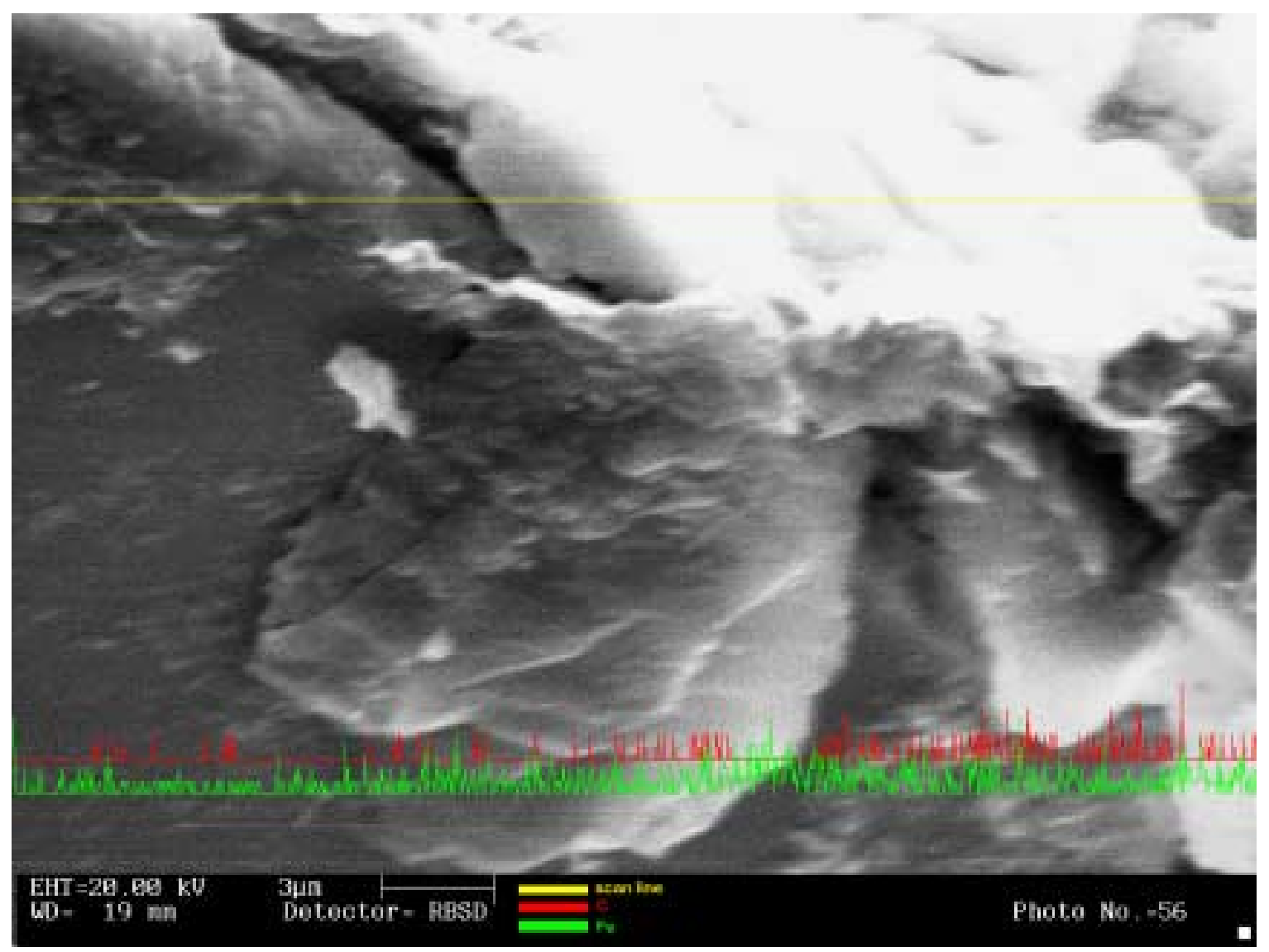

Abbildung 73: REM/EDX-Scan (C: rot/Fe: grün) des mit Brenzkatechin belegten Montmorillonits.

Die beiden Verteilungskurven für die Elemente Kohlenstoff und Eisen zeigen im Verlauf der Scan-Linie einen Anstieg der Intensitäten des Kohlenstoffs- und des Eisensignals von links nach rechts. Während in der linken Bildhälfte nur sehr schwache Kohlenstoffsignale zu beobachten sind, steigen diese nach rechts ab etwa der Bildmitte hin an. Hier ist nun eine relativ erhöhte Intensität des Kohlenstoffs zu erkennen, die Stärke des Eisensignals nimmt hingegen im Vergleich geringfügiger zu. Berücksichtigt man in dieser Aufnahme den allgemeinen Intensitätsanstieg der beiden Elementsignale aufgrund der Neigung des Großteils der Probenoberflächen zum Detektor hin, was auch durch den deutlichen Helligkeitsanstieg im Bild zu erkennen ist, so kann in dieser Aufnahme nur von einem erkennbaren, jedoch recht schwachen Zusammenhang zwischen Bereichen der verstärkten Akkumulation organischen Materials und dem Eisengehalt der unterliegenden mineralischen Oberfläche gesprochen werden.

Die Bedeutung der Tonmineralkanten als Ort der intensivsten Adsorption organischen Materials und der größten Aktivität der katalytischen Umwandlung adsorbierter Organika (VARADACHARI et al. 1995) wurde bereits in diesem Kapitel und im Kapitel 2.1.2 darge- 
stellt. Aufgrund der an Tonmineralkanten zugänglichen Metallatome der inneren Mineralstruktur und der vorwiegend an diesen Bereichen an die Tonminerale gebundenen Metalloxide und Metallhydroxide sind die Bedingungen für die Sorption organischer Substanzen und deren nachfolgende chemische Reaktion dort besonders vorteilhaft. So sollte als Konsequenz der dargestellten theoretischen Sachverhalte bei der Abtastung von Tonmineralkanten eine erhöhte Intensität bezüglich der Fe-Gehalte zu beobachten sein. Damit verbunden wäre folglich ebenso ein Intensitätsanstieg für das Element Kohlenstoff. In der REM-Aufnahme der folgenden Mikrobereichanalyse wurde die Scanlinie so gewählt, dass möglichst viele Kanten von Tonmineralplättchen und -flocken getroffen wurden. Die Übersicht über die Elementverteilung (Abbildung 74) weist hohe Intensitäten oberhalb des maximalen Anzeigebereichs für die Elemente Kohlenstoff, Sauerstoff, Natrium und Silizium auf. Als weitere Elemente sind Peaks für Aluminium, Magnesium, Chlor und Eisen zu beobachten.

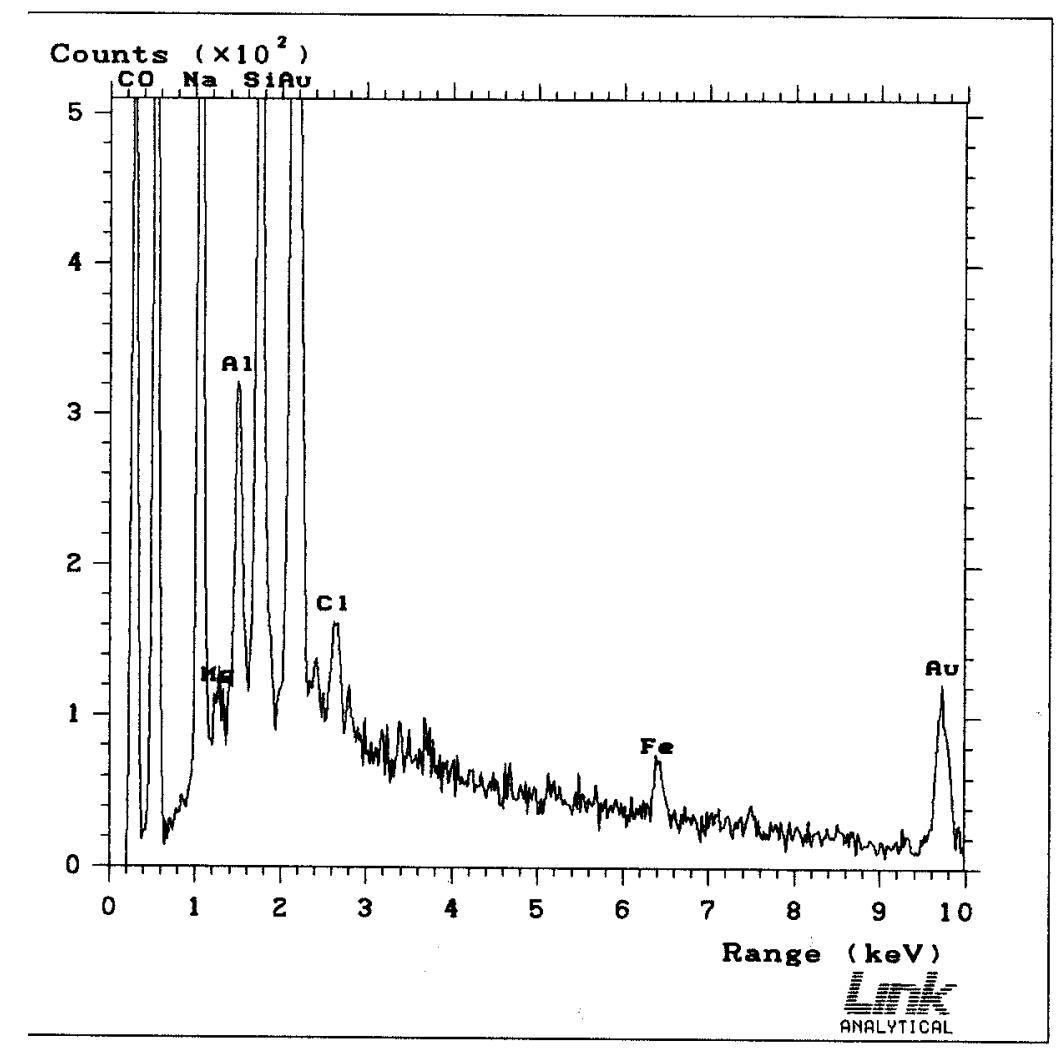

Abbildung 74: EDX-Scan des mit Brenzkatechin belegten Montmorillonits.

Zur Verdeutlichung der Rolle der Tonmineralkanten bei der Sorption und Umwandlung organischer Substanz wurden die folgenden REM-Aufnahmen (Abbildungen 75 und 76), bei denen es sich um den selben Probenausschnitt handelt, angefertigt. Die Scanlinie wurde jedoch in der ersten Aufnahme über möglichst viele Kanten gelegt, während sie in der zweiten Aufnahme etwas darunter über relativ ebene Flächen der Tonmineralplättchen bzw. -flocken verschoben wurde. 


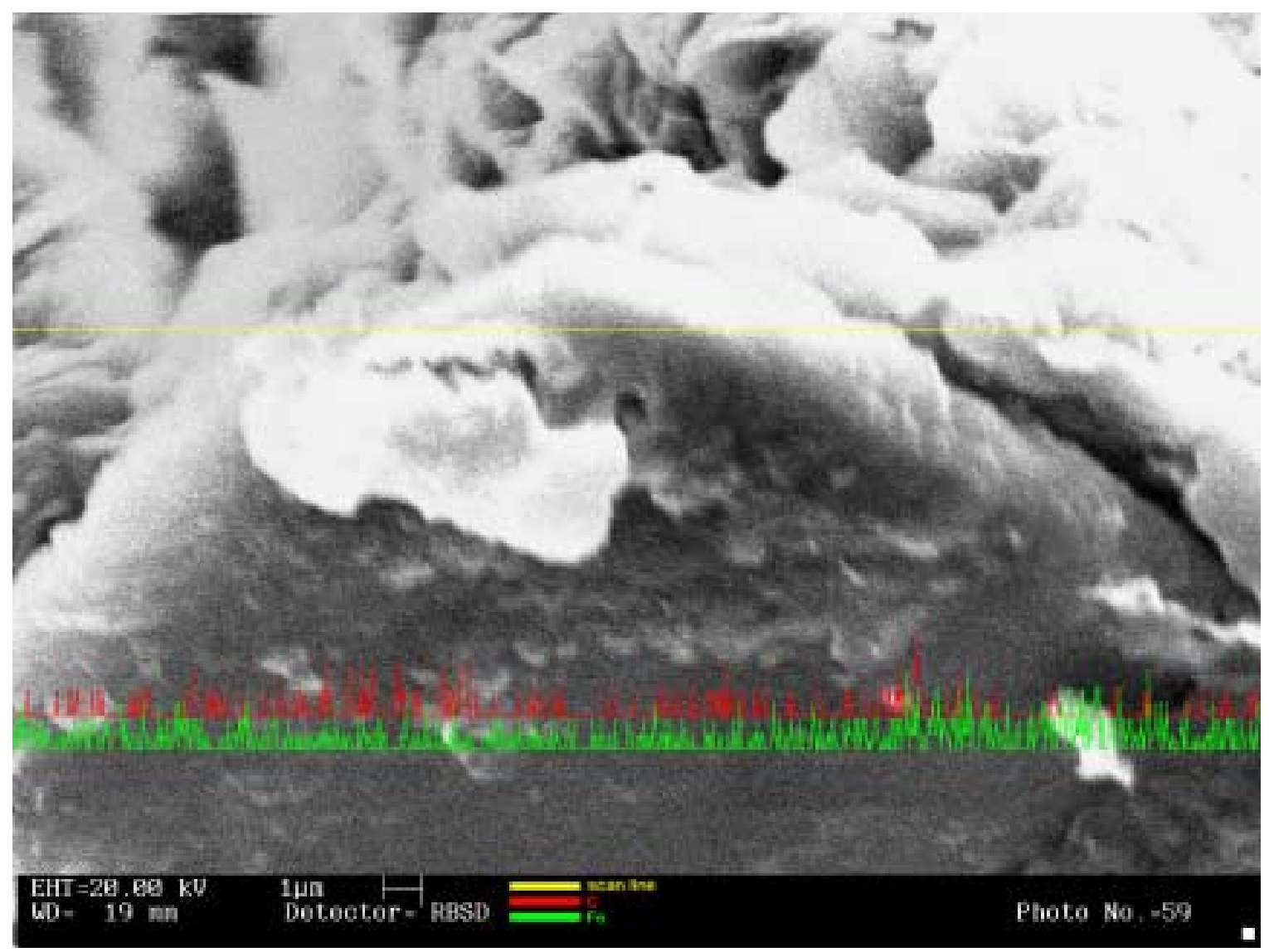

Abbildung 75: EDX/REM-Analyse des mit Brenzkatechin belegten Montmorillonits.

Die Elementverteilung von Kohlenstoff und Eisen in der ersten Aufnahme (Abbildung 75) zeigt, dass mit dem Abtasten der Tonmineralkanten nicht nur ein Anstieg des KohlenstoffSignals zu beobachten ist, sondern gleichzeitig eine Intensitätszunahme für das Eisen festgestellt werden kann. Besonders deutlich tritt dieser Zusammenhang auf der linken und auf der rechten Kante des zentralen Tonplättchens zutage. Weiterhin ist dieser Effekt auf der in der Bildmitte zu erkennenden Tonflocke, sowie im weiteren Verlauf der Scanlinie in der rechten Bildhälfte zu beobachten.

Im Gegensatz dazu weist die gleiche Aufnahme (Abbildung 76) mit der nur etwas nach unten verschobenen Scanlinie zwar noch deutliche Signale der beiden Elemente, insbesondere des Eisens, auf, aber der zuvor deutlich festzustellende Zusammenhang zwischen der Intensität des C-Signals und dem Fe-Signal ist hier nicht mehr zu erkennen. Darüber hinaus ist festzustellen, dass auf den relativ planaren Mineraloberflächen, hier in der Bildmitte der Aufnahme zu erkennen, C-Signale mit einer deutlich geringeren Intensität zu beobachten sind. Das lässt darauf schließen, dass auf den Ober- und Unterseiten der Tonmineralstapel relativ betrachtet weniger organisches Material adsorbiert wird als an den Kanten der Tonminerale. 


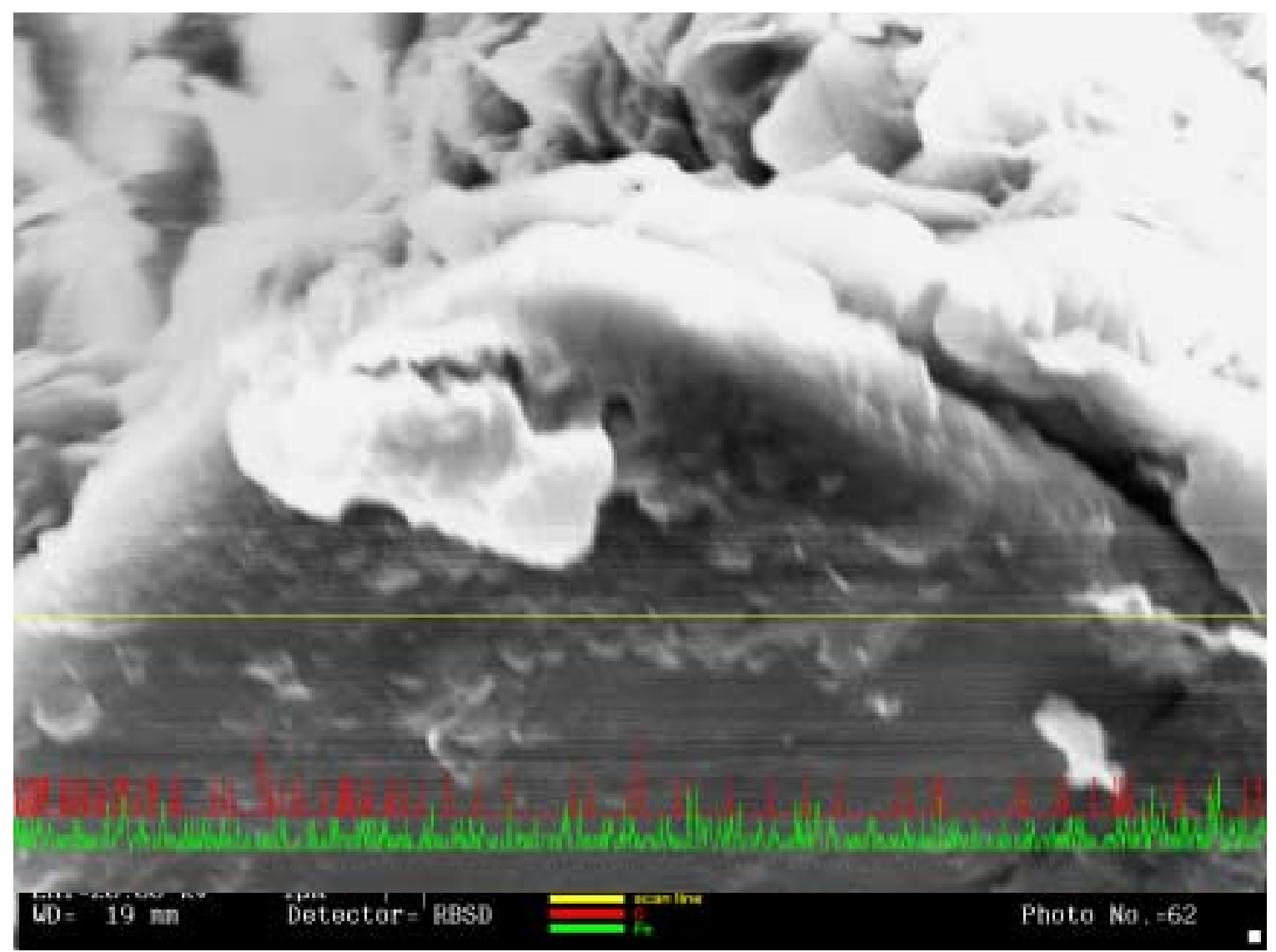

Abbildung 76: EDX-Analyse des mit Brenzkatechin belegten Montmorillonits (verschobene Scanlinie).

Aus den Ergebnissen dieser vergleichenden Untersuchungen kann somit abgeleitet werden, dass die organische Substanz an Tonmineraloberflächen vorwiegend an den Kanten der Minerale adsorbiert wird. Da die Mineralkanten die Hauptorte der Adsorption an Tonmineralen sind, kann folglich auch davon ausgegangen werden, dass sie auch die Zentren der chemischen Aktivität der Tonminerale darstellen.

\subsubsection{Diskussion der Resultate der Röntgenmikrobereichsanalysen}

Die Ergebnisse der durchgeführten Mikrobereichsanalysen bestätigen die durch die FEMAufnahmen aufgestellten Vermutungen, dass es sich bei den dort zu beobachtenden Strukturen auf den Oberflächen von Tonmineralen um organische Substanzen handelt. Es konnte zweifelsfrei nachgewiesen werden, dass durch Sublimation aus der Gasphase auf der Tonmineraloberfläche abgelegte Phenole wie Brenzkatechin und Pyrogallol bzw. deren Reaktionsprodukte sowohl auf Aggregat- als auch auf Pulverproben wiedergefunden werden konnten. Zugleich konnte beobachtet werden ,dass die auf der Oberfläche der Tonminerale auftretenden organischen Überzüge nicht nur sehr kleine organische Strukturen von wenigen Mikrometern Durchmesser, wie im Kapitel 4.2.3 beschrieben, bilden, sondern auch großflächige Überzüge von mehreren Zehnern bis Hundertern Mikrometer (Abbildung 62 entstehen. Auffallend in der Auswertung der Aufnahmen, dass die Bildung der organischen Überzüge nicht gleichmäßig verteilt über ein ganzes Tonmineralteilchen erfolgt, sondern Reaktionen nur an 
diskreten Stellen ablaufen (BIRKEL et al. 2000c). Dies steht im Widerspruch zur gängigen Lehrmeinung, das die Tonminerale in den obersten Horizonten der Böden gleichmäßig von einer Schicht organischen Kohlenstoffs überzogen sind. Mit Hilfe der Röntgenmikrobereichsanalyse konnten erste Hinweise auf die möglichen Ursachen für die inhomogene Verteilung der Organika gefunden werden. Das Verteilungsmuster des Eisens zeigt ebenfalls eine heterogene Verteilung an. So zeigen insbesondere die Kanten der Tonminerale deutlich höhere Intensitäten für das Element Eisen als benachbarte Oberflächen von Tonmineralstapeln. Die Untersuchungen deuten darauf hin, dass das Auftreten der organischen Überzüge mit, an der Tonmineraloberfläche vorkommendem Eisen korreliert. Damit wäre nicht nur ein Hinweis auf die bevorzugten Sorptionsplätze organischer Substanz, die Tonmineralkanten gegeben, sondern zusätzlich die zentralen Orte der chemischen Aktivität der Tonminerale bestimmt. Das Eisen als Bestandteil der Mineralstruktur der Tonminerale bzw. als adsorbiertes Eisenoxidund -hydroxid nicht alleine für die Sorption und Reaktion von organischem Material an Tonmineralen verantwortlich ist, zeigen die Analysenergebnisse, die das Auftreten organischer Überzüge auch bei schwachen Signalintensitäten des Eisens belegen. Dies steht im Widerspruch zur Literatur (WANG \& HUANG 2000), in der eine Reaktivität mineralischer Oberflächen hauptsächlich von elementarem Eisen und Aluminium bzw. von gebundenen Metalloxiden und -hydroxiden wie z.B. Eisen und Mangan abhängig ist (WHELAN \& SIMS 1995). Neben den Metalloxiden und -hydroxiden muss es noch weitere Aktivitätszentren an den Tonmineraloberflächen geben, die organische Verbindungen durch abiotische, physikochemische Prozesse zu transformieren vermögen. Mehrere Autoren führen die reaktiven Eigenschaften der Tonminerale auf deren Bronsted- bzw. Lewis-Azidität zurück. Andere Forschungen weisen auf die Möglichkeit hin, dass Tonminerale durch Charge-Transfer-Komplexe von adsorbierter organischer Substanz mit dieser reagieren können und es über die Initiierung von radikalischen Systemen zur chemischen Veränderung der organischen Verbindungen kommen kann (Kapitel 2.1.2). Für die radikalische Oxidation von an mineralischen Oberflächen adsorbierter organischer Substanz spricht die „Vergesellschaftung“ von Kohlenstoff- und Eisen bzw. Mangan. Nach VOUDRIAS \& REINHARD (1986) kann die Bildung von Radikalen an mineralischen Oberflächen in mehreren verschiedenen Reaktionspfaden erfolgen. Durch Adsorption von Sauerstoff oder Wasser (atmosphärisch) werden Hydroxyl- bzw. Hydroxyperoxylradikale durch Ladungstransfer vom Sorbat zum Sorbens gebildet.

Dem kritischen Leser dieses Kapitels mag sich die Frage stellen, ob es sich bei den nachgewiesen organischen Substanzen nicht lediglich um Überzüge, bestehend aus dem jeweiligen sublimierten Phenol handelt, welches an die bevorzugten Sorptionsplätze andockt. Mit den Ergebnissen der Mikrobereichsanalysen allein kann die Frage, ob hier adsorbierte Ausgangssubstanz oder durch die chemische Aktivität der Tonminerale gebildete Umwandlungsprodukte diese organischen Überzüge bilden, nicht beantwortet werden. Dazu ist auf die, im weiteren Verlauf dieser Arbeit dargestellten Ergebnisse der chemischen und insbesondere der spektroskopischen Analysen (Kapitel 4.6 und 4.7) zu verweisen, jedoch spricht bisher vieles dafür, dass hier erste Hinweise für die Oxidation und Polymerisation von, an Tonmineralen sorbier- 
ten, organischen Substanzen durch adsorbierte Fe-Oxide und/oder Fe-Hydroxide bzw. strukturellem Eisen gegeben sind.

\subsection{Qualitative Nachweise mit nasschemischen Methoden}

Nach der Extraktion der organischen Überzüge der Belegung von Montmorilloniten mit Phenolen wurden vor den geplanten spektroskopischen Untersuchungen nasschemische Analysen zur Charakterisierung der in den Extrakten enthaltenen Verbindungen und funktionellen Gruppen durchgeführt. Ausgewählt wurden Tests, die spezifisch auf die, in den Extrakten zu erwartenden Verbindungen (Phenole, allgem. $\mathrm{C}=\mathrm{O}$-Bindungen, Aldehyde/Ketone) ansprechen.

\subsubsection{Nachweis von Phenolen}

Der Nachweis von Phenolen erfolgt durch die Zugabe von Eisen(III)Chlorid $\left(\mathrm{FeCl}_{3}\right)$ in eine ethanolische Lösung der entsprechenden Proben (EHLERS 1989). Das $\mathrm{Fe}^{3+}$-Ion bildet über die phenolischen Hydroxylgruppen farbige Fe-Phenol-Komplexe (Abbildung 77). Dazu werden $0,5 \mathrm{ml}$ der Probenlösung mit Ethanol versetzt und 0,5 bis $1 \mathrm{ml} 1 \%$-ige $\mathrm{FeCl}_{3}$-Lösung zugegeben.

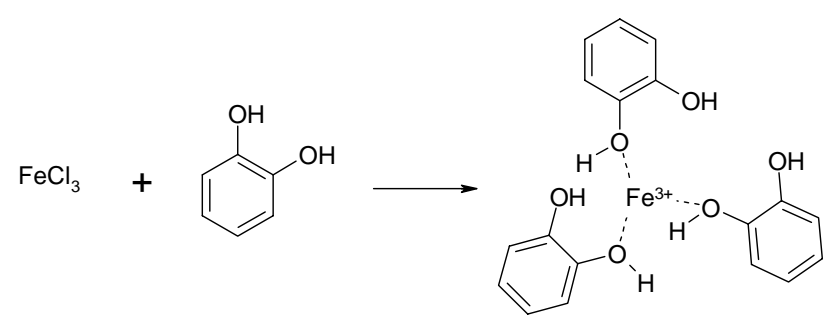

Abbildung 77: Komplexbildung von $\mathrm{Fe}^{3+}$ mit Brenzkatechin.

In Anwesenheit von Phenolen zeigt sich eine blaue bis violette, zum Teil auch gelbe Färbung der Lösung. Die Resultate für die gewonnenen Extraktlösungen sind in Tabelle 6 dargestellt.

Tabelle 6: Ergebnisse des Phenol-Nachweises mit $\mathrm{FeCl}_{3}$-Lösung.

\begin{tabular}{|c|l|c|}
\hline Proben-Nr. & \multicolumn{1}{|c|}{ Bezeichnung } & Farbe \\
\hline 1 & Na-Montmorillonit + Brenzkatechin 1 & schwarz \\
\hline 2 & Na-Montmorillonit + Brenzkatechin 2 & gelb-braun, schwärzlich \\
\hline 3 & Na-Montmorillonit + Phenanthren 1 & leuchtend gelb \\
\hline 4 & Na-Montmorillonit + 2,6-Dimethylphenol 1 & leuchtend hellgelb \\
\hline 5 & Na-Montmorillonit + Pyrogallol 2 & - \\
\hline 6 & Na-Montmorillonit + Phenanthren 2 & gelb-braun \\
\hline 7 & Na-Montmorillonit + 2,6-Dimethylphenol & gelb \\
\hline 8 & Na-Montmorillonit + Guajacol & gelb \\
\hline 9 & Na-Montmorillonit + 2,6-Dimethylphenol 2 & schwarz \\
\hline 10 & Na-Montmorillonit + Pyrogallol 1 & schwarz \\
\hline 11 & Na-Montmorillonit + Phenanthren + Brenzkatechin 1 & \\
\hline 12 & Na-Montmorillonit + Phenanthren + Brenzkatechin 2 &
\end{tabular}


Es ist zu beobachten, dass in allen Proben, die zuvor mit Phenolen belegt wurden, auch noch nach der Reaktion Phenole nachgewiesen werden konnten. Die Reaktionen, die zur Bildung farbiger Überzüge auf den Tonmineraloberflächen führten haben keine vollständige Umsetzung der Phenole bewirkt.

\subsubsection{Nachweis von Carbonylgruppen}

Im Kapitel 2.2.5 wurde bereits dargelegt, dass durch radikalische Prozesse eine Oxidation der Phenole durch die katalytische Reaktion an Tonmineralen bewirkt werden kann. Als Reaktionsprodukte sind somit vor allem chinoide Verbindungen, Aldehyde und Ketone, charakterisiert durch Carbonylgruppen $(-\mathrm{C}=\mathrm{O})$, anzunehmen. Um die extrahierten organischen Lösungen auf diese funktionelle Gruppen zu testen, wurden zwei weitere Nachweisreaktionen durchgeführt.

\subsubsection{Nachweis von Aldehyden und Ketonen}

Der Nachweis von Carbonylgruppen von Aldehyden bzw. von Ketonen erfolgte mit fuchsinschwefliger Säure (Nachweis nach Schiff). Fuchsin gehört zu den Triphenylmethanfarbstoffen und hat in der Normalform eine rote Farbe. Wird zum Fuchsin schweflige Säure zugesetzt, so wird die chinoide Struktur des Farbstoffs zur farblosen Leukoform umgewandelt. Aldehyde und Ketone bewirken eine Oxidation der Leukoform und es entsteht wieder die Normalform mit einer Rosa- bis Violettfärbung der Probe. Zur Herstellung des Schiff'schen Reagenzes werden 0,1 g Fuchsin in $100 \mathrm{ml}$ destilliertem Wasser gelöst und bis zur Entfärbung der Lösung Schwefeldioxid $\left(\mathrm{SO}_{2}\right)$ eingeleitet. Die Herstellung von $\mathrm{SO}_{2}$ erfolgt durch Reaktion von $\mathrm{NaHSO}_{3}$ mit $\mathrm{H}_{2} \mathrm{SO}_{4}$ :

$$
\mathrm{NaHSO}_{3}+\mathrm{H}_{2} \mathrm{SO}_{4} \rightarrow \mathrm{SO}_{2} \uparrow+\mathrm{NaHSO}_{4}+\mathrm{H}_{2} \mathrm{O}
$$

2 Tropfen der zu testenden Lösung werden mit $2 \mathrm{ml} \mathrm{Reagenz}$ versetzt und in einem verschließbaren Reagenzglas geschüttelt. Sind Aldehyde oder Ketone in der Lösung vorhanden, so tritt schon nach wenigen Sekunden eine rosa bis violette Färbung auf. Bei schwer löslichen Aldehyden und solchen mit einem hohen Molekulargewicht können bis zum Farbeintritt mehrere Minuten verstreichen (Tabelle 7 aufgeführt).

Tabelle 7: Nachweis von Aldehyden und Ketonen mit Schiff'schem Reagenz.

\begin{tabular}{|c|l|c|}
\hline Proben-Nr. & \multicolumn{1}{|c|}{ Bezeichnung } & Farbe \\
\hline 1 & Na-Montmorillonit + Brenzkatechin 1 & rosa \\
\hline 2 & Na-Montmorillonit + Brenzkatechin 2 & gelblich-rosa \\
\hline 3 & Na-Montmorillonit + Phenanthren 1 & klar \\
\hline 4 & Na-Montmorillonit + 2,6-Dimethylphenol 1 & leicht rosa \\
\hline 5 & Na-Montmorillonit + Pyrogallol 2 & klar \\
\hline 6 & Na-Montmorillonit + Phenanthren 2 & klar \\
\hline 7 & Na-Montmorillonit + 2,6-Dimethylphenol & klar \\
\hline 8 & Na-Montmorillonit + Guajacol & klar \\
\hline 9 & Na-Montmorillonit + 2,6-Dimethylphenol 2 & klar \\
\hline 10 & Na-Montmorillonit + Pyrogallol 1 & klar \\
\hline 11 & Na-Montmorillonit + Phenanthren + Brenzkatechin 1 & gelblich-rosa \\
\hline 12 & Na-Montmorillonit + Phenanthren + Brenzkatechin 2 & gelblich-rosa \\
\hline
\end{tabular}


Carbonylgruppen von Aldehyden konnten eindeutig in den Proben 1, 2, 4, 11 und 12 nachgewiesen werden. Die anderen Proben zeigten keine Verfärbungen. Nach einer mehrstündigen Reaktionszeit trat jedoch bei allen Proben, die Phenole enthielten, ein Farbeintritt auf (Tabelle 8).

Tabelle 8: Nachweis von Aldehyden und Ketonen nach mehrstündiger Reaktionszeit.

\begin{tabular}{|c|l|c|}
\hline Proben-Nr. & \multicolumn{1}{|c|}{ Bezeichnung } & Farbe \\
\hline 1 & Na-Montmorillonit + Brenzkatechin 1 & violett, intensiv \\
\hline 2 & Na-Montmorillonit + Brenzkatechin 2 & gelblich-rosa \\
\hline 3 & Na-Montmorillonit + Phenanthren 1 & flieder \\
\hline 4 & Na-Montmorillonit + 2,6-Dimethylphenol 1 & rosa \\
\hline 5 & Na-Montmorillonit + Pyrogallol 2 & rosa \\
\hline 6 & Na-Montmorillonit + Phenanthren 2 & rosa \\
\hline 7 & Na-Montmorillonit + 2,6-Dimethylphenol & klar, leicht rosa \\
\hline 8 & Na-Montmorillonit + Guajacol & leicht rosa \\
\hline 9 & Na-Montmorillonit + 2,6-Dimethylphenol 2 & leicht rosa \\
\hline 10 & Na-Montmorillonit + Pyrogallol 1 & rosa-violett \\
\hline 11 & Na-Montmorillonit + Phenanthren + Brenzkatechin 1 & rosa-violett \\
\hline 12 & Na-Montmorillonit + Phenanthren + Brenzkatechin 2 & \\
\hline
\end{tabular}

Die Transformation von Phenolen durch abiotische Katalyse an Tonmineralen führte zur Entstehung von Aldehyden bzw. Ketonen. In der Extraktlösung der Belegung des Montmorillonits mit Phenanthren konnten hingegen keine Carbonylgruppen nachgewiesen werden.

\subsubsection{Nachweis von Carbonylgruppen mit Dinitrophenylhydrazin}

Für den Nachweis mit Dinitrophenylhydrazin wurden 0,5 g 2,4-Dinitrophenylhydrazin in 50 $\mathrm{ml} 50$ \%-iger Schwefelsäure unter Kühlen gelöst. Von diesem Reagenz wurden $3 \mathrm{ml}$ in $6 \mathrm{ml}$ destilliertem Wasser verdünnt. $1 \mathrm{ml}$ Probenlösung wurden mit Isopropanol versetzt, geschüttelt und dem Reagenz zugesetzt. Durch Ausfallen eines gelben bis orangefarbenen Niederschlags (Abbildung 78) werden Carbonylgruppen nachgewiesen (Tabelle 9).

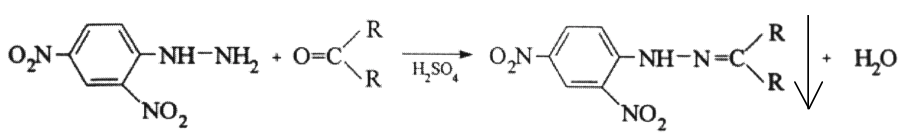

Abbildung 78: Nachweisreaktion von Carbonylgruppen.

Tabelle 9: Nachweis von Carbonylgruppen.

\begin{tabular}{|c|l|c|}
\hline Proben-Nr. & \multicolumn{1}{|c|}{ Bezeichnung } & Farbe des Niederschlags \\
\hline 1 & Na-Montmorillonit + Brenzkatechin 1 & orange \\
\hline 2 & Na-Montmorillonit + Brenzkatechin 2 & orange \\
\hline 3 & Na-Montmorillonit + Phenanthren 1 & kein Niederschlag \\
\hline 4 & Na-Montmorillonit + 2,6-Dimethylphenol 1 & gelborange \\
\hline 5 & Na-Montmorillonit + Pyrogallol 2 & kein eindeutiger Niederschlag \\
\hline 6 & Na-Montmorillonit + Phenanthren 2 & kein Niederschlag \\
\hline 7 & Na-Montmorillonit + 2,6-Dimethylphenol & kein Niederschlag \\
\hline 8 & Na-Montmorillonit + Guajacol & kein Niederschlag \\
\hline 9 & Na-Montmorillonit + 2,6-Dimethylphenol 2 & leichter Niederschlag \\
\hline 10 & Na-Montmorillonit + Pyrogallol 1 & kein Niederschlag \\
\hline 11 & Na-Montmorillonit + Phenanthren + Brenzkatechin 1 & hellorange \\
\hline 12 & Na-Montmorillonit + Phenanthren + Brenzkatechin 2 & hellorange \\
\hline
\end{tabular}


Bei den Proben 1, 2, 4, 11 und 12 sind deutliche Ausfällungen des spezifischen Farbkomplexes zu beobachten. Sie weisen auf die Existenz von Carbonylverbindungen im extrahierten Probenmaterial hin und bestätigen die Oxidation der phenolischen Grundkörper zu chinoiden Verbindungen. Bei den übrigen Phenolen konnten keine bzw. keine eindeutigen Nachweisreaktionen erkannt werden. Als Gründe hierfür sind, wie beim Nachweis von Aldehyden und Ketonen mit dem Schiff'schen Reagenz, zu kurze Reaktionszeiten bis zur Aufnahme der Resultate zu nennen. Beobachtungen nach einstündiger Wirkzeit resultierten im Auftreten von gelben Niederschlägen in allen phenolhaltigen Proben. In den aus der Belegung mit Phenanthren extrahierten Proben konnten zu keiner Zeit Carbonylgruppen nachgewiesen werden.

\subsubsection{Diskussion der Ergebnisse}

Die als Vorproben zu den nachfolgenden spektroskopischen Untersuchungen (Kapitel 4.6/4.7) durchgeführten nasschemischen Nachweise konnten die im Kapitel 2.2.5 angenommenen Transformationsprodukte von Phenolen schon allein anhand der eingetretenen Nachweisreaktionen eine chemische Umwandlung der eingesetzten Ausgangssubstanzen nachgewiesen werden. Durch das Auftreten von Carbonylbindungen als funktionelle Gruppen kann die Bildung der entsprechenden Reaktionsprodukte durch oxidative Prozesse an Tonmineralen zu Chinonen bzw. chinoiden Verbindungen angenommen werden.

Aus den Ergebnissen dieser ersten Analysen bleibt zudem festzuhalten, dass Phenanthren als Vertreter der polyzyklischen aromatischen Kohlenwasserstoffe keine, mit dieser Methodik erkennbaren Reaktionen durch abiotisch induzierte Katalyse zeigt. 


\subsection{High Performance Liquid Chromatographie}

Das Prinzip der Chromatographie beruht darauf, dass die einzelnen Bestandteile einer Probe in einer mobilen Phase (Fließmittel) in unterschiedlichen Geschwindigkeiten durch eine stationäre Phase transportiert werden. Die stationäre Phase besteht aus dichtgepackten porösen Teilchen (Kieselgele), die ein hohes Verhältnis von Oberfläche zu Volumen bilden. Die variierenden Geschwindigkeiten beruhen auf der unterschiedlich starken Sorption der Substanzen an der Oberfläche der stationären Phase. Die durch Adsorption hervorgerufene Bindung von Substanzen an Oberflächen kann physikalischer oder chemischer Natur sein. Die Chromatographie macht sich die physikalische Bindung von Molekülen an Oberflächen zunutze, bei der es sich um ein reines Festhalten der Stoffe ohne deren chemische Veränderung handelt. Die Wirkung der stationären Phase als Adsorbens geht auf polare Silanolgruppen an der Oberfläche des Silikagels zurück. Von diesen hydrophilen Hydroxyl-Gruppen der oberflächenständigen Siliziumatome gehen Anziehungskräfte aus, die die Probesubstanzen für kurze Zeit sorbieren. Je polarer die Substanzen, desto stärker werden sie an der Oberfläche des Kieselgels retardiert und um so später verlassen sie die Trennsäule. Diese Art der Chromatographie, die Normalphasen-Chromatographie dient zur Trennung unpolarer bis mittelpolarer Verbindungen. Um unpolare bis polare Komponenten mit Hilfe der Chromatographie zu trennen, kommt ein Verfahren zur Anwendung, dass als Reversed-Phase-Chromatographie (RPC) bezeichnet wird. In diesem Falle ist die stationäre Phase unpolarer als die mobile Phase (z. B. Alkohol/Wasser). Hierzu wird die polare Oberfläche der Silikagele durch chemische Modifikation zu einer unpolaren Oberfläche verändert. Dabei werden die polaren Hydroxylgruppen durch unpolare funktionelle Gruppen ersetzt (z.B. n-Octadecyl), der hydrophobe Charakter nimmt mit wachsender Kettenlänge des Restes zu. An einer RP-Oberfläche werden Substanzen um so stärker festgehalten, je geringer ihre Löslichkeit in Wasser ist. Daraus ergeben sich eine kurze Adsorptionsdauer für polare Verbindungen und längere Retentionszeiten für unpolare Substanzen. Somit erhöhen sich die Retentionszeiten von Verbindungen mit zunehmender Hydrophobizität und auch mit zunehmendem Kondensationsgrad der zu trennenden Substanzen. Die Wahl des Eluenten ist von großer Bedeutung für die Auftrennung von Substanzgemischen. Die Lösemittelmoleküle konkurrieren mit den Probemolekülen um die aktiven Gruppen an der Oberfläche der stationären Phase. Je stärker das Fließmittel selbst von der stationären Phase sorbiert wird, desto größer ist seine Elutionskraft; besitzt das Fließmittel selbst eine starke Affinität zur stationären Phase, dann werden die Probenmoleküle leicht vom Adsorbens gelöst und weitertransportiert. Die Polarität des Lösemittels wird durch die eluotrope Reihe beschrieben. Wasser gilt bei der Normalphasen-Chromatographie als stärkstes Elutionsmittel, bei der RP-Chromatographie hingegen stellt es jedoch die schwächste mobile Phase und verlängert mit steigendem Anteil am Eluenten die Retentionszeiten. So bewirkt eine Zunahme des Wassergehaltes um 10\% eine Verdopplung der Retention, die Trennung von Substanzen wird daher schärfer (KRAATZ 1995). Im nachfolgend beschriebenen Analyseverfahren wird der Anteil des Wassers in der mobilen Phase im Verlauf einer Messung kontinuier- 
lich herabgesetzt. Die Veränderung der Fließmittelstärke (Gradienten) wird als oft einzige Möglichkeit zur Auftrennung von Gemischen sehr unterschiedlicher Retention verwendet. Das Ergebnis einer chromatographischen Analyse wird durch ein Chromatogramm dargestellt. Bei vollständiger Trennung von Substanzen wird für jede Komponente eine Kurve (Peak), annähernd einer Gauß'schen Kurve, aufgezeichnet.

\subsubsection{Analytische und präparative Chromatographie}

Das chromatographische Trennprinzip beruht wie eingangs geschildert in der Trennung von Substanzgemischen durch deren unterschiedliche Transportgeschwindigkeiten aufgrund sorptiver Vorgänge am Füllmaterial der Säule. Eine qualitative Analytik ist mit einem solchen System somit nur insoweit möglich, dass man Referenzmaterial (Standards) chromatographiert und dessen Retentionszeiten, das ist die Zeit, welche vom Einspritzen der Probe in das System bis zur Signaldetektion am Detektor vergeht, mit denen der Peaks der Substanzgemische vergleicht. Weitere qualitative Aussagen bei Verwendung der Reversed-PhaseChromatographie können noch über die Retentionszeit selbst gegeben werden. Je kürzer die Retentionszeit einer Substanz desto polarer ist diese und umso länger eine Substanz braucht, um den Detektor zu erreichen desto unpolarer ist sie.

Zum Einsatz kamen zwei verschiedene Trennungsverfahren. Um einen Überblick über die Retentionszeiten der Referenzproben und der extrahierten Überzüge und somit über die Anzahl der entstandenen Reaktionsprodukte zu erhalten wurde zuerst mit einer analytischen Trennsäule gearbeitet. Diese zeichnet sich durch eine hohe Empfindlichkeit bei geringen Probenkonzentrationen und Probenmenge $(10 \mu \mathrm{l})$ aus. Zur Gewinnung und Aufkonzentrierung von einzelnen Probenfraktionen wurde eine präparatives Trennverfahren angewendet, wobei deutlich höhere Probenmengen $(100 \mu 1)$ aufgegeben werden konnten und so eine effektivere Fraktionierung mit höheren Konzentrationen möglich wurde.

Beim verwendeten HPLC-System handelt es sich um eine Anlage des Instituts und Museums für Geologie und Paläontologie der Universität Göttingen, bestehend aus einer zentralen Steuer- und Pumpeneinheit (Controller 616, Pump 600 von Waters) mit vorgeschaltetem Degasser, einem Säulenofen und UV-Detektor (Waters 486). Als Probenaufgabesystem wurde ein Autosampler von Thermo Separations verwendet, zur Gewinnung der einzelnen Fraktionen wurde seitens des Teilprojekts A6 ein Fraktionensammler angeschafft (Gilson FC 203B). Zur Aufzeichnung und Aufbereitung der Chromatogramme wurde das Programmpaket „Millennium 32" der Fa. Waters eingesetzt. Als analytische Trennsäule wurde eine Reversed-PhaseSäule (250×4,6 mm), gefüllt mit ODS-Hypersil, Körnungsdurchmesser $5 \mu \mathrm{m}$ (Fa. Chromatographie-Service) benutzt. Zum Schutz vor eventuellen Verunreinigungen war eine Vorsäule $(10 \times 4,6 \mathrm{~mm})$ vorgeschaltet. Der Eluent bestand aus im Ultraschallbad entgastem Methanol (LiChroSolv, Merck) 75\% - Wassergemisch, gepuffert mit 0,1 M KOH und 0,1 $\mathrm{M} \mathrm{KH}_{2} \mathrm{PO}_{4}$ (Merck) auf $\mathrm{pH} 7,0$ bei einer konstanten Temperatur von $20^{\circ} \mathrm{C}$ und einem Fluss von 0,8 $\mathrm{ml} / \mathrm{min}$. Die chromatographische Trennung des Probenmaterials erfolgte isokratisch. Die zu- 
vor durch Extraktion gewonnenen Lösungen der Reaktionsprodukte wurden nach Filtration durch einen 0,45 $\mu \mathrm{m}$ Polyamidfilter in $1 \mathrm{ml}$ Braunglasfläschen mit Teflonsepten abgefüllt und in den Autosampler gegeben. Die Trennungsläufe dauerten je nach Probenlösung zwischen 15 und $30 \mathrm{~min}$. Die Detektion erfolgte bei einer Wellenlänge von $254 \mathrm{~nm}$ (Ultraviolett).

Zur Sammlung und Aufkonzentrierung der entstandenen Reaktionsprodukte wurde eine präparative Trennsäule (ODS-Hypersil, Körnungsdurchmesser $10 \mu \mathrm{m}, 250 \times 8 \mathrm{~mm}$ ) der Fa. Chromatographie-Service verwendet. Der Eluent bestand aus im Ultraschallbad entgastem Methanol (LiChroSolv, Merck) 75\% - Wassergemisch, gepuffert mit 0,1 M KOH und 0,1 M $\mathrm{KH}_{2} \mathrm{PO}_{4}$ (Merck) auf $\mathrm{pH} 7,0$ bei einer konstanten Temperatur von $20^{\circ} \mathrm{C}$ und einem Fluss von 2,0 $\mathrm{ml} / \mathrm{min}$. Die Trennung der einzelnen Peaks erfolgte isokratisch, wo eine bessere Trennung nicht zur erreichen war wurde ein Gradientenprogramm (Tabelle 10) gewählt.

Tabelle 10: Instrumenten-Konfiguration und Gradientenprogramm.

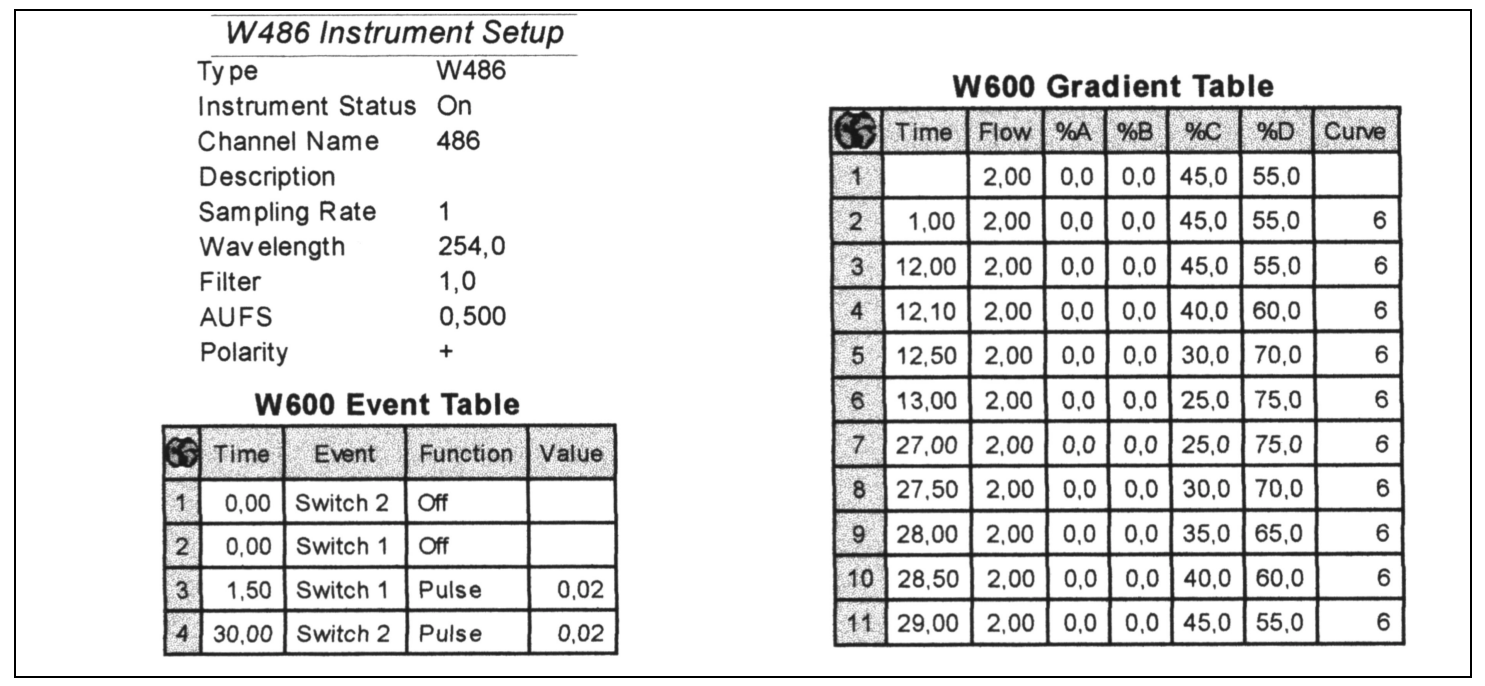

Die Laufzeiten der Fraktionierungsvorgänge wurden nach den verschiedenen Substanzgemischen ausgerichtet und beanspruchten zwischen 15 und 40 min. Die aufgetrennten Substanzen wurden mit dem an das HPLC-System gekoppelten Fraktionensammler für weitere Analysen separat aufgefangen, so dass einen weitgehend automatisierte Abarbeitung des Probenmaterials gewährleistet wurde.

\subsubsection{Ergebnisse der HPLC-Analysen}

Die anfänglich durchgeführten chromatographischen Trennungen hatten die Aufgabe das zu erwartende Gemisch aus Reaktionsprodukten aufzutrennen, um die Zahl der entstandenen Substanzen zu bestimmen. Die folgenden Chromatogramme zeigen, dass es durch die abiotische Reaktion von Phenolen an Tonmineraloberflächen zur Bildung mehrerer Reaktionsprodukte gekommen ist.

\subsubsection{Reaktionsprodukte des Brenzkatechins}

Das Chromatogramm der nach der Belegung mit Brenzkatechin von der Tonmineraloberfläche extrahierten Überzüge zeigt neben dem Peak für das Brenzkatechin mit einer Retentions- 
zeit von ca. 6 min eine Vielzahl weiterer Peaks die Retentionszeiten von 2,6 min bis 13 min aufweisen. Der Peak bei 2,6 min wird durch das Methanol hervorgerufen, in welches das extrahierte Probenmaterial aufgenommen wurde. Dieses als Lösemittelpeak bezeichnete Signal wir durch den raschen Konzentrationsanstieg des Methanols im Eluenten hervorgerufen und kann zur Ermittlung der Totzeit, dass ist der Zeitraum der vom Einspritzen der Probe bis zum Erreichen der Detektorzelle vergeht, herangezogen werden. Von der Anzahl der entstandenen Fraktionen ist dieser Peak somit abzuziehen.

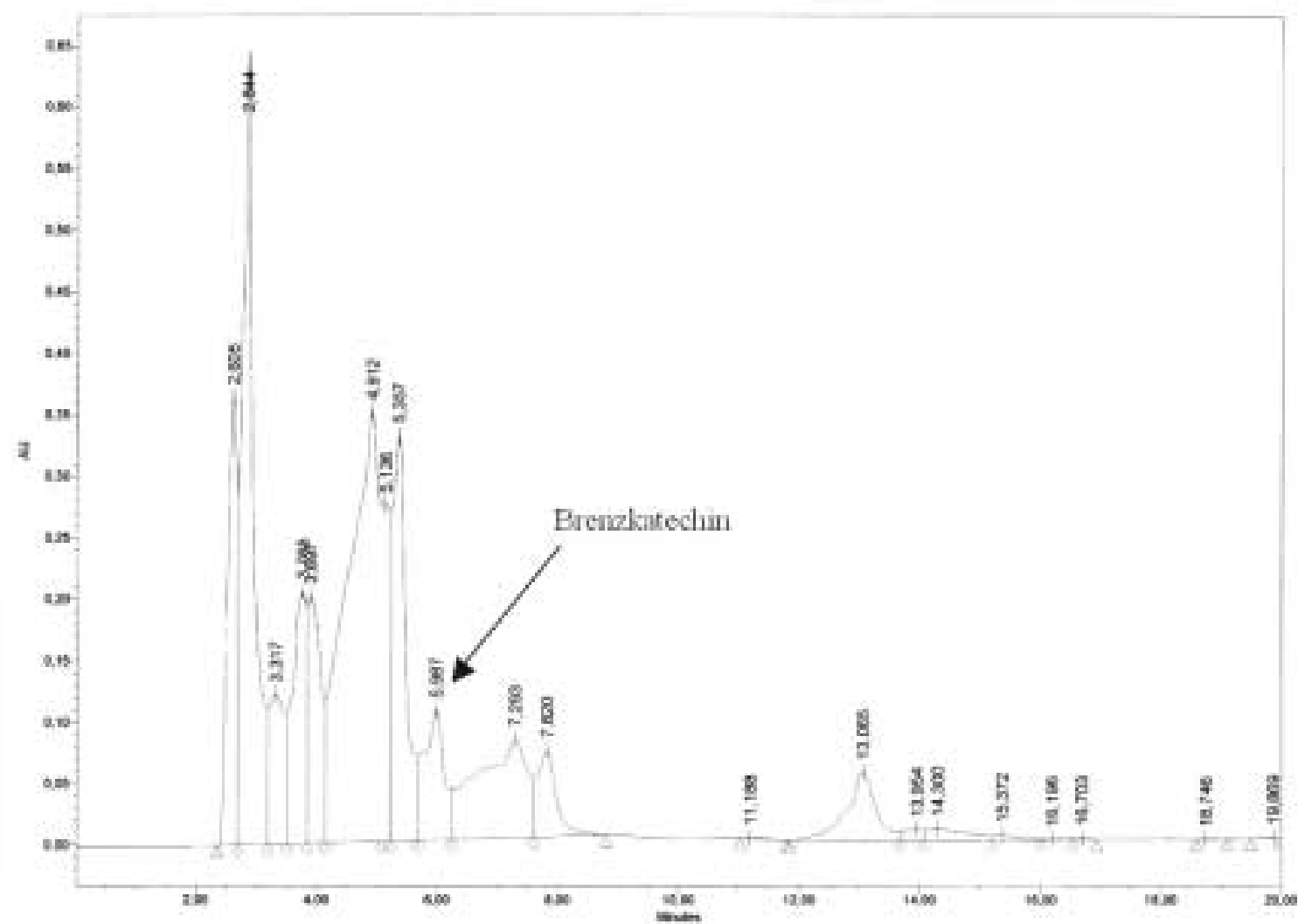

Abbildung 79: Chromatogramm extrahierter Überzüge nach Belegung mit Brenzkatechin.

Zwischen dem Lösemittelpeak (2,6 min) und dem Peak für das noch in der Probe verbliebene chemisch unveränderte Brenzkatechin (5,98 min) treten weitere Peaks unterschiedlicher Intensität auf. Diese liegen bei 2,8, 3,3, 3,8, 4,9, 5,1 und 5,4 min. Aufgrund der kürzeren Retentionszeiten als die des Brenzkatechins kann bei Verwendung einer unpolaren Reversed-PhaseSäule somit daraus geschlossen werden, dass die detektierten Substanzen eine geringere Molekülgröße bzw. höhere Polarität als die Ausgangssubstanz aufweisen. Das Entstehen polarerer Verbindungen kann durch die Bildung von alkoholischen $\mathrm{OH}-$ bzw. Carboxylgruppen hervorgerufen werden (Kapitel 4.6 bzw. 4.7). Verbindungen mit längeren Retentionszeiten als die der Ausgangssubstanz sind im Chromatogramm bei 7,3, 7,8, 13,1 und ca. 14,3 min zu beobachten, wobei durch die schlechte Auflösung des letzten Peaks zu erkennen ist, dass die isokratische Trennmethode hier an ihre Grenzen stößt. Die Retentionszeiten dieser Peaks lassen darauf schließen, dass die entstandenen Verbindungen, bei denen es sich auch um Molekülbruchstücke handeln kann, größer als die Ausgangssubstanz sind bzw. eine niedrigere Po- 
larität aufweisen und daher aufgrund häufigerer Sorption am Säulenfüllmaterial langsamer transportiert werden. In einer homologen Reihe steigt die Festhaltekraft am Säulenmaterial mit steigender Kohlenstoffzahl. Die Reihenfolge verläuft in Richtung zunehmender Festhaltekräfte von Carbonsäuren über Alkohole, Phenole, Amine, Ether, Aldehyde und Ketone schließlich bis zu Aliphaten. Aus dieser Reihe kann somit abgeleitet werden, dass es durch die Oxidation des Brenzkatechins an der Oberfläche des Montmorillonits neben der Bildung von polareren Verbindungen (Carbonsäuren) zur Entstehung sowohl höhermolekularer Verbindungen bzw. zur Bildung von Ethern, Aldehyden, Ketonen (Chinonen) oder Aliphaten gekommen sein kann (Kapitel 4.6, 4.7). Entgegen ersten Vermutungen führt die abiotische Reaktion von Brenzkatechin auf der Oberfläche von Tonmineralen jedoch nicht zur Entstehung eines einzelnen Reaktionsprodukts, es konnten insgesamt 10 neue Verbindungen nachgewiesen werden.

\subsubsection{HPLC-MS der Reaktionsprodukte des Brenzkatechins}

Zur Bestimmung der Molekülmassen und somit zur Identifikation der entstandenen Reaktionsprodukte der Belegung des Montmorillonits mit Brenzkatechin wurde in Kooperation mit der Arbeitsgruppe Organische Chemie der Universität Oldenburg verschiedene massenspektrometrische Untersuchungen durchgeführt. Unter anderem wurden die in Göttingen erzielten Ergebnisse mit einem an ein Massenspektrometer gekoppeltem HPLC-System (HPLC-ACPI-MS, mit RP-C 18 -Säule (Merck Lichrospher 100, $5 \mu \mathrm{m}, 250 x 4 \mathrm{~mm}$ ), Methanol/Wasser-Gemisch im Verhältnis 25:75) nachgebildet. Für die massenspektrometrische Analyse der Reaktionsprodukte des Brenzkatechins konnten erste Ergebnisse erzielt werden, für die übrigen Phenole stehen die Daten noch aus.

Für das extrahierte Probenmaterial der Belegung von Montmorillonit mit Brenzkatechin konnten im Bereich von 0 bis 10 min 7 Peaks detektiert werden (Tabelle 11).

Tabelle 11: Ergebnisse der HPLC-MS-Analyse der extrahierten Überzüge der Belegung mit Brenzkatechin.

\begin{tabular}{|c|c|l|}
\hline Retentionszeit (min) & Masse $\mathbf{( m / z )}$ & \multicolumn{1}{c|}{ mögliche Zuordnung } \\
\hline 2,17 & 309 & Pentacenedion, Warfarin \\
\hline 2,28 & 161 & Naphtalendiol, Ethanon, 1,2-Dihydroxynaphtalen \\
\hline 3,45 & $215,247,827$ & $\begin{array}{l}\text { 215: Benzoesäure, Methanon, Benzylmethoxyether } \\
247: \text { Tetrahydroxybenzophenon }\end{array}$ \\
\hline 3,60 & 309 & \\
\hline 3,87 & 293 & \\
\hline 4,29 & 141 & $\begin{array}{l}\text { Cyclohexadiendion, Methoxyresorcinol, Methoxyhydroquino- } \\
\text { ne, 1,2-Benzendiol }\end{array}$ \\
\hline 5,53 & 215 & \\
\hline
\end{tabular}

Aus der Tabelle 11 kann entnommen werden, dass, wie bereits durch die Interpretation des Chromatogramms angenommen, durch die Reaktion des Brenzkatechins an der Tonmineraloberfläche zum einen eine Oxidation des Phenols eingetreten ist. Andererseits zeigen die detektierten Molekülmassen im Vergleich zum Brenzkatechin mit einer Masse von $110 \mathrm{u}$, dass es zur Bildung von Dimeren bzw. Oligomeren gekommen ist, da Massen mit mehr als $220 \mathrm{u}$ zu beobachten sind. 


\subsubsection{Reaktionsprodukte des Pyrogallols}

Die chromatographische Trennung der extrahierten Reaktionsprodukte des mit Pyrogallol belegten Montmorillonits (Abbildung 80) weist ebenfalls die Entstehung von 10 neu auftretenden Verbindungen nach. Neben dem Peak des Lösemittels bei 2,9 min ist im Unterschied zum Chromatogramm des Brenzkatechins festzustellen, dass nur ein Peak eine kürzere Retentionszeit als das Pyrogallol (3,83 min) aufweist und somit einer Verbindung mit einer geringeren Molekülgröße bzw. höheren Polarität zugeordnet werden kann.

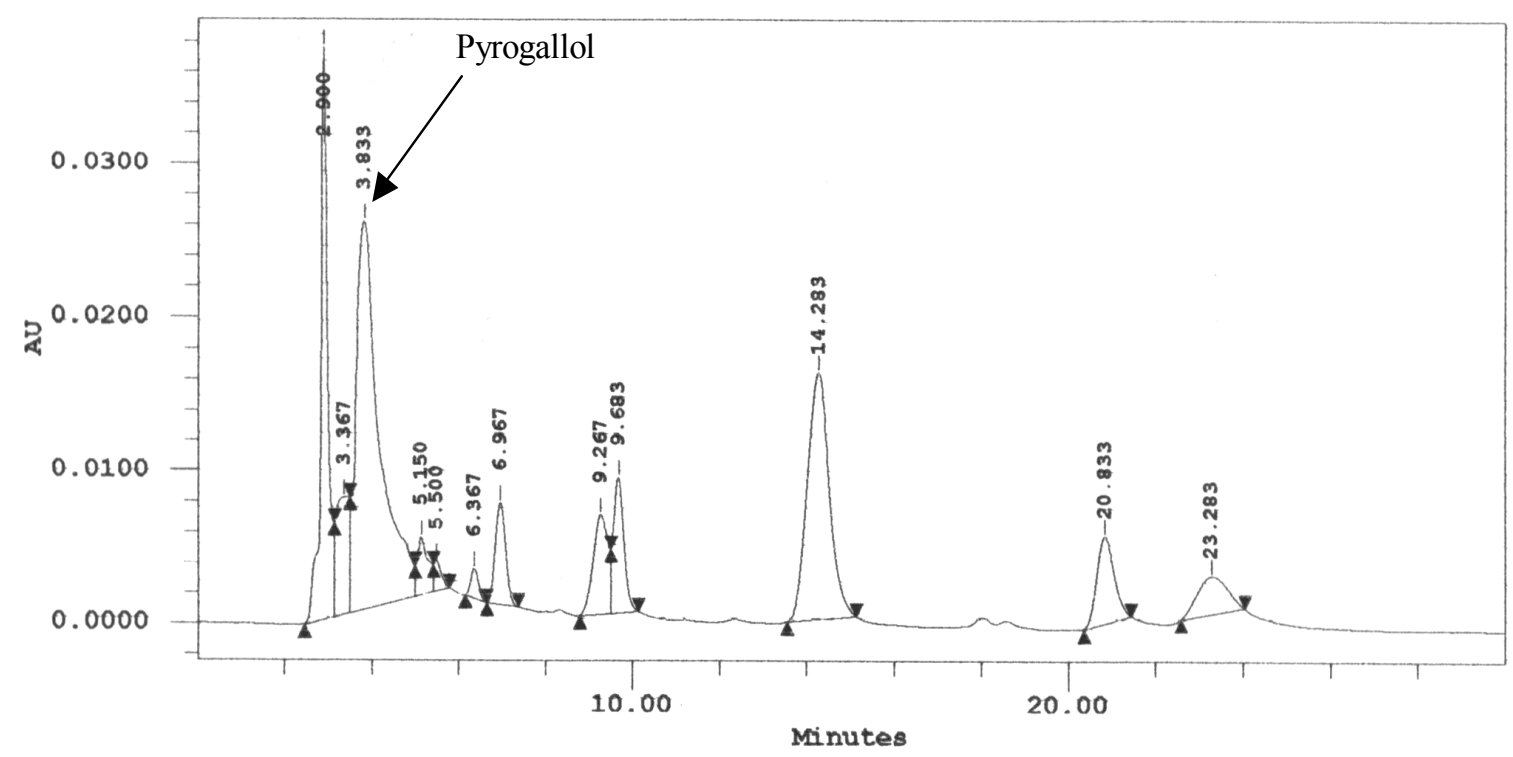

Abbildung 80: Chromatogramm der extrahierten Überzüge des mit Pyrogallol belegten Montmorillonits.

Die Retentionszeiten der übrigen Verbindungen sind zum Teil deutlich länger als die der Ausgangssubstanz (5,2, 5,5, 6,4, 6,9, 9,3, 9,7, 14,3, 20,8 und 23,3 min). Daraus kann nach der vorab dargestellten homologen Reihe der Zunahme der Festhaltekräfte abgeleitet werden, dass die Reaktion des Pyrogallols an der Tonmineraloberfläche zur Bildung von Reaktionsprodukten mit stärkeren Festhaltekräften als die des dreiwertigen Phenols geführt hat. Es sind in diesem Falle Reaktionsprodukte mit einer niedrigeren Polarität bzw. größeren Molekülmasse entstanden (Ether, Aldehyde, Ketone und Aliphate). Daraus kann eine Oxidation des Pyrogallols zu Chinonen bzw. eine Polymerisation des Phenols bzw. seiner Oxidationsprodukt(e) angenommen werden (Kapitel 4.6/4.7). Insbesondere die im Vergleich zur Ausgangssubstanz relativ langen Retentionszeiten von 14,3, 20,8 und 23,3 min lassen darauf schließen, dass es durch Verknüpfung mehrerer Phenolmoleküle zur Bildung eines aromatischen Oligmers bzw. Polymers gekommen sein kann. Die Intensität dieser Peaks im Vergleich zur Ausgangssubstanz und den übrigen Signalen deutet auf eine Bevorzugung der Bildung der entsprechenden Reaktionsprodukte und somit zu unpolareren bzw. höhermolekularen Verbindungen hin. 


\subsubsection{Reaktionsprodukte des 2,6-Dimethylphenols}

Das Chromatogramm der extrahierten Überzüge des mit 2,6-Dimethylphenol belegten Montmorillonits (Abbildung 81) weist neben dem Peak für das Lösemittel (2,7 min) und der Ausgangssubstanz bei 16,96 min weitere 10 neu hinzutretende Substanzen auf.

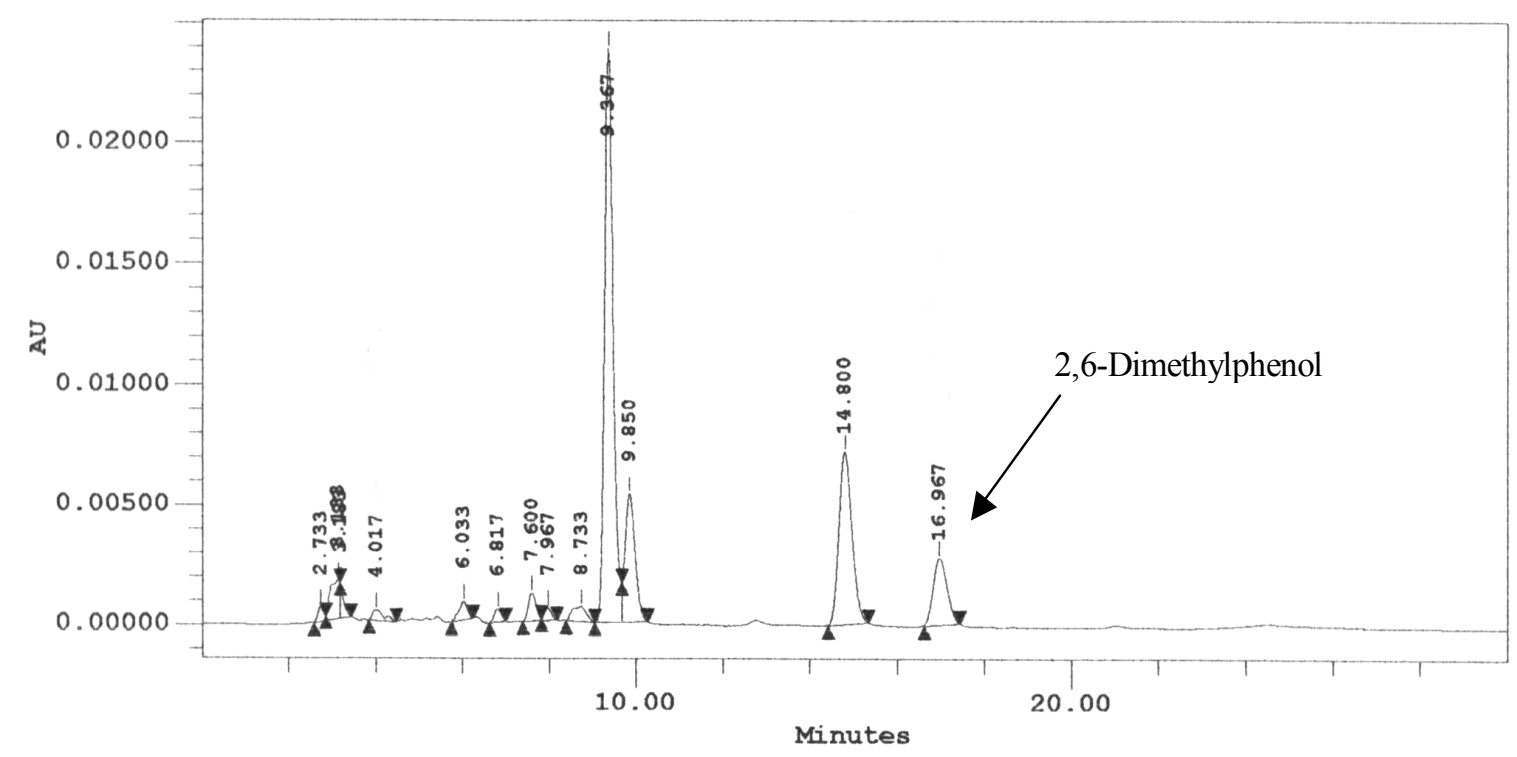

Abbildung 81: Chromatogramm extrahierter Überzüge des mit 2,6-Dimethylphenol belegten Montmorillonits.

Im Unterschied zu den vorangegangenen Chromatogrammen des Brenzkatechins und des Pyrogallols ist zu erkennen, dass alle neu auftretenden Peaks kürzere Retentionszeiten als die des 2,6-Dimethylphenols aufweisen (3,2, 4,0, 6,0, 6,8, 7,6, 8,0, 8,7, 9,4, 9,9 und 14,8 min). Entsprechend der homologen Reihe der Festhaltekräfte kann somit davon ausgegangen werden, dass die Reaktion des Phenols an der Oberfläche des Montmorillonits die Entstehung von Verbindungen mit einer höheren Polarität bzw. einer geringeren Molekülgröße zur Folge hat. Die relativ hohe Intensität des Peaks bei 9,4 min sowie 9,9 und 14,8 min zeigt, dass die Reaktion des 2,6-Dimethylphenols bevorzugt in Richtung der Bildung von Verbindungen dieser Peaks entsprechend abgelaufen ist und so vorwiegend diese Reaktionsprodukte gebildet worden sind.

\subsubsection{Diskussion der Ergebnisse}

Die dargestellten Ergebnisse der chromatographischen Trennung der von den Tonmineralen extrahierten organischen Überzüge konnten nachweisen, dass es durch die Reaktivität der Tonminerale zur abiotischen Reaktion der auf den Tonmineraloberflächen sublimierten Organika gekommen ist und durch diese Aktivität eine Vielzahl von Substanzen mit verschiedenen chemischen Eigenschaften entstanden sind.

Im Falle der Belegung des Montmorillonits mit Brenzkatechin zeigen die Ergebnisse, dass vorwiegend Reaktionsprodukte mit kürzeren Retentionszeiten, d. h. mit einer höheren Polarität, entstanden sind. Die massenspektrometrischen Analysen bestätigen dies und weisen zu- 
sätzlich die schon vorab vermutete Polymerisation der aromatischen Strukturen zu Dimeren bzw. Oligomeren nach.

Die Reaktion von Pyrogallol resultiert in der bevorzugten Bildung von Reaktionsprodukten mit längeren Retentionszeiten als das Phenol und lässt somit die Entstehung von Verbindungen mit unpolarerem Charakter bzw. höheren Molekülmassen erkennen.

Für die Belegung des Tons mit 2,6-Dimethylphenol kann festgehalten werden, dass dessen Reaktion an der Tonmineraloberfläche zur Entstehung von Verbindungen mit kürzeren Retentionszeiten als die Ausgangssubstanz geführt hat, was wiederum auf Substanzen mit polareren Eigenschaften schließen lässt. Außerdem kann auch in diesem Falle beobachtet werden, dass die Reaktion bevorzugt in Richtung der Bildung von drei Verbindungen abläuft.

Um die extrahierten Proben, bei denen es sich um Substanzgemische handelt besser zu charakterisieren bzw. identifizieren zu können, wurde mit Hilfe einer semipräparativen Säule und eines Fraktionensammlers die einzelnen Fraktionen voneinander getrennt gesammelt und einer IR-spektroskopischen Untersuchung zugeführt. Die gewonnenen Ergebnisse sind in Kapitel 4.6 dargestellt.

\subsection{Fourier-Transform Infrarot Spektroskopie}

Die Bedeutung der Infrarot-Spektroskopie (FTIR) beruht im hohen Informationsgehalt der durch sie aufgenommenen Spektren untersuchter Substanzen (AROCENA et al. 1995, KAISER \& ZECH 1997). Die Lage und Intensität der Absorptionsbanden einer spektroskopierten Substanz sind außerordentlich stoffspezifisch. IR-Spektren können ähnlich wie Fingerabdrücke zur Identifizierung einer Substanz herangezogen werden (GÜNZLER \& HEISE 1996). Für die Identifizierung von Substanzen sind insbesondere jene Absorptionsbanden geeignet, die dem Kohlenstoffgerüst des Moleküls zuzuordnen sind. Solche Absorptionsbanden sind häufig im Bereich zwischen 1000 bis $1500 \mathrm{~cm}^{-1} \mathrm{zu}$ finden, dieser Wellenzahlenbereich wird auch als „Fingerprint-Bereich“ bezeichnet.

\subsubsection{Physikalische Grundlagen der Infrarot-Spektroskopie}

Der Wellenlängenbereich der Infrarot-Strahlung liegt zwischen $1 \mathrm{~mm}$ und $1 \mu \mathrm{m}$. In der IRSpektroskopie ist die Angabe der Wellenlänge jedoch nicht gebräuchlich, statt dessen hat sich die Wellenzahl als Größe durchgesetzt. Die Wellenzahl $v$ ist der reziproke Wert der Wellenlänge mit der Einheit $\mathrm{cm}^{-1}$ :

$$
v=\frac{1}{\lambda[\mathrm{cm}]}=\frac{1 \times 10^{4}}{\lambda[\mu \mathrm{m}]}
$$

Für die Verwendung der Wellenzahl als Messgröße spricht ihre Proportionalität zur Frequenz $v$ des elektromagnetischen Wechselfeldes, sie steht mit der Wellenzahl $v$ in folgender Beziehung (GÜNZLER \& HEISE 1996): 


$$
v=\frac{v}{c} c m^{-1}
$$

wobei: Wellenzahl $=\frac{\text { Frequenz }}{\text { Lichtgeschwindigkeit }}$.

Zum Verständnis, wie infrarote Strahlung auf Moleküle wirkt, muss zuvor deren Aufbau erklärt werden. Moleküle bestehen aus mindestens zwei Atomen, die durch Wechselwirkung zwischen den Elektronen der äußeren Umlaufbahnen auf bestimmten Abständen gehalten werden. Im Molekülverband sind Atome in ganz definierter räumlicher Lage relativ zueinander angeordnet. Die Abstände der Atome werden durch die Summe aller Kräfte festgelegt, die zwischen allen Atomen wirken. Durch Energieaufnahme können die Atome zu Schwingungen um ihre Gleichgewichtslage angeregt werden (GÜNZLER \& HEISE 1996). Durch infrarote Strahlung im Wellenlängenbereich zwischen $780 \mathrm{~nm}$ und $50 \mathrm{~mm}$, was Wellenzahlen zwischen 12800 und $200 \mathrm{~cm}^{-1}$ entspricht, werden Moleküle angeregt. Elektromagnetische Strahlung kann jedoch nur von einem Molekül absorbiert werden, wenn mit der anzuregenden Schwingung der betroffenen Atomgruppe eine Dipolmomentänderung verbunden ist. Dipolmomente entstehen bei asymmetrischer Verteilung von Ladungen der Atome in einem Molekül oder wenn die Ladungsverteilung durch Schwingungsbewegungen der Atome eine Asymmetrie aufweist. Moleküle können Schwingungs- und Rotationsbewegungen ausführen, wobei zweiatomige Moleküle lediglich eine einzige Schwingungsbewegung ausführen können. Bei mehratomigen Molekülen kann sich jedes einzelne Atom in den drei Richtungen des Raumes bewegen. Somit werden zur Beschreibung eines solchen Moleküls $3 \times N$ Raumkoordinaten benötigt, wobei $\mathrm{N}$ die Zahl der Atome wiedergibt. Teilweise erfolgt jedoch keine Verschiebung der Atome relativ zueinander, sondern bei drei Bewegungen findet eine Bewegung in derselben Richtung unter gleichzeitiger Veränderung der Lage des Masseschwerpunktes statt. Diese Bewegungen werden als Translationsschwingungen bezeichnet und sind IR-inaktiv, da sie keine Wechselwirkung mit elektromagnetischer Strahlung eingehen. Daneben erzeugen drei weitere Schwingungskombinationen eine Rotation des Moleküls um den Masseschwerpunkt. Somit ergibt sich die eigentliche Anzahl der Schwingungen nach der Formel: $Z=3 \times N-6$. Hieraus kann die Anzahl der Schwingungen eines Moleküls berechnet werden, die unabhängig voneinander angeregt werden können. Jeder dieser als Normalschwingungen bezeichneten Bewegungen kann eine bestimmte Schwingungsfrequenz zugeordnet werden. Im folgenden werden die möglichen Schwingungsformen von Molekülen am Beispiel eines dreiatomigen linearen Moleküls erläutert, welches vier Schwingungen auszuführen vermag. Bei der Schwingungsform $v_{1}$ bewegen sich die beiden äußeren Atome symmetrisch in Valenzrichtung vom Zentralatom hin bzw. von ihm weg. Bei gleicher Masse der beiden Atome entspricht der Masseschwerpunkt dem Zentralatom, aus diesem Grund führt dieses keine Bewegung aus. Durch die symmetrische Valenzschwingung erfährt das Molekül keine Änderung des Dipolmomentes und kann somit durch die elektromagnetische Strahlung im Infrarot nicht angeregt werden, diese Schwingung ist daher IR-inaktiv. 


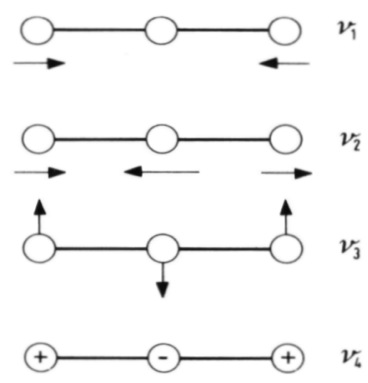

Abbildung 82: Mögliche Schwingungsformen eines dreiatomigen, linearen Moleküls (GÜNZLER \& HEISE 1996).

Bei der Schwingungsform $v_{2}$ bewegen sich die beiden äußeren Atome gleichsinnig und somit asymmetrisch in Beziehung zum Zentralatom, welches eine Gegenbewegung ausführt, womit der Masseschwerpunkt erhalten bleibt. Die asymmetrische Valenzschwingung ist IR-aktiv. Bei der durch die Schwingungsform $v_{3}$ beschriebenen Bewegung der Atome senkrecht zur Valenzrichtung, wobei die beiden äußeren Atome gleichsinnig schwingen und das Zentralatom in entgegengesetzter Richtung schwingen. Diese Schwingung bewirkt eine Veränderung in der Gleichgewichtslage des $180^{\circ}$ betragenden Valenzwinkels. Aus diesem Grunde wird diese Schwingung als Deformationsschwingung bezeichnet. Der entstehende Dipolmoment hat zur Folge, dass dieser Schwingungstyp IR-aktiv ist. Die Schwingungsform $v_{4}$ entspricht im Prinzip der Schwingungsform $v_{3}$, die Bewegungsrichtung der Atome ist lediglich um $90^{\circ}$ aus der Ebene heraus gedreht. Diese Deformationsschwingung ist ebenfalls IR-aktiv. Lineare Moleküle mit unsymmetrischer Massen- und Ladungsverteilung verfügen über ein permanentes Dipolmoment, ihre beiden Valenzschwingungen sind daher IR-aktiv (Abbildung 83).

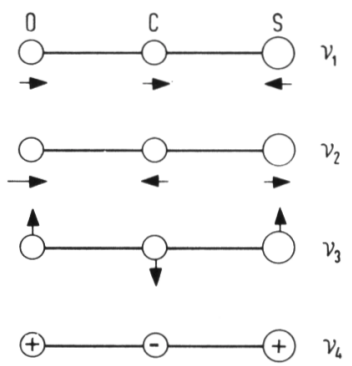

Abbildung 83: Mögliche Schwingungsformen eines dreiatomigen, linearen Moleküls mit unsymmetrischer Massenverteilung (GÜNZLER \& HEISE 1996).

Nicht lineare dreiatomige Moleküle können aufgrund ihres Aufbaus 3 Normalschwingungen ausführen (Abbildung 84). 


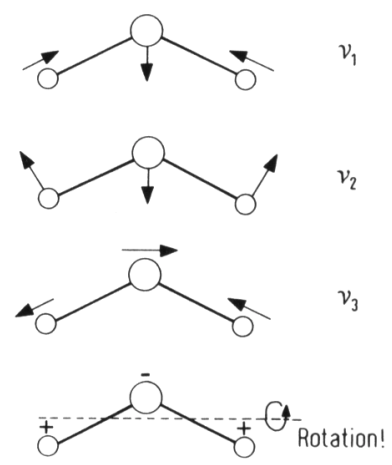

Abbildung 84: Mögliche Schwingungsformen eines dreiatomigen, gewinkelten Moleküls (GÜNZLER \& HEISE 1996).

Neben den Schwingungsformen der Normalschwingung, der Valenzschwingungen und der Deformationsschwingungen treten noch weitere Schwingungen als Absorptionsbanden in IRSpektren auf. Oberschwingungen sind charakterisiert durch ein Vielfaches der Frequenz der Grundschwingungen. Sie sind in ihrer Intensität stark gegenüber den Grundschwingungen verringert, jedoch sind in IR-Spektren die Obertöne von $\mathrm{CH}$-und $\mathrm{OH}-$ Valenzschwingungen im Bereich zwischen 2800 und $3600 \mathrm{~cm}^{-1}$ zu beobachten. Die Oberschwingungen von Grundschwingungen im Bereich kleinerer Wellenzahlen liegen ebenfalls in diesem Bereich und sind somit von den Grundschwingungen nicht leicht zu unterscheiden. Des weiteren können Kombinationsschwingungen auftreten. Diese entstehen dadurch, dass unterschiedliche Grundschwingungen gleichzeitig angeregt werden. Sie treten regelmäßig bei Substitutionsprodukten des Benzols im Wellenzahlenbereich zwischen 1600 und $2000 \mathrm{~cm}^{-1}$ auf (GÜNZLER \& HEISE 1996). Zusammenfassend kann festgehalten werden, dass Obertöne von Grundschwingungen und Kombinationsschwingungen durch ihr Auftreten neben den für die Interpretation wichtigen Absorptionsbanden die Auswertung von IR-Spektren komplizieren können.

Von großer Bedeutung für die Schwingungsspektroskopie ist die Symmetrie von Molekülen, da diese deren IR-Aktivität bzw. IR-Inaktivität bestimmen. Moleküle weisen Symmetrieelemente auf, die bei Symmetrieoperationen zu berücksichtigen sind, die eine Molekülstruktur wieder in sich selbst überführen. Die Anzahl aller Symmetrieoperationen eines Moleküls bilden eine Gruppe, die bei den Schwingungsbewegungen eines Moleküls als Punktgruppen bezeichnet werden. Während der Schwingungen bleibt zumindest ein Punkt, das Symmetriezentrum in einem Molekül ohne Bewegung. So weist das Benzol $\mathrm{C}_{6} \mathrm{H}_{6}$, Kernstruktur der in dieser Arbeit verwendeten Organika, 9 Symmetrieelemente auf. Bei einer Substitution des Benzols erfährt das Derivat normalerweise eine Reduzierung der Symmetrie. Bei monoSubstituierung entsteht eine Phenylgruppe, deren Schwingungen alle IR-aktiv werden. Die Entartung, so werden mit gleicher Frequenz erfolgende Schwingungen bezeichnet, entfällt und damit steigt die Anzahl der insgesamt aktiven Schwingungen von 20 auf 30 an (Tabelle 12). 
Tabelle 12: Verteilung der Symmetrierassen von Normalschwingungen des Benzols und monosubstituierter Derivate (nach GÜNZLER \& HEISE 1996).

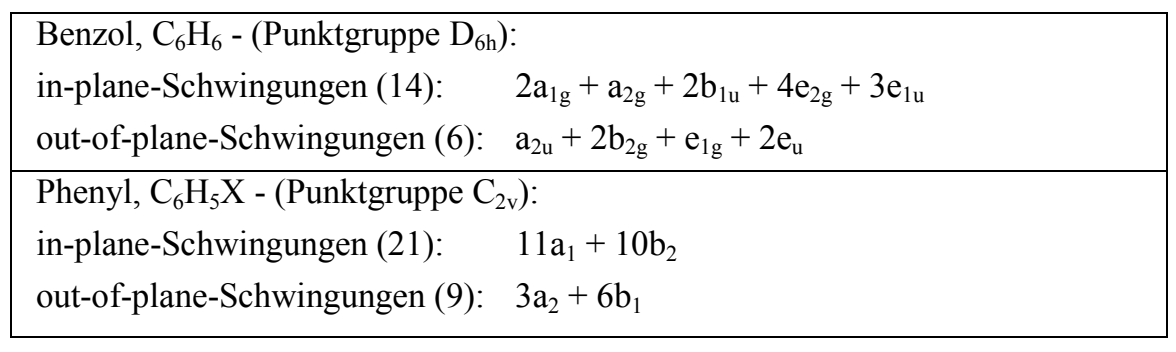

Mit zunehmender Zahl der Substitution des Benzolrings ist mit einer Erhöhung der IR-aktiven Schwingungen zu rechnen, was bei der Auswertung von IR-Spektren zu berücksichtigen ist.

\subsubsection{FT-IR-Spektrometer}

Zur nachfolgend beschriebenen qualitativen Untersuchung der durch die Reaktionen von aromatischen Verbindungen an Tonmineraloberflächen gewonnenen Proben(-gemische) wurde ein Fourier-Transform-Infrarot-Spektrometer 1600 von Perkin Elmer verwendet. Die Funktionsweise von FTIR-Spektrometern unterscheidet sich wesentlich von dispersiven IRSpektrometern, welche die infrarote Strahlung durch Prismen oder Beugungsgitter zerlegen und die Probe sukzessive mit den verschiedenen Wellenlängen abtasten. Beim FT-IR wird die Strahlung durch einen halbdurchlässigen Strahlteiler in zwei Strahlen aufgespalten, ein Teilstrahl wird auf die Probe gelenkt, dort reflektiert und erzeugt nach Rekombination mit dem zweiten Teilstrahl Interferenzen. Durch die simultane Abtastung aller Wellenlängen werden so Interferogramme gebildet, diese werden über die mathematische Fourier-Transformation des Interferogramms zu Absorptions- bzw. Reflexionsspektren umgerechnet. Die Vorteile des FTIR-Spektrometers gegenüber dispersiven IR-Spektrometern liegen in der gleichzeitigen Messung aller Wellenlängen. Die Verwendung eines FTIR-Spektrometers bietet kürzere Messzeiten, eine theoretische bis um 200-2.000fache höhere Empfindlichkeit und ein deutlich besseres Signal-/Rauschverhältnis, eine höhere Wellenzahlstabilität bei längeren Messungen und einen zu vernachlässigenden Streustrahlungsanteil (GÜNZLER \& HEISE 1996). Die Abtastrate für FTIR-Spektrometer liegt bei einer Auflösung von $1 \mathrm{~cm}^{-1}$ bei etwa $10 \mathrm{Scans} / \mathrm{min}$. Damit können sehr schnell Messungen mit hoher Auflösung über ein breites Spektrum auch bei Proben, die sehr geringe Mengen an zu spektroskopierenden Substanzen enthalten, durchgeführt werden (WHITE 1986).

\subsubsection{Präparation der Proben für die FT-IR-Spektroskopie}

Die Durchführung der infrarotspektroskopischen Untersuchungen war ursprünglich auf direktem Wege geplant, d. h. die mit organischen Überzügen versehenen Tonmineralproben sollten ohne vorherige Extraktion mit organischen Lösemitteln ,in situ“ analysiert werden. Der Grund hierfür war die Vermeidung der Bildung von Artefakten durch aggressive Extraktionsverfahren bzw. Lösemittel (Kapitel 4.1.6). Entsprechende Messreihen in Vorversuchen führ- 
ten jedoch aufgrund der zu geringen Konzentration der entstandenen Reaktionsprodukte nur zu sehr aussageschwachen Resultaten (BIRKEL 1997, KAISER \& ZECH 1997). Aus diesem Grunde wurden größere Mengen (2-4 g) mit Phenolen bzw. PAK belegten Montmorillonits mit Methanol extrahiert und anschließend durch Trocknung im Vakuum schonend aufkonzentriert, so dass eine zumeist ausreichende Probenkonzentration erreicht wurde.

Die so gewonnenen extrahierten Lösungen der Reaktionsprodukte wurden mittels Rotationsverdampfer (Büchi Rotavapor RE 111) unter Vakuum ( $40^{\circ} \mathrm{C}, 250$ mbar) bis zum Feststoff eingeengt um das als Lösemittel eingesetzte Methanol zu entfernen und damit keine störenden Absorptionsbanden des Lösungsmittels die Auswertung der IR-Spektren beeinträchtigen konnten. Das hygroskopische Kaliumbromid (Uvasol, Merck) wurde bei $105^{\circ} \mathrm{C}$ mindestens $12 \mathrm{~h}$ getrocknet um störende Wasserbanden zu vermeiden. Jeweils 1-1,5 mg der gewonnenen Feststoffmenge wurden mit je $300 \mathrm{mg}$ Kaliumbromid (KBr) in einer Schwingmühle (Perkin Elmer) $10 \mathrm{~s}$ gemörsert und unter Vakuum, zur Entfernung eventuell noch vorhandener Restfeuchte, zu einer Tablette mit einem Druck von etwa 0,75 GPa gepresst. Die Aufnahme der IR-Spektren erfolgte im Wellenzahlenbereich zwischen 4000 und $450 \mathrm{~cm}^{-1}$ an einem FT-IRSpektrometer von Perkin Elmer. Jedes Spektrum setzt sich aus 64 einzelnen Scans zusammen. Die Aufbereitung der Spektren erfolgte mit dem Programm „Spectrum Lite“ von Perkin Elmer. Um eine bessere Auswertbarkeit zu erreichen wurden alle Spektren basislinienkorrigiert. Je nach Bedarf erfolgte eine Korrektur durch einen Rauschfilter (block average), um so auftretendes Zittern im Spektrum, zumeist aufgrund enthaltener Restfeuchte im Probenmaterial bzw. Kaliumbromid, zu verringern. Zum besseren Vergleich der IR-Spektren wurden diese zwischen 4000 und $450 \mathrm{~cm}^{-1}$ auf einen Transmissionsbereich von 0 bis $100 \%$ normalisiert.

\subsubsection{Qualitative Interpretation der IR-Spektren}

Die Identifikation von organischen, insbesondere von aromatischen Verbindungen in der IRSpektroskopie erfolgt durch Zuordnungen charakteristischer, die Eigenschaften der Verbindungen bestimmenden funktionellen Gruppen; diesen werden Gruppenfrequenzen zugewiesen. Anhand der typischen Banden dieser Gruppenfrequenzen können schon nach kurzer Betrachtung viele organische Substanzklassen, wie beispielsweise Alkane, Alkene, Aromaten, Alkohole, Ketone, Aldehyde (GÜNZLER \& HEISE 1996) identifiziert werden. Weiterhin ist eine Unterscheidung von aromatischen Verbindungen gegenüber aliphatischen Substanzen möglich. Einen Überblick über die Verteilung der Gruppenfrequenzen im Spektrum des Infrarot gibt die folgende Abbildung (85). 


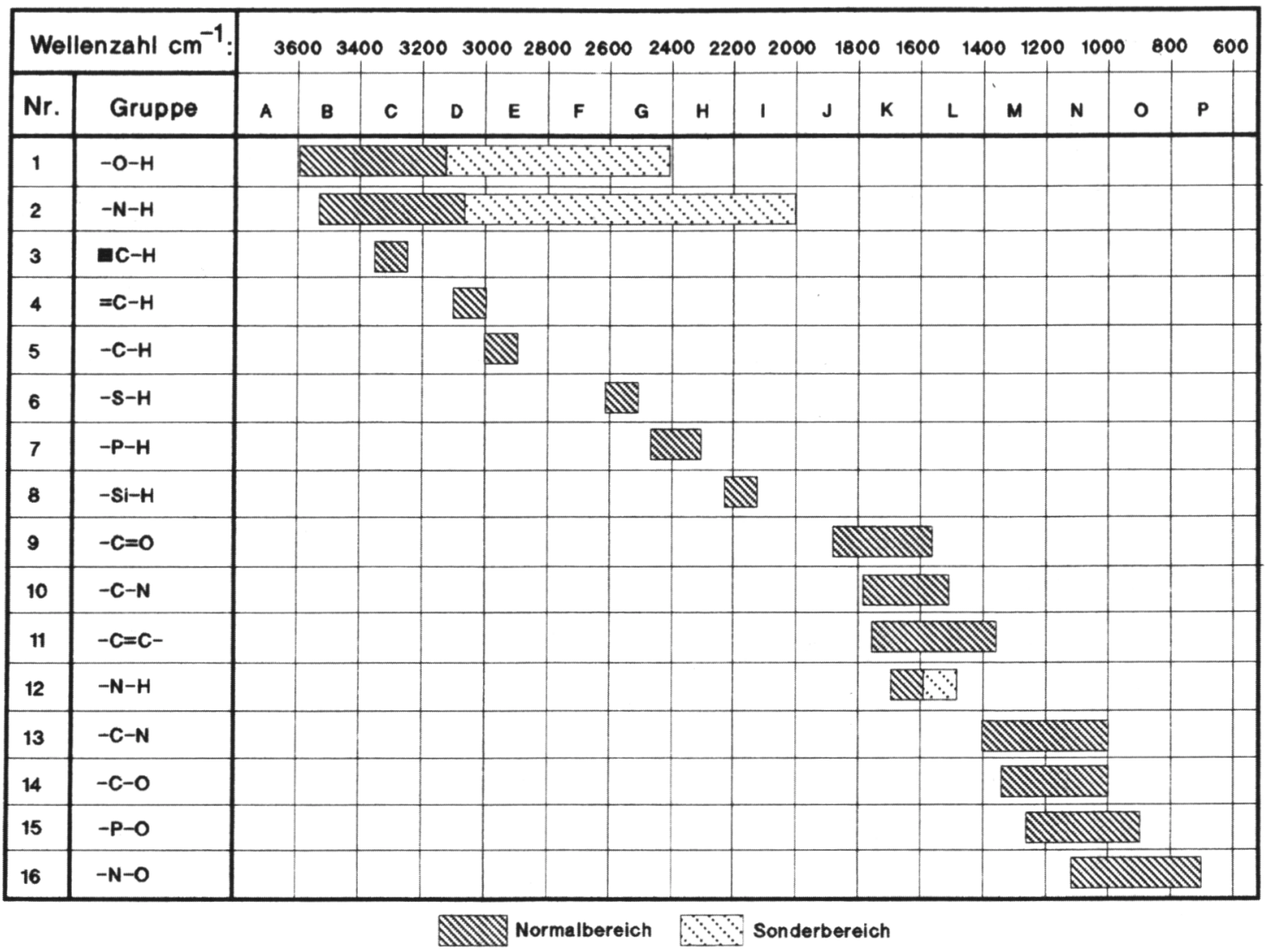

Abbildung 85: Gruppenfrequenzen im IR-Spektrum (GOTTWALD \&WACHTER 1997).

Die Absorptionsbereiche von Mehrfachbindungen sind deutlich voneinander getrennt, bei komplizierter aufgebauten Molekülen kann jedoch eine Überlappung von Banden im Bereich $\operatorname{der} \mathrm{C}=\mathrm{C}$ - und $\mathrm{C}=\mathrm{O}$-Doppelbindungen auftreten.

\subsubsection{Absorptionsbereiche organischer Verbindungen}

Die durchgeführten Messungen der Proben lassen schon durch die Verwendung von Aromaten als Ausgangssubstanzen auch in den Spektren Kennzeichen aromatischer Verbindungen erwarten. Diese weisen im IR-Spektrum charakteristische Absorptionsbereiche auf. Hier sind vor allem die Banden der C-C-Schwingungen des aromatischen Rings bei 1585-1600 $\mathrm{cm}^{-1}$, $1430-1500 \mathrm{~cm}^{-1}$ und bei $700 \mathrm{~cm}^{-1} \mathrm{zu}$ nennen. Die Banden im Bereich zwischen 1500 und 1600 $\mathrm{cm}^{-1}$ fallen meist zu einer einzigen Bande zusammen, durch Konjugation mit $\mathrm{C}=\mathrm{O}-, \mathrm{C}=\mathrm{N}-$, $\mathrm{C}=\mathrm{C}$-, $\mathrm{NO}_{2}$-Bindungen sowie mit Elementen wie $\mathrm{Cl}$ oder Si kann jedoch eine Aufspaltung der Bande hervorgerufen werden (GÜNZLER \& HEISE 1996). Die Substituierung des aromatischen Rings beeinflusst zudem die Banden der C-C-Schwingungen im Bereich zwischen $1430-1500 \mathrm{~cm}^{-1}$. Hier sind zumeist ebenfalls zwei Banden $\mathrm{zu}$ beobachten, eine Bande bei $1470 \mathrm{~cm}^{-1}$, die andere zwischen 1465 und $1430 \mathrm{~cm}^{-1}$. Durch para-Substituierung verschiebt sich die zweite Bande zu 10-20 cm höheren Wellenzahlen gegenüber andersartig substituierten Aromaten. Zudem kann bei der $1500 \mathrm{~cm}^{-1}$-Bande auf die Art der Substituenten geschlos- 
sen werden. So erhöhen elektronenabgebende Gruppen deren Intensität, während elektronenakzeptierende Gruppen die Ausbildung dieser Bande schwächen oder gar verhindern. Der Bereich um $700 \mathrm{~cm}^{-1}$ kann weitere Hinweise auf die Art der Substitution des Aromats geben. Bei mono- und meta-disubstituierten Aromaten tritt zwischen 675 und $710 \mathrm{~cm}^{-1}$ eine Bande hoher Intensität auf, bei asymmetrisch-trisubstituierten Aromaten liegt diese Bande im Bereich 690$730 \mathrm{~cm}^{-1}$. Die Bande für ortho- und para-disubstituierte Derivate des Benzols liegt ebenfalls in diesem Bereich (WHITE 1986). Zu beachten ist jedoch, dass die Absorptionsbande nur bei sich voneinander unterscheidenden Substituenten beobachtet werden kann, während sie bei gleichen Substituenten fehlt (GÜNZLER \& HEISE 1996). Die C-H-Streckschwingungen $(v(\mathrm{C}-\mathrm{H}))$ der an die sechs C-Atome des aromatischen Rings gebundenen H-Atome liegen im Bereich zwischen 3000 und $3100 \mathrm{~cm}^{-1}$. Daneben treten Absorptionsbanden durch Deformationsschwingungen zwischen 1000 und $1400 \mathrm{~cm}-1$ (,Fingerprint-Bereich“) und zwischen 675 und $1000 \mathrm{~cm}^{-1}$ auf.

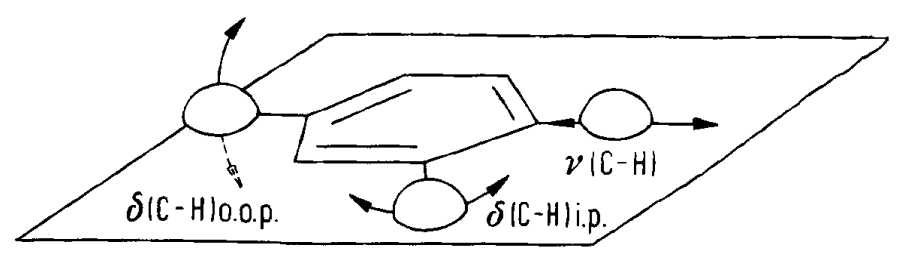

Abbildung 86: C-H-Schwingungsformen am aromatischen Ring (GÜNZLER \& HEISE 1996).

Die im Bereich zwischen 3000 und $3100 \mathrm{~cm}^{-1}$ absorbierenden H-Atome sind nicht aromatenspezifisch und aufgrund der in diesem Bereich liegenden Absorptionen von Wasserstoffbrücken schwer nachweisbar. Die C-H-Deformationsschwingungen (d(C-H) o.o.p.) im Bereich zwischen 660 und $910 \mathrm{~cm}^{-1}$ weisen eine gegenseitige Beeinflussung auf, die starke Kopplung benachbarter C-H-Gruppen wird von Substituenten unterbrochen. Somit zeigen die Absorptionsbanden in diesem Bereich die Stellung der Substituenten am aromatischen Ring auf (Tabelle 13).

Tabelle 13: Bandenlagen substituierter Benzolderivate
\begin{tabular}{|c|c|}
\hline Substitution & Wellenzahl $\left(\mathbf{c m}^{-1}\right)$ \\
\hline mono- & $751 \pm 15$ \\
\hline ortho- & $751 \pm 7$ \\
\hline meta- & $782 \pm 9$ \\
\hline para- & $817 \pm 13$ \\
\hline
\end{tabular}

Im Bereich zwischen 1600 und $2000 \mathrm{~cm}^{-1}$ treten Oberschwingungen der C-HDeformationsschwingungen auf, diese ergeben für den Substitutionstyp des Aromats hinsichtlich der Stellung der Substituenten und des Substitutionsgrades charakteristische Spektren (GÜNZLER \& HEISE 1996). Die vorab beschriebenen, für aromatische Ringe so typischen 
Absorptionsbanden lassen diese im Spektrum sehr schnell erkennen.

Die Absorptionsbereiche von organischen Verbindungen, speziell die funktioneller Gruppen, sind neben den Banden der aromatischen Grundstrukturen für die Auswertung von IRSpektren von großer Bedeutung, da durch sie auf die eigentliche Zusammensetzung organischer Substanzen geschlossen werden kann (NIEMEYER et al. 1992, PARKER \& FROST 1996). Die folgende Tabelle (14) gibt einen Überblick der für die weitere Interpretation unerlässlichen Absorptionsbereiche organischer funktioneller Gruppen.

Tabelle 14: Absorptionsbanden von Huminstoffen (nach Stevenson 1994).

\begin{tabular}{|c|c|}
\hline Frequenz $\left(\mathrm{cm}^{-1}\right)$ & Zuordnung \\
\hline $3400-3300$ & OH-Streckschwingung (stretching), N-H stretching \\
\hline $2940-2900$ & aliphatische C-H-Streckschwingung \\
\hline $1725-1720$ & $\mathrm{C}=\mathrm{O}$ stretching von $\mathrm{COOH}$ und Ketonen \\
\hline $1660-1630$ & $\begin{array}{l}\mathrm{C}=\mathrm{O} \text { stretching von Amiden, Chinonen und/oder } \mathrm{C}=\mathrm{O} \text { von wasserstoffverbrückten, } \\
\text { konjugierten Ketonen }\end{array}$ \\
\hline $1620-1600$ & aromatische $\mathrm{C}=\mathrm{C}$, starke $\mathrm{H}$-Brücken zwischen $\mathrm{C}=\mathrm{O}$ konjugierter Ketone \\
\hline $1590-1517$ & $\begin{array}{l}\text { symmetrisches stretching von } \mathrm{COO}^{-}, \mathrm{N}-\mathrm{H}-\text { Deformationsschwingung, } \mathrm{C}=\mathrm{N} \text { stret- } \\
\text { ching }\end{array}$ \\
\hline $1460-1450$ & aliphatisches $\mathrm{C}-\mathrm{H}$ \\
\hline $1400-1390$ & $\begin{array}{l}\text { OH-Deformationsschwingung und } \mathrm{C}-\mathrm{O} \text { stretching phenolischer } \mathrm{OH}-\mathrm{Gruppen}, \mathrm{C}-\mathrm{H}- \\
\text { Deformationsschw. von } \mathrm{CH}_{2} \text { - und } \mathrm{CH}_{3} \text {-Gruppen, antisymmetrisches stretching von } \\
\mathrm{COO}^{-}\end{array}$ \\
\hline $1280-1200$ & $\begin{array}{l}\mathrm{C}-\mathrm{O} \text { stretching und } \mathrm{OH}-\text { Deformationsschw. von } \mathrm{COOH}, \mathrm{C}-\mathrm{O} \text { stretching von Ary- } \\
\text { lethern }\end{array}$ \\
\hline $1170-950$ & $\begin{array}{l}\text { C-O stretching von Polysacchariden oder ähnlicher Substanzen, Si-O silikatischer } \\
\text { Verunreinigungen }\end{array}$ \\
\hline
\end{tabular}

\subsubsection{Auswertung der IR-Spektren}

Die IR-Spektren der Phenole Brenzkatechin, Pyrogallol, 2,6-Dimethylphenol, des polyzyklischen aromatischen Kohlenwasserstoffs Phenanthren sowie die extrahierten organischen Überzüge aller Belegungsvarianten wurden aufgenommen und ausgewertet.

\subsubsection{Brenzkatechin}

Die beiden Absorptionsbanden bei 3425 und $3325 \mathrm{~cm}^{-1}$ beschreiben die Streckschwingungen $(\mathrm{n}(\mathrm{O}-\mathrm{H}))$ phenolischer OH-Gruppen, sie bewirken ein breites Absorptionsmaximum zwischen 3705 und $3125 \mathrm{~cm}^{-1}$ (Abbildung 87). Die Absorptionsbande bei $3045 \mathrm{~cm}^{-1}$ charakterisiert neben dem breiten Absorptionsbereich der OH-Bindung auftretende Bande der $\mathrm{vC}-\mathrm{H}-\mathrm{Bindung}$ des aromatischen Ringes, sie liegt im allgemeinen im Bereich zwischen 3100 und $3000 \mathrm{~cm}^{-1}$. 


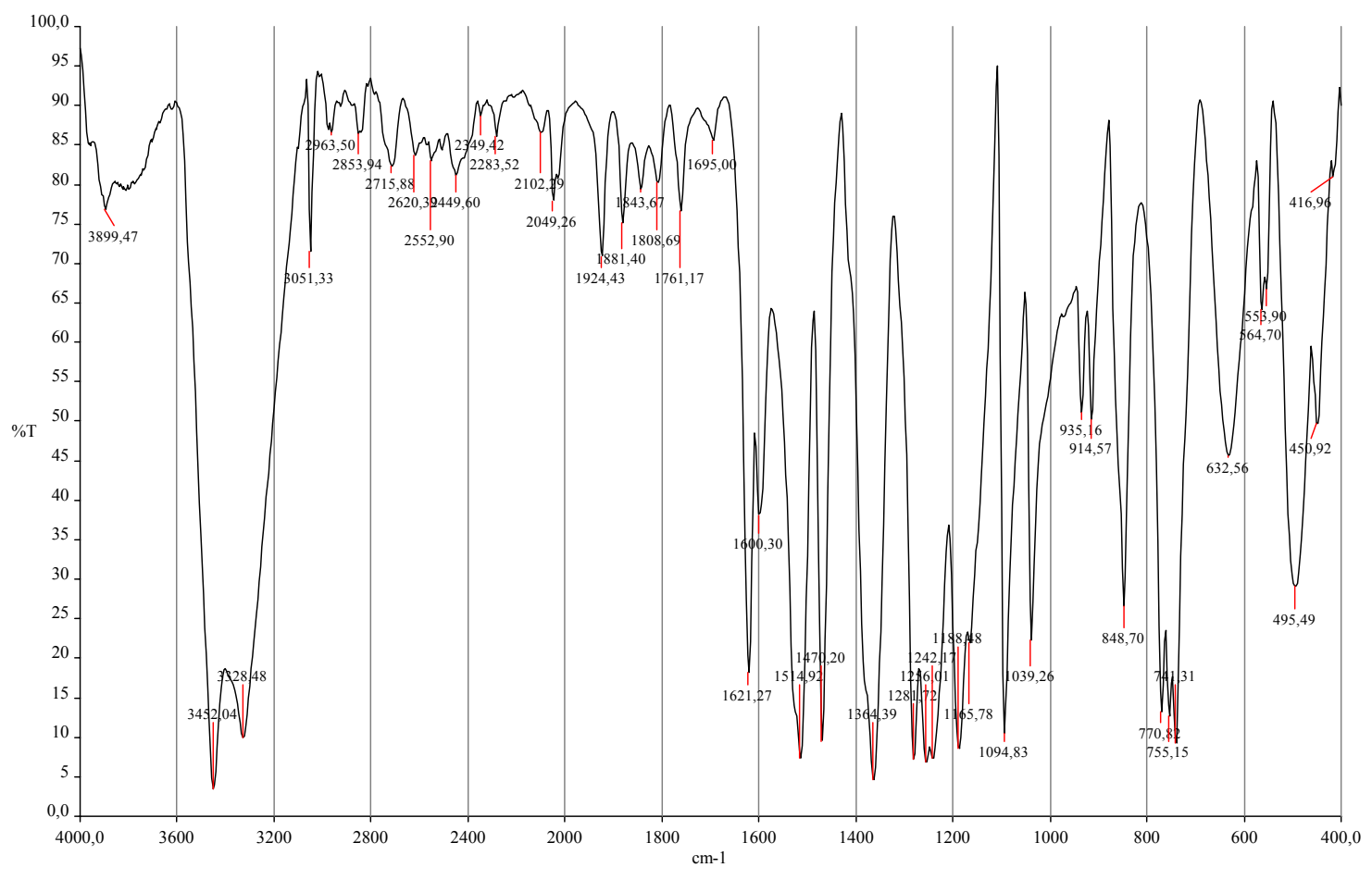

Abbildung 87: IR-Spektrum von Brenzkatechin.

Die im Bereich zwischen 2000 und $1600 \mathrm{~cm}^{-1}$ liegenden Absorptionsbanden, die bei Substitutionsprodukten des Benzols durch Oberschwingungen auftreten, ergeben sich aus einer Kombination von Deformationsschwingungen $(\delta \mathrm{C}-\mathrm{H})$ der $\mathrm{C}-\mathrm{H}-\mathrm{Gruppen}$ aromatischer Ringe (GÜNZLER \& HEISE 1983). Die Banden bei $1605 \mathrm{~cm}^{-1}, 1515 \mathrm{~cm}^{-1}$ und $1470 \mathrm{~cm}^{-1}$ beschreiben die $v C=C$-Bindung (Streckschwingungsbanden) des aromatischen Ringes, sie sind die charakteristischen Merkmale für die Anwesenheit eines Aromats. Der Bereich zwischen 1430 $\mathrm{cm}^{-1}$ und $1000 \mathrm{~cm}^{-1}$ stellt den sogenannten Fingerprint-Bereich dar. In ihm liegen die für die Identifizierung einzelner Substanzen wichtigen Schwingungen der Einfachbindungen der Cund O-Atome. Bei $1370 \mathrm{~cm}^{-1}$ befindet sich die Absorptionsbande der $\mathrm{OH}-$ Deformationsschwingung der phenolischen OH-Gruppen. Zwischen 1335 und $1165 \mathrm{~cm}^{-1}$ finden sich Banden der Aryl-OH-Gruppen, sie sind meist in mehrere Maxima aufgespalten: 1280 $\mathrm{cm}^{-1}, 1240 \mathrm{~cm}^{-1}, 1175 \mathrm{~cm}^{-1}$. Hier zeigen sich jedoch Überschneidungen mit den Absorptionsbanden der C-O-Streckschwingungen ( $v \mathrm{C}-\mathrm{O}$ ), welche im Bereich von 1260-1180 $\mathrm{cm}^{-1}$ liegen. Die im Spektrum auftretenden zusätzlichen Banden im Bereich der C-HDeformationsschwingungen ( $\delta \mathrm{C}-\mathrm{H}$ o.o.p.) zwischen 1100 und $800 \mathrm{~cm}^{-1}: 1090 \mathrm{~cm}^{-1}, 1030 \mathrm{~cm}^{-1}$ und $840 \mathrm{~cm}^{-1}$ werden hervorgerufen durch „X-sensitive bands“. Diese entstehen wenn Deformationsschwingungen des aromatischen Rings mit C-X-Streckschwingungen der Substituenten koppeln. Das X steht in diesem Falle stellvertretend für verschiedene Atome (GÜNZLER \& HEISE 1996). Der Bereich zwischen $910 \mathrm{~cm}^{-1}$ und $660 \mathrm{~cm}^{-1}$ treten Absorptionsbanden auf, welche die Art der Substitution des aromatischen Ringes wiedergeben. Die Substitutionsbanden für Brenzkatechin würden entsprechend der Substituierung im Bereich von $770-730 \mathrm{~cm}^{-1}$ liegen (Tabelle 13). Dies gilt jedoch nicht für gleiche Substituenten, in diesem Falle ist aus 
dem Spektrum keine Substituierung ablesbar. Die in diesem Bereich auftretenden Banden im Bereich von $760 \mathrm{~cm}^{-1}$ sind als Deformationsschwingungen ( $\delta$ (Ring)) des aromatischen Rings anzusprechen.

\subsubsection{Pyrogallol}

Im Wellenzahlenbereich zwischen $3600 \mathrm{~cm}^{-1}$ und $2800 \mathrm{~cm}^{-1}$ ist eine, sich aus mindestens drei Banden zusammensetzende breite Absorptionsbande zu beobachten. In diesem Frequenzbereich treten die charakteristischen Valenzschwingungen (vO-H) von Phenolen auf. Die Breite dieser Banden ist abhängig vom Assoziationsgrad, d. h. von der Anzahl der Wasserstoffbrücken. Phenole, deren $\mathrm{OH}-$ Gruppen im festen oder flüssigen Zustand nicht abgeschirmt sind, zeigen im IR-Spektrum eine sehr breite und intensive Absorption mit einem Maximum bei $3300 \mathrm{~cm}^{-1}$. Da Pyrogallol über drei OH-Gruppen verfügt, zeigen diese zusätzlich durch ihre $\mathrm{H}-$ Verbrückung diesen breiten Absorptionsbereich an. Dadurch ist der Schwingungsbereich der Valenzschwingung von C-H in aromatischen Systemen $(\mathrm{vC}-\mathrm{H})$, die eine charakteristische schmale Bande mittlerer Bandenstärke aufweist und im Wellenzahlenbereich zwischen 3100 $\mathrm{cm}^{-1}$ und $3000 \mathrm{~cm}^{-1}$ liegt teilweise überdeckt, eine häufig auftretende Aufspaltung dieser Bande ist nicht erkennbar. Die zu beobachtende Schulter bei $3053 \mathrm{~cm}^{-1}$ weist jedoch eindeutig das Auftreten dieser Schwingungsform nach. Der Bereich zwischen $2700 \mathrm{~cm}^{-1}$ und $1700 \mathrm{~cm}^{-1}$ weist hier lediglich einige schwache Banden auf und sind keiner Bindungsform zuzurechnen. In diesem Absorptionsbereich treten Kombinationsbanden auf, die aus einer Kombination des ein- oder mehrfachen Frequenzbetrages von zwei oder mehr Normalschwingungen hervorgehen (GÜNZLER \& HEISE 1996).

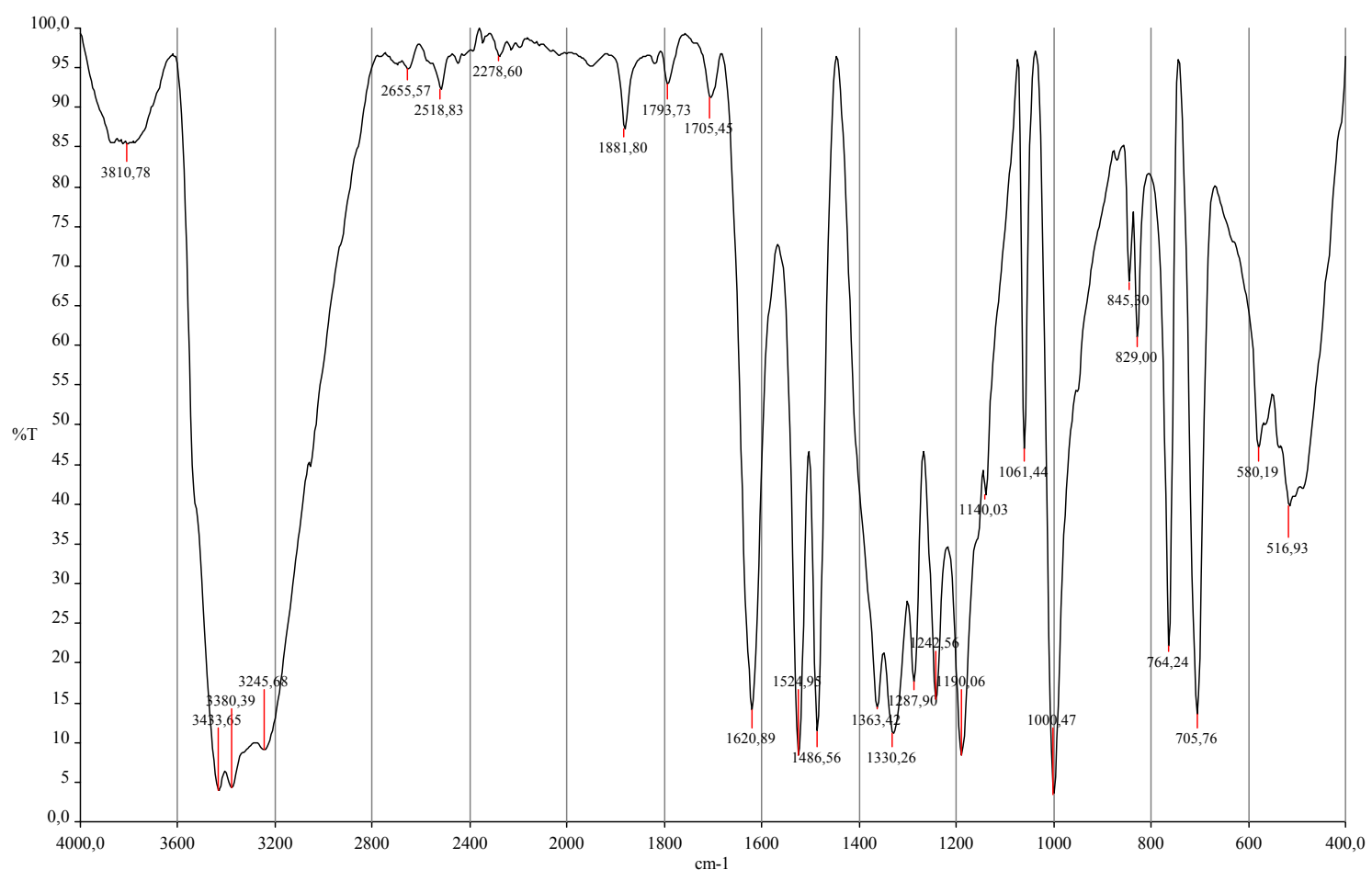

Abbildung 88: IR-Spektrum von Pyrogallol. 
Im längerwelligen Frequenzbereich sind die, für die Valenzschwingung von C-C-Bindungen ( $\vee C-C)$ der aromatischen Gerüsts zu erkennen. Diese weisen zumeist eine Gruppe von drei oder vier schmalen Banden zwischen $1620 \mathrm{~cm}^{-1}$ und $1460 \mathrm{~cm}^{-1}$ auf. Als Skelettschwingungen des aromatischen Systems des Pyrogallols sind hier die Banden bei $1620 \mathrm{~cm}^{-1}, 1524 \mathrm{~cm}^{-1}$ und $1486 \mathrm{~cm}^{-1} \mathrm{zu}$ beobachten. Im Wellenzahlenbereich zwischen $1400 \mathrm{~cm}^{-1}$ und $1200 \mathrm{~cm}^{-1}$ treten die in-plane-Deformationsschwingungen der O-H-Gruppen $(\delta \mathrm{O}-\mathrm{H})$ auf. Oft wir diese sehr breite Bande aus einem Multiplett mehrerer Banden gebildet und wie im Falle der Valenzschwingungen der $\mathrm{OH}-$ Gruppen ist dieser Absorptionsbereich sehr stark von Anzahl und Stärke der H-Brücken in der Verbindung beeinflusst. Die drei Banden bei $1363 \mathrm{~cm}^{-1}, 1330$ $\mathrm{cm}^{-1}$ und $1287 \mathrm{~cm}^{-1}$ sind den $\delta \mathrm{O}-\mathrm{H}-\mathrm{Schwingungen} \mathrm{des} \mathrm{Pyrogallols} \mathrm{zuzuordnen.} \mathrm{Die} \mathrm{Valenz-}$ schwingungen der C-O-Bindungen (vC-O) von Phenolen (Aryl-OH) liegen zwischen 1260 $\mathrm{cm}^{-1}$ und $1180 \mathrm{~cm}^{-1}$ und sind im Spektrum durch die Banden bei $1242 \mathrm{~cm}^{-1}$ und $1190 \mathrm{~cm}^{-1}$ vertreten. Durch diesen relativ engen Bereich kann zwischen C-O-Schwingungen phenolischer Herkunft und dem breiteren Absorptionsbereich alkoholischer Valenzschwingungen kann somit leicht unterschieden werden. Der in Richtung langwelligeren Frequenzen sich rechts anschließende Wellenzahlenbereich beschreibt die out-of-plane-Deformationsschwingungen der C-H-Bindungen $(\delta \mathrm{C}-\mathrm{H})$ zwischen $900 \mathrm{~cm}^{-1}$ und $650 \mathrm{~cm}^{-1}$. Diese Bande wird durch die Anzahl der benachbarten H-Atome am aromatischen Ring beeinflusst und dient somit zur Bestimmung des Substitutionstyps (Substitutionsmusterbanden). Darüber hinaus bewirken jedoch in diesem Bereich auftretende, durch Aryl-OH induzierte „X-sensitive Banden“ eine Interpretation dieser Banden. Die meta-Substitution der drei OH-Gruppen am Benzolring wird durch die Banden bei $764 \mathrm{~cm}^{-1}$ und $705 \mathrm{~cm}^{-1}$ nachgewiesen (Abbildung 89).

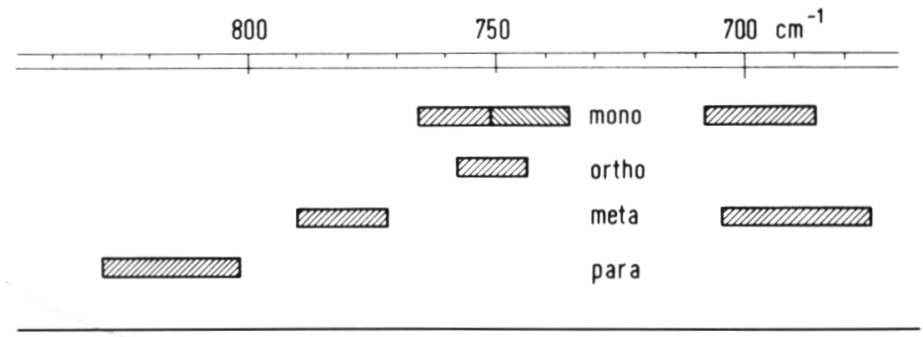

Abbildung 89: Substitutionsbanden (8C-H o.o.p.) von Benzolderivaten (GÜNZLER \& HEISE 1996).

$\mathrm{Ab} 700 \mathrm{~cm}^{-1}$ treten noch Banden auf, die einen Einbruch der Basislinie zeigen und ein Charakteristikum für alle Alkohole und Phenole darstellt (GOTTWALD \& WACHTER 1997).

\subsubsection{2,6-Dimethylphenol}

Das IR-Spektrum des 2,6-Dimethylphenols zeigt eine intensive Absorption bei $3411 \mathrm{~cm}^{-1}$. Diese Bande ist der Valenzschwingung der O-H-Bindung zuzuordnen und weist aufgrund der Substitution des aromatischen Rings mit lediglich einer OH-Gruppe eine schwächere Ausprägung als in den Spektren der vorab beschriebenen Phenole auf. Dadurch kann die durch die Absorptionsbande der OH-Gruppen zumeist verdeckte Bande der Valenzschwingung der C- 
H-Bindung bei $3040 \mathrm{~cm}^{-1}$ eindeutig bestimmt werden. Die auftretenden Banden bei $2969 \mathrm{~cm}^{-}$ 1, $2941 \mathrm{~cm}^{-1}, 2915 \mathrm{~cm}^{-1}$ und $2854 \mathrm{~cm}^{-1}$ weisen das Auftreten von Methylgruppen nach. Die Valenzschwingung ( $v \mathrm{C}-\mathrm{H})$ tritt überwiegend als Dublette auf, wobei die Bande höherer Wellenzahl die antisymmetrische Schwingung und die etwas niedrigere Zahl die symmetrische Schwingung beschreibt.

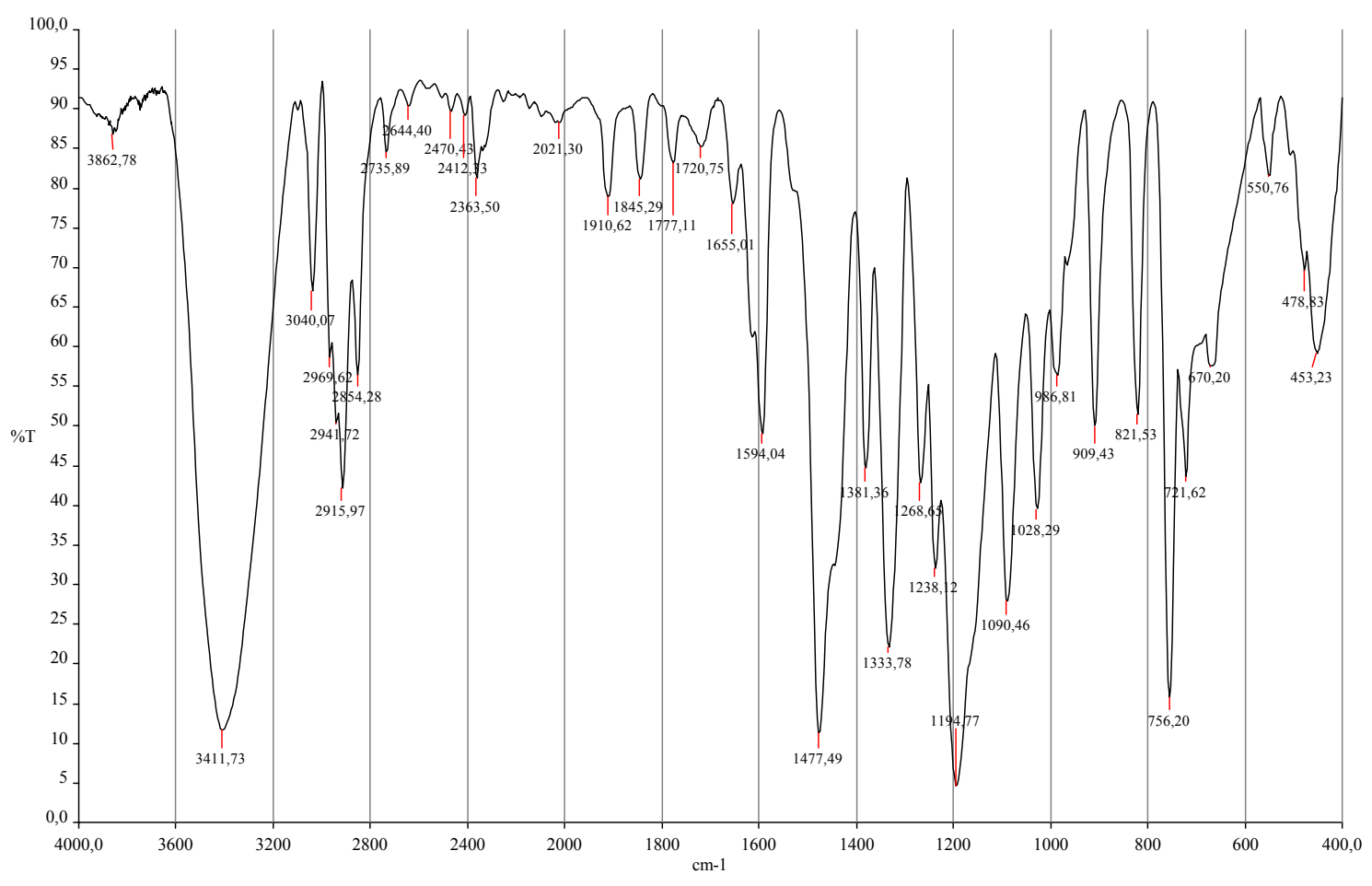

Abbildung 90: IR-Spektrum von 2,6-Dimethylphenol (KBr).

Die Banden bei $1910 \mathrm{~cm}^{-1}, 1845 \mathrm{~cm}^{-1}$ und $1777 \mathrm{~cm}^{-1}$ stellen Oberschwingungsbanden von Aromaten dar und werden aufgrund ihrer charakteristischen Erscheinung als Aromatenfinger bezeichnet. Sie stellen somit eine gute Identifikationshilfe dar (GOTTWALD \& WACHTER 1997). Bei $1613 \mathrm{~cm}^{-1}, 1594 \mathrm{~cm}^{-1}$ und $1477 \mathrm{~cm}^{-1}$ sind die charakteristischen Valenzschwingungen der C-C-Bindungen ( $\mathrm{vC}-\mathrm{C})$ des aromatischen Systems zu beobachten. Überlagert werden diese Banden jedoch von den Deformationsschwingungen $(\delta \mathrm{C}-\mathrm{H})$ der Methylgruppen des Phenols zwischen $1480 \mathrm{~cm}^{-1}$ und $1350 \mathrm{~cm}^{-1}$, was anhand der Schulter bei $1443 \mathrm{~cm}^{-1}$ und der Bande bei $1381 \mathrm{~cm}^{-1} \mathrm{zu}$ erkennen ist. Weiterhin treten die Deformationsschwingungen der $\mathrm{O}-\mathrm{H}-$ Gruppe $(\delta \mathrm{O}-\mathrm{H})$ in diesem Wellenzahlenbereich zwischen $1450 \mathrm{~cm}^{-1}$ und $1200 \mathrm{~cm}^{-1}$ hinzu. Die zumeist als Multiplett ausgebildete Bande weist mit Wellenzahlen zwischen 1410$1320 \mathrm{~cm}^{-1}$ assoziierte phenolische OH-Gruppen, hier $1333 \mathrm{~cm}^{-1}$ sowie zwischen 1300-1250 $\mathrm{cm}^{-1}$ freie phenolische OH-Gruppen, hier $1268 \mathrm{~cm}^{-1}$ nach. Nachfolgend werden dem Wellenzahlenbereich zwischen $1260 \mathrm{~cm}^{-1}$ und $970 \mathrm{~cm}^{-1}$ die Valenzschwingung von C-O-Bindungen (vC-O) zugeordnet, die häufig als Dublett ausgebildet sind und im Falle phenolischer C-OBindungen im Bereich zwischen 1260-1180 $\mathrm{cm}^{-1}$ liegen. Im Spektrum sind dieser Schwingungsform die Banden bei $1238 \mathrm{~cm}^{-1}$ und $1194 \mathrm{~cm}^{-1}$ zuzuordnen. Die Absorptionsbanden zwischen $1090 \mathrm{~cm}^{-1}$ und $900 \mathrm{~cm}^{-1}$ beschreiben die Deformationsschwingung $(\delta \mathrm{C}-\mathrm{H})$ der C-H- 
Bindungen. Im Bereich der Substitutionsbanden zwischen $900 \mathrm{~cm}^{-1}$ und $650 \mathrm{~cm}^{-1} \mathrm{kann}$ durch die auftretenden Banden bei $821 \mathrm{~cm}^{-1}, 756 \mathrm{~cm}^{-1}$ und $721 \mathrm{~cm}^{-1}$ die tri-Substitution des aromatischen Rings nachgewiesen werden.

\subsubsection{Extrahierte Überzüge des mit Brenzkatechin belegten Montmorillonits}

Das IR-Spektrum der extrahierten Überzüge des mit Brenzkatechin belegten Montmorillonits weist einen deutlichen Unterschied zum IR-Spektrum des Standards auf. Zur besseren Darstellung sind jeweils das Spektrum des Standards und das der extrahierten Probe gegenüber gestellt (Abbildung 91). Beim Vergleich der Spektren muss jedoch berücksichtigt werden, dass bei den Analysen große Unterschiede in der Konzentration auftraten, was sich in der Ausprägung der Absorptionsbanden enorm bemerkbar macht.

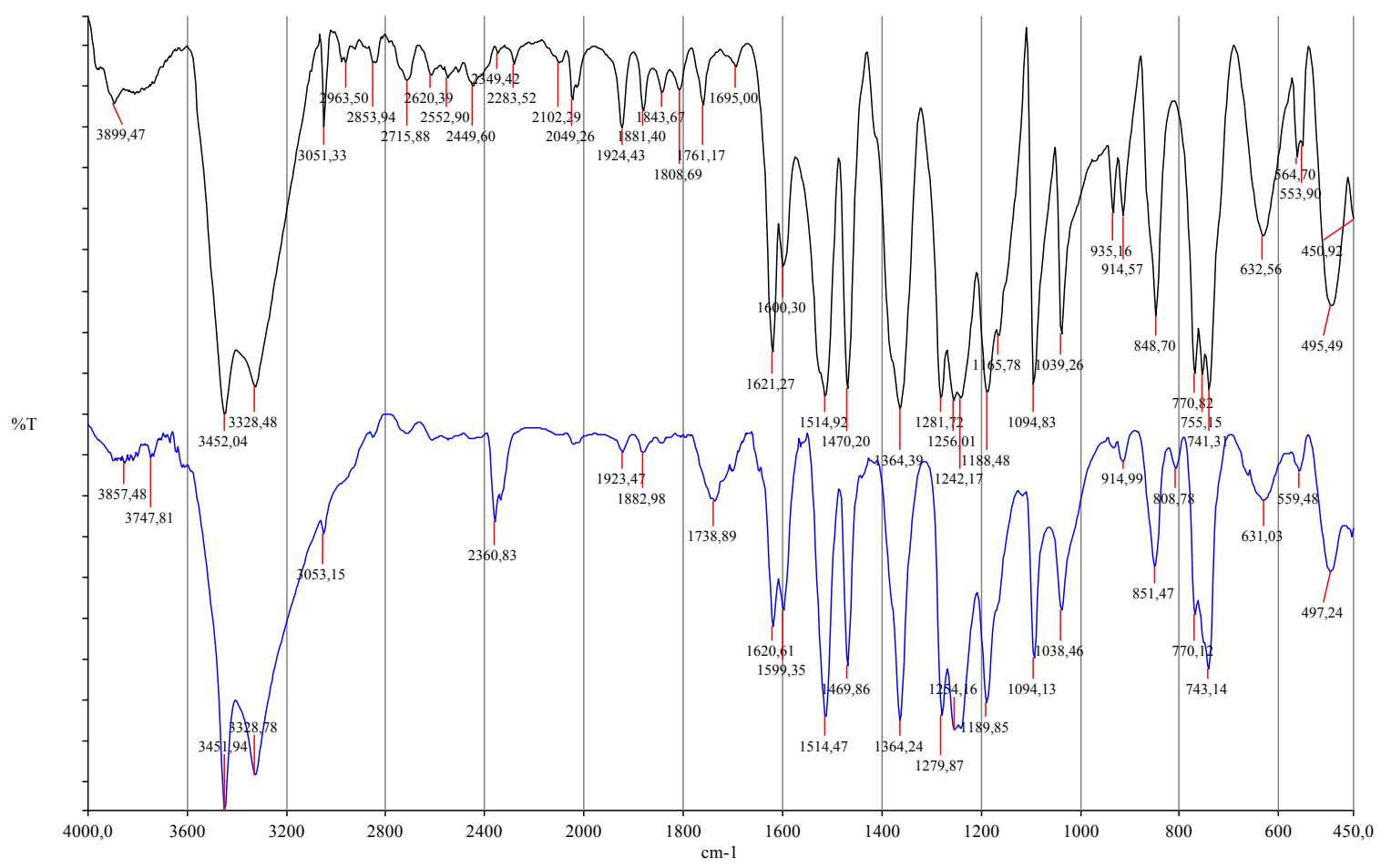

Abbildung 91: IR-Spektrum der extrahierten Überzüge des mit Brenzkatechin belegten Montmorillonits (schwarz: Brenzkatechin Standard; blau: extrahierte Überzüge).

Im Vergleich zum Brenzkatechin tritt eine, aufgrund der geringen Konzentration der bzw. des entstandenen Reaktionsprodukte(s), gut zu erkennende Absorptionsbande bei $1739 \mathrm{~cm}^{-1}$ auf. Dieser Bereich charakterisiert die Valenzschwingungen von Carbonylverbindungen. Diese, im sonst nur wenig beanspruchten Gebiet zwischen 1800 und $1650 \mathrm{~cm}^{-1} \mathrm{zu}$ beobachtende Bande zeigt, dass die Reaktion des Brenzkatechins an der Tonmineraloberfläche zur chemischen Transformation des Phenols geführt hat. Die Vermutung, dass es sich bei der gebildeten Verbindung um ein Oxidationsprodukt des Brenzkatechins handelt ist naheliegend. Die Interpretation wird jedoch durch die Überlagerung der Spektreninformation der Reaktionsprodukte mit denen der zum großen Teil noch enthaltenen unveränderten Ausgangssubstanz erschwert. Aus diesem Grunde wurden die von der Tonmineraloberfläche extrahierten Verbindungsge- 
mische durch eine halbautomatisierte Fraktionierung mittels HPLC (Kapitel 4.5) voneinander getrennt. Anschließend erfolgte dann die qualitative IR-spektroskopische Untersuchung. Für die Belegung des Montmorillonits mit Brenzkatechin ergaben sich so bis zu 10 Fraktionen. Die einzelnen IR-Spektren werden im Folgenden der besseren Übersichtlichkeit wegen in zwei Abbildungen (Abbildungen 92 und 93) dargestellt.

\subsection{Fraktionen 1-5 der chromatographisch getrennten Reaktionsprodukte}

Die IR-Spektren der Fraktionen 1-5 zeigen eine intensive Absorption im Valenzschwingungsbereich der O-H-Gruppen zwischen $3600 \mathrm{~cm}^{-1}$ und $3100 \mathrm{~cm}^{-1}$, wobei die Spektren der Fraktionen 3 und 4 ein deutliches Maximum bei etwa 3450-3400 $\mathrm{cm}^{-1}$ aufweisen.

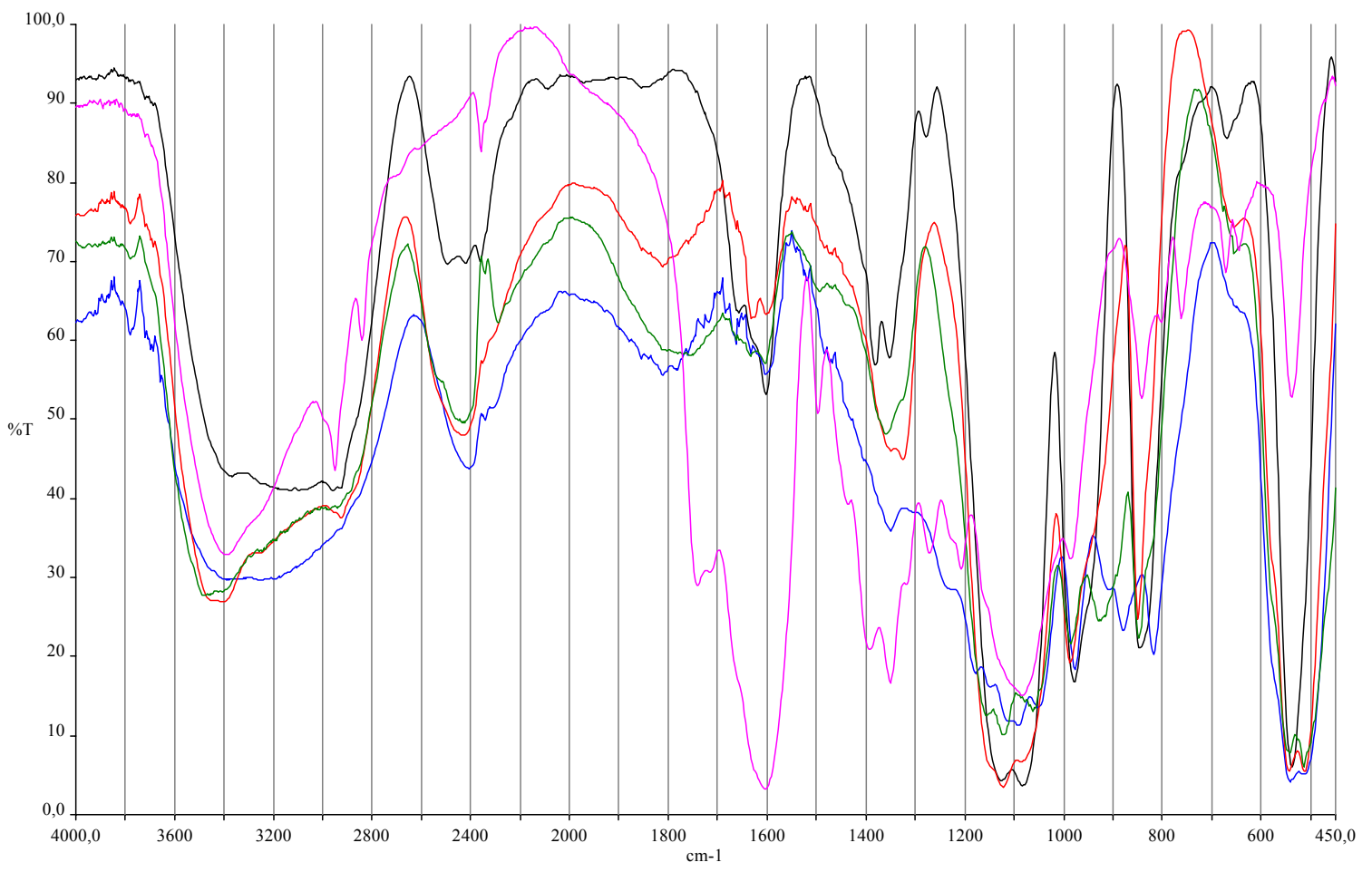

Abbildung 92: IR-Spektren der Fraktionen 1-5 (schwarz: Fraktion 1, blau: Fraktion 2, rot: Fraktion 3, grün: Fraktion 4, rosa: Fraktion 5).

Zusammen mit dem Spektrum der Fraktion 1 zeigen diese Spektren bei $2923 \mathrm{~cm}^{-1}$ mit der Valenzschwingung (vC-H) das Auftreten von aromatischen Methylgruppen an, wobei das Spektrum der Fraktion 1 eine zusätzliche Bande bei $2961 \mathrm{~cm}^{-1}$ aufweist und somit auch als Streckschwingung einer Methoxylgruppe $\left(\mathrm{R}-\mathrm{O}-\mathrm{CH}_{3}\right)$ interpretiert werden kann. Die in allen Spektren zu beobachtende Absorptionsbande bei etwa $2400 \mathrm{~cm}^{-1}$ bis $200 \mathrm{~cm}^{-1}$ ist auf die atmosphärische Inkompensation von $\mathrm{CO}_{2}$ bei Proben- und Hintergrundspektrum zurückzuführen. Dies wird durch die schwache Bande bei $669 \mathrm{~cm}^{-1}$ bestätigt. Die breite, mit einem Maximum bei $1813 \mathrm{~cm}^{-1}$ auftretende Bande $(\mathrm{vC}=\mathrm{O})$ weist die Existenz der Carbonylgruppen von Carbonsäuren nach. Dafür sprechen auch die breite $\mathrm{OH}-\mathrm{Bande}$ und die als Kontrollbanden geltenden Banden (vC-O oder $\delta \mathrm{OH}$ ) zwischen 1440 bis $1210 \mathrm{~cm}^{-1}$, eine intensive Bande im Bereich von 1315 bis $1075 \mathrm{~cm}^{-1}$ und eine weitere Bande ( $\left.\mathrm{OOH}-\mathrm{O}\right)$ bei etwa 960 bis $880 \mathrm{~cm}^{-1}$. Aus dem 
Auftreten der Valenzschwingung der Carbonylbindung zwischen 1800 und $1740 \mathrm{~cm}^{-1}$ ist zu erkennen, dass monomere Formen der Carbonsäuren in einem Teil der Proben enthalten sind. Eine Assoziation der Substituentenreste hätte eine Verschiebung der Absorption in Richtung niedrigerer Frequenzen (1740-1650 $\mathrm{cm}^{-1}$ ) zur Folge (GOTTWALD \& WACHTER 1997). In den Fraktionen 2 bis 4 sind somit Carbonsäuren enthalten, während diese in der ersten Fraktion nicht nachzuweisen sind. Deutlich hiervon zu unterscheiden ist das Spektrum der Fraktion 5 , hier ist eine intensive Bande $1742 \mathrm{~cm}^{-1}$ und eine etwas schwächere Bande bei $1714 \mathrm{~cm}^{-1} \mathrm{zu}$ beobachten. Absorptionsbanden im Bereich zwischen 1765 und $1645 \mathrm{~cm}^{-1}$ sind der Valenzschwingung $(\mathrm{vC}=\mathrm{O})$ der Carbonylgruppen von Aldehyden bzw. Ketonen zuzuordnen. Chinone als aromatische Ketone werden somit auch dieser Verbindungsklasse zugeschlagen. Eine breite Bande zwischen 1680 und $1570 \mathrm{~cm}^{-1}$ tritt in allen Fraktionen auf. Gemeinsam mit der Absorptionsbande im Bereich von $1470 \mathrm{~cm}^{-1}$ beschreibt sie die Valenzschwingung (vC-C) im aromatischen Ringskelett und weist somit Aromate in den Fraktionen, insbesondere in Fraktion 5, nach. Der Bereich zwischen 1450 und $1250 \mathrm{~cm}^{-1}$ ist der Deformationsschwingung ( $\delta \mathrm{O}-$ $\mathrm{H})$ der $\mathrm{OH}-G r u p p e n$ zuzuordnen und sind in den Spektren der Fraktionen 1 bis 5 enthalten. Unterschiede der Spektren von Fraktion 2 und 4 treten im Vergleich zu den übrigen Fraktionen bei $1230 \mathrm{~cm}^{-1}$ auf, diese Bande ist nur dort zu beobachten und kann aufgrund ihrer schmalen Ausprägung der Valenzschwingung ( $\mathrm{vC}-\mathrm{O})$ von aromatischen Ethern zugeschrieben werden. Die breite Bande zwischen 1200 und $1020 \mathrm{~cm}^{-1}$ charakterisiert die Streckschwingung (vC-O) von Alkoholen und Phenolen und ist bei allen Fraktionen durch mehrere Bandenlagen in diesem Bereich vertreten. Während im höheren Frequenzbereich von 1260 bis $1180 \mathrm{~cm}^{-1}$ auftretende Absorptionen den C-O-Schwingungen von Phenolen zuzuordnen sind, bedeuten kleinere Wellenzahlen alkoholische C-O-Schwingungen. Die im Spektrum der Fraktion 5 zu beobachtende Bande mit einem Maximum bei $1080 \mathrm{~cm}^{-1}$ ist der Valenzschwingung (vC-C) in Ketonen zuzuordnen, diese Schwingungen werden durch die unmittelbare Nähe mit der Carbonylgruppe aktiviert (GOTTWALD \& WACHTER 1997). Die in den IR-Spektren aller fünf Fraktionen auftretende Bande bei $980 \mathrm{~cm}^{-1}$ kann durch die out-of-planeDeformationsschwingung $(\delta \mathrm{C}-\mathrm{H})$ von $\mathrm{C}-\mathrm{H}$ in Alkenen hervorgerufen werden. Ebenso wie bei der Bande bei $927 \mathrm{~cm}^{-1}$ (Fraktion 4) erscheint eine eindeutige Zuordnung schwierig. Im sich zu kleineren Wellenzahlen hin anschließenden Bereich unterscheiden sich die einzelnen Fraktionen sehr deutlich voneinander. Dieser Bereich der Substitutionsmusterbanden von Aromaten $\left(900-650 \mathrm{~cm}^{-1}\right)$ wird durch die Deformationsschwingung $(\delta \mathrm{C}-\mathrm{H})$ aus der Ebene des Rings heraus hervorgerufen und zeigt allgemein eine sehr breite Bande. Hieraus kann die Substitution des aromatischen Gerüsts abgelesen werden (Tabelle 15). 
Tabelle 15: Substitutionsmusterbanden der Fraktionen 1-5.

\begin{tabular}{|c|c|c|}
\hline Fraktion & Wellenzahl $\left(\mathbf{c m}^{-1}\right)$ & Substitution \\
\hline 1 & 848 & $1,4-$ \\
\hline 2 & $909,879,815$ & $1,3-$ \\
\hline 3 & 848 & $1,4-$ \\
\hline 4 & 848,824 & $1,4-$ \\
\hline 5 & $842,803,760,672$ & $1,3-; 1,2-$ \\
\hline
\end{tabular}

Die Unterschiede der IR-Spektren der einzelnen Fraktionen in diesem Bereich sind signifikant, jedoch können die Absorptionsbanden nicht eindeutig und nur sehr schwer einem bestimmten Substitutionstyp zugeordnet werden. Aus diesem Grunde sollten die Inhalte der obigen Tabelle nur mit Vorsicht und als Hinweise einer strukturellen Veränderung des aromatischen Systems im Vergleich zur Ausgangssubstanz (1,2-substituiert) angesehen werden.

\subsection{Fraktionen 6-10 der chromatographisch getrennten Reaktionsprodukte}

Die IR-Spektren der Fraktionen 6-10 weisen, wie die bei den Fraktionen 1-5 eine breite, intensive Absorption im Bereich der Streckschwingungen der OH-Gruppen (vO-H) mit einem Maximum bei $3400 \mathrm{~cm}^{-1}$ und einer zweiten Bande bei $3250 \mathrm{~cm}^{-1}$ auf. Diese ist besonders in den Fraktionen 7 und 10 ausgeprägt. In Fraktion 9 ist diese Bande nach etwa $3200 \mathrm{~cm}^{-1}$ verschoben. Die Breite dieser Bande ist bestimmt durch den Assoziationsgrad, d. h. durch die Anzahl der Wasserstoffbrücken.

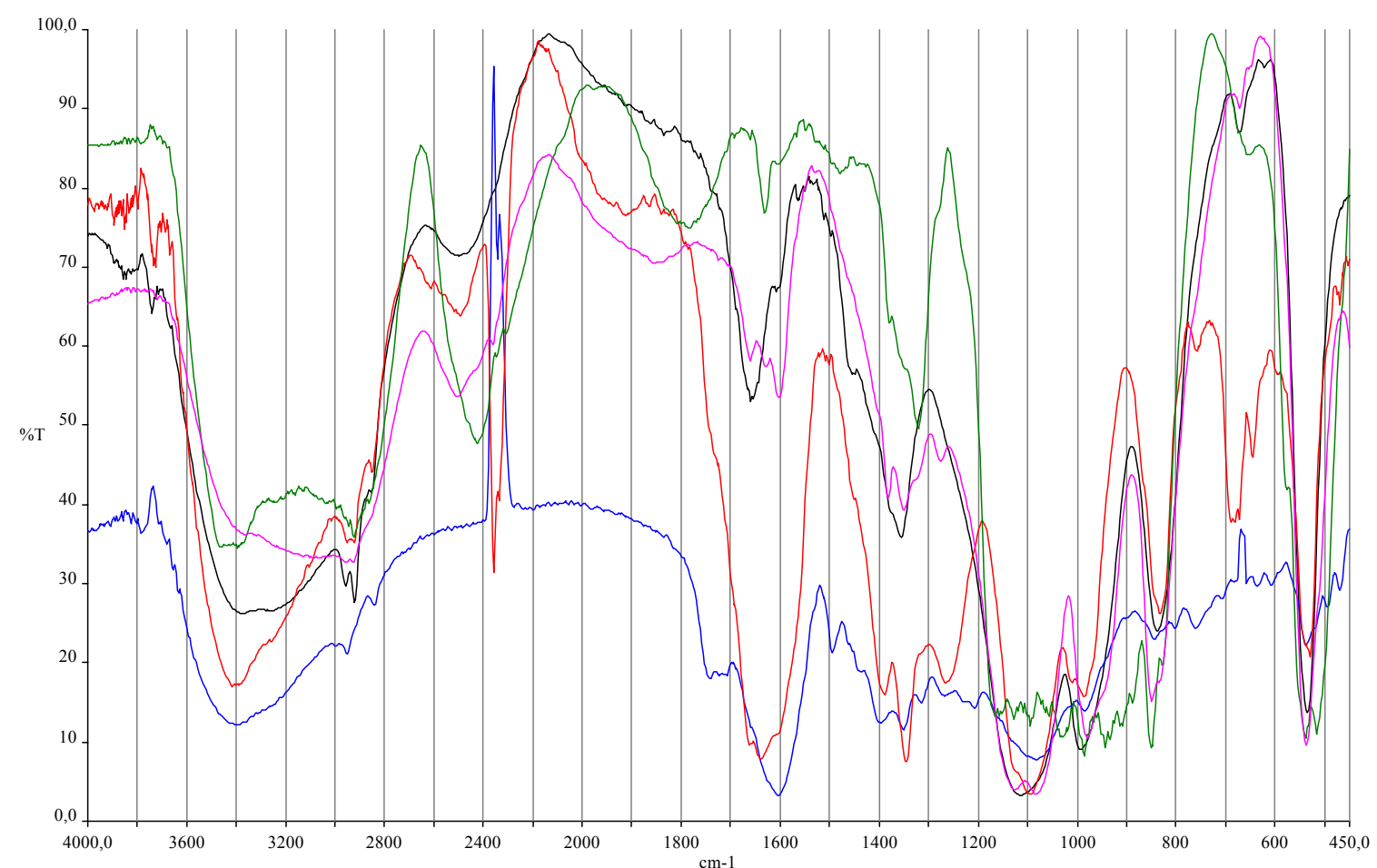

Abbildung 93: IR-Spektren der Fraktionen 6-10 (blau: Frakt. 6, rot: Frakt. 7, grün: Frakt. 8, rosa: Frakt. 9 , schwarz: Frakt. 10).

Eine Verschiebung zu kleineren Wellenzahlen (3250-3200 $\left.\mathrm{cm}^{-1}\right)$ und eine breite Absorptionsbande deutet auf assoziierte bzw. polymere $\mathrm{OH}-\mathrm{Gruppen}$ hin, während freies $\mathrm{OH}$ eine scharfe 
Bande zwischen 3650 bis $3590 \mathrm{~cm}^{-1}$ und phenolisches $\mathrm{OH}$ eine intensive Bande von 3560 bis $3400 \mathrm{~cm}^{-1}$ hervorruft. Gleichfalls tritt in allen IR-Spektren, besonders gut zu erkennen bei den Fraktionen 6 und 10 die Bande der Valenzschwingung von $\mathrm{C}-\mathrm{H}(\mathrm{vC}-\mathrm{H})$ in aromatischen Systemen auf. Die Banden dieser Schwingung sind aufgespalten und liegen bei $2956 \mathrm{~cm}^{-1}, 2917$ $\mathrm{cm}^{-1}$ und $2851 \mathrm{~cm}^{-1}$. Zwischen $2450 \mathrm{~cm}^{-1}$ und $2350 \mathrm{~cm}^{-1}$ sind wiederum die Banden für atmosphärisches Kohlendioxid zu beobachten. Der Wellenzahlenbereich zwischen 1850 und 1700 $\mathrm{cm}^{-1}$ ist bei den IR-Spektren der Fraktionen 7 und 8 von einer relativ breiten Absorptionsbande geprägt und dem Auftreten von Carboxylgruppen zuzuordnen, insbesondere Fraktion 9 zeigt eine intensive Bande mit einem Maximum bei $1783 \mathrm{~cm}^{-1}$ (siehe Fraktionen 2-5). Die Absorptionsmaxima der Banden von $\mathrm{C}=\mathrm{O}$-Bindungen in den Fraktionen 6 und 7 liegen bei 1742, 1725, 1705 und $1663 \mathrm{~cm}^{-1}$. Somit wird das Vorkommen von Ketonen bzw. Chinonen nachgewiesen werden. Im Gegensatz dazu treten bei den Spektren der Fraktionen 9 und 10 deutliche Unterschiede auf. Während im Spektrum der Fraktion 9 keine signifikante Absorption zu beobachten ist, tritt bei Fraktion 10 keine Bande mehr in diesem Bereich auf. Daraus ist zu schließen, dass in diesen Fraktionen keine Carbonsäuren enthalten sind. Im Wellenzahlenbereich zwischen etwa 1680 und $1600 \mathrm{~cm}^{-1}$ treten in 6,7 und 8 Banden auf, während im Bandenbereich zwischen 1620 und $1460 \mathrm{~cm}^{-1}$, dem Valenzschwingungsbereich (vC-C) in aromatischen Systemen, insbesondere bei $1500 \mathrm{~cm}^{-1}$ keine oder nur sehr schwache Banden zu erkennen sind. Dies spricht dafür, dass Aromaten in diesen Fraktionen nur zu äußerst geringen Anteilen bzw. gar nicht enthalten sind. Die Dominanz der Banden oberhalb von $1600 \mathrm{~cm}^{-1}$, zwischen $1650 \mathrm{~cm}^{-1}$ (Fraktion 10), 1640 bis $1632 \mathrm{~cm}^{-1}$ (Fraktion 7, 8, 9) und $1610 \mathrm{~cm}^{-1}$ (Fraktion 6,9) weist die Streckschwingung $(\mathrm{vC}=\mathrm{C})$ von konjugierten Alkenen nach. Diese Absorption ist normalerweise als schmale Bande im Spektrum zu beobachten. Eine breite und starke Ausprägung dieser Bande (1650-1615 $\left.\mathrm{cm}^{-1}\right)$ spricht für die Konjugation der Doppelbindung mit einer Carbonylgruppe $(\mathrm{C}=\mathrm{O})$ (GOTTWALD \& WACHTER 1997). Das Fehlen von Absorptionsbanden aromatischer Skelettschwingungen und das Auftreten von Schwingungen von Alkenen deutet auf die Bildung von Reaktionsprodukten hin, die durch die Aufspaltung des aromatischen Gerüstes der Ausgangssubstanz entstanden sind.

Der Bereich zwischen 1500 und $1300 \mathrm{~cm}^{-1}$ ist bei den Fraktionen 6-10 von einer allgemeinen Zunahme der Absorption gekennzeichnet. Die hier auftretenden Banden beschreiben die Deformationsschwingung $(\delta \mathrm{C}-\mathrm{H})$ von Methyl und Methylengruppen. Bei mehreren Methylgruppen an einem Kohlenstoffatom kommt es zur Aufspaltung der Bande. Dies ist bei den IRSpektren der Fraktionen 9 (Dublett bei 1380 und $1350 \mathrm{~cm}^{-1}$ ) und 10 (Dublett bei 1378 und $1358 \mathrm{~cm}^{-1}$ ) zu beobachten. In den Spektren der Fraktionen 6 und 7 sind diese Banden nicht eindeutig auszumachen. Fraktion 8 zeigt nur eine schwache Ausprägung dieser Banden, die Information wird durch eine dominante Bande bei $1321 \mathrm{~cm}^{-1}$ überdeckt. Hierbei handelt es sich um die Absorption durch die Deformationsschwingung ( $\delta \mathrm{O}-\mathrm{H}$ in-plane). Der sich nach rechts anschließende Bandenbereich von etwa 1200 bis $1050 \mathrm{~cm}^{-1}$ Informationen von verschiedenen Bindungen beinhalten. Durch das Auftreten der Bande der Valenzschwingung 
$(\mathrm{C}=\mathrm{O})$ zwischen 1800 und $1650 \mathrm{~cm}^{-1}$ gemeinsam mit starken Banden in diesem Bereich werden die Hinweise auf die Existenz von Carboxylgruppen in diesen Fraktionen durch in diesem Bereich erscheinende Kontrollbanden erhärtet. Diese Banden charakterisieren die Valenzschwingungen $(\mathrm{VC}-\mathrm{O})$ sowie die Deformationsschwingung $(\gamma \mathrm{OH})$ in Carbonsäuren und treten als Kombination zweier Banden auf (Fraktion 8, 9). Bestätigt wird dies durch eine weitere diagnostische Bande zwischen 960 und $880 \mathrm{~cm}^{-1}$, die die Deformationsschwingung ( $\left.8 \mathrm{OH}-\mathrm{O}\right)$ o.o.p.) beschreibt und in den Fraktionen 6 bis 9 ebenfalls gut zu erkennen ist. Das IRSpektrum der Fraktion 10 fehlt die diagnostische Bande der Carbonsäure, die Absorption bei 1116 und $996 \mathrm{~cm}^{-1}$ ist somit der Valenzschwingung (vC-O) der C-O-Gruppe (Alkohole, Ether) zuzuordnen. Für den Bereich der Substitutionsmusterbanden ist keine eindeutige Zuordnung aufgrund der Verteilung und teilweise schwachen Ausprägung der Banden durchzuführen.

4.6.6.5 Extrahierte Überzüge des mit Pyrogallol belegten Montmorillonits

Der direkte Vergleich der IR-Spektren der extrahierten Überzüge des mit Pyrogallol belegten Montmorillonits (blau) mit der Reinsubstanz (schwarz) zeigt mehrere wesentliche Unterschiede:

1. die Bande im Absorptionsbereich der OH-Gruppen (3600-3200 $\mathrm{cm}^{-1}$ ) ist deutlich verbreitert, was auf eine Zunahme des Assoziationsgrades schließen lässt

2. es treten Banden von Valenzschwingungen (vC-H) von Methylgruppen bei 2922 und $2853 \mathrm{~cm}^{-1}$ auf. Dies kann als Methylierung der Substituenten am aromatischen Ring interpretiert werden

3. eine breite, intensive Bande mit einem Maximum bei $1723 \mathrm{~cm}^{-1}$ weist das Auftreten von Valenzschwingungen $(\mathrm{v} C=\mathrm{O})$ von Carbonylgruppen nach

4. der Wellenzahlenbereich zwischen 1500 und $1000 \mathrm{~cm}^{-1}$ zeigt eine deutlich höhere Absorption durch Überlagerung einer Vielzahl von Banden

5. der Bereich der Substitutionsmusterbanden lässt eine Änderung der Substitution der bzw. des gebildeten Reaktionsprodukte(s) erkennen.

Um eine bessere Differenzierung und Charakterisierung der extrahierten organischen Überzüge zu erhalten, wurde auch hier eine Fraktionierung des Probenmaterials durchgeführt. Durch chromatographische Trennung konnten 6 Fraktionen voneinander getrennt werden. In Abbildung 95 sind die IR-Spektren der einzelnen Fraktionen zur Verdeutlichung der Unterschiede dargestellt. Der Absorptionsbereich der Valenzschwingung der OH-Gruppen erstreckt sich bei den IR-Spektren der Fraktionen 1, 3-6 von 3600 bis 3270 $\mathrm{cm}^{-1}$ mit einem Maximum bei $3420 \mathrm{~cm}^{-1}$. Dieser Bereich ist im Spektrum der Fraktion 2 noch breiter ausgeprägt. Dies lässt auf eine erhöhte Zahl von assoziierten, über H-Brücken verbundene $\mathrm{OH}-\mathrm{Gruppen}$ schließen. 


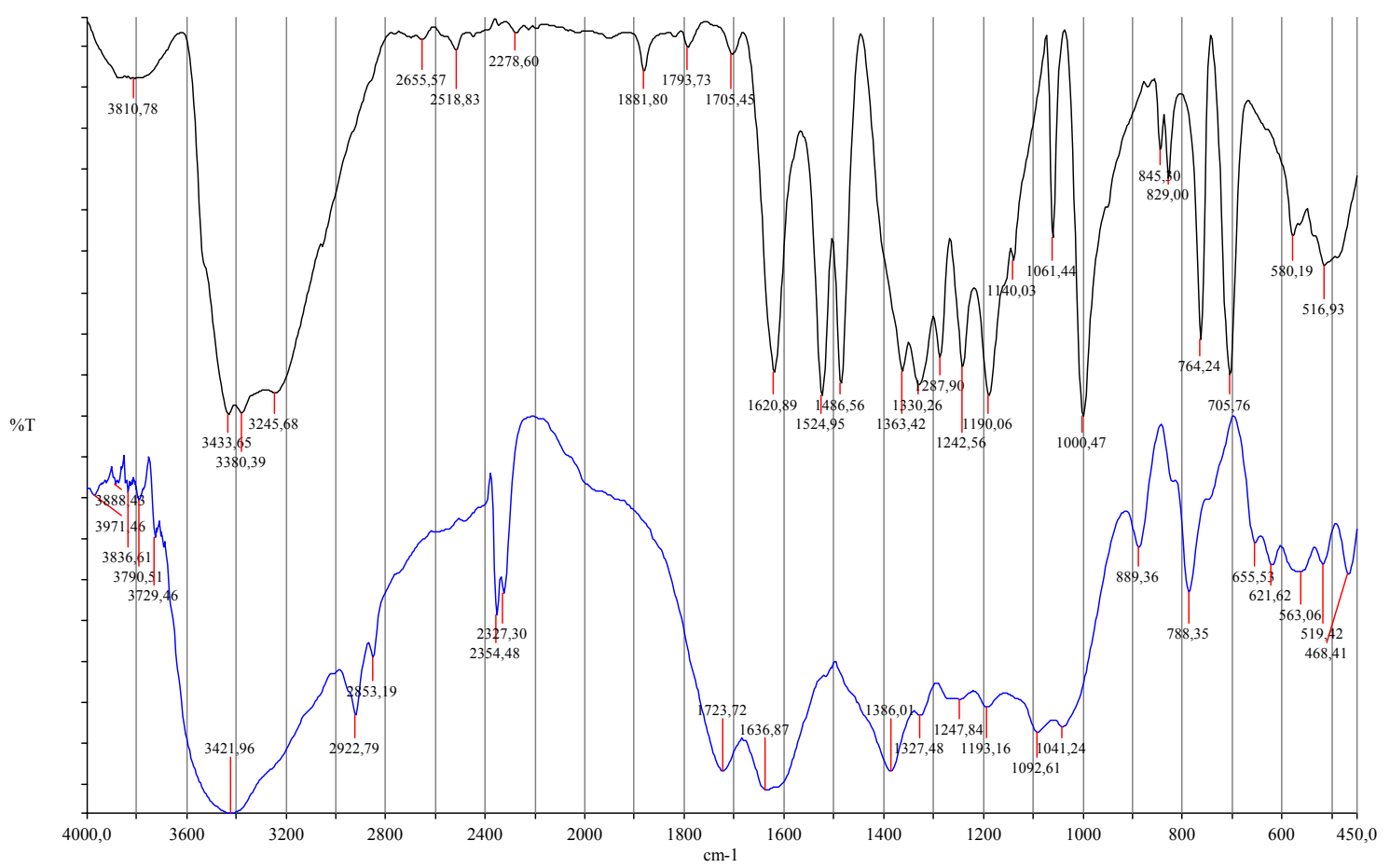

Abbildung 94: IR-Spektrum der extrahierten Überzüge des mit Pyrogallol belegten Montmorillonits (schwarz: Pyrogallol Standard; blau: extrahierte Überzüge).

Bei 2925 und $2854 \mathrm{~cm}^{-1}$ weisen die Fraktionen 1, 3 bis 6 Absorptionsbanden der Valenzschwingung $(v \mathrm{C}-\mathrm{H})$ von Methylgruppen auf. Bei diesen handelt es sich aufgrund der Bandenlage um aromatisches $-\mathrm{CH}_{3}$.

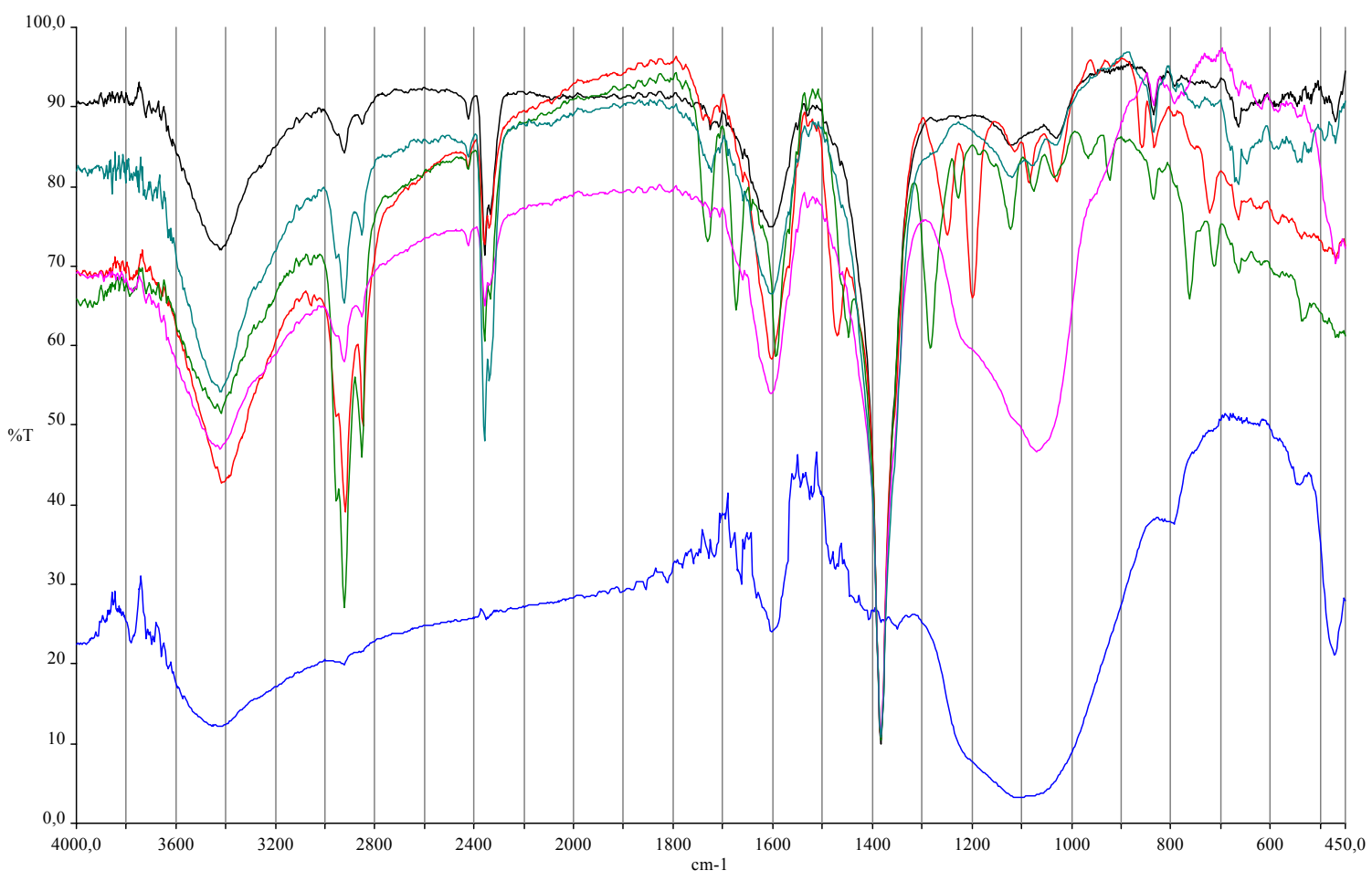

Abbildung 95: IR-Spektren der Fraktionen 1-5 (schwarz: Fraktion 1, blau: Fraktion 2, rot: Fraktion 3, grün: Fraktion 4, rosa: Fraktion 5, türkis: Fraktion 6). 
Als Störsignal tritt bei $2355 \mathrm{~cm}^{-1}$ eine Absorption durch atmosphärisches $\mathrm{CO} 2$ auf, daneben ist eine geringe Restfeuchte des Probenmaterials anhand des zittrigen Verlaufs der Spektren im Bereich zwischen 3800 bis $3600 \mathrm{~cm}^{-1}$ und 2000 bis $1500 \mathrm{~cm}^{-1}$ festzustellen. Im Schwingungsbereich der Carbonylgruppen sind in den Spektren der einzelnen Fraktionen deutliche Unterschiede zu erkennen. Während in den Fraktionen 1, 2 und 5 keine Absorptionsbanden zu beobachten sind, zeigen die Fraktionen 3 und 6 schwache Banden, welche $\mathrm{C}=\mathrm{O}-\mathrm{Bindungen}$ nachweisen. Diese liegen bei 1739 und $1725 \mathrm{~cm}^{-1}$ (Fraktion 3) sowie $1725 \mathrm{~cm}^{-1}$ (Fraktion 6). Aufgrund der fehlenden diagnostischen Merkmale für Carbonsäuren - es treten weder eine sehr breite Bande der Streckschwingung (vO-H) von OH-Gruppen zwischen 3550 und 2500 $\mathrm{cm}^{-1}$ (OH-Vibration der Carboxylgruppe (WANG \& HUANG 2000) und auch keine Kontrollbanden zwischen 1440-1210 $\mathrm{cm}^{-1}$ ( $\left.\mathrm{vC}-\mathrm{O}\right), 1315-1075(\delta \mathrm{OH}) \mathrm{cm}^{-1}$ sowie zwischen 960$880 \mathrm{~cm}^{-1}(\delta \mathrm{OH}-\mathrm{O})$ auf. Somit ist davon auszugehen, dass die $\mathrm{C}=\mathrm{O}-\mathrm{Gruppen}$ in diesen Fraktionen den Ketonen zuzuordnen sind, wofür auch die Lage der Kontrollbanden zwischen 1330 und $1075 \mathrm{~cm}^{-1}$ hindeutet. Die Oberschwingung bei $3450 \mathrm{~cm}^{-1}$, die ebenfalls als diagnostisches Merkmal von Ketonen gilt, fällt hier mit den Streckschwingungen $(v \mathrm{OH})$ der $\mathrm{OH}-$ Gruppen zusammen. Das Maximum der Absorption liegt jedenfalls bei $3420 \mathrm{~cm}^{-1}$. In Fraktion 4 ist die Bandenlage deutlicher ausgeprägt: zwei Banden bei 1730 und $1675 \mathrm{~cm}^{-1}$ weisen Carbonylgruppen nach. Durch die Verschiebung der zweiten Bande in den langwelligeren Frequenzbereich mit gleichzeitigem Auftreten der diagnostischen Bandenlagen der Streckschwingung (vC-C) von Aromaten zwischen 1600 und $1400 \mathrm{~cm}^{-1}$ ist auf ein aromatisches Keton in dieser Fraktion zu schließen. Die Fraktionen 1, 2 und 5 zeigen keine Absorption die dem Auftreten von $\mathrm{C}=\mathrm{O}$-Gruppen zugerechnet werden kann. Neben den kurzwelligen Valenzschwingungen der OH-Gruppen sind in den Fraktionen 1 und 5 keine Banden, die aromatischen Strukturen zugeordnet werden können zu beobachten. Die Bande bei $1597 \mathrm{~cm}^{-1}$ ist charakteristisch für die Valenzschwingung $(\mathrm{vC}=\mathrm{C})$ von Alkenen. Die daneben auftretende Bande bei $1384 \mathrm{~cm}^{-1}$ weist Methyl- bzw. Methylengruppen $(\delta \mathrm{C}-\mathrm{H})$ nach. In diesen Fraktionen sind somit nur aliphatische Verbindungen enthalten. Durch die breite Bande zwischen 1240 und $970 \mathrm{~cm}^{-1}$, die durch die Absorption der Streckschwingung (vC-O) von C-O-Bindungen hervorgerufen wird, kann davon ausgegangen werden, dass in der Fraktion 5 lediglich ein aliphatischer Alkohol enthalten ist. Das IR-Spektrum der Fraktion 2 ist aufgrund der Bandenlage im Bereich zwischen 1650 und $1400 \mathrm{~cm}^{-1}$ nicht so eindeutig zu interpretieren: Wie in Fraktion 5 ist eine breite Bande der Valenzschwingung ( $v \mathrm{C}-\mathrm{O})$ zu beobachten, was auf alkoholische Strukturen hindeutet. Durch die unklare Bandenlage im Absorptionsbereich der Streckschwingung ( $\mathrm{VC}-\mathrm{C}$ ) von Aromaten ist jedoch in diesem Falle keine einwandfreie $\mathrm{Zu}-$ ordnung, ob es sich in dieser Fraktion um Aliphate oder Aromate handelt, möglich. Eine Bestimmung des Substitutionstyps ist in allen Fraktionen aufgrund der schwachen Ausprägung der Banden ebenfalls nicht praktikabel. 
4.6.6.6 Extrahierte Überzüge des mit 2,6-Dimethylphenol belegten Montmorillonits

Das IR-Spektrum des Gesamtextraktes des mit 2,6-Dimethylphenol belegten Montmorillonits weist im Vergleich mit dem Spektrum des reinen Phenols deutliche Unterschiede auf (Abbildung 96).

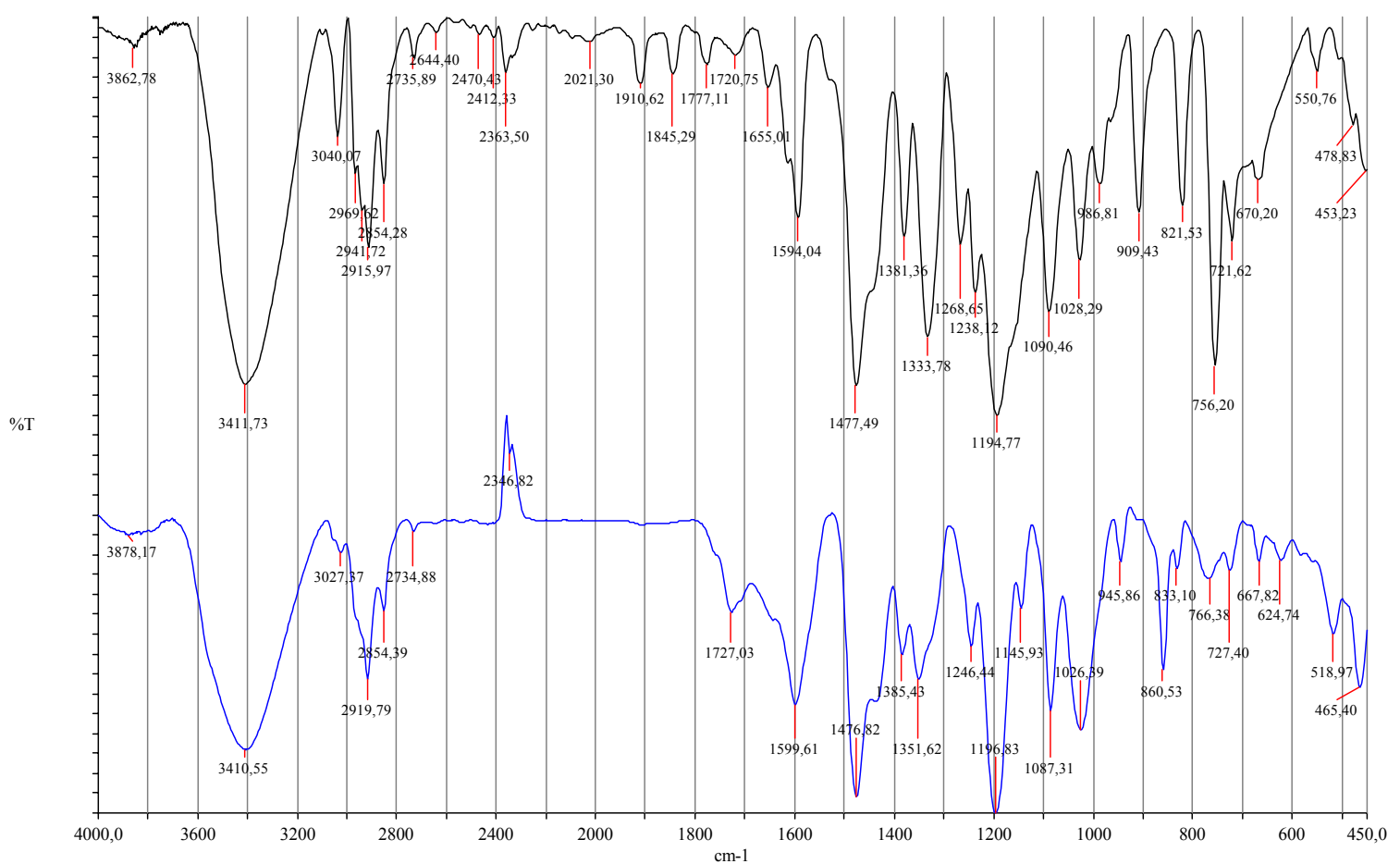

Abbildung 96: IR-Spektrum der extrahierten Überzüge des mit 2,6-Dimethylphenol belegten Montmorillonits (schwarz: Pyrogallol Standard; blau: extrahierte Überzüge).

Trotz der wesentlichen Übereinstimmung im Aussehen der beiden Spektren zeigen sich die deutlichsten Unterscheidungsmerkmale im Bereich der Absorption der Carbonylgruppen und in der Lage der Substitutionsmusterbanden. Mit der breiten Bande bei $1727 \mathrm{~cm}^{-1} \mathrm{kann}$ eine $\mathrm{C}=\mathrm{O}$-Bindung $(\mathrm{v} \mathrm{C}=\mathrm{O})$ nachgewiesen werden. Der weitere Verlauf der beiden Spektren weist große Ähnlichkeiten auf, ab etwa $1000 \mathrm{~cm}^{-1}$ treten jedoch wieder deutliche Unterschiede zu Tage, die darin zu begründen sind, dass sich die Substitution des aromatischen Systems verändert hat. Statt der scharfen Banden bei 909, 821 und $756 \mathrm{~cm}^{-1}$ beim 2,6-Dimethylphenol treten im Spektrum der extrahierten Überzüge diese Banden bei 860 und $766 \mathrm{~cm}^{-1}$ auf.

Da jedoch auch bei diesem Gesamt-Spektrum eine Überdeckung von weiterer Information durch unveränderte Ausgangssubstanz naheliegend ist, wurde folglich auch in diesem Falle eine Fraktionierung der extrahierten Überzüge durchgeführt. Mit Hilfe der chromatographischen Trennung konnten 13 Fraktionen gewonnen werden, in Abbildung 97 und 98 werden die IR-Spektren der einzelnen Fraktionen dargestellt. 


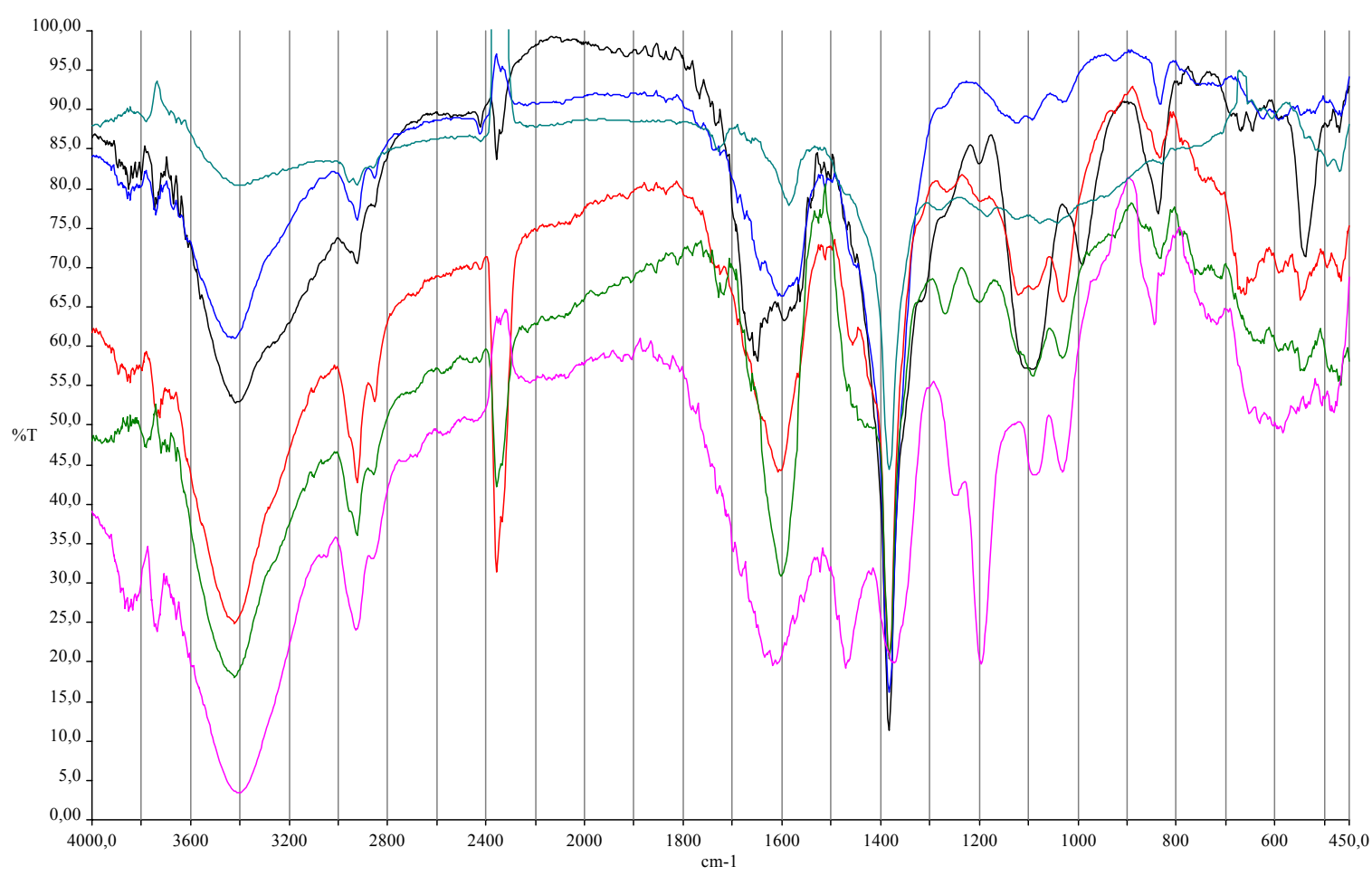

Abbildung 97: IR-Spektren der Fraktionen 1-6 (schwarz: Fraktion 1, blau: Fraktion 2, rot: Fraktion 3, grün: Fraktion 4, rosa: Fraktion 5, türkis: Fraktion 6).

Die IR-Spektren der Fraktionen 1-5 zeigen Absorptionsbanden für die Valenzschwingung (vO-H) der OH-Gruppen mit einem Maximum bei $3420 \mathrm{~cm}^{-1}$, diese Bande ist im Spektrum der Fraktion 6 hingegen schwach ausgeprägt, was auf einen nur geringen Gehalt an $\mathrm{OH}-$ Gruppen zurück geführt werden kann. Andererseits ist diese Aussage jedoch auch durch die schwache Intensität des Spektrums insgesamt zu relativieren. Eine weitere Gemeinsamkeit aller Spektren ist das Auftreten von Streckschwingungen ( $\mathrm{vC}-\mathrm{H})$ der C-H-Bindung in Methylbzw. Methylengruppen, die bei 2926 und $2857 \mathrm{~cm}^{-1}$ erscheinen und aufgrund ihrer Lage im Spektrum entweder aromatischen $\mathrm{CH}_{3}$ - bzw. aliphatischen $\mathrm{CH}_{2}$-Gruppen zuzuordnen sind. Die bei etwa $2360 \mathrm{~cm}^{-1} \mathrm{zu}$ beobachtende Bande weist wie in den vorangegangenen Spektren atmosphärisches $\mathrm{CO}_{2}$ nach und ist für die Interpretation der Spektren von keinerlei Bedeutung. Der weitere Bandenverlauf weist bis zu einer Wellenzahl von etwa $1800 \mathrm{~cm}^{-1}$ in allen Spektren keine Absorptionen auf. Ab $1800 \mathrm{~cm}^{-1}$ ist jedoch im Spektrum der Fraktion 5 eine intensive Bande der Streckschwingung von Carbonylgruppen $(\mathrm{vC}=\mathrm{O})$ mit einem Maximum bei etwa $1620 \mathrm{~cm}^{-1} \mathrm{zu}$ erkennen. Aufgrund dieses Merkmals, zusammen mit den auftretenden

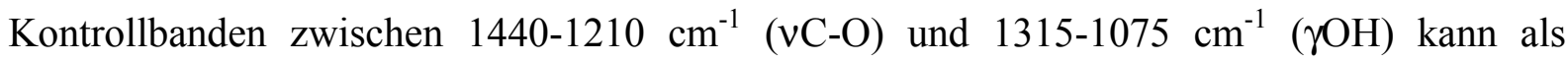
Hauptbestandteil dieser Fraktion eine Carbonsäure angenommen werden. Die Verteilung der Banden der Valenzschwingung ( $\mathrm{vC}-\mathrm{O}$ ) mit einer Absorptionsbande bei 1384 und $1196 \mathrm{~cm}^{-1}$ und durch die fehlende, nur bei dimeren Carbonsäuren vorhandene Bande der Deformationsschwingung $(\delta \mathrm{OH}-\mathrm{O})$ des $\mathrm{OH}$ der Carboxylgruppe wird eine monomere Carbonsäure nachgewiesen. In den Fraktionen 2, 3 und 4 werden durch die Banden zwischen 1730 und 1720 $\mathrm{cm}^{-1}$ ebenfalls Valenzschwingungen $(\mathrm{v} \mathrm{C}=\mathrm{O})$ von Carbonyl- bzw. Carboxylgruppen (WANG 
\& HUANG 2000) festgestellt. Aufgrund der Verteilung der im langwelligeren Bereich nachfolgenden Banden können jedoch keine Carbonsäuren in diesen Fraktionen nachgewiesen werden. Gleichfalls fehlen die für aromatische Strukturen charakteristischen drei Banden zwischen 1600 und $1400 \mathrm{~cm}^{-1}$. Daher kann von aliphatischen Aldehyden in diesen Fraktionen ausgegangen werden. Dieses Ergebnis bedeutet, dass der aromatische Ring des 2,6Dimethylphenols durch die Reaktion an der Tonmineraloberfläche aufgespaltet wurde. Fraktion 1 zeigt keine Absorption im Bereich der Carbonylgruppen, die Gesamtcharakteristika des Spektrums lässt als Resultat der Interpretation lediglich auf Aliphate mit OH-Gruppen sowie $\mathrm{CH}_{3}$ - bzw. $\mathrm{CH}_{2}$-Gruppen schließen. Die Auswertung des IR-Spektrums der Fraktion 6 ergibt ebenfalls eine erkennbare Absorption im Bereich der $\mathrm{C}=\mathrm{O}$-Bindungen bei $1729 \mathrm{~cm}^{-1}$, durch die geringe Intensität des Spektrums sind jedoch weitere Aussagen zur Struktur der in dieser Fraktion enthaltenen Verbindung nicht mit der erforderlichen Sicherheit möglich.

Die IR-Spektren der Fraktionen 7-13 zeigen im Verlauf der Spektren insgesamt ein sehr einheitliches Bild. Zu den Fraktionen 10, 11 und 13 können Aussagen nur mit großen Einschränkungen getroffen werden. Aufgrund ihrer geringen Signalstärke ist es nicht zulässig, Interpretationen, die über den Nachweis von Absorptionsbanden durch Valenzschwingungen von OH-Gruppen zwischen 3600 und $3200 \mathrm{~cm}^{-1}$ mit einem Maximum bei $3385 \mathrm{~cm}^{-1}$, auftretende Methyl- bzw. Methylengruppen bei 2924 und $2858 \mathrm{~cm}^{-1}$, Valenzschwingungen $(\mathrm{VC}=\mathrm{C})$ von konjugierten Doppelbindungen in Alkenen, der Deformationsschwingung $(\delta \mathrm{O}-\mathrm{H})$ bei 1383 und $1350 \mathrm{~cm}^{-1}$ sowie der Streckschwingung (vC-O) von C-O-Bindungen hinausgehen darzustellen.

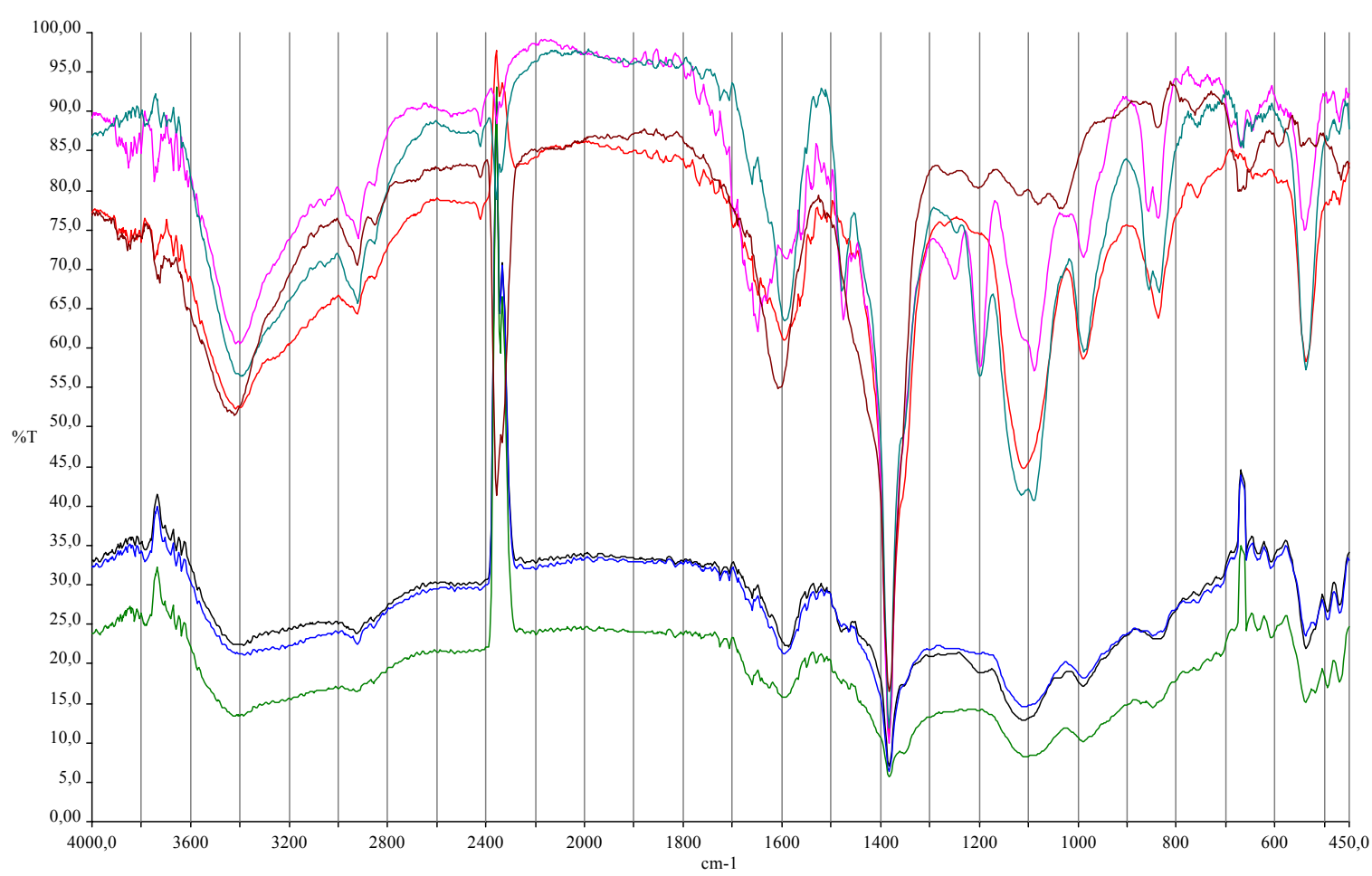

Abbildung 98: IR-Spektren der Fraktionen 7-13 (rosa: Fraktion 7, türkis: Fraktion 8, braun: Fraktion 9, schwarz: Fraktion 10, blau: Fraktion 11, rot: Fraktion 12, grün: Fraktion 13). 
In den übrigen Fraktionen $(7,8,9,12)$ sind aufgrund der höheren Güte der Spektren weitreichendere Aussagen möglich. Im Wellenzahlenbereich zwischen 3600 und $1800 \mathrm{~cm}^{-1}$ sind keine Unterschiede zu den übrigen Spektren zu erkennen, ab etwa $1780 \mathrm{~cm}^{-1}$ ist jedoch insbesondere im Spektrum der Fraktion 12 eine Zunahme der Absorption zu beobachten. Diese Bande fällt bis zum einem Absorptionsmaximum bei $1597 \mathrm{~cm}^{-1}$ stetig ab, wobei in dieser breiten Bande kleinere, in diese zum Teil integrierte Banden deutlich zu erkennen sind. Deren Maxima liegen bei 1767, 1734 und $1700 \mathrm{~cm}^{-1}$ und weisen wiederum eindeutig die Existenz von Carbonylgruppen nach. Die diagnostischen Kontrollbanden der Carbonsäuren bei 1440-1210 $\mathrm{cm}^{-1}$ und 1315-1075 $\mathrm{cm}^{-1}$ sind im Spektrum dieser Fraktion zu erkennen. Somit kann in Fraktion 12 eine Carbonsäure als Hauptbestandteil angenommen werden. Jedoch aufgrund der fehlenden, für aromatische Strukturen charakteristischen Signale und der nicht auftretenden Bande zwischen 960 und $880 \mathrm{~cm}^{-1}$ kann diese Verbindung als monomere, aliphatische Carbonsäure identifiziert werden. In den Fraktionen 7 und 8 kann von identischen Verbindungen ausgegangen werden, da die Spektren eine vollständige Übereinstimmung aufweisen. Als Grund für das, bereits bei der Fraktionierung mehrerer Lösungen verschiedener Ausgangssubstanzen, insbesondere bei direkt benachbarten Fraktionen, beobachteten Phänomens kann die Verschleppung von Probenmaterial durch die, auf extrem hohe Empfindlichkeit des automatischen Fraktionensammlers eingestellte Signaldetektion, die bei leichten Abweichungen im Verlauf der chromatographischen Trennung zur Auslassung eines Peaks führen kann, herangezogen werden. Der Vollständigkeit wegen wurden diese Fraktionen jedoch nicht zusammengefasst, sondern weiter aufgeführt. Trotzdem kann daneben eine qualitative Aussage zu diesen beiden Fraktionen getroffen werden. In dieser Fraktion sind im Vergleich mit Fraktion 12 ebenfalls Banden für Carbonylbindungen zu beobachten, die gemeinsam mit den zugehörigen diagnostischen Kontrollbanden Carbonsäuren nachweisen. Daneben sind aber vorwiegend Charakteristika aliphatischer Bindungstypen zu erkennen.

Das IR-Spektrum der Fraktion 9 zeigt zwar auch eine zunehmende Absorption ab etwa 1800 $\mathrm{cm}^{-1}$, vergleicht man jedoch diese Fraktion mit den vorab geschilderten Ergebnissen, so ist schnell zu erkennen, dass hier die bei Carbonsäuren auftretenden Banden völlig fehlen bzw. für eine eindeutige Identifizierung viel zu schwach ausgeprägt sind. Carbonylbindungen können nachgewiesen werden, aber in diesem Falle ist die $\mathrm{C}=\mathrm{O}$-Bindung einem aliphatischen $\mathrm{Al}$ dehyd (1740-1720 $\mathrm{cm}^{-1}$ ) zuzuordnen, da die diagnostischen Banden von aromatischen Systemen ebenfalls fehlen und die intensive Bande bei $1383 \mathrm{~cm}^{-1}$ die Gerüstschwingung (vC-C) der Aldehyde bestätigt. Aufgrund der relativen Breite der Bande der Valenzschwingung der C-O-Bindung von Aldehyden im Spektrum kann keine Aussage getroffen werden, ob es sich bei diesem Aldehyd daneben um ein ungesättigtes $\left(1705-1660 \mathrm{~cm}^{-1}\right)$ oder auch um ein konjugiertes (1700-1680 $\mathrm{cm}^{-1}$ ) Aldehyd handelt. Die Lage der Absorptionsbanden deutet auf eine Verteilung beide Formen hin. Die breite Absorptionsbande mit einem Maximum bei $1608 \mathrm{~cm}^{-}$ ${ }^{1}$ weist mit der Valenzschwingung $(\mathrm{vC}=\mathrm{C})$ konjugierte Doppelbindungen von Alkenen nach. Aus diesen Spektreninformationen kann die Aufspaltung des aromatischen Gerüsts des 2,6- 
Dimethylphenols hin zu aliphatischen Strukturen beobachtet werden.

4 6.6.7 Extrahierte Überzüge des mit Phenanthren bzw. Brenzkatechin und Phenanthren belegten Montmorillonits

Das um einen Polyzyklischen Aromatischen Kohlenwasserstoff (PAK) erweiterte Stoffspektrum der Untersuchungen der abiotischen Transformation organischer Huminsäurebausteine bzw. organischer Schadstoffe ergab nach der Durchführung infrarot-spektroskopischer Analysen keine Resultate, die eine Umwandlung des Phenanthrens in andere chemische Verbindungen bzw. das Auftreten neuer Bindungstypen erkennen ließen. Im dargestellten IR-Spektrum der extrahierten Überzüge des mit Phenanthren (blau) als auch mit Brenzkatechin und Phenanthren belegten Montmorillonits (rot) sind im Vergleich zum Spektrum der Reaktionsprodukte des Brenzkatechins keine Unterschiede hinsichtlich neu auftretender, fehlender oder verschobener Absorptionsbanden zu beobachten.

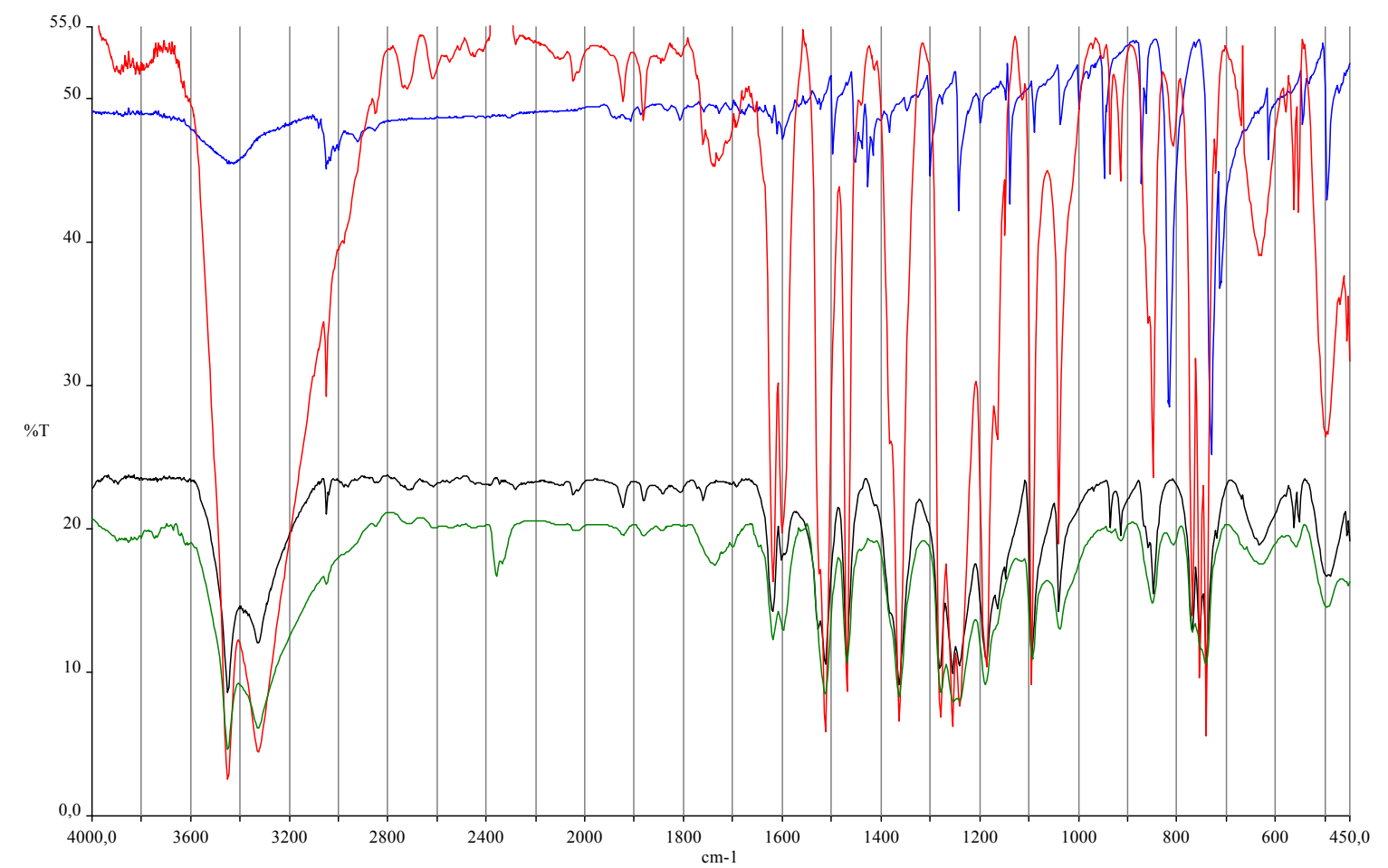

Abbildung 99: IR-Spektren von Brenzkatechin (schwarz), Phenanthren (blau) und der extrahierten Überzüge des mit Brenzkatechin (grün) sowie mit Brenzkatechin und Phenanthren belegten Montmorillonits (rot).

Mit dieser spektroskopischen Methode konnten keine Hinweise auf die im Kapitel 2.2 angestellten Überlegungen gefunden werden, dass die an Tonmineraloberflächen gemeinsam adsorbierten Brenzkatechin und Phenanthren durch die Bildung von organischen Radikalen, auf die die abiotische Umwandlung der Phenole zurückzuführen ist, auch zu einer Transformation des PAK führen kann. Die Lage der Absorptionsbanden der Spektren des Brenzkatechins und die des mit Phenol und PAK belegten Tons sind identisch. Dieses Resultat kann im wesentlichen auf drei Gründe zurückgeführt werden:

1. durch organische (phenolische) Radikale kann keine Reaktion des Phenanthrens initi- 
iert werden

2. die gebildeten Reaktionsprodukte des Phenanthrens konnten nicht von der Tonmineraloberfläche extrahiert werden und so einer Analyse zugeführt werden

3. die Konzentration der entstandenen Umwandlungsprodukte war nicht ausreichend, um einen Niederschlag in den untersuchten Proben zu finden.

Die erste These erscheint plausibel, jedoch führten die Untersuchungen mittels ${ }^{13} \mathrm{C}$-NMRSpektroskopie (Kapitel 4.7) zu anderen, weitaus positiveren Ergebnissen. Aus den dort erhaltenen Resultaten kann auch die unter 2. vorgestellte Überlegung verneint werden, da die der ${ }^{13} \mathrm{C}$-NMR-Spektroskopie zugeführten Proben nach der gleichen Methode extrahiert wurden wie die der FTIR-spektroskopischen Analysen. Somit bleibt als Ursache der negativen Ergebnisse dieser Untersuchungsreihe nur die Begründung, dass die gewonnene Probenkonzentration für eine erfolgreiche Analyse, trotz hoher Ausgangskonzentrationen des Phenols und des PAK, zu gering war und wohl zudem eine zu starke Überdeckung der eventuell vorhandenen Information durch das Phenol bzw. dessen Reaktionsprodukte vorlag.

\subsubsection{Vergleich der IR-Spektren mit Spektren bodenbürtiger Fulvosäuren}

\subsubsection{IR-Absorptionsbereiche von Fulvo- und Huminsäuren}

Die charakteristischen Hauptabsorptionsbanden von Fulvo- und Huminsäuren sind nach STEVENSON (1994) die Regionen um $3300 \mathrm{~cm}^{-1}$, die der Absorption wasserstoffverbrückter OH-Gruppen zuzuordnen sind. Weitere typische Banden liegen bei $2900 \mathrm{~cm}^{-1}$ (aliphatische CH-Streckschwingungen, aliphatische Seitenketten eines aromatischen Kerns (WANG \& HUANG 2000), $1720 \mathrm{~cm}^{-1}$ ( $\mathrm{C}=\mathrm{O}-$ Streckschwingungen von Carboxylgruppen und Ketonen), $1610 \mathrm{~cm}^{-1}$ (aromatische $\mathrm{C}=\mathrm{C}$ - sowie $\mathrm{H}$-gebundene $\mathrm{C}=\mathrm{O}$-Bindungen) und $1250 \mathrm{~cm}^{-1}$ (C-OStreck- und $\mathrm{OH}-$ Deformationsschwingungen von $\mathrm{COOH}-\mathrm{Gruppen})$. Zusätzliche schwache Absorptionsbanden treten bei etwa $1500 \mathrm{~cm}^{-1}$ (aromatische $\mathrm{C}=\mathrm{C}$-Bindungen), $1460 \mathrm{~cm}^{-1}$ (CH-Deformationsschwingungen von $\mathrm{CH}_{2-}$ und $\mathrm{CH}_{3}$-Gruppen), $1390 \mathrm{~cm}^{-1}$ (O-HDeformationsschwingungen, $\mathrm{CH}_{3}$-Bindungsschwingungen oder $\mathrm{C}-\mathrm{O}$-Streckschwingungen) auf. Fulvo- und Huminsäuren zeigen sehr ähnliche IR-Spektren, das wesentliche Unterscheidungsmerkmal von Fulvo- zu Huminsäuren ist die höhere Intensität der Bande bei $1720 \mathrm{~cm}^{-1}$. Dies liegt im erhöhten Anteil von Carboxylgruppen begründet, außerdem ist die Absorptionsbande bei $1600 \mathrm{~cm}^{-1} \mathrm{zu}$ höheren Frequenzen hin (bei $1640 \mathrm{~cm}^{-1}$ ) verschoben. Eine Übersicht der in Huminstoffen auftretenden charakteristischen Absorptionsbanden zeigt Tabelle 14.

Vergleiche von IR-Spektren der in dieser Arbeit analysierten Reaktionsprodukte mit denen bodenbürtiger Fulvosäuren weisen weitreichende Übereinstimmungen in Auftreten und Lage der Absorptionsbanden auf (Abbildungen 100-102). Diese großen Ähnlichkeiten weisen zuerst einmal darauf hin, dass natürliche Fulvo- bzw. Huminsäuren zu einem hohen Anteil aus aromatischen (phenolischen) Strukturen aufgebaut sind. 


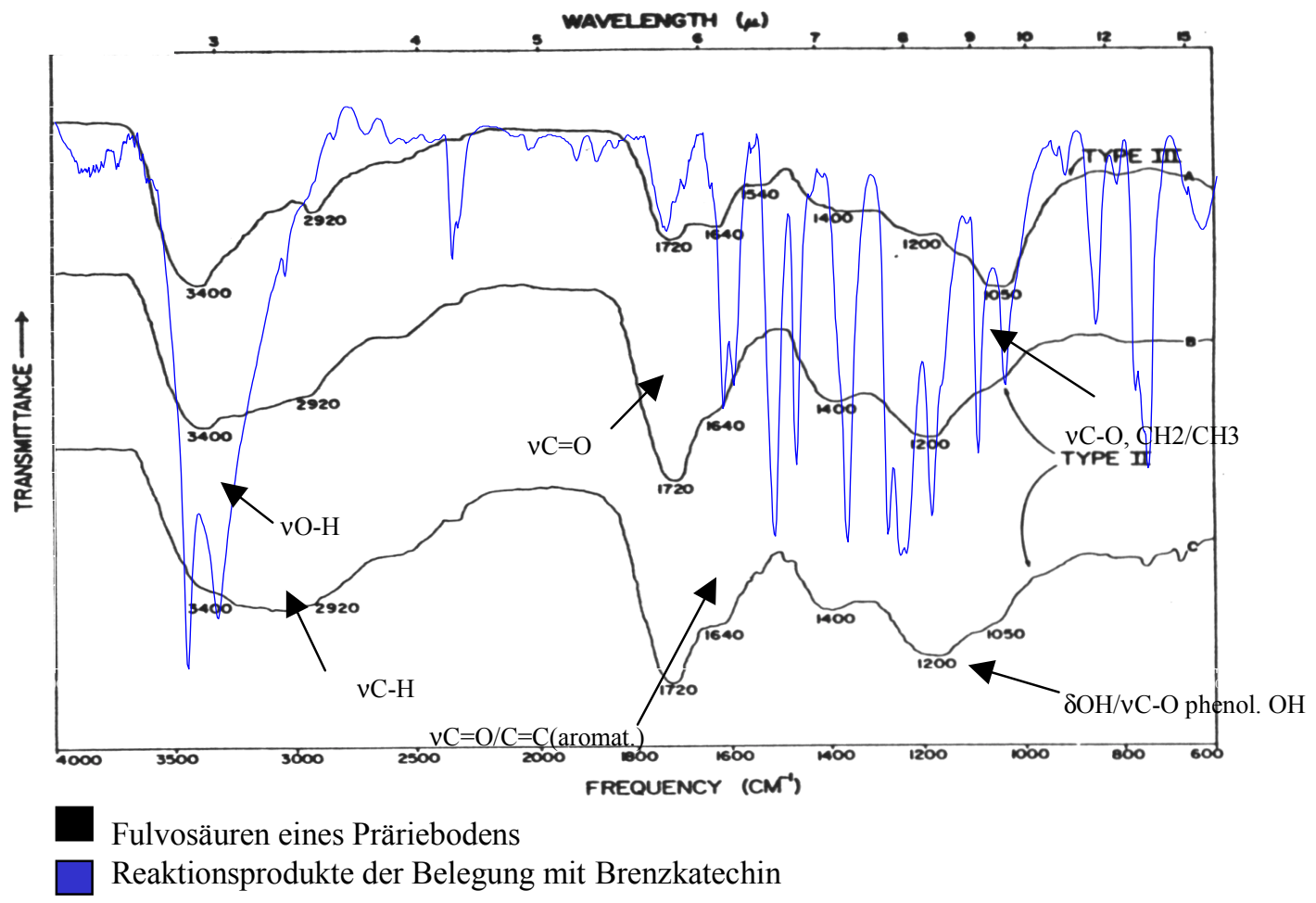

Abbildung 100: Vergleich der IR-Spektren bodenbürtiger Fulvosäuren (Stevenson 1994, verändert) mit den gewonnenen Reaktionsprodukten des Brenzkatechins.

Das IR-Spektrum der extrahierten Überzüge des mit Brenzkatechin belegten Montmorillonits weist sehr deutliche Übereinstimmungen mit den Positionen der Absorptionsbanden der Fulvosäuren auf. Der Gesamteindruck der Spektren lässt erkennen, dass Fulvosäuren zum Teil aus aromatischen Strukturen aufgebaut sein müssen. Die große Ähnlichkeit, insbesondere im Auftreten und der Position der Bande der Streckschwingung von Carbonylgruppen $\left(1725 \mathrm{~cm}^{-}\right.$ ${ }^{1}$ ), weist auf einen gemeinsamen chemischen Aufbau der Fulvosäuren mit den extrahierten Verbindungen hin. Leichte Verschiebungen in der Lage der Absorptionsbanden im Vergleich zu gemessenen Werten können auf Effekten, hervorgerufen durch das verwendete Löse- bzw. Extraktionsmittel beruhen. So weist STEVENSON (1994) darauf hin, dass die Verwendung methanolischer Lösemittel eine Methylierung der zu analysierenden Substanzen zur Folge haben kann, was in einem Anstieg der entsprechenden Absorptionsbanden (C-HStreckschwingung bei $2900 \mathrm{~cm}^{-1}$ ), der Valenzschwingung der $\mathrm{C}=\mathrm{O}-\mathrm{Gruppen}$ von Carbonylverbindungen $\left(1720 \mathrm{~cm}^{-1}\right)$ sowie der C-H-Deformationsschwingung von Methylgruppen ($\mathrm{CH}_{3}$ bei $1460 \mathrm{~cm}^{-1}$ ) zu beobachten ist. Zusätzlich erfolgt eine Verschärfung der Bande bei $1250 \mathrm{~cm}^{-1}$ (C-O-Streckschwingung und $\mathrm{OH}$-Deformationsschwingung von $\mathrm{COOH}$ ). Die $\mathrm{Ab}$ sorptionsbande bei $1725 \mathrm{~cm}^{-1}$ ist nach KAISER \& ZECH (1997) der Streckschwingung protonierter Carboxylgruppen zuzuordnen. Tritt diese Bande in Huminsäuren auf, sollte eine vorsichtige Interpretation erwogen werden, da diese Absorption auch durch die Degradation von Estern während der Extraktion gebildet werden kann. Ursprünglich ist diese Absorptionsbande in höherfrequenten Bereichen zwischen 1785 und $1735 \mathrm{~cm}^{-1} \mathrm{zu}$ beobachten und korreliert mit den Carbonylgruppen von Estern (AROCENA et al. 1995). 
Noch weitreichendere Ähnlichkeiten und damit Hinweise auf Gemeinsamkeiten im chemischen Aufbau zeigt der Vergleich des IR-Spektrums der extrahierten Reaktionsprodukte des Pyrogallols mit den aus der Literatur entnommenen Spektren.

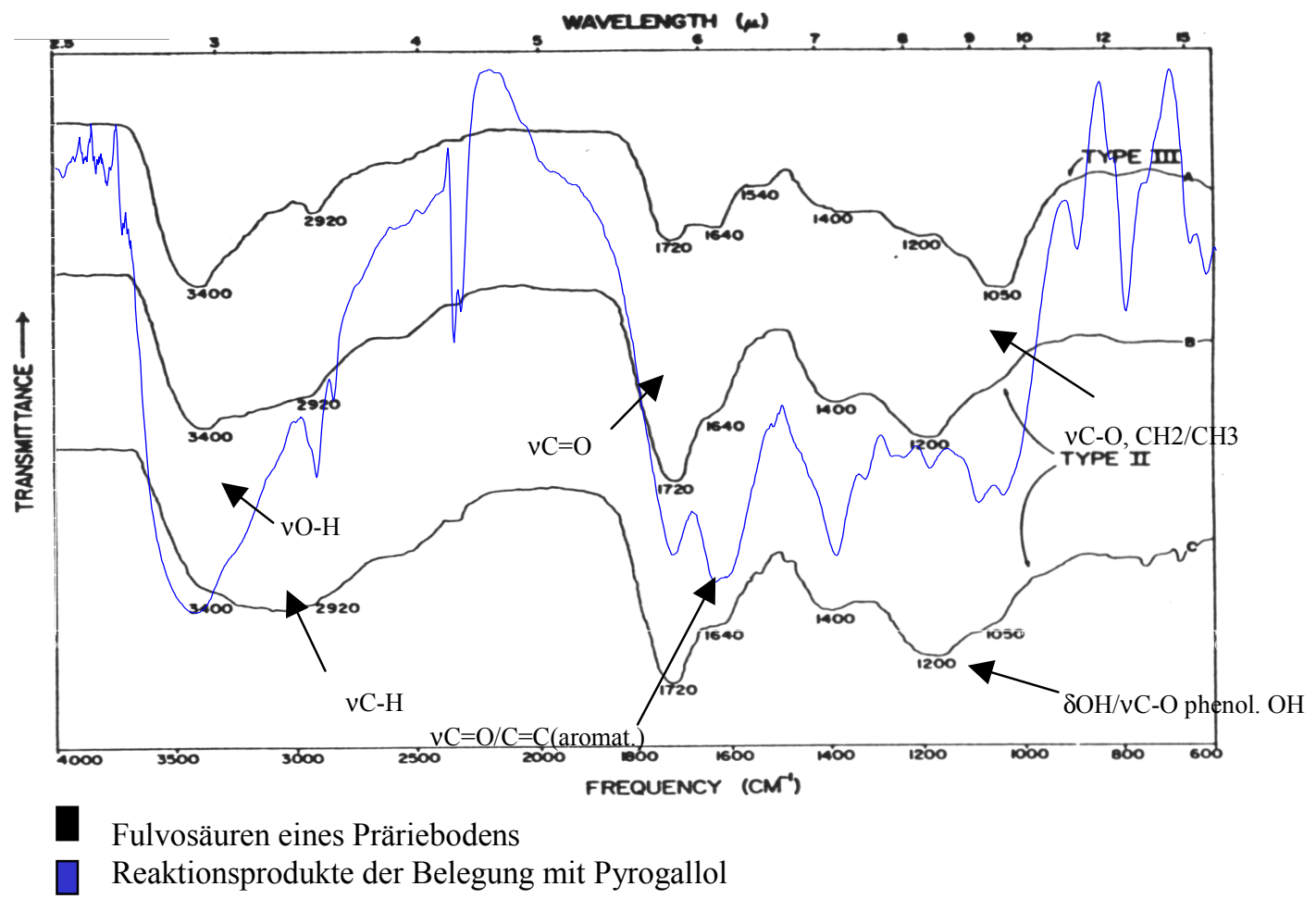

Abbildung 101: Vergleich der IR-Spektren bodenbürtiger Fulvosäuren (Stevenson 1994, verändert) mit den gewonnenen Reaktionsprodukten des Pyrogallols.

Vor allem im Bereich der Streckschwingung von OH-Gruppen (3400-3300 $\left.\mathrm{cm}^{-1}\right)$, der Valenzschwingung von Carbonylgruppen $\left(1720 \mathrm{~cm}^{-1}\right)$, des Stretchings der $\mathrm{C}=\mathrm{C}$-Bindungen von Aromaten $\left(1640 \mathrm{~cm}^{-1}\right)$, der $\mathrm{OH}$-Deformations- und der $\mathrm{C}$-O-Streckschwingung phenolischer $\mathrm{OH}-$ Gruppen $\left(1400 \mathrm{~cm}^{-1}\right)$ sowie der C-O-Valenz- und OH-Deformationsschwingung von Carboxylgruppen $\left(1200 \mathrm{~cm}^{-1}\right)$ zeigen sich derart große Übereinstimmungen, dass hier von der Bildung sehr ähnlicher Verbindungen ausgegangen werden kann.

Auch der Vergleich des IR-Spektrums des 2,6-Dimethylphenols mit den Spektren von Fulvosäuren bestätigt die, in den vorangegangenen Betrachtungen gefundenen weitreichenden Übereinstimmungen in Verteilung und Lage der Absorptionsbanden. Neben den mit höherer Intensität auftretenden Banden von Methylgruppen, die auf die chemische Zusammensetzung des Phenols zurückzuführen sind, liegen die Gemeinsamkeiten der Spektren wiederum im Bereich der Streckschwingung der OH-Gruppen (3500-3300 $\mathrm{cm}^{-1}$ ), der Valenzschwingung der $\mathrm{C}=\mathrm{O}$-Gruppen $\left(1720 \mathrm{~cm}^{-1}\right)$ und der Streckschwingung der $\mathrm{C}=\mathrm{C}$-Bindungen aromatischer Strukturen $\left(1640 \mathrm{~cm}^{-1}\right)$. 


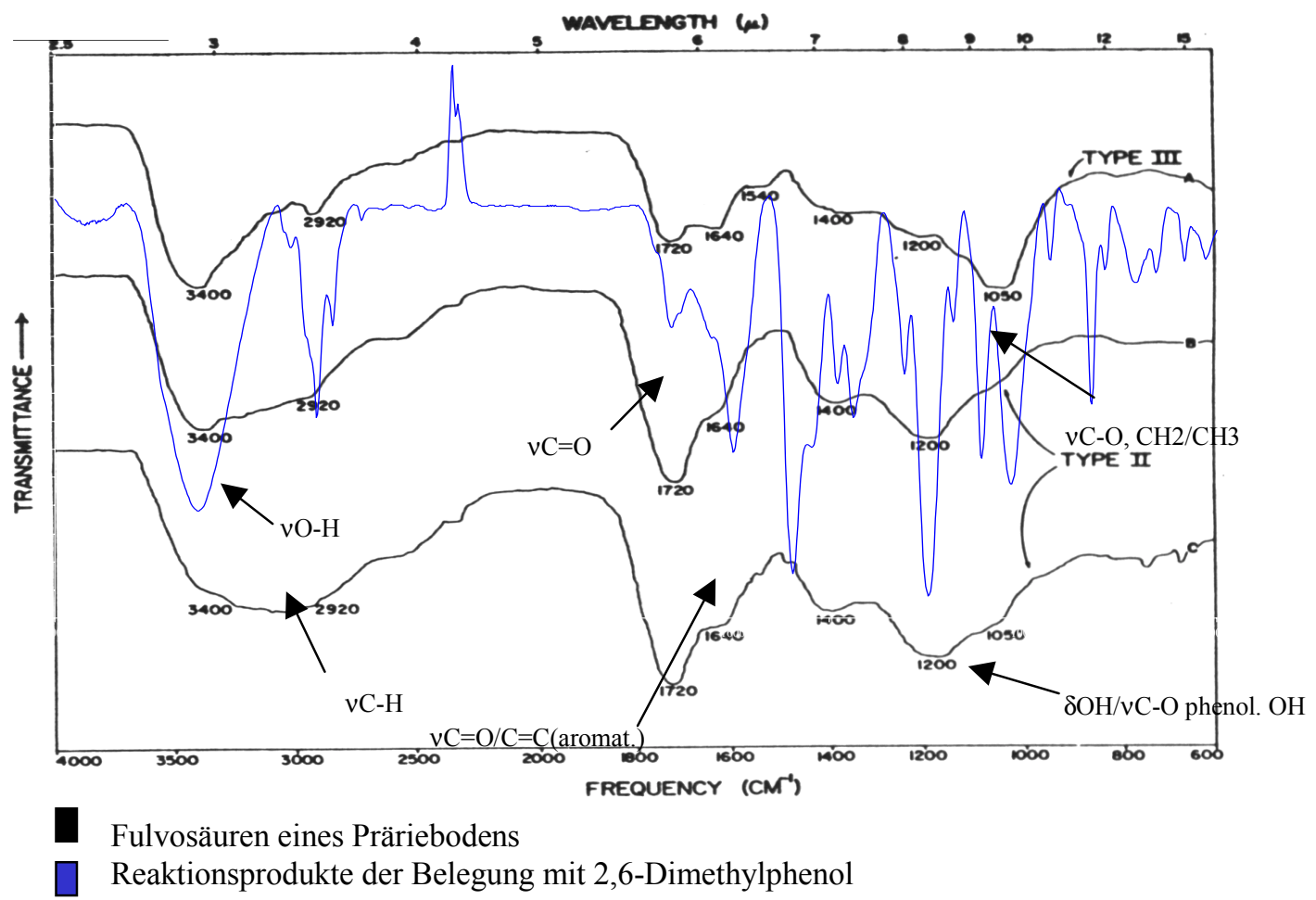

Abbildung 102: Vergleich der IR-Spektren bodenbürtiger Fulvosäuren (Stevenson 1994, verändert) mit den gewonnenen Reaktionsprodukten des 2,6-Dimethylphenols.

Weiterhin sind gemeinsame Bandenlagen im Bereich der Deformationsschwingung von $\mathrm{OH}$ und der C-O-Streckschwingung phenolischer OH-Gruppen $\left(1400 \mathrm{~cm}^{-1}\right)$ sowie bei Valenzschwingung von C-O bzw. OH-Deformationsschwingung der Carboxylgruppen $\left(1200 \mathrm{~cm}^{-1}\right)$ zu beobachten.

\subsubsection{Diskussion der IR-spektroskopischen Untersuchungen}

Als Ergebnisse der durchgeführten Untersuchungen ist festzuhalten, dass die Reaktion der Phenole an den Tonmineraloberflächen zur Entstehung mehrerer Verbindungen, die stark voneinander unterscheidende Eigenschaften aufweisen, geführt hat.

Beim mit Brenzkatechin belegten Montmorillonit wird dieses durch die Reaktion an der Tonmineraloberfläche teilweise zu einem (o-Benzo-)Chinon oxidiert. Dies lässt sich durch die im Spektrum zu beobachtenden, gegenüber dem Referenzspektrum neu auftretenden Absorptionsbanden von $\mathrm{C}=\mathrm{O}$-Bindungen nachweisen. Eine Kondensation zu Verbindungen größerer Molekülmasse kann durch die Zunahme des Polymerisationsgrades angenommen werden. Dies zeigt sich durch den Anstieg des Gehaltes an H-Brücken durch Assoziation aromatischer Ringstrukturen und durch Zunahme von Substituierungen der Aromate. Daneben ist die Entstehung weiterer chemischer Bindungstypen zu beobachten. Es konnten Carboxylgruppen als auch aliphatische Alkohole und Alkene identifiziert werden. Außerdem konnte nachgewiesen werden, dass die Reaktion von Brenzkatechin an der Oberfläche von Tonmineralen zur Bildung mehrerer Reaktionsprodukte geführt hat. Es wurden funktionelle Gruppen detektiert, die 
nicht nur auf die Oxidation des Phenols schließen lassen. Darüber hinaus wurde durch das Auftreten von Carboxylgruppen Carbonsäuren nachgewiesen. Zusätzlich belegen die Absorptionsbanden für aliphatische Methyl- bzw. Methylengruppen eine Aufspaltung der aromatischen Strukturen der Ausgangssubstanz.

$\mathrm{Zu}$ einer weitgehenden Übereinstimmung führten auch die Resultate der Analyse der extrahierten Überzüge des mit Pyrogallol belegten Tons. Es konnten Carbonylbindungen, sowohl von Carbonsäuren als auch von Ketonen identifiziert werden. Des weiteren waren Anteile aliphatischer Strukturen zu beobachten. Das IR-Spektrum weist im Bereich $1.725 \mathrm{~cm}^{-1}$ eine Bande für $\mathrm{C}=\mathrm{O}$-Doppelbindungen auf. Das Fehlen der charakteristischen Banden für aromatische Strukturen (Wellenzahlenbereich 1600-1480 $\mathrm{cm}^{-1}$, Obertonschwingungen und Kombinationsbanden zwischen 2000 und $1800 \mathrm{~cm}^{-1}$ ) deutet darauf hin, dass durch die Reaktion an der Tonmineraloberfläche zum Aufbrechen der aromatischen Strukturen bis hin zur Entstehung von Alkanen und Alkenen geführt hat. Gemeinsam mit Auftreten von $\mathrm{C}=\mathrm{O}$-Doppelbindungen ist daraus abzuleiten, dass Keto-Verbindungen gebildet worden sind.

Die abiotische Reaktion des 2,6-Dimethylphenols an der Oberfläche von Tonmineralen führte zur Bildung verschiedener Bindungstypen bzw. Verbindungen. Neben Absorptionsbanden, die das Auftreten von $\mathrm{C}=\mathrm{O}$-Doppelbindungen nachweisen, sind auch beim 2,6Dimethylphenol Bande zu beobachten, die durch das Erscheinen charakteristischer Signale von Methyl- bzw. Methylengruppen und Alkenen deutliche Hinweise darauf geben, dass durch die Reaktivität des Tons eine Aufspaltung des aromatischen Rings des Phenols eingetreten ist. Die Lage der Absorptionsbanden der Carbonylgruppen lässt zudem erkennen, dass neben Carbonsäuren auch aliphatische Aldehyde durch Ringspaltung und Oxidation des 2,6Dimethylphenols entstanden sind.

Die Untersuchungen der neben den Phenolen zusätzlich mit Phenanthren versetzten Proben mittels FT-IR-Spektroskopie führte leider zu keinen verwertbaren Ergebnissen.

Die reaktiven Prozesse an der Oberfläche von Tonmineralen führen zu Verbindungen, die den in Böden vorkommenden Fulvosäuren sehr ähnlich sein müssen. Durch radikalische, oxidative Vorgänge werden die, als Modellsubstanzen für Huminsäurebausteine verstandenen, Phenole in ihrer Struktur chemisch so verändert, dass ihre IR-Spektren große Gemeinsamkeiten mit denen natürlicher organischer Substanz aufweisen. Dies ist einerseits ein weiteres Indiz dafür, dass die Huminstoffbildung in Böden auch durch die oxidative Polymerisation von Phenolen beruht, da auch Fulvo- und Huminsäuren hochmolekulare Verbindungen darstellen. Weiterhin kann somit gezeigt werden, dass die, bisher mikrobieller bzw. enzymatischer Aktivität zugerechneten Prozesse der Huminstoffgenese auch auf abiotischem Wege durch physikochemische Reaktionen an mineralischen Oberflächen, hier Tonmineralen ablaufen können. Somit kann die derzeit geltende Theorie der Huminstoffgenese, die die Bildung von Huminstoffen u.a. durch Polykondensation von Phenolen allein aufgrund mikrobieller Aktivität (STEVENSON 1994) erklärt, um einen abiotischen Reaktionspfad erweitert werden. 


\section{$4.7 \quad{ }^{13}$ C-NMR Spektroskopie}

Die NMR-Spektroskopie wird vorwiegend bei der Strukturaufklärung von organischen Molekülen eingesetzt. Insbesondere die ${ }^{13} \mathrm{C}$-NMR-Spektroskopie ist hier von sehr großer Bedeutung (HERZOG \& MESSERSCHMIDT 1995) und erfährt auch als Analysemethode in der Strukturaufklärung organischer Substanz und Huminstoffe von Böden eine zunehmende Beachtung (DEC \& BOLLAG 2000, FRÜND \& LÜDEMANN 1994, GOLCHIN 1994, GRANDJEAN \& LAZLO 1995, HAYES 1991, HEMMINGA \& BUURMAN 1997, KINCHESH et al. 1995, KÖGEL-KNABNER 1997, PRESTON 1996, RANDALL et al. 1997).

\subsubsection{Theorie der ${ }^{13}$ C-NMR-Spektroskopie}

Atomkerne verfügen über einen Kern- oder Eigendrehimpuls P (Kernspin). Dieser Drehimpuls ist gequantelt, er kann nicht beliebige Werte annehmen:

$$
P=\sqrt{I(I+1) \eta}
$$

h: Planck'sches Wirkungsquantum

I: Drehimpuls- oder Kernspinquantenzahl (Kernspin)

Mit dem Drehimpuls P ist ein magnetisches Moment $\mu$ verknüpft. Die beiden vektoriellen Größen sind proportional zueinander:

$$
\mu=\gamma P
$$

$\gamma$ : Proportionalitätskonstante

Die Proportionalitätskonstante ist eine charakteristische Konstante für jedes Isotop der verschiedenen Elemente und wird als gyromagnetisches Verhältnis bezeichnet. Von ihr hängt die Nachweisempfindlichkeit eines Kernes ab. Das magnetische Moment ergibt sich aus den vorangegangenen Gleichungen:

$$
\mu=\gamma \sqrt{I(I+1)} \eta
$$

Hieraus ergibt sich ,dass Atome mit gerader Massen- oder Kernladungszahl, wie das Kohlenstoff-Isotop ${ }^{12} \mathrm{C}$ und das Sauerstoff-Isotop ${ }^{16} \mathrm{O}$, kein magnetisches Kernmoment haben und somit die wichtigsten Bausteine organischer Verbindungen durch die NMR-Spektroskopie nicht nachweisbar sind. Atome mit ungeraden Kernladungszahlen hingegen, wie ${ }^{1} \mathrm{H},{ }^{13} \mathrm{C}$ und ${ }^{17} \mathrm{O}$ weisen einen Kernspin auf. Die Bewegung elektrischer Ladung verursacht ein magnetisches Feld, verläuft der Strom in einem geschlossenen Kreis, so ist ein magnetisches Dipolmoment mit ihm verknüpft. Bei der Kernresonanz-Spektroskopie werden Übergänge zwischen verschiedenen Energieniveaus induziert, in dem Kerne mit einer elektromagnetischen Welle der richtigen Frequenz $v_{1}$ bestrahlt werden. Die magnetische Komponente der Welle tritt dabei mit den Kerndipolen in Wechselwirkung:

$$
h v_{1}=\Delta E,
$$

wobei $\Delta E=\eta \eta B_{0}$.

$\mathrm{B}_{0}$ : magnetische Flussdichte $[\mathrm{T}]$ 
Ein Magnetfeld B übt auf einen Körper mit einem magnetischen Dipol $\mu$ ein Drehmoment D aus. Der Körper ist versucht, sich parallel zur Magnetfeldrichtung zu stellen:

$$
D=\mu \times B
$$

Ist der Dipol selbst in einer Drehbewegung, so resultiert eine Präzession um die Richtung des Magnetfeldes, diese Bewegung des Kernspins wird Larmor-Präzession genannt. Ist nun die eingestrahlte Frequenz identisch, d.h. in Resonanz mit der Larmor-Frequenz des Kernspins, so können Energieübergänge induziert werden.

Übergänge vom energieärmeren ins energiereichere Niveau entsprechen einer Energieabsorption, umgekehrt eine Energieemission. Jeder Übergang ist mit der Umkehr der KernspinOrientierung verbunden (FRIEBOLIN 1992). Wegen des Besetzungsüberschusses im energieärmeren Niveau überwiegt die Energieabsorption aus dem eingestrahlten Zusatzfeld. Dies wird als Signal gemessen, wobei die Intensität des Signals dem Besetzungsunterschied proportional, somit auch proportional der Gesamtzahl der Spins in der Probe, also der Konzentration ist.

Resonanzbedingung:

$$
v_{L}=v_{1}=\left|\frac{\gamma}{2 \pi}\right| B_{0}
$$

vL: Larmor-Frequenz

Alle Kerne in einer Probe werden gleichzeitig durch einen Hochfrequenz-Impuls angeregt. Zum Nachweis der ${ }^{13} \mathrm{C}$-Resonanzen wird mit einer Resonanz-Frequenz von 50,3 $\mathrm{MHz}$ gepulst. Die Pulslänge beträgt einige $\mu$ s. Nach Abschaltung des Impulses ist der Magnetisierungsvektor aus der Gleichgewichtslage ausgelenkt. Durch Relaxation kehrt das Spinsystem in den Gleichgewichtszustand zurück. Am Empfänger aufgenommene Abklingkurve ist die Abnahme der Quermagnetisierung und wird als freier Induktionsabfall (engl. Free Induction Decay - FID) bezeichnet. Bei einer Probe von Kernen mit verschiedenen Resonanzfrequenzen überlagern sich die Abklingkurven und interferieren. Diese Interferogramme enthalten den gesamten Informationsgehalt eines Spektrums, also Resonanzfrequenzen und Intensitäten. Diese Spektren können jedoch nicht direkt analysiert werden, da der Mensch gewohnt ist ein Spektrum in der Frequenzdomäne zu interpretieren und nicht in der Zeitdomäne. Durch die Fourier-Transformation werden beide Spektren ineinander verrechnet:

$$
f(\omega)=f(t) e^{i \omega t} d t
$$

$\mathrm{f}(\mathrm{t})$ : Spektrum der Zeitdomäne

$\mathrm{f}(\omega)$ : Spektrum der Frequenzdomäne

Die Intensität eines einzelnen FIDs ist meistens so schwach, dass die Signale nach der Fourier-Transformation im Verhältnis zum Rauschen sehr klein sind. Dies gilt vor allem bei Kernen mit geringer Empfindlichkeit und geringer natürlicher Häufigkeit wie den ${ }^{13} \mathrm{C}$-Kernen sowie schwach konzentrierte Proben. Deswegen werden die FIDs vieler Impulse im Rechner aufsummiert, akkumuliert und erst danach transformiert. Bei der Akkumulation mittelt sich das statistisch auftretende, elektronische Rauschen zum Teil heraus, während der Beitrag der 
Signale stets positiv ist und sich deshalb aufsummiert. Insbesondere bei geringer Probenkonzentration werden die FIDs, über einen Zeitraum von mehreren Tagen können dies mehrere hunderttausend sein, akkumuliert (FRIEBOLIN 1992).

Die Resonanzen des beobachteten Kerns werden in charakteristischer Weise von seiner Umgebung beeinflusst. Bei der Untersuchung von Molekülen sind Kerne immer von Elektronen und anderen Atomen umgeben. Als Folge ist das am Kernort wirkende Magnetfeld $\mathrm{B}_{\text {eff }}$ stets kleiner als das angelegte Feld $\mathrm{B}_{0}$, somit sind die Kerne abgeschirmt. Dieser Effekt ist klein, aber messbar:

$$
B_{\text {eff }}=B_{0}-\sigma B_{0}=(1-\sigma) B_{0}
$$

$\sigma$ : Abschirmungskonstante (dimensionslos)

Wird für schwere Atome höher, da die Abschirmung mit zunehmender Elektronenzahl größer wird. Die Resonanzbedingung geht somit über in:

$$
v_{1}=\frac{\gamma}{2 \pi}(1-\sigma) B_{0}
$$

Die Resonanzfrequenz $v_{1}$ ist zum Abschirmungsterm (1- $\sigma$ ) proportional, daraus sich, dass chemisch nicht-äquivalente Kerne unterschiedlich abgeschirmt sind und im Spektrum getrennte Resonanzsignale liefern.

In der NMR-Spektroskopie gibt es keinen absoluten Maßstab, da Resonanzfrequenz und magnetische Flussdichte entsprechend der Resonanzbedingung miteinander verknüpft sind. Aus diesem Grunde verwendet man einen relativen Maßstab, man misst FrequenzDifferenzen $\Delta v$ zwischen Resonanzsignalen der Probe und der einer Referenzsubstanz. Als Referenz (innerer Standard) wird zumeist Tetramethylsilan (TMS) verwendet. Seine Eignung rührt von seinen idealen spektroskopischen als auch chemischen Eigenschaften her. Es verfügt über zwölf äquivalente, stark abgeschirmte Protonen, man erhält ein scharfes, von den meisten anderen Resonanzsignalen deutlich getrenntes Signal am rechten Spektrenrand. Zudem ist TMS chemisch inert, magnetisch isotrop und assoziiert nicht. Die Referenzierung der ${ }^{13} \mathrm{C}$-Spektren mit TMS funktioniert aufgrund der geringen Signalstärke nicht mit TMS, sondern zumeist über das Lösemittelsignal.

Aus der Resonanz-Frequenz der Probe vSubstanz und der Resonanz-Frequenz vReferenz kann man eine dimensionslose Größe berechnen, die chemische Verschiebung $\delta$.

$$
\delta=\frac{v_{\text {Subs } \tan z}-v_{\mathrm{Re} \text { ferenz } z}}{v_{\operatorname{Re} \text { feren } z}} \times 10^{6}
$$

Bei dieser werden alle Linienlagen durch Fourier-Transformation berechnet und die Frequenz-Differenzen direkt ausgegeben. Der Faktor $10^{6}$ wird zur Vereinfachung der Zahlenwerte verwendet und deshalb in parts per million (ppm) angegeben (KALINOWSKI et al. 1984). Durch Unterdrückung der C-H-Kopplung durch Protonen-Breitband-Entkopplung können ${ }^{13} \mathrm{C}$-Signale um bis zu 200\% an Intensität zunehmen. Dieser Effekt wird als NuclearOverhauser-Enhancement (NOE) bezeichnet. Dieser Effekt beruht auf der Sättigung der ${ }^{13} \mathrm{C}$ ${ }^{1}$ H-Resonanzen durch den Protonen-Entkoppler. Dadurch verändert sich das Besetzungsver- 
hältnis der Energieniveaus von ${ }^{13} \mathrm{C}$-Kernen. Der energieärmere Zustand wird stärker besetzt. Die beeinflussten Kerne können daher mehr Energie aufnehmen und abgeben, das kommt einer Intensitätssteigerung im resultierenden NMR-Spektrum gleich. Nur protonentragende ${ }^{13} \mathrm{C}$ Kerne erfahren so eine Intensitätssteigerung (PRESTON 1996). Der Effekt wirkt nur über eine C-H-Bindung oder bei extremer Nähe zweier Kerne aufgrund der stereochemischen Verhältnisse. Der Effekt ist der Grund für ein im Vergleich zum Signal der Carbonylgruppe höheres C-H-Signal:

$$
\left.\left.\mathrm{NOE} E_{\left(-\mathrm{CH}_{2-}\right)}\right\rangle \mathrm{NOE} E_{\left.-\mathrm{CH}_{3}-\right)}\right\rangle N O E_{(=\mathrm{C}-\mathrm{H}-)} .
$$

Aufgrund der geringen natürlichen Häufigkeit des ${ }^{13} \mathrm{C}$-Isotops, der Anteil beträgt lediglich $1,1 \%$, sind höhere Probenmengen notwendig. Für ein gutes Spektrum sind so $50 \mathrm{mg}$ Substanz vonnöten. Deuterierte Lösemittel mit natürlichem ${ }^{13} \mathrm{C}$-Gehalt kommen hier zum Einsatz, da die Bindung zwischen ${ }^{13} \mathrm{C}$ und Deuterium keinen Nuclear-Overhauser-Enhancement-Effekt hervorruft und somit eine geringere Intensität des Lösemittelsignals auftritt.

\subsubsection{Chemische Verschiebungen in $\operatorname{der}^{13} \mathrm{C}$-NMR-Spektroskopie}

${ }^{13} \mathrm{C}$-Resonanzen erstrecken sich über einen Verschiebungsbereich von 200 ppm. Die Spektren enthalten im Normalfall für jedes ${ }^{13} \mathrm{C}$-Atom ein getrenntes Signal. Es gilt, diese Signale den richtigen ${ }^{13} \mathrm{C}$-Atomen des Moleküls zuzuordnen. Die ${ }^{13} \mathrm{C}$-Verschiebungsbereiche organischer Verbindungen zeigen, dass viele kohlenstoffhaltige funktionelle Gruppen in ${ }^{13} \mathrm{C}-\mathrm{NMR}$ Spektren an typischen Verschiebungswerten erkennbar sind (Abbildung 103).

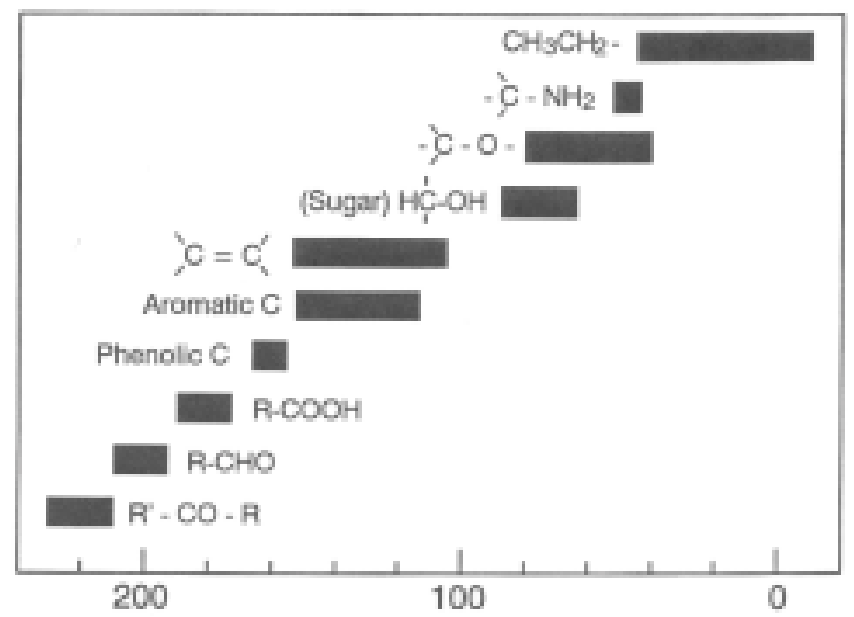

Abbildung 103: 13C-Verschiebungen funktioneller Gruppen (STEVENSON 1994).

Die chemischen Verschiebungen von ${ }^{13} \mathrm{C}$-Atomen unverzweigter linearer und verzweigter Kohlenwasserstoffe liegen im Bereich von 60 ppm, die Verschiebung nimmt in der Reihenfolge $\mathrm{CH}_{3}<\mathrm{CH}_{2}<\mathrm{CH}<\mathrm{C}$ zu. Die Signale von Ether-Methoxy-Bindungen treten zwischen 55 und 62 ppm, von Ester-Methoxy-Gruppen bei 52 ppm auf. Von Bedeutung für eine Interpretation ist, dass Methyl-Signale bei $20 \mathrm{ppm}$ auch $\mathrm{zu}$ Methylgruppen an $\mathrm{CX}$ - oder $\mathrm{C}=\mathrm{C}$ Doppelbindungen gehören können. Die chemischen Verschiebungen für Alkene liegen im Be- 
reich von 75 bis 175 ppm, bei Einbeziehung zyklischer und polyzyklischer Verbindungen (Aromate). Alkine absorbieren im Bereich von 60 bis 95 ppm.

Im Gegensatz zu ${ }^{1} \mathrm{H}$-NMR-Spektren unterscheiden ${ }^{13} \mathrm{C}$-Verschiebungen im allgemeinen nicht zwischen Aromaten/Heteroaromaten einerseits und Alkanen andererseits (BREITMAIER 1992). Die Signale von aromatischen Kohlenwasserstoffen sind in einem engen Bereich zwischen 100 und 150 ppm zu beobachten. Einen großen Einfluss auf die Verschiebungswerte spielen die Substituenten am aromatischen System. So bewirken Substituenten am Benzolring wie z. B. eine als Elektronendonator fungierende $\mathrm{OCH}_{3}$-Gruppe die Abschirmung des $\mathrm{C}$ Atoms, daraus resultieren kleinere Verschiebungswerte. Elektronenakzeptoren als Substituenten hingegen bewirken eine umgekehrte Polarisierung der Doppelbindungen und damit größere Verschiebungen. Ein Elektronendonator am Benzolring entschirmt das $\alpha$-Atom, in orthound para-Stellung (oder vergleichbaren Positionen in Heteroaromaten) schirmt er dagegen ab. Elektronenakzeptoren zeigen in ortho- und para-Positionen größere Verschiebungen. Die meta-Stellung eines Substituenten hingegen weist weder bei Elektronendonatoren bzw. Elektronenakzeptoren keinen signifikanten Einfluss aus (BREITMAIER 1992).

Die Signale für Carbonyl-Verbindungen liegen im Bereich von 150 bis 220 ppm. KetoCarbonyl-Gruppen erscheinen als Singuletts zwischen 190 und 220 ppm, Signale von Aldehyden zwischen 185 und 205 ppm überlappen mit dem Keto-Carbonyl-Bereich, sie unterscheiden sich jedoch durch CH-Dublett-Signale auf. Die Carbonyl-C-Atome von Chinonen treten zwischen 180 und 190 ppm auf. Die $\mathrm{C}=\mathrm{O}$-Bindungen der Carbonsäuren und ihrer Derivate sind zwischen 160 und $180 \mathrm{ppm}$ zu beobachten. Zu beachten ist allerdings, dass die ${ }^{13} \mathrm{C}$ Signale von Phenoxy-C-Atomen, Carbonaten, Harnstoffen, Oximen und anderen Iminen um 160 ppm liegen (BREITMAIER 1992, VIRKKI 1993). Eine Übersicht über die chemischen Verschiebungen von ${ }^{13} \mathrm{C}$-Kernen organischer Verbindungen bietet Tabelle 16.

Tabelle 16: ${ }^{13} \mathrm{C}-$ Verschiebungen von Fulvo- und Huminsäuren (nach STEVENSON 1994).

\begin{tabular}{|c|l|}
\hline Verschiebung [ppm] & \multicolumn{1}{c|}{ Zuordnung } \\
\hline $0-50$ & unsubstituiertes, aliphatisches C (Alkane, Fettsäuren) \\
\hline $50-60$ & Methoxyl-C \\
\hline $60-110$ & aliphatisches C-O (Kohlehydrate) \\
\hline $110-160$ & $\begin{array}{l}\text { aromatisches C, aus unsubstituiertem und alkyl-substituiertem aromatischen C } \\
(110-150 \text { ppm) und phenolischem C (150-160 ppm), Alkene }\end{array}$ \\
\hline $160-190$ & C von Carboxylgruppen und COO \\
\hline $190-200$ & C=O von Ketogruppen von Estern und Amiden \\
\hline
\end{tabular}




\subsubsection{Präparation der Proben für die ${ }^{13} \mathrm{C}$-NMR-Spektroskopie}

Die gewonnenen extrahierten Lösungen der Reaktionsprodukte wurden mittels Rotationsverdampfer (Büchi Rotavapor RE 111, Vakuumpumpe Laboport KNF-Neuberger) unter Vakuum ( $40^{\circ} \mathrm{C}, 250$ mbar) bis zum Feststoff eingeengt. Von diesem Rückstand wurden 20-50 mg in $500 \mu 1$ deuteriertem Dimethylsulfoxid (DMSO-d6, Cambridge Isotope Labs) aufgenommen, in Standard-NMR-Probenröhrchen (Wilmad) gefüllt und diese verschlossen. Bis zur Messung wurden die Proben lichtgeschützt und gekühlt $\left(6^{\circ} \mathrm{C}\right)$ aufbewahrt.

Die ${ }^{13} \mathrm{C}$-NMR spektroskopischen Analysen wurden am Max-Planck-Institut für Experimentelle Medizin Göttingen an einem NMR-Spektrometer (Bruker Aspect 3000, 50/200 MHz) vorgenommen.

\subsection{4 ${ }^{13} \mathrm{C}$-NMR spektroskopische Untersuchungen}

Bisher wurden Untersuchungen zur Grenzflächenreaktivität durch abiotische Katalyse von Huminsäurebausteinen an Tonmineralen durchgeführt. Dazu wurden Montmorillonite $\left(\mathrm{Na}^{+}-\right.$ und $\mathrm{Ca}^{2+}$-Montmorillonit) durch Sublimation mit drei Phenolen, Brenzkatechin, Pyrogallol und 2,6-Dimethylphenol als Ligninbausteine belegt. Nach Extraktion der entstandenen, farbigen Reaktionsprodukte wurden diese bisher mit chromatographischen (HPLC) und spektroskopischen (FTIR, ${ }^{13}$ C-NMR) Methoden untersucht, um die Stoffe qualitativ zu analysieren. Die Auswertung der NMR-Spektren erfolgte durch Nutzung von Spektren-Datenbanken SDBS, Universität Potsdam), „Increment 99“ (PC-Programm zur Berechnung von chemischen Verschiebungen) und Fachliteratur (EISENER 1983, FRIEBOLIN 1992, GRANDJEAN \& LAZLO 1995, HERZOG \& MESSERSCHMIDT 1995, KALINOWSKI et al. 1984, KNICKER \& LÜDEMANN 1995, KÖGEL-KNABNER 1997a,b, THENG et al. 1998, VIRKKI 1993, WAIS 1995, WANG \& HUANG 2000).

\subsubsection{Brenzkatechin}

Das NMR-Spektrum für das Brenzkatechin zeigt neben dem Septett für das Lösungsmittel Dimethylsulfoxid (DMSO) drei weitere Peaks bei 115,7 ppm, 119,3 ppm und 145,3 ppm. Diese chemischen Verschiebungen liegen im Verschiebungsbereich der Aromaten. Zuordnungen der chemischen Verschiebungen sind Tabelle $17 \mathrm{zu}$ entnehmen.

Tabelle 17: Brenzkatechin, Verschiebungswerte und Zuordnungen (Quelle: SDBS).

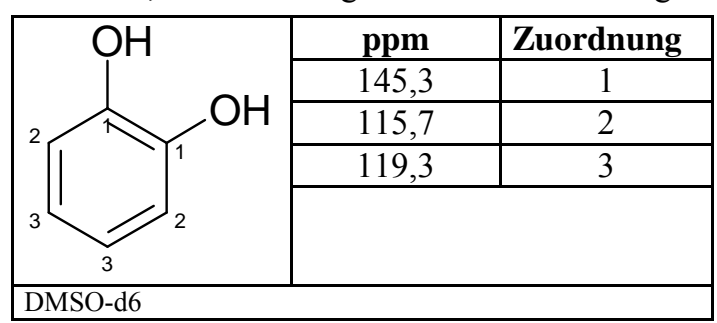

Der Peak bei 145,3 ppm (Abbildung 104) repräsentiert die C-OH-Bindung im aromatischen System (C-Atome 1), die chemische Verschiebung von 119,3 ppm gibt die Bindung $\mathrm{C}=\mathrm{C}$ der 
C-Atome (3) an. Die Verschiebung von 115,7 ppm charakterisiert die C-Bindung der Atome (2).

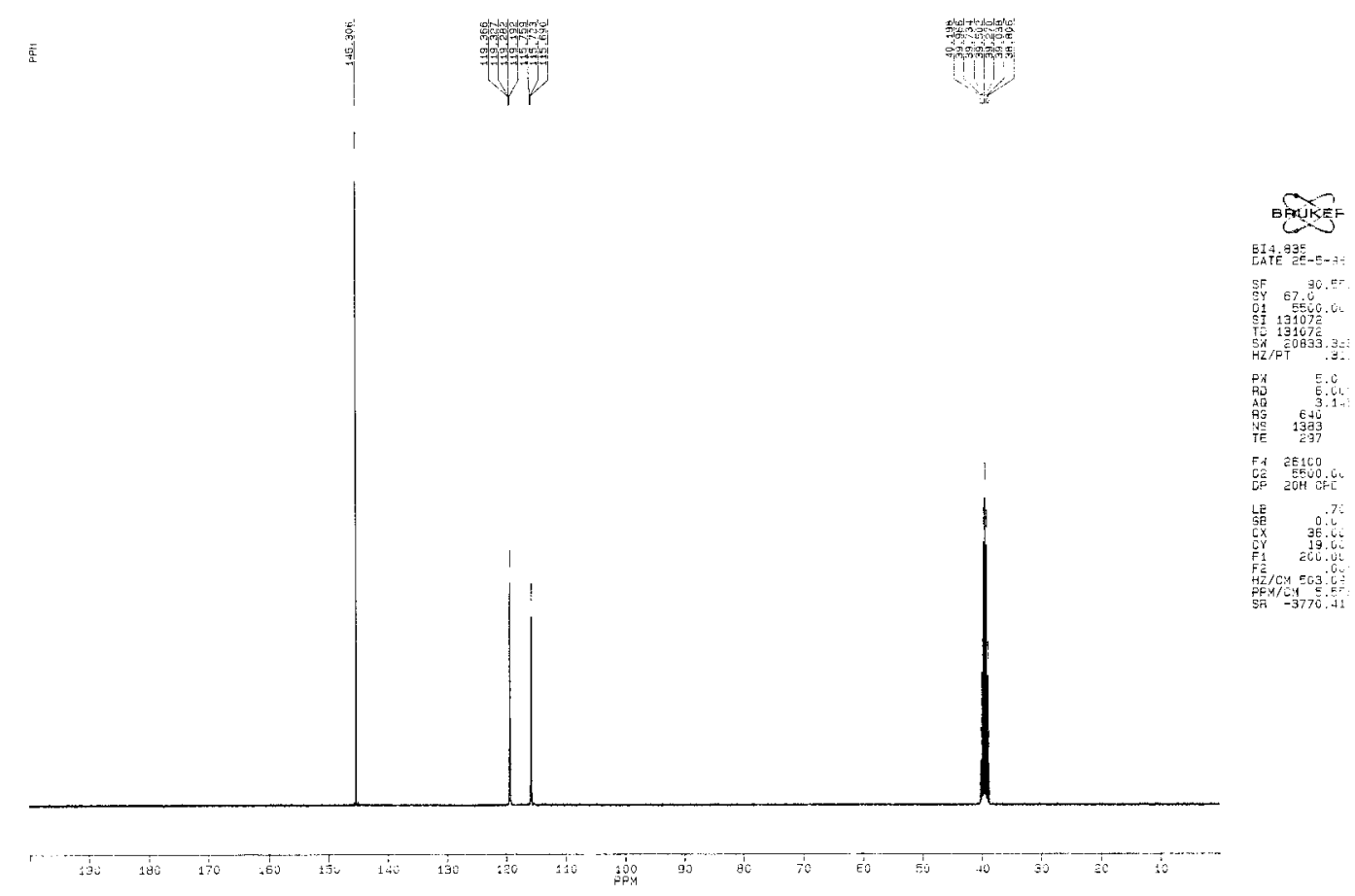

Abbildung 104: ${ }^{13} \mathrm{C}-\mathrm{NMR}$ Spektrum von Brenzkatechin.

\subsubsection{Pyrogallol}

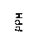

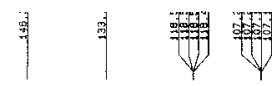

in
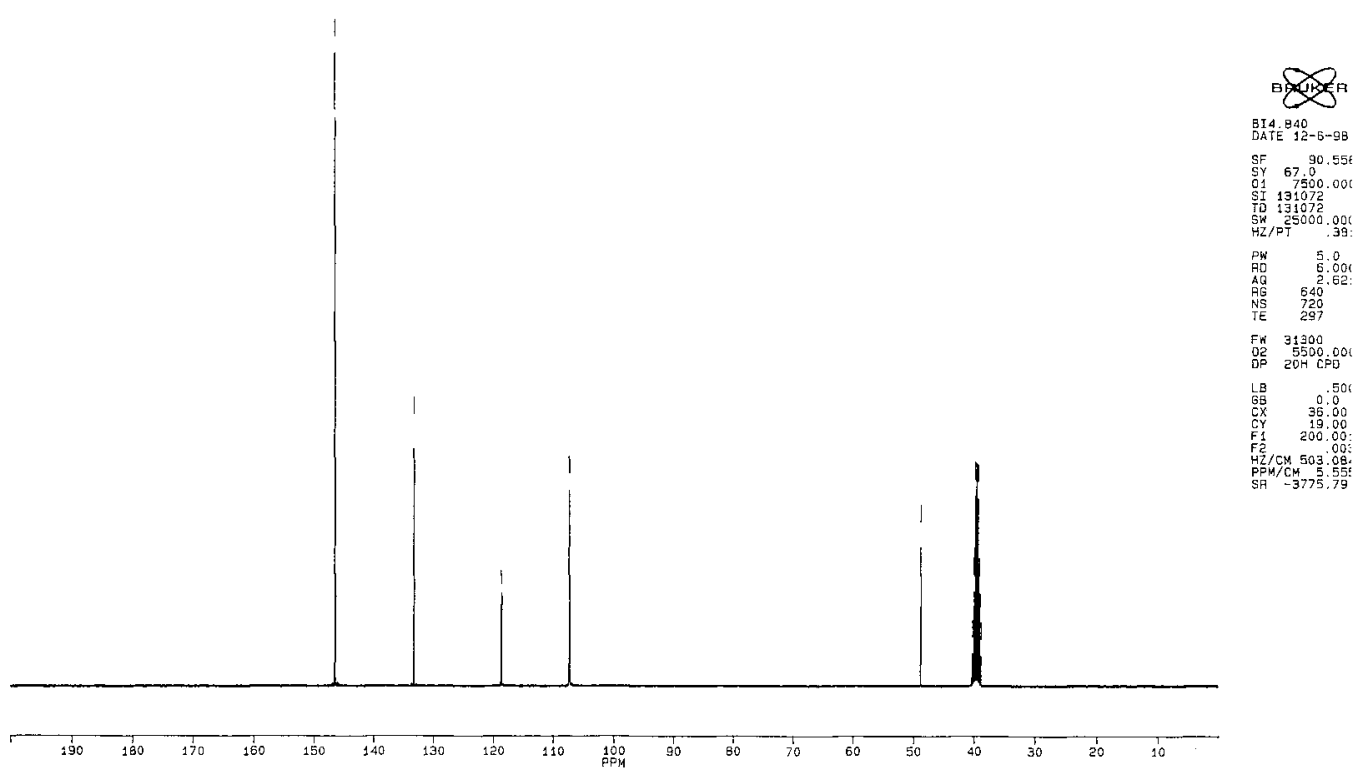

Abbildung 105: ${ }^{13} \mathrm{C}-\mathrm{NMR}$ Spektrum von Pyrogallol. 
Die chemische Verschiebung von 146,3 ppm (Abbildung 105) charakterisiert die C-OHBindung der Atome (C1). Die Bindung der zweiten OH-Gruppe wird durch den Peak bei 133,2 ppm repräsentiert. Der Verschiebungswert bei 118,5 ppm beschreibt die beiden CBindungen (4) und der Peak bei 107,2 ppm die $\mathrm{C}=\mathrm{C}$-Bindung (3).

Tabelle 18: Pyrogallol, Verschiebungswerte und Zuordnungen (Quelle: SDBS).

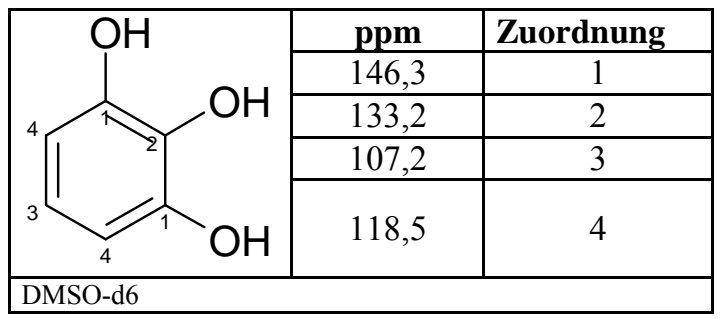

\subsubsection{2,6-Dimethylphenol}

Die chemische Verschiebung bei 153,3 ppm beschreibt die C-OH-Bindung am aromatischen Ring des Phenols (Abbildung 106). Der Wert von 124,3 ppm charakterisiert die beiden Methylgruppen um die OH-Gruppe in ortho-Position (3). Die Verschiebung bei 128,3 ppm beschreibt die beiden C-Atome (C-C-Bindungen, 2). Der Peak bei 119,2 ppm charakterisiert das C-Atom (4) des aromatischen Rings, dass dem C-Atom mit der OH-Gruppe gegenüberliegt. Die Verschiebung bei 16,6 ppm beschreibt die beiden Methylgruppen $\left(-\mathrm{CH}_{3}, 5\right)$ des Phenols (Tabelle 19). Der Verschiebungswert von 48,7 ppm stammt vom Methanol, mit dem die Substanzen von den Tonmineralen extrahiert wurden.

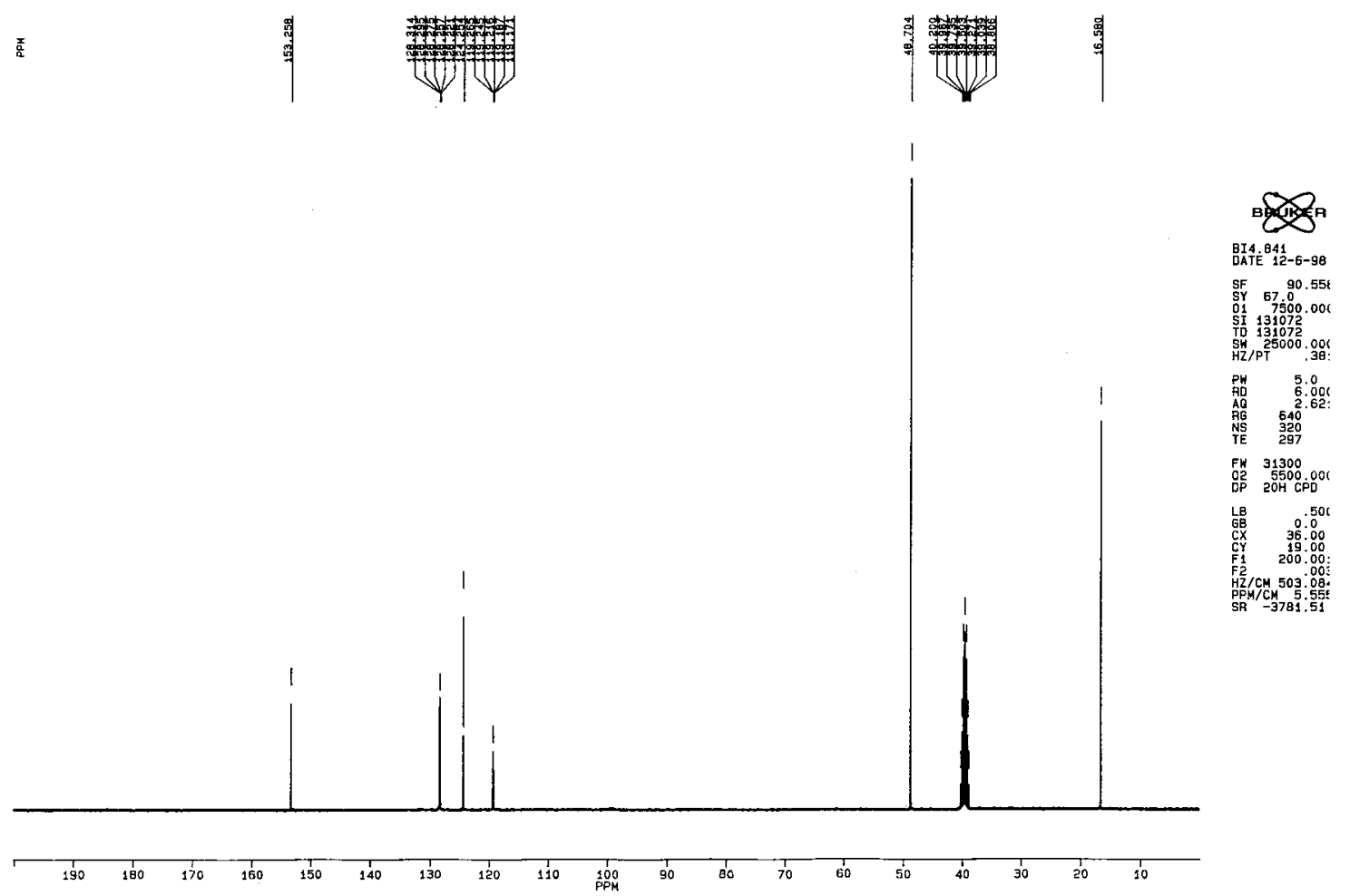

Abbildung 106: ${ }^{13} \mathrm{C}-\mathrm{NMR}$ Spektrum von 2,6-Dimethylphenol. 
Tabelle 19: 2,6-Dimethylphenol, Verschiebungswerte und Zuordnungen (Quelle: SDBS).

\begin{tabular}{|c|c|c|}
\hline \multirow{6}{*}{$\mathrm{H}_{3} \stackrel{5}{\mathrm{C}}$} & \multirow{2}{*}{$\begin{array}{c}\text { ppm } \\
153,3 \\
\end{array}$} & \multirow{2}{*}{$\begin{array}{c}\text { Zuordnung } \\
1\end{array}$} \\
\hline & & \\
\hline & 128,3 & 2 \\
\hline & 124,3 & 3 \\
\hline & 119,2 & 4 \\
\hline & 16,6 & 5 \\
\hline
\end{tabular}

\subsubsection{Phenanthren}

Das Phenanthren ist als ein Vertreter der Polyzyklischen Aromatischen Kohlenwasserstoffe (PAK) lediglich aus drei Benzolringen aufgebaut. Somit weist es keine Substituenten auf, daher sind nur Informationen über die C-C- bzw. C-H-Bindungen im NMR-Spektrum enthalten. Die chemischen Verschiebungen sind aus Tabelle $20 \mathrm{zu}$ entnehmen.

Tabelle 20: Zuordnung der chemischen Verschiebungen für Phenanthren (Quelle: SDBS).

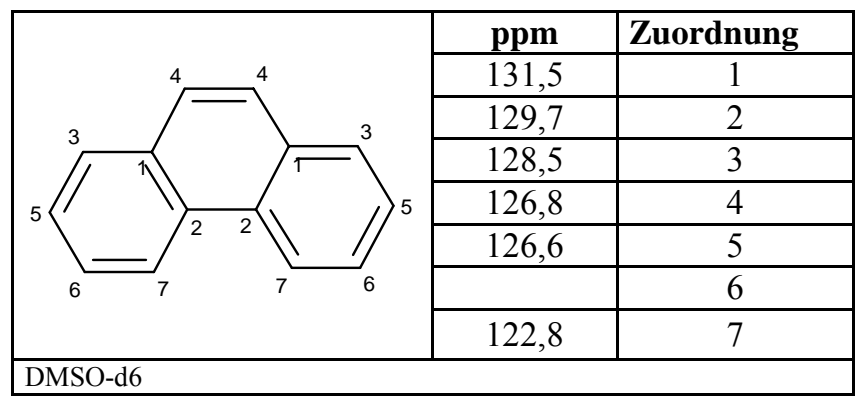

$\frac{1}{2}$
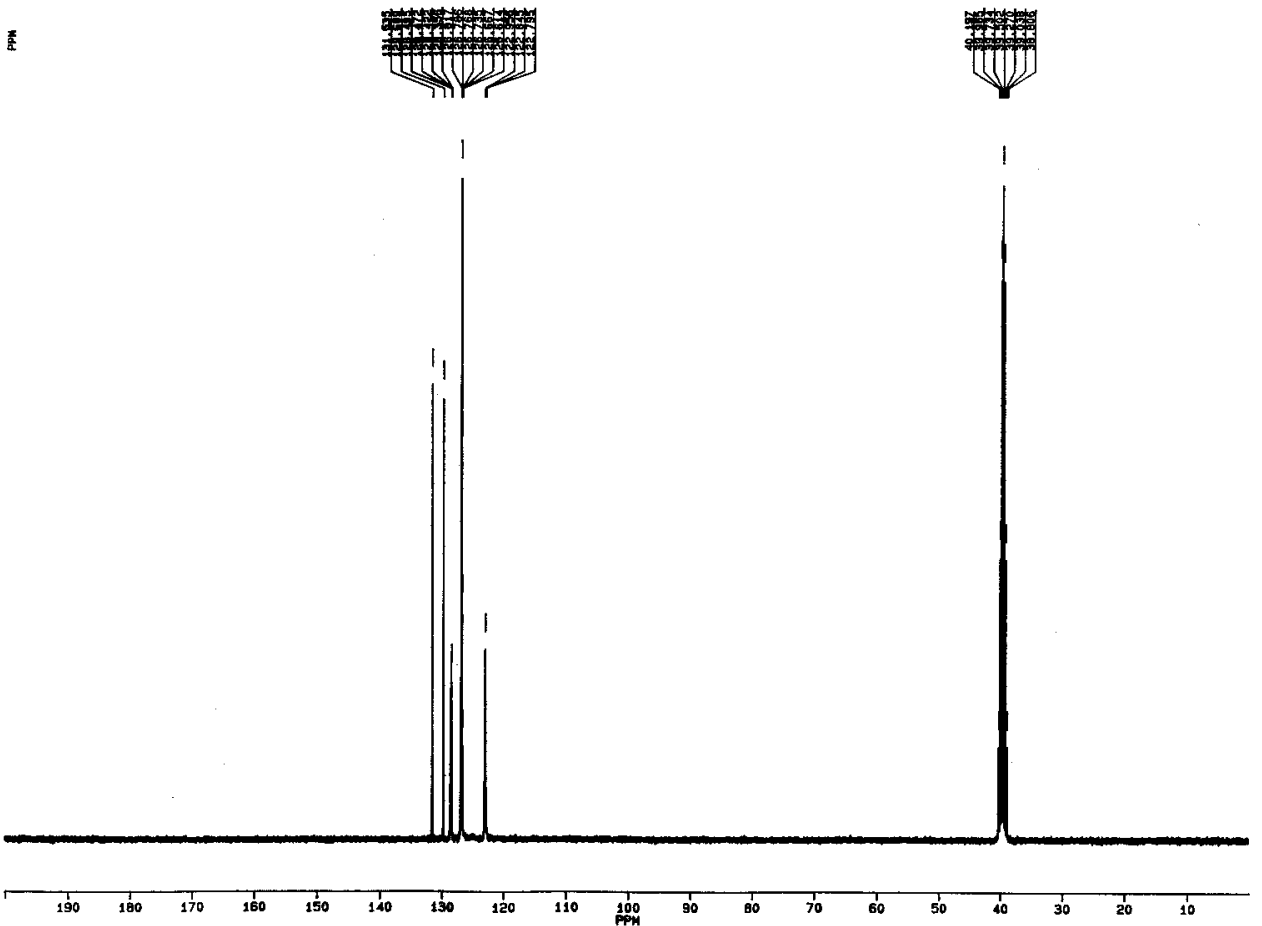


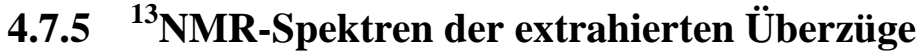

\subsubsection{Brenzkatechin}

Das ${ }^{13} \mathrm{C}$-NMR-Spektrum der extrahierten Überzüge der Belegung des Montmorillonits mit Brenzkatechin weist neben den Signalen für das Phenol eine Vielzahl von chemischen Verschiebungen auf, die eine Reaktion des auf die Tonmineraloberfläche sublimierten Phenols nachweisen.

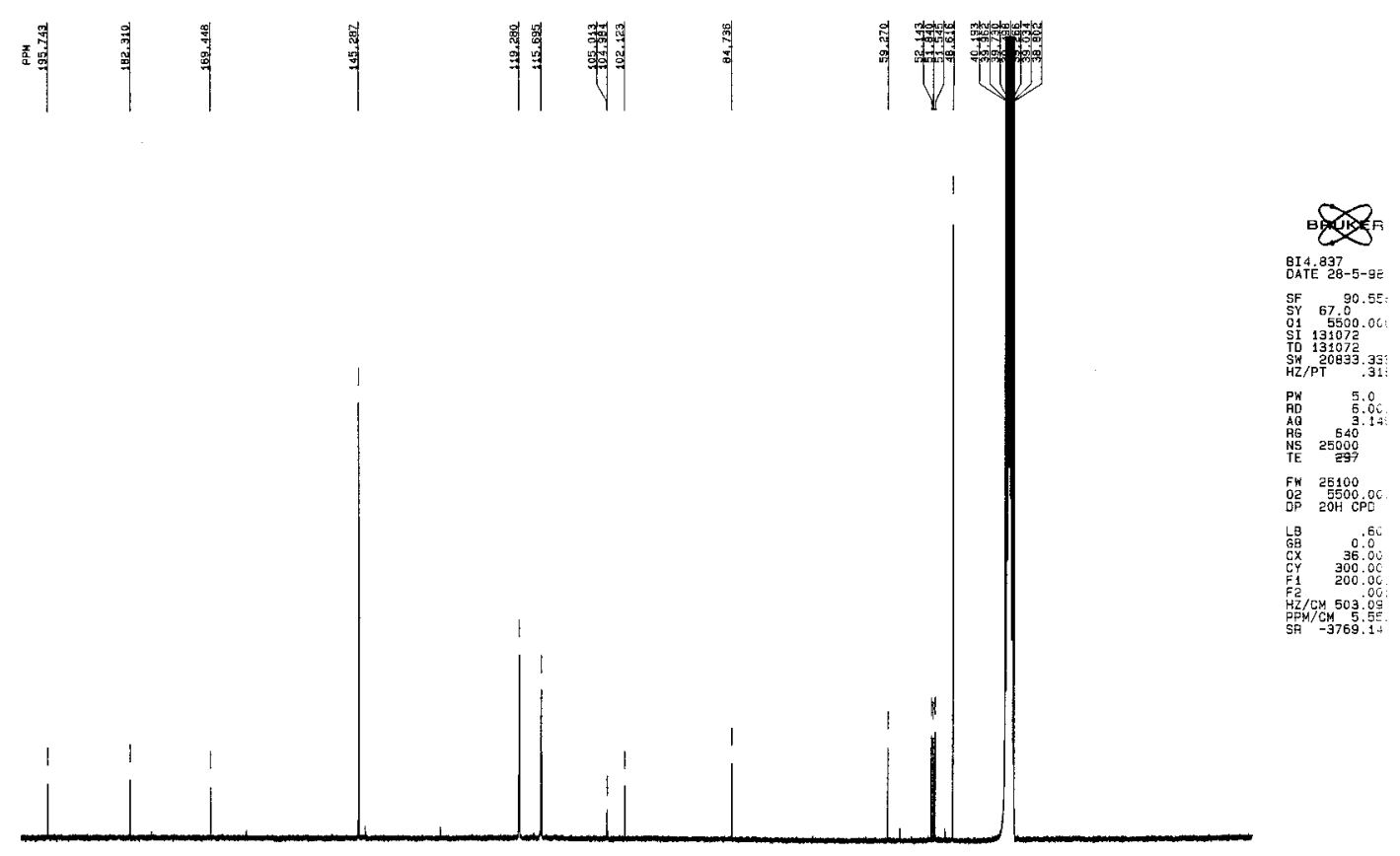

Abbildung 108: ${ }^{13} \mathrm{C}-\mathrm{NMR}$ Spektrum extrahierter Überzüge des mit Brenzkatechin belegtem Na-Montmorillonits.

Eine Übersicht über die Verteilung der Peaks und ihre Zuordnung gibt Tabelle 21. Die auffälligsten Signale sind die hohen, im linken Bereich des Spektrums erkennbaren Verschiebungswerte. Chemische Verschiebungen oberhalb von 160 ppm bis 220 ppm sind charakteristisch für Carbonylverbindungen. Das Signal bei 195,7 ppm liegt im Zuordnungsbereich der Aldehyde und Ketone (KALINOWSKI et al. 1984). Der auftretenden Peak bei 183,5 ppm als auch das Signal bei 170,2 ppm beschreiben ebenfalls das Auftreten von C=O-Bindungen von Carboxylgruppen (KÖGEL-KNABNER 1997). Der höhere Wert liegt im Verschiebungsbereich der Chinone (180 ppm) während das niedrigere Signal die Existenz von Carboxylgruppen von Carbonsäuren nachweist (FRIEBOLIN 1992). Die Werte bei 145,8 ppm, 120,4 ppm und 116,4 ppm beschreiben hier die zuvor zugeordneten Peaks des Phenols (Abbildung 104). Daneben weist das Spektrum in diesem Verschiebungsbereich weitere zusätzliche Peaks auf, die der Bindung von Sauerstoff am aromatischen System zuzuordnen sind (133,2 und 144,9 ppm). Dadurch kann eine Veränderung des Aromats nachgewiesen werden. Im Bereich der Gerüststruktur der Aromaten zwischen 100 und 130 ppm sind weitere Signale zu beobachten. Die Peaks bei 118,1 ppm, 105,4 ppm und 102,8 ppm resultieren aus einer Veränderung der 
Substituierung des aromatischen Rings. Besondere Beachtung gilt hier Signal bei 118 ppm, der einem protonierten aromatischen C-Atom zugeordnet wird (KÖGEL-KNABNER 1997). Diese Werte werden in der Regel neben substituierten C-Atomen eines Rings beobachtet und sind unsubstituierten C-Atomen des aromatischen Systems zuzuordnen. Der zu erkennende Peak bei 85,8 ppm beschreibt das C-Atom in $\mathrm{R}_{3}-\mathrm{C}$-O-Bindungen, kann aber auch Dreifachbindungen von C-Atomen ((Cyclo-)Alkine) zugeordnet werden (BREITMAIER 1992). Das Auftreten dieses Signals kann am plausibelsten als Bestandteil eines Substituenten erklärt werden, während eine Interpretation in Richtung Umsetzung des aromatischen Gerüsts zu (Cyclo-)-Alkinen durch die bereits zugeordneten Verschiebungswerte als sehr unwahrscheinlich erscheint. Zwischen 50 und 80 ppm treten Signale für C-Atome in $\mathrm{O}-\mathrm{CH}_{2}$ bzw. O- $\mathrm{CH}_{3}-$ Bindungen auf. Diese Region ist charakteristisch für Verbindungen, die über Hydroxylgruppen verbundene C-Atome enthalten (WERSHAW et al. 1996). Das gemeinsame Auftreten von O-Alkylbindungen und Verschiebungswerten im Bereich von $145 \mathrm{ppm}$ deutet auf die Substituierung des aromatischen Rings mit $\mathrm{OCH}_{3}$-Gruppen hin.

Tabelle 21: Übersicht der chemischen Verschiebungen.

\begin{tabular}{|c|c|c|c|c|}
\hline \multicolumn{2}{|c|}{$\begin{array}{l}\text { Standard } \\
\text { Brenzkatechin } \\
\end{array}$} & \multirow[t]{20}{*}{$\begin{array}{l}\text { Montmorillonit } \\
\text { belegt mit }\end{array}$} & \multicolumn{2}{|c|}{ Brenzkatechin } \\
\hline ppm & Zuordnung & & ppm & Zuordnung \\
\hline 39,5 & DMSO-d6 & & 39,5 & DMSO-d6 \\
\hline \multirow[t]{8}{*}{49,5} & Methanol & & 49,5 & Methanol \\
\hline & & & 51,8 & $\mathrm{O}-\mathrm{CH}_{3}$ \\
\hline & & & 52,4 & $\mathrm{O}-\mathrm{CH}_{3}$ \\
\hline & & & 53 & $\mathrm{O}-\mathrm{CH}_{3}$ \\
\hline & & & 59,8 & $\mathrm{O}-\mathrm{CH}_{3}$ \\
\hline & & & 85,8 & $\mathrm{C}-\mathrm{OH}$ \\
\hline & & & 102,8 & Alkene \\
\hline & & & 105,4 & Alkene \\
\hline \multirow[t]{2}{*}{115,7} & Ring (C3,C6) & & 116,4 & Ring (C3,C6) \\
\hline & & & 118,1 & Ring \\
\hline \multirow[t]{3}{*}{119,3} & Ring (C4,C5) & & 120,4 & Ring (C4,C5) \\
\hline & & & 133,2 & Ring-OH \\
\hline & & & 144,9 & Ring-OH \\
\hline \multirow[t]{4}{*}{145,3} & Ring-OH $(\mathrm{C} 1, \mathrm{C} 2)$ & & 145,8 & Ring-OH $(\mathrm{C} 1, \mathrm{C} 2)$ \\
\hline & & & 170,2 & Carboxyl, -COOH \\
\hline & & & 183,5 & Chinon, $-\mathrm{C}=\mathrm{O}$ \\
\hline & & & 196,9 & Aldehyd, $-\mathrm{C}=\mathrm{O}$ \\
\hline
\end{tabular}

Da im Verschiebungsbereich von 0 bis 50 ppm keine Peaks zu beobachten sind, kann davon ausgegangen werden, dass es durch die Reaktion des Brenzkatechins am Tonmineral nicht zu dessen Aufspaltung in kurzkettige Kohlenwasserstoffbruchstücke gekommen ist. Vielmehr ist davon auszugehen, dass Teile des Brenzkatechins durch die Aktivität des Montmorillonits oxidiert wurden. Das entsprechende Oxidationsprodukt des Brenzkatechins ist das orthoBenzochinon. Durch die Reaktion kann es zur Methylierung einer bzw. beider OH-Gruppen des Phenols gekommen sein. Dafür spricht das Auftreten von Peaks im Bereich der ${\mathrm{O}-\mathrm{CH}_{3}-}^{-}$ Gruppen und das gleichzeitige Auftreten der Verschiebung um 145 ppm. Das Spektrum weist deutliche Übereinstimmungen mit dem Spektrum des 1,2-Cyclohexandion auf (Abbildung 
109).

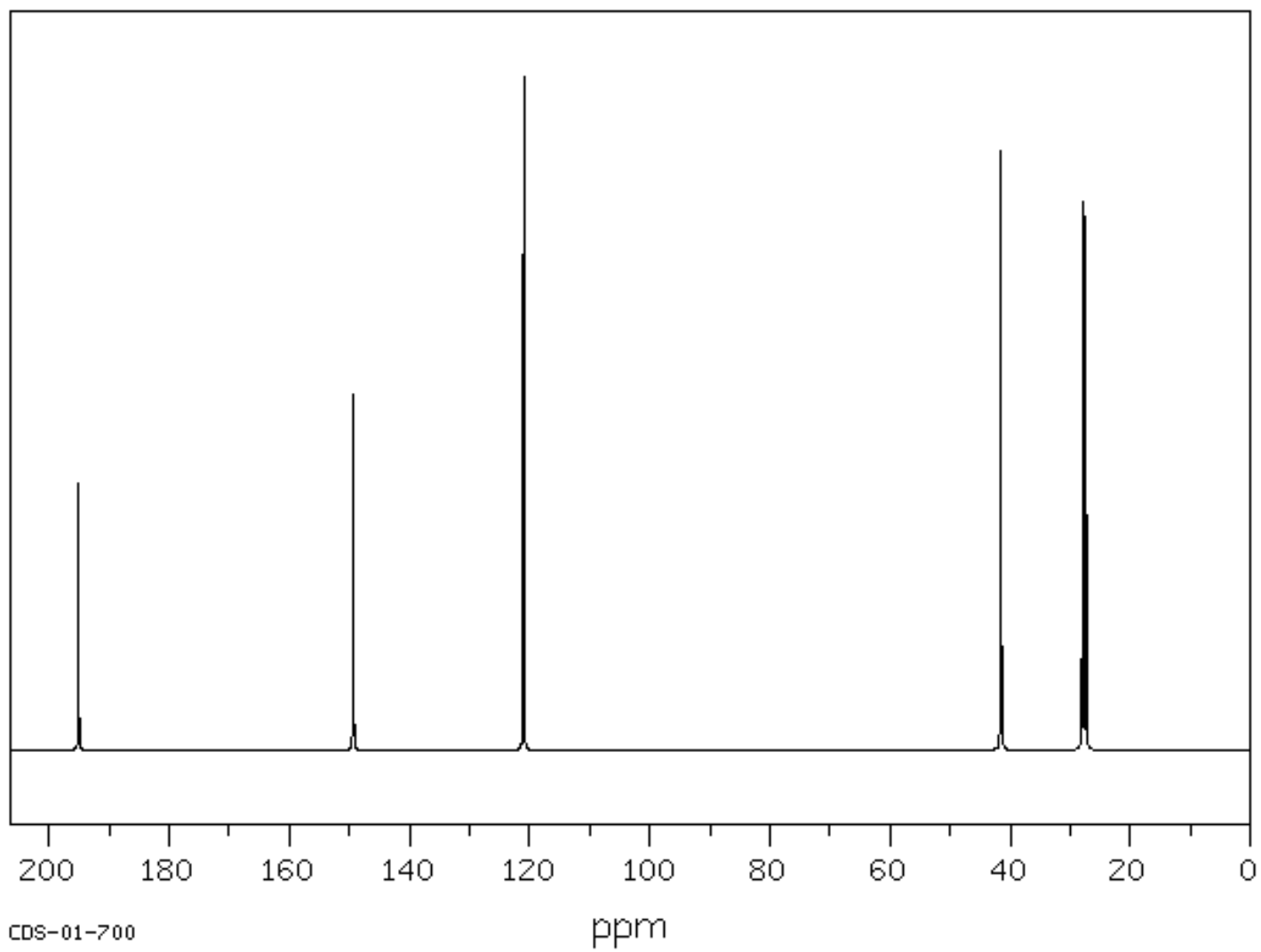

Abbildung 109: ${ }^{13}$ C-NMR Spektrum des 1,2-Cyclohexandions (Quelle: SDBS).

Tabelle 22: Übersicht der chemischen Verschiebungen und ihre Zuordnungen (Quelle: SDBS).

\begin{tabular}{|c|c|c|}
\hline \multirow[t]{7}{*}{$\mathrm{OH}$} & ppm & Zuordnung \\
\hline & 194,72 & 1 \\
\hline & 147,91 & 2 \\
\hline & 118,9 & 3 \\
\hline & 37,49 & 4 \\
\hline & 23,61 & $5^{*}$ \\
\hline & 23,05 & 6* \\
\hline
\end{tabular}

Dafür, dass diese Verbindung entstanden ist spricht einerseits die Struktur der Ausgangssubstanz, die Abstammung vom Brenzkatechin ist gut $\mathrm{zu}$ erkennen. Andererseits ist die $\mathrm{C}=\mathrm{O}$ Doppelbindung am aromatischen Ring aus der Oxidation des Phenols entstanden. Die Grundstruktur der Chinone, den Oxidationsprodukten der Phenole, sind aus einem Kohlenstoffring mit sechs C-Atomen und mindestens einem Sauerstoff-Atom aufgebaut, welches mit dem Kohlenstoff über eine Doppelbindung verbunden ist. Durch die Vielzahl der auftretenden Peaks kann aber auch einerseits davon ausgegangen werden, dass es zur Bildung mehrerer, verschiedener Reaktionsprodukte gekommen ist. Das es zur Bildung von Cyclohexandion gekommen ist wird zusätzlich durch die Auswertung der infrarotspektroskopischen Untersuchungen gestützt (Kapitel 4.6). Es besteht die Möglichkeit, dass aus der Ausgangssubstanz verschiedene Chinone gebildet worden sein können. Neben dem bereits dargestellten 1,2Cyclohexandion ist die Bildung von o-Benzochinon anzunehmen (Abbildung 110). 
<smiles>O=C1C=CC=CC1=O</smiles>

Abbildung 110: 1,2-Benzochinon.

Andererseits besteht die Möglichkeit der Entstehung höhermolekularer Verbindungen durch eine oxidative Polymerisation der phenolischen bzw. chinoiden Komponenten (Kapitel 2.2). Somit ist davon auszugehen, dass in einem ersten Schritt der Oxidation des Brenzkatechins in zu einem Chinon bzw. zu einer chinoiden Verbindung erfolgt ist. Anschließend tritt eine Verkettung der Ringstrukturen miteinander ein. Diese Zusammenlagerung von Oxidationsprodukten führt über die Bildung von Dimeren, Oligomeren schließlich zur Entstehung von Polymeren (Kapitel 2.2.5). Die chemische Transformation des Brenzkatechins zu organischen Substanzen mit anderen Substituenten wurde mittels FTIRSpektroskopie bereits nachgewiesen. Die Reaktion von Brenzkatechin an der Tonmineraloberfläche führte zur Bildung von Verbindungen mit Carboxyl-, Chinon- und Aldehydgruppen (Tabelle 21). Zusätzlich treten Peaks von O-Alkyl- und Alkenkohlenstoffverbindungen auf. Durch das Auftreten dieser Peaks, insbesondere durch den das Chinon repräsentierende Signal konnte die chemische Umwandlung des Brenzkatechins zum entsprechenden Oxidationsprodukt durch die Aktivität des Tonminerals nachgewiesen werden.

\subsubsection{Pyrogallol}

Das ${ }^{13}$ C-NMR-Spektrum der extrahierten Überzüge des mit Pyrogallol sublimierten Montmorillonits weist neben den bereits zugeordneten Verschiebungswerten des Phenols eine Vielzahl neuer Peaks auf. Auch hier treten neue Verschiebungen im Bereich von 150 bis 220 ppm auf, die den $\mathrm{C}=\mathrm{O}$-Doppelbindungen (Carbonylverbindungen) zuzuordnen sind. Im Bereich der aromatischen Strukturen (100-130 ppm) und der O- $\mathrm{CH}_{3}-$ Gruppen (50-90 ppm) treten ebenfalls zusätzliche Verschiebungen auf (Tabelle 23). Im Unterschied zum ${ }^{13} \mathrm{C}-\mathrm{NMR}$ Spektrum der Reaktionsprodukte des Brenzkatechins (Abbildung 104) sind im Verschiebungsbereich zwischen 0 und 40 ppm, der die $\mathrm{CH}_{2}$ - und $\mathrm{CH}_{3}$-Gruppen repräsentiert, zusätzlich auftretende Peaks zu beobachten. Das Auftreten von Signalen in diesem Verschiebungsbereich kann als Hinweis für die Aufspaltung eines Teils des aromatischen Gerüsts des Pyrogallols in kurzkettige, nichtaromatische Verbindungen interpretiert werden. Andererseits können in diesem Verschiebungsbereich auftretende Signale kombiniert mit den im Aromatenbereich auftretenden Verschiebungen als Veränderungen in der Substituierung des Aromaten interpretiert werden. 
Tabelle 23: Übersicht der chemischen Verschiebungen.

\begin{tabular}{|c|c|c|c|c|}
\hline \multicolumn{2}{|c|}{$\begin{array}{l}\text { Standard } \\
\text { Pyrogallol }\end{array}$} & \multirow[t]{22}{*}{$\begin{array}{l}\text { Montmorillonit } \\
\text { belegt mit }\end{array}$} & \multicolumn{2}{|c|}{ Pyrogallol } \\
\hline \multirow[t]{6}{*}{ ppm } & Zuordnung & & ppm & Zuordnung \\
\hline & & & 16,9 & $\mathrm{C}-\mathrm{CH}_{3}$ \\
\hline & & & 29,4 & $\mathrm{C}-\mathrm{CH}_{3}$ \\
\hline & & & 31,7 & $\mathrm{C}-\mathrm{CH}_{2}$ \\
\hline & & & 34,3 & $\mathrm{C}-\mathrm{CH}_{2}$ \\
\hline & & & 36,4 & $\mathrm{C}-\mathrm{CH}_{2}$ \\
\hline 39,5 & DMSO-d6 & & 39,5 & DMSO-d6 \\
\hline \multirow[t]{5}{*}{49,5} & Methanol & & 49,5 & Methanol \\
\hline & & & 53,1 & $\mathrm{O}-\mathrm{CH}_{3}$ \\
\hline & & & 55,2 & $\mathrm{O}-\mathrm{CH}_{3}$ \\
\hline & & & 61,5 & $\mathrm{O}-\mathrm{CH}_{3}$ \\
\hline & & & 70,2 & O-Alkyl (HC-OH) \\
\hline 107,2 & Ring (C4) & & 106,1 & Ring (C4) \\
\hline \multirow[t]{3}{*}{118,5} & Ring (C3) & & 116,1 & Ring (C3) \\
\hline & & & 124,8 & aromat. Ring \\
\hline & & & 126,3 & aromat. Ring \\
\hline 133,2 & Ring-OH (C2) & & 132,1 & Ring-OH (C2) \\
\hline \multirow[t]{4}{*}{146,3} & Ring-OH (C1) & & 144,9 & Ring-OH (C1) \\
\hline & & & 152,5 & Ring-O \\
\hline & & & 158,6 & Ring-O \\
\hline & & & 162,5 & $\mathrm{C}=\mathrm{O}$ \\
\hline
\end{tabular}

Die erkennbaren Peaks im Bereich der Aromaten (100 bis $150 \mathrm{ppm})$ und das Signal der $\mathrm{C}=\mathrm{O}-$ Doppelbindung bei 162,5 ppm zeigen jedoch, dass das aromatische System des Phenols eine Veränderung in Richtung Oxidation und Polymerisation hin zu höhermolekularen Verbindungen erfahren hat (WANG \& HUANG 2000): es treten neben den der Ausgangssubstanz Pyrogallol eindeutig zuzuordnenden Verschiebungen für den aromatischen Ring bei 106,1 und 116,1 ppm sowie für die C-O-Bindung bei 132,1 ppm und 144,9 ppm neue Peaks auf, die auf die Entstehung ausgehend vom Pyrogallol neu gebildeter Reaktionsprodukte hindeuten. Durch die nachweisbaren O-Alkylverbindungen zwischen 53,1 ppm, 55,2 ppm und 61,5 ppm kann eine zumindest teilweise Methylierung der ursprünglichen Substituenten angenommen werden. An diese Substituenten können auch weitere $\mathrm{CH}_{2}-$ bzw. $\mathrm{CH}_{3}$-Gruppen (31,7 ppm, 34,3 ppm, 36,4 ppm bzw. 16,9, ppm, 29,4 ppm), zum Teil auch über eine HC-OH-Bindung bei 70,2 ppm gebunden sein. Eine Bindung dieser Substituenten an den aromatischen Ring kann über die Sauerstoffatome erfolgen, deren Bindung durch die beiden neuen Signale bei 152,5 ppm und 158,6 ppm beschrieben sind. Das gleichzeitige Auftreten von Signalen bei 106 ppm und 153 ppm ist charakteristisch für Sinapyl-Monomere (WERSHAW et al. 1996). Dies würde die Bildung von Alkoxylgruppen am Benzolring nachweisen, die, je höher ihre Anzahl ein umso größeres Oxidationspotential aufweisen (KIRK \& FARELL 1987). Die Oxidation des Phenols lässt sich anhand des Signals für eine Carbonylbindung bei 162,5 ppm feststellen. Es kann, analog zur Transformation des Brenzkatechins, von einer partiellen Bildung chinoider Reaktionsprodukte des Pyrogallols ausgegangen werden. 


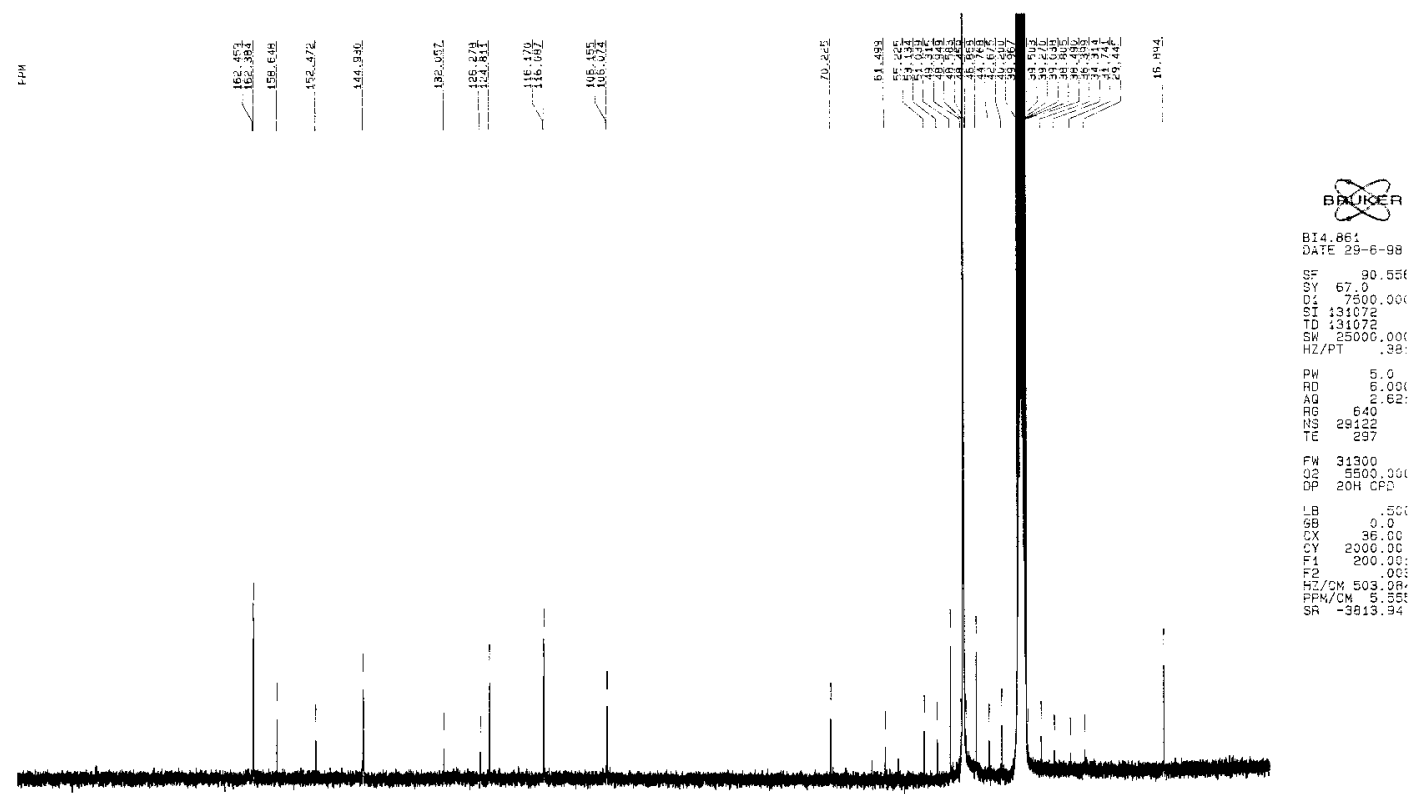

Abbildung 111: ${ }^{13} \mathrm{C}-\mathrm{NMR}$ Spektrum der extrahierten Überzüge des mit Pyrogallol belegten Ca-Montmorillonits.

Eine Besonderheit weist die Untersuchung der Reaktionsprodukte des Pyrogallols im Vergleich zu den beiden anderen Phenolen auf. Die bereits im Kapitel 4.1 beschriebene Problematik der nur unvollständigen Extraktion der Reaktionsprodukte des auf die Tonmineraloberfläche sublimierten Pyrogallols lässt erkennen, dass lediglich ein kleiner Teil der entstandenen Reaktionsprodukte erfasst und der Analytik zugeführt werden konnte. Die Färbung der extrahierten Lösung wies nur eine blass gelbliche Färbung auf, der Großteil der stark braun gefärbten Reaktionsprodukte verblieb auf dem Ton und ließ sich trotz der Verwendung aggressiver Extraktionsmethoden nicht ablösen. Aufgrund der sehr starken Bindung der entstandenen Reaktionsprodukte an die mineralische Komponente ist auf deren chemisorptive Festlegung zu schließen. Dieses Gesamtverhalten der entstandenen, nicht extrahierbaren Reaktionsprodukte weist somit auf Parallelen mit bodenbürtiger, nicht-extrahierbarer organischer Substanzen hin (BIRKEL \& NIEMEYER 1998b).

\subsubsection{2,6-Dimethylphenol}

Das ${ }^{13}$ C-NMR-Spektrum der durch Sublimation von 2,6-Dimethylphenol auf Na- bzw. CaMontmorillonit gewonnenen dunkel orangeroten Überzüge weist neben den wiederzufindenden chemischen Verschiebungen für das Phenol eine Vielzahl von neu auftretenden Signalen auf (Tabelle 24). Die chemischen Verschiebungen reichen über den gesamten Verschiebungsbereich von den Alkanen über die $\mathrm{C}=\mathrm{C}$ - und $\mathrm{C}$-OH-Bindungen der Aromate bis hin zu den $\mathrm{C}=\mathrm{O}$-Bindungen. 
Tabelle 24: Übersicht der chemischen Verschiebungen.

\begin{tabular}{|c|c|c|c|c|}
\hline \multicolumn{2}{|c|}{$\begin{array}{l}\text { Standard } \\
\text { 2,6-Dimethylphenol }\end{array}$} & \multirow[t]{25}{*}{$\begin{array}{l}\text { Montmorillonit } \\
\text { belegt mit }\end{array}$} & \multicolumn{2}{|c|}{ 2,6-Dimethylphenol } \\
\hline ppm & Zuordnung & & ppm & Zuordnung \\
\hline \multirow[t]{4}{*}{16,5} & $\mathrm{C}-\mathrm{CH}_{3}$ & & 16,6 & $\mathrm{C}-\mathrm{CH}_{3}$ \\
\hline & & & 24 & $\mathrm{C}-\mathrm{CH}_{3}$ \\
\hline & & & 27 & $\mathrm{C}-\mathrm{CH}_{3}$ \\
\hline & & & 35,2 & $\mathrm{C}-\mathrm{CH}_{2}$ \\
\hline 39,5 & DMSO-d6 & & 39,5 & DMSO-d6 \\
\hline \multirow[t]{6}{*}{49,5} & Methanol & & 49,5 & Methanol \\
\hline & & & 57 & $\mathrm{O}-\mathrm{CH}_{3}$ \\
\hline & & & 77 & $\mathrm{O}-\mathrm{CH}$ \\
\hline & & & 83 & $\mathrm{C}-\mathrm{O}$ \\
\hline & & & 93 & $\mathrm{C} \equiv \mathrm{C}$ \\
\hline & & & 103,5 & $\mathrm{C}=\mathrm{C}$ \\
\hline \multirow[t]{2}{*}{119,1} & Ring (C4) & & 120 & Aromat, $\mathrm{C}=\mathrm{C}$ \\
\hline & & & 124 & Aromat, $\mathrm{C}=\mathrm{C}$ \\
\hline \multirow[t]{3}{*}{124,2} & Ring-CH3 $(\mathrm{C} 2, \mathrm{C} 6)$ & & 124,3 & Ring $(\mathrm{C} 2, \mathrm{C} 6)$ \\
\hline & & & 124,5 & Aromat, $\mathrm{C}=\mathrm{C}$ \\
\hline & & & 125,3 & Aromat, $\mathrm{C}=\mathrm{C}$ \\
\hline \multirow[t]{6}{*}{128,3} & Ring (C3,C5) & & & \\
\hline & & & 136 & Aromat \\
\hline & & & 138 & Aromat \\
\hline & & & 148 & Ring-OH \\
\hline & & & 151 & Ring-OH \\
\hline & & & 152,6 & Ring-OH \\
\hline 153,3 & Ring-OH (C1) & & 153,1 & Ring-OH $(\mathrm{C} 1)$ \\
\hline
\end{tabular}

Im Spektrum wiederzufinden sind auch hier die Signale der Ausgangssubstanz, bei 16,6 ppm die Signale der beiden Methylgruppen.

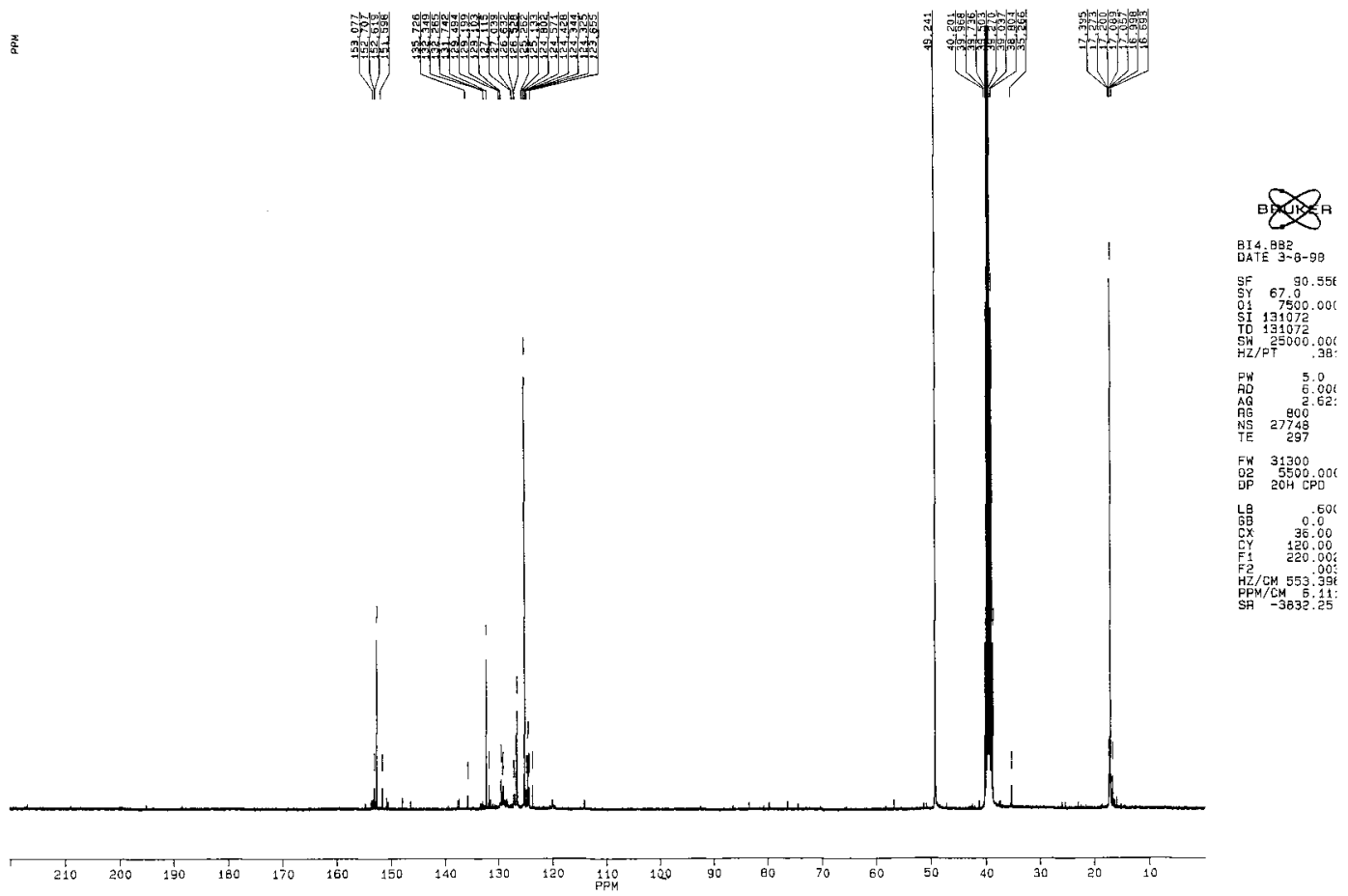

Abbildung 112: ${ }^{13}$ C-NMR Spektrum der extrahierten Überzüge des mit 2,6-Dimethylphenol belegten NaMontmorillonits. 
Bei 119,1 ppm treten die C=C-Bindungen des aromatischen Rings, bei 124,2 ppm die Ring-CBindung der beiden $\mathrm{CH}_{3}$-Substituenten sowie bei 153,3 ppm die C-O-Bindung der $\mathrm{OH}$ Gruppe an den Benzolring auf (Tabelle 24).

Die große Vielzahl neu hinzugekommener Signale sind, wie in Abbildung 112 ersichtlich, aufgrund ihrer schwachen Ausprägung nur sehr schwer zu erkennen bzw. zuzuordnen. Aus diesem Grunde wurde der Verschiebungsbereich zwischen 160 und 120 ppm zu besseren Interpretation gespreizt. Die folgende Abbildung stellt die nun deutlich besser erkennbaren Verschiebungswerte dar (Abbildung 113).

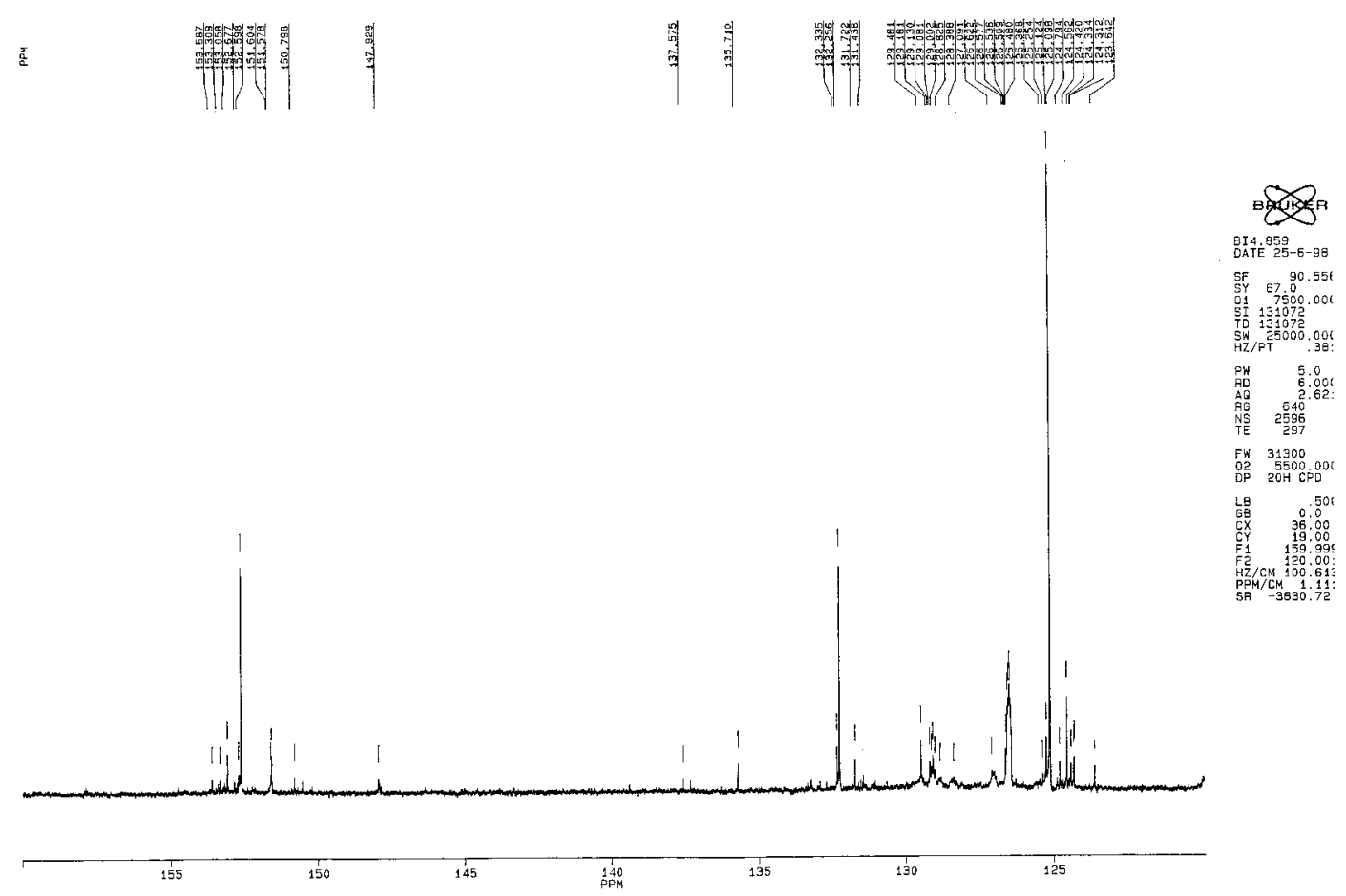

Abbildung 113: ${ }^{13} \mathrm{C}-\mathrm{NMR}$ Spektrum der extrahierten Überzüge des mit 2,6-Dimethylphenol belegten NaMontmorillonits (Ausschnittvergrößerung).

Wie bei den vorab beschriebenen ${ }^{13} \mathrm{C}-\mathrm{NMR}-$ Spektren von Brenzkatechin und Pyrogallol führte die Reaktion von 2,6-Dimethylphenol zur Bildung verschiedener Reaktionsprodukte. Eine Übersicht über die detektierten Verschiebungen und ihre Zuordnungen gibt Tabelle 25. Die große Zahl der detektierten Signale steht für die Vielzahl der entstandenen C-Bindungen. Es treten mehrere Signale für Methylgruppen bei 17 ppm, 17,4 ppm, 26 ppm und 27 ppm auf. Die Verschiebung bei 35,2 ppm beschreibt eine $\mathrm{CH}_{2}$-Bindung. Die Peaks bei 57 und 75 ppm sind Signale für O-Alkylbindungen $\left(\mathrm{O}-\mathrm{CH}_{2}\right.$ bzw. O-CH). Zwischen 76 und 80 ppm können nichtaromatische C-O-Bindungen nachgewiesen werden. Die Verschiebungen bei 84 ppm, 87 ppm und 92,5 ppm beschreiben Dreifachbindungen von Kohlenstoffatomen (Alkine) bzw. Allene. 
Tabelle 25: Chemische Verschiebungen des Extraktes des mit 2,6-Dimethylphenol belegten Na-Montmorillonits (entnommen aus Abb. 112+Abb. 113).

\begin{tabular}{|c|c|c|c|}
\hline ppm & Zuordnung & ppm & Zuordnung \\
\hline 217 & $\mathrm{C}=\mathrm{O}$, Ketone & 126,6 & $\mathrm{C}=\mathrm{C}$, Aromat \\
\hline 209 & $\mathrm{C}=\mathrm{O}$, Ketone & 126,5 & $\mathrm{C}=\mathrm{C}$, Aromat \\
\hline 196 & $\mathrm{C}=\mathrm{O}$, Ketone & 124,8 & $\mathrm{C}=\mathrm{C}$, Aromat \\
\hline 188 & $-\mathrm{CHO}$, Aldehyde & 124,5 & $\mathrm{C}=\mathrm{C}$, Aromat \\
\hline 155 & $\mathrm{C}-\mathrm{O}$, Aromat & 124,3 & $\mathrm{C}=\mathrm{C}$, Aromat \\
\hline 153,6 & $\mathrm{C}-\mathrm{O}$, Aromat & 123,6 & $\mathrm{C}=\mathrm{C}$, Aromat \\
\hline 153,3 & $\mathrm{C}-\mathrm{O}$, Aromat & 120 & $\mathrm{C}=\mathrm{C}$, Aromat \\
\hline 153 & $\mathrm{C}-\mathrm{O}$, Aromat & 92,5 & $-\mathrm{C} \equiv \mathrm{C}-$, Alkin oder $\mathrm{C}=\mathrm{C}=\mathrm{C}$, Allen \\
\hline 152,7 & $\mathrm{C}-\mathrm{O}$, Aromat & 87 & $-\mathrm{C} \equiv \mathrm{C}-$, Alkin oder $\mathrm{C}=\mathrm{C}=\mathrm{C}$, Allen \\
\hline 152,6 & $\mathrm{C}-\mathrm{O}$, Aromat & 84 & $-\mathrm{C} \equiv \mathrm{C}-$, Alkin oder $\mathrm{C}=\mathrm{C}=\mathrm{C}$, Allen \\
\hline 151,6 & $\mathrm{C}-\mathrm{O}$, Aromat & 80 & $-\mathrm{C}-\mathrm{O}-$ \\
\hline 150,8 & $\mathrm{C}-\mathrm{O}$, Aromat & 76 & $-\mathrm{C}-\mathrm{O}-$ \\
\hline 147,9 & $\mathrm{C}-\mathrm{O}$, Aromat & 75 & $\mathrm{O}-\mathrm{CH}$ \\
\hline 147 & $\mathrm{C}-\mathrm{O}$, Aromat & 57 & $\mathrm{O}-\mathrm{CH} \mathrm{H}_{2}$ \\
\hline 137,6 & $\mathrm{C}=\mathrm{C}$, Aromat & 35,2 & $-\mathrm{CH},-\mathrm{CH}$ \\
\hline 135,7 & $\mathrm{C}=\mathrm{C}$, Aromat & 27 & $-\mathrm{CH}_{3}$ \\
\hline 132,3 & $\mathrm{C}=\mathrm{C}$, Aromat & 26 & $-\mathrm{CH}_{3}$ \\
\hline 131,7 & $\mathrm{C}=\mathrm{C}$, Aromat & 17,4 & $-\mathrm{CH}_{3}$ \\
\hline 129,5 & $\mathrm{C}=\mathrm{C}$, Aromat & 17 & $-\mathrm{CH}_{3}$ \\
\hline 129,2 & $\mathrm{C}=\mathrm{C}$, Aromat & 16,7 & $-\mathrm{CH}_{3}$ \\
\hline 129,1 & $\mathrm{C}=\mathrm{C}$, Aromat & & \\
\hline 127,1 & $\mathrm{C}=\mathrm{C}$, Aromat & & \\
\hline 127 & $\mathrm{C}=\mathrm{C}$, Aromat & & \\
\hline
\end{tabular}

Der mit 16 detektierten Signalen stark repräsentierte Verschiebungsbereich der aromatischen C-Bindungen liegt zwischen $120 \mathrm{ppm}$ und 137,6 ppm. Es folgen die aromatischen C-OBindungen mit zehn Peaks zwischen 147 ppm und 155 ppm. Bei 188 ppm ist das Signal für die Carbonylbindung eines Aldehyds zu beobachten. Die chemischen Verschiebungen bei 196 ppm, 209 ppm und 217 ppm beschreiben die C=O-Doppelbindungen von Ketogruppen. Aus der Vielzahl dieser Signale kann entnommen werden, dass das 2,6-Dimethylphenol durch die Sublimation auf den Ton chemisch stark verändert wurde. Die Bildung einer hohen Zahl von Reaktionsprodukten kann angenommen werden. Insbesondere die vielen Signale im C-Cbzw. C-O-Bindungsbereich der Aromaten sprechen für eine vielfältige Transformation des Phenols hinsichtlich der Substituierung und der Bindung einzelner Aromate zu höhermolekularen Verbindungen. Das Auftreten von Carbonylbindungen zwischen 188 ppm und 217 ppm weist die teilweise Oxidation der Ausgangssubstanz mit der Bildung chinoider Strukturen nach. Es ist davon auszugehen, dass die Reaktion von 2,6-Dimethylphenol an der Tonmineraloberfläche zur Bildung von Oxidationsprodukten geführt hat, die dann z. B. durch radikalische Prozesse zu Verbindungen mit höheren Massengewichten reagiert haben. So beschreiben IKEDA et al. (1996) als Reaktionsprodukt der enzymatischen Katalyse von 2,6Dimethylphenol durch Peroxidasen eine oxidative Polymerisation des Phenols zu polymeren Verbindungen, die vorwiegend aus einer Mischung von Phenylen- und Oxyphenyleneinheiten zusammensetzen. Hauptreaktionsprodukt ist hierbei das Poly(2,6-dimethyl-1,4-oxyphenylen)(Polyphenylenoxid) (PPO), ein Polymer aufgebaut aus 2,6-Dimethylphenol-Monomeren (Abbildung 114). 


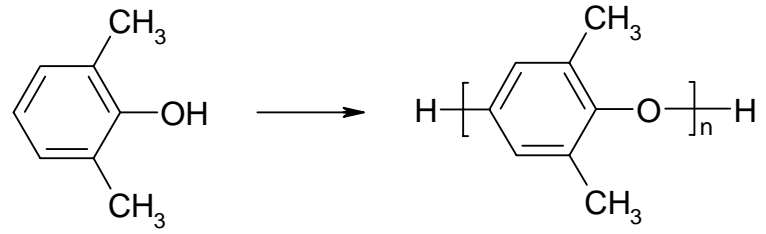

Abbildung 114: Bildung von PPO aus 2,6-Dimethylphenol (nach IKEDA et al. 1996).

Durch oxidative Kopplung von zwei Molekülen des Phenols an der Position 4 des Monomers wurde bei Katalyse mit dem Enzym Laccase 3,5,3',5'-Tetramethyl-4,4'-diphenochinon (DPQ) gebildet (IKEDA et al. 1996).<smiles>CC1=CC(=C2C=C(C)C(=O)C(C)=C2)C=C(C)C1=O</smiles>

Abbildung 115: Oxidatives Polymerisationsprodukt (DPQ) aus 2,6-Dimethylphenol (IKEDA et al. 1996).

Diese Verbindung weist eine Masse von 241 auf. Die im Rahmen dieser Arbeit gemessenen Massenspektren des mit 2,6-Dimethylphenol belegten Na-Montmorillonits bestätigen diesen Wert (Kapitel 4.8.3). Daraus ist abzuleiten, dass durch die Reaktion des Phenols an der Tonmineraloberfläche unter anderem das DPQ gebildet wurde. Das von IKEDA et al. 1996 mit Hilfe der ${ }^{13} \mathrm{C}$-NMR-Spektroskopie bestimmte Reaktionsprodukt der Peroxidase-katalysierten Polymerisation von 2,6-Dimethylphenol (Abbildung 116) ergab folgende Struktur und Zuordnung (Tabelle 26):

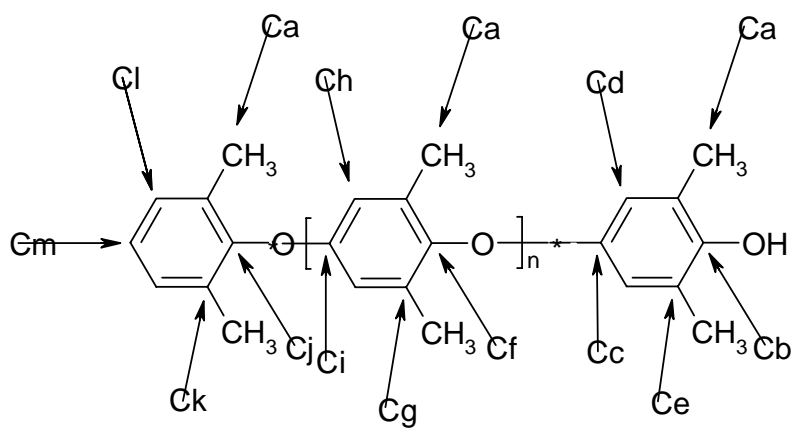

Abbildung 116: Polymer aus 2,6-Dimethylphenol-Monomeren (IKEDA et al. 1996).

Tabelle 26: Chemische Verschiebungen des Reaktionsproduktes und Zuordnungen.

\begin{tabular}{|c|c|c|c|}
\hline Chemische Verschiebung (ppm) & Zuordnung & Chemische Verschiebung (ppm) & Zuordnung \\
\hline $16,6-16,8$ & $\mathrm{Ca}$ & 132,7 & $\mathrm{Cg}$ \\
\hline 114,1 & $\mathrm{Cd}$ & 145,6 & $\mathrm{Cf}$ \\
\hline 114,5 & $\mathrm{Ch}$ & 146,4 & $\mathrm{Cb}$ \\
\hline 124,4 & $\mathrm{Cc}$ & 151,5 & $\mathrm{Cj}$ \\
\hline 125,0 & $\mathrm{Cm}$ & 154,5 & $\mathrm{Ce}$ \\
\hline 129,0 & $\mathrm{C} 1$ & 154,8 & $\mathrm{Ci}$ \\
\hline 131,6 & $\mathrm{Ck}$ & & \\
\hline
\end{tabular}

Diese Verschiebungswerte für das Polymerisationsprodukt werden auch in den ${ }^{13} \mathrm{C}-\mathrm{NMR}$ - 
Spektren des extrahierten Probenmaterials wiedergefunden. Somit ist davon auszugehen, dass neben anderen Reaktionsprodukten diese Verbindung durch die Kopplung mehrerer 2,6Dimethylphenol-Monomere aneinander gebildet worden ist (SAWHNEY et al. 1984).

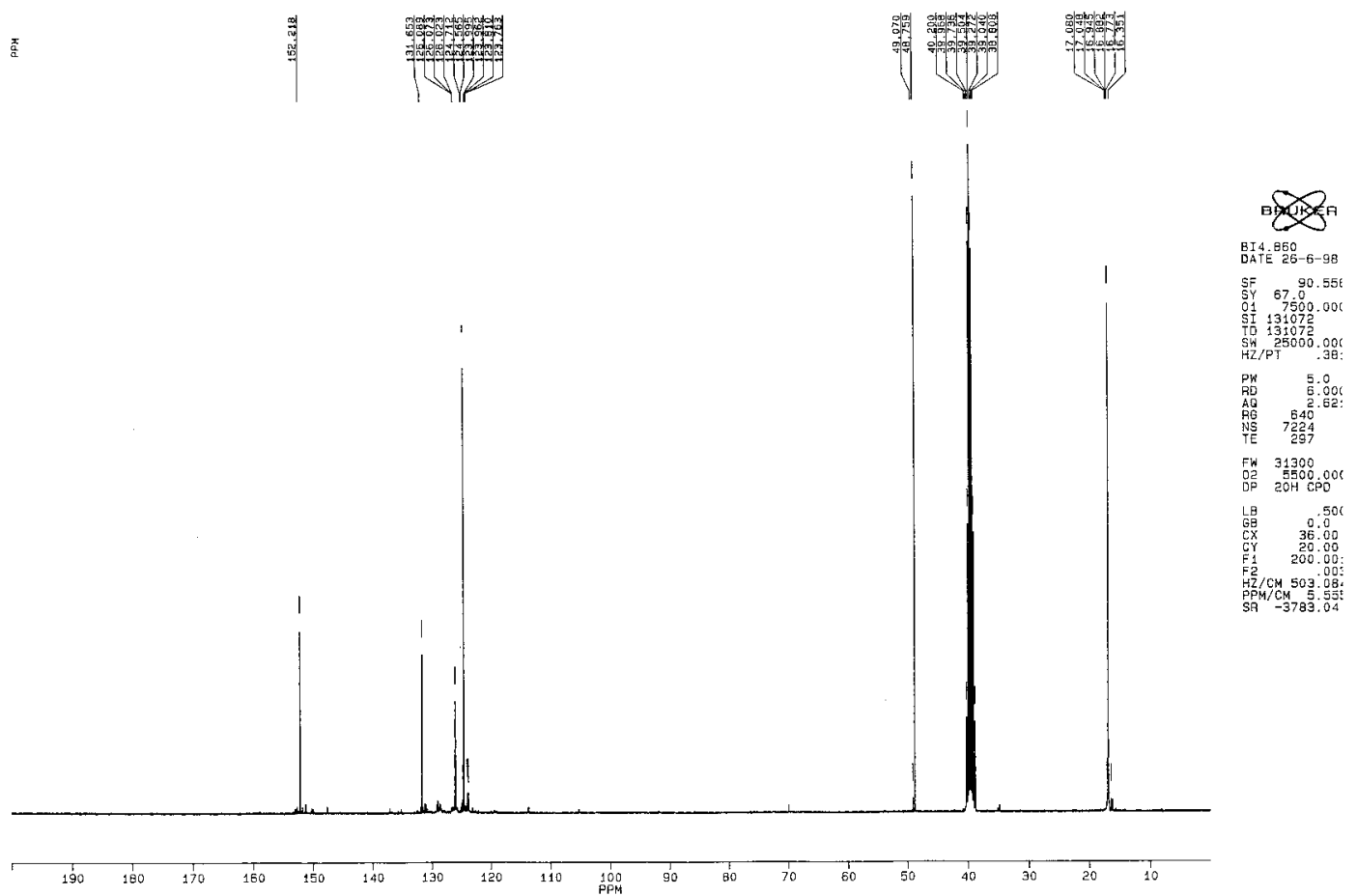

Abbildung 117: ${ }^{13} \mathrm{C}-\mathrm{NMR}-$ Spektrum der Extrakte des mit 2,6-Dimethylphenol belegten Ca-Montmorillonits.
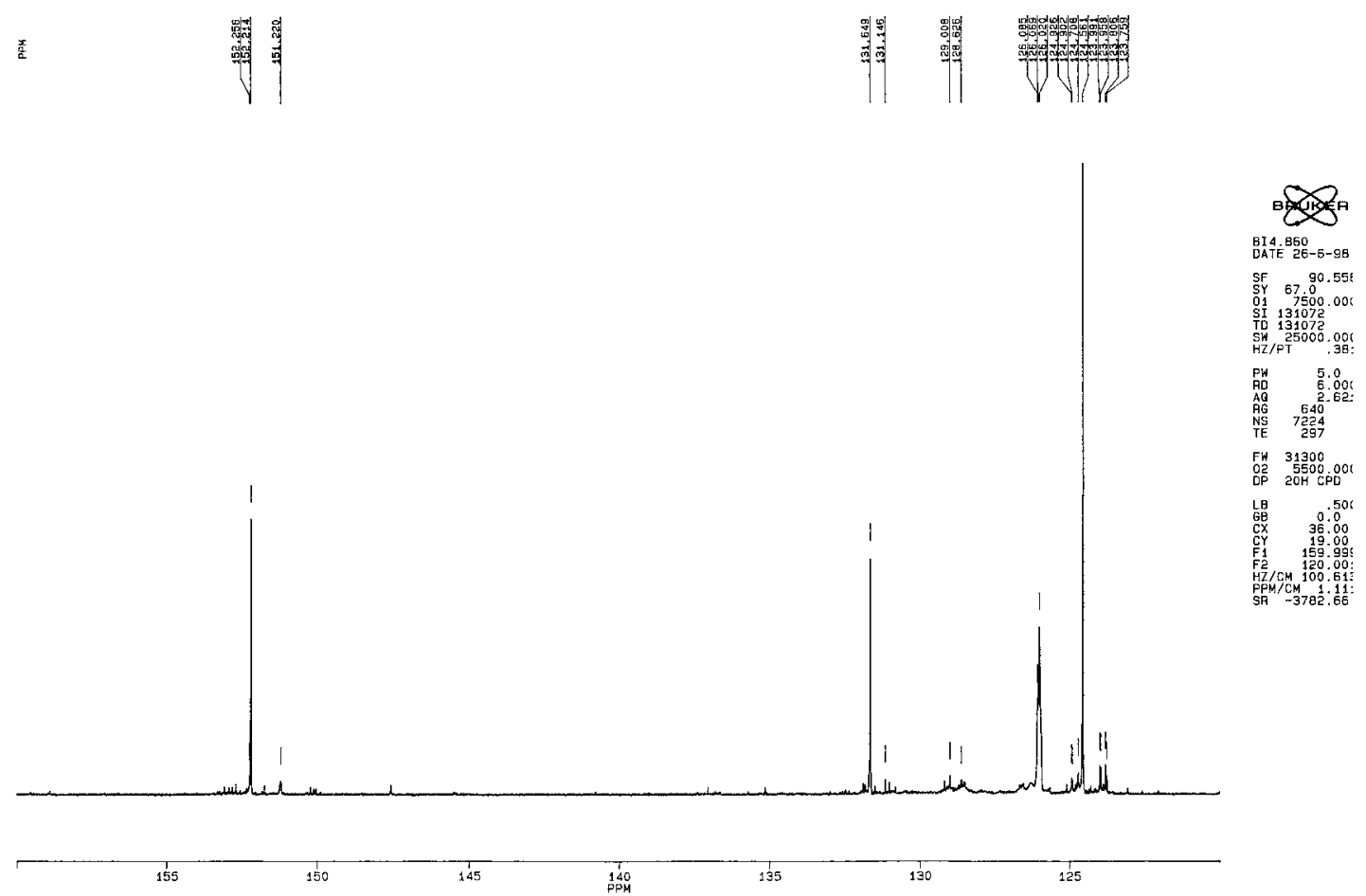

Abbildung 118: ${ }^{13} \mathrm{C}-\mathrm{NMR}-$ Spektrum der extrahierten Überzüge des mit 2,6-Dimethylphenol belegten CaMontmorillonits (Ausschnittvergrößerung). 
Die ${ }^{13} \mathrm{C}$-NMR-Spektren des mit 2,6-Dimethylphenol belegten Ca-Montmorillonits (Abbildungen 117 und 118) weisen im Vergleich mit denen belegten Na-Montmorillonits keine signifikanten Unterschiede auf. Die Unterschiede in der Reaktivität der Tonminerale bzw. der Bildung unterschiedlicher Reaktionsprodukte aufgrund unterschiedlicher Kationenbelegung des Tonminerals konnten durch diese Methode und Analysen nicht bestätigt werden. Die geringeren Intensitäten der Signale bei den Proben mit Ca-Montmorillonit beruhen auf einer Schwankung in der Probenkonzentration. Durch die angewendete Methode der Belegung der Tonminerale mit Organika (Kapitel 4.1) war eine Einhaltung immer exakt gleicher aufsublimierter Mengen nicht zu erreichen.

\subsubsection{Phenanthren}

Ziel der Untersuchungen war, neben Modellsubstanzen für Huminstoffe wie die verwendeten Phenole, zusätzlich noch eine weitere Substanzklasse in diese Untersuchungen einzubeziehen. Diese sollte als charakteristische Gruppe anthropogen in die Umwelt eingebrachter organischer Schadstoffe anzusehen sein. Aus diesem Grunde wurde als Vertreter der Polyzyklischen Aromatischen Kohlenwasserstoffe (PAK) das Phenanthren gewählt (Kapitel 2.2). Die Belegung auf der Tonminerale erfolgte im Falle des Phenanthrens, aufgrund des hohen Schmelzpunktes, nicht wie bei den Phenolen durch Sublimation aus der Gasphase, sondern durch Aufbringung methanolischer Phenanthren-Lösung auf den Ton (Kapitel 4.1).

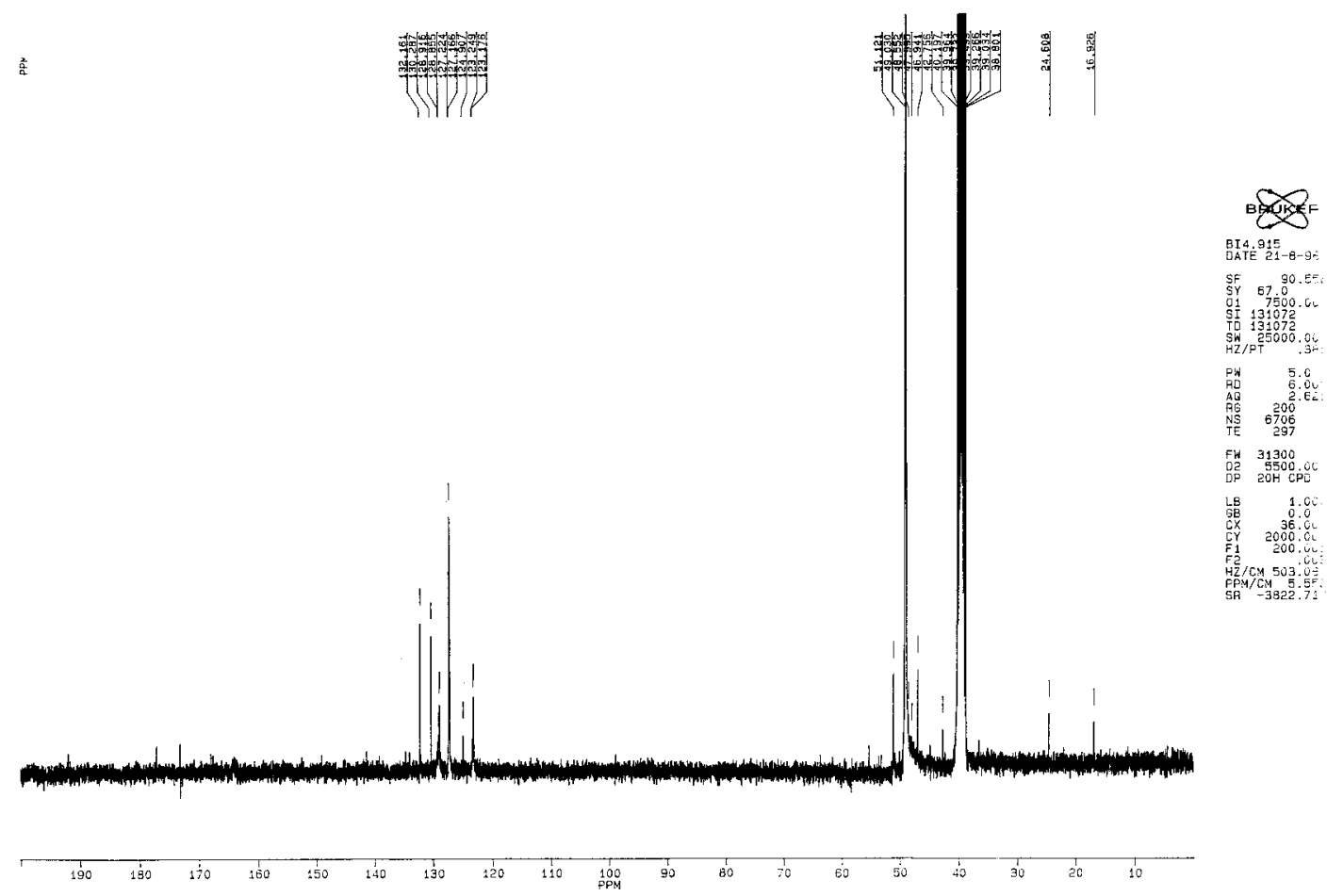

Abb. 119: ${ }^{13} \mathrm{C}-\mathrm{NMR}-$ Spektrum von Na-Montmorillonit belegt mit Phenanthren.

Die weitere Verfahrensweise wurde entsprechend der Belegung mit Phenolen beibehalten. Es sollte untersucht werden, ob auch höher kondensierte, chemisch relativ träge organische Ver- 
bindungen, neben den bereits vielfach untersuchten biologischen Abbau- und Transformationsprozessen, auf abiotischem Wege durch die Reaktivität der Tonminerale in ihrer Zusammensetzung verändert werden.

Die im Folgenden präsentierten Resultate zeigen, dass eine, wenn auch sehr schwache Reaktion des auf die Oberfläche der Tonminerale aufgebrachten Phenanthrens stattgefunden eingetreten ist (Abbildungen 119 und 120).Neben den Peaks für die C-C- und $\mathrm{C}=\mathrm{C}$-Bindungen des Phenanthrens (Abbildung 107) treten Signale für Methylgruppen (Verschiebungsbereich 16 bis 25 ppm) auf. Das Auftreten von Signalen für $\mathrm{CH}_{3}$-gruppen kann einerseits als Hinweis auf die Methylierung von Substituenten des Aromats gedeutet werden. Andererseits kann die Reaktion des Phenanthrens an der Tonmineraloberfläche auch zur teilweisen Aufspaltung der aromatischen Strukturen geführt haben. Weiterhin konnten $\mathrm{O}-\mathrm{CH}_{3}-\mathrm{Bindungen} \mathrm{bei} 55,5 \mathrm{ppm}$ nachgewiesen werden.

Tabelle 27: Übersicht der chemischen Verschiebungen und Zuordnungen.

\begin{tabular}{|c|c|c|c|c|}
\hline \multicolumn{2}{|c|}{$\begin{array}{l}\text { Standard } \\
\text { Phenanthren }\end{array}$} & \multirow[t]{19}{*}{$\begin{array}{l}\text { Montmorillonit } \\
\text { belegt mit }\end{array}$} & \multicolumn{2}{|c|}{ Phenanthren } \\
\hline \multirow[t]{3}{*}{ ppm } & Zuordnung & & ppm & Zuordnung \\
\hline & & & 16,9 & $-\mathrm{CH}_{3}$ \\
\hline & & & 24,6 & $-\mathrm{CH}_{3}$ \\
\hline 39,5 & DMSO-d6 & & 39,5 & DMSO-d6 \\
\hline \multirow[t]{2}{*}{49,5} & Methanol & & 49,5 & Methanol \\
\hline & & & 55,5 & $\mathrm{O}-\mathrm{CH}_{3}$ \\
\hline 122,8 & Ring (C7) & & 123,3 & Ring (C7) \\
\hline 126,6 & Ring (C5) & & 124,9 & Ring (C5) \\
\hline 126,8 & Ring (C4) & & 127,2 & Ring (C4) \\
\hline 128,4 & Ring (C3) & & 128,9 & Ring (C3) \\
\hline 129,7 & Ring (C2) & & 130,3 & Ring (C2) \\
\hline \multirow[t]{7}{*}{131,5} & Ring (C1) & & 132,2 & Ring (C1) \\
\hline & & & 133,5 & Ring-OH \\
\hline & & & 134,5 & Ring-OH \\
\hline & & & 141,5 & Ring-OH \\
\hline & & & 173 & $\mathrm{C}=\mathrm{O}$, Carboxyl \\
\hline & & & 176,5 & $\mathrm{C}=\mathrm{O}$ \\
\hline & & & 192 & $\mathrm{C}=\mathrm{O}$, Chinon \\
\hline
\end{tabular}

Im Bereich aromatischer Strukturen sind Signale von C-OH-Bindungen bei 133,5 ppm, 134,5 ppm und 141,5 ppm zu beobachten. Außerdem treten Carbonylverbindungen, erkennbar durch die chemischen Verschiebungen im Bereich der $\mathrm{C}=\mathrm{O}$-Doppelbindungen zwischen bei 173 ppm, 176,5 ppm und 192 ppm auf. Das Auftreten von $\mathrm{O}_{-} \mathrm{CH}_{3^{-}}, \mathrm{C}-\mathrm{OH}-$ und insbesondere $\mathrm{C}=\mathrm{O}$-Signalen im ${ }^{13} \mathrm{C}$-NMR-Spektrum zeigt, dass es neben der unveränderten Ausgangssubstanz zur Entstehung neuer Substanzen gekommen ist. Diese neu gebildeten Verbindungen deuten stark auf eine Oxidation der aromatischen Struktur des Polyzyklischen Aromatischen Kohlenwasserstoffs hin.

Das ${ }^{13} \mathrm{C}$-NMR-Spektrum des mit Phenanthren belegten Ca-Montmorillonits zeigt ebenfalls eine, wenn auch schwache, Reaktion des PAKs an. Durch das hohe Signalrauschen aufgrund der langen Analysedauer ist eine eindeutige Zuordnung der Peaks erschwert. Die Verlängerung der Messzeit war hinsichtlich der sehr geringen Konzentration in der Probe unumgäng- 
lich. Im Gegensatz zum vorangegangenen Spektrum kann hier Auftreten von Methylgruppen nicht eindeutig beschrieben werden. Bei dem im Spektrum sichtbaren Verschiebungswert bei 46,6 ppm scheint es sich lediglich um ein Satellitensignal des Methanols aufgrund der langen Dauer des Scans zu handeln. Weiterhin ist kein Signal im Bereich der $\mathrm{O}-\mathrm{CH}_{3}$-Bindungen zu erkennen, stattdessen tritt ein deutlich zu erkennender Peak bei 84 ppm, also im Bereich der C-OH-Bindungen auf, der im Spektrum des mit Phenanthren belegten Na-Montmorillonits nicht zu beobachten war.

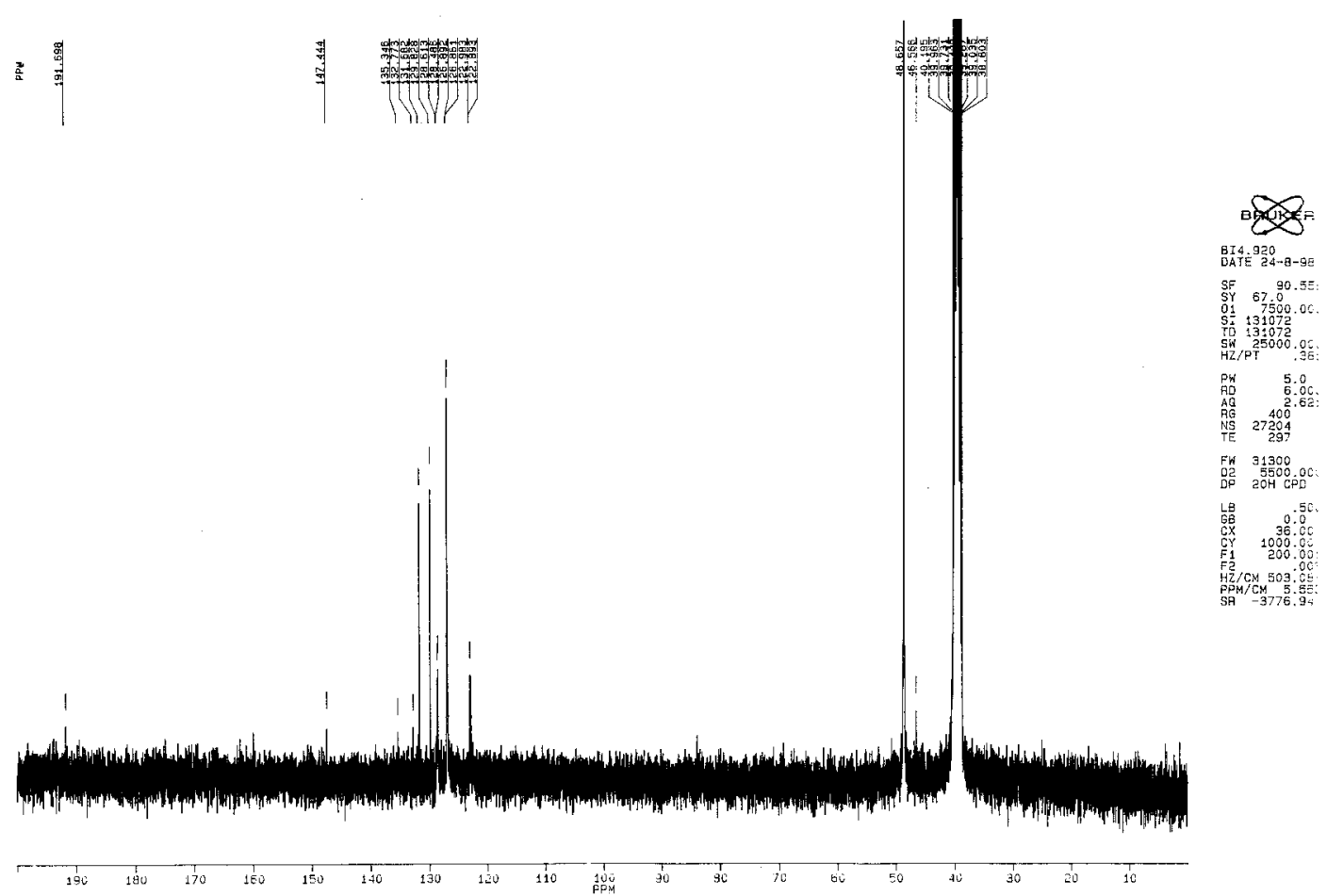

Abbildung 120: ${ }^{13}$ C-NMR-Spektrum von Ca-Montmorillonit belegt mit Phenanthren.

Neben den eindeutig zuzuordnenden Verschiebungswerten der $\mathrm{C}-\mathrm{C}$ - und der $\mathrm{C}=\mathrm{C}$-Bindungen des aromatischen Systems des Phenanthrens sind auch in diesem Spektrum wiederum Signale für aromatische C-OH-Bindungen bei 132,8 ppm, 135,3 ppm und 147,4 ppm und damit eine Substituierung des aromatischen Systems zu beobachten (Tabelle 28). Die im Verschiebungsbereich ab 160 ppm zu beobachtenden Peaks weisen das Auftreten von $\mathrm{C}=\mathrm{O}$ Doppelbindungen nach. Somit kann die Oxidation des PAK zu Verbindungen, die deutliche Übereinstimmungen mit den Reaktionsprodukten der auf die Tonmineraloberfläche sublimierten Phenole zeigen, angenommen werden. Von besonderer Bedeutung sind hier die auftretenden Signale im Verschiebungsbereich der $\mathrm{C}-\mathrm{OH}$ - und der $\mathrm{C}=\mathrm{O}$-Bindungen. Naheliegend ist die Vermutung, dass die Reaktion an der Tonmineraloberfläche zur Oxidation des PAK, entsprechend den Phenolen, geführt hat. 
Tabelle 28: Übersicht der chemischen Verschiebungen und ihre Zuordnungen.

\begin{tabular}{|c|c|c|c|c|}
\hline \multicolumn{2}{|c|}{$\begin{array}{l}\text { Standard } \\
\text { Phenanthren }\end{array}$} & \multirow[t]{17}{*}{$\begin{array}{l}\text { Montmorillo- } \\
\text { nit belegt mit }\end{array}$} & \multicolumn{2}{|c|}{ Phenanthren } \\
\hline ppm & Zuordnung & & ppm & Zuordnung \\
\hline 39,5 & DMSO-d6 & & 39,5 & DMSO-d6 \\
\hline \multirow[t]{2}{*}{49,5} & Methanol & & 49,5 & Methanol \\
\hline & & & 84 & $-\mathrm{C}-\mathrm{OH}$ \\
\hline 122,8 & Ring (C7) & & 122,9 & Ring (C7) \\
\hline 126,6 & Ring (C5) & & 126,9 & Ring (C5) \\
\hline 126,8 & Ring (C4) & & 128,4 & Ring (C4) \\
\hline 128,4 & Ring (C3) & & 128,6 & Ring (C3) \\
\hline 129,7 & Ring (C2) & & 129,8 & Ring (C2) \\
\hline \multirow[t]{7}{*}{131,5} & Ring (C1) & & 131,7 & Ring (C1) \\
\hline & & & 132,8 & Ring-OH \\
\hline & & & 135,3 & Ring-OH \\
\hline & & & 147,4 & Ring-OH \\
\hline & & & 160 & $\mathrm{C}=\mathrm{O}$, Carboxyl \\
\hline & & & 176,5 & $\mathrm{C}=\mathrm{O}$ \\
\hline & & & 191,7 & $\mathrm{C}=\mathrm{O}$, Chinon \\
\hline
\end{tabular}

Die entsprechenden mikrobiellen Abbau- bzw. Oxidationsprodukte des Phenanthrens (CERNIGLIA 1993) sind unter anderem das 9-Phenanthrol, das 9,10-Phenanthrenchinon und die Diphensäure (Abbildungen 121, 122, 123). Die zugehörigen ${ }^{13} \mathrm{C}-\mathrm{NMR}-$ Spektren zeigen weitreichende Übereinstimmungen zu den in den Tabellen 29-31 aufgeführten Verschiebungswerten.

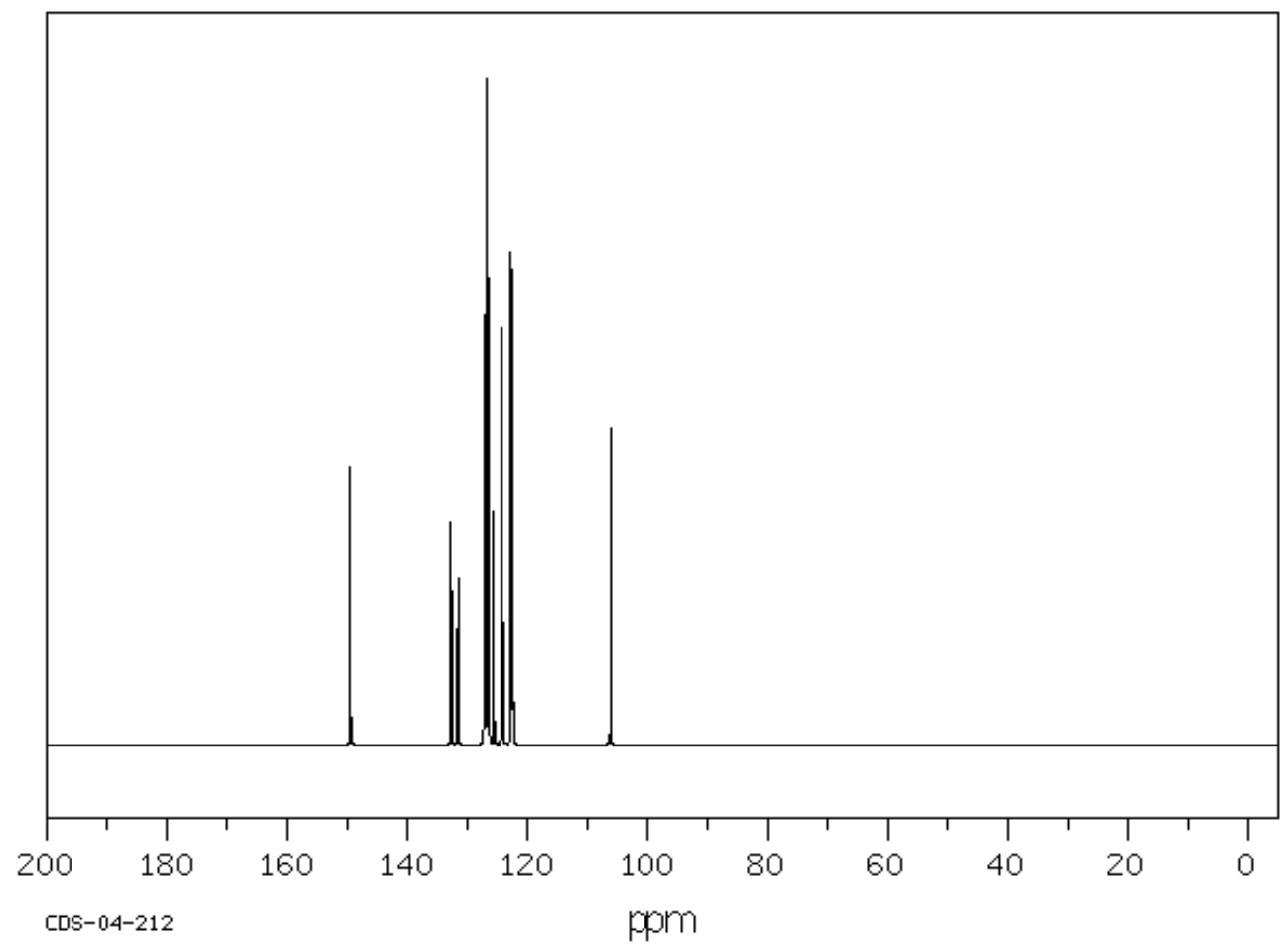

Abbildung 121: ${ }^{13} \mathrm{C}-\mathrm{NMR}-$ Spektrum des 9-Phenanthrols (Quelle: SDBS). 
Tabelle 29: Verschiebungswerte des 9-Phenanthrols und Zuordnungen.

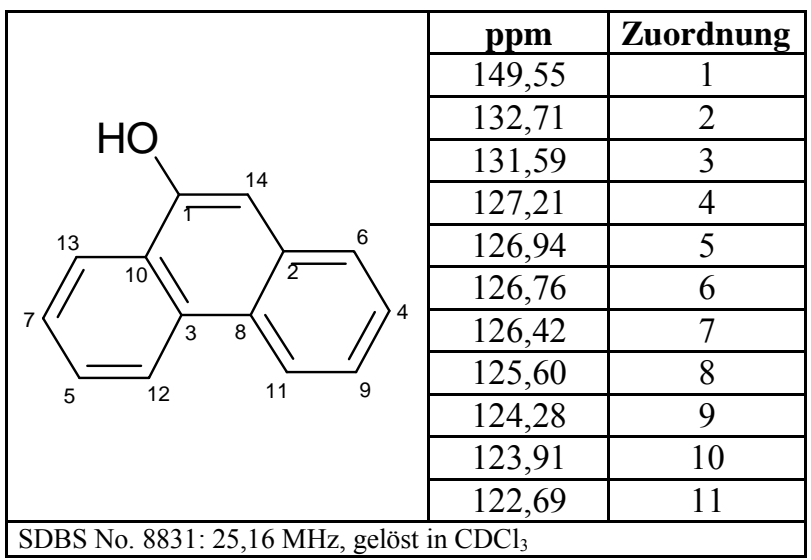

Über den Weg der Hydroxylierung (Kapitel 2.2) erfährt das relativ reaktionsträge Phenanthren hier eine Transformation, die der des mikrobiellen Abbaus durch Bodenbakterien und Bodenpilzen ähnelt.

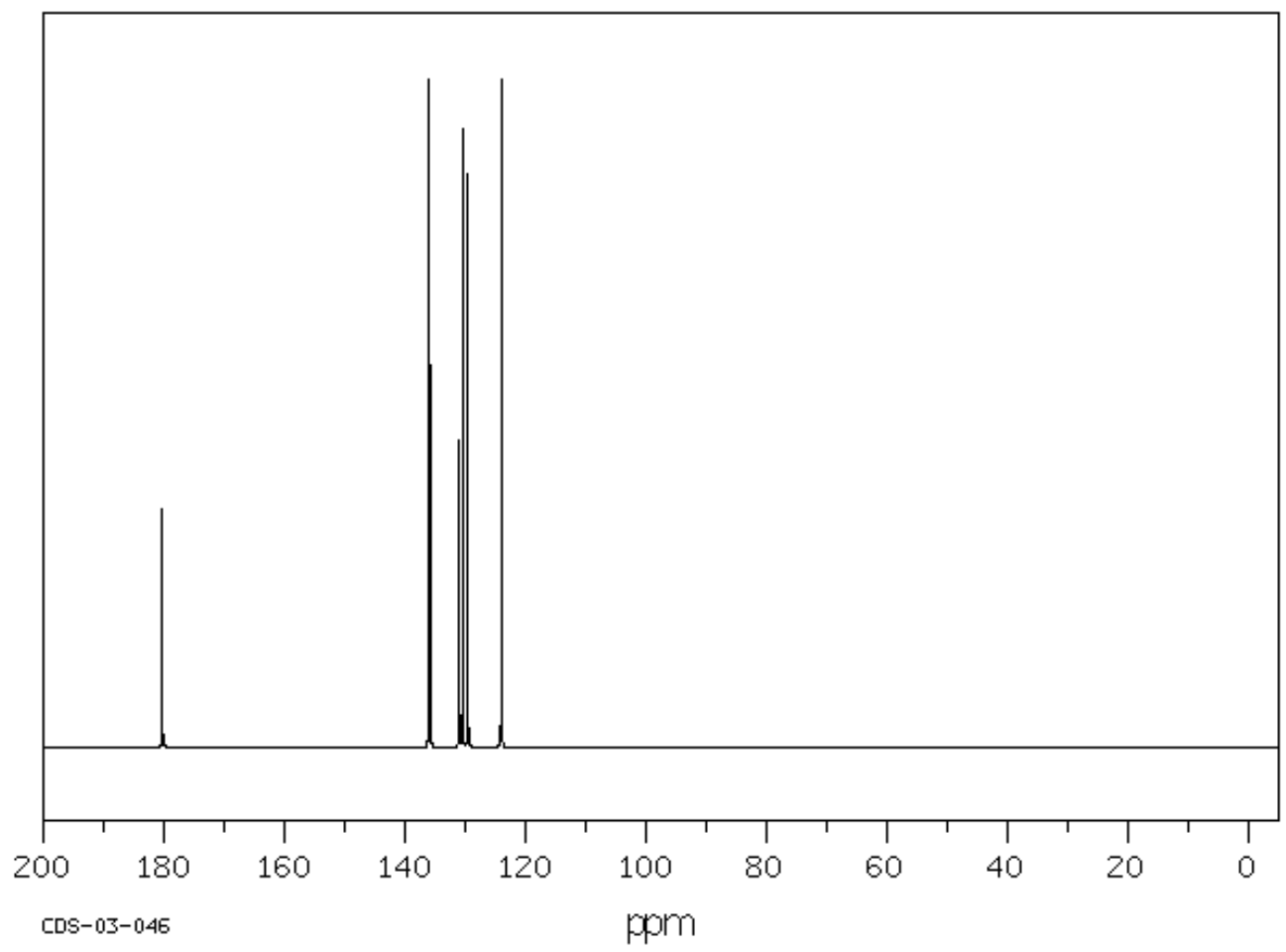

Abbildung 122: ${ }^{13}$ C-NMR-Spektrum von 9,10-Phenanthrenchinon (Quelle: SDBS).

Der Metabolismus lässt zuerst das 9-Phenanthrol, dann das 9,10-Phenanthrenchinon und schließlich die Diphensäure entstehen (HAMMEL et al. 1992). 
Tabelle 30: Verschiebungswerte des 9,10-Phenanthrenchinons und ihre Zuordnungen.

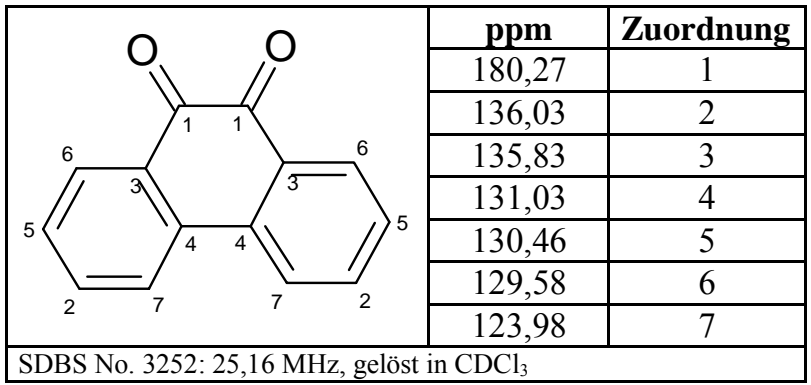

Neben diesen drei Reaktionsprodukten des oxidativen Abbaus von Phenanthren wird durch das Enzym Dioxygenase das Methoxyphenanthren gebildet. Dafür das diese Verbindung auch durch abiotische Reaktion an der Tonmineraloberfläche gebildet wurde, spricht das bereits erwähnte Auftreten eines Signals bei $55,5 \mathrm{ppm}$, welches charakteristisch für $\mathrm{OCH}_{3}-$ Bindungen ist.

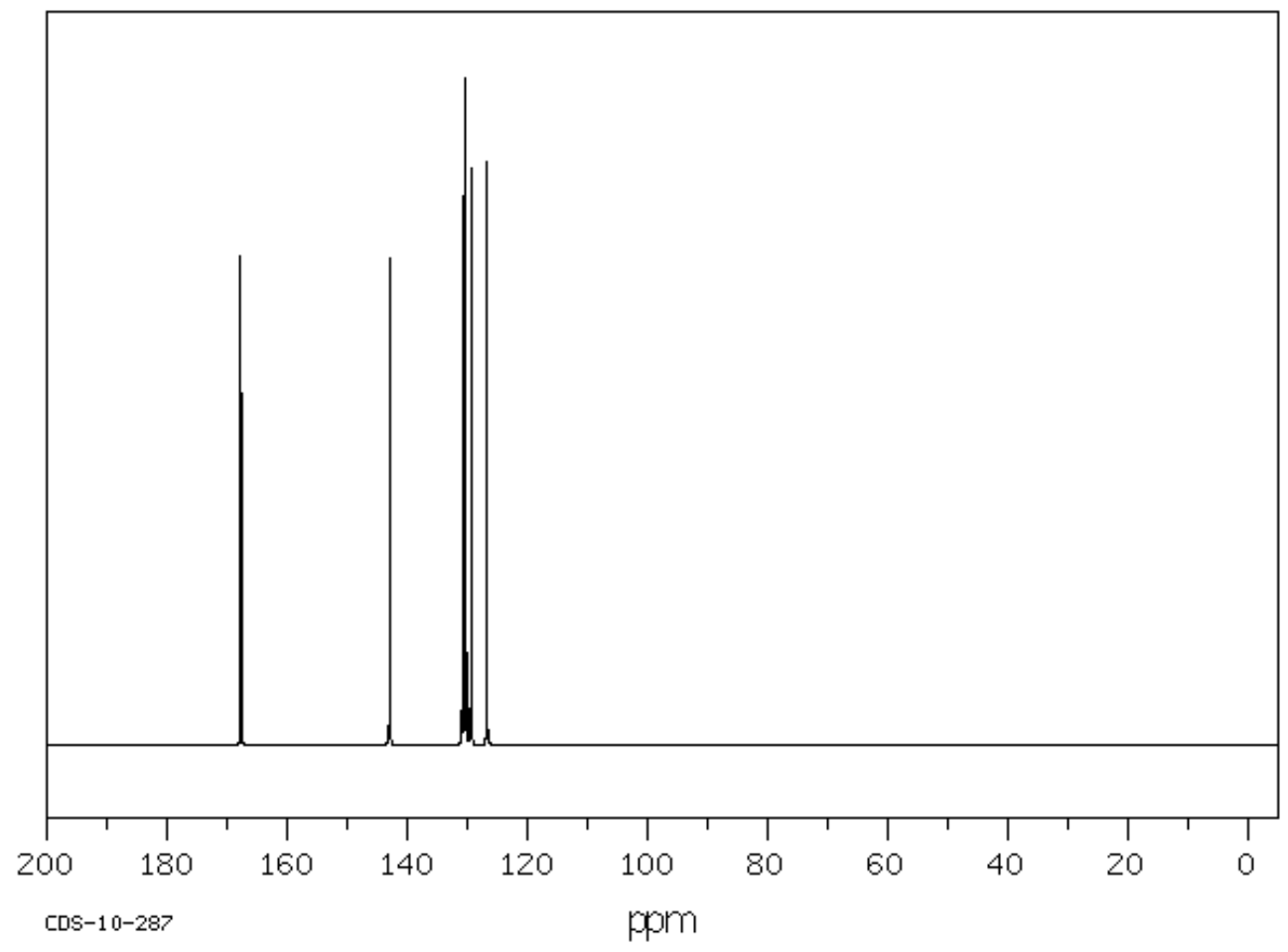

Abbildung 123: ${ }^{13}$ C-NMR-Spektrum von Diphensäure (Quelle: SDBS).

Tabelle 31: Chemische Verschiebungen der Diphensäure und Zuordnung.

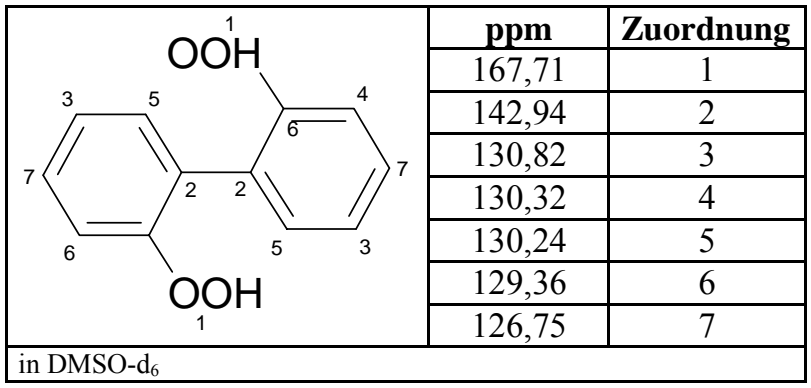


Die mikrobiellen Abbauprozesse führen zur teilweisen Spaltung der aromatischen Gerüststrukturen. Der Metabolismus verläuft über die Bildung von Phthalaten und Pyruvaten und kann im Idealfalle die vollständige Mineralisation $\left(\mathrm{CO}_{2}\right)$ erreichen.

Zusammenfassend kann festgestellt werden, dass die katalytischen Eigenschaften der Tonminerale eine Transformation des reaktionsträgen polyzyklischen aromatischen Kohlenwasserstoffs Phenanthren herbeizuführen. Die dargestellten und ausgewerteten ${ }^{13} \mathrm{C}-\mathrm{NMR}$-Spektren weisen eine Umwandlung der Ausgangssubstanz $\mathrm{zu}$ entsprechenden Oxidationsprodukten nach. Es konnten trotz der teilweise sehr schwachen Signalstärken deutliche Hinweise auf die Entstehung dem biotischen Abbau des Phenanthrens vergleichbarer Verbindungen beobachtet werden.

Die Belegung von Tonmineralen mit aromatischen polyzyklischen Kohlenwasserstoffen führte durch die katalytischen Eigenschaften der Tone im Falle des in dieser Arbeit verwendeten Phenanthrens zur abiotischen Bildung von entsprechenden Oxidationsprodukten bzw. zur teilweisen Aufspaltung der aromatischen Strukturen des PAK. Als Resultat dieser chemischen Transformation bleibt festzuhalten, dass die entstandenen Reaktionsprodukte aufgrund ihrer Zusammensetzung, gegenüber eventuell nachfolgenden Reaktionen mit organischen Komponenten, wesentlich empfindlicher als das reaktionsträge Phenanthren sind. Um diese Ergebnisse nachzuprüfen wurden weitere Analysen durchgeführt, die zum Ziel hatten genau diese Überlegung der erhöhten Reaktivität des Phenanthrens bzw. dessen Transformationsprodukte in Gegenwart von Phenolen sowie deren Umsetzungsprodukte als Modell für die Einbindung organischer Schadstoffe in die organische Substanz des Bodens zu untersuchen.

Bei Belegung des Montmorillonits mit Phenanthren als einem Vertreter der polyzyklischen aromatischen Kohlenwasserstoffe zusätzlich zur Belegung mit Phenolen, lässt sich anhand des ${ }^{13} \mathrm{C}-\mathrm{NMR}$ Spektrums erkennen, dass neben den bereits beschriebenen Reaktionsprodukten neue Peaks, die O-Alkyl-, C=C-Bindungen, aromatischen Strukturen und Carbonylverbindungen zuzuordnen sind, auftreten. Dies weist darauf hin, dass es auf dem mit Pyrogallol und Phenanthren belegten $\mathrm{Na}^{+}$-Montmorillonit zu einer abiotisch induzierten chemischen Reaktion der beiden Substanzen gekommen ist.

Die Ausdehnung der Versuche auf nicht bis gering reaktive organische Stoffe wie PAK konnte in ersten Ergebnissen zeigen, dass durch die Reaktivität der Tonminerale mit adsorbierten Phenolen und Phenanthren die Bildung von neuen Verbindungen mit entsprechenden funktionellen Gruppen und Strukturen eintritt (BIRKEL et al. 1998b, BIRKEL et al. 2000a).

\subsubsection{Brenzkatechin und Phenanthren}

Im Gegensatz zur beobachtbaren, aber recht schwachen Reaktion des auf die Tonmineraloberfläche sublimierten PAK (Abbildungen 119 und 120) war auch bei der gemeinsamen Belegung des Tons mit Phenanthren und mit Brenzkatechin eine intensive braun-schwarze Verfärbung des Tons zu beobachten. Entgegen ersten Annahmen, dass es sich in diesem Falle nur um die farbigen Reaktionsprodukte der, schon bei der Belegung mit dem Phenol gebildeten Verbindungen handelt, konnte durch die Auswertung der folgenden ${ }^{13} \mathrm{C}-\mathrm{NMR}-\mathrm{Spektren}$ ge- 
zeigt werden, dass neben den bereits im ${ }^{13} \mathrm{C}$-NMR-Spektrum der Reaktionsprodukte der abiotischen Transformation des Brenzkatechins ausgewerteten Verschiebungswerte, weitere Signale von verschiedenen C-Bindungen auftreten. Daraus kann die Bildung weiterer Reaktionsprodukte nachgewiesen werden (Tabelle 32).

Tabelle 32: Übersicht der chemischen Verschiebungen und ihre Zuordnungen.

\begin{tabular}{|c|c|c|c|c|c|c|}
\hline \multicolumn{2}{|c|}{$\begin{array}{l}\text { Standards } \\
\text { Brenzkatechin } \\
\text { Phenanthren }\end{array}$} & \multirow[t]{2}{*}{$\begin{array}{l}\text { Montmorill } \\
\text { onit belegt } \\
\text { mit }\end{array}$} & \multicolumn{2}{|c|}{ Brenzkatechin } & \multicolumn{2}{|r|}{$\begin{array}{c}\text { Brenzkatechin + } \\
\text { Phenanthren }\end{array}$} \\
\hline $\mathrm{ppm}$ & Zuordnung & & $\mathrm{ppm}$ & Zuordnung & $\mathrm{ppm}$ & Zuordnung \\
\hline & & & & & 11,4 & $\mathrm{C}-\mathrm{CH}_{3}$ \\
\hline & & & & & 14,4 & $\mathrm{C}-\mathrm{CH}_{3}$ \\
\hline & & & & & 23,2 & C-CH ${ }_{3}$ \\
\hline & & & & & 24,1 & C- $-\mathrm{CH}_{3}$ \\
\hline & & & & & 29,2 & $\mathrm{C}-\mathrm{CH}_{3}$ \\
\hline & & & & & 30,1 & C-CH ${ }_{2}$ \\
\hline & & & & & 30,6 & C-CH ${ }_{2}$ \\
\hline \multirow[t]{3}{*}{39,5} & DMSO-d6 & & 39,5 & DMSO-d6 & 39,5 & DMSO-d6 \\
\hline & & & & & 43,5 & $\mathrm{C}-\mathrm{CH}_{2}$ \\
\hline & & & & & 45,5 & $\mathrm{C}-\mathrm{CH}_{2}$ \\
\hline \multirow[t]{15}{*}{49,5} & Methanol & & 49,5 & Methanol & 49,5 & Methanol \\
\hline & & & 51,8 & $\mathrm{O}-\mathrm{CH}_{3}$ & & \\
\hline & & & 52,4 & $\mathrm{O}-\mathrm{CH}_{3}$ & & \\
\hline & & & 53 & $\mathrm{O}-\mathrm{CH}_{3}$ & & \\
\hline & & & & & 54,5 & $\mathrm{O}-\mathrm{CH}_{3}$ \\
\hline & & & & & 56,5 & $\mathrm{O}-\mathrm{CH}_{3}$ \\
\hline & & & & & 58 & $\mathrm{O}-\mathrm{CH}_{3}$ \\
\hline & & & 59,8 & $\mathrm{O}-\mathrm{CH}_{3}$ & & \\
\hline & & & & & 68,4 & $\mathrm{CH}_{2}-\mathrm{OH}$ \\
\hline & & & 85,8 & $\mathrm{C}-\mathrm{OH}$ & 85,8 & $\mathrm{C}-\mathrm{OH}$ \\
\hline & & & & & 101,5 & Alkene \\
\hline & & & 102,8 & Alkene & 102,8 & Alkene \\
\hline & & & 105,4 & Alkene & 105,4 & Alkene \\
\hline & & & & & 111,1 & Aromat $\mathrm{C}=\mathrm{C}$ \\
\hline & & & & & 114,3 & Aromat $\mathrm{C}=\mathrm{C}$ \\
\hline \multirow{2}{*}{115,7} & Ring (C3,C6) & & 116,4 & Ring (C3,C6) & 116,5 & Ring (C3,C6) \\
\hline & & & 118,1 & Aromat $\mathrm{C}=\mathrm{C}$ & 118 & Aromat $\mathrm{C}=\mathrm{C}$ \\
\hline 119,3 & Ring (C4,C5) & & 120,4 & Ring (C4,C5) & 120,4 & Ring (C4,C5) \\
\hline 122,8 & Ring (C7) & & & & 123 & Ring \\
\hline 126,6 & Ring (C5) & & & & 124 & Aromat $\mathrm{C}=\mathrm{C}$ \\
\hline 126,8 & Ring (C4) & & & & 127,5 & Ring (C4,C5) \\
\hline 128,4 & Ring (C3) & & & & 129,5 & Ring (C3) \\
\hline 129 & Ring (C2) & & & & 130,5 & Ring (C2) \\
\hline \multirow[t]{3}{*}{131} & Ring (C1) & & & & 132,4 & Ring (C1) \\
\hline & & & 133,2 & Ring-OH & & \\
\hline & & & 144,9 & Ring-OH & 144,9 & Ring-OH \\
\hline \multirow[t]{5}{*}{145,3} & Ring-OH $(\mathrm{C} 1, \mathrm{C} 2)$ & & 145,8 & Ring-OH $(\mathrm{C} 1, \mathrm{C} 2)$ & 145,8 & Ring-OH $(\mathrm{C} 1, \mathrm{C} 2)$ \\
\hline & & & & & 168 & $\mathrm{C}=\mathrm{O}$, Carboxyl \\
\hline & & & 170,2 & Carboxyl & 170,2 & Carboxyl \\
\hline & & & 183,5 & Chinon & 183,5 & Chinon \\
\hline & & & 196,9 & Aldehyd & 196,9 & Aldehyd \\
\hline
\end{tabular}

Im Vergleich zum ${ }^{13} \mathrm{C}$-NMR-Spektrum der Reaktionsprodukte des Brenzkatechins - dessen Verschiebungswerte wurden aus Gründen der Übersichtlichkeit hier nochmals dargestellt fallen in diesem Spektrum (Abbildung 124) besonders die Vielzahl von Signalen im Ver- 
schiebungsbereich der Methylgruppen $\left(-\mathrm{CH}_{3}\right)$ zwischen 11,4 ppm und 29,2 ppm auf. Zusätzlich sind Peaks für $\mathrm{C}-\mathrm{CH}_{2}$-Bindungen ab $30 \mathrm{ppm}$ bis $50 \mathrm{ppm}$ zu beobachten. Zwischen 55 und $58 \mathrm{ppm}$ werden mehrere $\mathrm{O}-\mathrm{CH}_{3}$-Bindungen detektiert. Signale in diesem Verschiebungsbereich repräsentieren Methyl-C von Methylgruppen, die an aromatische Ringe gekoppelt sind (WERSHAW et al. 1996). Der Wert bei 68,4 ppm weist die Existenz von $\mathrm{CH}_{2}-\mathrm{OH}-\mathrm{Bindungen}$ nach. Diese hohe Zahl an Signalen, die charakteristisch für die Methylierung bzw. Alkylierung der anfangs ausschließlich vorhandenen aromatischen Substanzen sind, zeigt, dass die chemischen Strukturen der beiden Ausgangskomponenten - diese enthalten zu Beginn keine der nun nachgewiesenen Bindungstypen (Substituenten) - weitgehend verändert wurden. Das es weiterhin zu einer Veränderung der aromatischen Strukturen gekommen sein muss, kann durch das Auftreten neuer Verschiebungswerte im Zuordnungsbereich der Aromaten zwischen 100 und 130 ppm belegt werden: bei 111,1 ppm, 114,3 ppm und 124 ppm sind neu auftretende Signale für $\mathrm{C}=\mathrm{C}$-Bindungen von aromatischen Systemen zu beobachten. Von Bedeutung erscheint in diesem Verschiebungsbereich, dass das Signal bei 126,6 ppm, welches der $\mathrm{C}=\mathrm{C}$-Bindung in Position $\mathrm{C} 5$ zugeordnet ist (Tabelle 20) in diesem Spektrum nicht mehr nachgewiesen werden kann. Daraus kann geschlossen werden, dass die aromatische Struktur des Phenanthrens an dieser Position verändert worden ist. Dies kann durch die Substituierung des Gerüsts erfolgen. Als weiteres Indiz für diese Annahme ist das Auftreten der Signale von O-Alkylbindungen.

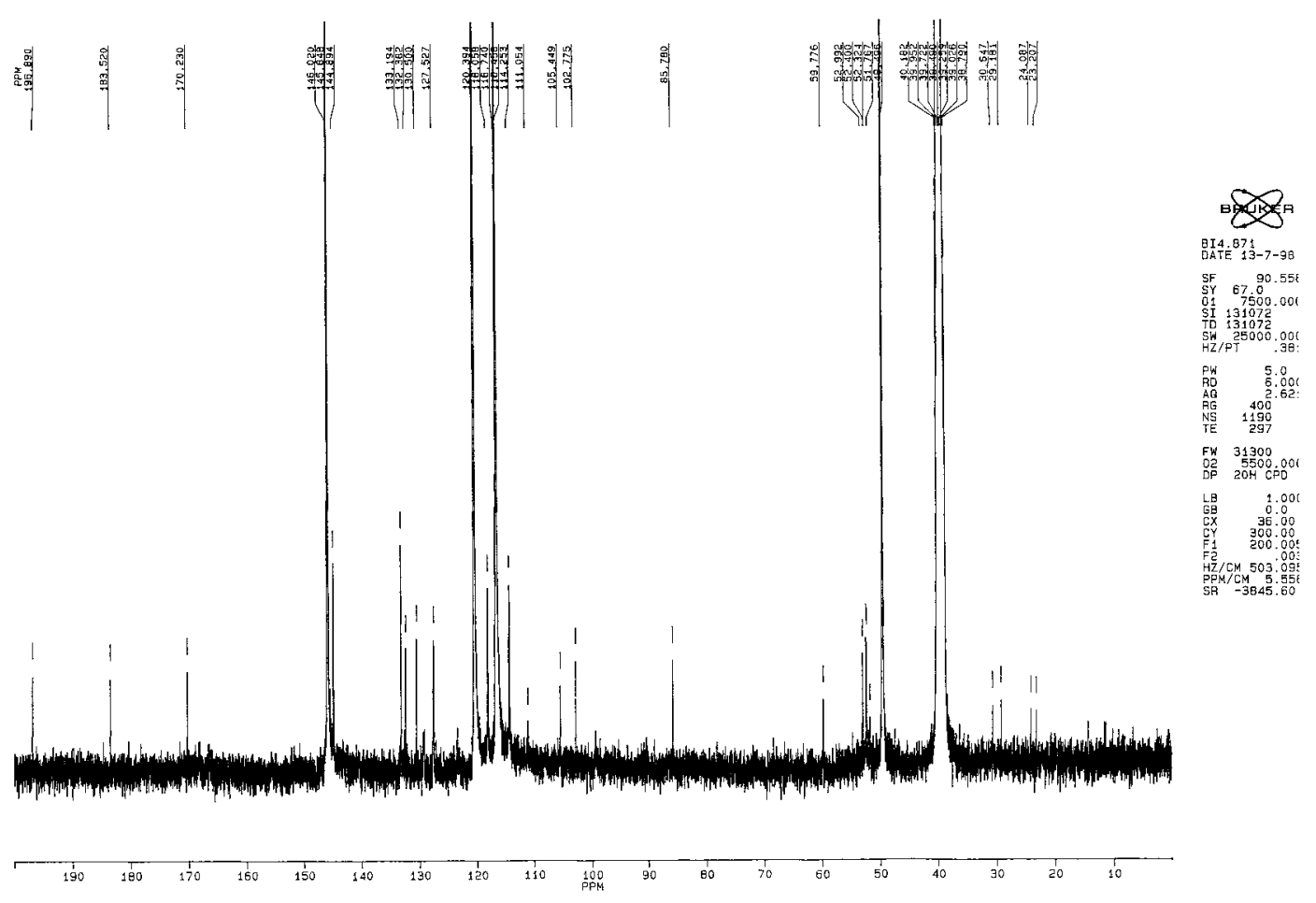

Abbildung 125: Na-Montmorillonit belegt mit Phenanthren und Brenzkatechin.

Die Oxidation des Phenanthrens kann in Gegenwart des Brenzkatechins zur Kopplung des polyzyklischen aromatischen Kohlenwasserstoffs mit dem Phenol über die neu entstandenen 
Substituenten, hier die im Spektrum nachgewiesenen $\mathrm{C}_{-} \mathrm{CH}_{2^{-}}(43,5 \mathrm{ppm}, 45,5 \mathrm{ppm})$ bzw. $\mathrm{CH}_{2}$-O-Gruppen $(68,4 \mathrm{ppm})$ führen.

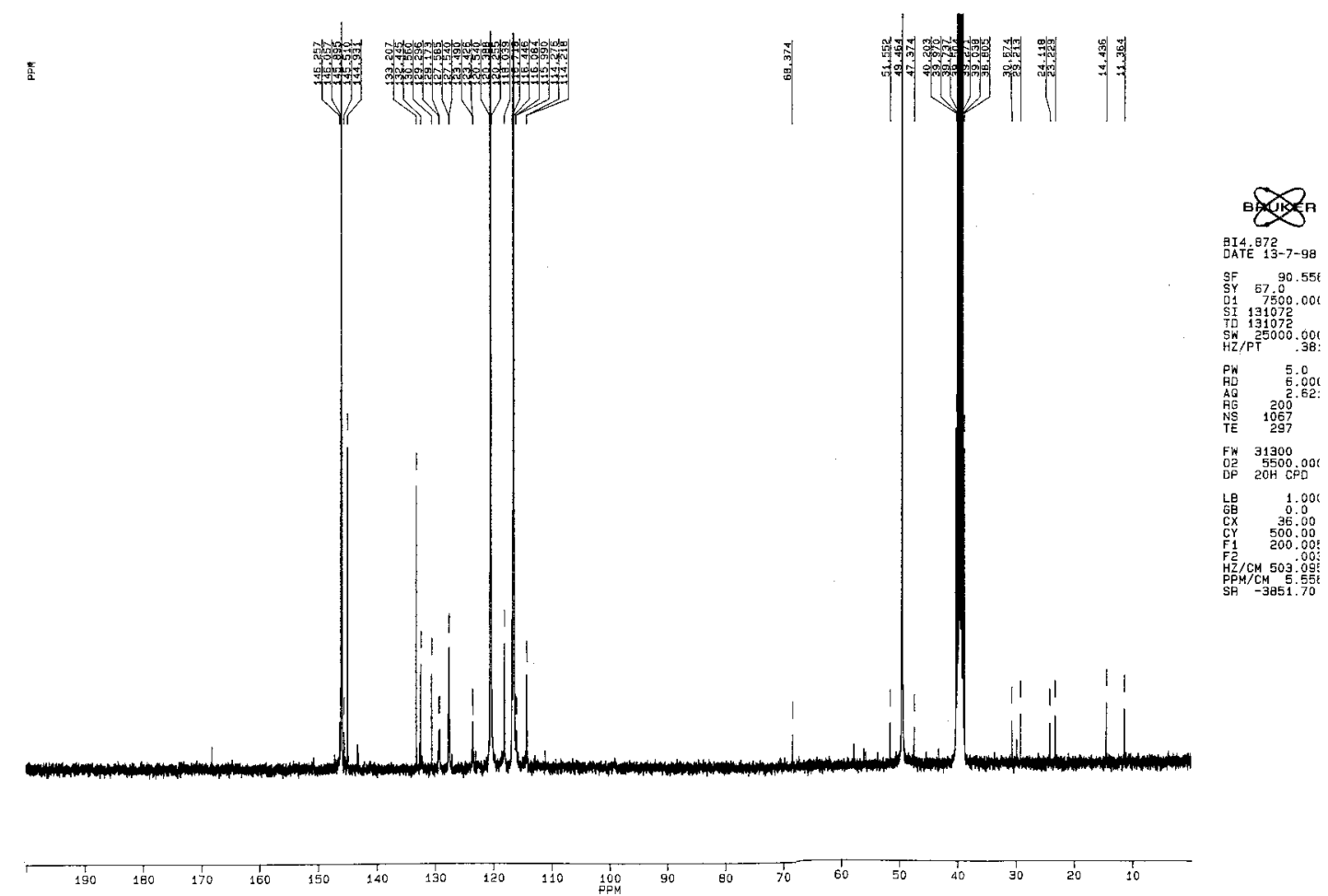

Abbildung 126: ${ }^{13} \mathrm{C}-\mathrm{NMR}$ Spektrum der extrahierten Überzüge des mit Phenanthren und Brenzkatechin belegten Ca-Montmorillonits.

Das Signal bei 168 ppm charakterisiert eine Carbonylbindung $(\mathrm{C}=\mathrm{O})$. Der Verschiebungsbereich zwischen 160 und 175 ppm ist speziell den Carboxylgruppen zuzuordnen. Das Auftreten solcher Bindungen weist, wie bereits bei der Charakterisierung der Reaktionsprodukte des Phenanthrens (Kapitel 2.2) dargestellt, die Oxidation des Ausgangssubstrates nach. Es hat den Anschein, dass diese Oxidationsprozesse in Anwesenheit eines organischen Reaktionspartners (hier: Phenol) zu einer verstärkten Umwandlung des polyzyklischen aromatischen Kohlenwasserstoffs führen. Durch die Eigenschaften des Brenzkatechins, i. e. seine Fähigkeit stark reaktive phenolische Radikale zu bilden, die umgebende Phenolmoleküle bzw. weitere organische Verbindungen zu oxidieren vermögen, ist nicht nur die oxidative Modifikation des Phenanthrens und Kopplung an das Phenol bzw. dessen chinoide Oxidationsprodukte anzunehmen, sondern auch der Einbau des PAK bzw. seiner Oxidationsprodukte in höherpolymere Verbindungen gegeben. Somit ist davon auszugehen, dass der Kontakt des Phenanthrens mit Brenzkatechin an der Tonmineraloberfläche die im Kapitel 2.2 angenommene These der Modifikation organischer Schadstoffe und ihre Festlegung in die organische Substanz des Bodens eine Bestätigung erfahren hat.

\subsubsection{Pyrogallol und Phenanthren}

Die Sublimation von Pyrogallol und Phenanthren auf die Tonmineraloberfläche zeigt, wie be- 
reits bei der Belegung mit dem Phenol, eine intensive Braunfärbung des Tons. Die Analyse der Extrakte dieser Überzüge ergab im ${ }^{13} \mathrm{C}$-NMR Spektrum im Vergleich zu den bereits dargestellten Ergebnissen der Reaktionsprodukte des Pyrogallols (Kapitel 4.1.4) einige charakteristische Signale neu auftretender C-Bindungstypen.

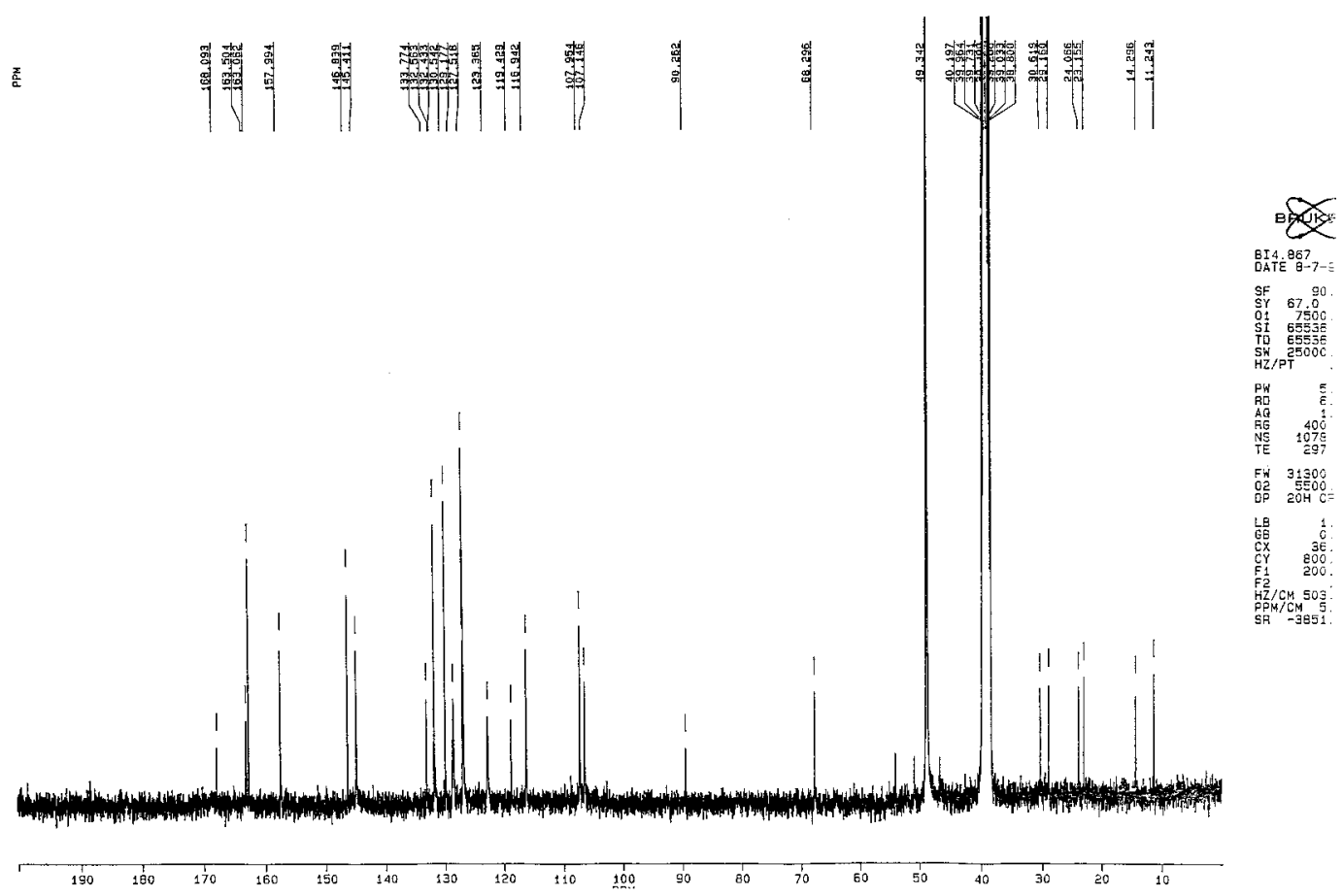

Abbildung 127: ${ }^{13}$ NMR-Spektrum Extrakte von Ca-Montmorillonit belegt mit Phenanthren und Pyrogallol.

Die in Tabelle 33 aufgelisteten Verschiebungswerte und deren Zuordnungen zeigen neben den, der besseren Übersicht wegen nochmals beschriebenen Peaks des Pyrogallols bzw. seiner Reaktionsprodukte wiederum eine Vielzahl von Signalen, die sich über den gesamten Bereich des Spektrums erstrecken. Wie im vorangegangenen Kapitel fällt wiederum die Vielzahl von Signalen der Methylbindungen auf. Diese liegen im Bereich von 11,1 ppm bis 24,1 ppm. Der Grund für das Auftreten von Methylgruppen kann, wie bereits dargestellt in der Substitution des Phenanthrens begründet als auch durch die teilweise Aufspaltung der aromatischen Strukturen herbeigeführt worden sein. Für die Bildung von Substituenten am Ringsystem der PAK spricht auch das Auftreten der $\mathrm{CH}_{2}-\mathrm{OH}$-Bindung bei 68,3 ppm sowie vorbehaltlich der Nachweis von O-Alkyl-Bindungen. Dieses Signal reicht jedoch mit 90,3 ppm auch in den Verschiebungsbereich der Dreifachbindung von C-Atomen (Alkine) hinein und kann somit nicht eindeutig einer Alkylbindung zugeordnet werden. Im Verschiebungswertebereich aromatischer $\mathrm{C}=\mathrm{C}$-Bindungen sind neben den Peaks für die Gerüstinformation des Pyrogallols zwei weitere Signale zu erkennen. 
Tabelle 33: Übersicht der chemischen Verschiebungen und ihre Zuordnungen.

\begin{tabular}{|c|c|c|c|c|c|c|}
\hline \multicolumn{2}{|c|}{$\begin{array}{l}\text { Standards } \\
\text { Pyrogallol } \\
\text { Phenanthren } \\
\end{array}$} & \multirow[t]{39}{*}{$\begin{array}{l}\text { Bentonit } \\
\text { belegt mit }\end{array}$} & \multicolumn{2}{|r|}{ Pyrogallol } & \multicolumn{2}{|c|}{ Pyrogallol + Phenanthren } \\
\hline \multirow[t]{10}{*}{ ppm } & Zuordnung & & $\mathrm{ppm}$ & Zuordnung & $\mathrm{ppm}$ & Zuordnung \\
\hline & & & & & 11,2 & $\mathrm{C}-\mathrm{CH}_{3}$ \\
\hline & & & 16,9 & $\mathrm{C}-\mathrm{CH}_{3}$ & 14,3 & $\mathrm{C}-\mathrm{CH}_{3}$ \\
\hline & & & & & 23,2 & $\mathrm{C}-\mathrm{CH}_{3}$ \\
\hline & & & & & 24,1 & $\mathrm{C}-\mathrm{CH}_{3}$ \\
\hline & & & 29,4 & $\mathrm{C}-\mathrm{CH}_{3}$ & 29,2 & $\mathrm{C}-\mathrm{CH}_{3}$ \\
\hline & & & & & 30,6 & $\mathrm{C}-\mathrm{CH}_{2}$ \\
\hline & & & 31,7 & $\mathrm{C}-\mathrm{CH}_{2}$ & & \\
\hline & & & 34,3 & $\mathrm{C}-\mathrm{CH}_{2}$ & & \\
\hline & & & 36,4 & $\mathrm{C}-\mathrm{CH}_{2}$ & & \\
\hline 39,5 & DMSO-d6 & & 39,5 & DMSO-d6 & 39,5 & DMSO-d6 \\
\hline \multirow[t]{7}{*}{49,5} & Methanol & & 49,5 & Methanol & 49,5 & Methanol \\
\hline & & & 53,1 & $\mathrm{O}-\mathrm{CH}_{3}$ & & \\
\hline & & & 55,2 & $\mathrm{O}-\mathrm{CH}_{3}$ & & \\
\hline & & & 61,5 & $\mathrm{O}-\mathrm{CH}_{3}$ & & \\
\hline & & & & & 68,3 & $-\mathrm{CH}_{2}-\mathrm{OH}$ \\
\hline & & & 70,2 & O-Alkyl (HC-OH) & & \\
\hline & & & & & 90,3 & O-Alkyl o. Alkin \\
\hline \multirow[t]{2}{*}{107,2} & Ring (C4) & & 106,1 & Ring (C4) & 107,1 & Ring (C4) \\
\hline & & & & & 107,9 & Ring, Alken \\
\hline \multirow[t]{2}{*}{118,5} & Ring (C3) & & 116,1 & Ring (C3) & 116,9 & Ring (C3) \\
\hline & & & & & 119,4 & Ring \\
\hline \multirow[t]{2}{*}{122,8} & Ring (C7) & & & & 123,4 & Ring (C7) \\
\hline & & & 124,8 & aromat. Ring & & \\
\hline 126,6 & Ring (C5) & & 126,3 & aromat. Ring & & \\
\hline 126,8 & Ring (C4) & & & & 127,5 & Ring (C4,C5) \\
\hline 128,4 & Ring (C3) & & & & 129,2 & Ring (C3) \\
\hline 129 & Ring (C2) & & & & 130,5 & Ring (C2) \\
\hline 131 & Ring (C1) & & & & 132,4 & Ring (C1) \\
\hline \multirow[t]{2}{*}{133,2} & Ring-OH (C2) & & 132,1 & Ring-OH (C2) & 132,6 & Ring-OH (C2) \\
\hline & & & & & 133,8 & Ring-OH \\
\hline \multirow[t]{7}{*}{146,3} & Ring-OH (C1) & & 144,9 & Ring-OH (C1) & 145,4 & Ring-OH (C1) \\
\hline & & & & & 146,8 & Ring-O \\
\hline & & & 152,5 & Ring-O & & \\
\hline & & & 158,6 & Ring-O & 158 & Ring-O \\
\hline & & & 162,5 & $\mathrm{C}=\mathrm{O}$ & 163,1 & $\mathrm{C}=\mathrm{O}$, Carboxyl \\
\hline & & & & & 163,5 & $\mathrm{C}=\mathrm{O}$, Carboxyl \\
\hline & & & & & 168,1 & $\mathrm{C}=\mathrm{O}$, Carboxyl \\
\hline
\end{tabular}

Die chemische Verschiebung bei 107,9 ppm beschreibt mit einer neu auftretenden $\mathrm{C}=\mathrm{C}$ Bindung ebenso wie bei 119,4 ppm eine Veränderung in der aromatischen Struktur der beiden Ausgangssubstanzen. Die bei der Belegung mit Pyrogallol aber ohne Phenanthren detektierten Verschiebungswerte von 124,8 ppm und 126,3 ppm sind hier nicht mehr zu beobachten. Dies ist ein Hinweis darauf, dass die Anwesenheit des PAK auch die Entstehung der Reaktionsprodukte des Phenols beeinflusst und zumindest teilweise andere Verbindungen gebildet werden. Im Bereich der aromatischen C-O-Bindungen wurden neue Signale bei 133,8 ppm und 146,8 ppm festgestellt. Dabei handelt es sich normalerweise um an aromatische Strukturen $(\mathrm{C}=\mathrm{C}$ Bindungen) gebundene $\mathrm{OH}-$ Gruppen. Einen weiteren Beweis für die Beeinflussung der Transformation von Phenolen in Anwesenheit von Phenanthren liefert der fehlende Verschie- 
bungswert der C-O-Bindung bei 152,5 ppm. Gemeinsam mit den nicht mehr auftretenden aromatischen $\mathrm{C}=\mathrm{C}$-Bindungen bei $124,8 \mathrm{ppm}$ und 126,3 ppm kann daraus abgeleitet werden, dass die durch Reaktion des Pyrogallols an der Tonmineraloberfläche entstandene Verbindung in Gegenwart des Phenanthrens mit diesem bzw. mit dessen Transformationsprodukten zu einer neuen Substanz reagiert, die entsprechend veränderte Verschiebungswerte und damit $\mathrm{C}$-Bindungen enthält. Diese modifizierten Strukturen werden durch die erwähnten aromatischen $\mathrm{C}=\mathrm{C}$-Bindungen von 107,9 ppm und 119,4 ppm sowie durch die C-O-Bindungen bei 133,8 ppm und 146,8 ppm nachgewiesen. Mit den Peaks bei 163,5 ppm und 168,1 ppm wurden $\mathrm{C}=\mathrm{O}$-Doppelbindungen, speziell Carboxylgruppen detektiert. Diese Signale sind weder bei den Reaktionsprodukten des Pyrogallols noch bei denen des Phenanthrens zu beobachten, der Peak bei 168,1 ppm wurde jedoch nach der gemeinsamen Belegung des Tons mit Phenanthren und Brenzkatechin nachgewiesen. Somit kann davon ausgegangen werden, dass auch hier die gemeinsame Reaktion des PAK mit dem Phenol zur Entstehung gleicher CBindungen und damit teilweise gleicher Reaktionsprodukte geführt hat.

Zusammenfassend bleibt festzuhalten, dass der Kontakt von Phenanthren mit dem Phenol die oxidative Transformation des PAK fördert und zugleich die der Umsetzungsprodukte des Pyrogallols stark beeinflusst. Dieses Ergebnis stützt die These, dass beide Substanzen bzw. ihre Reaktionsprodukte miteinander zu höhermolekularen Verbindungen reagieren. Auch die Resultate der Reaktion von Brenzkatechin mit Phenanthren bestätigen diese Überlegungen. Die Substanzen reagieren zum Teil miteinander zu neuen chemischen Verbindungen, jedoch sind die entsprechenden Ergebnisse im Falle der Reaktion von Pyrogallol mit Phenanthren deutlich stärker ausgeprägt. Es ist davon auszugehen, dass die Reaktion von Pyrogallol mit Phenanthren zur Entstehung von einem oder mehreren Reaktionsprodukten, hierfür sprechen auch die Ergebnisse der HPLC-Analysen (Kapitel 4.5), unter (An-)Kopplung des Phenols bzw. seiner Reaktionsprodukte an das aromatische System des PAK geführt haben. Die teilweise Aufspaltung aromatischer Strukturen kann mit Hilfe der neu auftretenden Signale für Methylgruppen zwischen 10 und 25 ppm interpretiert werden, da diese häufig mit den auch neu hinzugekommenen O-Alkyl-Bindungen als Resultate der Spaltung aromatischer Systeme verbunden sind.

\subsubsection{Diskussion der Ergebnisse der ${ }^{13} \mathrm{C}$-NMR spektroskopischen Untersuchungen}

Die ${ }^{13} \mathrm{C}$-NMR-spektroskopischen Untersuchungen der extrahierten Reaktionsprodukte der Belegung von Tonmineralen mit Phenolen und einem PAK wurden mit dem Ziel durchgeführt, genauere Hinweise auf die strukturelle Veränderung der Organika bzw. auf die Zusammensetzung der entstandenen Reaktionsprodukte der sublimierten Ausgangssubstanzen zu gewinnen und wenn möglich die ersten, mit Hilfe der FT-IR-Spektroskopie erzielten Resultate zu überprüfen und zu erhärten. Diese Zielsetzung konnte - trotz einiger analysentechnischer Schwierigkeiten, wie vor allem die zu schwachen Probenkonzentrationen aufgrund sehr geringer Ausbeute an verwertbarer Substanz und die daraus resultierenden langen Analysenzei- 
ten mit den damit verbundenen Nachteilen im Signal-/Rauschverhältnis der Spektren - weitgehend erreicht werden. Die Auswertung der ${ }^{13} \mathrm{C}$-NMR Spektren der von den Tonmineralen extrahierten Überzüge ergab eine Vielzahl von chemischen Veränderungen an den auf die Tonminerale sublimierten Ausgangssubstanzen. Bei allen drei Phenolen (Brenzkatechin, Pyrogallol und 2,6-Dimethylphenol) konnte eine strukturelle Transformation durch oxidative Prozesse nachgewiesen werden. Außerdem sind, insbesondere bei der Analyse der Verschiebungswerte des 2,6-Dimethylphenols, Kopplungsvorgänge der einzelnen aromatischen Monomere hin zu höhermolekularen Einheiten beobachtet worden. Dadurch konnte gezeigt werden, dass Tonminerale als mineralische, abiotische Komponente des Bodens in der Lage sind, an sie adsorbierte organische Substanz durch physikochemische Prozesse zu katalysieren. Weiterhin sind Reaktionsprodukte entstanden, die zumindest vergleichbar mit den Metaboliten biotischer Abbau- bzw. Transformationsprozesse organischer (Schad-)Stoffe sind. Das beobachtete Spektrum der gebildeten Verbindungen reicht, wie vorab beschrieben, von Methylgruppen, O-Alkyl-Bindungen, Aromaten bis hin zum Nachweis von Carbonylbindungen von Carboxylgruppen, Aldehyden und Chinonen. Daraus kann geschlossen werden, dass es neben einer Zunahme der Substituierung der Aromaten zur Veränderung der aromatischen Strukturen der Ausgangssubstanzen und schließlich zur Oxidation der Phenole bzw. des Polyzyklischen Aromatischen Kohlenwasserstoffs gekommen sein muss. Außerdem ist eine, zumindest teilweise Aufspaltung aromatischer Strukturen durch das Auftreten von MethylBindungen belegt worden (BIRKEL \& NIEMEYER 1998b). Das Ziel mit Hilfe der ${ }^{13} \mathrm{C}-\mathrm{NMR}$ Spektroskopie eine vollständige Strukturaufklärung der entstandenen Reaktionsprodukte der auf die Tonminerale sublimierten Organika zu betreiben und die, die Überzüge färbenden Substanzen eindeutig zu identifizieren, konnte nicht ganz erreicht werden. Die Gründe hierfür sind vor allem die systembedingte relative Unempfindlichkeit der ${ }^{13} \mathrm{C}-\mathrm{NMR}$ Spektroskopie. Die schon sehr geringe Ausbeute an extrahiertem Probenmaterial war gerade ausreichend, um jeweils Übersichtsspektren des Gesamtsubstanzgemisches aufzunehmen. Auch aus diesem Grunde war eine eindeutige Strukturaufklärung nicht möglich. Die als Lösung dieses Problems durchgeführte Fraktionierung der Probengemische durch chromatographische Trennverfahren (Kapitel 4.5 und 4.6) erzielte gute Resultate, die jedoch aufgrund der extrem geringen Konzentrationen in den einzelnen Fraktionen sowie aus systemtechnischen Gründen keine, für die ${ }^{13} \mathrm{C}$-NMR-Spektroskopie ausreichenden Probenmengen zu liefern vermochten. Optimale Methoden wie die Abtrennung großer Probenvolumina mit Hilfe der FlüssigChromatographie standen leider nicht zur Verfügung. Es bleibt jedoch festzuhalten, dass die angewendete Analysenmethode trotz der erwähnten Nachteile, zu eindeutigen Ergebnissen, insbesondere unter Zuhilfenahme IR-spektroskopischer und massenspektrometrischer Verfahren hinsichtlich der Transformation und der Zusammensetzung der entstandenen Reaktionsprodukte geführt hat. 


\subsection{Massenspektrometrische Untersuchungen}

Die massenspektrometrische Untersuchung der von den Tonmineraloberflächen extrahierten Organika zielt auf eine bessere und genauere Charakterisierung der abiotisch gebildeten, insbesondere der beobachteten polymerisierten Reaktionsprodukte (BICKE \& METZGER 1993, KRÜGER 1995, WU \& ODOM 1998, POTTHAST et al. 1999). Durch die Untersuchungen mittels Infrarot-Spektroskopie und ${ }^{13} \mathrm{C}-\mathrm{NMR}-$ Spekroskopie konnten nur eingeschränkt Aussagen zur Zusammensetzung und zum Aufbau. Dies liegt in der Problematik der Überlagerung von Spektreninformationen durch die extrahierten Substanzgemische begründet. Die chromatographische Trennung der Gemische gestaltete sich als recht schwierig und resultierte zum großen Teil in einer zu geringen Ausbeute an gewonnenen Substanzen. Mit Hilfe der Massenspektrometrie können die extrahierten Substanzgemische, bestehend aus der Ausgangssubstanz und den entstandenen Reaktionsprodukten, direkt, anhand ihrer Massengewichte bestimmt werden. Zur Anwendung kamen zwei Analyseverfahren: Matrix Assisted Laser Desorption Ionisation Time of Flight Mass Spectrometry (MALDI-TOF-MS) Gaschromatographie-MS (GC-MS), Atmospheric Pressure Chemical Ionisation (APCI) und HPLC-MS. Die Analysen wurden aufgrund der Kooperationen mit Herrn Dr. Habicher (MALDI) von der Abteilung Organische Chemie der TU Dresden sowie mit der Arbeitsgruppe Organische Chemie, Prof. Dr. Metzger der Universität Oldenburg von Herrn Dipl.Chemiker Griep-Raming durchgeführt.

Die dargestellten Ergebnisse stellen Resultate erster, probeweise durchgeführter Untersuchungen dar. Sie konnten aufgrund projekttechnischer Gründe noch nicht vollständig durchgeführt werden, wurden aber aufgrund der Bedeutung ihrer Ergebnisse schon in diese Arbeit aufgenommen.

\subsubsection{Matrix Assisted Laser Desorption Ionization Time of Flight Mass Spectrometry}

Bei diesem Untersuchungsverfahren handelt es sich um eine leistungsfähige Methode, die angenommene Bildung von kondensierten Polymeren zu analysieren. MALDI-TOF-MS wird erfolgreich als recht neue Methode zur Bestimmung der Massen großer Biomoleküle und synthetischer Polymere angewendet (BAHR et al. 1994, KARAS \& BAHR 1996, PASCH et al. 1996). Mit dieser Methode können Massen bis zu $300 \mathrm{kDa}$ nachgewiesen werden (WU \& ODOM 1998), andere Quellen nennen - in Abhängigkeit vom Analysator - bis zu 500 kDa, routinemäßig 5 bis $100 \mathrm{kDa}$ (Polymere, Biomoleküle, Komplexe, Enzyme) (KARAS \& BAHR 1996).

\subsubsection{Funktionsprinzip des MALDI-Flugzeitmassenspektrometers}

Das Target in das Massenspektrometer eingeschleust. Für die Bestrahlung der Probe wird in der Regel ein Stickstofflaser (Wellenlänge: 337 nm, Pulsbreite: 3-4 ns) verwendet. Durch den Beschuss mit Laserlicht werden Matrix- und Probenmoleküle ins Vakuum desorbiert und ionisiert (Abbildung 128). 


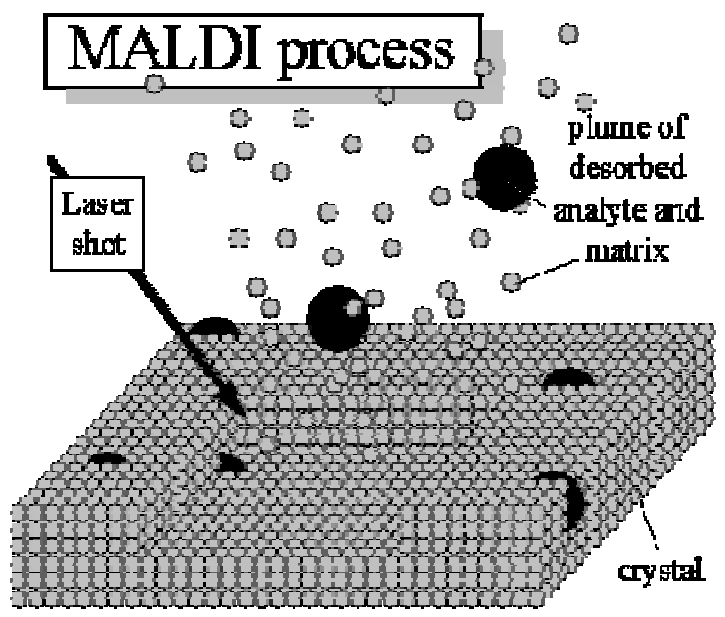

Abbildung 128: Prozess der Desorption und Ionisation beim MALDI (Quelle: Abteilung Massenspektrometrie, Organische Chemie Uni Bielefeld).

Der Prozess der Ionisierung ist noch nicht vollständig verstanden. Die Desorption der vergleichsweise kleinen Matrixmoleküle gelingt durch das lokale Erhitzen mit Laserlicht. Dabei werden die größeren Analytmoleküle mitgerissen. Die zweite Funktion des Lasers neben der Desorption ist die Photoionisation der Matrixmoleküle (KARAS \& BAHR 1997). Durch die elektronische Anregung wird eine Photo-Ionisation der Matrixmoleküle $\left(\mathrm{MH}^{\mathrm{n}^{*}}\right)$ induziert; im positiven Ionenmodus führt dies zu Elektronenabstraktion und daher zu positiv geladenen Matrixradikalen (1). Durch Stoß mit diesen wird auf ein ungeladenes Matrix (2a) - oder Analytmolekül (A) (2b) ein Proton übertragen. Hierbei entstehen zum einen protonierte Matrixbzw. Analytmoleküle und zum anderen werden ungeladene, deprotonierte Matrixradikale gebildet. Die protonierten Matrixmoleküle können nun ihrerseits Protonen auf ungeladene Analytmolekülen transferieren und somit ebenso die gewünschten Analytsignalen zum Ergebnis haben (3):

(1) $\mathrm{MH}^{\mathrm{n}^{*}} \rightarrow$ $\mathrm{MH}^{+\bullet}+\mathrm{e}^{-}$

(2a) $\mathrm{MH}^{+\bullet}+$

$\mathrm{A} \quad \rightarrow$

$[\mathrm{A}+\mathrm{H}]^{+}+$

$[\mathrm{M}-\mathrm{H}]^{\bullet}$

(2b) $\mathrm{MH}^{+\bullet}+$

$\mathrm{MH} \rightarrow$

$[\mathrm{MH}+\mathrm{H}]^{+}+$

(3) $[\mathrm{MH}+\mathrm{H}]^{+}$

$\mathrm{A} \quad \rightarrow \quad[\mathrm{A}+\mathrm{H}]^{+}$

$+$

[MH].

Die Ionisierung von Analytmolekülen (A) durch elektronisch angeregte Matrixmoleküle (MHn*) im MALDI-Prozess (positiver Ionenmodus). Die MALDI-Technik lässt sich mit einem Time-of-Flight (TOF)-Analysator oder einem Fourier-Transform-Massenspektrometer koppeln. Die mit der MALDI-Ionisationsmethode erzeugten Molekülionen können grundsätzlich mit diversen Massenanalysatoren analysiert werden. Als gepulste Desorptions/Ionisationsmethode bietet sich jedoch MALDI für die Kopplung zu einem Flugzeitmassenspektrometer (Time-of-flight mass spectrometer, TOF-MS) an, da diese Art des Massenanalysators einen diskontinuierlichen Ionenstrahl erfordert. Bei einem Flugzeitmassenspektrometers ist - im Vergleich zu vielen anderen Massenanalysatoren - ein sehr großer, zugänglicher Massenbereich gegeben. Weiterhin kann das Spektrum in extrem kurzen Analy- 
sezeiten aufgenommen werden, da alle gebildeten Ionen innerhalb ihrer sehr kurzen Flugzeit registriert werden können. Eine Akkumulation über viele Einzelspektren ermöglicht die Verbesserung des Signal-Rausch-Verhältnisses (PFENNINGER 2000).

Im Hochvakuum $\left(<10^{-4} \mathrm{~Pa}\right)$ wird die Probe mit gepulstem Laserlicht von wenigen Nanosekunden Dauer bestrahlt. Daraufhin kann die Desorption und Ionisation der Analytmoleküle erfolgen. Die Ionen gelangen nach der Beschleunigung in die feldfreie Driftstrecke, an deren Ende (Abbildung 129) sich entweder ein Reflektor zur Verbesserung der Massenauflösung oder direkt der Detektor befindet. Dabei dient die Flugzeit der im elektrischen Feld beschleunigten Ionen bis zum Auftreffen auf den Detektor zur Bestimmung ihres Verhältnisses von Masse zu Ladung (m/z).

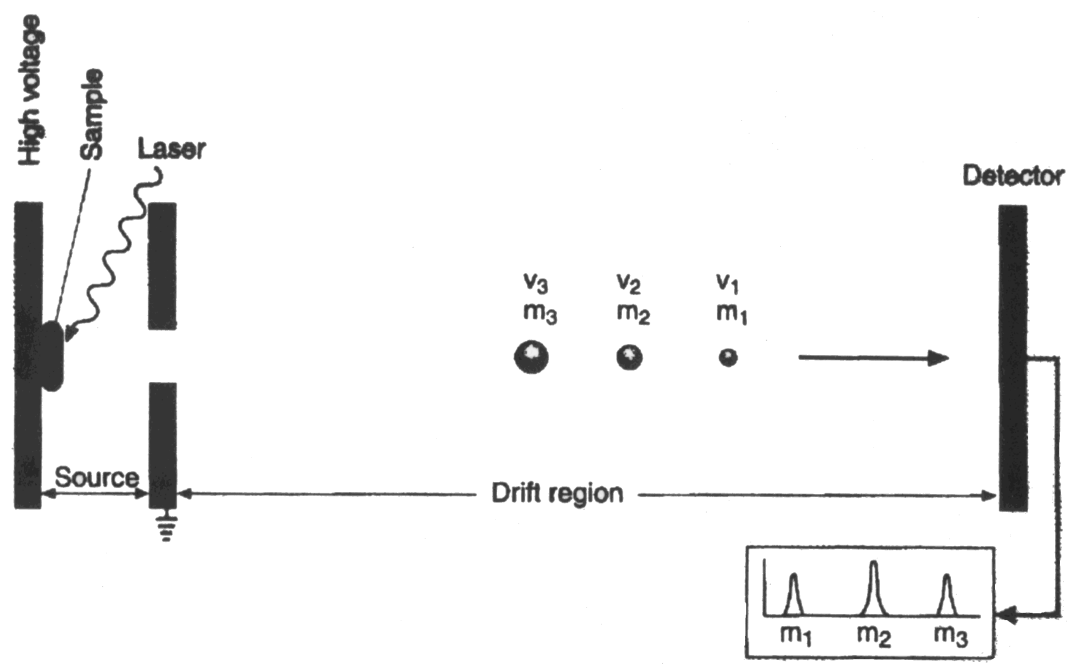

Abbildung 129: Schematische Darstellung eines TOF-Massenspektrometers (WU \& ODOM 1998).

Eine Kalibrierung der Massenskala mit bestmöglicher Massengenauigkeit erfolgt über die Bestimmung von Eichsubstanzen bekannter Massen. In den meisten Anwendungsfällen wird eine Massengenauigkeit von bis zu 100 ppm erreicht, die ebenso mit einer verzögerten Extraktion der MALDI-Ionen auf $<10$ ppm gesteigert werden kann. Für eine MALDI-Analyse werden in der Regel pMol bis fMol Substanzmenge benötigt; in einzelnen Fällen kann die Empfindlichkeit sogar auf weniger als 10 amol beziffert werden.

\subsubsection{Probenpräparation}

Der betreffende Analyt wird in einem niedrig siedenden Lösungsmittel (Wasser, Methanol, Acetonitril, Aceton, Hexan etc.) gelöst. Die Probenaufarbeitung mit nicht flüchtigen Salzen ist zu vermeiden. Es sollten flüchtige Salze wie Ammoniumformiat, Ammoniumacetat, Ammoniumhydrogencarbonat verwendet werden. Wenn möglich, sollten die Proben angesäuert werden, dazu sind leicht flüchtige organische Säuren wie Essigsäure oder Trifluoressigsäure geeignet). Die Konzentration sollte zwischen ca. $10^{-6}-10^{-8}$ mol/l liegen (Lösung A). Die Matrix (z.B. 2,5-Dihydroxybenzoesäure (DHB), Sinapinsäure, Ferulasäure, 1,8,9-Trihydroxy- 
anthracen, 4-Nitroanilin, etc.) wird in einem ähnlichen Lösungsmittel gelöst mit einer Konzentration von 5 bis $10 \mathrm{mg} / \mathrm{ml}$ (Lösung B). Die Lösungen A und B werden im Verhältnis 1:1 gemischt. Von der Mischung wird $1 \mu \mathrm{l}$ auf den MALDI-Probenträger aufgetragen. Die Lösung wird langsam getrocknet, so dass sich ein Kristallfilm auf dem Target bildet. Als Matrix dienen in der Regel kleine, aromatische Moleküle, die folgende Mindestvoraussetzungen erfüllen müssen (PFENNIGER 2000):

- starke Absorption der eingestrahlten Laserlichtwellenlänge (UV oder IR),

- Vakuumstabilität (geringe Sublimationsrate),

- Inertie gegenüber dem Analyt,

- Löslichkeit in einem verdampfbaren Lösungsmittel.

Neben der Absorption des Laserlichts ist es eine weitere Funktion der Matrix, die Probe zu verdünnen. Ein 100- bis 1000-facher molarer Überschuss der Matrix liefert die besten Ergebnisse; als Grund dafür wird die Aufhebung intermolekularer Wechselwirkungen zwischen den Analytmolekülen angesehen (PFENNINGER 2000). Die Konzentrationen der Analytlösungen liegen typischerweise bei $10^{-5}$ bis $10^{-8} \mathrm{~mol} / \mathrm{l}$, so dass Matrixkonzentrationen von $10^{-2}$ bis $10^{-6}$ mol/l zum Einsatz kommen. Bei der Oberflächenpräparation erfolgt die Trocknung in zwei Schritten; zunächst wird eine geringes Volumen der Matrixlösung (typischerweise $1 \mu \mathrm{l}$ ) auf den Probenteller aufgebracht und zur vollständigen Trocknung eingedampft. Auf die entstandene (fein-)kristalline Oberfläche wird Analytlösung aufgebracht und wiederum getrocknet. Um ein Auflösen der Matrix im Analytlösungsmittel zu vermeiden, darf die Matrix darin nicht bzw. in nur sehr geringem Maß löslich sein.

\subsubsection{Atmospheric Pressure Chemical Ionization}

Atmospheric Pressure Chemical Ionization (APCI) ist ein Verfahren, durch chemische Ionisation von gelöstem Probenmaterial (Lösemittel: Methanol) bei Atmosphärendruck durch direkte Injektion in ein CI-Plasma Ionen durch Ion-Molekül-Reaktionen zu produzieren.

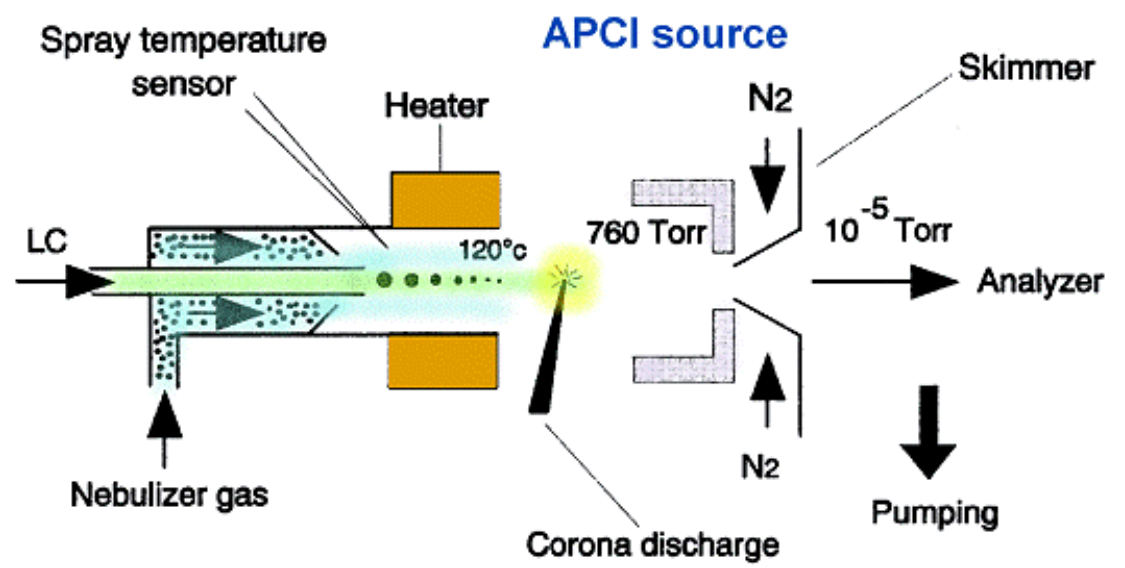

Abbildung 130: Funktionsprinzip eines APCI (Quelle: Mass Spectrometry Facility, Institut für Organische Chemie, Universität Heidelberg) 
Das Plasma wird durch eine elektrische Entladung zwischen Spray-Kapillare und Gegenelektrode aufrecht erhalten. Die entstandenen Ionen gelangen in den Massenanalysator durch Verwendung mehrerer Skimmer-Ebenen (Abbildung 130). Ein Gegenstrom von trockenem und vorgeheiztem Stickstoff wird zur Entfernung des Lösemittels verwendet.

\subsubsection{Gas-Chromatographie}

Die Untersuchungen der Proben mittels Gas-Chromatographen mit gekoppeltem Massenspektrometer (GC-MS) wurden freundlicherweise ebenfalls von Herrn Griep-Raming durchgeführt. Dazu wurde ein GC-MS-System mit Elektronenstoßionisation (EI) sowie mit chemischer Ionisation (CI: Reaktandgas: iso-Butan) verwendet.

\subsubsection{Ergebnisse der Untersuchungen durch MALDI-TOF-MS}

Die massenspektrometrischen Untersuchungen der extrahierten organischen Überzüge der mit Brenzkatechin belegten Montmorillonite mittels MALDI wurden von Herrn PD Dr. Habicher, Organische Chemie der Universität Dresden durchgeführt. Das Probenmaterial wurde auf zwei verschiedene Matrices aufgetragen (A1: 1,8,9-Trihydroxyanthracen, W2: 4-Nitroanilin), Methanol wurde als Lösemittel verwendet. Mit einem MALDI-TOF-MS (Kratos Kompact MALDI III) wurde bei einer Wellenlänge von $337 \mathrm{~nm}$ desorbiert/ionisiert und anschließend die massenspektrometrische Analyse durchgeführt.

\subsubsection{Brenzkatechin auf Na-Montmorillonit}

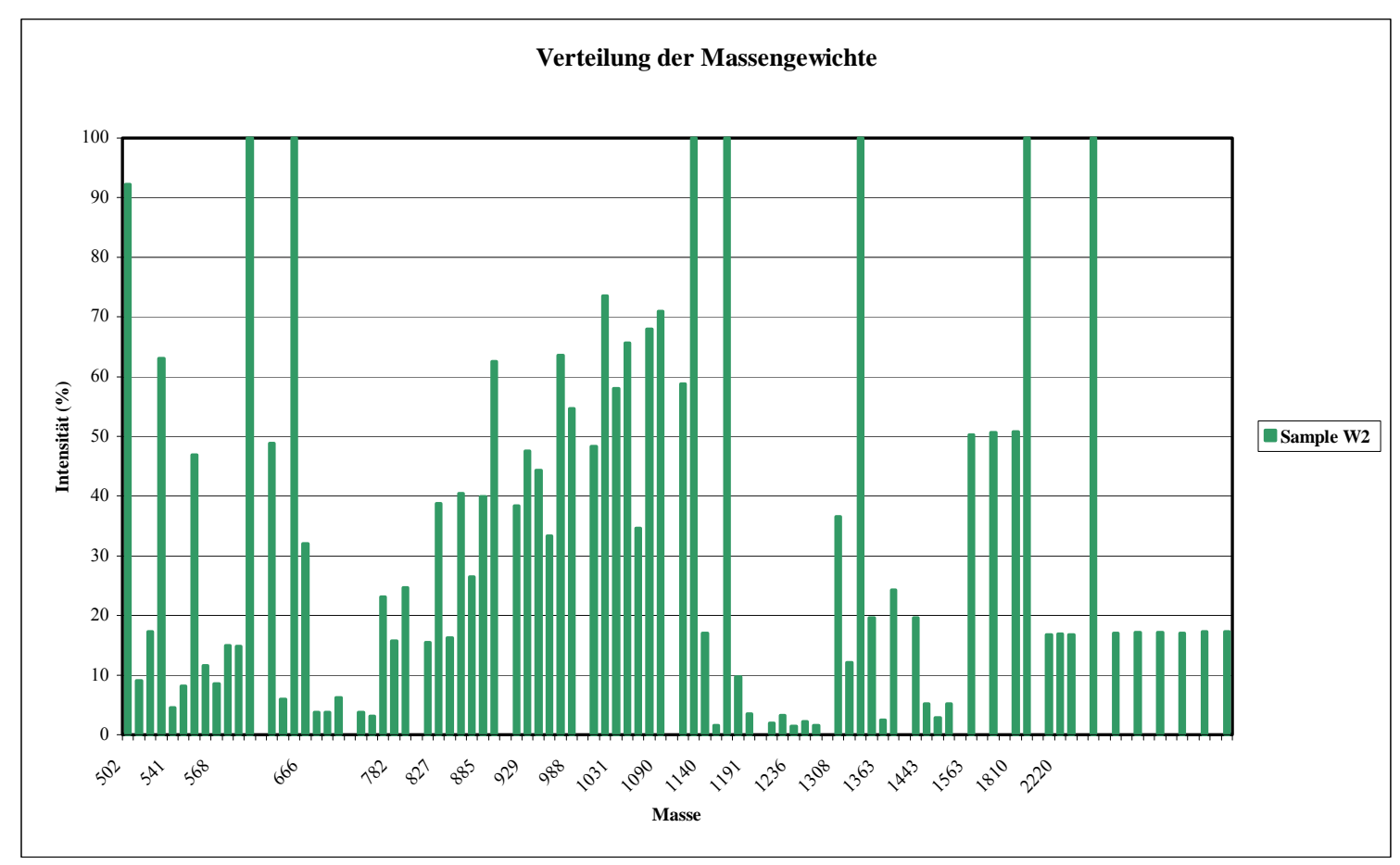

Abbildung 131: Verteilung der Massengewichte des mit Brenzkatechin belegten Na-Montmorillonits. 
Die Bestimmung der Molmassen der entstandenen Reaktionsprodukte der abiotischen Reaktion von Brenzkatechin an Tonmineraloberflächen zeigte Massenspektren der extrahierten Lösungen, die eine große Spannweite aufweisen. Die gemessenen Massen erstrecken sich bei der Probe W2 (Na-Montmorillonit) von 502 bis zu 3823 Da (Abbildung 131). Auffällig ist die deutliche Scharung von Peaks zwischen 813 und 1104 Da, wobei die detektierten Massen jeweils Inkremente von 13 bis $16 \mathrm{Da}$, mit einem Schwerpunkt bei $14 \mathrm{Da}$, aufweisen (Tabelle 34: grün unterlegter Bereich).

Tabelle 34: Übersicht der Massengewichte und Signalintensitäten der Probe W2.

\begin{tabular}{|c|c|c|c|c|c|}
\hline \multicolumn{6}{|l|}{ Probe W2 } \\
\hline Masse [Da] & Intensität [\%] & Masse [Da] & Intensität [\%] & Masse [Da] & Intensität [\%] \\
\hline 502 & 92,2 & 870 & 26,51 & 1308 & 36,5 \\
\hline 512 & 9,1 & 885 & 39,9 & 1329 & 12,2 \\
\hline 522 & 17,3 & 899 & 62,47 & 1342 & 100 \\
\hline 529 & 63,1 & 913 & 38,32 & 1351 & 19,6 \\
\hline 541 & 4,5 & 929 & 47,51 & 1363 & 2,4 \\
\hline 546 & 8,2 & 944 & 44,36 & 1385 & 24,3 \\
\hline 552 & 46,9 & 957 & 33,33 & 1430 & 19,7 \\
\hline 563 & 11,6 & 973 & 63,52 & 1443 & 5,2 \\
\hline 568 & 8,5 & 988 & 54,59 & 1472 & 2,9 \\
\hline 573 & 15 & 1002 & 48,29 & 1481 & 5,2 \\
\hline 581 & 14,9 & 1016 & 73,49 & 1563 & 50,3 \\
\hline 591 & 100 & 1031 & 58,01 & 1644 & 50,6 \\
\hline 610 & 48,9 & 1045 & 65,62 & 1810 & 50,8 \\
\hline 623 & 5,9 & 1061 & 34,65 & 1832 & 100 \\
\hline 638 & 100 & 1075 & 67,98 & 2172 & 16,8 \\
\hline 666 & 32 & 1090 & 70,87 & 2220 & 16,9 \\
\hline 683 & 3,7 & 1104 & 58,79 & 2289 & 16,8 \\
\hline 688 & 3,8 & 1121 & 100 & 2365 & 100 \\
\hline 694 & 6,2 & 1140 & 17,1 & 2631 & 17 \\
\hline 710 & 3,7 & 1149 & 1,6 & 2902 & 17,2 \\
\hline 731 & 3,1 & 1162 & 100 & 3190 & 17,2 \\
\hline 752 & 23,1 & 1177 & 9,7 & 3222 & 17,1 \\
\hline 782 & 15,75 & 1191 & 3,5 & 3791 & 17,3 \\
\hline 796 & 24,67 & 1209 & 2 & 3823 & 17,3 \\
\hline 813 & 15,49 & 1221 & 3,2 & & \\
\hline 827 & 38,8 & 1236 & 1,4 & & \\
\hline 841 & 16,3 & 1253 & 2,2 & & \\
\hline 856 & 40,42 & 1297 & 1,5 & & \\
\hline
\end{tabular}

Diese Massengewichte entsprechen, bei Beschränkung der Betrachtung auf die organischen Elemente Kohlenstoff, Wasserstoff und Sauerstoff, einer CH-Gruppe (13 Da), einer $\mathrm{CH}_{2}$ Gruppe (14 Da), einer $\mathrm{CH}_{3}$-Gruppe (15 Da) bzw. einem Sauerstoffatom (16 Da). Diese Ergebnisse können dahingehend interpretiert werden, dass durch den Beschuss mit dem Laserstrahl des MALDI in der Probe vorhandene hochpolymere Verbindungen in kleinere Einheiten zerfallen, wobei jeweils eine $\mathrm{CH}-, \mathrm{CH}_{2}$ - oder $\mathrm{CH}_{3}$-Gruppe bzw. ein Sauerstoffatom abgespalten wird. Die um diese Bruchstücke reduzierten Polymere werden anschließend durch 
das Massenspektrometer erfasst. Versucht man aus den Massegewichten der jeweils abgespaltenen Bruchstücke auf die Struktur der polymeren Ausgangssubstanz zu schließen, so erscheint es am wahrscheinlichsten, dass es sich dabei um langkettige aliphatische Verbindungen handelt. Durch die Bestrahlung dieser Aliphate werden Glieder der langkettigen Verbindung abgespalten. Eine Darstellung des Zerfalls zeigt Abbildung 132.

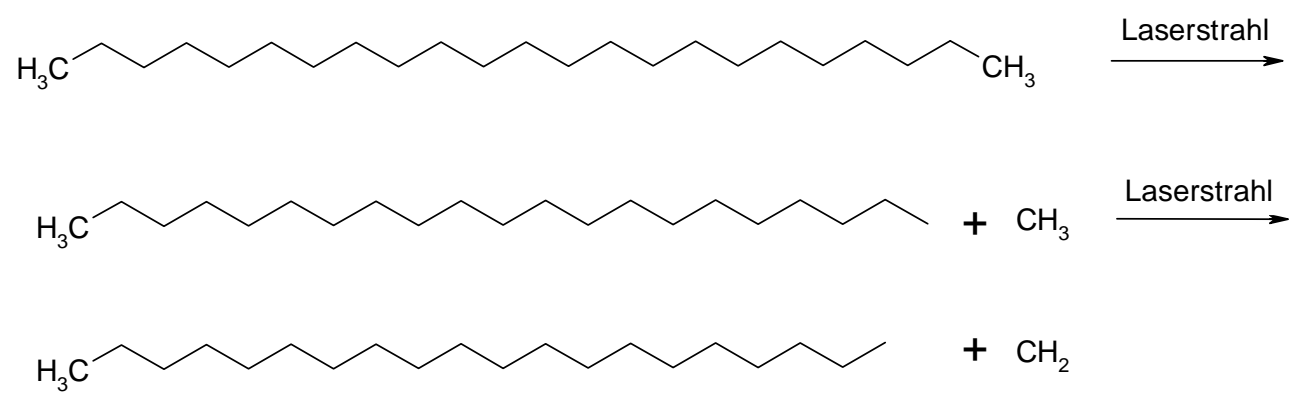

Abbildung 132: Zerfall langkettiger Aliphate durch Laserbeschuss.

Darüber hinaus ist jedoch auch, ausgehend von hochpolymeren aromatischen Reaktionsprodukten der Belegung von Montmorillonit mit Brenzkatechin, die Zerstörung aromatischer Strukturen durch Beschuss mit einer hochenergetischen Strahlungsquelle möglich, wobei als direkte Folge wiederum Molekülbruchstücke mit geringen Massegewichten abgespalten werden können und die um diese Anteile reduzierten Verbindungen detektiert werden.

Die Reaktion des Brenzkatechins an der Oberfläche von Tonmineralen führt somit nicht nur zur Bildung hochpolymerer Substanzen mit einem breiten Massenspektrum, es ist weiterhin davon auszugehen, dass zumindest ein Teil der gebildeten Polymere eine relativ geringe Stabilität aufweisen und durch äußere Einflüsse, wie beispielsweise durch Bestrahlung mit Licht, wiederum in kleinere Einheiten zerfallen können. $\mathrm{Ob}$ und in wie weit solche Prozesse in situ auch an der Tonmineraloberfläche stattfinden und dadurch eine ständige Neu- bzw. Umbildung des adsorbierten organischen Materials an der mineralischen Oberfläche erfolgt und damit ein Beitrag zur Bildung von immer höher polymerisiertem Material geleistet wird, ist durch weitere Untersuchungen zu klären.

\subsubsection{Brenzkatechin auf Ca-Montmorillonit}

Die Belegung des Phenols Brenzkatechin auf einen Ca-Montmorillonit und die anschließende Analyse der extrahierten organischen Überzüge wurde zusätzlich vorgenommen, um den etwaigen Einfluss der Wertigkeit der Zwischenschicht-Kationen eines Tonminerals (Kapitel 2.1) auf die Entstehung von Reaktionsprodukte und die gebildeten Molekülgrößen zu überprüfen. Aus den Resultaten der zuvor durchgeführten spektroskopischen Untersuchungen konnte festgestellt werden, dass das Zwischenschicht-Kation, war es nun $\mathrm{Na}^{+}$oder $\mathrm{Ca}^{2+}$ keine Auswirkung auf die nachgewiesenen funktionellen Gruppen hatte (Kapitel 4.6/4.7). 
Die detektierten Massen erstrecken sich im Falle der Probe A1 (Ca-Montmorillonit) von 512 auf bis zu 3652 Da (Abbildung 133). Auffallend ist hier nicht nur der zu beobachtende weite Wertebereich, sondern auch die hohen Werte der detektierten Massengewichte der gebildeten Verbindungen. Damit kann eindeutig nachgewiesen werden, dass die Reaktion des Brenzkatechins an der Oberfläche des Ca-Montmorillonits zu einer intensiven Polymerisation des Phenols an der mineralischen Oberfläche geführt hat. Nimmt man vereinfachend an, dass es, ausgehend vom Brenzkatechin mit einer Molmasse von $110 \mathrm{u}$, zur Kopplung (aromatischer) phenolischer bzw. chinoider Strukturen (Kapitel 2.2) gekommen ist, so ergibt sich daraus die Bildung von Polymeren mit 5- bis zu mehr als 30-fachem Molekulargewicht.

Abbildung 133: Massenspektrum des mit Brenzkatechin belegten Ca-Montmorillonits.

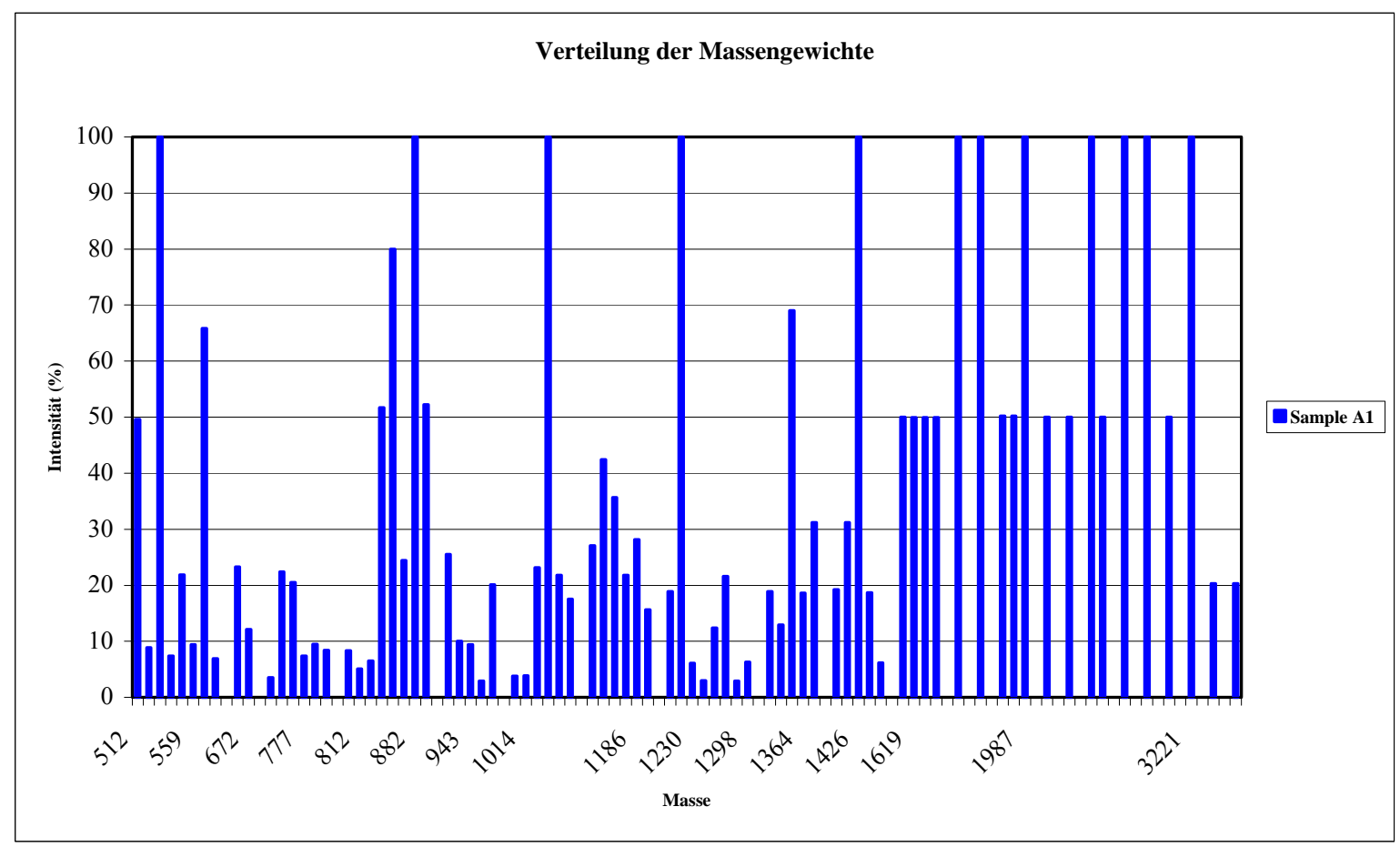

Die Verteilung der Massengewichte in Abbildung 133 zeigt hohe Signalintensitäten bei den Werten 527, 882, 1050, 1216, 1426, 1725, 1810, 1987, 2212, 2365, 2722 und 3221 Da. Mittlere Intensitäten zwischen 40 und $70 \%$ werden bei den Massen 512, 571, 837, 898, 1340, 1619, 1643, 1679, 1908, 1953, 2094, 2183, 2267 und 2864 Da beobachtet. Die Verbindungen mit diesen Massengewichten können als Hauptreaktionsprodukte der abiotischen Reaktion von Brenzkatechin auf der Oberfläche eines Ca-Montmorillonits angesprochen werden. Eine detaillierte Übersicht der detektierten Massengewichte und der zugehörigen Signalintensitäten bietet Tabelle 35 . 
Tabelle 35: Übersicht der Massengewichte und Signalintensitäten der Probe A1.

\begin{tabular}{|c|c|c|c|c|c|}
\hline Probe A1 & \multicolumn{7}{|c|}{} \\
\hline Masse [Da] & Intensität [\%] & Masse [Da] & Intensität [\%] & Masse [Da] & Intensität [\%] \\
\hline 512 & 49,6 & 882 & 100 & 1419 & 31,2 \\
\hline 516 & 8,9 & 898 & 52,22 & 1426 & 100 \\
\hline 527 & 100 & 912 & 25,56 & 1471 & 18,7 \\
\hline 536 & 7,4 & 928 & 10 & 1481 & 6,2 \\
\hline 550 & 21,9 & 943 & 9,44 & 1605 & 50 \\
\hline 559 & 9,4 & 975 & 2,9 & 1619 & 49,9 \\
\hline 571 & 65,8 & 990 & 20,1 & 1643 & 49,9 \\
\hline 590 & 6,9 & 1001 & 3,8 & 1679 & 49,9 \\
\hline 613 & 23,3 & 1014 & 3,9 & 1725 & 100 \\
\hline 672 & 12,1 & 1028 & 23,2 & 1810 & 100 \\
\hline 727 & 3,5 & 1050 & 100 & 1908 & 50,2 \\
\hline 754 & 22,4 & 1071 & 21,8 & 1953 & 50,2 \\
\hline 768 & 20,5 & 1249 & 12,4 & 1987 & 100 \\
\hline 777 & 7,4 & 1262 & 21,6 & 2094 & 50 \\
\hline 781 & 9,5 & 1276 & 2,9 & 2183 & 50 \\
\hline 792 & 8,4 & 1298 & 6,3 & 2212 & 100 \\
\hline 806 & 8,3 & 1319 & 18,9 & 2267 & 50 \\
\hline 812 & 5,1 & 1329 & 12,9 & 2365 & 100 \\
\hline 826 & 6,5 & 1340 & 69 & 2722 & 100 \\
\hline 837 & 51,67 & 1364 & 18,6 & 2864 & 50 \\
\hline 853 & 80 & 1383 & 31,2 & 3221 & 100 \\
\hline 866 & 24,44 & 1401 & 19,2 & 3456 & 20,3 \\
\hline & & & & 3652 & 20,3 \\
\hline & & & & & \\
\hline
\end{tabular}

\subsubsection{Ergebnisse der Untersuchungen mittels GC-MS}

\subsubsection{Brenzkatechin}

Die Analysen der extrahierten Organika des mit Brenzkatechin belegten Na-Montmorillonits ergaben den Nachweis von mindestens neun verschiedenen Substanzen. Neben einem hohen Anteil an nicht umgesetzter Ausgangssubstanz (Brenzkatechin) mit einer Masse von $110 \mathrm{u}$ treten Verbindungen mit höheren Massen auf: Diese reichen von 178, 214, 279, 355, 445, 519 bis 593 u. Die Massen zeigen, dass eine Polymerisation des Brenzkatechins eingetreten ist. Die Identifikation der Verbindungen anhand der Molekülmassen erfolgte durch Auswertung von entsprechenden Datenbanken im Internet. Die genutzten Datenbanken sind zum Einen das „NIST Chemistry Webbook“ des National Institute of Standards and Technology (NIST) in den Vereinigten Staaten (http://www.nist.gov) sowie das „Integrated Spectral Data Base System for Organic Compounds“ (SDBS) des National Institute of Materials and Chemical Research in Japan (http://www.sdbs.jp). Die nachfolgend aufgelisteten Ergebnisse der Datenbankrecherchen erheben keinen Anspruch auf Vollständigkeit. Es wurden lediglich die Resultate aufgenommen, die von ihrer chemischen Zusammensetzung bzw. von der der Ausgangssubstanz her in Frage kamen.

Für die detektierten Molmassen wurden in diesen Datenbanken die folgenden Entsprechungen 
gefunden:

Molmasse $110 \mathrm{u}$ : Brenzkatechin (m/w: 110,1)

Molmasse $178 \mathrm{u}$ : nach Mitteilung von Herrn Griep-Raming handelt es sich Phenanthren. Aufgrund des Reaktionsweges des Brenzkatechins ist das Vorkommen von Phenanthren lediglich als Verunreinigung möglich. Somit ist von anderen Reaktionsprodukten auszugehen, es kann sich um Verbindungen handeln, die aus der Kopplung von zwei aromatischen Systemen hervor gegangen sind, zudem ist die Möglichkeit gegeben, dass es sich beim entstandenen Reaktionsprodukt um eine Verbindung handelt, die aus der Oxidation und Spaltung des aromatischen Systems des Phenols resultieren. Beispiele für solche Verbindungen sind in Tabelle 36 dargestellt.

Tabelle 36: Ergebnisse für Reaktionsprodukte mit der Molmasse 178 (Quelle: NIST).

\begin{tabular}{|l|l|l|}
\hline $\begin{array}{l}\text { Dimethyl D-tartrate } \\
\text { Formula: } \mathrm{C}_{6} \mathrm{H}_{10} \mathrm{O}_{6} \\
\text { Molecular Weight: 178.14 }\end{array}$ & $\begin{array}{l}\text { D-Gluconic acid, } \delta \text {-lactone } \\
\text { Formula: } \mathrm{C}_{6} \mathrm{H}_{10} \mathrm{O}_{6}\end{array}$ \\
Molecular Weight: 178.14 & $\begin{array}{l}\text { cis-2-Methoxycinnamic acid } \\
\text { Formula: } \mathrm{C}_{10} \mathrm{H}_{10} \mathrm{O}_{3} \\
\text { CAS Number: 90-80-2 }\end{array}$ \\
Molecular Weight: 178.18 \\
CAS Number: 14737-91-8
\end{tabular}

Die Resultate der Datenbankrecherche für Verbindungen mit der Molmasse $178 \mathrm{u}$ ergaben sowohl aromatische Verbindungen als auch nicht aromatische Substanzen, die aus einer oxidativen Reaktion des Brenzkatechins hervorgegangen sein können. Es konnten chinoide Strukturen (Tabelle 36: Glukonsäure) gefunden werden. Daneben zeigen viele der Verbindungen Reste eines durch Ringspaltung zerstörten aromatischen Ringes, der an einen intakten Benzolring gebunden ist. Diese Ergebnisse bestätigen die bisherigen Überlegungen und Resultate hinsichtlich der oxidativen Spaltung aromatischer Strukturen und der Kopplung von zwei Aromaten bzw. deren Bruchstücke aneinander. Weiterhin belegen sie die aus den IRund ${ }^{13} \mathrm{C}$-NMR-spektroskopischen erhaltenen Resultate der Entstehung von Carbonyl- und 
Carboxylgruppen (Kapitel 4.6/4.7).

Molmasse $214 \mathrm{u}$ :

Die in den Datenbanken gefundenen Entsprechungen zu Verbindungen mit der Molmasse 214 $\mathrm{u}$ sind in Tabelle 37 aufgelistet. Sie zeigen Aromate, die aus mindestens zwei Benzolringen bestehen und zumeist über die Sauerstoffatome des Aromats miteinander verbunden sind. Die aufgeführten Beispiele zeigen wiederum Ester und Carbonsäuren. Dies würde die mit spektroskopischen Methoden gewonnenen Ergebnisse somit bestätigen.

Tabelle 37: Resultate der Datenbankrecherche für Verbindungen mit der Molmasse 214.

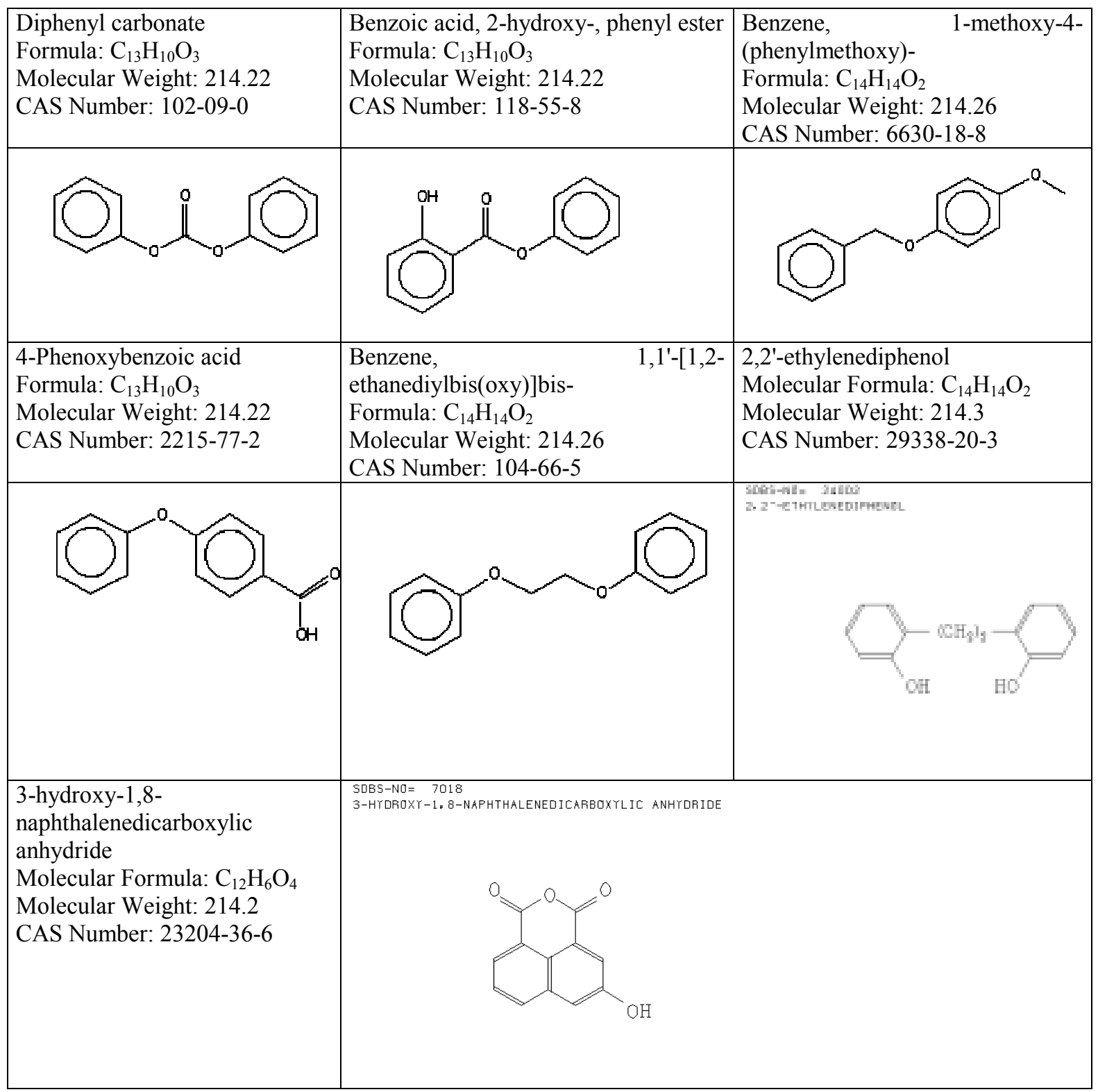

Molmasse $279 \mathrm{u}$ : Hierbei handelt es sich um Phthalsäureester, diese stammen aus den verwendeten Lösemitteln. Diese Erklärung ist aufgrund des Vorkommens in den extrahierten Lösungen der Belegung mit Brenzkatechin als auch mit 2,6-Dimethylphenol plausibel.

In den verwendeten Datenbanken konnten zu den Molmassen von 355, 445, 519 und $593 \mathrm{u}$ unter der Bedingung, dass die entstandenen Verbindungen nur aus Kohlenstoff, Sauerstoff 
und Wasserstoff aufgebaut sind - keine entsprechenden Einträge gefunden werden. Es kann jedoch aus dem Molgewicht abgeleitet werden, dass es sich bei diesen Verbindungen um Reaktionsprodukte, zusammengesetzt aus mehreren Aromaten, handelt. Die Addition der Molmasse der Ausgangssubstanz (Brenzkatechin: $110 \mathrm{u}$ ) gibt darauf deutliche Hinweise. So setzt sich die Verbindung mit der Molmasse von $355 \mathrm{u}$ unter anderem aus drei Benzolringen, jene mit einer Masse von $445 \mathrm{u}$ aus vier, jene mit 519 u unter anderem ebenfalls mindestens aus vier Benzolringen zusammen und die Verbindung mit 593 besteht unter anderem aus zumindest fünf Ringen.

\subsubsection{2,6-Dimethylphenol}

Für die extrahierten Überzüge des mit 2,6-Dimethylphenol belegten Na-Montmorillonits wurden Molmassen mit $122 \mathrm{u}$ (nicht umgesetztes Phenol), 242, 279 und 256 u nachgewiesen. Bei der Substanz mit der Masse von 279 handelt es sich, wie vorab dargestellt um Phthalsäureester aus den verwendeten Lösemitteln. Die Verbindung mit der Molmasse von $242 \mathrm{u}$ entspricht einer Verbindung, die sich aus zwei 2,6-Dimethylphenol-Molekülen über die $\mathrm{OH}$ Gruppe des Phenols verbunden sind. Daraus ergibt sich auch die Verringerung der Molmasse um zwei Wasserstoffatome (242 u statt $244 \mathrm{u}$ ). Die Bildung von Dimeren und Polymeren aus der oxidativen Reaktion von 2,6-Dimethylphenol an Oberflächen von industriellen Katalysatoren sowie durch enzymatische Aktivität wird in chemisch-technischen Synthesen zur Herstellung von Kunststoffen angewendet (IKEDA et al. 1996). Die folgende Abbildung (134) zeigt den Polymerisationsvorgang.

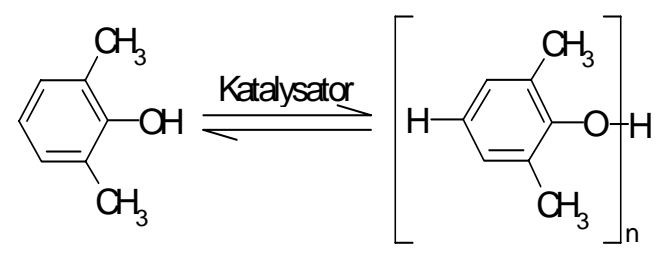

Abbildung 134: Polymerisation von 2,6-Dimethylphenol (nach IKEDA et al. 1996).

Die detektierte Verbindung mit der Molmasse von 256 u stellt ebenfalls ein Reaktionsprodukt der oxidativen Kopplung von zwei Phenol-Molekülen dar. Die Abfrage der Datenbanken ergab folgende Resultate (Tabelle 38). 
Tabelle 38: Verbindungen mit der Molmasse 256.

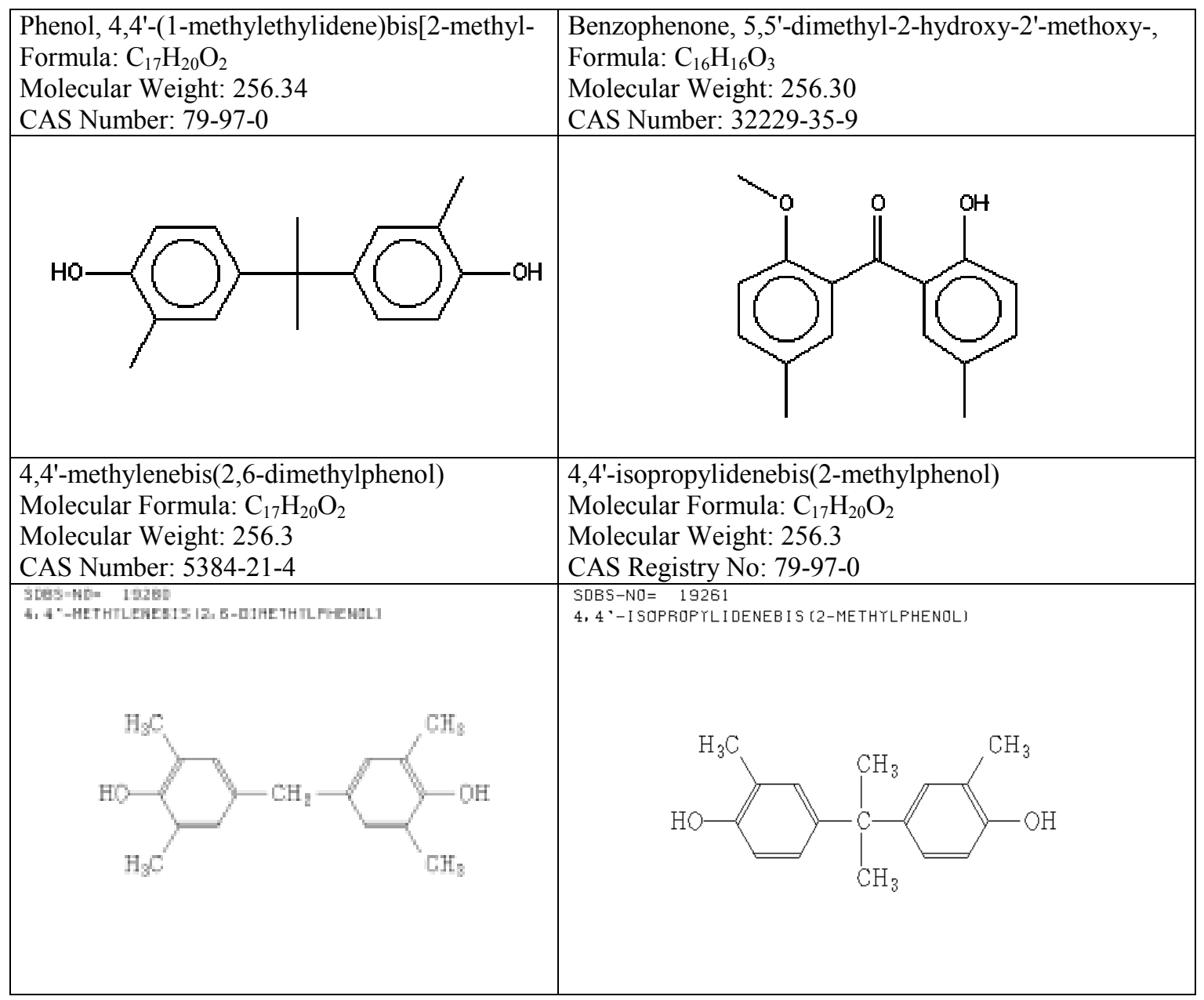

Die in der Tabelle 38 dargestellten Resultate der Datenbankabfrage bestätigen die aus der Literatur bzw. mit spektroskopischen Methoden erzielten Ergebnisse. Sie zeigen Verbindungen, die aus der Kopplung von zwei Phenol-Molekülen aufgebaut und über eine Methylgruppe bzw. direkt am aromatischen Ring über eine C-C-Bindung miteinander verbunden sind.

\subsubsection{Ergebnisse der Untersuchungen mit APCI}

Die Analysen mittels APCI wurden an zwei Phenolen, Brenzkatechin und 2,6Dimethylphenol durchgeführt.

\subsubsection{Brenzkatechin}

Die massenspektrometrischen Untersuchungen mit APCI führten im Falle der extrahierten Überzüge des mit Brenzkatechin belegten Montmorillonits zu detektierten Molmassen von 168, 214, 216, 246, 322, 370 und 662 u. Die zugehörigen Verbindungen wurden wiederum durch Eingabe der Molmassen und des Elementspektrums in die erwähnten Datenbanken ermittelt. Für die detektierten Massen wurden folgende Verbindungen aufgelistet (es ist anzumerken, dass die Ergebnisse der Datenbankrecherchen keinen Anspruch auf eine vollständige Auflistung aller Verbindungen mit entsprechender Masse erheben): 
Verbindungen mit der Molmasse $168 \mathrm{u}$ sind in Tabelle 39 dargestellt. Die gefundenen Substanzen bestehen aus ein bis zwei aromatischen Ringen, es handelt sich um Oxidationsprodukte von Phenolen, darunter ein Chinon (2,6-Dimethoxybenzochinon), eine Carbonsäure (Benzoesäure), Dibenzofuran als Kopplungsprodukt von zwei Benzolringen und ein Cyclohexandion. Diese Verbindungen stellen beispielhaft dar, um welche Verbindunge(n) es sich durch der durch abiotische Reaktion an der Tonmineraloberfläche handeln könnte.

Tabelle 39: Verbindungen mit der Molmasse $168 \mathrm{u}$.

\begin{tabular}{|l|l|}
\hline $\begin{array}{l}\text { 2,6-Dimethoxybenzoquinone } \\
\text { Formula: } \mathrm{C}_{8} \mathrm{H}_{8} \mathrm{O}_{4}\end{array}$ & $\begin{array}{l}\text { Benzoic acid, 4-hydroxy-3-methoxy- } \\
\text { Molecular Weight: 168.15 }\end{array}$ \\
Formula: $\mathrm{C}_{8} \mathrm{H}_{8} \mathrm{O}_{4}$ \\
Molecular Weight: 168.15 \\
CAS Registry Number: 121-34-6
\end{tabular}

Für die Molmasse 214 u können die Ergebnisse der GC-MS-Analysen herangezogen werden. Die Ergebnisse der Recherche zeigt Tabelle 40. Man kann aus den Ergebnissen anhand der obigen Beispiele ableiten, dass es sich bei der detektierten Substanz mit der Masse 214 u um eine Verbindung, bestehend aus bis zu drei Ringen handeln kann. Dabei handelt es sich jedoch nicht um ein Kopplungsprodukt aus zwei Phenol-Molekülen, die um 6 u geringere Molmasse zeigt, dass sechs Wasserstoffatome abgetrennt wurden. 
Tabelle 40: Verbindungen mit der Molmasse $214 \mathrm{u}$.

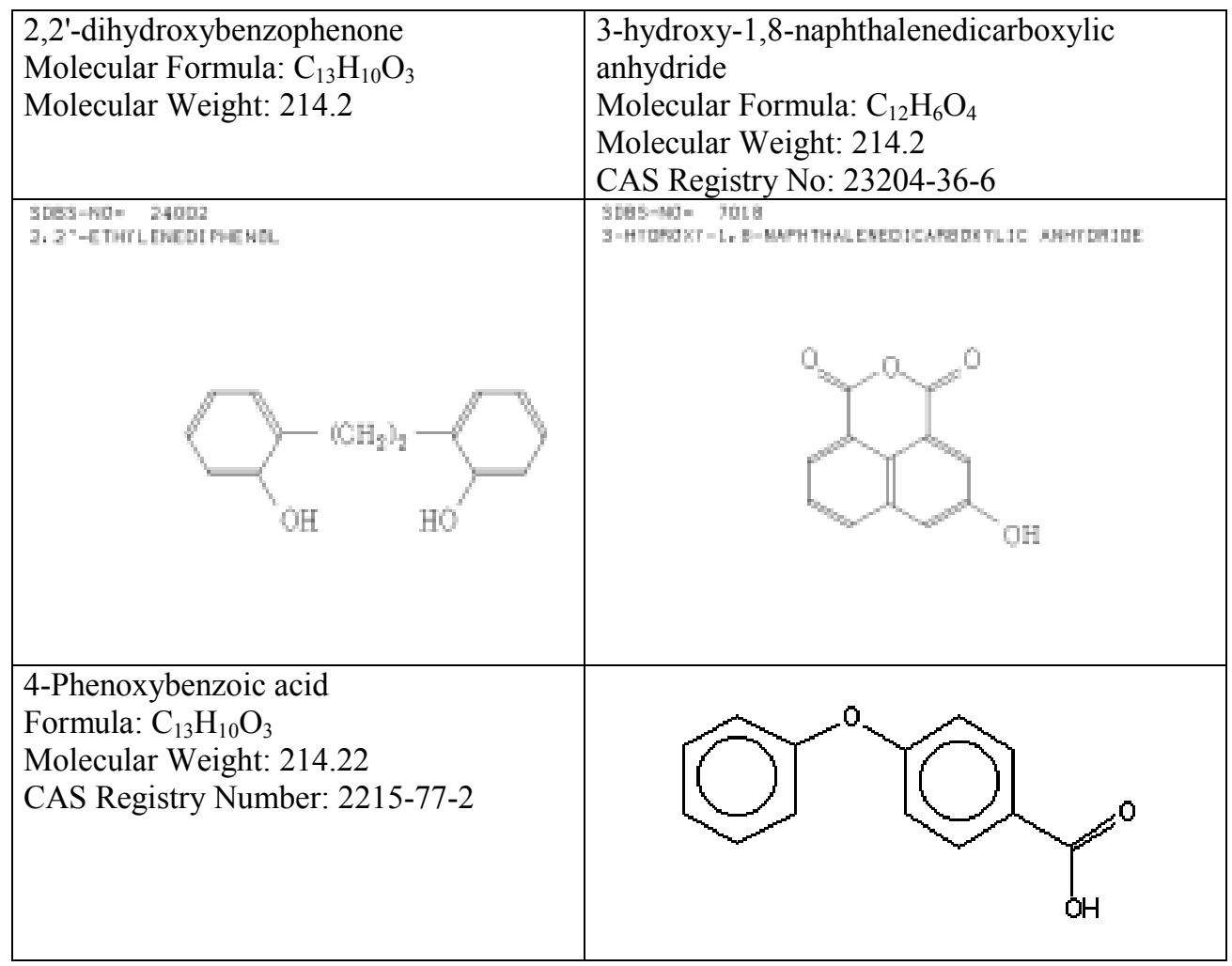

Ein der Molmasse von $216 \mathrm{u}$ entsprechender Eintrag konnte in den Datenbanken nicht ermittelt werden. Es ist jedoch davon auszugehen, dass es sich um ein Kopplungsprodukt bzw. Oxidationsprodukt aus zwei Phenol-Molekülen handeln kann, wobei hier eine Reduzierung um vier H-Atome zusätzlich angenommen werden kann.

Beispiele für Verbindungen mit der Molmasse $246 \mathrm{u}$ sind in Tabelle 41 aufgeführt. Bei der Verbindung mit dieser Masse kann wiederum eine Substanz, bestehend aus zwei PhenolMolekülen angenommen werden. Die unterschiedliche Masse im Vergleich zu einem Dimer mit der Molmasse 218 u kann durch eine abweichende Konfiguration zweier zusätzlicher $\mathrm{CH}_{2}$-Gruppen (28 u) herrühren über die beide Phenol-Moleküle verbunden sein könnten. Schließlich können jedoch auch höher kondensierte Verbindungen mit der entsprechenden Molmasse entstanden sein. Die weiterhin gefundenen Entsprechungen zu den Molmassen sind keine Polymerisationsprodukte des Brenzkatechins, sondern es handelt sich hierbei um nichtaromatische, aliphatische Säuren und Ester. Die als Spalt- bzw. Zerfallprodukte der Reaktion von Phenolen an Tonmineraloberflächen auftreten. Die große Vielzahl dieser Verbindungen konnte im Rahmen dieser Arbeit nicht dargestellt werden. 
Tabelle 41: Verbindungen mit der Molmasse $246 \mathrm{u}$.

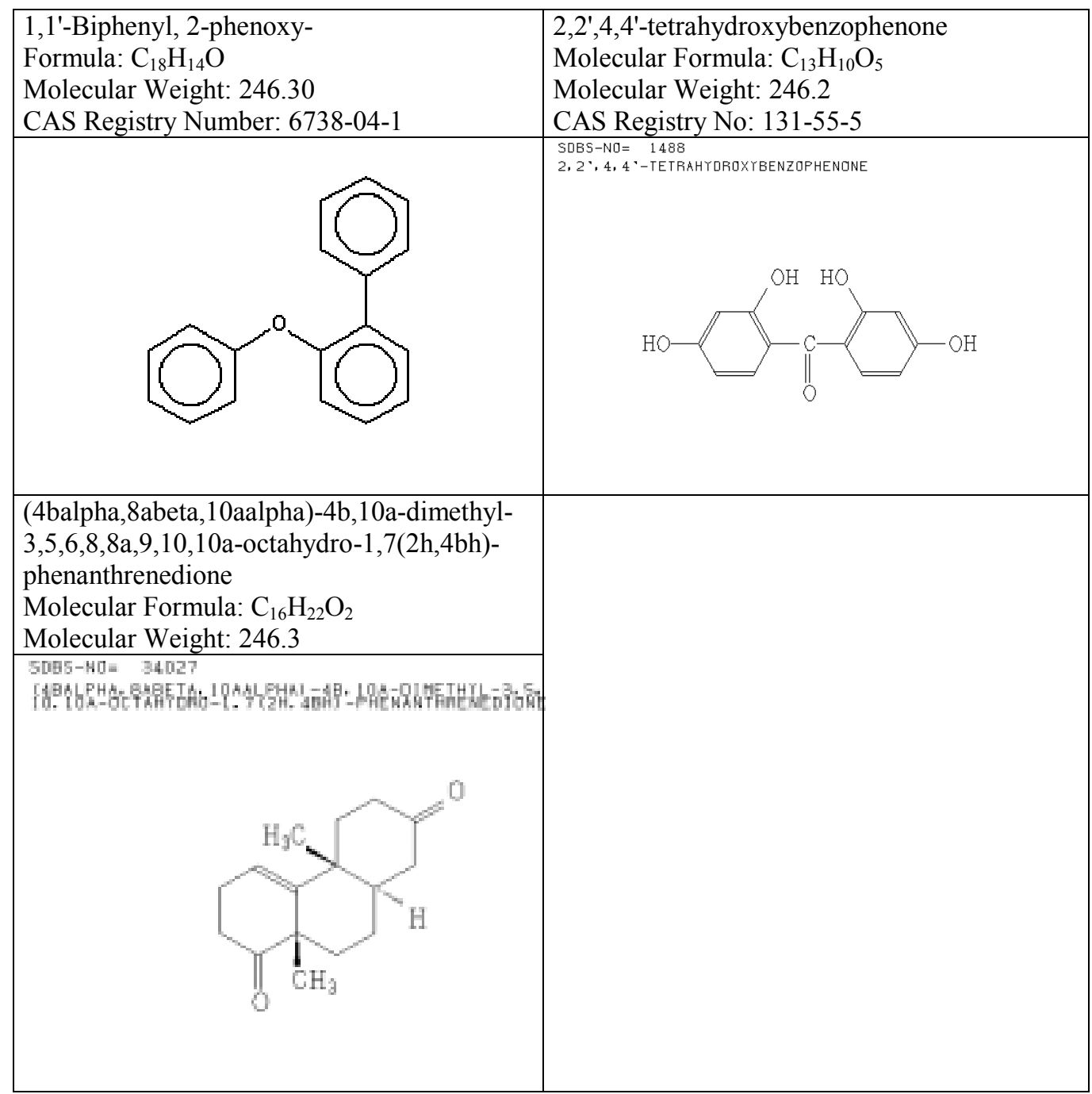

Bei der Verbindungen mit der Molmasse 322 bzw. 370 u handelt es sich wahrscheinlich um ein Trimer, bestehend aus drei Phenol-Molekülen bzw. deren Oxidationsprodukte. Darüber hinaus gehende Beispiele sind in Tabelle 42 und 43 aufgeführt.

Tabelle 42: Verbindungen mit der Molmasse $322 \mathrm{u}$.

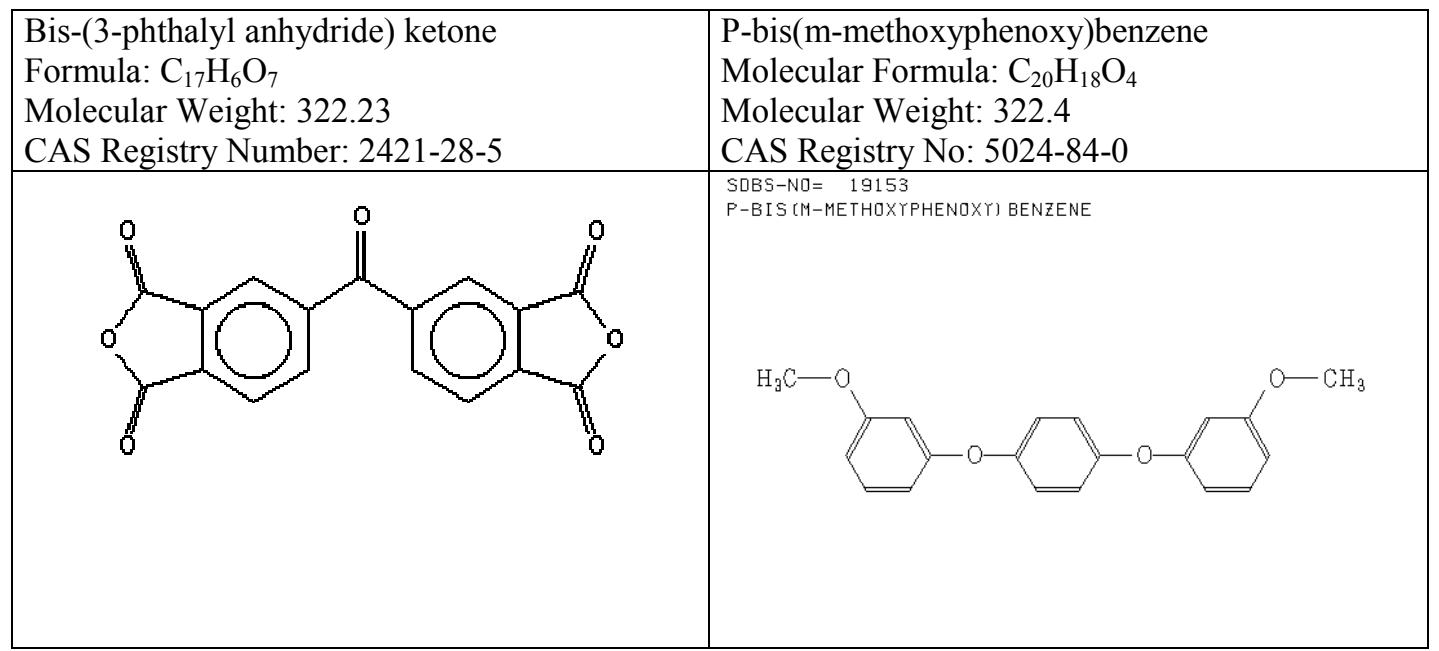


Tabelle 43: Verbindungen mit der Molmasse $370 \mathrm{u}$.

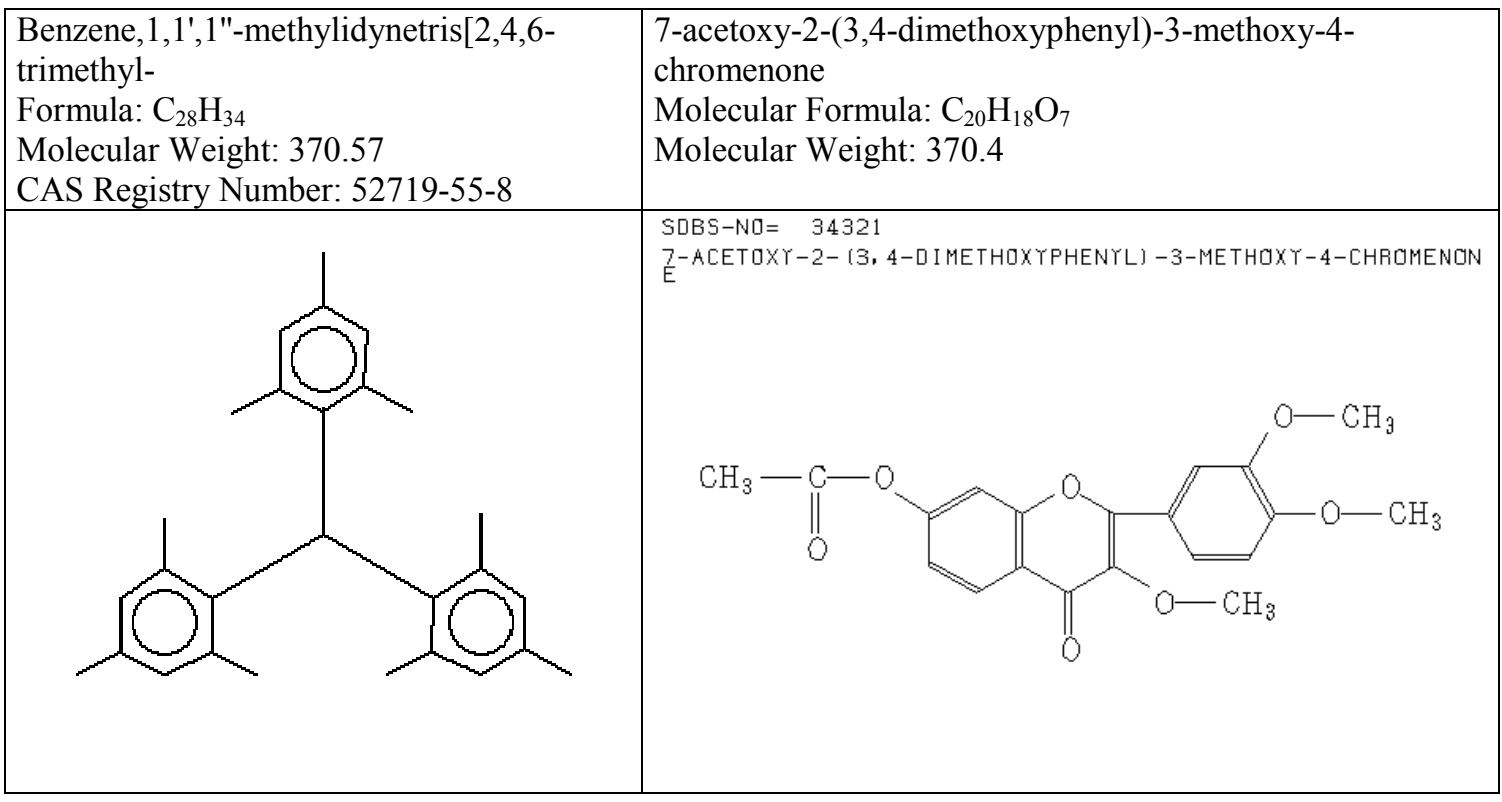

Die Beispiele verdeutlichen, dass es sich bei den entstandenen Verbindungen um immer höher kondensierte Substanzen, gebildet aus Molekülen der Ausgangssubstanz bzw. deren Oxidationsprodukte handeln muss. Dies wird noch weiter verdeutlicht durch die Verbindung mit der Masse von $662 \mathrm{u}$. Bei dieser Verbindung wird angenommen, dass es sich um ein Polymer aus sechs Brenzkatechin-Molekülen bzw. deren Oxidationsprodukte handelt $(6 \times 110,1 \mathrm{u}=660,6$ u).

\subsubsection{2,6-Dimethylphenol}

Die Analysen der extrahierten Überzüge der Belegung eines Na-Montmorillonits mit 2,6Dimethylphenol wiesen Verbindungen mit folgenden Molmassen nach: 226, 240, 254, 360, 374, 480, 495, 600, 720 und 840 u. Auffallend ist hier die Regelmäßigkeit der auftretenden Molmassen. So erhöhen sich die Massen von 240 über 360, 480, 600, 720 bis zu 840 u jeweils in Inkrementen mit einer Massenzunahme von $120 \mathrm{u}$. So entspricht die Verbindung mit der Molmasse von $240 \mathrm{u}$ einem Dimer (3,3',5,5'-Tetramethyl(bi-2,5-cyclohexadien-1-yliden)4,4'-dion) aus zwei miteinander verbundenen 2,6-Dimethylphenol-Molekülen (Abbildung $135)$.

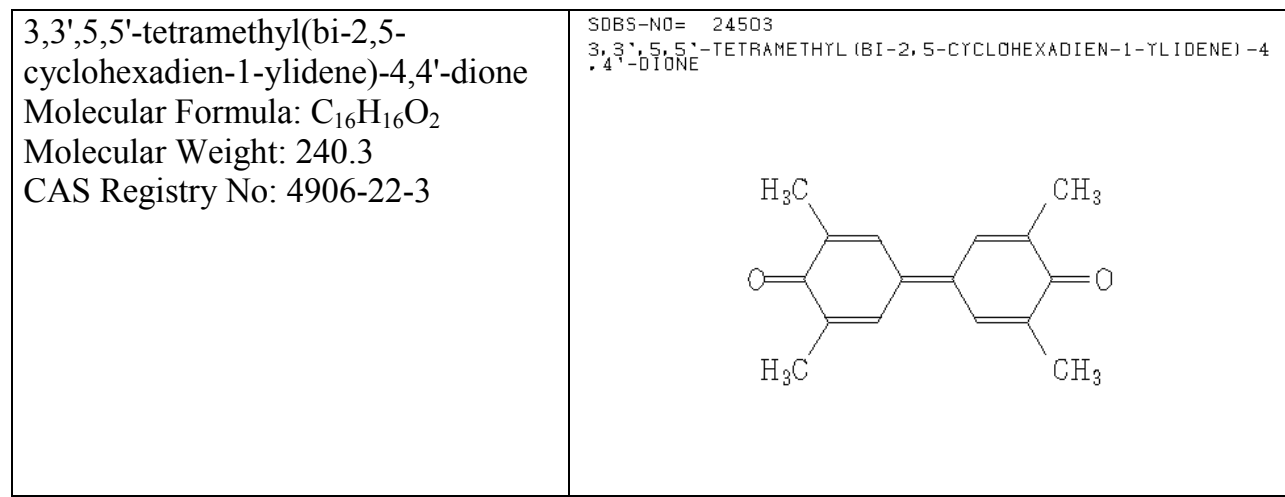

Abbildung 135: Dimer aus zwei 2,6-Dimethylphenol-Molekülen mit der Molmasse 240 u (Quelle: SDBS). 
Diese Masse entspricht genau einem 2,6-Dimethylphenol-Molekül, abzüglich zweier Wasserstoffatome. Die Phenolmoleküle binden über die Hydroxy-Gruppe des Phenols bzw. über das der OH-Gruppe gegenüberliegende C-Atom des aromatischen Rings. Der Polymerisationsprozess des 2,6-Dimethylphenols erfolgt nach IKEDA et al. (1996) und POTTHAST et al. (1999) durch Kopplung einzelner Phenol-Moleküle über die phenolische OH-Gruppe (Abbildung 136).

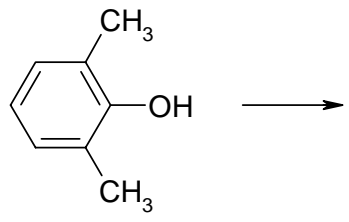

$122 \mathrm{u}$

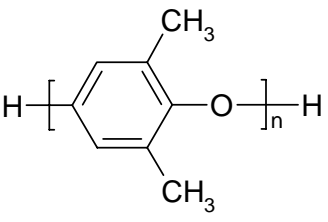

$120 \mathrm{u}$

Abbildung 136: Polymerisation des 2,6-Dimethylphenols (nach IKEDA et al. 1996).

Somit kann eine schrittweise Polymerisation des 2,6-Dimethylphenols mit der Molmasse 122 u nachgewiesen werden (Abbildung 137).<smiles>Cc1cccc(C)c1O</smiles>

2,6-Dimethylphenol (122 u)<smiles>Cc1cc(Oc2c(C)cccc2C)cc(C)c1O</smiles>

Dimer $(240 \mathrm{u})$<smiles>Cc1cc(C)c(Oc2cc(C)c(Oc3cc(C)c(O)c(C)c3)c(C)c2)c(C)c1</smiles>

Trimer $(360 \mathrm{u})$<smiles>Cc1cc(C)cc(Oc2c(C)cc(Oc3c(C)cc(Oc4c(C)cc(C)c(O)c4C)cc3C)cc2C)c1</smiles>
Tetramer $(480 \mathrm{u})$<smiles>Cc1cc(C)c(Oc2cc(C)c(Oc3cc(C)c(Oc4cc(C)c(Oc5cc(C)c(O)c(C)c5)c(C)c4)c(C)c3)c(C)c2)c(C)c1</smiles>

Pentamer $(600 \mathrm{u})$<smiles>Cc1cc(Oc2c(C)cc(Oc3c(C)cc(Oc4c(C)cc(Oc5c(C)cc(Oc6c(C)cc(C)c(O)c6C)cc5C)cc4C)cc3C)cc2C)cc(C)c1O</smiles><smiles>Cc1cc(Oc2cc(C)c(Oc3cc(C)c(Oc4cc(C)c(Oc5cc(C)c(Oc6cc(C)c(Oc7cc(C)c(O)c(C)c7)c(C)c6)c(C)c5)c(C)c4)c(C)c3)c(C)c2)cc(C)c1O</smiles>

Heptamer (840 u)

Abbildung 137: Polymerisationsprozess des 2,6-Dimethylphenols vom Dimer zum Heptamer. 
Die weiteren Verbindungen mit den Molmassen von 226, 254, 374 und 495 u zeigen, analog zu den zuvor beschriebenen Regelmäßigkeiten, einen deutlichen Zusammenhang. Addiert bzw. subtrahiert man von den Massegewichten der Polymere des 2,6-Dimethylphenols jeweils $14 \mathrm{u}$, so erhält man die betreffenden Werte. Das Molekulargewicht von $14 \mathrm{u}$ entspricht, bleibt man den drei Konstituenten Kohlenstoff, Sauerstoff und Wasserstoff - einem Kohlenstoff und zwei Wasserstoffatomen - und somit einer $\mathrm{CH}_{2}$-Gruppe. Zusätzlich fällt auf, dass bei Addition von $120 \mathrm{u}$, dies entspricht einem Phenolbaustein, ab der Verbindung mit der Molmasse von $254 \mathrm{u}$ sich wiederum die Werte 374 und 495 u errechnen lassen. Daraus ist zu schließen, dass die Reaktion des 2,6-Dimethylphenols an der Oberfläche des Montmorillonits nicht nur zur Bildung von Polymeren aus Phenolbausteinen mit der Molmasse $120 \mathrm{u}$ geführt hat, sondern zudem Verbindungen entstanden sind, die, aufbauend auf der Verbindung mit der Molmasse $254 \mathrm{u}$, sich dann wiederum zu Oligomeren mit 2,6-Dimethylphenol-Molekülen (120 u) verbinden und so Verbindungen mit den Molmassen 374 und $495 \mathrm{u}$ entstehen.

Das Reaktionsprodukt mit der Molmasse $254 \mathrm{u}$ stellt ein Dimer dar, welches anstelle von zwei gekoppelten 2,6-Dimethylphenol-Molekülen zusätzlich noch eine $\mathrm{CH}_{2}$-Gruppe einbindet (Strukturvorschlag, siehe Abbildung 138).

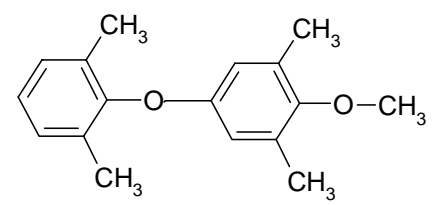

Abbildung 138: Bildung eines Dimers aus 2,6-Dimethylphenol mit der Molmasse $254 \mathrm{u}$.

Aus diesem Dimer entstehen durch Anbindung weiterer Phenolmoleküle Oligomere mit den nachgewiesenen Molmassen von 374 und 495 u (Abbildung 139).

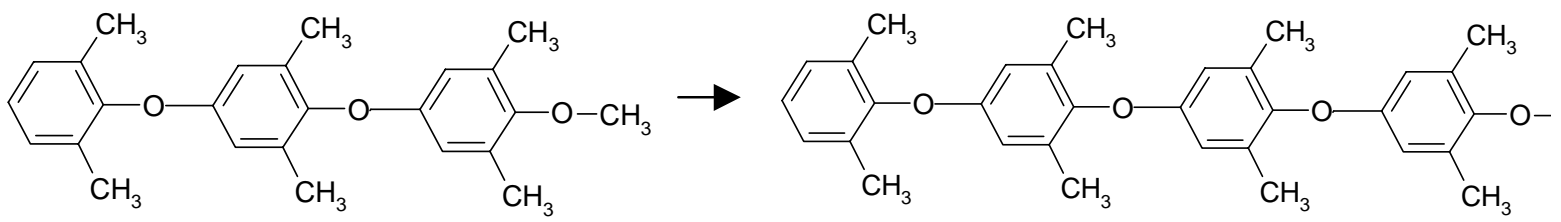

Molmasse $374 \mathrm{u}$

Molmasse $495 \mathrm{u}$

Abbildung 139: Bildung von Oligomeren mit den Molmassen 374 und $495 \mathrm{u}$.

Die Polymerisation von 2,6-Dimethylphenol wird in chemisch-technischen Synthesen genutzt, um Polymere mit ausgezeichneten chemischen und physikalischen Eigenschaften zu produzieren. Dazu wurden Kupfer/Amin-Katalysatoren verwendet. In neueren Arbeiten wurde untersucht, ob auch Bio-Katalysatoren eine Polymerisation des 2,6-Dimethylphenols bewirken können. Dazu wurden Enzyme, wie Laccase und Peroxidasen verwendet (KURIOKA et al. 1998), deren Aktivitäten hinsichtlich der Polymerisation von Phenolen und Alkylphenolen mit Reaktionsprodukten von mehreren Tausend Molekülmassen bekannt sind (IKEDA et al. 1996, UAYAMA et al. 1998). Die durchgeführten massenspektrometrischen Untersuchungen zeigen, dass auch durch die katalytische Aktivität mineralischer Oberflächen, wie in die- 
sem Falle die Tonminerale, ein Polymerisationsprozess der Kopplung von 2,6Dimethylphenol-Molekülen zu beobachten ist.

\subsubsection{Diskussion der Ergebnisse der massenspektrometrischen Untersuchungen}

Die durchgeführten massenspektrometrischen Untersuchungen konnten eine Vielzahl von Informationen zur Aufklärung der Zusammensetzung der durch abiotische Katalyse von Phenolen an Tonmineraloberflächen liefern.

\subsubsection{MALDI-TOF-MS}

Die Analysen des, durch Sublimation von Brenzkatechin auf verschiedenen Montmorilloniten und anschließende Extraktion gewonnenen organischen Probenmaterials mit MALDI-TOFMS zeigen breite Massenspektren mit Massengewichten zwischen 500 und 3600 bzw. 3800 Da. Diese Resultate belegen, dass es durch die abiotische Reaktion des Phenols auf der Oberfläche von Tonmineralen zu einer Polymerisation des Brenzkatechins zu Oligomeren bis hin zu Polymeren gekommen ist. Betrachtet man diese Ergebnisse zusammen mit den Resultaten der EDX-Analysen (Kapitel 4.3), so ist ersichtlich, dass es sich bei den in den REMAufnahmen erkennbaren, auf der mineralischen Oberfläche aufsitzenden Überzügen tatsächlich um polymerisiertes organisches Material handelt, welches durch katalytische Prozesse aus einfachen aromatischen Verbindungen auf der Oberfläche von Tonmineralen gebildet worden sind.

Die Analysen mittels MALDI zeigen sehr deutlich, dass das durch Sublimation auf der Oberfläche von Tonmineralen abgelegte Phenol eine chemische Veränderung erfahren hat. Diese Reaktion drückt sich dadurch aus, dass Verbindungen mit hohen Molekulargewichten entstanden sind. Somit konnte die Hypothese, dass durch physikochemische Prozesse einfache organische Verbindungen durch die Aktivität der Tonminerale zu Polymeren reagieren weiter erhärtet werden. Wie zuvor schon, durch die Resultate der spektroskopischen Untersuchungen vermutet und schließlich durch EDX-Analysen auch beobachtet werden konnte, sind Verbindungen, die in ihrem Molekulargewicht ein Vielfaches der Ausgangssubstanz repräsentieren, entstanden: das Massenspektrum erstreckt sich etwa von 5-fachen bis zum über 30-fachen der Molmasse des Brenzkatechins. Die Entstehung von organischen Polymeren aus einer einfachen aromatischen Verbindung konnte somit auch mit dieser Methode nachgewiesen werden.

Als Ergebnis der massenspektrometrischen Untersuchung der organischen Überzüge eines mit Brenzkatechin belegten Ca-Montmorillonits zeigte sich im Unterschied zur Analyse der extrahierten Organika des ebenfalls mit Brenzkatechin belegten Na-Montmorillonits ein Unterschied in der Verteilung der Massengewichte. Im Gegensatz zur Probe W2 (NaMontmorillonit) zeigt die Probe A1 (Ca-Montmorillonit) deutlich mehr Signale mit höheren Intensitäten im Bereich höherer Massengewichte. Es konnten somit für den Ton mit einem Zwischenschicht-Kation von niedrigerer Wertigkeit größere Mengen stärker polymerisierter Verbindungen nachgewiesen werden. Betrachtet man die absoluten Werte, so ist hingegen 
festzustellen, dass die Massengewichte der extrahierten Probelösung eine breitere Spanne einnehmen und mit der Molmasse von 3823 Da eine um 171 Da schwerere Verbindung entstanden ist. Die Unterschiede in der qualitativen Zusammensetzung des Probenmaterials sind signifikant. Während in Probe W2 eine Häufung der detektierten Massengewichte zwischen 856 und 1162 Da liegt, treten in der Probe A1 vermehrt Verbindungen mit Massen ab 1605 Da auf.

\subsubsection{Gaschromatographie-MS}

Die Untersuchungen von Tonmineralen extrahierten Organika mittels GC-MS und APCI bestätigten einerseits die mit MALDI-TOF-MS erzielten Ergebnisse bezüglich der Polymerisation von Phenolen an Tonmineraloberflächen. Zusätzlich konnten jedoch, detailliertere Aussagen zur qualitativen Zusammensetzung der entstandenen Reaktionsprodukte, insbesondere im Bereich niedriger Massegewichte (122 bis 593 u) gewonnen werden, die bei den Untersuchungen mittels MALDI nicht erfasst wurden. Die Auswertung von Datenbanken ergab eine Vielzahl von Substanzen mit entsprechenden Molmassen, die durch eine oxidative Umsetzung der Phenole zu chinoiden Strukturen und Carbonsäuren gebildet werden. Außerdem konnte schon bei diesen niedrigen Massegewichten eine Aneinanderlagerung von Aromaten beobachtet werden, womit eine beginnende Polymerisation der phenolischen Komponenten zu erkennen ist. Dieser Prozess konnte anhand der Kopplung von zwei 2,6-DimethylphenolMolekülen sehr anschaulich dargestellt werden. Als Resultat der gaschromatographischen Analysen bleibt festzuhalten, dass die durch die Aktivität der Tonminerale induzierte Reaktion von Phenolen zu einem einzigen entsprechenden Reaktionsprodukt geführt hat, sondern, wie schon durch die vorangegangenen Ergebnisse der spektroskopischen Untersuchungen (Kapitel 4.6/4.7) vermutet, zu einer Vielzahl von Umbauprodukten geführt hat.

\subsubsection{APCI}

Die Analysenergebnisse der Chemischen Ionisation bei Atmosphärendruck zeigten für die extrahierten organischen Überzüge des Brenzkatechins, ausgehend von dessen Molmasse, eine Zunahme der Massengewichte von 168 u bis zu 662 u. Dies ist mit einer oxidativen Polymerisation des Phenols zum Dimer (216 u) bis hin zum Hexamer (662 u) zu erklären. Das Auftreten verschiedener Massen liegt in der Bildung unterschiedlicher Reaktionsprodukte begründet. Die durch Datenbankrecherchen ermittelten Verbindungen entsprechender Molmassen zeigen beispielhaft zu welchen Verbindungen die Reaktion des Phenols an der Tonmineraloberfläche geführt haben kann. Bestätigt werden auch mit dieser Methode die spektroskopischen Analyseergebnisse, die das Auftreten von Carbonyl- und Carboxylgruppen nachgewiesen haben.

Von besonderer Bedeutung waren die Resultate des extrahierten Probenmaterials der Belegung von Montmorillonit mit 2,6-Dimethylphenol. In diesem Falle konnte aufgrund der detektierten Molmassen eine Polymerisation des Phenols durch Kopplung einzelner Moleküle beobachtet werden. Durch das Auftreten von Massen, die jeweils um den Inkrementwert von 
$120 \mathrm{u}$ anstiegen, konnte die Bildung von Polymeren aus Phenol-Molekülen vom Dimer bis hin zum Oligomer, bestehend aus 8 Molekülen beobachtet werden. Dieses Ergebnis ist daher von besonderer Bedeutung, da diese Reaktion des 2,6-Dimethylphenols bisher nur in chemisch-technischen Verfahren unter Einsatz von Katalysatoren (Kupfer/Amin bzw. Enzyme) beobachtet werden konnte. Das Tonminerale als Hauptbestandteile der mineralischen Fraktion des Bodens zu solch heftigen Reaktionen fähig sind, wurde bisher in diesem Ausmaß noch nicht festgestellt. Durch diese Beobachtungen den katalytischen Eigenschaften von Tonmineralen eine völlig neue Bedeutung im Hinblick auf den Auf-, Ab- und Umbauprozesse, sowohl von natürlicher organischer Substanz als auch von anthropogen in Böden eingebrachte organischen Schadstoffen zuzumessen. 


\section{Schlussfolgerungen}

Die vorliegende Arbeit liefert am Beispiel von drei Phenolen und einem Vertreter der polyzyklischen aromatischen Kohlenwasserstoffe einen Beitrag zum Verständnis des Verhaltens von organischen Modellsubstanzen für Huminstoffmonomere bzw. von organischen Umweltchemikalien im Boden. Aus den Ergebnissen der Untersuchungen lassen sich neben den Erkenntnissen über die abiotische Aktivität von Tonmineralen und deren Einfluss auf die Bildung von tonorganischen Komplexen und die Genese von Huminstoffen auch Aussagen über Reaktionsmechanismen und Bindungsformen ableiten. An dieser Stelle soll darüber hinaus die methodische Herangehensweise bewertet und auf die Relevanz sowie die Konsequenzen der Ergebnisse eingegangen werden.

\subsection{Methodik}

Einführend ist anzumerken, dass zur Erreichung der in der Zielsetzung (Kapitel 3) postulierten Absichten zu Beginn dieser Arbeit, aufgrund der Komplexität der Fragestellung, keine festgelegten Untersuchungs- und Analysenvorschriften bestanden. Erst durch den sukzessiven Ausbau und Verbesserung der Untersuchungsmethoden konnten die gewonnenen Resultate erzielt werden.

Die Belegung von Phenolen auf die Oberfläche von Tonmineralen durch Sublimation aus der Gasphase bzw. die Aufbringung methanolischer Lösung eines polyzyklischen aromatischen Kohlenwasserstoffes können als gut geeignete Verfahren zur Kontamination mineralischer Oberflächen mit Organika angesehen werden. Mit dieser Methode konnten auf einfachem Wege und ohne die Problematik nasschemischer Ansätze (Kapitel 4.1) mineralische Oberflächen mit Organika belegt und die so gewonnenen organischen Überzüge in situ durch rasterelektronenmikroskopische Systeme sowie durch energiedispersive Röntgenmikrobereichsanalyse untersucht werden. Diese Methode bietet weiterhin den Vorteil, bei Verwendung eines geeigneten FTIR-Spektrometers, die sich auf den Oberflächen von Tonmineralen befindenden Überzüge direkt einer qualitativen Analyse, ohne den Umweg der Extraktion und Probenaufbereitung mit den damit verbundenen Risiken der Bildung von Artefakten, zuführen zu können.

Die Extraktion mit Methanol erwies sich als effizientes und schonendes Verfahren, um die organischen Überzüge von den mineralischen Oberflächen zu trennen. Lediglich sehr stark chemisorptiv gebundene Reaktionsprodukte, wie im Falle der Belegung von Montmorilloniten mit Pyrogallol, konnten mit dieser Methode nicht vollständig extrahiert werden.

Durch die Anwendung rasterelektronenmikroskopischer Methoden und energiedispersiver Röntgenmikrobereichsanalysen konnten die Gestalt, Größe und die Verteilung organischer Überzüge auf der Oberfläche von Tonmineralen qualitativ bestimmt werden (BIRKEL et al. 2000c). Darüber hinaus konnten Rückschlüsse auf bevorzugte Bindungsplätze der Organika auf der Oberfläche und damit auch auf die Reaktionsmechanismen (Kapitel 5.2) gezogen 
werden.

Die chromatographische Detektion der extrahierten Substanzgemische ergab einen Überblick über die Anzahl der gebildeten Fraktionen innerhalb der Lösungen. Weitere qualitative Aussagen waren mit dieser Methode jedoch nur sehr eingeschränkt möglich. Die Verwendung eines HPLC-Systems zur präparativen Trennung größerer Mengen der Einzelfraktionen für die FTIR- und ${ }^{13} \mathrm{C}$-NMR-spektroskopischen Untersuchungen erwies sich aufgrund der so gewonnenen, sehr geringen Probenkonzentrationen als recht problematisch. Mit Hilfe eines Flüssigchromatographie-Systems wäre hier eine deutlich effektivere Probenausbeute zu erzielen. Durch die Kopplung einer HPLC mit einem Massenspektrometer, wie in dieser Arbeit in Kapitel 4.5.2.2 dargestellt, entfallen diese probentechnischen Unzulänglichkeiten jedoch weitestgehend.

Mit den durchgeführten infrarot- und ${ }^{13} \mathrm{C}-\mathrm{NMR}$-spektroskopischen Analysen konnten, insbesondere durch letztere Methode, detaillierte Aussagen zum Auftreten charakteristischer, funktioneller Gruppen getroffen werden. Die Anwendung beider Verfahren erwies sich als vorteilhaft, da die hohe Empfindlichkeit der FTIR-Spektroskopie, zur Überlagerung von Spektreninformationen neigt und damit die Interpretation erschwert. Die ${ }^{13} \mathrm{C}-\mathrm{NMR}$-Spektroskopie, die systembedingt eine relativ unempfindliche Methode darstellt und daher größere Probenmengen beansprucht (Kapitel 4.7.1), gleicht dieses Manko durch eine detailliertere und übersichtlichere Strukturinformation des Probenmaterials mehr als aus. Somit wurde eine optimale Ergänzung beider Methoden erreicht.

Die im Rahmen dieser Arbeit begonnenen massenspektrometrischen Untersuchungen dienten der Überprüfung der schon zuvor gewonnenen Informationen und der Ergänzung bezüglich des Aufbaus und der Eigenschaften der durch abiotische Katalyse gebildeten Reaktionsprodukte. Die Anwendung von massenspektrometrischen Methoden erwies sich zur Identifizierung der Reaktionsprodukte sowie zur Charakterisierung der an Tonmineralen ablaufenden Reaktionsmechanismen als diesbezüglich sehr aussagekräftige Verfahren (BIRKEL et al. 2000b).

\subsection{Verhalten von Organika an Tonmineralen}

Durch die durchgeführten Untersuchungen zur abiotischen Transformation aromatischer Verbindungen an Tonmineralen konnten die in Kapitel 3 gesteckten Ziele weitgehend erreicht werden.

Die Belegung von Na- und Ca-Montmorilloniten mit Phenolen als Modellsubstanzen von Huminsäuren führte zu einer deutlichen Reaktion der sublimierten Organika auf den Oberflächen der Tonminerale, die sich in einer intensiven Verfärbung der Tonoberflächen niederschlug. Diese Verfärbungen sind ein erstes Indiz dafür, dass durch den Kontakt der Phenole mit der mineralischen Oberfläche ein katalytischer Prozess initiiert wurde, der zur chemischen Umsetzung der Aromate geführt hat. Für diese Reaktion ist jedoch Sauerstoff unbedingt notwendig, da in vorab durchgeführten Untersuchungen (BIRKEL 1997) gezeigt werden konnte, 
dass in einer sauerstofffreien Atmosphäre keine Verfärbung der mit Organika belegten Mineraloberflächen eintritt. Im Falle der Belegung der Tone mit einem polyzyklischen aromatischen Kohlenwasserstoff konnte jedoch keine, schon mit bloßem Auge erkennbare, Reaktion beobachtet werden. Daraus kann jedoch nicht geschlossen werden, dass es auf diesem Wege nicht zu einer abiotischen Transformation von PAK an Tonmineralen kommt.

Die Untersuchungen der mit Organika belegten Pulver- und Aggregatpräparate aus Montmorillonit mit Hilfe der Feldemissions-Rasterelektronenmikroskopie (Kapitel 4.2) zeigten neu auftretende Strukturen auf den Mineraloberflächen mit einer schleierförmigen bis watteartigen Morphologie. Die Größe der Objekte reicht von wenigen hundert Nanometern bis zu mehreren Mikrometern Durchmesser. Auffallend ist die heterogene Verteilung der Objekte auf der Oberfläche. Bisher wurde in der Literatur (Kapitel 2.2 und 4.3) eine gleichmäßige Verteilung organischer Substanz bei organomineralischen Komplexen angenommen. Das angewendete Belegungsverfahren sollte aufgrund der mit Phenolen gesättigten Atmosphäre für eine vollständige und homogene Bedeckung der Tonoberflächen sorgen. Daraus kann geschlossen werden, dass der Aufbau und die Struktur der Oberfläche der Tonminerale die entscheidende Einflussgröße der physikalisch-chemischen Transformation der auf ihr adsorbierten organischen Verbindungen sein muss.

Zur Überprüfung der vorangegangenen Beobachtungen wurde mit einem an ein Rasterelektronenmikroskop gekoppelten EDX-System die Verteilung der Elemente Kohlenstoff, Silizium und Eisen auf den mit Organika belegten Tonmineralen gemessen. Als Ergebnisse des Elementmappings von Kohlenstoff und Silizium konnten die zuvor beobachteten Strukturen als eindeutig organischen Ursprungs nachgewiesen werden. Auch bei diesen Untersuchungen war eine heterogene Verteilung der organischen Überzüge zu erkennen. Die neben der Messung des Kohlenstoffsignals erfolgte gleichzeitige Detektion des Elements Eisen ergab eine schwache, jedoch erkennbare Korrelation beider Elemente (BIRKEL et al. 2000b). Die Kumulation von Signalen organischen Materials und von Eisen zeigt einerseits, dass die Adsorption organischer Substanzen vorrangig an Oberflächen stattfindet, an denen Eisen in Form von an den Tonmineralen adsorbierten Fe-Oxiden oder Fe-Hydroxiden bzw. als Bestandteil der Mineralstruktur des Tons zugänglich ist. Darüber hinaus ist ersichtlich, dass das an das Eisen adsorbierte organische Material eine chemische Transformation dahingehend durchlaufen hat, dass nicht nur die Bildung großflächiger Überzüge eingesetzt hat, sondern es zudem zur Entstehung hochmolekularer organischer Strukturen gekommen ist. Die Resultate dieser Analysen weisen die Bildung von Ladungstransferkomplexen zwischen Eisen und den sublimierten organischen Verbindungen nach, die in Umgebungsatmosphäre unter Bildung von Hydroxylradikalen schließlich zur Entstehung organischer Radikale (Kapitel 2.2.1) und damit zur oxidativen Polymerisation von Phenolen auf der Oberfläche von Tonmineralen führen (Abbildung 140). 


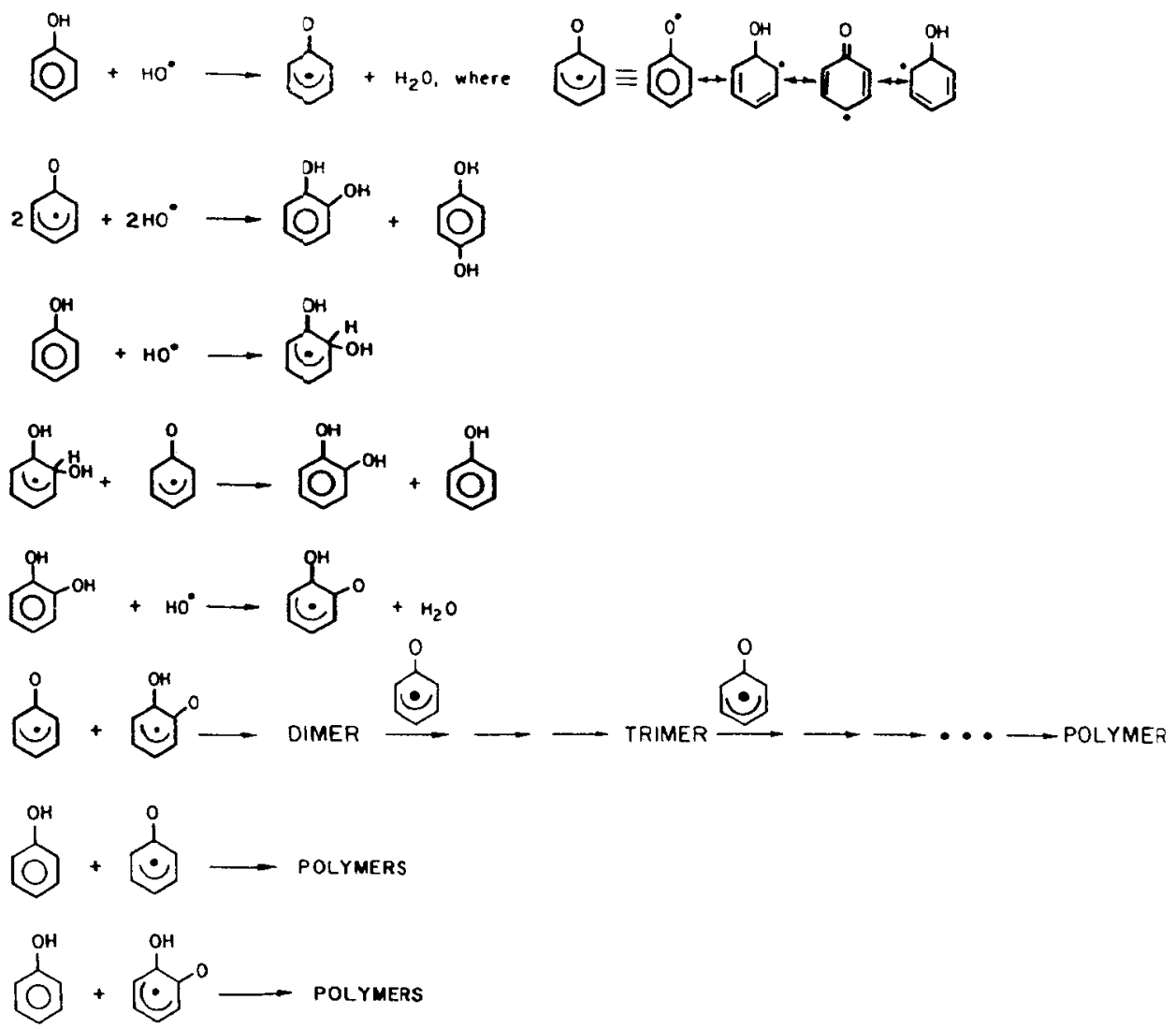

Abbildung 140: Bildung phenolischer Radikale und deren anschließende Polymerisation (VOUDRIAS \& REINHARD 1986).

Die im Anschluss an die Extraktion der organischen Überzüge zur ersten Charakterisierung der gebildeten Substanzen durchgeführten Nachweisreaktionen bestätigen die im Kapitel 2.2 dargestellte Oxidation der Phenole zu chinoiden Verbindungen durch den positiven Nachweis von entsprechenden funktionellen Aldehyd- bzw. Ketogruppen $(-\mathrm{C}=\mathrm{O})$. Somit konnte nachgewiesen werden, dass die Belegung der Tonminerale mit Phenolen zu deren Oxidation geführt hat.

Durch Analysen der Extraktionslösungen mit Hilfe der HPLC konnte gezeigt werden, dass bei allen auf der Tonmineraloberfläche sublimierten Phenolen zahlreiche Reaktionsprodukte entstanden sind, die sowohl höhere als auch niedrigere Polaritäten aufweisen (BECKMANN et al. 2000, BIRKEL et al. 2000a). Die mit einem HPLC-MS-System gewonnenen Ergebnisse zeigen, dass durch die abiotische Umsetzung des Brenzkatechins an der Tonmineraloberfläche ausschließlich höhermolekulare Verbindungen entstanden sind.

Die zur Strukturaufklärung angewendete FT-IR-Spektroskopie bestätigte die mit den Nachweisreaktionen erfasste Bildung von Carbonylbindungen $(-\mathrm{C}=\mathrm{O})$ und damit die Oxidation der Phenole zu ihren entsprechenden chinoiden Oxidationsprodukten. Daneben konnte durch die Auswertung der jeweiligen Gesamt-Spektren, sowie der mit einem HPLC-System und gekoppeltem Fraktionensammler spektroskopierten Einzelfraktionen, zahlreiche weitere Bindungen und funktionelle Gruppen nachgewiesen werden. Es treten Carboxylgruppen und aliphatische Alkohole und Alkene im extrahierten Probenmaterial auf, zudem konnte eine Polymerisation 
des organischen Materials durch ein Ansteigen der H-Brückenbindungen festgestellt werden. Diese bei allen untersuchten Phenolen beobachtete Verteilung der Bindungstypen bzw. funktionellen Gruppen weist neben der Oxidation und Polymerisation der Phenole bzw. ihrer Reaktionsprodukte zusätzlich auf eine teilweise Aufspaltung der aromatischen Strukturen in aliphatische Verbindungen (Alkane, Alkene, Alkohole und Carbonsäuren) und damit auf die Zerstörung des aromatischen Rings der Phenole hin. Die weitreichenden Übereinstimmungen der IR-Spektren von natürlichen Fulvo- und Huminsäuren mit den Spektren der von den Tonmineralen extrahierten organischen Überzüge verdeutlichen, dass durch abiotische Transformation einfacher aromatischer Verbindungen höhermolekulare Reaktionsprodukte entstehen, die in ihrer chemischen Zusammensetzung zumindest sehr große Ähnlichkeiten aufweisen.

Mit Hilfe der ${ }^{13} \mathrm{C}$-NMR-Spektroskopie wurden die Resultate der FT-IR-Spektroskopie verifiziert und es konnten noch detailliertere Informationen über die Zusammensetzung der extrahierten Organika gewonnen werden. Neben den bereits durch FT-IR-Spektroskopie nachgewiesenen Carbonyl- und Carboxylgruppen, die eine Oxidation der Phenole bestätigen, wurden O-Alkylgruppen und Signale neu auftretender aromatischer Strukturen beobachtet, durch die die Entstehung neuer (polymerer) Aromate angenommen werden kann. Von besonderer Bedeutung sind die zusätzlich erzielten Ergebnisse der Belegung von Tonmineralen mit einem Phenol und einem PAK. Sie zeigen, dass das Phenanthren in Anwesenheit eines Phenols (Brenzkatechin und Pyrogallol) im Gegensatz zur Belegung ohne Phenol eine chemische Transformation dahingehend erfährt, dass eine Alkylierung des PAK und somit eine Veränderung der aromatischen Strukturen beobachtet werden konnte (BIRKEL et al. 2000a). Diese Ergebnisse sind in zweierlei Hinsicht von Bedeutung. Zum Einen konnte somit die abiotische Transformation von polyzyklischen aromatischen Kohlenwasserstoffen an Tonmineralen nachgewiesen werden. Darüber hinaus wird der Reaktionsweg, der nach den Resultaten der EDX-Analysen durch Ladungstransfer-Reaktionen mit anschließender Bildung von Radikalen induziert wird, hiermit bestätigt und fortgeführt: die Entstehung phenolischer Radikale (Kapitel 2.2.5) führt zur Oxidation ebenfalls auf der Tonmineraloberfläche adsorbierter, normalerweise reaktionsträger bzw. inerter Organika.

Die durchgeführten massenspektrometrischen Untersuchungen (APCI, GC/MS) zeigten für die mit den Phenolen Brenzkatechin bzw. 2,6-Dimethylphenol belegten Montmorillonite eine Polymerisation zu Verbindungen mit Massegewichten, die mehr als das sechsfache bzw. achtfache der Masse der Ausgangssubstanz erreichten. Substanzen mit noch höherer Teilchengröße wurden mit Hilfe von MALDI nachgewiesen. Sie reichten im Falle der Reaktionsprodukte der mit Brenzkatechin belegten Montmorillonite von 500 bis über 3800 Da. Diese Ergebnisse ergänzen und bestätigen die bisherigen Resultate der REM/EDX-Analysen, bei denen das Aufwachsen organischer Strukturen an mineralischen Oberflächen beobachtet werden konnte.

\subsection{Relevanz und Konsequenzen der erzielten Ergebnisse}

Die in dieser Arbeit durchgeführten Untersuchungen hatten zum Ziel, die Bedeutung von 
Tonmineralen bei der Transformation organischer Schadstoffe und ihre Rolle bei der Genese von Huminstoffen zu beleuchten. Dazu wurden umfangreiche Labor- und Analysemethoden angewendet. Deren zahlreiche Ergebnisse lassen sich dahingehend zusammenfassen, dass Tone im Boden eine nicht zu vernachlässigende Rolle bei der chemischen Umsetzung von adsorbierten organischen Verbindungen innehaben. Die als Huminsäure-Vorstufen anzusprechenden Phenole reagieren durch physikalisch-chemische Prozesse zu Verbindungen, die eine völlig andere chemische Eigenschaften als die Ausgangssubstanzen aufweisen und darüber hinaus zu höhermolekularen Verbindungen polymerisieren, deren Zusammensetzung deutliche Übereinstimmungen mit natürlichen Huminstoffen zeigen. Es kommt teilweise zu einer chemisorptiven Festlegung von Reaktionsprodukten der Belegung von Tonmineralen mit Phenolen, die Parallelen zur Bindung natürlicher Huminstoffe an die mineralische Matrix in Böden aufweisen. Somit kann und muss die Genese von Huminstoffen, die laut derzeit vorherrschender Lehrmeinung biotisch induziert ist (Kapitel 2.2) zusätzlich durch einen abiotischen Reaktionspfad erweitert werden (Abbildung 141).

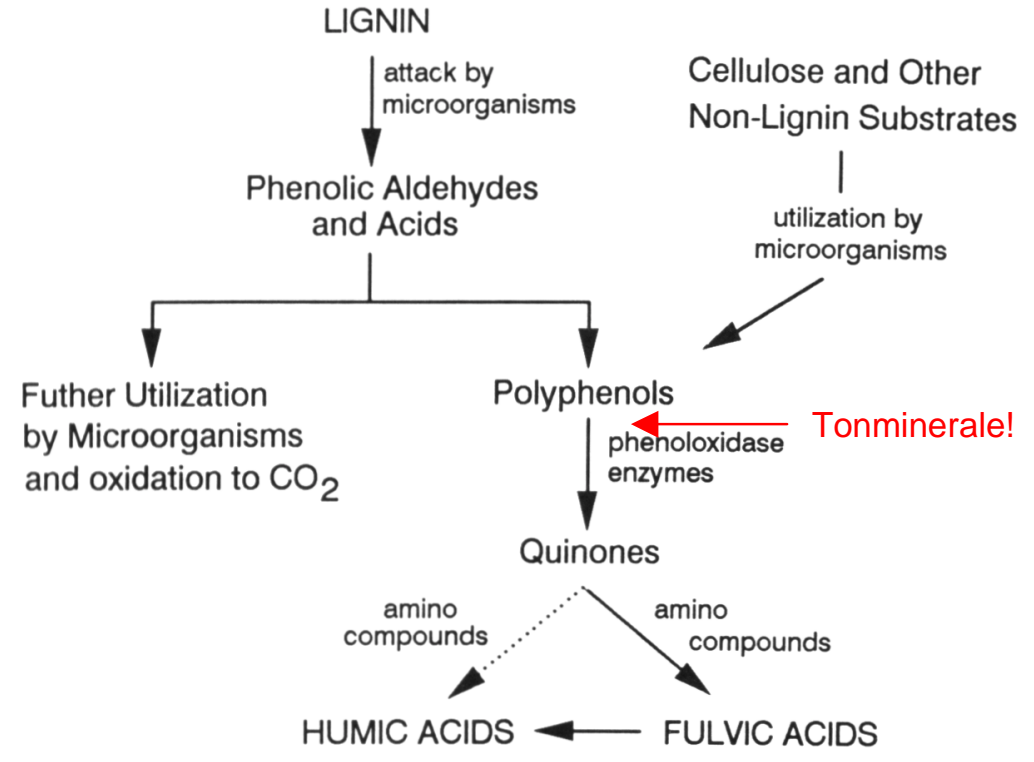

Abbildung 141: Theorie der Entstehung von Huminstoffen (nach Stevenson 1994).

Montmorillonite ,aktivieren“ die an sie sorbierte organische Substanz und leiten darüber hinaus auch eine Transformation chemisch relativ reaktionsträger Stoffe (i.e. polyzyklische aromatische Kohlenwasserstoffe) ein und führen somit zur Festlegung der Xenobiotika in die organische Substanz. Tonminerale zeigen das Vermögen, natürliche, quasinatürliche und xenobiotische organische Verbindungen chemisch zu transformieren und damit einen Beitrag zur Umsetzung und Festlegung von Organika in Böden zu leisten. Die Bildung organischer Substanz und die Degradation von Xenobiotika kann somit nicht mehr allein auf biologische bzw. photochemische Prozesse zurückgeführt werden. 


\section{Zusammenfassung}

Ziel dieser Arbeit war, die Bedeutung von Tonmineralen in Böden im Hinblick auf die abiotische Transformation organischer Substanz - natürlicher und anthropogener Herkunft - zu erfassen. Da humuschemische Untersuchungen und Aussagen zur Struktur von natürlicher organischer Substanz in Böden aufgrund der Komplexität von Huminstoffen nur sehr eingeschränkt möglich sind, wurde es als sinnvoll erachtet, das zu untersuchende System (Tonmineral-organische Substanz) zu vereinfachen. Um konkrete Aussagen zur Aktivität von Tonmineralen und zur Umsetzung von Organika zu erhalten wurden definierte Lagerstättentone mit einfachen aromatischen Verbindungen belegt. Phenole kommen sowohl natürlich als Degradationsprodukte des Lignins und Huminsäure-Vorstufen als auch als Schadstoffe in Böden vor. Zusätzlich wurde Phenanthren als Vertreter vorwiegend anthropogenen Ursprungs ausgewählt.

Die Phenole reagieren auf Tonmineralen zu farbigen Überzügen, die sich auf deren Oberflächen als heterogen verteilte, flächenhafte und watteartige Strukturen von bis zu mehreren Mikrometern Durchmesser präsentieren. Ein Zusammenhang zwischen der an Tonmineraloberflächen sorbierten organischen Substanz und dem Auftreten von Eisen konnte nachgewiesen werden. Damit wurden stichhaltige Hinweise auf den, die katalytische Aktivität der Tonminerale mit hervorrufenden, Reaktionsmechanismus gefunden. Durch Ladungstransferkomplexe von adsorbierter organischer Substanz zum Eisen kommt es zur Bildung von Radikalen, die eine Oxidation der Organika bewirken. Darüber hinaus polymerisieren die Oxidationsprodukte zu Verbindungen mit höheren Molekulargewichten. Infrarotspektren der abiotisch gebildeten Reaktionsprodukte der Phenole weisen große Übereinstimmungen mit Spektren natürlicher Huminstoffe auf. Als charakteristische funktionelle Gruppen der Reaktion der Phenole sind Chinone, Carbonsäuren und Alkane zu nennen. Neben der Polymerisation von Aromaten treten auch Spaltprodukte aromatischer Systeme auf. Phenanthren erfährt nur in Gegenwart von Phenolen eine chemische Transformation, was wiederum auf den Prozess der Oxidation von Organika durch phenolische Radikale hindeutet und als ein einfaches Modell des Umbaus bzw. Einbaus von Xenobiotika in die organische Substanz von Böden aufgefasst werden kann.

Es konnte gezeigt werden, dass Tonminerale über die Fähigkeit verfügen, analog den biotischen Prozessen der Transformation von Xenobiotika bzw. der Bildung von Huminstoffen, organische Verbindungen abiotisch zu modifizieren. Damit vermögen sie einen Beitrag zur Genese der Huminstoffe bzw. zur Degradation und Festlegung organischer Schadstoffe in Böden zu leisten. 


\section{Literatur}

Adams, J.M.; Clapp, T.V.; Clement, D.E. (1983): Catalysis by montmorillonites.- Clay Minerals, 18, S. 411-421.

Alexander, M. (1995): How toxic are toxic chemicals in soil?- Environmental Science \& Technology, 29, H. 11, S. 2713-2717.

Andreux, F.: Genesis and properties of humic molecules.- Aus: Bommeau, M. (ed.): Constitutents and properties of soils. London, New York 1982, S. 109-139.

Anonym (1995): Ion-exchanged pillared clays: A new class of catalysts for selective catalytic reduction of NO by hydrocarbons and by ammonia.- Journal of Catalysis, 155, S. 414417.

Araki, T., Tanaka, N., Hinokimori, T., Hotta, K., Tateishi, K., Kubo, Y., Yamaguchi, T., Watanabe, K., Fukuda, H., Asa, H. (1992): Non-classical urea polymers. Part XIV. Some new properties of $\mathrm{Cu}$ (II) ion encircled by bis-branched oligomeric urea ligand: properties associated with catalysis for oxidative coupling of phenols.- Journal of Molecular Catalysis, 75, S. 21-40.

Arocena, J.M., Pawluk, S., Dudas, M.J., Gajdostik, A. (1995): In situ investigation of soil organic matter aggregates using infrared microscopy.- Canadian Journal of Soil Science, 75 , S. 327-332.

Aust, S.D. (1995): Mechanisms of degradation by white rot fungi.- Environmental Health Perspectives, H. 103 (5), S. 59-61.

Bahr, U., Karas, M., Hillenkamp, F. (1994): Analysis of biopolymers by Matrix-assisted Laser Desorption-Ionization (MALDI) mass spectrometry.- Fresenius' journal of analytical chemistry, H. 348, S. 783-791.

Backhaus, W.; Klumpp, E.; Schwuger, M.J.: Adsorption of aromatic hydrocarbons and nonionic surfactant on clay and silica surfaces.- Aus: Elsen, A.; Grobet, P.; Keung, M. (eds.): Euroclay'95-Clays and Clays Material Sciences. Book of Abstracts. Leuven 1995, S. 134.

Ballantine, J.A.; Purnell, J.H.; Thomas, J.M. (1983): Organic reactions in a clay microenvironment.- Clay Minerals, 18, S. 347-356.

Balogh, M., Laszlo, P., Pandey, G. (1994): Organic chemistry using clays.- Angewandte Chemie, 21, H. 106, S. 2317.

Barkow, A. (1994): Energiedispersive Röntgenmikroanalyse am REM.- Studienarbeit FH Mannheim.

Bart, J.C. (1979): Formation of polymeric species in the interlayer of bentonite.- Clays and Clay Minerals, 27, H. 6, S. 429-432. 
Beckmann, S., Birkel, U., J. Niemeyer \& M. Zai (2000): Reaktionen und Verlagerung von organischen Schadstoffen in der ungesättigten Zone von Böden.- Mitteilungen der Deutschen Bodenkundlichen Gesellschaft, 92, 31-33.

Bedioui, F. (1995): Zeolite-encapsulated and clay-intercalated metal porphyrin, phtalocyanine and Schiff-base complexex as models for biomimetic oxidation catalysts: An overview.Coordination Chemistry Reviews, H. 144, S. 39-68.

Bekassy, S., Cseri, T., Horvath, M., Farkas, J., Figueras, F. (1998): Low temperature selective catalytic oxidation and nitration using environmentally friendly reagents.- New Journal of Chemistry, S. 339-342.

Bezalel, L., Hadar, Y., Cerniglia, C. E. (1997): Enzymatic mechanisms involved in phenanthrene degradation by the white rot fungus Pleurotus ostreatus.- Applied and Environmental Microbiology, 63, H. 7, S. 2495-2501.

Bicke, C., Metzger, J.O. (1993): Matrixunterstützte Laserdesorptions-Massenspektrometrie von Ligninen.- GIT Fachzeitschrift Labor, H. 2, S. 77-83.

Bigi, F.; Chesini, L.; Maggi, R.; Sartori, G. (1999): Montmorillonite KSF as an inorganic, water stable, and reusable catalyst for the Knoevenagel synthesis of coumarin-3carboxylic acids.- Journal of Organic Chemistry, 64, S. 1033-1035.

Birkel, U. (1997): Tonminerale als Katalysatoren der abiotischen Transformation und Interaktion des Huminsäuremonomers Brenzkatechin mit dem organischen Schadstoff p-Chloranilin.- unveröffentlichte Diplomarbeit, Trier.

Birkel, U., J. Niemeyer (1998): Tonminerale als Katalysatoren bei der Umwandlung von organischen Verbindungen.- Zeitschrift für Umweltchemie und Ökotoxikologie, 10 (6), 345-352.- Zeitschrift für Umweltchemie und Ökotoxikologie, 10 (6), 345-352.

Birkel, U., Niemeyer, J., Seeger, B. \& G. Gerold (1998): ${ }^{13}$ C-NMR spektroskopische Untersuchungen zur abiotischen Reaktion von Phenolen und PAK an Tonmineralen.Mitteilungen der Deutschen Bodenkundlichen Gesellschaft, 88, 163-166.

Birkel, U., J. Niemeyer (1999): Montmorillonit-katalysierte Bildung von Vorstufen gebundener Rückstände aus Brenzkatechin und p-Chloranilin.- Chemie der Erde, 59, 47 55 .

Birkel, U., Gallé, T., Niemeyer, J. \& S. Thees (1999): Preparation and reactivity of organicinorganic model colloids for analysis in soil chemistry.- (accepted in Colloids and Surfaces A).

Birkel, U., Vieth, A. \& J. Niemeyer (2000a): Montmorillonit-katalysierter Einbau von polyzyklischen Aromaten in Modellhuminstoffe.- In: Abt. Bodenkunde, Universität Trier (Hrsg.): Festschrift für Dietmar Schröder zum 60. Geburtstag (= Trierer Bodenkundliche Schriften, Band 1), Selbstverlag der Abteilung Bodenkunde, Universität Trier). 
Birkel, U., Gerold, G. \& J. Niemeyer (2000b): Abiotic Reactions of Organics on Clay Mineral Surfaces.- (accepted in Proceedings of the Third International Symposium of the Working Group M.O. "Interactions of Soil Minerals with Organic Components and Microorganisms" of the International Union of Soil Sciences, Naples-Capri, Italy, May 22-26, 2000).

Birkel, U., Dathe, A., Eins, S., Gerold, G. \& J. Niemeyer (2000c): Grenzflächenreaktivität, Aufbau und Zerfall von Mikroaggregaten.- Abschlußbericht des Teilprojekts A6 des Sonderforschungsbereiches 468 ,Wechselwirkungen an geologischen Grenzflächen“ der Deutschen Forschungsgemeinschaft (im Druck).

Blume, H.-P. (Hrsg.): Handbuch des Bodenschutzes.- Landsberg/Lech 1992.

Bollag, J.-M., Myers, C. J., Minard, R. D. (1992): Biological and chemical interactions of pesticides with soil organic matter.- The Science of the Total Environment, H. 123/124, S. 205-217.

Bollag, J.-M., Dec, J., Huang, P. M. (1998): Formation mechanisms of complex organic structures in soil habitats.- Advances in Agronomy, H. 63, S. 237-266.

Bosetto, M.; Arfaioli, P.; Ristori, G.G.; Fusi, P. (1994): Influence of some homoionic clays on the formation of melanoidinic compounds from glucose and tryptophan.- Fresenius Environment Bulletin, H. 3, S. 371-376.

Bosetto, M.; Arfaioli; P.; Ristori, G.G.; Fusi, P. (1995): Formation of melanin-type compounds from L-tryptophan on $\mathrm{Ca}$ - and $\mathrm{Al}$-saturated clays.- Fresenius Environment Bulletin, H. 4, S. 369-374.

Bosetto, M.; Arfaioli, P.; Pantani, O.L.; Ristori, G.G. (1997): Study of the humic-like compounds formed from L-tyrosine on homoionic clays.- Clay Minerals, H. 32, S. 341349.

Boyd, S.A., Mortland, M.M. (1986): Radical formation and polymerization of chlorophenols and chloroanisole on Copper(II)-Smectite.- Environmental Science \& Technology, 20, S. 1056-1058.

Brannon, J.M., Price, C.B., Hayes, C. (1998): Abiotic transformation of TNT in montmorillonite and soil suspensions under reducing conditions.- Chemosphere, 36, $\mathrm{H}$. 6, S. 1453-1462.

Breen, C., Adams, J.M., Riekel, C. (1985): Review of the diffusion of water and pyridine in the interlayer space of montmorillonite: Relevance to kinetics of catalytic reactions in clays.- Clays and Clay Minerals, 33, H. 4, S. 275-284.

Breitmaier, E. (1992): Vom NMR-Spektrum zur Strukturformel organischer Verbindungen. Stuttgart.

Brindley, G.W; Lemaitre, J.: Thermal, oxidation and reduction reactions of clay minerals.Aus: Newman, A.C.D. (ed.): Chemistry of clays and clay minerals. London 1987 (= Mineralogical Society Monograph No. 6), S. 319-370. 
Brown, D.R., Rhodes, C.N.: Acid-treated and ion-exchanged montmorillonite catalysts. Dependence of Activity on Composition.- Aus: Elsen, A., Grobet, P., Keung, M. (eds.): Euroclay'95-Clays and Clays Material Sciences. Book of Abstracts. Leuven 1995, S. 189-190.

Brown, D. R., Rhodes, C. N. (1997): Bronsted and Lewis acid catalysis with ion-exchanged clays.- Catalysis Letters, H. 45, S. 35-40.

Bujdak, J., Rode, B.M. (1996): The effect of smectite composition on the catalysis of peptide bond formation.- Journal of Molecular Evolution, H. 43, S. 326-333.

Bujdak, J., Son, H.L., Rode, B.M. (1996): Montmorillonite catalyzed peptide bond formation:. The effect of exchangeable cations.- Journal of Inorganic Chemistry, H. 63, S. 119-124.

Buondonno, A.: Models of organo-mineral aggregates with polyphenols. 2. Stability of bentonite-Ca-gallotannin interaction products.- Aus: Senesi, N.; Miano, T.M. (eds.): Humic substances in the global environment and implications on human health. 1994, S. 1101-1106.

Cairns-Smith, A.G. (ed.): Clay minerals and the origin of life. Cambridge 1986.

Campelo, J.M. (1994): Spanish sepiolite clay as a new heterogenous catalyst for the tetrahydropyranylation of alcohols and phenols.- Synthetic Communications, 24, H. 10, S. 1345-1350.

Casillas, R. P., Crow, S. A., Heinze, T. M., Deck, J., Cerniglia, C. E. (1996): Initial oxidative and subsequent conjugative metabolites produced during the metabolism of phenanthrene by fungi.- Journal of Industrial Microbiology, H. 16, S. 205-215.

Cativiela, C. (1993a): Relationship between solvent effects and catalyst activation method in a clay-catalysed Diels-Alder reaction. In: Journal of Molecular Catalysis, 79, S. 305-310.

Cativiela, C. (1993b): Clay-catalysed asymmetric Diels-Alder reaction of cyclopentadiene with chiral acrylates.- Tetrahedron: Asymmetry, 4, H. 2, S. 223-228.

Cerniglia, C.E.: Microbial Transformation of Aromatic Hydrocarbons. Aus: Atlas, R.M. (ed.): Petroleum Microbiology. New York 1984, S. 99-128.

Cerniglia, C.E. (1993): Biodegradation of polycyclic aromatic hydrocarbons.- Current Opinion in Biotechnology, H. 4, S. 331-338.

Chahi, A., Larque, P., Frere, Y., Gramain, P., Weber, F. (1996): The use of surfactants for clay dispersion in organic matter-rich soil: Preliminary examination.- Soil Science Society of America Journal, 161, H. 11, S. 761-769.

Choudry, G.G. (1983): Humic Substances. New York.

Clark, P.D., Kirk, A., Yee, G.K. (1995): An improved synthesis of Benzo[b]thiopene and its derivatives using modified montmorillonite clay catalysts.- Journal of Organic Chemistry, H. 60, S. 1936-1938. 
Collins, M.J., Bishop, A.N., Farrimond, P. (1995): Sorption by mineral surfaces: Rebirth of the classical condensation pathway for kerogen formation?- Geochimica and Cosmochimica Acta, 59, H. 11, S. 2387-2391.

Corma, A., Wojciechowski, B.W. (1985): The chemistry of catalytic cracking.- Catalysis Reviews, 27, H. 1, S. 29-150.

Cornélis, A., Laszlo, P.: Preparative organic chemistry using clays. Aus: Setton, E. (eds.): Chemical reactions in organic and inorganic constrained systems. 1986, S. 213-228.

Cozens, F.L., Gessner, F., Scaiano, J.C. (1993): Spontaneous carbocation generation on clays.- Langmuir, H. 9, S. 874-876.

Cseri, T., Bekassy, S., Figueras, F., Cseke, E., De Menorval, L.-C., Dutartre, R. (1995): Characterization of clay-based $\mathrm{K}$ catalysts and their application in Friedel-Crafts alkylation of aromatics.- Applied Catalysis A: General, H. 132, S. 141-155.

D'Acqui, L.P.; Daniele, E.; Fornasier, F.; Radaelli, L.; Ristori, G.G. (1998): Interaction between clay microstructure, decomposition of plant residues and humification.European Journal of Soil Science, H. 49, S. 579-587.

Dec, J., Bollag, J.-M. (2000): Phenoloxidase-mediated interactions of phenols and anilines with humic Materials.- Journal of Environmental Quality, H. 29, S. 665-676.

Do, J.-S., Yeh, W.-C. (1996): Paired electrooxidative degradation of phenol with in situ electrogenerated hydrogen peroxide and hypochlorite.- Journal of Applied Electrochemistry, 26, S. 673-678.

Dragun, J., Helling, C.S. (1985): Physicochemical and structural relationships of organic chemicals undergoing soil- and clay-catalyzed free-radical oxidation.- Soil Sience, 139, H. 2, S. 100-111.

Dunnivant, F.M., Schwarzenbach R.P. (1992): Reduction of substituted nitrobenzenes in aqueous solutions containing natural organic matter.- Environmental Science \& Technology, H. 26, S. 2133-2141.

Ehlers, E. (1989): Chemie II. Neckarsulm.

Eisener, W. (1983): Untersuchungen zur Genese und Strukturaufklärung von Huminsäuren.Dissertation, Tübingen.

Emundts, J. (1997): Zur Optimierung der Wiederfindungsraten von Schadstoffen in Bodengemischen am Beispiel der Verteilung von polycyclischen aromatischen Kohlenwasserstoffen.- Dissertation, Göttingen.

Evangelou, V.P. (1998): Environmental Soil and water chemistry. Principles and applications.- New York.

Evanko, C.R., Dzombak, D.A. (1999): Surface complexation modeling of organic acid sorption to goethite.- Journal of Colloid and Interface Science, H. 214, S. 189-206. 
Faguy, P.W., Lucas, R.A., Ma, W. (1995): An FT-IR-ATR spectroscopic study of the spontaneous polymerization of pyrrole in iron-exchanged montmorillonite.- Colloids and Surfaces, H. 105, S. 105-112.

Fiedler, H.J., Everts, H. (1975): Energiedispersive Röntgen-Mikroanalyse, Grundlagen und Instrumente.-

Filip, Z., Flaig, W., Rietz, E. (1981): Oxidation of some phenolic substances as influenced by clay minerals.- International Clay Conference, S. 91-96.

Flaig, W.: Generation of model chemical precursors.- Aus: Frimmel, F.H., Christman, R.F. (Hrsg.): Humic Substances and their Role in the Environment. 1988. Berlin.

Friebolin, H. (1992): Ein- und zweidimensionale NMR-Spektroskopie.- Weinheim.

Fripiat, J.J.; Cruz-Cumplido, M.I.: Clays as catalysts for natural processes.- Annual Review of Earth and Planetary Sciences 2, S. 239-256.

Fründ, R., Lüdemann, H.-D.: Characterization of soil organic matter with light-speed CPMAS-NMR-spectroscopy.- Aus: Senesi, N., Miano, T.M. (eds.): Humic substances in the global environment and implications on human health. 1994, S. 161-165.

Golchin, A. (1994): Study of free and occluded particilate organic matter in soils by solid state ${ }^{13} \mathrm{C}$ CP/MAS NMR spectroscopy and scanning electron microscopy.- Soil Biology and Biochemistry, 32, S. 285-309.

Gottwald, W., Wachter, G. (1997): IR-Spektroskopie für Anwender.- Weinheim (=Die Praxis der instrumentellen Analytik).

Grandjean, J., Laszlo, P.: Structure and dynamics of polar molecules at a clay interface: a multinuclear magnetic resonance study.- Aus: Elsen, A., Grobet, P., Keung, M. (eds.): Euroclay'95 - Clays and Clays Material Sciences. Book of Abstracts. Leuven 1995, S. 8-31.

Grauer, R. (1989): Zur Koordinationschemie der Huminstoffe.- Technischer Bericht. PSI.

Gu, B., Doner, H. (1992): The interaction of polysaccharides with Silver Hill illite.- Clays and Clay Minerals, H. 40 (2), S. 151-156.

Günzler, H., Heise, M.H. (1996): IR-Spektroskopie: Eine Einführung.- Weinheim.

Hammel, K.E. (1992): Oxidative degradation of phenanthrene by the ligninolythic fungus Phanerochaete chrysosporium.- Applied and Environmental Microbiology, 58, H. 6, S. 1832-1838.

Hancock, P., Dean, J.R. (1997): Extraction and fate of phenols in soil.- Analytical Communications, H. 34, S. 377-379.

Hasselmann, N.: Über den Mechanismus des Kohlehydrateinbaus in Huminstoffen.- Aus: Müller-Wegener, U. (Hrsg.): Organische Inhaltsstoffe des Bodens. 1994 
Hatzinger, P.B., Alexander, M. (1995): Effect of aging of chemicals in soil on their biodegradability and extractability.- Environmental Science \& Technology, 29, H. 2, S. 537-545.

Hauke, V., Trendel, J.M., Albrecht, P. (1994): Spiro-tripentenes from clay-catalysed rearrangement of hopenes: NMR structural elucidation and occurence in a recent sediment.- Tetrahedron Letters, 35, H. 14, S. 2227-2230.

Hay, A.S. (1967): Aromatic polyethers.- Advances in polymer science, 4, S. 496-527.

Hayes, M.H.B.: Concepts of the origins, composition, and structures of humic substances.Aus: Wilson, W.S. (ed.): Advances in soil organic matter research. 1991

Heimann, R.B. (1993): Bronsted acidification observed during hydrothermal treatment of calcium montmorillonite.- Clays and Clay Minerals, 41, H. 6, S. 718-725.

Heinrichs, T. (o.J.): Arbeitsanleitung REM.- Labor IGDL, Universität Göttingen.

Helsen, J. (1982): Clay Minerals as solid acids and their catalytic properties.- Journal of Chemical Education, 59, H. 12, S. 1063-1065.

Hemminga, M. A., Buurman, P. (1997): Editorial: NMR in soil science.- Geoderma, H. 80, S. 221-224.

Hermosin, M.C.; Martin, P.; Cornejo, J. (1993): Adsorption mechanisms of monobutyltin in clay minerals.- Environmental Science \& Technology, 27, S. 2606-2611.

Herzog, W.-D., Messerschmidt, M. (1995): NMR-Spektroskopie für Anwender. Weinheim 1995. (= Die Praxis der instrumentellen Analytik.)

Huang, P.M.: Role of soil minerals in transformations of natural organics in soil.- Aus: Bollag, J.-M.; Stotzky, G. (eds.): Soil Biochemistry. New York, Basel 1990, S. 29-115.

Ikeda, R., Sugihara, J., Uyama, H., Kobayashi, S. (1996): Enzymatic oxidative polymerization of 2,6-dimethylphenol.- Macromolecules, H. 29, S. 8702-8705.

Ionescu, M., Mihis, A.B. (1997): The mechanism of chain growth in oxidative polycondensation of phenols.- Macromolecules Symp., H. 122, S. 249-256.

Isaacson, P.J., Sawhney, B.L. (1983): Sorption and transformation of phenols on clay surfaces. effect of exchangeable cations.- Clay Minerals, H. 18, S. 253-265.

Izumi, Y., Urabe, K., Onaka, M. (1992): Zeolithe, clay and heteropoly acid in organic reactions.- Tokyo, Weinheim, New York.

Jasmund, K., Lagaly, G. (1993): Tonminerale und Tone: Struktur, Eigenschaften, Anwendungen und Einsatz in Industrie und Umwelt.- Darmstadt.

Jnaneshwara, G.K., Deshpande, V.H., Bedekar, A.V. (1999): Clay catalyzed conversion of 2,2-disubstituted malononitriles to 2-oxazolines. Towards unnatural amino acids.Journal of Chemical Research, S. 1219-1227. 
Johnston, C.T.: Sorption of organic compounds on clay minerals.- Aus: Senesi, N., Miano, T.M. (eds.): Humic substances in the global environment and implications on human health. 1994.

Kaiser, K., Zech, W. (1997): Natural organic matter sorption on different mineral surfaces studied by DRIFT spectroscopy.- Sciences of Soils, 2.

Kalinowski, H.-O., Berger, S., Braun, S. (1984): ${ }^{13}$ C-NMR-Spektroskopie.

Kannan, P., Pitchumani, K. (1997): Clay-catalysed radical addition of aliphatic thiols to styrene.- Catalysis Letters, 45, 271-273.

Kantam, M. L., Kavita, B., Figueras, F. (1998): Oxidative coupling of 2-naphtols catalysed by clays-supported iron catalysts.- Catalysis Letters, H. 51, S. 113-115.

Karas, M., Bahr, U. (1996): Matrix-Assisted Laser Desorption-Ionization (MALDI) Mass Spectrometry of Biological Molecules.- NATO ASI series / C, Bd. 475, S. 33-50.

Karas, M., Bahr, U. (1997): Matrix-Assisted Laser Desorpition-Ionization (MALDI) Mass Spectrometry: Principles and Applications.- NATO: NATO ASI series / C., Bd. 504, S. 33-54.

Keren, R., Sparks, D.L. (1995): The role of edge surfaces in flocculation of 2:1 clay minerals.- Soil Science Society of America Journal, 59, S. 430-435.

Kinchesh, P., Randall, E. W., Zick, K. (1994): Magnetic susceptibility effects in imaging: distortion-free images of plant tissue in soil.- Magnetic Resonance Imaging, H. 12, S. 305-307.

Kirk, T.K., Farell, R.L. (1987): Enzymatic "combustion": The microbiological degradation of lignin.- Annual Review of Microbiology, 41, S. 465-505.

Klöpffer, W. (1996): Verhalten und Abbau von Umweltchemikalien. Physikalisch-chemische Grundlagen.- Landsberg. (= Angewandter Umweltschutz.)

Knicker, H., Lüdemann, H.-D. (1995): N-15 and C-13 CPMAS and solution NMR studies of $\mathrm{N}-15$ enriched plant material during 600 days of microbial degradation.- Organic Geochemistry, 23, H. 4, S. 329-341.

Koch,R. (1995): Umweltchemikalien: physikalisch-chemische Daten, Toxizitäten, Grenz- und Richtwerte, Umweltverhalten.- Weinheim.

Kögel-Knabner, I. (1997a): ${ }^{13} \mathrm{C}$ and ${ }^{15} \mathrm{~N}$ NMR spectroscopy a tool in soil organic matter studies.- Geoderma, H. 80, S. 243-270.

Kögel-Knabner, I. (1997b): Forest soil organic matter.

Köhler, A. (1992): Zur Problematik der biologischen Verfügbarkeit von polycyclischen aromatischen Kohlenwasserstoffen untersucht am Beispiel des Phenanthrenabbaus.Dissertation, Stuttgart. 
Komadel, P., Janek, M., Madejova, J., Weekes, A., Breen, C. (1997): Acidity and catalytic activity of mildly acid-treated Mg-rich montmorillonite and hectorite.- Journal of the Chemical Society: Faraday Transactions, 93, H. 23, S. 4207-4210.

Kowalska, M., Güler, H., Cocke, D.L. (1994): Interactions of clay minerals with organic pollutants.- The Science of the Total Environment, 141, S. 223-240.

Kraatz, M. (1995): Untersuchungen zum Einfluss von bodenmikrobiologischen Aktivitäten und Humifizierungsprozessen auf die Schadstoffdynamik während der mikrobiologischen on-site-Sanierung und Rekultivierung eines mit Polyzyklischen Aromatischen Kohlenwasserstoffen (PAK) belasteten Altstandortes.- Dissertation, Trier.

Kretzschmar, R.; Hesterberg, D.; Sticher, H. (1997): Effects of adsorbed humic acid on surface charge and flocculation of kaolinite.- Soil Science Society of America Journal, 61, S. 101-108.

Krüger, R.-P. (1995): MALDI-TOF-MS synthetischer Polymere.- GIT-Fachzeitschrift Labor, H. 3, S. 189-195.

Kunc, F., Stotzky, G. (1977): Acceleration of aldehyde decomposition in soil by montmorillonite.- Soil Science, 124, H. 3, S. 167-172.

Kurioka, H., Uyama, H., Kobayashi, S. (1998): Peroxidase-catalyzed dispersion polymerization of phenol derivatives.- Polymer Journal, H. 30 (6), S. 526-529.

Kürschner, U., Seefeld, V., Parlitz, B., Geßner, W., Lieske, H. (1998): Catalytic activity and acidity of Al pillared clays and zeolites in different hydrocarbon reactions.- Reaction Kinetics Catalysis Letters, H. 65(1), S. 17-23.

Lagaly, G. (1994): Bentonites: adsorbents of toxic substances.- Progress in Colloid \& Polymer Science, H. 95, S. 61-72.

Larson, R.A., Hufnal, J.M. (1980): Oxidative polymerization of dissolved phenols by soluble and insoluble inorganic species.- Limnology Oceanography, 25, H. 3, S. 505-512.

Li, Y.; Gupta, G. (1994): Adsorption/desorption of hydrocarbons on clay minerals.Chemosphere, 28, H. 3, S. 627-638.

Li, T.-S., Li, A.-X. (1998): Montmorillonite clay catalysis. Part 10. K-10 and KSF-catalysed acylation of alcohols, phenols, thiols and amines: Scope and limitation.- Journal of the Chemical Society, Perkin Transactions 1, S. 1913-1917.

Li, T.-S., Zhang, Z.-H., Yang, F., Fu, C.-G. (1998): Montmorillonite clay catalysis. Part 7. An environmentally friendly procedure for the synthesis of coumarins via Pechmann condensation of phenols with ethyl acetoacetate.- Journal of Chemical Research, H. 5, S. 38-39.

Lovley, D.R., Coates, J.D., Blunt-Harris, E.L., Phillips, E.J.P., Woodward, J.C. (1996): Humic substances as electron acceptors for microbial respiration.- Nature, H. 382, S. 445-448. 
Lovley, D.R., Fraga, J.L., Blunt-Harris, E.L., Hayes, L.A., Phillips, E.J.P., Coates, J.D. (1998): Humic substances as a mediator for microbially catalyzed metal reduction.- Acta Hydrochimica Hydrobiologica, 26, H. 3, S. 152-157.

Maiti, A.K., Biswas, G.K., Bhattacharyya, P. (1993): Montmorillonite clay-catalysed regioselective ring opening of oxiranes with thiophenol: A simple synthesis of Bhydroxy sulfides.- Journal of Chemical Research, S. 325.

Marschner, B. (1997): Chemische und biologische Einflussfaktoren der PAK- und PCBMobilisierung im Boden.- Berlin (= Bodenökologie und Bodengenese. Heft 25).

McBride, M.B. (1985): Surface reaction of 3,3',5,5'-tetramethyl benzidine on hectorite.Clays and Clay Minerals, 33, H. 6, S. 510-516.

McBride, M.B. (1994): Environmental chemisty of soils.- New York, Oxford 1994.

Miller, D.M., Buettner, G.R., Aust, S.D. (1990): Transition metals as catalysts of autooxidation reactions.- Free Radical Biology \& Medicine, H. 8, S. 95-108.

Morikawa, Y. (1993): Catalysis by metal ions interlaced in layer lattice silicates.- Advances in Catalysis, 39, S. 303-327.

Morill, L.G. (1982): Organic compounds in soils: Sorption, degradation and persistence.- Ann Arbor.

Mortland, M.M. (1970): Clay-organic complexes and interactions.- Advances in Agronomy, 22. S. 75-116.

Mortland, M.M.: Surface reactions of low-molecular-weight organics with soil components.Aus: Banin, A.; Kafkaki, U. (eds.): Agrochemicals in Soils. 1980. S. 67-72.

Mortland, M.M.: Mechanisms of adsorption of nonhumic organic species by clays.- Aus: Soil Science Society of America (eds.): Interaction of soil minerals with natural organics and microbes. Madison 1986 (=SSSA Spec. Pub. 17), S. 59-76.

Müncnerova, D., Augustin, J. (1994): Fungal metabolism and detoxification of polycyclic aromatic hydrocarbons: A review.- Bioresource Technology, 48, H. 2, S. 97-106.

Naidja, A., Huang, P. M., Bollag, J.-M. (1998): Comparison of reaction products from the transformation of catechol catalyzed by birnessite or tyrosinase.- Soil Science Society of America Journal, H. 62, S. 188-195.

Naidja, A., Huang, P.M., Bollag, J.-M. (2000): Enzyme-clay interactions and their impact on transformations of natural and anthropogenic organic compounds in soil.- Journal of Environmental Quality, H. 29, S. 677-691.

Nakazawa, H.; Yamada, H.; Hashizume, H.: The upper crust origin of life, a hypothesis based on miscellaneous roles of clays in chemical evolution.- Aus: Elsen, A.; Grobet, P.; Keung, M. (eds.): Euroclay'95-Clays and Clays Material Sciences. Book of Abstracts. Leuven 1995, S. 200-201. 
Newman, A.C.D. (1987): Chemistry of clays and clay minerals London (= Mineralogical Society Monograph No. 6).

Niemeyer, J., Chen, Y., Bollag, J.-M. (1992): Characterization of humic acids, composts and peat by diffuse reflectance Fourier-Transform Infrared Spectroscopy.- Soil Science Society of America Journal, 56, H. 135-140.

Nowara, A., Burhenne, J., Spiteller, M. (1997): Binding of fluoroquinolone carboxylic acid derivatives to clay minerals.- Journal of Agriculture and Food Chemistry, H. 45, S. 1459-1463.

Occelli, M.L.: Surface and catalytic properties of some pillared clays.- Aus: Schultz, L.G.; Van Olpen, H.; Mumpton, F.A. (eds.): Proceedings of the International Clay Conference, Denver 1985. Bloomington 1987, S. 319-323.

Ohashi, H., Nakazawa, H. (1996): The microstructure of humic acid-montmorillonite composites.- Clay Minerals, H. 31, S. 347-354.

Ortega-Calvo, J.-J., Saiz-Jimenez, C. (1998): Effect of humic fractions and clay on biodegradation of phenanthrene by a Pseudomonas fluorescens strain isolated from soil.Applied and Environmental Microbiology, 64, H. 8, S. 3123-3126.

Ortego, J.D., Kowalska, M., Cocke, D.L. (1991): Interactions of montmorillonite with organic compounds - Adsorptive and catalytic properties.- Chemosphere, 22, H. 8, S. 769-798.

Pal, S., Bollag, J.-M., Huang, P.M. (1994): Role of abiotic and biotic catalysts in the transformation of phenolic compounds through oxidative coupling reactions.- Soil Biology and Biochemistry, 26, H. 7, S. 813-820.

Pal, T., Pal, A. (1996): Oxidative phenol coupling: A key step for the biomimetic synthesis of many important natural products.- Current Science, H. 71 (2), S. 106-108.

Park, J.-W., Dec, J., Kim, J.-E., Bollag, J.-M. (1999): Effect of humic constituents on the transformation of chlorinated phenols and anilines in the presence of oxireductase enzymes or birnessite.- Environmental Science \& Technology, H. 33, S. 2028-2034.

Park, J.-W., Dec, J., Kim, J.-E., Bollag, J.-M. (1999): Dehalogenation of xenobiotics as a consequence of binding to humic materials.- Archives of Environmental Contamination and Toxicology, 38, S. 405-410.

Park, J.-W., Dec, J., Kim, J.-E., Bollag, J.-M. (2000): Transformation of chlorinated phenols and anilines in the presence of humic acid.- Journal of Environmental Quality, 29, S. 214-220.

Parker, R.W.; Frost, R.L. (1996): The application of DRIFT spectroscopy to the multicomponent analysis of organic chemicals adsorbed on montmorillonite.- Clays and Clay Minerals, 44, H. 1, S. 32-40.

Parker, A., Rae, J.E. (eds.): Environmental Interaction of Clays. Clays and the Environment. Berlin, Heidelberg, New York 1998. 
Pasch, H. (1996): Functionality analysis of polymers by MALDI-MS.- Polymer Preprints, 37, S. 319-320.

Pfenniger, A. (2000): Massenspektrometrische Untersuchungen von Oligosacchariden aus humaner Milch.- Dissertation, Frankfurt.

Pinnavaia, T.J. (1983): Intercalated clay catalysts.- Science, 220, H. 4595, S. 365-371.

Pinnavaia, T.J.; Mortland, M.M.: Clays as catalysts.- Aus: Cairns-Smith, A.G. (ed.): Clay minerals and the origin of life. Cambridge 1986, S. 130-135.

Ponde, D. E., Deshpande, V. H., Bulbule, V. J., Sudalai, A., Gajare, A. S. (1998): Selective catalytic transesterification, transthiolesterification, and protection of carbonyl compounds over natural kaolinitic clay.- Journal of Organic Chemistry, H. 63, S. 10581063.

Pospisil, F.: Oxidation von Phenolen, deren Interaktionen und Synthese von Huminsäuren.Aus: Müller-Wegener, U. (Hrsg.): Organische Inhaltsstoffe des Bodens 1994.

Potthast, A., Rosenau, T., Koch, H., Fischer, K. (1999): The reaction of phenolic model compounds in the laccase-mediator system (LMS) - investigations by matrix assisted laser desorption ionization time-of-flight mass spectrometry (MALDI-TOF-MS).Holzforschung, H. 53, S. 175-180.

Poupaert, J.H., Bukuru, J., Gozzo, A. (1999): Clay (montmorillonite K10) catalysis of the Michael addition of a,b-unsaturated carbonyl compounds to indoles. The beneficial role of alcohols.- Monatshefte für Chemie, H. 130, S. 929-932.

Preston, C.M. (1996): Applications of NMR to soil organic matter analysis. history and prospects.- Soil Science Society of America Journal, 161, H. 3, S. 144-166.

Pusch, R.: Transport of radionuclides in smectite clay.- Aus: Parker, A. \& Rae, J.E. (eds.) Environmental Interactions of Clay. Berlin 1998.

Randall, E. W., Mahieu, N., Ivanova, G. I. (1997): NMR studies of soil, soil organic matter and nutrients: spectroscopy and imaging.- Geoderma, H. 80, S. 307-325.

Righi, D.; Dinel, H.; Schulten, H. R.; Schnitzer, M. (1995): Characterization of clay-organicmatter complexes resistant to oxidation by peroxide.- European Journal of Soil Science, H. 46, S. 423-429.

Ruggiero, P.: Abiotic transformations of organic xenobiotics in soils. A compounding factor in the assessment of bioavailability.- Aus: Baveye, P. et al. (eds.): Bioavailability of organic xenobiotics in the environment. Dordrecht 1999, S. 159-205.

Rupert, P.J.; Granquist, W.T.; Pinnavaia, T.J.: Catalytic properties of clay minerals.- Aus: Newman, A.C.D. (ed.): Chemistry of clays and clay minerals. London 1987 (= Mineralogical Society Monograph No. 6), S. 275-318. 
Sack, U., Heinze, T. M., Deck, J., Cerniglia, C. E., Cazau, M. C., Fritsche, W. (1997): Novel metabolites in phenanthrene and pyrene transformation by Aspergillus niger.- Applied and Environmental Microbiology, H. 63, S. 2906-2909.

Saleh, S.A., Mahmoud, S.S. (1998): Catalytic debutylation of tert-butylphenols by some natural clays.- Reaction Kinetics Catalysis Letters, 64, H. 2, S. 373-380.

Sanger, L.J., Anderson, J.M., Little, D., Bolger, T. (1997): Phenolic and carbohydrate signatures of organic matter in soils developped under grass and forest plantations following changes in land use.- European Journal of Soil Science, 48, S. 311-317.

Sartori, G., Maggi, R., Bigi, F., Arienti, A., Casnati, G. (1992): Regiochemical control in the oxidative coupling of metal phenolates. Highly selective synthesis of symmetric, hydroxylated biaryls.- Tetrahedron Letters, 33, H. 16, S. 2207-2210.

Sartori, G., Maggi, R., Bigi, F., Arienti, A., Casnati, G. (1992): Oxidative coupling of dichloroaluminium phenolates. Highly selective synthesis of hydroxylated bi- and tetraaryls.- Tetrahedron Letters, 48, H. 43, S. 9483-9494.

Sawhney, B.L., Kozloski, R.K., Isaacson, P.J., Gent, M.P.N. (1984): Polymerization of 2,6dimethylphenol on smectite surfaces.- Clays and Clay Minerals, 32, H. 2, S. 108-114.

Sawhney, B.L. (1985): Vapor-phase sorption and polymerization of phenols by smectite in air and nitrogen.- Clays and Clay Minerals, 33, H. 2, S. 123-127.

Schachtschabel, P., Blume, H.-P., Hartge, K.-H., Schwertmann, U. (1992): Lehrbuch der Bodenkunde.- Stuttgart.

Scheunert, I. (1999): Entstehung und Freisetzung von nicht-extrahierbaren Rückständen im Boden.- Chemie in unserer Zeit, 33, H. 1, S. 59-60.

Schmidt, P.F. (1994): Praxis der Rasterelektronenmikroskopie und Mikrobereichsanalyse.Renningen-Malmsheim (= Kontakt und Studium, Bd. 444).

Schnitzer, M.: Organic matter characterization.- Aus: ASA-SSSA (ed.): Methods of Soil Analysis. Part 2-Chemical and Microbiological Properties. Madison 1982. (=Agronomy Monograph. 9) S. 581-594.

Schoonheydt, R.A.: Clay Mineral Surfaces.- Aus: Vaughn, D.J., Pattrick, R.A.D. (eds.): Mineral Surfaces.- London 1995, S. 303-332.

Schulten, H.R., Schnitzer, M. (1997): Chemical model structures for soil organic matter and soils.- Soil Science, 162, H. 2, S. 115-130.

Schultz, L.G., Van Olpen, H., Mumpton, F.A. (eds.): Proceedings of the International Clay Conference, Denver 1985.- Bloomington 1987.

Schumacher, B. (1995): Huminstoffsysteme und mikrobielle Eigenschaften rekultivierter Böden des Rheinischen Braunkohlereviers unter verschiedener Erstnutzung und ihre Interaktion mit einem polyzyklischen aromatischen Kohlenwasserstoff.- Dissertation, Trier. 
Servagent-Noinville, S., Revault, M., Quiquampoix, H., Baron, M.-H. (2000): Conformational changes of bovine serum albumin induced by adsorption on different clay surfaces: FTIR Analysis.- Journal of Colloid and Interface Science, H. 221, S. 273 283.

Sharma, G.V.M., Ramanaiah, K.C.V., Krishnudu, K. (1994): Clay montmorillonite in carbohydrates: Use of "claysil" as an efficient heterogenous catalyst for the intermolecular ferrier reactions leading to 1,6-anhydro rare saccharides.- Tetrahedron: Asymmetry, 5, H. 10, S. 1905-1908.

Shevchenko, S.M., Bailey, G.W. (1998): Modelling sorption of soil organic matter on mineral surfaces: wood-derived polymers on mica.- Supramolecular Science, 5, H. 1-2, S. $143-$ 157.

Shevchenko, S.M., Bailey, G.W., Akim, L.G. (1999): The conformational dynamics of humic polyanions in model organic and organo-mineral aggregates.- Theochem, H. 460, S. 179-190.

Shindo, H., Huang, P.M. (1985a): The catalytic power of inorganic components in the abiotic synthesis of hydroquinone-derived humic polymers.- Applied Clay Science, H. 1, S. 71 81.

Shindo, H., Huang, P.M. (1985b): Catalytic polymerization of hydroquinone by primary minerals.- Soil Sience, 139, H. 6, S. 505-511.

Shindo, H.: Significance of Mn(IV) and Fe(III) oxides in the synthesis of humic acids from phenolic compounds.- Aus: Senesi, N., Miano, T.M. (eds.): Humic substances in the global environment and implications on human health. 1994, S. 361-365.

Solomon, D.H. (1968): Clay minerals as electron acceptors and/or electron donors in organic reactions.- Clays and Clay Minerals, 16, S. 31-39.

Solomon, D.H., Hawthorne, D.G. (1983): Chemistry of pigments and fillers.- New York.

Soma, Y., Soma, M. (1989): Chemical reactions of organic compounds on clay surfaces.Environmental Health Perspectives, H. 88, S. 205-214.

Son, H.L., Suwannachot, Y., Bujdak, J., Rode, B.M. (1998): Salt-induced peptide formation from amino acids in the presence of clays and related catalysts.- Inorganica Chimica Acta, H.272, S. 89-94.

Stevenson, F.J. (1994): Humus Chemistry. New York, Chichester, Brisbane, Toronto, Singapore 1994.

Sutherland, J.B. (1992): Detoxification of polycyclic aromatic hydrocarbons by fungi.Journal of Industrial Microbiology, 9, S. 53-62.

Tadjerpisheh, N. (1986): Katalytische und adsorptive Eigenschaften der Tonminerale.Fortschritte der Mineralogie, 67, H. 1, S. 176. 
Tateiwa, J., Nishimura, T., Uemura, S. (1996): $\mathrm{Al}^{3+}$-exchanged montmorillonite as an effective solid catalyst for selective synthesis of alkylphenols and bisphenols.Chemistry Letters, S. 59-60.

Theng, B.K.G. (1976): Interactions between montmorillonite and fulvic acid.- Geoderma, 15, S. 243-251.

Theng, B.K.G.: Clay-activated organic reactions.- International Clay Conference 1981, S. 197-239.

Theng, B.K.G.; Newman, R.H.; Whitton, J.S. (1998): Characterization of an alkylammoniummontmorillonite-phenanthrene intercalation complex by carbon-13 nuclear magnetic resonance spectroscopy.- Clay Minerals, 33, S. 221-229.

Thiem, A. (1994): Der Einfluss von Tensiden auf die Mobilisierung und den mikrobiellen Abbau von Polyzyklischen Aromatischen Kohlenwasserstoffen.- Dissertation, Hamburg.

Thompson, T.D., Moll, W.F. (1973): Oxidative power of smectites measured by hydroquinone.- Clays and Clay Minerals, 21, S. 337-350.

Tipton, T., Gerdom, L.E. (1992): Polymerization and transalkylation reactions of toluene on $\mathrm{Cu}(\mathrm{II})$-montmorillonite.- Clays and Clay Minerals, 40, H. 4, S. 429-435.

Tributh, H., Lagaly, G. (1986): Aufbereitung und Identifizierung von Boden- und Lagerstättentonen. I. Aufbereitung der Proben im Labor.- GIT-Fachzeitschrift Labor, H. 6, S. 524-529.

Uyama, H., Ikeda, R., Sugihara, J., Kobayashi, S. (1998): Enzymatic polymerization of natural phenol derivatives to a new class of polyphenols.- Polymer Preprints, 39, H. 2, S. 106-107.

Vaccari, A. (1998): Preparation and catalytic properties of cationic and anionic clays.Catalysis Today, H. 41, S. 53-71.

Vaccari, A. (1999): Clays and catalysis: A promising future.- Applied Clay Science, H. 14, S. 161-198.

Varadachari, C.; Mondal, A.H.; Ghosh, K. (1995): The influence of crystal edges on clayhumus complexation.- Soil Sience, 159, H. 3, S. 185-190.

Velu, S., Swamy, C.S. (1996): Alkylation of phenol with 1-propanol and 2-propanol over catalysts derived from hydrotalcite-like anionic clays.- Catalysis Letters, H. 40, S. $265-$ 272.

Veeman, W. S. (1997): Nuclear magnetic resonance, a simple introduction to the principles and applications.- Geoderma, H. 80, S. 225-242.

Violante, A., Arienzo, M., Sannino, F., Colombo, C., Piccolo, A., Gianfreda, L. (1999): Formation and characterization of $\mathrm{OH}-\mathrm{Al}$-humate-montmorillonite complexes.- Organic Geochemistry, 30, S. 461-468. 
Virkki, L. (1993): Structural characterization of chlorolignins by spectroscopic and liquid chromatographic methods and a comparison with humic substances.- Dissertation, Jyraskyla.

Voudrias, E.A., Reinhard, M.: Abiotic organic reactions at mineral surfaces.- Aus: Davis, J., Hayes, K. (eds.): Geochemical processes at mineral surfaces. Washington 1986, S. $462-$ 486.

Wais, A. (1995): Using ${ }^{13} \mathrm{C}-\mathrm{NMR}$ spectroscopy to evaluate the binding mechanism of bound pesticide residues in soils. 1. Solution High Resolution NMR Spectroscopy.- Journal of Environmental Science and Health, Bd. 30, H. 1, S. 1-25.

Waksman, S.A. (1932): Humus.- Baltimore.

Walzak, M.J., Davidson, R., Biesinger, M. (1998): The use of XPS, FTIR, SEM/EDX, Contact Angle, and AFM in the characterization of coatings.- Journal of Materials Engineering and Performance, 7, S. 317-323.

Wang, M.C.; Huang, P.M.: Structural role of polyphenols in influencing the ring cleavage and related chemical reactions as catalysed by nontronite. Aus: Senesi, N.; Miano, T.M. (Hrsg.): Humic substances in the global environment and implications on human health. o.O. (Elsevier) 1994. S. 173-179.

Wang, M.C., Huang, P.M. (1987): Catalytic polymerization of hydroquinone by nontronite.Canadian Journal of Soil Science, H. 67, S. 867-875.

Wang, M.C., Huang, P.M. (2000): Characteristics of pyrogallol-derived polymers formed by catalysis of oxides.- Soil Science, H. 165, S. 737-747.

Wang, T.S.C., Wu, L.S. (1977): Clay minerals as heterogeneous catalysts in preparation of model humic substances.- Zeitschrift für Pflanzenernährung und Bodenkunde, 140, S. 669-676.

Wang, T.S.C. (1983): Catalytic synthesis of humic substances by natural clays, silts and soils.- Soil Sience, 135, H. 6, 350-360.

Wang, T.S.C., Wang, M.-C., Huang, P.M. (1983): Catalytic synthesis of humic substances by using aluminas as catalysts.- Soil Sience, 136, H. 4, S. 226-230.

Wang, T.S.C.: The role of soil minerals in the abiotic polymerization of phenolic compounds and formation of humic substances.- Aus: Soil Science Society of America (eds.): Interaction of soil minerals with natural organics and microbes. Madison 1986 (=SSSA Spec. Pub. 17), S. 251-277.

Whelan, G.; Sims, R.C. (1995): Mn-catalyzed oxidation of multiple-ringed aromatics.Hazardous Waste \& Hazardous Materials, 12, H. 3, S. 243-254.

Wershaw, R.L., Llaguno, E.C., Leenheer, J.A. (1996): Mechanism of formation of humus coatings on mineral surfaces. 3. Composition of adsorbed organic acids from compost 
leachate on alumina by solid-state ${ }^{13}$ C-NMR.- Colloids and Surfaces /A, H. 108, S. 213 224.

White, J.L., Roth, B.: Infrared Spectrometry.- Soil Science Society of America, S. 291-330.

Wu, K.W., Odom, R.W. (1998): Characterizing synthetic polymers.- Analytical Chemistry News \& Features, S. 456A-461A.

Yariv, S. (1996): Thermo-IR-spectroscopy analysis of the interactions between organic pollutants and clay minerals.- Thermochimica Acta, 274, S. 1-35.

Ziechmann, W., Müller-Wegener, U. (1990): Bodenchemie.- Mannheim.

Ziechmann, W. (1996): Huminstoffe und ihre Wirkungen.- Heidelberg, Berlin, Oxford.

Zielke, R.C.; Pinnavaia, T.J. (1988): Modified clays for the adsorption of environmental toxicants: Binding of chlorophenols to pillared, delaminated and hydroxy-interlayered smectites.- Clays and Clay Minerals, 36, H. 5, S. 403-408.

Zubkova, T.A. (1989): Catalytic functions of clay minerals in soils.- Soviet Soil Science, 21, H. 4, S. 59-71.

Online-Quellen im World-Wide-Web (WWW):

"NIST Chemistry Webbook" des National Institute of Standards and Technology (NIST) der USA (http://www.nist.gov).

"Integrated Spectral Data Base System for Organic Compounds" (SDBS) des National Institute of Materials and Chemical Research in Japan (http://www.sdbs.jp). 


\section{Curriculum Vitae}

\section{Ulf Birkel}

Martinstraße 4

54340 Riol

\section{Persönliche}

Angaben:

Ausbildung:

$1974-1978$

1978 - 1988

20. Juni 1988

Juli 1988 - September 1989

Oktober 1989 - Dezember 1996

\subsubsection{2}

August/September 1994

13. Januar 1997

01. Februar 2001

\section{Beschäftigungs-}

verhältnisse:
April 1997 - Juni 2000

Oktober 2000 - Dezember 2001

\section{März 1968}

Trier

verheiratet

deutsch

Grundschule Trier-Biewer

Friedrich-Spee-Gymnasium Trier

Abitur

Grundwehrdienst in Pinneberg, Büchel und Goose Bay, Kanada

Studium der Angewandten Physischen Geographie mit den Nebenfächern Bodenkunde und Fernerkundung an der Universität Trier bestandene Diplomvorprüfung

Praktika bei TECUS - Gesellschaft für Technik und Consulting im Umweltschutz, Saarbrücken und beim Landesamt für Umweltschutz, Saarbrücken Diplom in Angewandter Physischer Geographie mit den Nebenfächern Bodenkunde und Fernerkundung Promotion zum Dr.rer.nat. an der Geowissenschaftlichen Fakultät der Georg-AugustUniversität Göttingen über ,Transformation organischer Schadstoffe und abiotische Bildung von Huminstoffen in Böden durch oberflächeninduzierte Reaktionen an Tonmineralen“"

Wissenschaftlicher Mitarbeiter im Sonderforschungsbereich 468 ,Wechselwirkungen an geologischen Grenzflächen“ der Deutschen Forschungsgemeinschaft am Geographischen Institut, Abteilung Landschaftsökologie der Georg-August-Universität Göttingen Wissenschaftlicher Mitarbeiter im DFG-Projekt „Grenzflächenreaktivität, Aufbau und Zerfall von Bodenaggregaten" (GE 431/14-1) der Abteilung Landschaftsökologie, Göttingen 